\title{
Constructing Saturated Guanidinum Heterocycles by Cycloaddition of $N$-Amidinyliminium Ions with Indoles
}

Tyler K. Allred, ${ }^{a}$ Michael B. Shaghafi, ${ }^{a}$ Pan-Pan Chen, ${ }^{\mathrm{b}}$ Quan Tran, ${ }^{\mathrm{b}}$ K. N. Houk, ${ }^{\text {* }}$ and Larry E. Overman $^{\text {a* }}$

${ }^{a}$ Department of Chemistry, University of California, Irvine, California 92697-2025

b Department of Chemistry \& Biochemistry, University of California, Los Angeles, California 90095-1569

leoverma@uci.edu

Supporting Information - Table of Contents

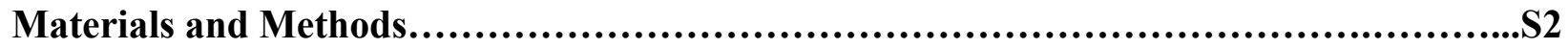

Experimental Procedures.......................................................................

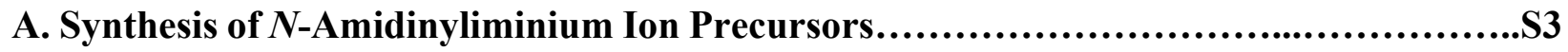

B. Synthesis of Indole Dienophiles...................................................S14

C. Summary of Reaction Optimization Experiments...................................S24

D. General Procedure for $N$-Amidinyliminium Ion Cyclizations (NAIC) with Indoles.......S27

E. Scope of Indole Dienophiles..........................................................

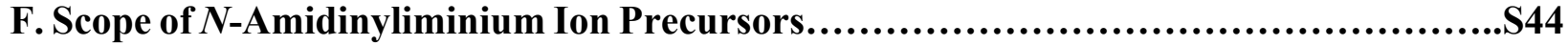

G. Large Scale Cycloaddition Procedure............................................................................S49

H. Deprotection of NAIC Adduct...................................................S50

I. Unsuccessful or Low Yielding Indole Dienophile Examples...........................S51

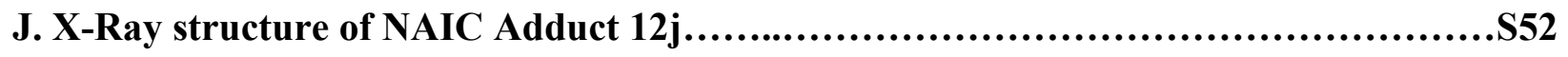

Computational Procedures............................................................S54

K. Computational Methods.................................................................S54

L. Origins of Regioselectivity for the Cycloaddition of A and C..........................S55

M. Table of Energies..................................................................S56

N. Cartesian Coordinates of the Structures...........................................S57

References.........................................................................

Spectral Data........................................................................S74 


\section{Materials and Methods:}

Unless stated otherwise, reactions were conducted in oven-dried glassware under an atmosphere of argon. Tetrahydrofuran (THF), 1,2-dimethoxyethane (DME), dimethylformamide (DMF), toluene, dichloromethane $\left(\mathrm{CH}_{2} \mathrm{Cl}_{2}\right)$, methanol $(\mathrm{MeOH})$ and triethylamine were dried by passage through activated alumina. All other commercial reagents were used as received unless otherwise noted. Reaction temperatures were controlled using a temperature modulator, and unless stated otherwise, reactions were performed at $\mathrm{rt}$ ( $\mathrm{rt}$, approximately $23^{\circ} \mathrm{C}$ ). Thin-layer chromatography (TLC) was conducted with silica gel 60 F254 pre-coated plates, $(0.25 \mathrm{~mm})$ and visualized by exposure to UV light $(254 \mathrm{~nm})$ or by $p$-anisaldehyde, ceric ammonium molybdate (CAM), and potassium permanganate $\left(\mathrm{KMnO}_{4}\right)$ staining. Silica gel 60 (particle size $0.040-0.063 \mathrm{~mm}$ ) was used for flash column chromatography. ${ }^{1} \mathrm{H}$ NMR spectra were recorded at 500 or $600 \mathrm{MHz}$ and are reported relative to deuterated solvent signals. Data for ${ }^{1} \mathrm{H}$ NMR spectra are reported as follows: chemical shift $(\delta \mathrm{ppm})$, multiplicity, coupling constant $(\mathrm{Hz})$, and integration. ${ }^{13} \mathrm{C} \mathrm{NMR}$ spectra were recorded at 125 or $150 \mathrm{MHz}$. Data for ${ }^{13} \mathrm{C}$ NMR spectra are reported in terms of chemical shift. IR spectra were recorded on a ThermoFisher Nicolet iS5 FT-IR spectrometer with an iD7 ATR accessory and are reported in terms of frequency of absorption $\left(\mathrm{cm}^{-1}\right)$. High-resolution mass spectra were obtained from the UC Irvine Mass Spectrometry Facility with a Micromass LCT spectrometer. X-Ray data collection was obtained from the UC Irvine X-Ray Crystallography Facility with a Bruker SMART APEX II diffractometer. The substituted 3-methylindoles used in this study are available from commercial sources or were prepared from the general procedures reported below.

Abbreviations commonly used are: IPA (isopropyl alcohol), Hex (hexanes), DMAP (4dimethylaminopyridine); For others, see: JOC Standard Abbreviations and Acronyms: http://pubs.acs.org/paragonplus/submission/joceah/joceah_abbreviations.pdf. 


\section{Experimental Procedures}

\section{A. Synthesis of N-Amidinyliminium Ion Precursors}

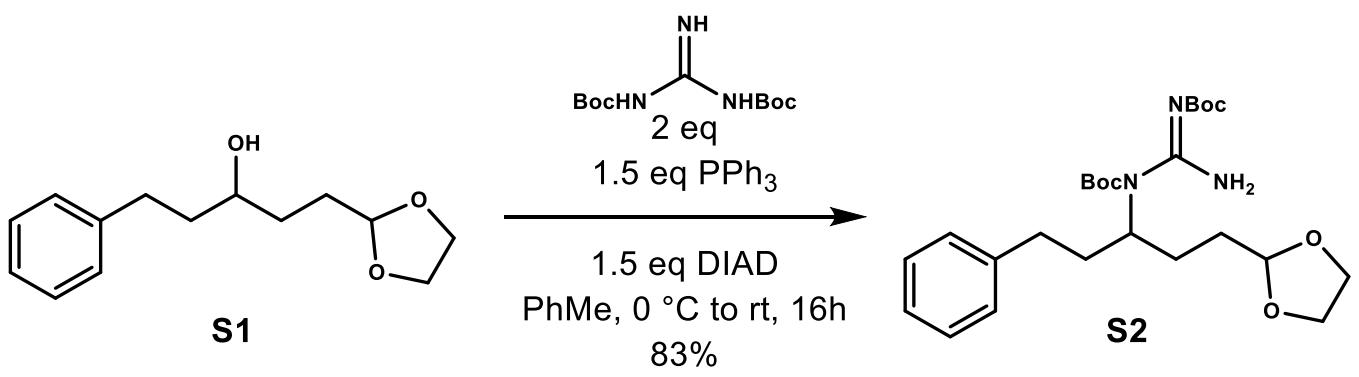

Preparation of 2-Phenethyl-5-phenylsulfanylpyrrolidine-1-carboxamidine Hydrochloride (S2): A flame dried $50 \mathrm{~mL}$ round-bottom flask was charged with a stir bar and alcohol S1 (1.00 g, $4.23 \mathrm{mmol})$ in toluene $(32 \mathrm{~mL}, 0.15 \mathrm{M})$. 1,3-bis(tert-butoxycarbonyl)guanidine (2.19 $\mathrm{g}, 8.46$ mmol, 2 equiv) and $\mathrm{PPh}_{3}$ (1.66 g, $6.35 \mathrm{mmol}, 1.5$ equiv) were added and the mixture was stirred at room temperature until a clear, colorless solution was obtained. The reaction was then cooled to $0{ }^{\circ} \mathrm{C}$ and a solution of diisopropyl azodicarboxylate $(1.25 \mathrm{~mL}, 6.35 \mathrm{mmol}, 1.5$ equiv) in toluene $(10 \mathrm{~mL})$ was then added over 5-10 min. After the addition was complete, stirring was continued at $0{ }^{\circ} \mathrm{C}$ for $2.5 \mathrm{~h}$, at which time the bath was removed and the reaction mixture was allowed to stir overnight $(\sim 16 \mathrm{~h})$ at room temperature. The reaction was then concentrated and the crude residue was diluted with EtOAc $(100 \mathrm{~mL})$. A $10 \%$ citric acid solution $(40 \mathrm{~mL})$ saturated with $\mathrm{NaCl}$ was added and the aqueous layer was extracted with EtOAc $(3 \times 100 \mathrm{~mL})$. The combined organic layers were then washed once with brine $(50 \mathrm{~mL})$, dried over $\mathrm{Na}_{2} \mathrm{SO}_{4}$, decanted and concentrated in vacuo to provide a yellow oil. The crude residue was purification by flash column chromatography $\left(\mathrm{SiO}_{2}\right.$, $20: 1 \rightarrow 4: 1 \rightarrow 1: 1 \mathrm{Hex} /$ EtOAc) to provide protected guanidine $\mathbf{S 2}(1.68 \mathrm{~g}, 83 \%)$ as a colorless amorphous powder, whose characterization data matched literature values. ${ }^{1}$

This intermediate was converted to $\mathrm{N}$-Amidiniyliminium precursor $\mathbf{1 0}$ according to the published procedure. ${ }^{1}$ 


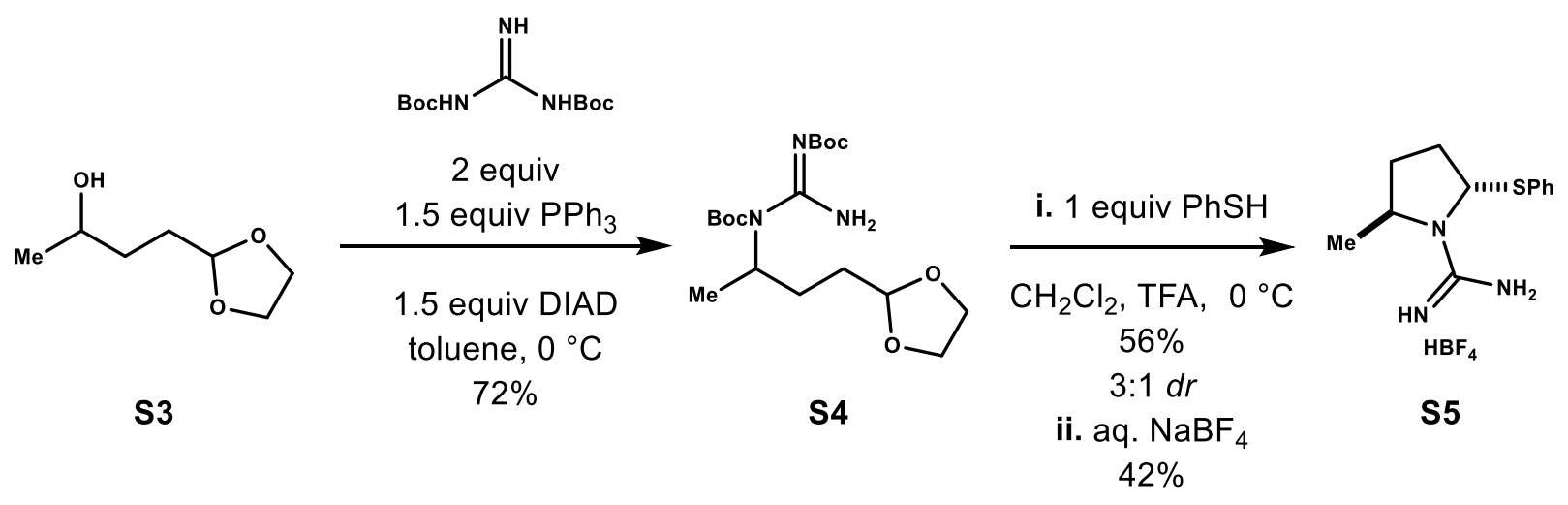

Protected Guanidine S5: A flame dried $250 \mathrm{~mL}$ round bottom flask was charged with alcohol $\mathbf{S 3}^{3}$ (1.59 g, 70\% purity, $7.63 \mathrm{mmol}$ ), bis-Boc-guanidine (3.86 g, $14.88 \mathrm{mmol}, 1.95$ equiv), and $\mathrm{PPh}_{3}\left(3.00 \mathrm{~g}, 11.46 \mathrm{mmol}, 1.5\right.$ equiv) in toluene $(50 \mathrm{~mL}, 0.15 \mathrm{M})$. The mixture was cooled to $0{ }^{\circ} \mathrm{C}$ and DIAD ( $2.25 \mathrm{~mL}, 11.46 \mathrm{mmol}, 1.5$ equiv) was added slowly. The reaction was then allowed to warm slowly to room temperature overnight $(16 \mathrm{~h})$. The solvent was removed in vacuo and the residue was diluted with EtOAc $(100 \mathrm{~mL})$ and washed with $10 \%$ citric acid solution saturated with $\mathrm{NaCl}(50 \mathrm{~mL})$. The aqueous layer was washed with EtOAc $(3 \times 50 \mathrm{~mL})$ and the combined organic layers were then washed with brine $(100 \mathrm{~mL})$, dried over $\mathrm{Na}_{2} \mathrm{SO}_{4}$, filtered, and concentrated. The crude residue was purified by flash column chromatography $\left(\mathrm{SiO}_{2}, 1: 0 \rightarrow 4: 1 \mathrm{Hex} / \mathrm{EtOAc}\right)$ to afford the protected guanidine $\mathbf{S 4}(2.12 \mathrm{~g}, 72 \%)$ as a colorless oil.

${ }^{1} \mathbf{H}$ NMR $\left(600 \mathrm{MHz}, \mathrm{CDCl}_{3}\right) \delta 9.41$ (bs, 1H), 9.21 (bs, $\left.1 \mathrm{H}\right), 5.38$ (ddt, $\left.J=6.6,9.6,13.1 \mathrm{~Hz}, 1 \mathrm{H}\right)$, $4.85(\mathrm{t}, J=4.5 \mathrm{~Hz}, 1 \mathrm{H}), 3.98-3.90(\mathrm{~m}, 2 \mathrm{H}), 3.87-3.79(\mathrm{~m}, 2 \mathrm{H}), 2.02-1.90(\mathrm{~m}, 1 \mathrm{H}), 1.73-1.63(\mathrm{~m}$, $2 \mathrm{H}), 1.57-1.50(\mathrm{~m}, 1 \mathrm{H}), 1.53(\mathrm{~s}, 9 \mathrm{H}), 1.48(\mathrm{~s}, 9 \mathrm{H}), 1.30(\mathrm{~d}, J=6.7 \mathrm{~Hz}, 3 \mathrm{H})$

${ }^{13}$ C NMR $\left(150 \mathrm{MHz}, \mathrm{CDCl}_{3}\right) \delta 164,0,161.6,155.5, .3,83.8,78.7,64.9,50.4,30.9,29.0,28.4$, $28.2,28.1,19.5$

IR (thin film) 3392, 3004, 2970, 2942, 1737, 1642, 1609, 1455, 1417, 1366, 1300, 1247, 1229, $1217,1206,1146,1085 \mathrm{~cm}^{-1}$

HRMS (ESI-TOF) m/z: $[\mathrm{M}+\mathrm{H}]^{+}$calcd for $\left[\mathrm{C}_{18} \mathrm{H}_{34} \mathrm{~N}_{3} \mathrm{O}_{6}\right]^{+} 388.2448$, found 388.2462

Preparation of N-Amidinyliminium Ion Precursor S5: A flame dried $100 \mathrm{~mL}$ round bottom flask was charged with protected guanidine $\mathbf{S} 4(1.98 \mathrm{~g}, 5.11 \mathrm{mmol})$ and thiophenol $(0.52 \mathrm{~mL}, 5.08$ mmol, 0.99 equiv) in $\mathrm{CH}_{2} \mathrm{Cl}_{2}(10 \mathrm{~mL})$. The solution was cooled to $0{ }^{\circ} \mathrm{C}$ and TFA $(10 \mathrm{~mL}, 0.25 \mathrm{M}$ final concentration) was added slowly. The reaction was then allowed to warm slowly to room temperature overnight (16h). The reaction was concentrated under reduced pressure and the crude residue was purified by flash column chromatography $\left(\mathrm{SiO}_{2}, 1: 0 \rightarrow 9: 1 \mathrm{CH}_{2} \mathrm{Cl}_{3} / \mathrm{MeOH}\right)$ to afford the TFA salt $(1.00 \mathrm{~g}, 56 \%, 3: 1 d r)$ as a light blue solid.

The purified salt $(350.1 \mathrm{mg}, 1.00 \mathrm{mmol})$ was then dissolved in $\mathrm{CHCl}_{3}(30 \mathrm{~mL})$ and washed with saturated aqueous $\mathrm{NaBF}_{4}(4 \times 30 \mathrm{~mL})$. The organic layer was then washed with $\mathrm{H}_{2} \mathrm{O}(30 \mathrm{~mL})$ and then concentrated. The solids were then azeotroped with toluene $(3 \times 20 \mathrm{~mL})$ to afford $\mathbf{S 5}$ (135 
$\mathrm{mg}, 42 \%$ ) as a light brown solid and 4:1 mixture of diastereomers. This material was used without further purification.

\section{Characterization of TFA salt}

${ }^{1} \mathbf{H}$ NMR $\left(600 \mathrm{MHz}, \mathrm{CDCl}_{3}\right) \delta$ 7.50-7.44 (m, 2H), 7.44-7.34 (m, 3H), $5.03(\mathrm{~d}, J=6.8 \mathrm{~Hz}, 0.25 \mathrm{H})$, $4.98(\mathrm{dd}, J=3.6,6.4 \mathrm{~Hz}, 0.75 \mathrm{H}), 4.11(\mathrm{sext}, J=6.2 \mathrm{~Hz}, 1 \mathrm{H}), 2.59-2.49(\mathrm{~m}, 0.25 \mathrm{H}), 2.35-2.14(\mathrm{~m}$, $2.5 \mathrm{H}), 2.01-1.92(\mathrm{~m}, 0.25 \mathrm{H}), 1.83-1.75(\mathrm{~m}, 0.75 \mathrm{H}), 1.59(\mathrm{dd}, J=6.4,12.6 \mathrm{~Hz}, 0.25 \mathrm{H}), 1.20(\mathrm{~d}, J$ $=6.3 \mathrm{~Hz}, 0.75 \mathrm{H}), 1.05(\mathrm{~d}, J=6.2 \mathrm{~Hz}, 2.25 \mathrm{H})$

${ }^{13} \mathrm{C}$ NMR (150 MHz, $\mathrm{CDCl}_{3}$, signals of TFA counter ion not included) $\delta$ (major) 156.2, 135.3, $129.87,129.84,129.75,68.7,55.6,32.8,31.3,19.4$ (minor) 155.3, 134.6, 130.7, 129.9, 129.5, $66.6,55.3,32.5,30.0,18.6$

IR (thin film) 3336, 3162, 2978, 2931, 2853, 1660, 1584, 1459, 1438, 1198, 1181, $1139 \mathrm{~cm}^{-1}$

HRMS (ESI-TOF) m/z: [M] $]^{+}$calcd for $\left[\mathrm{C}_{12} \mathrm{H}_{18} \mathrm{~N}_{3} \mathrm{~S}\right]^{+} 236.1221$, found 236.1223

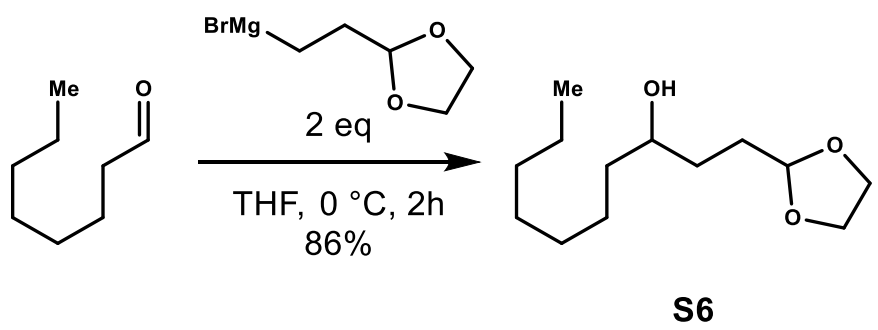

1-(1,3-dioxolan-2-yl)decan-3-ol (S6): To a suspension of Mg turnings (1.878 g, $77.28 \mathrm{mmol}, 4$ equiv) in THF (55 mL), a small crystal of $\mathrm{I}_{2}$ was added as well as 2-(2-bromoethyl)-1,3-dioxolane $(0.25 \mathrm{~mL})$. The mixture was gently warmed with a heat gun until the color dissipated and initiation was observed. Then the remaining bromide ( $4.25 \mathrm{mmol}, 38.33 \mathrm{mmol}$ total, $\sim 2$ equiv) as a solution in THF $(18 \mathrm{~mL})$ was added dropwise at a rate to maintain a gentle reflux. The reaction was allowed then allowed to cool to room temperature and stirred for 2 hours. Then octanal $(3.12 \mathrm{~mL}, 20 \mathrm{mmol}$, 1 equiv) was added dropwise at room temperature to the vigorously stirred Grignard solution. The reaction was stirred for 2 hours at room temperature and then the reaction was quenched by the addition of saturated aqueous $\mathrm{NH}_{4} \mathrm{Cl}(100 \mathrm{~mL})$. The mixture was extracted with $\mathrm{Et}_{2} \mathrm{O}(100 \mathrm{~mL})$ and the aqueous layer was washed with $\mathrm{Et}_{2} \mathrm{O}(2 \times 50 \mathrm{~mL})$. The combined organic layeres were dried over $\mathrm{MgSO}_{4}$, filtered, and concentrated to a yellow oil. The crude residue was purified by flash column chromatography $\left(\mathrm{SiO}_{2}\right.$ neutralized with $5 \% \mathrm{Et}_{3} \mathrm{~N}$ in Hexanes, 1:0 $\rightarrow 4: 1 \rightarrow 1: 1$ Hexanes/EtOAc) to afford alcohol $\mathbf{S 6}(3.97 \mathrm{~g}, 86 \%)$ as a colorless oil.

${ }^{1} \mathbf{H}$ NMR $\left(600 \mathrm{MHz} \mathrm{CDCl}_{3}\right) \delta 4.88(\mathrm{t}, J=4.5 \mathrm{~Hz}, 1 \mathrm{H}), 4.00-3.93(\mathrm{~m}, 2 \mathrm{H}), 3.88-3.82(\mathrm{~m}, 2 \mathrm{H})$, $3.63-3.57$ (m, 1H), 2.14 (bs, 1H), 1.86-1.71 (m, 3H), 1.66-1.59 (m, 1H), 1.54-1.46 (m, 1H), 1.45$1.38(\mathrm{~m}, 3 \mathrm{H}), 1.31-1.22(\mathrm{~m}, 8 \mathrm{H}), 0.86(\mathrm{t}, J=7.0 \mathrm{~Hz}, 3 \mathrm{H})$

${ }^{13} \mathrm{C}$ NMR $\left(150 \mathrm{MHz}, \mathrm{CDCl}_{3}\right) \delta 104.6,71.6,65.0,64.9,37.6,31.9,31.4,30.1,29.7,29.3,25.8$, $22.7,14.1$ 
IR (thin film) 3409, 2954, 2924, 2854, 1456, 1409, 1142, 1032, 966, $942 \mathrm{~cm}^{-1}$

HRMS (ESI-TOF) m/z: $[\mathrm{M}+\mathrm{Na}]^{+}$calcd for $\left[\mathrm{C}_{13} \mathrm{H}_{26} \mathrm{O}_{3} \mathrm{Na}\right]^{+} 253.1780$, found 253.1780

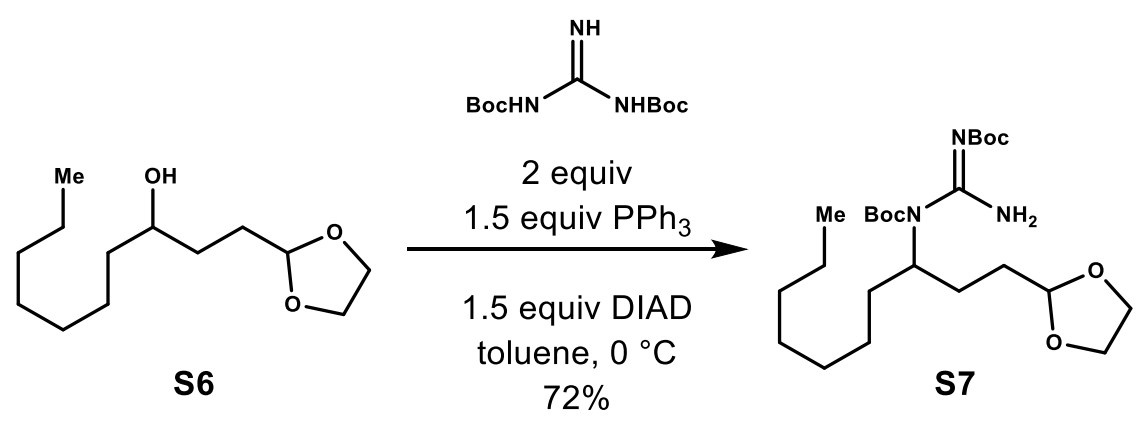

Protected Guanidine S7: A $100 \mathrm{~mL}$ flame dried round bottom flask was charged with a stir bar and alcohol S6 (1.019 g, $4.42 \mathrm{mmol})$ in toluene $(30 \mathrm{~mL}, 0.15 \mathrm{M})$. 1,3-bis(tertbutoxycarbonyl)guanidine ( $2.29 \mathrm{~g}, 8.84 \mathrm{mmol}, 2$ equiv) and $\mathrm{PPh}_{3}(1.73 \mathrm{~g}, 6.62 \mathrm{mmol}, 1.5$ equiv) were added and the mixture was stirred at room temperature for $10 \mathrm{~min}$. The reaction was then cooled to $0{ }^{\circ} \mathrm{C}$ and a solution of diisopropyl azodicarboxylate $(1.30 \mathrm{~mL}, 6.64 \mathrm{mmol}, 1.5$ equiv) was added dropwise. After the addition was complete, the reaction was then allowed to slowly warm to room temperature overnight $(\sim 16 \mathrm{~h})$. The reaction was then concentrated and the crude residue was diluted with EtOAc $(50 \mathrm{~mL})$. A $10 \%$ citric acid solution $(40 \mathrm{~mL})$ saturated with $\mathrm{NaCl}$ was added and the aqueous layer was extracted with EtOAc $(3 \times 50 \mathrm{~mL})$. The combined organic layers were then washed once with brine $(50 \mathrm{~mL})$, dried over $\mathrm{Na}_{2} \mathrm{SO}_{4}$, filtered and concentrated in vacuo to provide a yellow oil. The crude residue was purified by flash column chromatography $\left(\mathrm{SiO}_{2}, 20: 1 \rightarrow 4: 1 \rightarrow 1: 1 \mathrm{Hex} / \mathrm{EtOAc}\right)$ to provide protected guanidine $\mathbf{S} 7(1.1673 \mathrm{~g}, 56 \%)$ as a colorless oil

${ }^{1} \mathbf{H}$ NMR $\left(600 \mathrm{MHz}, \mathrm{CDCl}_{3}\right) \delta 9.33(\mathrm{bs}, 2 \mathrm{H}), 5.37-5.25(\mathrm{~m}, 1 \mathrm{H}), 4.86(\mathrm{t}, J=4.7 \mathrm{~Hz}, 1 \mathrm{H}), 3.97-$ $3.90(\mathrm{~m}, 2 \mathrm{H}), 3.86-3.78(\mathrm{~m}, 2 \mathrm{H}), 1.97-1.87(\mathrm{~m}, 1 \mathrm{H}), 1.83-1.75(\mathrm{~m}, 1 \mathrm{H}), 1.74-1.61(\mathrm{~m}, 3 \mathrm{H}), 1.56-$ $1.49(\mathrm{~m}, 1 \mathrm{H}), 1.52(\mathrm{~s}, 9 \mathrm{H}), 1.47(\mathrm{~s}, 9 \mathrm{H}), 1.31-1.13(\mathrm{~m}, 10 \mathrm{H}), 0.86(\mathrm{t}, J=7.1 \mathrm{~Hz}, 3 \mathrm{H})$

${ }^{13}$ C NMR $\left(150 \mathrm{MHz}, \mathrm{CDCl}_{3}\right) \delta 164.0,162.0,155.6,104.5,83.7,78.5,64.9,54.5,33.8,31.9,30.6$, $29.6,29.2,28.4,28.2,28.1,27.9,26.2,22.7,14.1$

IR (thin film) 3381, 2955, 2928, 2857, 1707, 1639, 1609, 1506, 1367, 1300, 1271, 1248, 1146, $1101 \mathrm{~cm}^{-1}$

HRMS (ESI-TOF) m/z: $[\mathrm{M}+\mathrm{Na}]^{+}$calcd for $\left[\mathrm{C}_{24} \mathrm{H}_{45} \mathrm{~N}_{3} \mathrm{O}_{6} \mathrm{Na}\right]^{+} 494.3206$, found 494.3201 


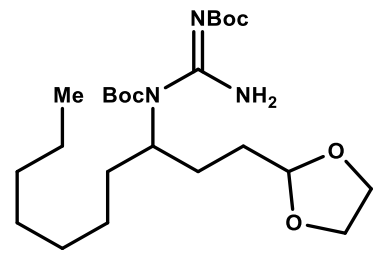

S7

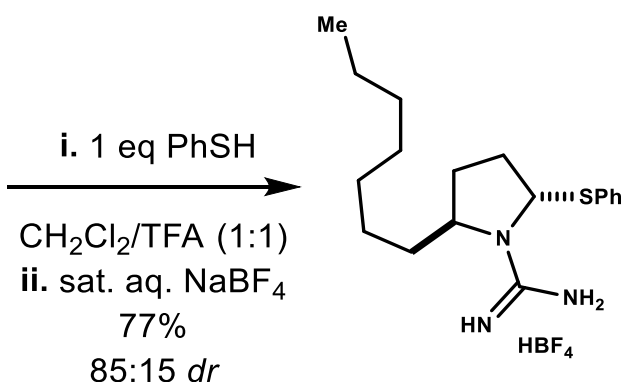

S8

Preparation of N-Amidinyliminium Ion Precursor S8: A flame dried $25 \mathrm{~mL}$ round bottom flask was charged with protected guanidine $\mathbf{S} 7(1.1495 \mathrm{~g}, 2.43 \mathrm{mmol})$ and thiophenol $(0.25 \mathrm{~mL}, 2.44$ mmol, 1 equiv) in $\mathrm{CH}_{2} \mathrm{Cl}_{2}(5 \mathrm{~mL}, 0.5 \mathrm{M})$. The solution was cooled to $0{ }^{\circ} \mathrm{C}$ and TFA $(5 \mathrm{~mL}, 0.25 \mathrm{M}$ final concentration) was added slowly. The reaction was then allowed to warm to room temperature overnight $(\sim 16 \mathrm{~h})$. The reaction was then concentrated to an orange oil. The crude residue was purified by flash column chromatography $\left(\mathrm{SiO}_{2}, 1: 0 \rightarrow 9: 1 \mathrm{CH}_{2} \mathrm{Cl}_{2} / \mathrm{MeOH}\right)$ to afford the intermediate TFA salt. The purified salt was then dissolved in $\mathrm{CHCl}_{3}(30 \mathrm{~mL})$ and washed with saturated aqueous $\mathrm{NaBF}_{4}(4 \times 30 \mathrm{~mL})$. The organic layer was then washed with $\mathrm{H}_{2} \mathrm{O}(30 \mathrm{~mL})$ and then concentrated. The solids were then azeotroped with toluene $(3 \times 20 \mathrm{~mL})$ to afford $\mathbf{S 8}(765.3$ $\mathrm{mg}, 77 \%$ ) as an off-white solid and 85:15 mixture of diastereomers.

${ }^{1}$ H NMR (600 MHz, CDCl $)$ $) \delta 7.51-7.45(\mathrm{~m}, 2 \mathrm{H}), 7.44-7.34(\mathrm{~m}, 3 \mathrm{H}), 6.45(\mathrm{bs}, 4 \mathrm{H}), 5.04(\mathrm{dd}, J$ $=3.7,6.8 \mathrm{~Hz}, 1 \mathrm{H}), 3.93-3.85(\mathrm{~m}, 0.85 \mathrm{H}), 3.83-3.77(\mathrm{~m}, 0.15 \mathrm{H}), 2.49-2.41(\mathrm{~m}, 0.15 \mathrm{H}), 2.37-2.27$ (m, 0.85H), 2.22-2.06 (m, 2H), 1.91-1.80 (m, 1H), 1.75-1.66 (m, 1H), 1.65-1.55 (m, 1H), 1.34$1.20(\mathrm{~m}, 7 \mathrm{H}), 1.19-1.10(\mathrm{~m}, 2 \mathrm{H}), 1.02-0.93(\mathrm{~m}, 1 \mathrm{H}), 0.88(\mathrm{t}, J=7.2 \mathrm{~Hz}, 2.55 \mathrm{H}), 0.85(\mathrm{t}, J=7.2$ $\mathrm{Hz}, 0.45 \mathrm{H})$

${ }^{13} \mathrm{C}$ NMR (150 MHz, $\left.\mathrm{CDCl}_{3}\right) \delta$ (major) 155.5, 135.2, 129.87, 129.85, 129.7, 68.6, 60.49, 33.2, $32.4,31.78,29.5,29.08,29.0,25.4,22.66,14.14$ (minor) 154.3, 134.8, 130.3, 129.82, 129.6, 66.3, $60.56,32.2,31.76,31.74,29.4,29.14,26.9,26.1,22.63,14.1$

IR (thin film) 3457, 3374, 3272, 2926, 2856, 1738, 1651, 1595, 1466, 1440, 1365, 1228, 1217 , $1204,1025 \mathrm{~cm}^{-1}$

HRMS (ESI-TOF) m/z: $[\mathrm{M}]^{+}$calcd for $\left[\mathrm{C}_{18} \mathrm{H}_{30} \mathrm{~N}_{3} \mathrm{~S}\right]^{+} 320.2160$, found 320.2149

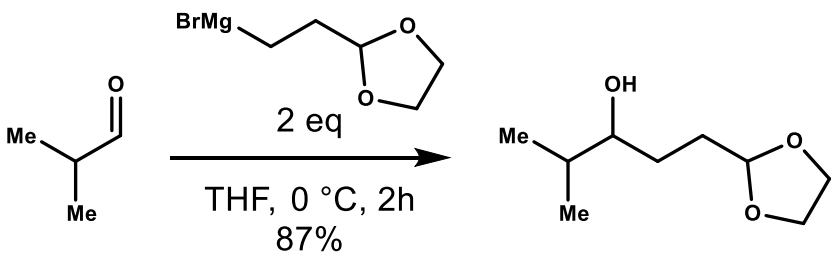

S9

1-(1,3-dioxolan-2-yl)-4-methylpentan-3-ol (S9): To a suspension of Mg turnings (1.878 g, 77.28 mmol, 4 equiv) in THF (55 mL), a small crystal of $\mathrm{I}_{2}$ was added as well as 2-(2-bromoethyl)-1,3- 
dioxolane $(0.25 \mathrm{~mL})$. The mixture was gently warmed with a heat gun until the color dissipated and initiation was observed. Then the remaining bromide ( $4.25 \mathrm{mmol}, 38.33 \mathrm{mmol}$ total, $\sim 2$ equiv) as a solution in THF $(18 \mathrm{~mL})$ was added dropwise at a rate to maintain a gentle reflux. The reaction was allowed then allowed to cool to room temperature and stirred for 2 hours. Then isobutyraldehyde ( $1.82 \mathrm{~mL}, 20 \mathrm{mmol}, 1$ equiv) was added dropwise at room temperature to the vigorously stirred Grignard solution. The reaction was stirred for 2 hours at room temperature and then the reaction was quenched by the addition of saturated aqueous $\mathrm{NH}_{4} \mathrm{Cl}(100 \mathrm{~mL})$. The mixture was extracted with $\mathrm{Et}_{2} \mathrm{O}(100 \mathrm{~mL})$ and the aqueous layer was washed with $\mathrm{Et}_{2} \mathrm{O}(2 \times 50 \mathrm{~mL})$. The combined organic layeres were dried over $\mathrm{MgSO}_{4}$, filtered, and concentrated to a yellow oil. The crude residue was purified by flash column chromatography $\left(\mathrm{SiO}_{2}\right.$ neutralized with $5 \% \mathrm{Et}_{3} \mathrm{~N}$ in Hexanes, $1: 0 \rightarrow 4: 1 \rightarrow 1: 1$ Hexanes/EtOAc) to afford alcohol S9 (3.03 g, 87\%) as a colorless oil.

${ }^{1} \mathbf{H}$ NMR $\left(600 \mathrm{MHz}, \mathrm{CDCl}_{3}\right) \delta 4.90(\mathrm{t}, J=4.5 \mathrm{~Hz}, 1 \mathrm{H}), 4.03-3.95(\mathrm{~m}, 2 \mathrm{H}), 3.90-3.82(\mathrm{~m}, 2 \mathrm{H})$, 3.40-3.34 (m, 1H), 2.05-1.99 (m, 1H), 1.89-1.83 (m, 1H), 1.83-1.75 (m, 1H), 1.69-1.60 (m, 2H), $1.53-1.45(\mathrm{~m}, 1 \mathrm{H}), 0.92(\mathrm{~d}, J=6.8 \mathrm{~Hz}, 3 \mathrm{H}), 0.91(\mathrm{~d}, J=6.8 \mathrm{~Hz}, 3 \mathrm{H})$

${ }^{13} \mathrm{C}$ NMR $\left(150 \mathrm{MHz}, \mathrm{CDCl}_{3}\right) \delta 104.6,76.5,65.0,64.9,33.8,30.5,28.3,18.8,17.5$

IR (thin film) 3435, 2958, 2931, 2875, 1470, 1447, 1409, 1367, 1141, $1034 \mathrm{~cm}^{-1}$

HRMS (ESI-TOF) m/z: $[\mathrm{M}+\mathrm{H}]^{+}$calcd for $\left[\mathrm{C}_{9} \mathrm{H}_{19} \mathrm{O}_{3}\right]^{+}$175.1334, found 175.1337

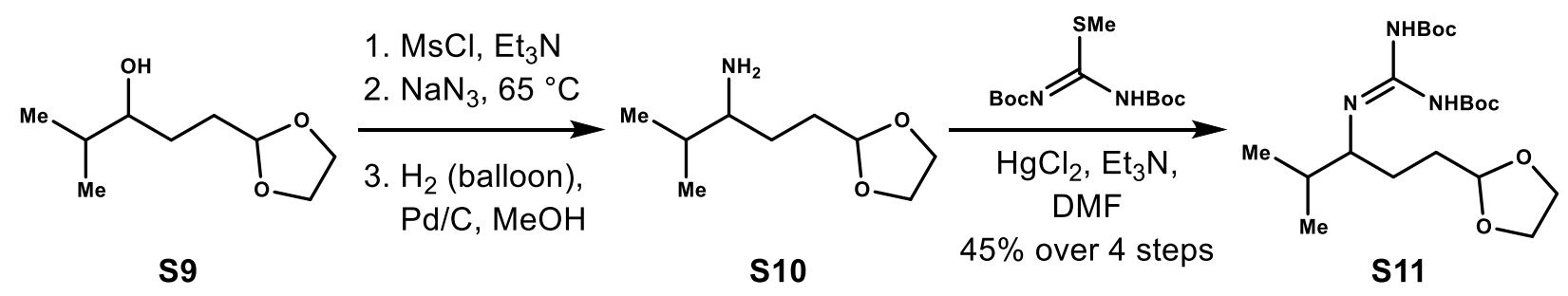

Preparation of Protected Guanidine S11: A $100 \mathrm{~mL}$ round bottom flask was charged with alcohol S9 $(511.2 \mathrm{mg}, 2.93 \mathrm{mmol})$ in $\mathrm{CH}_{2} \mathrm{Cl}_{2}(19 \mathrm{~mL}, 0.15 \mathrm{M})$. The solution was cooled to $0{ }^{\circ} \mathrm{C}$ and $\mathrm{Et}_{3} \mathrm{~N}$ ( $0.82 \mathrm{~mL}, 5.85 \mathrm{mmol}, 2$ equiv) was added followed by dropwise addition of $\mathrm{MsCl}(0.34$ $\mathrm{mL}, 4.39 \mathrm{mmol}, 1.5$ equiv). The reaction was then allowed to warm to room temperature and the mixture was stirred for 3 hours. The reaction was then diluted with sat. aq. $\mathrm{NaHCO}_{3}(40 \mathrm{~mL})$ and the mixture was extracted with $\mathrm{CH}_{2} \mathrm{Cl}_{2}(2 \times 30 \mathrm{~mL})$. The combined organics were then dried over $\mathrm{MgSO}_{4}$, filtered, and concentrated to a pale-yellow oil, which was used without further purification.

The crude mesylate was dissolved in DMF (15 mL, 0.2M) and $\mathrm{NaN}_{3}(380.4 \mathrm{mg}, 5.85 \mathrm{mmol}, 2$ equiv) was added. The mixture was then heated to $60{ }^{\circ} \mathrm{C}$ overnight $(\sim 16 \mathrm{~h})$. The reaction was cooled to room temperature and diluted with half saturated brine $(200 \mathrm{~mL})$. The mixture was then extracted with $\mathrm{Et}_{2} \mathrm{O}(3 \times 40 \mathrm{~mL})$ and the combined organics were dried over $\mathrm{MgSO}_{4}$, filtered, and concentrated to a yellow oil, which was used without further purification. 
The intermediate azide was dissolved in $\mathrm{MeOH}(15 \mathrm{~mL}, 0.2 \mathrm{M})$ and $\mathrm{Pd} / \mathrm{C}(10 \mathrm{wt} \%, 154.6 \mathrm{mg}$, $0.144 \mathrm{mmol}, 5 \mathrm{~mol} \%$ ) was added. The mixture was sparged with $\mathrm{H}_{2}$ for 20 minutes and then stirred under $\mathrm{H}_{2}$ (balloon) overnight ( $\sim 16 \mathrm{~h}$ ). The reaction was then filtered over celite and the solids were washed with $\mathrm{MeOH}(30 \mathrm{~mL})$. The filtrate was concentrated to afford intermediate amine $\mathbf{S 1 0}$ as a yellow oil (crude weight: $390.4 \mathrm{mg}$ ), which was used without further purification.

A $100 \mathrm{~mL}$ round bottom flask was charged with crude amine $\mathbf{S 1 0}$ (390.4 mg, $2.25 \mathrm{mmol}$ ), N,N'Di-Boc-S-methylisothiourea (724.9 mg, $2.49 \mathrm{mmol}, 1.1$ equiv) and $\mathrm{Et}_{3} \mathrm{~N}(1.26 \mathrm{~mL}, 8.99 \mathrm{mmol}, 4$ equiv) in DMF $(9 \mathrm{~mL}, 0.25 \mathrm{M})$. The mixture was stirred until homogenous then $\mathrm{HgCl}_{2}(666.7 \mathrm{mg}$, $2.45 \mathrm{mmol}, 1.09$ equiv) was added in one portion. The reaction was then stirred at room temperature overnight $(\sim 16 \mathrm{~h})$. The reaction was then diluted with EtOAc $(40 \mathrm{~mL})$, filtered over celite, and the solids were washed with EtOAc $(30 \mathrm{~mL})$. The filtrate was then washed with $\mathrm{H}_{2} \mathrm{O}(2$ $\mathrm{x} 50 \mathrm{~mL}$ ) and brine $(50 \mathrm{~mL})$. The organics were then dried over $\mathrm{MgSO}_{4}$, filtered, and concentrated. The crude residue was then purified by flash column chromatography $\left(\mathrm{SiO}_{2}, 1: 0 \rightarrow 9: 1 \rightarrow 4: 1\right.$ Hex/EtOAc) to afford protected guanidine $\mathbf{S 1 1}(553.8 \mathrm{mg}, 45 \%$ over 4 steps) as a colorless oil.

${ }^{1}$ H NMR $\left(600 \mathrm{MHz}, \mathrm{CDCl}_{3}\right) \delta 8.61(\mathrm{bs}, 1 \mathrm{H}), 4.86(\mathrm{t}, J=4.0 \mathrm{~Hz}, 1 \mathrm{H}), 4.19-4.11(\mathrm{~m}, 1 \mathrm{H}), 3.99-$ $3.91(\mathrm{~m}, 2 \mathrm{H}), 3.88-3.80(\mathrm{~m}, 2 \mathrm{H}), 1.90-1.80(\mathrm{~m}, 1 \mathrm{H}), 1.75-1.66(\mathrm{~m}, 3 \mathrm{H}), 1.57-1.41(\mathrm{~m}, 2 \mathrm{H}), 1.50$ (s, 9H), 1.49 (s, 9H), 0.93 (d, $J=6.8 \mathrm{~Hz}, 3 \mathrm{H}), 0.92(\mathrm{~d}, J=6.8 \mathrm{~Hz}, 3 \mathrm{H})$

${ }^{13} \mathbf{C}$ NMR $\left(150 \mathrm{MHz}, \mathrm{CDCl}_{3}\right) \delta 161.7,155.8,153.2,104.3,83.8,80.7,64.93,64.90,55.7,31.4$, $30.0,28.3,28.1,25.7,18.6,17.9$

IR (thin film) 3320, 3280, 2970, 2932, 2875, 1783, 1736, 1721, 1636, 1614, 1569, 1543, 1417, $1366,1313,1250,1229,1216,1121,1055 \mathrm{~cm}^{-1}$

HRMS (ESI-TOF) m/z: $[\mathrm{M}+\mathrm{H}]^{+}$calcd for $\left[\mathrm{C}_{20} \mathrm{H}_{38} \mathrm{~N}_{3} \mathrm{O}_{6}\right]^{+} 416.2761$, found 416.2780
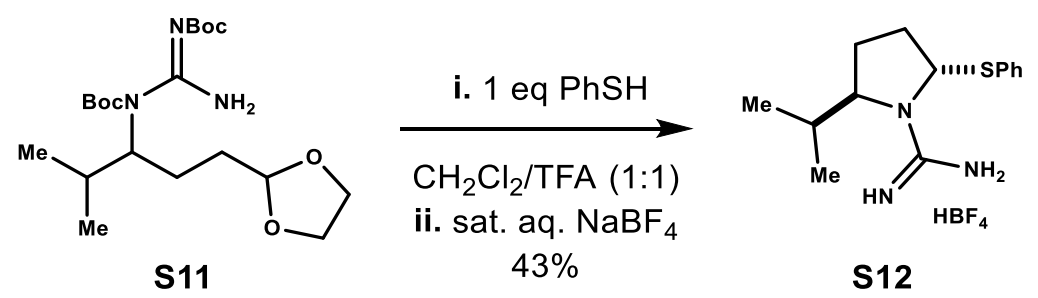

Preparation of N-Amidinyliminium Ion Precursor S12: A $25 \mathrm{~mL}$ round bottom flask was charged with protected guanidine $\mathbf{S 1 1}(334.4 \mathrm{mg}, 0.804 \mathrm{mmol})$ and thiophenol $(0.08 \mathrm{~mL}, 0.782$ mmol, 0.99 equiv) in $\mathrm{CH}_{2} \mathrm{Cl}_{2}(1.6 \mathrm{~mL})$. The solution was cooled to $0{ }^{\circ} \mathrm{C}$ and TFA $(1.6 \mathrm{~mL}, 0.25 \mathrm{M}$ final concentration) was added slowly. The reaction was then allowed to slowly warm to room temperature overnight $(\sim 16 \mathrm{~h})$. The reaction was then concentrated to an orange oil. The crude residue was purified by flash column chromatography $\left(\mathrm{SiO}_{2}, 1: 0 \rightarrow 9: 1 \mathrm{CH}_{2} \mathrm{Cl}_{2} / \mathrm{MeOH}\right)$ to afford the intermediate TFA salt as an orange oil. The isolated TFA salt was then dissolved in $\mathrm{CHCl}_{3}(30$ $\mathrm{mL})$ and washed with sat. aq. $\mathrm{NaBF}_{4}(3 \times 40 \mathrm{~mL})$. The organic layer was then washed with $\mathrm{H}_{2} \mathrm{O}$ $(40 \mathrm{~mL})$ and concentrated to off-white solids. The solids were then azeotroped with toluene $(3 \mathrm{x}$ $20 \mathrm{~mL})$ to afford $\mathbf{S 1 2}(122.3 \mathrm{mg}, 43 \%)$ as an off-white solid and as a single diastereomer. 
${ }^{1} \mathbf{H}$ NMR $\left(600 \mathrm{MHz}, \mathrm{CDCl}_{3}\right) \delta$ 7.51-7.44 (m, 2H), 7.41-7.35 (m, 3H), $6.8(\mathrm{bs}, 4 \mathrm{H}), 5.05(\mathrm{~d}, J=$ $5.8 \mathrm{~Hz}, 1 \mathrm{H}), 3.95(\mathrm{q}, J=6.5 \mathrm{~Hz}, 1 \mathrm{H}), 2.33-2.24(\mathrm{~m}, 1 \mathrm{H}), 2.19-2.07(\mathrm{~m}, 2 \mathrm{H}), 2.04-1.95(\mathrm{~m}, 1 \mathrm{H})$, $0.94(\mathrm{~d}, J=6.6 \mathrm{~Hz}, 3 \mathrm{H}), 0.88(\mathrm{~d}, J=6.6 \mathrm{~Hz}, 3 \mathrm{H})$

${ }^{13} \mathrm{C}$ NMR $\left(150 \mathrm{MHz}, \mathrm{CDCl}_{3}\right) \delta 156.3,134.0,130.9,129.9,129.4,70.2,65.3,32.7, .8,24.1,19.2$, 16.3

IR (thin film) 3433, 3372, 3260, 2964, 2922, 2851, 1659, 1585, 1467, 1439, $1023 \mathrm{~cm}^{-1}$

HRMS (ESI-TOF) m/z: [M] $]^{+}$calcd for $\left[\mathrm{C}_{14} \mathrm{H}_{22} \mathrm{~N}_{3} \mathrm{~S}\right]^{+} 264.1534$, found 264.1533

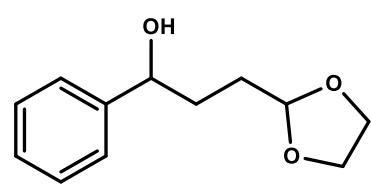

S13

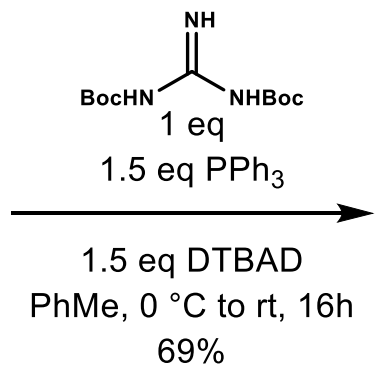

$69 \%$

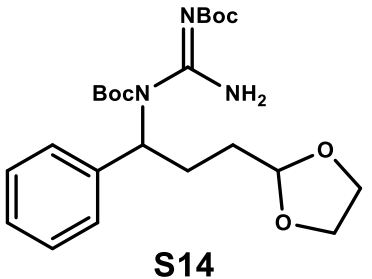

S14

Preparation of Protected Guanidine 14: A flame-dried $250 \mathrm{~mL}$ round-bottom flask was charged with $\mathrm{PPh}_{3}$ (5.40 g, $20.6 \mathrm{mmol}, 1.5$ equiv) and anhydrous toluene (42 mL). The flask was cooled to $0{ }^{\circ} \mathrm{C}$ and a solution of di-tert-butyl azodicarboxylate (DTBAD, $4.74 \mathrm{~g}, 20.6 \mathrm{mmol}, 1.5 \mathrm{equiv}$ ) in toluene $(35 \mathrm{~mL})$ was added. The subsequent yellow solution was allowed to stir for $30 \mathrm{~min}$. at 0 ${ }^{\circ} \mathrm{C}$, over which time a precipitate formed. A solution of 1,3-bis(tert-butoxycarbonyl)guanidine (3.57 g, $13.8 \mathrm{mmol}, 1$ equiv) and alcohol $\mathbf{S 1 3}^{4}(2.87 \mathrm{~g}, 13.8 \mathrm{mmol})$ in toluene $(63 \mathrm{~mL})$ was added. After stirring $10 \mathrm{~min}$. at $0{ }^{\circ} \mathrm{C}$, the cooling bath was removed and the solution was allowed to stir at room temperature overnight $(\sim 16 \mathrm{~h})$. The reaction was then concentrated and the crude residue was taken up in EtOAc $(100 \mathrm{~mL})$. This solution was then washed with a solution of $10 \%$ citric acid saturated with $\mathrm{NaCl}$. The aqueous layer was extracted with EtOAc $(3 \times 50 \mathrm{~mL})$ and the combined organics were dried with $\mathrm{Na}_{2} \mathrm{SO}_{4}$, filtered, and concentrated. The crude residue was purified by flash column chromatography $\left(\mathrm{SiO}_{2}, 20: 1 \rightarrow 4: 1 \mathrm{Hex} / \mathrm{EtOAc}\right)$ to provide $\mathbf{S 1 4}$ (4.30 g, $69 \%$ ) as a colorless powder.

${ }^{1} \mathbf{H}$ NMR $\left(600 \mathrm{MHz}, \mathrm{CDCl}_{3}\right) \delta 9.54$ (br s, $\left.1 \mathrm{H}\right), 9.32$ (br s, 1H), 7.31-7.27 (m, 1H), 7.25-7.20 (m, $3 \mathrm{H}), 7.18(\mathrm{t}, J=7.0 \mathrm{~Hz}, 1 \mathrm{H}), 6.67(\mathrm{dd}, J=6.6,9.5 \mathrm{~Hz}, 1 \mathrm{H}), 4.99(\mathrm{t}, J=4.6 \mathrm{~Hz}, 1 \mathrm{H}), 4.05-3.94$ $(\mathrm{m}, 2 \mathrm{H}), 3.92-3.81(\mathrm{~m}, 2 \mathrm{H}), 2.38-2.22(\mathrm{~m}, 2 \mathrm{H}), 1.96-1.85(\mathrm{~m}, 1 \mathrm{H}), 1.74-1.63(\mathrm{~m}, 1 \mathrm{H}), 1.49(\mathrm{~s}$, 9H), $1.16(\mathrm{~s}, 9 \mathrm{H})$

${ }^{13} \mathrm{C}$ NMR $\left(150 \mathrm{MHz}, \mathrm{CDCl}_{3}\right) \delta 164.1,162.0,155.2,142.3,128.1,126.5,126.2,104.6,84.1,78.9$, $65.1,54.9,30.6,28.6,27.8,25.7$

IR (thin film) 3377, 3053, 2979, 2884, 1711, 1638, 1608, 1498, 1267, 1248, $1146 \mathrm{~cm}^{-1}$

HRMS (ESI-TOF) m/z: $[\mathrm{M}+\mathrm{Na}]^{+}$calcd for $\left[\mathrm{C}_{23} \mathrm{H}_{35} \mathrm{~N}_{3} \mathrm{O}_{6} \mathrm{Na}\right]^{+} 472.2424$, found 472.2418 


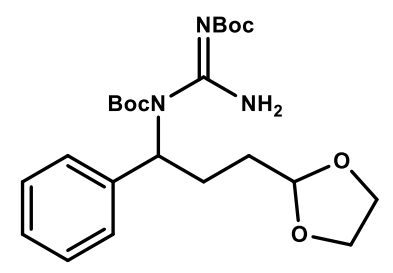

S14

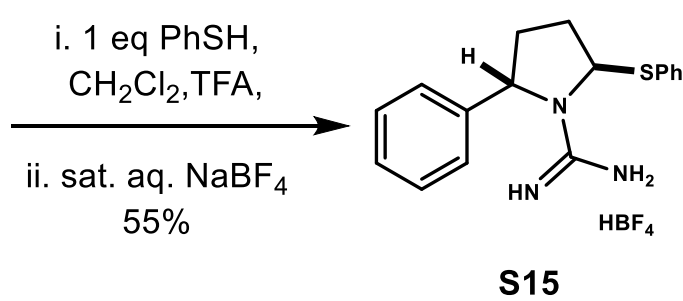

S15

Preparation of N-Amidinyliminium Precursor S15: A flame dried flask was charged with protected guanidine S14 (1.7403 g, $3.87 \mathrm{mmol})$ and thiophenol (0.4 mL, $3.87 \mathrm{mmol}, 1$ equiv) in $\mathrm{CH}_{2} \mathrm{Cl}_{2}(7.5 \mathrm{~mL}, 0.5 \mathrm{M})$ and the solution was cooled to $0{ }^{\circ} \mathrm{C}$. Trifluoroacetic acid $(7.5 \mathrm{~mL}, 0.25 \mathrm{M}$ final concentration) was added slowly and the mixture was allowed to warm slowly to room temperature overnight $(\sim 16 \mathrm{~h})$. The reaction was then concentrated under reduced pressure to afford a red-orange oil. The crude residue was purified by flash column chromatography $\left(\mathrm{SiO}_{2}, 1: 0 \rightarrow 9: 1\right.$ $\mathrm{CH}_{2} \mathrm{Cl}_{2} / \mathrm{MeOH}$ ) to afford the intermediate TFA salt as an orange oil. The intermediate trifluoroacetate salt was dissolved in $\mathrm{CHCl}_{3}(20 \mathrm{~mL})$. The solution was then washed with saturated aqueous $\mathrm{NaBF}_{4}(4 \times 30 \mathrm{~mL})$ and then $\mathrm{H}_{2} \mathrm{O}(30 \mathrm{~mL})$. The organic layer was concentrated and the resulting residue was dried by azeotropic removal of water with toluene $(2 \times 20 \mathrm{~mL})$ to afford pyrrolidine $\mathbf{S 1 5}(821.7 \mathrm{mg}, 55 \%$ ) as a colorless solid and a 4:1 mixture of diastereomers.

${ }^{1} \mathbf{H}$ NMR $\left(600 \mathrm{MHz}, \mathrm{CDCl}_{3}\right) \delta 7.58-7.52(\mathrm{~m}, 2 \mathrm{H}), 7.45-7.30(\mathrm{~m}, 6 \mathrm{H}), 7.09(\mathrm{~d}, J=7.4 \mathrm{~Hz}, 2 \mathrm{H})$, $5.40(\mathrm{~d}, J=6.9 \mathrm{~Hz}, 0.8 \mathrm{H}), 5.31(\mathrm{~d}, J=6.1 \mathrm{~Hz}, 0.2 \mathrm{H}), 4.92(\mathrm{~d}, J=8.5 \mathrm{~Hz}, 0.8 \mathrm{H}), 4.88(\mathrm{dd}, J=6.7$, $9.1 \mathrm{~Hz}, 0.2 \mathrm{H}), 2.60-2.50(\mathrm{~m}, 1 \mathrm{H}), 2.47-2.23(\mathrm{~m}, 1.4 \mathrm{H}), 2.21(\mathrm{dd}, J=6.4,13.5 \mathrm{~Hz}, 0.8 \mathrm{H}), 1.75$ $(\mathrm{dd}, J=6.5,12.6 \mathrm{~Hz}, 0.8 \mathrm{H})$

${ }^{13} \mathbf{C ~ N M R}\left(150 \mathrm{MHz}, \mathrm{CDCl}_{3}\right) \delta$ (major) 155.2, 138.0, 134.8, 130.02, 129.92, 129.87, 129.11, 126.2 , 125.1, 67.9, 63.7, 32.6, 31.5 (minor) 155.6, 137.9, 134.0, 130.8, 130.05, 129.8, 129.5, 129.2, $129.1,69.7,65.1,35.1,33.0$

IR (thin film) 3458, 3374, 3271, 3063, 2922, 2852, 1651, 1590, 1581, 1452, 1439, 1024, $912 \mathrm{~cm}^{-}$ 1

HRMS (ESI-TOF) m/z: [M] $]^{+}$calcd for $\left[\mathrm{C}_{17} \mathrm{H}_{20} \mathrm{~N}_{3} \mathrm{~S}\right]^{+} 298.1378$, found 298.1380

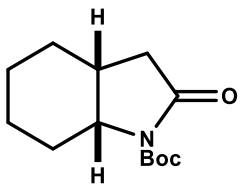

S16

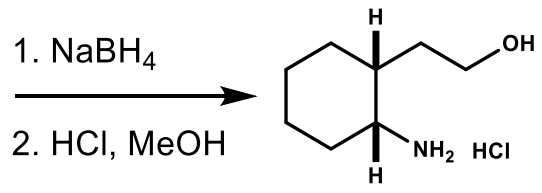

S17

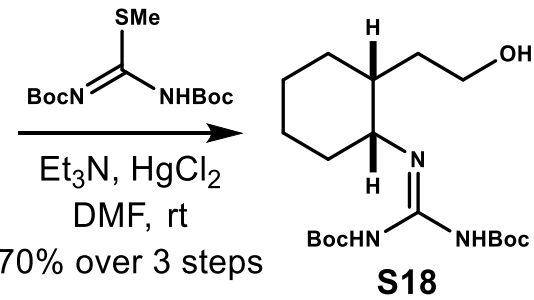

S18

Guanidine S18: A $250 \mathrm{~mL}$ round bottom flask was charged with lactam $\mathbf{S 1 6}^{5}$ (2.0 g, $\left.8.35 \mathrm{mmol}\right)$ as a solution in $\mathrm{MeOH} / \mathrm{H}_{2} \mathrm{O}(10: 1,55 \mathrm{~mL}, 0.15 \mathrm{M})$. The solution was cooled to $0{ }^{\circ} \mathrm{C}$ and $\mathrm{NaBH}_{4}$ ( $2.08 \mathrm{~g}, 54.9 \mathrm{mmol}, 6.6$ equiv) was added in two portions over 10 minutes (CAUTION: strong exotherm) and the mixture was stirred for 10 minutes at $0{ }^{\circ} \mathrm{C}$. The ice bath was then removed and 
the mixture was warmed to room temperature where it was stirred for $4 \mathrm{~h}$. The reaction was quenched by the addition of sat. $\mathrm{NH}_{4} \mathrm{Cl}(100 \mathrm{~mL})$. The methanol was then removed under vacuum and the resulting aqueous solution was extracted with EtOAc $(3 \times 50 \mathrm{~mL})$. The organics were then dried over $\mathrm{MgSO}_{4}$, filtered, and concentrated to afford the intermediate protected amine as light orange foam, which was used without further purification.

A $100 \mathrm{~mL}$ round bottom flask was charged with $\mathrm{MeOH}(30 \mathrm{~mL})$ and cooled to $0{ }^{\circ} \mathrm{C}$. Then acetyl chloride ( $9 \mathrm{~mL}, 126.1 \mathrm{mmol}, 15$ equiv) was added dropwise. The mixture was stirred at $0{ }^{\circ} \mathrm{C}$ for 30 minutes. The crude protected amine was then added and the mixture was stirred at room temperature for $1 \mathrm{~h}$. The reaction was then concentrated to afford the intermediate $\mathrm{HCl}$ salt $\mathbf{S 1 7}$ as a brown foam.

A $100 \mathrm{~mL}$ round bottom flask was charged with the intermediate amine salt S17, N,N'-Di-Boc-Smethylisothiourea $\left(2.47 \mathrm{~g}, 8.50 \mathrm{mmol}, 1.02\right.$ equiv) and $\mathrm{Et}_{3} \mathrm{~N}$ (4.32 mL, $30.84 \mathrm{mmol}, 4$ equiv) in DMF $(30 \mathrm{~mL}, 0.25 \mathrm{M})$. The solution was stirred for 10 minutes until the mixture was homogenous and then $\mathrm{HgCl}_{2}$ (2.28 g, $8.41 \mathrm{mmol}, 1.01$ equiv) was added. The reaction was then stirred at room temperature overnight $(\sim 16 \mathrm{~h})$. The reaction was diluted with EtOAc $(60 \mathrm{~mL})$, filtered over celite, and the solids were washed with EtOAc $(30 \mathrm{~mL})$. The filtrate was then washed with $\mathrm{H}_{2} \mathrm{O}(2 \times 100$ $\mathrm{mL})$ and brine $(100 \mathrm{~mL})$. The organics were then dried over $\mathrm{MgSO}_{4}$, filtered, and concentrated to an orange foam. The crude residue was purified by flash column chromatography $\left(\mathrm{SiO}_{2}\right.$, $1: 0 \rightarrow 4: 1 \rightarrow 2: 1 \mathrm{Hex} /$ EtOAc) to afford protected guanidine $\mathbf{S 1 8}(2.25 \mathrm{~g}, 70 \%$ over 3 steps $)$ as a pale orange foam.

${ }^{1}$ H NMR $\left(600 \mathrm{MHz}, \mathrm{CDCl}_{3}\right) \delta 11.50(\mathrm{~s}, 1 \mathrm{H}), 8.74(\mathrm{~d}, J=8.8 \mathrm{~Hz}, 1 \mathrm{H}), 4.54(\mathrm{dd}, J=3.3,8.8 \mathrm{~Hz}$, $1 \mathrm{H}), 3.81-3.75(\mathrm{~m}, 1 \mathrm{H}), 3.69-3.61(\mathrm{~m}, 1 \mathrm{H}), 2.88(\mathrm{bs}, 1 \mathrm{H}), 1.82-1.68(\mathrm{~m}, 3 \mathrm{H}), 1.61-1.52(\mathrm{~m}$, $4 \mathrm{H}), 1.50(\mathrm{~s}, 9 \mathrm{H}), 1.48(\mathrm{~s}, 9 \mathrm{H}), 1.42-1.28(\mathrm{~m}, 3 \mathrm{H}), 1.26-1.18(\mathrm{~m}, 1 \mathrm{H})$

${ }^{13} \mathrm{C}$ NMR $\left(150 \mathrm{MHz}, \mathrm{CDCl}_{3}\right) \delta 163.6,156.1,153.4,83.2,79.2,62.0,49.1,38.7,35.3,30.8,28.5$, $28.3,28.2,28.0,25.4,21.0$

IR (thin film) 3321, 3281, 3139, 2979, 2930, 2858, 1791, 1717, 1637, 1613, 1574, 1479, 1455, $1416,1394,1367,1343,1324,1268,1250,1228,1157,1122,1057 \mathrm{~cm}^{-1}$

HRMS (ESI-TOF) m/z: $[\mathrm{M}+\mathrm{Na}]^{+}$calcd for $\left[\mathrm{C}_{19} \mathrm{H}_{35} \mathrm{~N}_{3} \mathrm{O}_{5} \mathrm{Na}\right]^{+} 408.2474$, found 408.2469

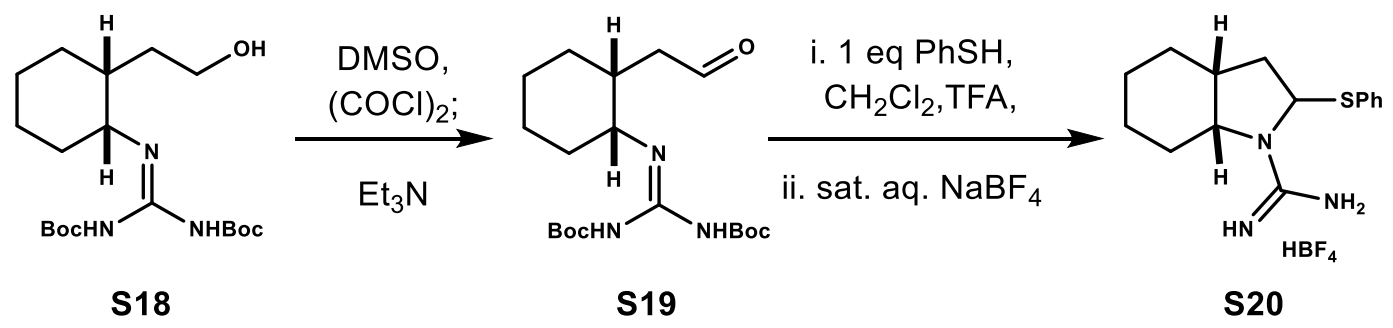

Preparation of N-Amidinyliminium Ion Precursor S20: A $250 \mathrm{~mL}$ round bottom flask was charged with a solution of DMSO $\left(0.55 \mathrm{~mL}, 7.74 \mathrm{mmol}, 2.4\right.$ equiv) in $\mathrm{CH}_{2} \mathrm{Cl}_{2}(80 \mathrm{~mL})$ and the mixture was cooled to $-78^{\circ} \mathrm{C}$. Oxalyl chloride $(0.33 \mathrm{~mL}, 3.84 \mathrm{mmol}, 1.2$ equiv) was added slowly 
and the reaction was stirred for $40 \mathrm{~min}$. Then a solution of alcohol $(1.247 \mathrm{~g}, 3.23 \mathrm{mmol})$ in $\mathrm{CH}_{2} \mathrm{Cl}_{2}$ $\left(60 \mathrm{~mL}, 0.02 \mathrm{M}\right.$ final concentration) was added slowly and the reaction was stirred for $40 \mathrm{~min}$. $\mathrm{Et}_{3} \mathrm{~N}$ ( $2.27 \mathrm{~mL}, 16.2 \mathrm{mmol}, 5$ equiv) was added to the reaction and the reaction was stirred at $-78{ }^{\circ} \mathrm{C}$ for 1 hour. The mixture was quenched with brine $(80 \mathrm{~mL})$ and warmed to room temperature. The mixture was diluted with $\mathrm{H}_{2} \mathrm{O}(50 \mathrm{~mL})$ and extracted with $\mathrm{CH}_{2} \mathrm{Cl}_{2}(2 \times 40 \mathrm{~mL})$. The organics were then dried over $\mathrm{MgSO}_{4}$, filtered, and concentrated to afford intermediate aldehyde $\mathbf{S 1 9}$ as a light orange foam, which was carried on without further purification.

A $100 \mathrm{~mL}$ round bottom flask was charged with crude aldehyde $\mathbf{S 1 9}$ and thiophenol $(0.33 \mathrm{~mL}$, $3.23 \mathrm{mmol}, 1$ equiv) in $\mathrm{CH}_{2} \mathrm{Cl}_{2}(6.5 \mathrm{~mL})$. The mixture was then cooled to $0{ }^{\circ} \mathrm{C}$ and then TFA $(6.5$ $\mathrm{mL}, 0.25 \mathrm{M}$ final concentration) was added slowly. The reaction was then allowed to warm to room temperature overnight $(\sim 16 \mathrm{~h})$. The reaction was then concentrated under reduced pressure and the crude residue was purified by flash column chromatography $\left(\mathrm{SiO}_{2}, 1: 0 \rightarrow 9: 1 \mathrm{CH}_{2} \mathrm{Cl}_{2} / \mathrm{MeOH}\right)$ to afford the intermediate TFA salt as an orange oil. The intermediate TFA salt was dissolved in $\mathrm{CHCl}_{3}(30 \mathrm{~mL})$. The solution was then washed with saturated aqueous $\mathrm{NaBF}_{4}(3 \times 30 \mathrm{~mL})$ and then $\mathrm{H}_{2} \mathrm{O}(30 \mathrm{~mL})$. The organic layer was concentrated and the resulting residue was dried by azeotropic removal of water with toluene ( $2 \times 20 \mathrm{~mL})$ to afford pyrrolidine $\mathbf{S 2 0}(383.1 \mathrm{mg}, 33 \%)$ as a colorless foam and a 2:1 mixture of diastereomers.

${ }^{1} \mathbf{H}$ NMR $\left(600 \mathrm{MHz}, \mathrm{CDCl}_{3}\right) \delta 7.53-7.36(\mathrm{~m}, 5 \mathrm{H}), 6.39(\mathrm{bs}, 4 \mathrm{H}), 5.02(\mathrm{~d}, J=6.2 \mathrm{~Hz}, 0.33 \mathrm{H})$, 4.94-4.88 (m, 0.66H), 3.92-3.85 (m, 0.33H), 3.84-3.76 (m, 0.66H), 2.66-2.57 (m, 0.33H), 2.46$2.37(\mathrm{~m}, 0.33 \mathrm{H}), 2.37-2.26(\mathrm{~m}, 1.98 \mathrm{H}), 2.18(\operatorname{app~d}, J=14.8 \mathrm{~Hz}, 0.33 \mathrm{H}), 2.09-2.02(\mathrm{~m}, 0.33 \mathrm{H})$, $1.74-1.58(\mathrm{~m}, 2.75 \mathrm{H}), 1.54-1.47(\mathrm{~m}, 0.33 \mathrm{H}), 1.45-1.35(\mathrm{~m}, 1.32 \mathrm{H}), 1.32-1.01(\mathrm{~m}, 1.98 \mathrm{H}), 0.98$ (q, $J=12.2 \mathrm{~Hz}, 0.66 \mathrm{H}), 0.08(\mathrm{q}, J=12.6 \mathrm{~Hz}, 0.66 \mathrm{H})$

${ }^{13} \mathbf{C}$ NMR $\left(150 \mathrm{MHz}, \mathrm{CDCl}_{3}\right) \delta$ (major) $154.8,136.5,130.4,129.9,129.8,66.1,59.4,35.7,35.4$, $26.3,25.1,22.87,19.8$ (minor) $154.5,134.1,130.6,129.5,127.9,66.3,59.3,35.8,34.3,26.2,24.9$, $22.88,20.1$

IR (thin film) 3457, 3378, 3275, 2930, 2860, 1651, 1595, 1530, 1469, 1439, 1285, $1022 \mathrm{~cm}^{-1}$

HRMS (ESI-TOF) m/z: $[\mathrm{M}]^{+}$calcd for $\left[\mathrm{C}_{15} \mathrm{H}_{22} \mathrm{~N}_{3} \mathrm{~S}\right]^{+} 276.1534$, found 276.1536 


\section{B. Synthesis of Indole Dienophiles}

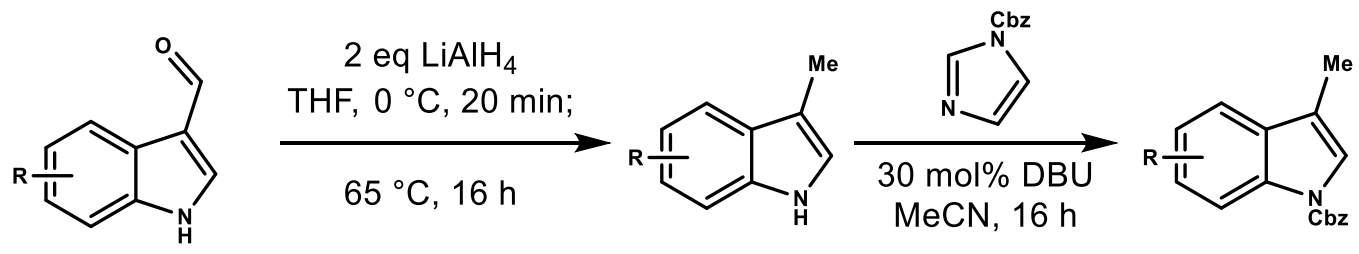

General Reduction Procedure for Indole Dienophile Synthesis: A flame dried round-bottom flask was charged with substituted 3-formyl indole (1 equiv) in THF $(0.2 \mathrm{M})$ and cooled to $0{ }^{\circ} \mathrm{C}$. $\mathrm{LiAlH}_{4}\left(2 \mathrm{eq}\right.$ ) was slowly added over $5 \mathrm{~min}$ and the reaction was then stirred at $0{ }^{\circ} \mathrm{C}$ for $20 \mathrm{~min}$. The flask was then equipped with a reflux condenser and the reaction was heated with a heating mantle to $65{ }^{\circ} \mathrm{C}$ for $16 \mathrm{hr}$. The reaction was then cooled to room temperature and then to $0{ }^{\circ} \mathrm{C}$. $\mathrm{H}_{2} \mathrm{O}$ (30 equiv) was added dropwise with vigorous stirring. The mixture was stirred for an additional 20 min and then diluted with $\mathrm{Et}_{2} \mathrm{O}$ (equal volume to initial THF). The solution was then filtered through a funnel containing a bottom layer of Celite ${ }^{\circledR}$ and top layer of $\mathrm{MgSO}_{4}$. The solids were then washed with $\mathrm{Et}_{2} \mathrm{O}(100 \mathrm{~mL})$ and the filtrate was concentrated to afford the crude intermediate substituted 3-methylindole, which was used directly in the next step.

General Protection Procedure for Indole Dienophile Synthesis: Indole protection utilized the following procedure adapted from Heller and coworkers. ${ }^{6}$ A flame dried round-bottom flask was charged with benzyl $1 \mathrm{H}$-imidazole-1-carboxylate ${ }^{7}$ (1.05 equiv) in $\mathrm{MeCN}(0.3 \mathrm{M})$. The crude substituted 3-methyl indole (1 equiv) was added followed by the DBU (50 mol\%). The reaction was then stirred overnight $(16 \mathrm{~h})$ at room temperature. The mixture was then diluted with aqueous $1 \mathrm{M} \mathrm{HCl}$ (equal volume to initial $\mathrm{MeCN}$ ) and $\mathrm{EtOAc}$ (equal volume to initial $\mathrm{MeCN}$ ). The aqueous layer was then washed EtOAc ( $3 \mathrm{x}$ equal volume to initial $\mathrm{MeCN}$ ) and the combined organic layers were dried over $\mathrm{Na}_{2} \mathrm{SO}_{4}$, filtered, and concentrated. The crude residue was purified by flash column chromatography $\left(\mathrm{SiO}_{2}, 1: 0 \rightarrow 9: 1 \rightarrow 4: 1 \mathrm{Hex} / \mathrm{EtOAc}\right)$ to afford protected indole dienophile. 


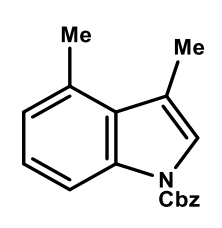

Benzyl 3,4-dimethyl-1H-indole-1-carboxylate (S21): According to the general reduction and protection procedures, 4-methyl- $1 H$-indole-3-carbaldehyde $(1.00 \mathrm{~g}, 6.28 \mathrm{mmol})$ was converted to benzyl 3,4-dimethyl-1H-indole-1-carboxylate (660 mg, 37\% over two steps), isolated as a clear oil.

${ }^{1} \mathbf{H}$ NMR $\left(500 \mathrm{MHz}, \mathrm{CDCl}_{3}\right) \delta 8.05(\mathrm{br} \mathrm{s}, 1 \mathrm{H}), 7.49-7.45(\mathrm{~m}, 2 \mathrm{H}), 7.44-7.35(\mathrm{~m}, 3 \mathrm{H}), 7.33(\mathrm{br} \mathrm{s}$, 1H), $7.17(\mathrm{t}, J=7.8 \mathrm{~Hz}, 1 \mathrm{H}), 6.97(\mathrm{~d}, J=7.3 \mathrm{~Hz}, 1 \mathrm{H}), 5.42(\mathrm{~s}, 2 \mathrm{H}), 2.67(\mathrm{~s}, 3 \mathrm{H}), 2.43(\mathrm{~s}, 3 \mathrm{H})$

${ }^{13}$ C NMR $\left(150 \mathrm{MHz}, \mathrm{CDCl}_{3}\right) \delta 150.7,136.3,135.4,131.4,129.5,128.8,128.6,128.4,124.62$, $124.57,122.7,118.3,113.1,68.4,19.8,13.4$

IR (thin film) 3125, 3035, 2960, 2930, 2251, 1730, 1609, 1574, 1425, 1255, 1108, 1063, $909 \mathrm{~cm}^{-}$ 1

HRMS (ESI-TOF) m/z: $[\mathrm{M}+\mathrm{Na}]^{+}$calcd for $\left[\mathrm{C}_{18} \mathrm{H}_{17} \mathrm{NO}_{2} \mathrm{Na}\right]^{+}$302.1157, found 302.1153

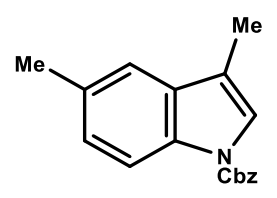

Benzyl 3,5-dimethyl-1 $H$-indole-1-carboxylate (S22): According to the general reduction and protection procedures, 5-methyl- $1 \mathrm{H}$-indole-3-carbaldehyde $(1.00 \mathrm{~g}, 6.28 \mathrm{mmol})$ was converted to benzyl 3,5-dimethyl-1H-indole-1-carboxylate ( $740 \mathrm{mg}, 42 \%$ over two steps), isolated as a clear oil.

${ }^{1} \mathbf{H}$ NMR $\left(500 \mathrm{MHz}, \mathrm{CDCl}_{3}\right) \delta 8.03$ (br s, 1H), 7.50-7.45 (m, 2H), 7.43-7.32 (m, 4H), $7.28(\mathrm{~s}$, $1 \mathrm{H}), 7.13(\mathrm{~d}, J=8.2 \mathrm{~Hz}, 1 \mathrm{H}), 5.43(\mathrm{~s}, 2 \mathrm{H}), 2.46(\mathrm{~s}, 3 \mathrm{H}), 2.23(\mathrm{~s}, 3 \mathrm{H})$

${ }^{13} \mathbf{C}$ NMR $\left(150 \mathrm{MHz}, \mathrm{CDCl}_{3}\right) \delta 150.9,135.5,133.7,132.3,131.7,128.8,128.6,128.4,125.9$, $122.5,119.0,117.2,114.8,68.4,21.4,9.7$

IR (thin film) 3032, 2919, 2859, 1728, 1603, 1455, 1399, 1356, 1289, 1246, 1222, 1196, 1079, $1025 \mathrm{~cm}^{-1}$

HRMS (ESI-TOF) m/z: $[\mathrm{M}+\mathrm{Na}]^{+}$calcd for $\left[\mathrm{C}_{18} \mathrm{H}_{17} \mathrm{NO}_{2} \mathrm{Na}\right]^{+}$302.1157, found 302.1158 


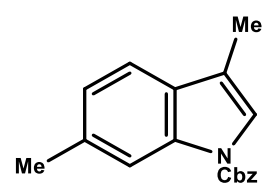

Benzyl 3,6-dimethyl-1H-indole-1-carboxylate (S23): According to the general reduction and protection procedures, 6-methyl- $1 H$-indole-3-carbaldehyde $(796.0 \mathrm{mg}, 5.00 \mathrm{mmol})$ was converted to benzyl 6-methoxy-3-methyl-1H-indole-1-carboxylate (1.29 g, 92\% over two steps), isolated as a colorless solid.

${ }^{1} \mathbf{H}$ NMR $\left(600 \mathrm{MHz}, \mathrm{CDCl}_{3}\right) \delta 8.02(\mathrm{bs}, 1 \mathrm{H}), 7.53-7.46(\mathrm{~m}, 2 \mathrm{H}), 7.44-7.34(\mathrm{~m}, 4 \mathrm{H}), 7.31$ (bs, 1H), 7.09 (d, $J=7.9 \mathrm{~Hz}, 1 \mathrm{H}), 5.43(\mathrm{~s}, 2 \mathrm{H}), 2.48(\mathrm{~s}, 3 \mathrm{H}), 2.23$ (d, $J=1.3 \mathrm{~Hz}, 3 \mathrm{H})$

${ }^{13} \mathrm{C}$ NMR $\left(150 \mathrm{MHz}, \mathrm{CDCl}_{3}\right) \delta 150.9,136.1,135.5,134.6,129.3,128.8,128.6,128.4,124.2$, $121.7,118.6,117.3,115.5,68.3,21.9,9.7$

IR (thin film) 3031, 2969, 2920, 2860, 1727, 1617, 1488, 1436, 1397, 1380, 1351, 1315, 1301, $1251,1202,1137,1087,1026,998 \mathrm{~cm}^{-1}$

Melting Point: $44-50{ }^{\circ} \mathrm{C}$

HRMS (ESI-TOF) m/z: $[\mathrm{M}+\mathrm{Na}]^{+}$calcd for $\left[\mathrm{C}_{18} \mathrm{H}_{17} \mathrm{NO}_{2} \mathrm{Na}\right]^{+} 302.1157$, found 302.1151

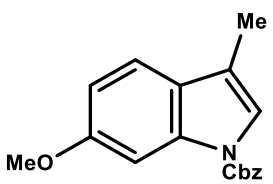

Benzyl 6-methoxy-3-methyl-1H-indole-1-carboxylate (S24): According to the general reduction and protection procedures, 6-methoxy- $1 H$-indole-3-carbaldehyde $(1.00 \mathrm{~g}, 5.71 \mathrm{mmol})$ was converted to benzyl 6-methoxy-3-methyl-1H-indole-1-carboxylate (1.25 g, 73\% over two steps), isolated as a yellow solid.

${ }^{1} \mathbf{H}$ NMR $\left(500 \mathrm{MHz}, \mathrm{CDCl}_{3}\right) \delta 7.74$ (br s, 1H), 7.51-7.45 (m, 2H), 7.43-7.33 (m, 4H), 7.28 (s, $1 \mathrm{H}), 6.88(\mathrm{dd}, J=2.0,8.5 \mathrm{~Hz}, 1 \mathrm{H}), 5.42(\mathrm{~s}, 2 \mathrm{H}), 3.83(\mathrm{~s}, 3 \mathrm{H}), 2.22(\mathrm{~s}, 3 \mathrm{H})$

${ }^{13} \mathrm{C}$ NMR $\left(150 \mathrm{MHz}, \mathrm{CDCl}_{3}\right) \delta 158.1,135.4,128.8,128.68,128.63,128.58,128.4,121.0,119.5$, $117.3,112.0,99.5,68.4,55.7,9.7$

IR (thin film) 3034, 2940, 2833, 1728, 1617, 1575, 1488, 1443, 1400, 1360, 1350, 1321, 1287 , $1258,1223,1143,1089,1040,1019 \mathrm{~cm}^{-1}$

Melting Point: $52-56^{\circ} \mathrm{C}$

HRMS (ESI-TOF) m/z: [M+Na $]^{+}$calcd for $\left[\mathrm{C}_{18} \mathrm{H}_{17} \mathrm{NO}_{3} \mathrm{Na}\right]^{+} 318.1106$, found 318.1104 


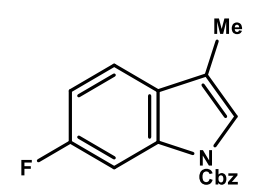

Benzyl 6-fluoro-3-methyl-1H-indole-1-carboxylate (S25): According to the general reduction and protection procedures, 6-fluoro- $1 H$-indole-3-carbaldehyde (500 $\mathrm{mg}, 3.35 \mathrm{mmol})$ was converted to benzyl 6-fluoro-3-methyl-1H-indole-1-carboxylate (660 mg, 70\% over two steps), isolated as a colorless solid.

${ }^{1}$ H NMR $\left(500 \mathrm{MHz}, \mathrm{CDCl}_{3}\right) \delta 7.90$ (br s, 1H), 7.51-7.45 (m, 2H), 7.44-7.29 (m, 5H), 7.01 (dt, $J$ $=2.1,9.1 \mathrm{~Hz}, 1 \mathrm{H}), 5.43(\mathrm{~s}, 2 \mathrm{H}), 2.23(\mathrm{~s}, 3 \mathrm{H})$

${ }^{13}$ C NMR $\left(150 \mathrm{MHz}, \mathrm{CDCl}_{3}\right) \delta 161.2(\mathrm{~d}, J=240.2 \mathrm{~Hz}), 150.7,135.1,128.8,128.79,128.5,127.8$, $122.6,119.7(\mathrm{~d}, J=10 \mathrm{~Hz}), 117.2,110.9(\mathrm{~d}, J=24.2 \mathrm{~Hz}), 102.7(\mathrm{~d}, J=28.6 \mathrm{~Hz}), 68.7,9.7$

IR (thin film) 3130, 2963, 2921, 2252, 1734, 1617, 1483, 1443, 1400, 1354, 1260, 1083, $909 \mathrm{~cm}^{-}$ 1

Melting Point: $39-42{ }^{\circ} \mathrm{C}$

HRMS (ESI-TOF) m/z: $[\mathrm{M}+\mathrm{H}]^{+}$calcd. for $\left[\mathrm{C}_{17} \mathrm{H}_{15} \mathrm{FNO}_{2}\right]^{+} 284.1087$, found 284.1088

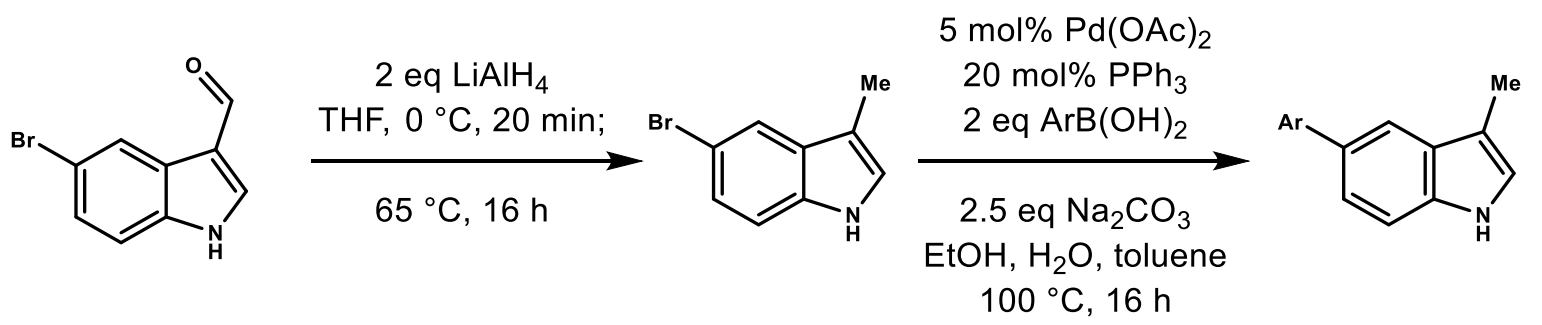

General Procedure for Suzuki Coupling: 5-bromo- $1 H$-indole-3-carbaldehyde $(2.0 \mathrm{~g}, 8.93$ mmol) was subjected to the general reduction procedure to afford 5-bromo-3-methyl- $H$-indole $(1.77 \mathrm{~g}, 95 \%)$ as a brown solid.

An adaption of the procedure reported by Giralt and coworkers was utilized to access 5-aryl-3methyl- $1 H$-indole derivatives. ${ }^{8}$ A flame dried $25 \mathrm{~mL}$ round-bottom flask was equipped with a reflux condenser and charged with $\mathrm{Na}_{2} \mathrm{CO}_{3}$ (2.5 equiv), Aryl Boronic Acid (2 equiv), 5-bromo-3methyl- $1 H$-indole ( 1 equiv), and $\mathrm{PPh}_{3}(20 \mathrm{~mol} \%)$ in a $1: 1: 1$ mixture of $\mathrm{EtOH} /$ toluene $/ \mathrm{H}_{2} \mathrm{O}(0.15 \mathrm{M}$ final concentation). The mixture was sparged with Argon with vigorous stirring for 15 minutes. Then $\mathrm{Pd}(\mathrm{OAc})_{2}(5 \mathrm{~mol} \%)$ was added and the mixture was heated with a heating mantle to $100{ }^{\circ} \mathrm{C}$ overnight $(\sim 16 \mathrm{~h})$. The reaction was then cooled to room temperature and concentrated. The black residue was then resuspended in 1:1 Hex/EtOAc and filtered through a $\mathrm{SiO}_{2}$ plug with 1:1 Hex/EtOAc. The filtrate was concentrated to afford the intermediate 3-methyl-5-aryl- $1 H$-indole derivative, which was used without further purification. 


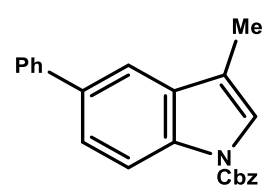

Benzyl 3-methyl-5-phenyl-1H-indole-1-carboxylate (S26): 3-methyl-5-bromo-1H-indole $(216.8 \mathrm{mg}, 1.03 \mathrm{mmol})$ was subjected to the general Suzuki coupling procedure with phenylboronic acid and the general protection procedure to afford benzyl 3-methyl-5-phenyl-1Hindole-1-carboxylate ( $251.9 \mathrm{mg}, 72 \%$ over two steps) as a colorless oil.

${ }^{1} \mathbf{H}$ NMR $\left(500 \mathrm{MHz}^{\mathrm{CDCl}} 3\right) \delta 8.19(\mathrm{bs}, 1 \mathrm{H}), 7.75-7.31(\mathrm{~m}, 13 \mathrm{H}), 5.45(\mathrm{~s}, 2 \mathrm{H}), 2.30(\mathrm{~s}, 3 \mathrm{H})$

${ }^{13} \mathrm{C}$ NMR $\left(125 \mathrm{MHz}, \mathrm{CDCl}_{3}\right) \delta 155.1,141.7,136.3,135.3,135.2,128.81,128.78,128.71,128.6$, $128.58,128.45,128.4,127.4,126.9,124.1,117.6,115.4,69.8,9.7$

IR (thin film) 3129, 3063, 3033, 2960, 2920, 2250, 1729, 1600, 1466, 1400, 1357, 1261, 1236, $1088,1025,909 \mathrm{~cm}^{-1}$

HRMS (ESI-TOF) m/z: [M] $]^{+}$calcd for $\mathrm{C}_{23} \mathrm{H}_{19} \mathrm{NO}_{2}{ }^{+} 341.1416$, found 341.1409

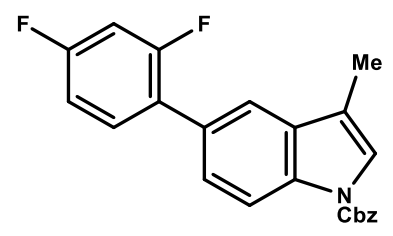

Benzyl 5-(2,4-difluorophenyl)-3-methyl-1H-indole-1-carboxylate (S27): 3-methyl-5-bromo$1 \mathrm{H}$-indole (423.4 mg, $2.01 \mathrm{mmol}$ ) was subjected to the general Suzuki coupling procedure with 2,4-difluorophenylboronic acid and the general protection procedure to afford benzyl 3-methyl-5(2,4-difluorophenyl)-1H-indole-1-carboxylate $(625.1 \mathrm{mg}, 82 \%$ over two steps) as a white solid.

${ }^{1} \mathbf{H}$ NMR $\left(600 \mathrm{MHz}, \mathrm{CDCl}_{3}\right) \delta 8.20(\mathrm{bs}, 1 \mathrm{H}), 7.60(\mathrm{~s}, 1 \mathrm{H}), 7.52-7.31(\mathrm{~m}, 8 \mathrm{H}), 7.00-6.88(\mathrm{~m}, 2 \mathrm{H})$, $5.45(\mathrm{~s}, 2 \mathrm{H}), 2.28(\mathrm{~s}, 3 \mathrm{H})$

${ }^{13}$ C NMR $\left(150 \mathrm{MHz}, \mathrm{CDCl}_{3}\right) \delta 163.0,162.9,161.34,161.26,160.66,160.58,159.0,158.9,150.8$, $135.3,135.2,131.8,131.78,131.75,131.7,129.7,128.8,128.7,128.63,128.58,128.46,128.38$, $125.87,125.85,125.78,125.6,123.1,119.6,117.5,115.2,111.58,111.55,111.44,111.41,104.5$, $104.3,104.2,68.6,9.7$

IR (thin film) 3031, 2970, 2945, 1727, 1613, 1508, 1466, 1454, 1397, 1380, 1356, 1274, 1256, $1227,1217,1138,1084,1024 \mathrm{~cm}^{-1}$

Melting Point: $68-72{ }^{\circ} \mathrm{C}$

HRMS (ESI-TOF) m/z: $[\mathrm{M}]^{+}$calcd for $\left[\mathrm{C}_{23} \mathrm{H}_{17} \mathrm{~F}_{2} \mathrm{NO}_{2}\right]^{+}$377.1227, found 377.1227 


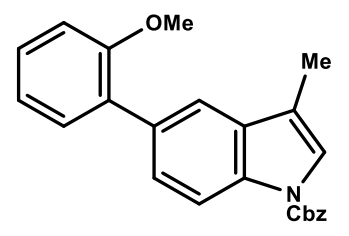

Benzyl 5-(2-methoxyphenyl)-3-methyl-1H-indole-1-carboxylate (S28): 3-methyl-5-bromo- $1 \mathrm{H}$ indole $(421.8 \mathrm{mg}, 2.01 \mathrm{mmol})$ was subjected to the general Suzuki coupling procedure with 2methoxyphenylboronic acid and general protection procedure to afford benzyl 3-methyl-5-(2,4difluorophenyl)-1 $H$-indole-1-carboxylate ( $665.1 \mathrm{mg}, 89 \%$ over two steps) as a colorless oil

${ }^{1} \mathbf{H}$ NMR $\left(600 \mathrm{MHz}, \mathrm{CDCl}_{3}\right) \delta 8.17$ (bs, 1H), $7.62(\mathrm{~s}, 1 \mathrm{H}), 7.53-7.46(\mathrm{~m}, 3 \mathrm{H}), 7.45-7.40(\mathrm{~m}, 3 \mathrm{H})$, 7.40-7.36 (m, 3H), 7.33 (t, $J=7.9 \mathrm{~Hz}, 1 \mathrm{H}), 7.05$ (t, $J=7.4 \mathrm{~Hz}, 1 \mathrm{H}), 7.01$ (d, $J=8.2 \mathrm{~Hz}, 1 \mathrm{H}), 5.45$ (s, $2 \mathrm{H}), 3.81(\mathrm{~s}, 3 \mathrm{H}), 2.27$ (s, 3H)

${ }^{13} \mathbf{C}$ NMR $\left(150 \mathrm{MHz}, \mathrm{CDCl}_{3}\right) \delta 156.6,135.4,133.3,131.5,131.22,131.20,128.8,128.7,128.64$, $128.60,128.44,128.40,126.5,122.7,120.9,120.0,117.7,114.7,111.3,68.5,55.7,9.7$

IR (thin film) 3032, 2937, 2833, 1727, 1598, 1579,1497, 1465, 1451, 1397, 1356, 1241, 1224 , $1179,1120,1086,1055,1025 \mathrm{~cm}^{-1}$

HRMS (ESI-TOF) m/z: $[\mathrm{M}+\mathrm{Na}]^{+}$calcd for $\left[\mathrm{C}_{24} \mathrm{H}_{21} \mathrm{NO}_{3} \mathrm{Na}\right]^{+}$394.1419, found 394.1436

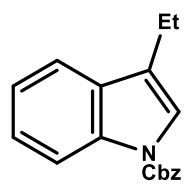

Benzyl 3-benzyl-1H-indole-1-carboxylate (S29): A $250 \mathrm{~mL}$ round bottom flask was charged with 3-acetyl-1H-indole $(1.01 \mathrm{~g}, 6.33 \mathrm{mmol})$ in THF $(63 \mathrm{~mL}, 0.1 \mathrm{M})$ and cooled to $0{ }^{\circ} \mathrm{C}$. Then LAH (645.4 mg, $17.00 \mathrm{mmol}, 2.6$ equiv) was added in two portions over 10 minutes. The reaction was then warmed to room temperature and stirred for 4 hours. The reaction was then cooled to 0 ${ }^{\circ} \mathrm{C}$ and $\mathrm{H}_{2} \mathrm{O}(0.6 \mathrm{~mL}), 15 \%$ w/v aqueous $\mathrm{NaOH}(0.6 \mathrm{~mL})$, and $\mathrm{H}_{2} \mathrm{O}(1.8 \mathrm{~mL})$ were sequentially added. The mixture was stirred for 30 minutes and then $\mathrm{MgSO}_{4}$ was added. The mixture was filtered and the solids were washed with $\mathrm{Et}_{2} \mathrm{O}(50 \mathrm{~mL})$. The filtrate was concentrated to afford 3ethyl-1 $H$-indole as a pale-yellow oil, which was used without further purification.

The crude 3-ethyl- $1 H$-indole was then subjected to the general protection procedure to afford benzyl 3-ethyl-1H-indole-1-carboxylate $(1.17 \mathrm{~g}, 66 \%$ over two steps), isolated as a pale-yellow oil.

${ }^{1} \mathbf{H}$ NMR $\left(600 \mathrm{MHz}, \mathrm{CDCl}_{3}\right) \delta 8.17(\mathrm{bs}, 1 \mathrm{H}), 7.53(\mathrm{~d}, J=7.7 \mathrm{~Hz}, 1 \mathrm{H}), 7.51-7.46(\mathrm{~m}, 2 \mathrm{H}), 7.45-$ $7.35(\mathrm{~m}, 4 \mathrm{H}), 7.32(\mathrm{t}, J=7.6 \mathrm{~Hz}, 1 \mathrm{H}), 7.25(\mathrm{t}, J=7.4 \mathrm{~Hz}, 1 \mathrm{H}), 5.44$ (s, 2H), 2.71 (dq, $J=1.1,7.5$ $\mathrm{Hz}, 2 \mathrm{H}), 1.32(\mathrm{t}, J=7.5 \mathrm{~Hz}, 3 \mathrm{H})$

${ }^{13}$ C NMR $\left(150 \mathrm{MHz}, \mathrm{CDCl}_{3}\right) \delta 151.0,135.4,130.8,128.8,128.7,128.6,128.5,124.6,124.1$, $122.7,121.3,119.1,115.3,68.5,18.2,13.5$ 
IR (thin film) 3033, 2964, 2932, 1727, 1608, 1498, 1435, 1395, 1356, 1316, 1304, 1236, 1219 , $1091,1054,1026,1017 \mathrm{~cm}^{-1}$

HRMS (ESI-TOF) m/z: $[\mathrm{M}+\mathrm{H}]^{+}$calcd. for $\left[\mathrm{C}_{18} \mathrm{H}_{18} \mathrm{NO}_{2}\right]^{+} 280.1338$, found 280.1349

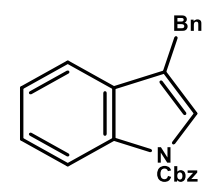

Benzyl 3-benzyl-1 $\boldsymbol{H}$-indole-1-carboxylate (S30): 3-benzyl-1 $H$-indole was prepared according to the method of Jia and Zhu. ${ }^{9}$ This material $(350 \mathrm{mg}, 1.69 \mathrm{mmol})$ was then subjected to the general protection procedure to afford benzyl 3-benzyl-1 $H$-indole-1-carboxylate (438 mg, 76\%), isolated as a pale-yellow solid.

${ }^{1}$ H NMR $\left(600 \mathrm{MHz}, \mathrm{CDCl}_{3}\right) \delta 8.17$ (bs, $\left.1 \mathrm{H}\right), 7.48(\mathrm{~d}, J=7.4 \mathrm{~Hz}, 2 \mathrm{H}), 7.44-7.37$ (m, 5H), 7.33$7.26(\mathrm{~m}, 5 \mathrm{H}), 7.24-7.18(\mathrm{~m}, 2 \mathrm{H}), 5.44$ (s, 2H), $4.04(\mathrm{~s}, 2 \mathrm{H})$

${ }^{13}$ C NMR $\left(150 \mathrm{MHz}, \mathrm{CDCl}_{3}\right) \delta 150.9,139.5,135.8,135.3,130.6,128.8,128.73,128.71,128.6$, $128.5,126.3,124.7,123.2,122.9,121.2,119.5,115.3,68.6,31.5$

IR (thin film) 3054, 3031, 2982, 2961, 2902, 2841, 2304, 1733, 1605, 1571, 1495, 1454, 1397, $1356,1262,1244,1081,1025 \mathrm{~cm}^{-1}$

Melting Point: $52-56{ }^{\circ} \mathrm{C}$

HRMS (ESI-TOF) m/z: $[\mathrm{M}+\mathrm{Na}]^{+}$calcd. for $\left[\mathrm{C}_{23} \mathrm{H}_{19} \mathrm{NO}_{2} \mathrm{Na}\right]^{+} 364.1313$, found 364.1307

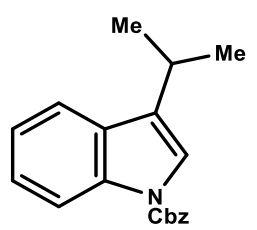

Benzyl 3-benzyl-1H-indole-1-carboxylate (S31): According to the procedure reported by Marinetti and coworkers, ${ }^{10}$ a $100 \mathrm{~mL}$ round bottom flask was charged with trichloroacetic acid (2.28 g, $13.97 \mathrm{mmol}, 0.95$ equiv) and $\mathrm{Et}_{3} \mathrm{SiH}(5.8 \mathrm{~mL}, 36.31 \mathrm{mmol}, 2.4$ equiv) in toluene $(7.5 \mathrm{~mL})$ and the mixture was heated with a heating mantle to $70{ }^{\circ} \mathrm{C}$. A solution of indole $(1.76 \mathrm{~g}, 15.06$ $\mathrm{mmol}$ ) and acetone ( $1.23 \mathrm{~mL}, 16.60 \mathrm{mmol}, 1.1 \mathrm{equiv})$ in toluene $(7.5 \mathrm{~mL}, 1 \mathrm{M}$ final concentration) was added via syringe pump over 1 hour. The reaction was stirred for an additional 30 minutes at $70{ }^{\circ} \mathrm{C}$ and then cooled to rt, where it was diluted with $10 \%$ w/v aqueous $\mathrm{K}_{2} \mathrm{CO}_{3}(50 \mathrm{~mL})$. The mixture was then extracted with EtOAc $(3 \times 50 \mathrm{~mL})$ and the organics were dried over $\mathrm{MgSO}_{4}$, filtered, and concentrated to afford 3-isopropyl-1H-indole as a red oil, which was used without further purification. 
The crude 3-ethyl- $1 H$-indole was then subjected to the general $\mathrm{Cbz}$ protection procedure to afford benzyl 3-isopropyl- $1 H$-indole-1-carboxylate ( $2.45 \mathrm{~g}, 55 \%$ over two steps), isolated as a paleyellow oil.

${ }^{1} \mathbf{H}$ NMR $\left(600 \mathrm{MHz}, \mathrm{CDCl}_{3}\right) \delta 8.18(\mathrm{bs}, 1 \mathrm{H}), 7.58(\mathrm{~d}, J=7.7 \mathrm{~Hz}, 1 \mathrm{H}), 7.51-7.46(\mathrm{~m}, 2 \mathrm{H}), 7.45-$ $7.34(\mathrm{~m}, 4 \mathrm{H}), 7.31(\mathrm{t}, J=7.6 \mathrm{~Hz}, 1 \mathrm{H}), 7.25(\mathrm{t}, J=7.5 \mathrm{~Hz}, 1 \mathrm{H}), 5.45$ (s, 2H), 3.12 (dh, $J=0.8,7.0$ $\mathrm{Hz}, 1 \mathrm{H}), 1.34(\mathrm{~d}, J=7.0 \mathrm{~Hz}, 6 \mathrm{H})$

${ }^{13}$ C NMR $\left(150 \mathrm{MHz}, \mathrm{CDCl}_{3}\right) \delta 151.0,135.4,130.2,129.1,128.8,128.7,128.6,128.5,124.5$, $122.6,120.3,119.5,115.4,68.5,25.3,22.6$

IR (thin film) 3029, 2970, 1737, 1455, 1396, 1365, 1305, 1230, 1217, $1059 \mathrm{~cm}^{-1}$

HRMS (ESI-TOF) m/z: $[\mathrm{M}+\mathrm{H}]^{+}$calcd. for $\left[\mathrm{C}_{19} \mathrm{H}_{20} \mathrm{NO}_{2}\right]^{+} 294.1494$, found 294.1498

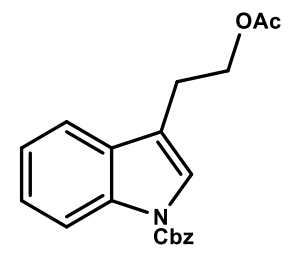

Benzyl 3-(2-acetoxyethyl)-1 $\boldsymbol{H}$-indole-1-carboxylate (S32): A $50 \mathrm{~mL}$ round bottom flask was charged with tryptophol $(819.1 \mathrm{mg}, 5.08 \mathrm{mmol})$ and pyridine $(10 \mathrm{~mL}, 0.5 \mathrm{M})$. Acetic anhydride $(0.66 \mathrm{~mL}, 6.98 \mathrm{mmol}, 1.4$ equiv) was added dropwise. The reaction was stirred at room temperature overnight $(\sim 16 \mathrm{~h})$. The reaction was then diluted with $\mathrm{H}_{2} \mathrm{O}(20 \mathrm{~mL})$ and vigorously stirred for 20 minutes. The mixture was then extracted with $\mathrm{CH}_{2} \mathrm{Cl}_{2}(2 \times 30 \mathrm{~mL})$ and the organics were then dried over $\mathrm{MgSO}_{4}$. The organics were then filtered through a $\mathrm{SiO}_{2}$ plug, the solids were washed with $\mathrm{CH}_{2} \mathrm{Cl}_{2}(100 \mathrm{~mL})$, and the filtrate was concentrated to afford the intermediate indole as a light orange oil.

The crude indole was then subjected to the general Cbz protection procedure to afford benzyl 3(2-acetoxyethyl)-1H-indole-1-carboxylate ( $1.52 \mathrm{~g}, 89 \%$ over two steps) as a colorless oil.

${ }^{1}$ H NMR $\left(600 \mathrm{MHz}, \mathrm{CDCl}_{3}\right) \delta 8.17$ (bs, $\left.1 \mathrm{H}\right), 7.56(\mathrm{~d}, J=7.7 \mathrm{~Hz}, 1 \mathrm{H}), 7.52-7.45(\mathrm{~m}, 3 \mathrm{H}), 7.44$ $7.36(\mathrm{~m}, 3 \mathrm{H}), 7.33(\mathrm{t}, J=7.6 \mathrm{~Hz}, 1 \mathrm{H}), 7.27$ (d, $J=7.6 \mathrm{~Hz}, 1 \mathrm{H}), 5.45$ (s, $2 \mathrm{H}), 4.35$ (t, $J=7.0 \mathrm{~Hz}$, $2 \mathrm{H}), 3.02(\mathrm{t}, J=7.0 \mathrm{~Hz}, 2 \mathrm{H}), 2.04(\mathrm{~s}, 3 \mathrm{H})$

${ }^{13} \mathbf{C}$ NMR $\left(150 \mathrm{MHz}, \mathrm{CDCl}_{3}\right) \delta 171.1,150.8,135.2,130.5,128.82,128.80,128.78,128.7,128.5$, $124.9,123.0,119.0,117.7,115.4,68.7,63.6,24.6,21.0$

IR (thin film) 3034, 2919, 2851, 1734, 1455, 1398, 1358, 1306, 1246, 1091, $1036 \mathrm{~cm}^{-1}$

HRMS (ESI-TOF) m/z: $[\mathrm{M}+\mathrm{Na}]^{+}$calcd. for $\left[\mathrm{C}_{20} \mathrm{H}_{19} \mathrm{NO}_{4} \mathrm{Na}\right]^{+} 360.1212$, found 360.1208 


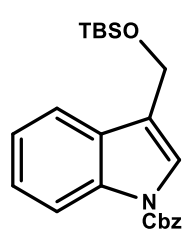

benzyl 3-(((tert-butyldimethylsilyl)oxy)methyl)-1H-indole-1-carboxylate (S33): A $25 \mathrm{~mL}$ round bottom flask was charged with benzyl 3-formyl-1H-indole-1-carboxylate ${ }^{11}$ (466 mg, 1.66 $\mathrm{mmol})$ in $\mathrm{MeOH}(2 \mathrm{~mL}, 0.83 \mathrm{M})$ and cooled to $0{ }^{\circ} \mathrm{C}$. $\mathrm{NaBH}_{4}(63.5 \mathrm{mg}, 1.67 \mathrm{mmol}, 1$ equiv) was added. The reaction was stirred for $1 \mathrm{~h}$ at $0{ }^{\circ} \mathrm{C}$ and then diluted with brine $(5 \mathrm{~mL})$. The mixture was extracted with EtOAc $(3 \times 10 \mathrm{~mL})$ and the organics were dried over $\mathrm{MgSO}_{4}$, filtered, and concentrated to a pale-yellow solid, which was used without further purification.

The crude alcohol was dissolved in $\mathrm{CH}_{2} \mathrm{Cl}_{2}(16 \mathrm{~mL}, 0.1 \mathrm{M})$ and imidazole $(227.9 \mathrm{mg}, 3.34 \mathrm{mmol}$, 2 equiv) was added followed by TBSCl (308.4 mg, $2.04 \mathrm{mmol}, 1.2$ equiv). The reaction was stirred at room temperature overnight $(\sim 16 \mathrm{~h})$. Then $\mathrm{MeOH}(0.2 \mathrm{~mL})$ was added and the mixture was stirred for 10 minutes. The reaction was then washed with $1 \mathrm{M}$ aqueous $\mathrm{HCl}(10 \mathrm{~mL})$ and the aqueous layer was extracted with $\mathrm{CH}_{2} \mathrm{Cl}_{2}(2 \times 20 \mathrm{~mL})$. The organics were dried over $\mathrm{MgSO}_{4}$, filtered, and concentrated. The crude residue was purified by flash column chromatography $\left(\mathrm{SiO}_{2}\right.$, $1: 0 \rightarrow 9: 1 \mathrm{Hex} /$ EtOAc) to afford the desired product $(608.3 \mathrm{mg}, 93 \%$ over two steps) as a paleyellow oil.

${ }^{1} \mathbf{H}$ NMR $\left(600 \mathrm{MHz}, \mathrm{CDCl}_{3}\right) \delta 8.16(\mathrm{bs}, 1 \mathrm{H}), 7.61-7.53(\mathrm{~m}, 2 \mathrm{H}), 7.50-7.45(\mathrm{~m}, 2 \mathrm{H}), 7.44-7.35$ $(\mathrm{m}, 3 \mathrm{H}), 7.32(\mathrm{t}, J=7.3 \mathrm{~Hz}, 1 \mathrm{H}), 7.26-7.22(\mathrm{~m}, 1 \mathrm{H}), 5.45(\mathrm{~s}, 2 \mathrm{H}), 4.85(\mathrm{~s}, 2 \mathrm{H}), 0.93(\mathrm{~s}, 9 \mathrm{H}), 0.11$ $(\mathrm{s}, 6 \mathrm{H})$

${ }^{13} \mathrm{C}$ NMR $\left(150 \mathrm{MHz}, \mathrm{CDCl}_{3}\right) \delta 150.9,135.9,135.3,129.4,128.8,128.7,128.4,124.8,122.9$, $122.5,122.1,119.7,115.3,68.6,58.0,26.0,18.5,-5.2$

IR (thin film) 2954, 2928, 2884, 2855, 1737, 1610, 1454, 1400, 1351, 1305, 1254, 1225, 1127 , $1087,1068,1025 \mathrm{~cm}^{-1}$

HRMS (ESI-TOF) m/z: $[\mathrm{M}+\mathrm{Na}]^{+}$calcd. for $\left[\mathrm{C}_{23} \mathrm{H}_{29} \mathrm{NO}_{3}\right]^{+} 418.1814$, found 418.1797

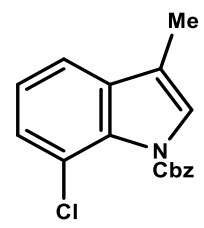

Benzyl 7-chloro-3-methyl-1H-indole-1-carboxylate (S34): According to the general reduction and protection procedures, 7-chloro- $1 H$-indole-3-carbaldehyde (483.2 $\mathrm{mg}, 2.69 \mathrm{mmol})$ was converted to benzyl 7-chloro-3-methyl-1H-indole-1-carboxylate $(584.2 \mathrm{mg}, 72 \%$ over two steps) as a pale-yellow oil.

${ }^{1} \mathbf{H}$ NMR $\left(600 \mathrm{MHz}, \mathrm{CDCl}_{3}\right) \delta 8.11$ (bs. 1H), 7.49 (d, J=7.9 Hz, 1H), 7.40-7.33 (m, 4H), 7.19 (d, $J=7.6 \mathrm{~Hz}, 1 \mathrm{H}), 7.06$ (t, $J=7.7 \mathrm{~Hz}, 1 \mathrm{H}), 7.03(\mathrm{bs}, 1 \mathrm{H}), 5.19$ (s, 2H), 2.34 (s, 3H) 
${ }^{13} \mathbf{C}$ NMR $\left(150 \mathrm{MHz}, \mathrm{CDCl}_{3}\right) \delta 155.1,135.2,133.5,129.8,128.64,128.59,128.4,122.3,121.3$, $119.9,117.5,116.5,112.9,69.8,9.8$

IR (thin film) 3064, 2917, 1733, 1623, 1489, 1453, 1436, 1393, 1344, 1325, 1264, 1201, 1077, $1048,947 \mathrm{~cm}^{-1}$

HRMS (ESI-TOF) m/z: $[\mathrm{M}]^{+}$calcd. for $\left[\mathrm{C}_{17} \mathrm{H}_{14} \mathrm{ClNO}_{2}\right]^{+} 299.0713$, found 299.0703

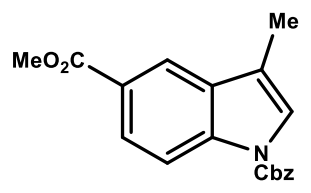

1-benzyl 5-methyl 3-methyl-1H-indole-1,5-dicarboxylate (S35): According to a modified procedure by Jia and Zhu, ${ }^{9}$ a solution of methyl 4-amino-3-iodobenzoate $(613.4 \mathrm{mg}, 2.21 \mathrm{mmol}$, 1.1 equiv) and DABCO (673.4 mg, $6.00 \mathrm{mmol}, 3$ equiv) in DMF $(10 \mathrm{~mL}, 0.2 \mathrm{M})$ were sparged with argon for 15 minutes. Then propionaldehyde $(0.15 \mathrm{~mL}, 2.09 \mathrm{mmol})$ and $\mathrm{Pd}(\mathrm{OAc})_{2}(22.4 \mathrm{mg}$, $0.1 \mathrm{mmol}, 5 \mathrm{~mol} \%)$ were added and the flask was sealed. The mixture was heated with a heating mantle to $90^{\circ} \mathrm{C}$ for $18 \mathrm{~h}$ and then cooled to room temperature. The mixture was diluted with brine $(100 \mathrm{~mL})$ and extracted with EtOAc $(3 \times 40 \mathrm{~mL})$. The organics were washed with brine $(50 \mathrm{~mL})$. The organics were then diluted with hexanes $(150 \mathrm{~mL})$ and flushed through a $\mathrm{SiO}_{2}$ plug with 1:1 Hex/EtOAc $(200 \mathrm{~mL})$. The filtrate was then concentrated to a green oil, which was used without further purification.

The crude indole was then subjected to the general Cbz protection procedure to afford 1-benzyl 5methyl 3-methyl- $1 H$-indole-1,5-dicarboxylate $(220.2 \mathrm{mg}, 33 \%$ over two steps) as a pale-yellow solid.

${ }^{1} \mathbf{H}$ NMR $\left(600 \mathrm{MHz}, \mathrm{CDCl}_{3}\right) \delta 8.23(\mathrm{~d}, J=0.8 \mathrm{~Hz}, 1 \mathrm{H}), 8.19(\mathrm{bs}, 1 \mathrm{H}), 8.02(\mathrm{~d}, J=8.6 \mathrm{~Hz}, 1 \mathrm{H})$, 7.48 (d, $J=7.2 \mathrm{~Hz}, 2 \mathrm{H}), 7.46-7.35$ (m, 4H), 5.44 (s, 2H), 3.95 (s, 3H), 2.29 (s, 3H)

${ }^{13} \mathrm{C}$ NMR $\left(150 \mathrm{MHz}, \mathrm{CDCl}_{3}\right)$ 167.6, 150.6, 138.3, 135.0, 131.4, 128.9, 128.6, 128.5, 126.0, 124.8, $123.6,121.4,117.9,114.9,68.9,52.1,9.6$

IR (thin film) 2949, 2919, 1712, 1614, 1443, 1396, 1352, 1288, 1266, 1218, 1118, 1070, 1024 , $986 \mathrm{~cm}^{-1}$

Melting Point: $74-76{ }^{\circ} \mathrm{C}$

HRMS (ESI-TOF) m/z: $[\mathrm{M}+\mathrm{H}]^{+}$calcd. for $\left[\mathrm{C}_{19} \mathrm{H}_{18} \mathrm{NO}_{4}\right]^{+} 324.1236$, found 324.1226 


\section{Summary of Reaction Optimization Experiments}

Table S1. Complete Summary of $\mathrm{N}$-Amidinyliminium Ion Cycloaddition with Indoles Reaction Optimization
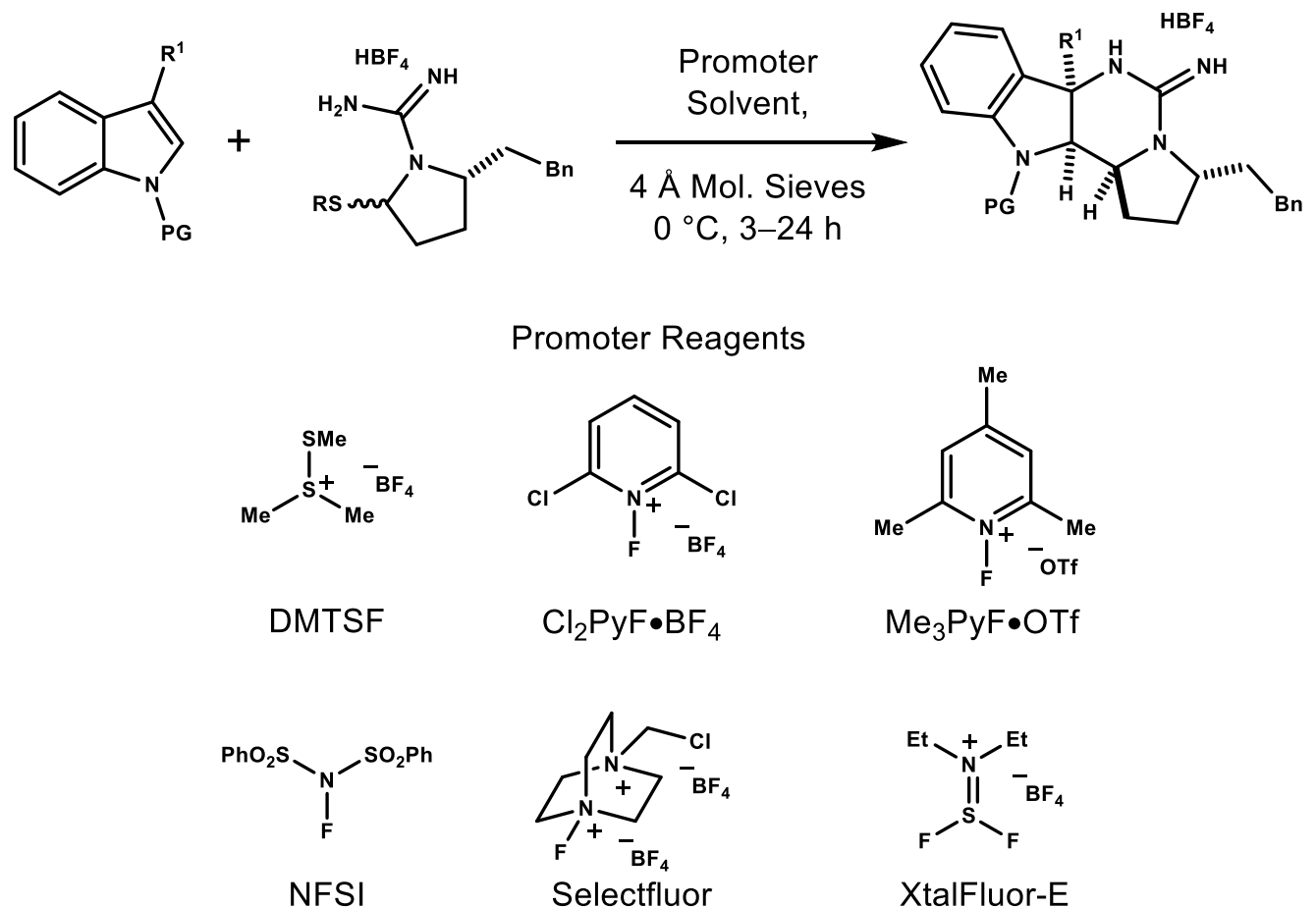

\begin{tabular}{|c|c|c|c|c|c|c|}
\hline Entry & $S R(m m o l)$ & $P G$ & $R^{l}$ & Promoter & Solvent & Yield \\
\hline 1 & $\mathrm{Ph}(0.1 \mathrm{mmol})$ & Me (1.5 equiv) & $\mathrm{H}$ & DMTSF (1 equiv) & $\mathrm{CH}_{2} \mathrm{Cl}_{2}(0.5 \mathrm{M})$ & $0 \%$ \\
\hline 2 & $\mathrm{Ph}(0.1 \mathrm{mmol})$ & $\mathrm{Me}$ ( 1.5 equiv) & $\mathrm{H}$ & $\begin{array}{l}\mathrm{Cl}_{2} \mathrm{PyF} \cdot \mathrm{BF}_{4} \\
(1 \text { equiv })_{4}\end{array}$ & $\mathrm{CH}_{2} \mathrm{Cl}_{2}(0.5 \mathrm{M})$ & $0 \%$ \\
\hline 3 & $\mathrm{Ph}(0.1 \mathrm{mmol})$ & $\mathrm{Me}(1.5$ equiv $)$ & $\mathrm{H}$ & $\begin{array}{l}\mathrm{Me}_{3} \mathrm{PyF} \cdot \mathrm{BF}_{4} \\
(1 \text { equiv) }\end{array}$ & $\mathrm{CH}_{2} \mathrm{Cl}_{2}(0.5 \mathrm{M})$ & $0 \%$ \\
\hline 4 & $\mathrm{Ph}(0.1 \mathrm{mmol})$ & Me (2 equiv) & $\mathrm{H}$ & $\begin{array}{c}\text { DMTSF } \\
\text { (1.05 equiv) }\end{array}$ & $\mathrm{CH}_{2} \mathrm{Cl}_{2}(0.15 \mathrm{M})$ & $0 \%$ \\
\hline 5 & $\mathrm{Ph}(0.1 \mathrm{mmol})$ & Me (2 equiv) & $\mathrm{H}$ & $\begin{array}{l}\mathrm{Cl}_{2} \mathrm{PyF} \cdot \mathrm{BF}_{4} \\
(1.05 \text { equiv) }\end{array}$ & $\mathrm{CH}_{2} \mathrm{Cl}_{2}(0.15 \mathrm{M})$ & $0 \%$ \\
\hline 6 & $\mathrm{Ph}(0.1 \mathrm{mmol})$ & Me (2 equiv) & $\mathrm{H}$ & $\begin{array}{l}\mathrm{Me}_{3} \mathrm{PyF} \cdot \mathrm{BF}_{4} \\
\text { (1.05 equiv) }\end{array}$ & $\mathrm{CH}_{2} \mathrm{Cl}_{2}(0.15 \mathrm{M})$ & $0 \%$ \\
\hline 7 & $\mathrm{Ph}(0.1 \mathrm{mmol})$ & Me (2 equiv) & $\mathrm{H}$ & $\begin{array}{l}\mathrm{Cl}_{2} \mathrm{PyF} \cdot \mathrm{BF}_{4} \\
(1.2 \text { equiv) }\end{array}$ & $\mathrm{CH}_{2} \mathrm{Cl}_{2}: \mathrm{MeCN}(4: 1,0.1 \mathrm{M})$ & $0 \%$ \\
\hline 8 & $\mathrm{Ph}(0.1 \mathrm{mmol})$ & Me (2 equiv) & $\mathrm{Me}$ & $\begin{array}{l}\mathrm{Cl}_{2} \mathrm{PyF} \cdot \mathrm{BF}_{4} \\
(1.2 \text { equiv) }\end{array}$ & $\begin{array}{c}\mathrm{CH}_{2} \mathrm{Cl}_{2}: \mathrm{MeCN}(4: 1 \\
0.05 \mathrm{M})\end{array}$ & $0 \%$ \\
\hline 9 & $\mathrm{Ph}(0.1 \mathrm{mmol})$ & Ac (2 equiv) & $\mathrm{H}$ & $\begin{array}{l}\mathrm{Cl}_{2} \mathrm{PyF} \cdot \mathrm{BF}_{4} \\
(1.2 \text { equiv) }\end{array}$ & $\mathrm{CH}_{2} \mathrm{Cl}_{2}: \mathrm{MeCN}(4: 1,0.1 \mathrm{M})$ & $0 \%$ \\
\hline 10 & $\mathrm{Ph}(0.1 \mathrm{mmol})$ & Ts ( 2 equiv) & $\mathrm{Me}$ & $\begin{array}{l}\mathrm{Cl}_{2} \mathrm{PyF} \cdot \mathrm{BF}_{4} \\
(1.2 \text { equiv) }\end{array}$ & $\begin{array}{c}\mathrm{CH}_{2} \mathrm{Cl}_{2}: \mathrm{MeCN}(4: 1, \\
0.05 \mathrm{M})\end{array}$ & $53 \%$ \\
\hline 11 & Et $(0.1 \mathrm{mmol})$ & Ts ( 2 equiv) & $\mathrm{Me}$ & $\begin{array}{l}\mathrm{Cl}_{2} \mathrm{PyF} \cdot \mathrm{BF}_{4} \\
(1.2 \text { equiv) }\end{array}$ & $\begin{array}{c}\mathrm{CH}_{2} \mathrm{Cl}_{2}: \mathrm{MeCN}(4: 1, \\
0.05 \mathrm{M})\end{array}$ & $50 \%$ \\
\hline 12 & PMP (0.05 mmol) & Ts ( 2 equiv) & $\mathrm{Me}$ & $\begin{array}{l}\mathrm{Cu}(\mathrm{OTf})_{2} \\
(1 \text { equiv })\end{array}$ & $\begin{array}{c}\mathrm{CH}_{2} \mathrm{Cl}_{2}: \mathrm{MeCN}(4: 1, \\
0.05 \mathrm{M}) \\
\end{array}$ & $0 \%$ \\
\hline
\end{tabular}




\begin{tabular}{|c|c|c|c|c|c|c|}
\hline 13 & 2-Py (0.05 mmol) & Ts ( 2 equiv) & $\mathrm{Me}$ & $\begin{array}{c}\mathrm{AgBF}_{4} \\
\text { (2 equiv) }\end{array}$ & $\mathrm{CH}_{2} \mathrm{Cl}_{2}(0.3 \mathrm{M})$ & $0 \%$ \\
\hline 14 & 2-Py $(0.05$ mmol $)$ & Ts (2 equiv) & $\mathrm{Me}$ & $\begin{array}{l}\text { AgOTf } \\
\text { ( } 2 \text { equiv) }\end{array}$ & $\mathrm{CH}_{2} \mathrm{Cl}_{2}(0.3 \mathrm{M})$ & $0 \%$ \\
\hline 15 & 2-Py (0.05 mmol) & Ts ( 2 equiv) & $\mathrm{Me}$ & $\begin{array}{l}\mathrm{Cu}(\mathrm{OTf})_{2} \\
(2 \text { equiv })\end{array}$ & $\mathrm{CH}_{2} \mathrm{Cl}_{2}(0.3 \mathrm{M})$ & $0 \%$ \\
\hline 16 & PMP (0.05 mmol) & Ts ( 2 equiv) & $\mathrm{Me}$ & $\begin{array}{l}\mathrm{Cu}(\mathrm{OTf})_{2} \\
(1 \text { equiv })\end{array}$ & $\mathrm{CH}_{2} \mathrm{Cl}_{2}(0.2 \mathrm{M})$ & $0 \%$ \\
\hline 17 & $\mathrm{Ph}(0.05 \mathrm{mmol})$ & Ts ( 2 equiv) & $\mathrm{Me}$ & $\begin{array}{l}\mathrm{Cu}(\mathrm{OTf})_{2} \\
(1 \text { equiv })\end{array}$ & $\mathrm{CH}_{2} \mathrm{Cl}_{2}(0.2 \mathrm{M})$ & $0 \%$ \\
\hline 18 & 2-Py (0.05 mmol) & Ts (2 equiv) & $\mathrm{Me}$ & $\begin{array}{l}\text { AgOTf } \\
\text { (1 equiv) }\end{array}$ & $\mathrm{CH}_{2} \mathrm{Cl}_{2}(0.1 \mathrm{M})$ & $0 \%$ \\
\hline 19 & PMP (0.1 mmol) & Ts ( 2 equiv) & $\mathrm{Me}$ & $\begin{array}{c}\mathrm{Cl}_{2} \mathrm{PyF} \cdot \mathrm{BF}_{4} \\
(1.1 \text { equiv) }\end{array}$ & $\begin{array}{c}\mathrm{CH}_{2} \mathrm{Cl}_{2}: \mathrm{MeCN}(4: 1, \\
0.05 \mathrm{M})\end{array}$ & $46 \%$ \\
\hline 20 & 2-Py (0.05 mmol) & Ts (2 equiv) & $\mathrm{Me}$ & $\begin{array}{c}\mathrm{Cl}_{2} \mathrm{PyF} \cdot \mathrm{BF}_{4} \\
(1.1 \text { equiv) }\end{array}$ & $\mathrm{CH}_{2} \mathrm{Cl}_{2}(0.15 \mathrm{M})$ & $0 \%$ \\
\hline 21 & 2-Py $(0.05$ mmol $)$ & Ts (2 equiv) & $\mathrm{Me}$ & $\begin{array}{c}\mathrm{Cl}_{2} \mathrm{PyF} \cdot \mathrm{BF}_{4} \\
(1.1 \text { equiv) }\end{array}$ & $\begin{array}{c}\mathrm{CH}_{2} \mathrm{Cl}_{2}: \mathrm{MeCN}(4: 1, \\
0.05 \mathrm{M})\end{array}$ & $0 \%$ \\
\hline 22 & $\mathrm{Ph}(0.1 \mathrm{mmol})$ & Ts ( 2 equiv) & $\mathrm{Me}$ & $\begin{array}{c}\mathrm{Cl}_{2} \mathrm{PyF} \cdot \mathrm{BF}_{4} \\
(1.1 \text { equiv) }\end{array}$ & THF:MeCN $(4: 1,0.2 \mathrm{M})$ & $0 \%$ \\
\hline 23 & $\mathrm{Ph}(0.1 \mathrm{mmol})$ & Ts ( 2 equiv) & $\mathrm{Me}$ & $\begin{array}{c}\mathrm{Cl}_{2} \mathrm{PyF} \cdot \mathrm{BF}_{4} \\
(1.1 \text { equiv) }\end{array}$ & PhMe:MeCN $(4: 1,0.2 \mathrm{M})$ & $39 \%$ \\
\hline 24 & $\mathrm{Ph}(0.1 \mathrm{mmol})$ & Ts ( 2 equiv) & $\mathrm{Me}$ & $\begin{array}{l}\mathrm{Cl}_{2} \mathrm{PyF} \cdot \mathrm{BF}_{4} \\
(1.1 \text { equiv) }\end{array}$ & DME:MeCN $(4: 1,0.2 \mathrm{M})$ & $0 \%$ \\
\hline 25 & $\mathrm{Ph}(0.1 \mathrm{mmol})$ & Ts ( 2 equiv) & $\mathrm{Me}$ & $\begin{array}{l}\mathrm{Cl}_{2} \mathrm{PyF} \cdot \mathrm{BF}_{4} \\
(1.1 \text { equiv) }\end{array}$ & DMF:MeCN $(4: 1,0.2 \mathrm{M})$ & $0 \%$ \\
\hline 26 & Ph (0.53 mmol) & Cbz (3 equiv) & Me & $\mathrm{Cl}_{2} \mathrm{PyF} \cdot \mathrm{BF}_{4}$ & $\mathrm{CH}_{2} \mathrm{Cl}_{2}: \mathrm{MeCN}(4: 1,0.1 \mathrm{M})$ & $85 \%$ \\
\hline 27 & Et $(0.1 \mathrm{mmol})$ & $\mathrm{Cbz}$ (2 equiv) & $\mathrm{Me}$ & $\begin{array}{l}\mathrm{Cl}_{2} \mathrm{PyF} \cdot \mathrm{BF}_{4} \\
(1.2 \text { equiv) }\end{array}$ & $\begin{array}{c}\mathrm{CH}_{2} \mathrm{Cl}_{2}: \mathrm{MeCN}(4: 1 \\
0.05 \mathrm{M})\end{array}$ & $42 \%$ \\
\hline 28 & PMP (0.1 mmol) & $\mathrm{Cbz}$ (2 equiv) & $\mathrm{Me}$ & $\begin{array}{l}\mathrm{Cl}_{2} \mathrm{PyF} \cdot \mathrm{BF}_{4} \\
(1.1 \text { equiv) }\end{array}$ & $\begin{array}{c}\mathrm{CH}_{2} \mathrm{Cl}_{2}: \mathrm{MeCN}(4: 1 \\
0.05 \mathrm{M}) \\
\end{array}$ & $32 \%$ \\
\hline 29 & $\mathrm{Ph}(0.1 \mathrm{mmol})$ & $\mathrm{Cbz}$ (3 equiv) & $\mathrm{Me}$ & $\begin{array}{l}\text { XtalFluor-E } \\
\text { (1.1 equiv) }\end{array}$ & $\begin{array}{c}\mathrm{CH}_{2} \mathrm{Cl}_{2}: \mathrm{MeCN}(4: 1, \\
0.25 \mathrm{M}) \\
\end{array}$ & $0 \%$ \\
\hline 30 & 4-FPh $(0.1 \mathrm{mmol})$ & $\mathrm{Cbz}$ (3 equiv) & $\mathrm{Me}$ & $\begin{array}{c}\mathrm{Cl}_{2} \mathrm{PyF} \cdot \mathrm{BF}_{4} \\
(1.1 \text { equiv) }\end{array}$ & $\begin{array}{c}\mathrm{CH}_{2} \mathrm{Cl}_{2}: \mathrm{MeCN}(4: 1, \\
0.25 \mathrm{M})\end{array}$ & $0 \%$ \\
\hline 31 & $\mathrm{Ph}(0.2 \mathrm{mmol})$ & $\mathrm{Cbz}$ (3 equiv) & $\mathrm{Me}$ & $\begin{array}{c}\text { NFSI } \\
\text { (1.1 equiv) }\end{array}$ & $\begin{array}{c}\mathrm{CH}_{2} \mathrm{Cl}_{2}: \mathrm{MeCN}(4: 1 \\
0.25 \mathrm{M})\end{array}$ & $0 \%$ \\
\hline 32 & $\mathrm{Ph}(0.2 \mathrm{mmol})$ & $\mathrm{Cbz}$ (3 equiv) & $\mathrm{Me}$ & $\begin{array}{l}\text { Selectfluor } \\
\text { (1.1 equiv) }\end{array}$ & $\begin{array}{c}\mathrm{CH}_{2} \mathrm{Cl}_{2}: \mathrm{MeCN}(4: 1, \\
0.25 \mathrm{M})\end{array}$ & $0 \%$ \\
\hline
\end{tabular}


Table S2. Optimization of Reaction Stoichiometry and Concentration
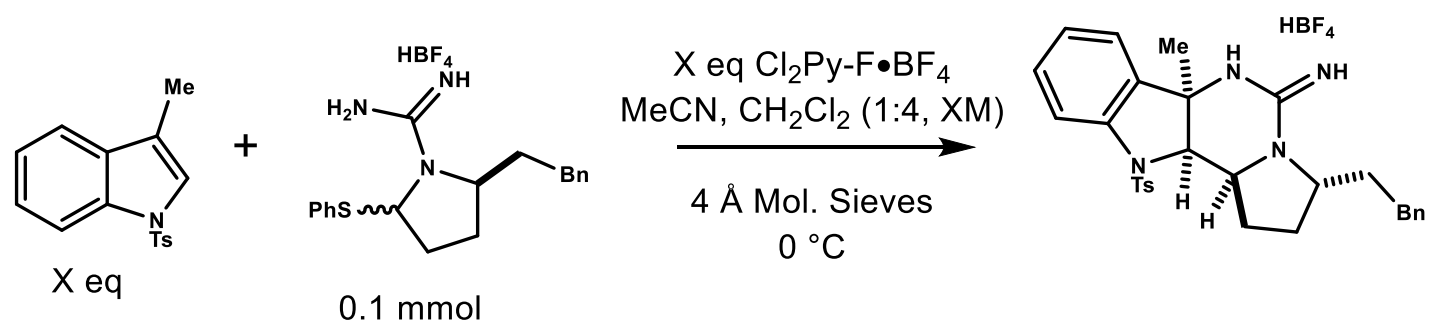

$0.1 \mathrm{mmol}$

\begin{tabular}{ccccc}
\hline Entry & Concentration & Indole Eq. & Promoter Eq & Yield \\
\hline 1 & 0.1 & 2 & 1.1 & $44 \%$ \\
\hline 2 & 0.25 & 2 & 1.1 & $51 \%$ \\
\hline 3 & 0.5 & 2 & 1.1 & $<20 \%$ \\
\hline 4 & 0.25 & 3 & 1.1 & $59 \%$ \\
\hline 5 & 0.25 & 1 & 1.1 & $31 \%$ \\
\hline 6 & 0.25 & 3 & 2 & $<20 \%$ \\
\hline 7 & 0.25 & 3 & 0.5 & $49 \%$ \\
\hline
\end{tabular}




\section{General Procedure for N-Amidinyliminium Ion Cyclizations}

A dram vial was charged with a stir bar and previously activated $4 \AA$ molecular sieves (100 mg). The system was placed under vacuum, flame-dried for several minutes, and then allowed to cool to room temperature. The system was then backfilled with argon and charged with the Namidinyliminium ion precursor salt (1 equiv) and the indole dienophile (3 equiv). A 4:1 $\mathrm{CH}_{2} \mathrm{Cl}_{2} / \mathrm{MeCN}(0.25 \mathrm{M})$ solution was added to the vial and the mixture was cooled to $0{ }^{\circ} \mathrm{C}$. $2,6-$ dichloro-1-fluoropyridinium tetrafluoroborate $(1.1 \mathrm{eq})$ was added in one portion. The mixture was stirred at $0{ }^{\circ} \mathrm{C}$ for 10 minutes and then allowed to warm to ambient temperature overnight. The mixture was then diluted with acetone $(10 \mathrm{ml})$, filtered, and the solids were washed with acetone $(10 \mathrm{~mL})$. The filtrate was concentrated in vacuo and the crude residue was purified by flash column chromatography $\left(\mathrm{SiO}_{2}, 1: 0 \rightarrow 9: 1 \mathrm{CH}_{2} \mathrm{Cl}_{2} / \mathrm{MeOH}\right)$. Product structure and relative configuration was assigned by ${ }^{1} \mathrm{H},{ }^{13} \mathrm{C}$ NMR, COSY, and HMQC NMR experiments and in some cases by X-ray crystallography. 


\section{E. Scope of Indole Dienophiles}

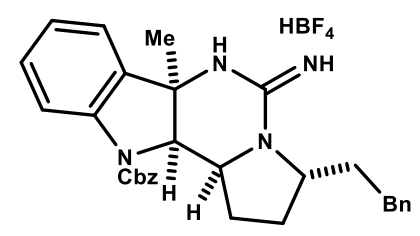

Adduct 12a: According to the representative procedure, $N$-amidinylpyrrolidine salt $10(221.2 \mathrm{mg}$, $0.535 \mathrm{mmol}$ ) and benzyl 3-methyl-1H-indole-1-carboxylate (422.7 $\mathrm{mg}, 1.59 \mathrm{mmol})$ was used as the dienophile. Purification by flash column chromatography $\left(\mathrm{SiO}_{2}, 1: 0 \rightarrow 95: 5 \mathrm{CH}_{2} \mathrm{Cl}_{2} / \mathrm{MeOH}\right)$ afforded adduct $\mathbf{1 2 a}(257.7 \mathrm{mg}, 85 \%)$ as a yellow solid.

${ }^{1} \mathbf{H}$ NMR $\left(600 \mathrm{MHz}, \mathrm{CDCl}_{3}\right) \delta 7.77(\mathrm{~s}, 1 \mathrm{H}), 7.53(\mathrm{~d}, J=7.5 \mathrm{~Hz}, 1 \mathrm{H}), 7.52-7.46(\mathrm{~m}, 1 \mathrm{H}), 7.42-$ $7.37(\mathrm{~m}, 5 \mathrm{H}), 7.25-7.18(\mathrm{~m}, 5 \mathrm{H}), 7.16(\mathrm{t}, J=7.4 \mathrm{~Hz}, 1 \mathrm{H}), 7.07(\mathrm{t}, J=7.5 \mathrm{~Hz}, 1 \mathrm{H}), 6.82-6.43(\mathrm{br}$ $\mathrm{s}, 2 \mathrm{H}), 5.32(\mathrm{~d}, J=12.1 \mathrm{~Hz}, 1 \mathrm{H}), 5.28(\mathrm{~d}, J=12.1 \mathrm{~Hz}, 1 \mathrm{H}), 4.82(\mathrm{~d}, J=5.3 \mathrm{~Hz}, 1 \mathrm{H}), 3.87-3.83$ $(\mathrm{m}, 1 \mathrm{H}), 3.63-3.59(\mathrm{~m}, 1 \mathrm{H}), 2.70-2.65(\mathrm{~m}, 1 \mathrm{H}), 2.56-2.53(\mathrm{~m}, 1 \mathrm{H}), 2.24-2.19(\mathrm{~m}, 1 \mathrm{H}), 2.16-2.14$ (m, 1H), 1.93-1.87 (m, 1H), $1.79(\mathrm{dd}, J=6.8,12.2 \mathrm{~Hz}, 1 \mathrm{H}), 1.69(\mathrm{~s}, 3 \mathrm{H}), 1.68-1.63(\mathrm{~m}, 2 \mathrm{H})$

${ }^{13} \mathrm{C}$ NMR $\left(150 \mathrm{MHz}, \mathrm{CDCl}_{3}\right) \delta 155.0,140.7,140.6,135.4,134.7,129.9,129.0,128.93,128.91$, $128.6,128.5,126.6,125.6,123.5,116.2,71.2,68.7,61.9,57.6,57.3,33.8,32.3,29.0,28.0,24.2$

${ }^{13} \mathrm{C}$ NMR $\left(150 \mathrm{MHz}, \mathrm{CD}_{2} \mathrm{Cl}_{2}\right) \delta 155.0,154.1,140.8,140.5,135.6,134.6,129.6,128.72,128.70$, $128.5,128.3,128.2,126.4,125.0,123.2,116.1,71.0,68.4,61.8,57.4,33.7,32.0,28.8,27.6,24.0$

IR (thin film) 3416, 2921, 1731, 1659, $1223 \mathrm{~cm}^{-1}$

Melting Point: $144-147^{\circ} \mathrm{C}$

HRMS (ESI-TOF) m/z: $[\mathrm{M}]^{+}$calcd for $\left[\mathrm{C}_{30} \mathrm{H}_{33} \mathrm{~N}_{4} \mathrm{O}_{2}\right]^{+} 481.2603$, found 481.2625

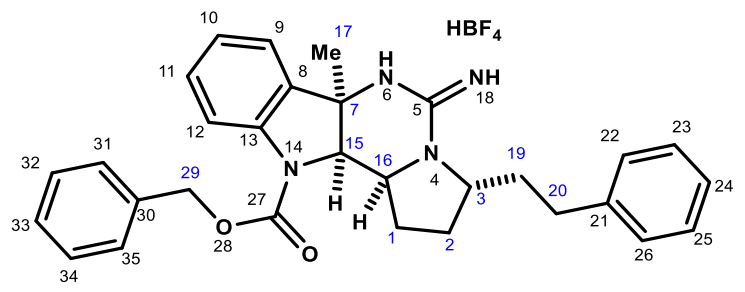

Assignment of Aliphatic Shifts (Solvent: $\mathrm{CDCl}_{3}$ )

\begin{tabular}{|c|c|c|c|c|c|}
\hline atom & ${ }^{13}$ C Shift & ${ }^{1} \mathrm{H}$ Shift & atom & ${ }^{13} \mathrm{C} \mathrm{Shift}$ & ${ }^{1} \mathrm{H}$ Shift \\
\hline 1 & 24.2 & $2.24-2.19(\mathrm{~m})$ & 17 & 28.0 & $1.69(\mathrm{~s})$ \\
\hline & & $2.16-2.14(\mathrm{~m})$ & 19 & 33.8 & $1.93-1.87(\mathrm{~m})$ \\
\hline 2 & 29.0 & $1.79(\mathrm{dd}, 6.8,12.2 \mathrm{~Hz})$ & & & $1.68-1.63(\mathrm{~m})$ \\
\hline & & $1.68-1.63(\mathrm{~m})$ & 20 & 32.3 & $2.70-2.65(\mathrm{~m})$ \\
\hline 3 & 57.6 & $3.63-3.59(\mathrm{~m})$ & & & $2.56-2.53(\mathrm{~m})$ \\
\hline 7 & 61.9 & NA & 29 & 68.7 & $5.32(\mathrm{~d}, 12.1 \mathrm{~Hz})$ \\
\hline 15 & 71.2 & $4.82(\mathrm{~d}, 5.3 \mathrm{~Hz})$ & & & $5.28(\mathrm{~d}, 12.1 \mathrm{~Hz})$ \\
\hline 16 & 57.3 & $3.87-3.83(\mathrm{~m})$ & \multicolumn{5}{|l}{}
\end{tabular}




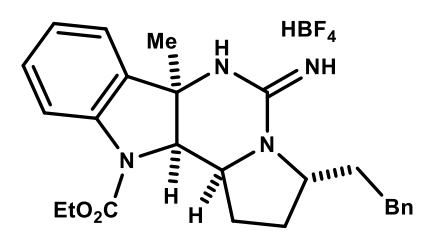

Adduct 12c: According to the representative procedure, $N$-amidinylpyrrolidine salt $10(55.1 \mathrm{mg}$, $0.133 \mathrm{mmol})$ and ethyl 3-methyl- $H$-indole-1-carboxylate $(81.2 \mathrm{mg}, 0.4 \mathrm{mmol})$ was used as the dienophile. Purification by flash column chromatography $\left(\mathrm{SiO}_{2}, 1: 0 \rightarrow 95: 5 \mathrm{CH}_{2} \mathrm{Cl}_{2} / \mathrm{MeOH}\right)$ afforded adduct $12 \mathrm{c}(45.9 \mathrm{mg}, 68 \%)$ as a yellow oil.

\section{Characterization of Major Product:}

${ }^{1} \mathbf{H}$ NMR (600 MHz, CD $\left.3 \mathrm{OD}\right) \delta 7.61(\mathrm{~d}, J=7.9 \mathrm{~Hz}, 1 \mathrm{H}), 7.34-7.20(\mathrm{~m}, 6 \mathrm{H}), 7.19-7.12(\mathrm{~m}, 2 \mathrm{H})$, $4.93(\mathrm{~d}, J=3.2 \mathrm{~Hz}, 1 \mathrm{H}), 4.35$ (q, $J=6.6 \mathrm{~Hz}, 2 \mathrm{H}), 4.07-4.00(\mathrm{~m}, 1 \mathrm{H}), 3.67-3.61(\mathrm{~m}, 1 \mathrm{H}), 2.69-$ $2.60(\mathrm{~m}, 1 \mathrm{H}), 2.59-2.50(\mathrm{~m}, 1 \mathrm{H}), 2.40-2.28(\mathrm{~m}, 2 \mathrm{H}), 2.04-1.94(\mathrm{~m}, 1 \mathrm{H}), 1.92-1.83(\mathrm{~m}, 1 \mathrm{H}), 1.80-$ $1.66(\mathrm{~m}, 2 \mathrm{H}), 1.71(\mathrm{~s}, 3 \mathrm{H}), 1.40(\mathrm{t}, J=6.7 \mathrm{~Hz}, 3 \mathrm{H})$

${ }^{13} \mathrm{C}$ NMR (150 MHz, CD $\left.{ }_{3} \mathrm{OD}\right) \delta 154.8,154.5,141.4,141.0,135.0,129.3,128.1,128.0,125.7$, $124.3,121.9,116.2,71.2,62.7,61.5,58.2,57.4,32.7,31.6,28.1,26.2,23.5,13.4$

IR (thin film) 3359, 3245, 2980, 2934, 1704, 1658, 1602, 1556, 1483, 1454, 1402, 1378, 1324, $1281,1248,1091,1019 \mathrm{~cm}^{-1}$

HRMS (ESI-TOF) m/z: $[\mathrm{M}]^{+}$calcd for $\left[\mathrm{C}_{25} \mathrm{H}_{31} \mathrm{~N}_{4} \mathrm{O}_{2}\right]^{+} 419.2447$, found 419.2429

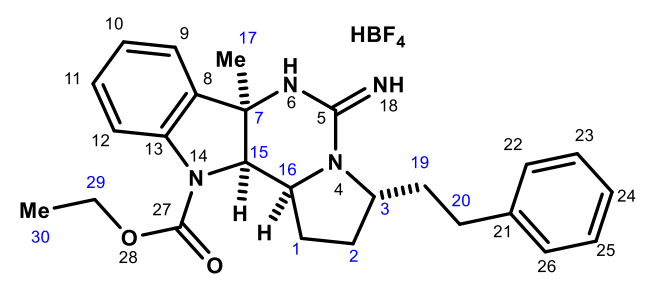

\section{Assignment of Aliphatic Shifts (Solvent: CD3OD)}

\begin{tabular}{|c|c|c|c|c|c|}
\hline atom & ${ }^{13} \mathrm{C}$ Shift & ${ }^{1} \mathrm{H}$ Shift & atom & ${ }^{13} \mathrm{C}$ Shift & ${ }^{1} \mathrm{H}$ Shift \\
\hline 1 & 23.5 & $2.40-2.28(\mathrm{~m})$ & 17 & 26.2 & $1.71(\mathrm{~s})$ \\
\hline 2 & 28.1 & $2.04-1.94(\mathrm{~m})$ & 19 & 32.7 & $1.92-1.83(\mathrm{~m})$ \\
\hline & & $1.80-1.66(\mathrm{~m})$ & & & $1.80-1.66(\mathrm{~m})$ \\
\hline 3 & 58.2 & $3.67-3.61(\mathrm{~m})$ & 20 & 31.6 & $2.69-2.60(\mathrm{~m})$ \\
\hline 7 & 61.5 & NA & & & $2.59-2.50(\mathrm{~m})$ \\
\hline 15 & 71.2 & $4.93(\mathrm{~d}, \mathrm{~J}=3.2 \mathrm{~Hz})$ & 29 & 62.7 & $4.35(\mathrm{q}, \mathrm{J}=6.6 \mathrm{~Hz})$ \\
\hline 16 & 57.4 & $4.07-4.00(\mathrm{~m})$ & 30 & 13.4 & $1.40(\mathrm{t}, \mathrm{J}=6.7 \mathrm{~Hz})$ \\
\hline
\end{tabular}




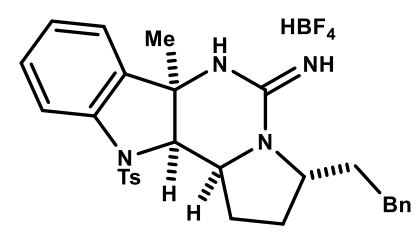

Adduct 12b: According to the representative procedure, $N$-amidinylpyrrolidine salt 10 (41.3 $\mathrm{mg}$, $0.1 \mathrm{mmol})$ and 3-methyl-1-tosyl- $1 \mathrm{H}$-indole $(85.6 \mathrm{mg}, 0.3 \mathrm{mmol})$ was used as the dienophile. Purification by flash column chromatography $\left(\mathrm{SiO}_{2}, 1: 0 \rightarrow 95: 5 \mathrm{CH}_{2} \mathrm{Cl}_{2} / \mathrm{MeOH}\right)$ afforded adduct 12b (35 mg, 59\%) as a yellow solid.

${ }^{1} \mathbf{H}$ NMR $\left(500 \mathrm{MHz}, \mathrm{CDCl}_{3}\right) \delta 7.80(\mathrm{~s}, \mathrm{br}, 1 \mathrm{H}), 7.67(\mathrm{~d}, J=8.3 \mathrm{~Hz}, 1 \mathrm{H}), 7.42(\mathrm{~d}, J=7.5 \mathrm{~Hz}, 1 \mathrm{H})$, 7.37 (d, $J=7.8 \mathrm{~Hz}, 2 \mathrm{H}), 7.31-7.11(\mathrm{~m}, 9 \mathrm{H}), 4.28$ (d, $J=4.5 \mathrm{~Hz}, 1 \mathrm{H}), 3.82-3.77(\mathrm{~m}, 1 \mathrm{H}), 3.61-$ $3.56(\mathrm{~m}, 1 \mathrm{H}), 3.00(\mathrm{dd}, J=7.5,13.0 \mathrm{~Hz}, 1 \mathrm{H}), 2.71-2.66(\mathrm{~m}, 1 \mathrm{H}), 2.59-2.53(\mathrm{~m}, 1 \mathrm{H}), 2.34(\mathrm{~s}, 3 \mathrm{H})$, 2.32-2.26 (m, 1H), 2.03-1.92 (m, 2H), 1.89-1.82 (m, 1H), 1.72-1.66 (m, 1H), $0.81(\mathrm{~s}, 3 \mathrm{H})$

${ }^{13}$ C NMR $\left(125 \mathrm{MHz}, \mathrm{CDCl}_{3}\right) \delta 155.3,145.5,140.9,140.6,136.5,132.7,130.3,130.0,128.9$, $128.6,127.8,127.5,126.6,124.1,117.9,73.9,63.1,57.7,57.5,33.7,32.4,29.0,27.2,24.0,21.8$

IR (thin film) 3172, 1665, 1359, $1088 \mathrm{~cm}^{-1}$

Melting Point: $185-187^{\circ} \mathrm{C}$

HRMS (ESI-TOF) m/z: $[\mathrm{M}]^{+}$calcd for $\left[\mathrm{C}_{29} \mathrm{H}_{34} \mathrm{~N}_{4} \mathrm{O}_{2} \mathrm{~S}\right]^{+} 501.2324$, found 502.2311

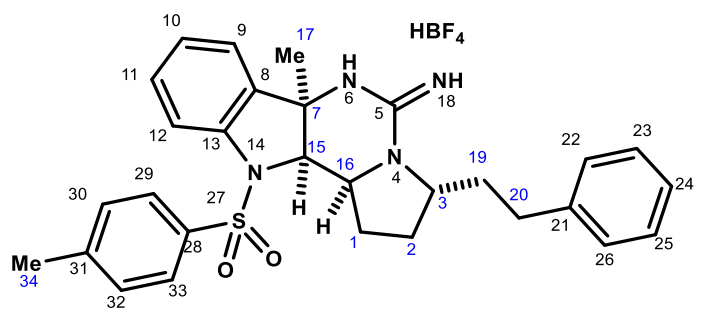

Assignment of Aliphatic Shifts (Solvent: $\mathrm{CDCl}_{3}$ )

\begin{tabular}{|c|c|c|}
\hline atom & ${ }^{13} \mathrm{C} \mathrm{Shift}$ & ${ }^{1} \mathrm{H}$ Shift \\
\hline 1 & 24.0 & $3.00(\mathrm{dd}, 7.5,13.0 \mathrm{~Hz}) ;$ \\
\hline & & $2.32(\mathrm{~m})$ \\
\hline 2 & 29.0 & $2.03-1.92(\mathrm{~m})$ \\
\hline 3 & 57.7 & $3.61-3.56(\mathrm{~m})$ \\
\hline 7 & 63.2 & NA \\
\hline 15 & 73.9 & $4.28(\mathrm{~d}, 4.5 \mathrm{~Hz})$ \\
\hline 16 & 57.7 & $3.82-3.77(\mathrm{~m})$ \\
\hline 17 & 27.2 & $0.81(\mathrm{~s})$ \\
\hline 19 & 33.7 & $1.89-1.82(\mathrm{~m})$ \\
\hline & & $1.72-1.66(\mathrm{~m})$ \\
\hline 20 & 32.4 & $2.71-2.66(\mathrm{~m})$ \\
\hline & & $2.59-2.53(\mathrm{~m})$ \\
\hline 34 & 21.8 & $2.34(\mathrm{~s})$ \\
\hline
\end{tabular}




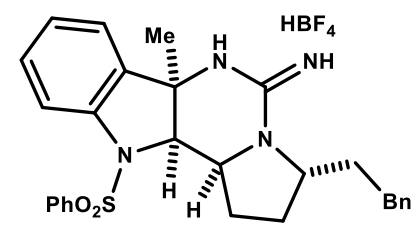

Adduct 12d: According to the representative procedure, $N$-amidinylpyrrolidine salt 10 (56.4 $\mathrm{mg}$, $0.136 \mathrm{mmol}$ ) and 3-methyl-1-(phenylsulfonyl)- $1 H$-indole $(111.5 \mathrm{mg}, 0.40 \mathrm{mmol})$ was used as the dienophile. Purification by flash column chromatography $\left(\mathrm{SiO}_{2}, 1: 0 \rightarrow 95: 5 \mathrm{CH}_{2} \mathrm{Cl}_{2} / \mathrm{MeOH}\right)$ afforded adduct $12 \mathrm{~d}(50.5 \mathrm{mg}, 64 \%)$ as a colorless foam.

${ }^{1} \mathbf{H}$ NMR $\left(600 \mathrm{MHz}, \mathrm{CDCl}_{3}\right) \delta 7.68(\mathrm{~d}, J=7.7 \mathrm{~Hz}, 1 \mathrm{H}), 7.54(\mathrm{t}, J=6.5 \mathrm{~Hz}, 1 \mathrm{H}), 7.48(\mathrm{~d}, J=7.1$ $\mathrm{Hz}, 2 \mathrm{H}), 7.36$ (t, $J=7.2 \mathrm{~Hz}, 2 \mathrm{H}), 7.36(\mathrm{~d}, J=7.0 \mathrm{~Hz}, 2 \mathrm{H}), 7.25-7.20(\mathrm{~m}, 2 \mathrm{H}), 7.20-7.12$ (m, $4 \mathrm{H}), 6.85(\mathrm{~s}, 1 \mathrm{H}), 6.19(\mathrm{bs}, 1 \mathrm{H}), 4.33-4.28(\mathrm{~m}, 1 \mathrm{H}), 3.84-3.77(\mathrm{~m}, 1 \mathrm{H}), 3.59-3.51(\mathrm{~m}, 1 \mathrm{H}), 3.00$ $(\mathrm{dd}, J=7.9,12.8 \mathrm{~Hz}, 1 \mathrm{H}), 2.70-2.62(\mathrm{~m}, 1 \mathrm{H}), 2.59-2.50(\mathrm{~m}, 1 \mathrm{H}), 2.36-2.21(\mathrm{~m}, 1 \mathrm{H}), 2.05-1.90$ (m, 2H), 1.88-1.73 (m, 3H), 1.71-1.58 (m, 1H), $0.76(\mathrm{~s}, 3 \mathrm{H})$

${ }^{13}$ C NMR $\left(150 \mathrm{MHz}, \mathrm{CDCl}_{3}\right) \delta 154.6,140.7,140.4,136.2,135.4,134.3,130.3,129.3,128.7$, $128.5,127.7,127.6,126.4,123.7,117.8,73.9,63.1,57.9,57.4,33.5,32.3,28.9,26.6,23.9$

IR (thin film) 3439, 3360, 3240, 3063, 3027, 2931, 2868, 1663, 1632, 1600, 1555, 1478, 1463, $1447,1358,1169,1086,1053,1027 \mathrm{~cm}^{-1}$

HRMS (ESI-TOF) m/z: $[\mathrm{M}]^{+}$calcd for $\left[\mathrm{C}_{28} \mathrm{H}_{31} \mathrm{~N}_{4} \mathrm{O}_{2} \mathrm{~S}\right]^{+} 487.2168$, found 487.2174

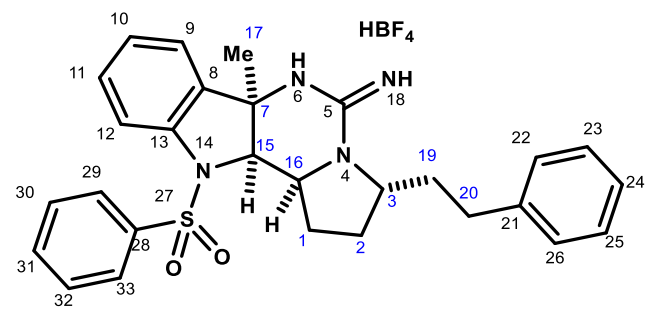

\section{Assignment of Aliphatic Shifts (Solvent: $\mathrm{CDCl}_{3}$ )}

\begin{tabular}{|c|c|c|}
\hline atom & ${ }^{13}$ C Shift & ${ }^{1} \mathrm{H}$ Shift \\
\hline 1 & 23.9 & $3.00(\mathrm{dd}, \mathrm{J}=7.9,12.8 \mathrm{~Hz})$ \\
\hline & & $2.36-2.21(\mathrm{~m})$ \\
\hline 2 & 28.9 & $2.05-1.90(\mathrm{~m})$ \\
\hline 3 & 57.9 & $3.59-3.51(\mathrm{~m})$ \\
\hline 7 & 63.1 & NA \\
\hline 15 & 73.9 & $4.33-4.28(\mathrm{~m})$ \\
\hline 16 & 57.4 & $3.84-3.77(\mathrm{~m}$ \\
\hline 17 & 26.6 & $0.76(\mathrm{~s})$ \\
\hline 19 & 33.5 & $1.88-1.73(\mathrm{~m})$ \\
\hline & & $1.71-1.58(\mathrm{~m})$ \\
\hline 20 & 32.3 & $2.70-2.62(\mathrm{~m})$ \\
\hline & & $2.59-2.50(\mathrm{~m})$ \\
\hline
\end{tabular}




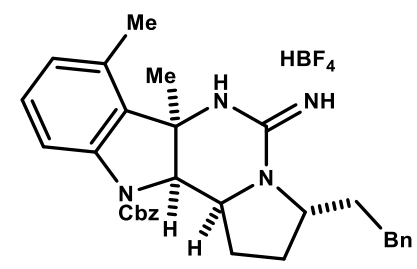

Adduct 12e: According to the representative procedure, $N$-amidinylpyrrolidine salt $10(55 \mathrm{mg}$, $0.132 \mathrm{mmol}$ ) and indole $\mathbf{S 2 1}(111 \mathrm{mg}, 0.396 \mathrm{mmol})$ was used as the dienophile. Purification by flash column chromatography $\left(\mathrm{SiO}_{2}, 1: 0 \rightarrow 97: 3 \mathrm{CH}_{2} \mathrm{Cl}_{2} / \mathrm{MeOH}\right)$ afforded adduct $12 \mathrm{e}(38 \mathrm{mg}, 49 \%)$ as a yellow oil.

${ }^{1}$ H NMR $\left(500 \mathrm{MHz}, \mathrm{CD}_{3} \mathrm{OD}\right) \delta 7.49(\mathrm{~d}, J=8.1 \mathrm{~Hz}, 1 \mathrm{H}), 7.46-7.42(\mathrm{~m}, 2 \mathrm{H}), 7.41-7.31(\mathrm{~m}, 3 \mathrm{H})$, 7.26-7.18 (m, 4H), 7.18-7.11 (m, 2H), $6.86(\mathrm{~d}, \mathrm{~J}=8.0 \mathrm{~Hz}, 1 \mathrm{H}), 5.32(\mathrm{~d}, J=12.1 \mathrm{~Hz}, 1 \mathrm{H}), 5.28$ $(\mathrm{d}, J=12.1 \mathrm{~Hz}, 1 \mathrm{H}), 4.84(\mathrm{~d}, J=6.1 \mathrm{~Hz}, 1 \mathrm{H}), 3.98-3.93(\mathrm{~m}, 1 \mathrm{H}), 3.64-3.56(\mathrm{~m}, 1 \mathrm{H}), 2.61$ (ddd, $J=5.4,11.6,13.2 \mathrm{~Hz}, 1 \mathrm{H}), 2.56-2.47(\mathrm{~m}, 1 \mathrm{H}), 2.43(\mathrm{~s}, 3 \mathrm{H}), 2.17-2.07(\mathrm{~m}, 1 \mathrm{H}), 2.03-1.94(\mathrm{~m}$, $1 \mathrm{H}), 1.93-1.80(\mathrm{~m}, 2 \mathrm{H}), 1.72(\mathrm{~s}, 3 \mathrm{H}), 1.71-1.59(\mathrm{~m}, 2 \mathrm{H})$

${ }^{13}$ C NMR (125 MHz, CD $\left.{ }_{3} \mathrm{OD}\right) \delta 155.4,155.3,143.0,142.5,137.4,135.2,133.0,130.8,129.9$, $129.8,129.7,129.6,129.5,128.6,127.2,115.3,72.9,69.4,63.2,59.6,58.5,34.2,33.0,29.5$, $25.9,25.3,18.2$

IR (thin film) 3404, 2517, 1644, $1453 \mathrm{~cm}^{-1}$

HRMS (ESI-TOF) $\mathrm{m} / \mathrm{z}$ : $[\mathrm{M}]^{+}$calcd for $\left[\mathrm{C}_{31} \mathrm{H}_{35} \mathrm{~N}_{4} \mathrm{O}_{2}\right]^{+}$495.2760, found 495.2776

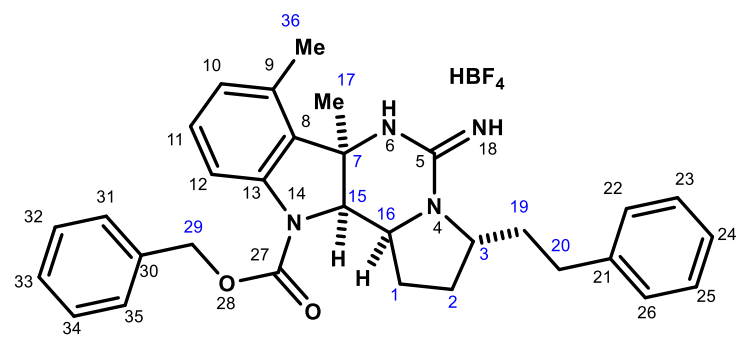

Assignment of Aliphatic Shifts (Solvent: CD,OD)

\begin{tabular}{|c|c|c|c|c|c|}
\hline atom & ${ }^{13} \mathrm{C}$ Shift & ${ }^{1} \mathrm{H}$ Shift & atom & ${ }^{13} \mathrm{C}$ Shift & ${ }^{1} \mathrm{H}$ Shift \\
\hline 1 & 25.3 & $2.17-2.07(\mathrm{~m})$ & 17 & 25.9 & $1.72(\mathrm{~s})$ \\
\hline & & $2.03-1.94(\mathrm{~m})$ & 19 & 34.2 & $1.93-1.80(\mathrm{~m})$ \\
\hline 2 & 29.5 & $1.93-1.80(\mathrm{~m})$ & & & $1.71-1.59(\mathrm{~m})$ \\
\hline & & $1.71-1.59(\mathrm{~m})$ & 20 & 33.0 & $2.61(\mathrm{ddd}, 5.4,11.6,13.2 \mathrm{~Hz})$ \\
\hline 3 & 59.6 & $3.64-3.56(\mathrm{~m})$ & & & $2.56-2.47(\mathrm{~m})$ \\
\hline 7 & 63.2 & NA & 29 & 69.4 & $5.32(\mathrm{~d}, 12.1 \mathrm{~Hz})$ \\
\hline 15 & 72.9 & $4.84(\mathrm{~d}, 6.1 \mathrm{~Hz})$ & & & $5.28(\mathrm{~d}, 12.1 \mathrm{~Hz})$ \\
\hline 16 & 58.5 & $3.98-3.93(\mathrm{~m})$ & 36 & 18.2 & $2.43(\mathrm{~s})$ \\
\hline
\end{tabular}




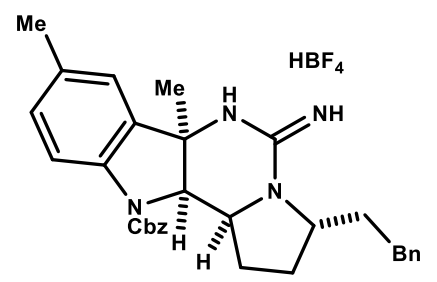

Adduct 12f: According to the representative procedure, $N$-amidinylpyrrolidine salt 10 (55 $\mathrm{mg}$, $0.132 \mathrm{mmol}$ ) and indole $\mathbf{S 2 2}$ (111 $\mathrm{mg}, 0.396 \mathrm{mmol}$ ) was used as the dienophile. Purification by flash column chromatography $\left(\mathrm{SiO}_{2}, 1: 0 \rightarrow 97: 3 \mathrm{CH}_{2} \mathrm{Cl}_{2} / \mathrm{MeOH}\right)$ afforded adduct $\mathbf{1 2 f}(47 \mathrm{mg}, 61 \%)$ as a colorless solid.

${ }^{1} \mathbf{H}$ NMR (500 MHz, CD 3 OD) $\delta$ 7.48-7.42 (m, 3H), 7.41-7.33 (m, 3H), 7.27-7.18 (m, 4H), 7.17$7.12(\mathrm{~m}, 1 \mathrm{H}), 7.11-7.06(\mathrm{~m}, 2 \mathrm{H}), 5.31(\mathrm{~s}, 2 \mathrm{H}), 4.91(\mathrm{~d}, \mathrm{~J}=5.0 \mathrm{~Hz}, 1 \mathrm{H}), 4.00-3.93(\mathrm{~m}, 1 \mathrm{H}), 3.63-$ $3.56(\mathrm{~m}, 1 \mathrm{H}), 2.60(\mathrm{ddd}, J=5.2,11.6,13.5 \mathrm{~Hz}, 1 \mathrm{H}), 2.55-2.46(\mathrm{~m}, 1 \mathrm{H}), 2.31(\mathrm{~s}, 3 \mathrm{H}), 2.28-2.14$ $(\mathrm{m}, 2 \mathrm{H}), 1.90-1.78(\mathrm{~m}, 2 \mathrm{H}), 1.74-1.60(\mathrm{~m}, 2 \mathrm{H}), 1.66(\mathrm{~s}, 3 \mathrm{H})$

${ }^{13}$ C NMR (125 MHz, CD 3 OD) $\delta$ 156.3, 155.9, 142.5, 140.4, 137.3, 136.5, 136.0, 131.4, 129.9, $129.8,129.7,129.6,129.5,127.2,123.8,117.5,73.0,69.5,63.0,59.7,58.8,34.2,33.1,29.5,27.7$, $24.9,21.1$

IR (thin film) 3413, 1703, 1660, 1265, $1091 \mathrm{~cm}^{-1}$

Melting Point: $132-134{ }^{\circ} \mathrm{C}$

HRMS (ESI-TOF) m/z: [M] $]^{+}$calcd for $\left[\mathrm{C}_{31} \mathrm{H}_{35} \mathrm{~N}_{4} \mathrm{O}_{2}\right]^{+} 495.2760$, found 495.2753

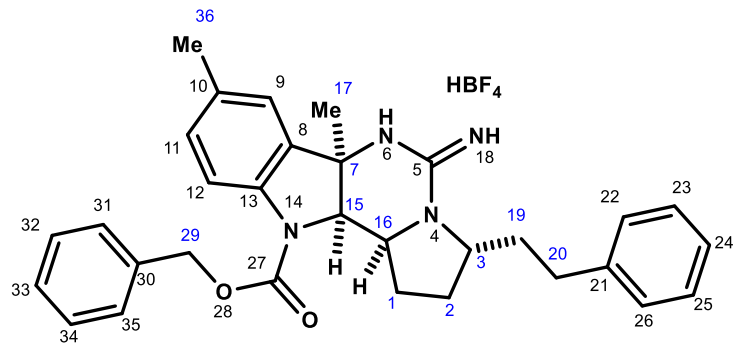

Assignment of Aliphatic Shifts (Solvent: CD $\left.{ }_{3} \mathrm{OD}\right)$

\begin{tabular}{|c|c|c|c|c|c|}
\hline atom & ${ }^{13} \mathrm{C}$ Shift & ${ }^{1} \mathrm{H}$ Shift & atom & ${ }^{13} \mathrm{C}$ Shift & ${ }^{1} \mathrm{H}$ Shift \\
\hline 1 & 24.9 & $2.28-2.14(\mathrm{~m})$ & 17 & 27.7 & $1.66(\mathrm{~s})$ \\
\hline 2 & 29.5 & $1.90-1.78(\mathrm{~m})$ & 19 & 34.2 & $1.90-1.78(\mathrm{~m})$ \\
\hline & & $1.74-1.60(\mathrm{~m})$ & & & $1.74-1.60(\mathrm{~m})$ \\
\hline 3 & 59.7 & $3.63-3.56(\mathrm{~m})$ & 20 & 33.1 & $2.60(\mathrm{ddd}, 5.2,11.6,13.5 \mathrm{~Hz})$ \\
\hline 7 & 63.0 & NA & & & $2.55-2.46(\mathrm{~m})$ \\
\hline 15 & 73.0 & $4.91(\mathrm{~d}, 5.0 \mathrm{~Hz})$ & 29 & 69.5 & $5.31(\mathrm{~s})$ \\
\hline 16 & 58.8 & $4.00-3.93(\mathrm{~m})$ & 36 & 21.1 & $2.31(\mathrm{~s})$ \\
\hline
\end{tabular}




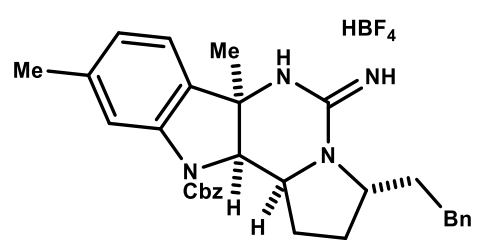

Adduct 12g: According to the representative procedure, $N$-amidinylpyrrolidine salt 10 (55.8 $\mathrm{mg}$, $0.135 \mathrm{mmol}$ ) and indole $\mathbf{S 2 3}$ (117.1 $\mathrm{mg}, 0.42 \mathrm{mmol}, 3$ equiv) were used. Purification of the crude residue by flash column chromatography $\left(\mathrm{SiO}_{2}, 1: 0 \rightarrow 9: 1 \mathrm{CH}_{2} \mathrm{Cl}_{2} / \mathrm{MeOH}\right)$ afforded adduct $\mathbf{1 2 g}$ $(64.1 \mathrm{mg}, 81 \%)$ as a brown foam.

${ }^{1} \mathbf{H}$ NMR (600 MHz, CD 3 OD) $\delta$ 7.50-7.46 (m, 2H), 7.45-7.36 (m, 4H), 7.29-7.24 (m, 2H), 7.23$7.20(\mathrm{~m}, 2 \mathrm{H}), 7.19-7.15(\mathrm{~m}, 2 \mathrm{H}), 6.96(\mathrm{~d}, J=7.7 \mathrm{~Hz}, 1 \mathrm{H}), 5.35(\mathrm{~d}, J=12.1 \mathrm{~Hz}, 1 \mathrm{H}), 5.32(\mathrm{~d}, J=$ $12.1 \mathrm{~Hz}, 1 \mathrm{H}), 4.92(\mathrm{~d}, J=5.0 \mathrm{~Hz}, 1 \mathrm{H}), 4.03-3.97(\mathrm{~m}, 1 \mathrm{H}), 3.66-3.60(\mathrm{~m}, 1 \mathrm{H}), 2.65-2.59(\mathrm{~m}, 1 \mathrm{H})$, 2.57-2.50 (m, 1H), 2.32 (s, 3H), 2.30-2.20 (m, 2H), 1.94-1.82 (m, 2H), 1.78-1.63 (m, 2H), 1.68 $(\mathrm{s}, 3 \mathrm{H})$

${ }^{13} \mathrm{C}$ NMR (150 MHz, CD 3 OD) $\delta 154.9,154.3,141.4,141.0,139.8,135.8,132.2,128.4,128.3$, $128.2,128.1,127.9,125.7,125.1,121.7,116.8,71.6,68.1,61.3,58.2,57.3,32.6,31.6,28.0,26.2$, $23.4,20.3$

IR (thin film) 3350, 3030, 2919, 2851, 2489, 1705, 1652, 1632, 1598, 1557, 1497, 1453, 1394, $1324,1300,1251,1220,1159,1117,1090,1027,974 \mathrm{~cm}^{-1}$

HRMS (ESI-TOF) m/z: $[\mathrm{M}]^{+}$calcd for $\left[\mathrm{C}_{31} \mathrm{H}_{35} \mathrm{~N}_{4} \mathrm{O}_{2}\right]^{+} 495.2760$, found 495.2753

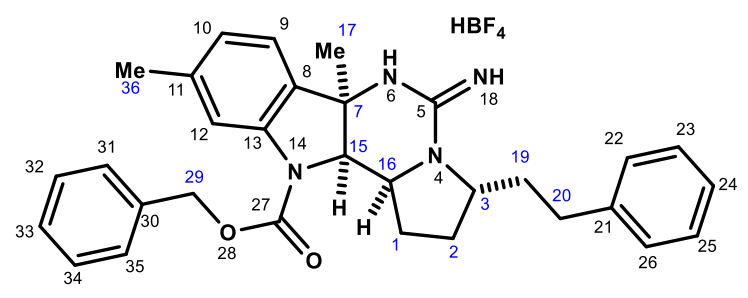

Assignment of Aliphatic Shifts (Solvent: CD3OD)

\begin{tabular}{|c|c|c|c|c|c|}
\hline atom & ${ }^{13} \mathrm{C}$ Shift & ${ }^{1} \mathrm{H}$ Shift & atom & ${ }^{13} \mathrm{C}$ Shift & ${ }^{1} \mathrm{H}$ Shift \\
\hline 1 & 23.4 & $2.30-2.20(\mathrm{~m})$ & 19 & 32.6 & $1.94-1.82(\mathrm{~m})$ \\
\hline 2 & 28.0 & $1.94-1.82(\mathrm{~m})$ & & & $1.78-1.63(\mathrm{~m})$ \\
\hline & & $1.78-1.63(\mathrm{~m})$ & 20 & 31.6 & $2.65-2.59(\mathrm{~m})$ \\
\hline 3 & 58.2 & $3.66-3.60(\mathrm{~m})$ & & & $2.57-2.50(\mathrm{~m}$ \\
\hline 7 & 61.3 & NA & 29 & 68.1 & $5.35(\mathrm{~d}, \mathrm{~J}=12.1 \mathrm{~Hz})$ \\
\hline 15 & 71.6 & $4.92(\mathrm{~d}, 5.0 \mathrm{~Hz})$ & & & $5.32(\mathrm{~d}, \mathrm{~J}=12.1 \mathrm{~Hz}$ \\
\hline 16 & 57.3 & $4.03-3.97(\mathrm{~m})$ & 36 & 20.3 & $2.32(\mathrm{~s})$ \\
\hline 17 & 26.2 & $1.68(\mathrm{~s})$ & \multicolumn{5}{|r}{} \\
\cline { 1 - 6 }
\end{tabular}




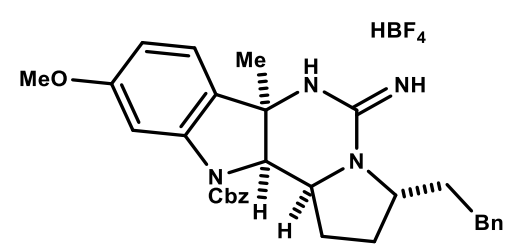

Adduct 12h: According to the representative procedure, $N$-amidinylpyrrolidine salt $10(55 \mathrm{mg}$, $0.132 \mathrm{mmol}$ ) and indole $\mathbf{S 2 4}(117 \mathrm{mg}, 0.396 \mathrm{mmol})$ was used as the dienophile. Purification by flash column chromatography $\left(\mathrm{SiO}_{2}, 1: 0 \rightarrow 95: 5 \mathrm{CH}_{2} \mathrm{Cl}_{2} / \mathrm{MeOH}\right)$ afforded adduct $\mathbf{1 2 h}(45 \mathrm{mg}$, $57 \%)$ as a green solid.

${ }^{1} \mathbf{H}$ NMR $\left(500 \mathrm{MHz}, \mathrm{CD}_{3} \mathrm{OD}\right) \delta 7.50(\mathrm{~d}, J=7.0 \mathrm{~Hz}, 2 \mathrm{H}), 7.46-7.41(\mathrm{~m}, 3 \mathrm{H}), 7.28(\mathrm{t}, J=7.5 \mathrm{~Hz}$, 2H), 7.24-7.17 (m, 5H), 6.69 (dd, J =2.3, 8.4 Hz, 1H), $5.35(\mathrm{~s}, 2 \mathrm{H}), 4.95(\mathrm{~d}, J=5.0 \mathrm{~Hz}, 1 \mathrm{H}), 4.03-$ $3.95(\mathrm{~m}, 1 \mathrm{H}), 3.73(\mathrm{~s}, 3 \mathrm{H}), 3.70-3.64(\mathrm{~m}, 1 \mathrm{H}), 2.65$ (ddd, $J=5.4,11.4,13.2 \mathrm{~Hz}, 1 \mathrm{H}), 2.56$ (ddd, $J=5.1,11.6,13.2 \mathrm{~Hz}, 1 \mathrm{H}), 2.36-2.29(\mathrm{~m}, 1 \mathrm{H}), 2.28-2.19(\mathrm{~m}, 1 \mathrm{H}) 1.96-1.86(\mathrm{~m}, 2 \mathrm{H}), 1.78-1.72$ $(\mathrm{m}, 2 \mathrm{H}), 1.70(\mathrm{~s}, 3 \mathrm{H})$

${ }^{13}$ C NMR (125 MHz, CD3OD) $\delta 162.5,156.3,155.7,143.9,142.3,136.9,129.84,129.82,129.80$, 129.6, 129.3, 128.3, 127.2, 124.0, 111.8, 103.5, 73.3, 69.7, 62.6, 59.5, 58.8, 56.1, 34.1, 33.0, 29.6, $27.9,24.9$

IR (thin film) 3415, 1708, 1661, 1265, $1093 \mathrm{~cm}^{-1}$

Melting Point: $124-126^{\circ} \mathrm{C}$

HRMS (ESI-TOF) m/z: $[\mathrm{M}]^{+}$calcd for $\left[\mathrm{C}_{31} \mathrm{H}_{35} \mathrm{~N}_{4} \mathrm{O}_{3}\right]^{+}$511.2709, found 511.2705

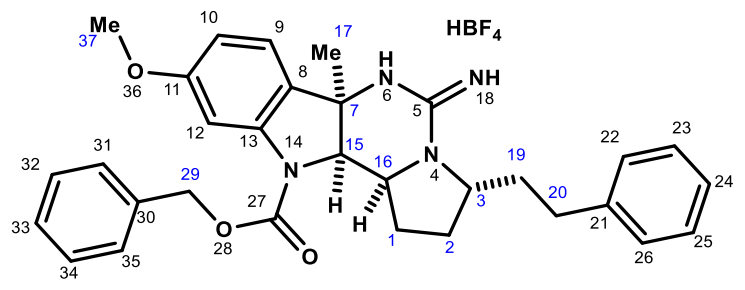

Assignment of Aliphatic Shifts (Solvent: CD3OD)

\begin{tabular}{|c|c|c|c|c|c|}
\hline atom & ${ }^{13} \mathrm{C}$ Shift & ${ }^{1} \mathrm{H}$ Shift & atom & ${ }^{13} \mathrm{C}$ Shift & ${ }^{1} \mathrm{H}$ Shift \\
\hline 1 & 24.9 & $2.36-2.29(\mathrm{~m})$ & 17 & 27.9 & $1.70(\mathrm{~s})$ \\
\hline & & $2.28-2.19(\mathrm{~m})$ & 19 & 34.1 & $1.96-1.86(\mathrm{~m})$ \\
\hline 2 & 29.6 & $1.96-1.86(\mathrm{~m})$ & & & $1.78-1.72(\mathrm{~m})$ \\
\hline & & $1.78-1.72(\mathrm{~m})$ & 20 & 33.0 & $2.65(\mathrm{ddd}, 5.4,11.4,13.2 \mathrm{~Hz})$ \\
\hline 3 & 59.5 & $3.70-3.64(\mathrm{~m})$ & & & $2.56(\mathrm{ddd}, 5.1,11.6,13.2 \mathrm{~Hz})$ \\
\hline 7 & 62.6 & NA & 29 & 69.7 & $5.35(\mathrm{~s})$ \\
\hline 15 & 73.3 & $4.95(\mathrm{~d}, 5.0 \mathrm{~Hz})$ & 37 & 56.1 & $3.73(\mathrm{~s})$ \\
\hline 16 & 58.8 & $4.03-3.95(\mathrm{~m})$ & \multicolumn{3}{|c}{} \\
\cline { 1 - 3 }
\end{tabular}




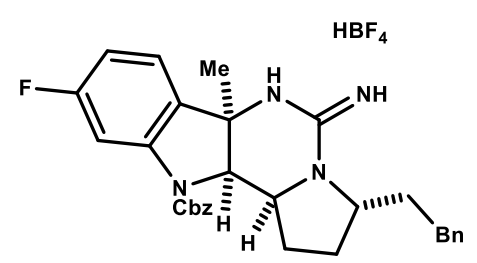

Adduct 12i: According to the representative procedure, $N$-amidinylpyrrolidine salt 10 (55 $\mathrm{mg}$, $0.132 \mathrm{mmol}$ ) and indole $\mathbf{S 2 5}(112 \mathrm{mg}, 0.396 \mathrm{mmol})$ was used as the dienophile. Purification by flash column chromatography $\left(\mathrm{SiO}_{2}, 1: 0 \rightarrow 97: 3 \mathrm{CH}_{2} \mathrm{Cl}_{2} / \mathrm{MeOH}\right)$ afforded adduct $12 \mathbf{i}(66 \mathrm{mg}, 86 \%)$ as a colorless solid.

${ }^{1}$ H NMR (500 MHz, CD 3 OD) $\delta$ 7.43-7.39 (m, 2H), 7.37-7.26 (m, 4H), 7.23-7.17 (m, 3H), 7.16$7.12(\mathrm{~m}, 2 \mathrm{H}), 7.09$ (t, $J=7.2 \mathrm{~Hz}, 1 \mathrm{H}), 6.79$ (dt, $J=2.4,8.9 \mathrm{~Hz}, 1 \mathrm{H}), 5.29(\mathrm{~d}, J=12.0 \mathrm{~Hz}, 1 \mathrm{H})$, $5.25(\mathrm{~d}, J=12.0 \mathrm{~Hz}, 1 \mathrm{H}), 4.89(\mathrm{~d}, J=5.0 \mathrm{~Hz}, 1 \mathrm{H}), 3.95-3.90(\mathrm{~m}, 1 \mathrm{H}), 3.61-3.55(\mathrm{~m}, 1 \mathrm{H}), 2.55$ (ddd, $J=5.1,11.7,13.0 \mathrm{~Hz}, 1 \mathrm{H}), 2.49-2.41(\mathrm{~m}, 1 \mathrm{H}), 2.23-2.09(\mathrm{~m}, 2 \mathrm{H}), 1.87-1.73(\mathrm{~m}, 2 \mathrm{H}), 1.67-$ $1.55(\mathrm{~m}, 2 \mathrm{H}), 1.61(\mathrm{~s}, 3 \mathrm{H})$

${ }^{13}$ C NMR $\left(125 \mathrm{MHz}, \mathrm{CD}_{3} \mathrm{OD}\right) \delta 164.00(\mathrm{~d}, J=245 \mathrm{~Hz}), 156.3,155.4,144.40(\mathrm{~d}, J=12 \mathrm{~Hz})$, 142.5, 137.1, 132.3 (d, $J=2 \mathrm{~Hz}), 129.9,129.6,129.5,127.2,124.9$ (d, $J=10 \mathrm{~Hz}), 112.4$ (d, $J=$ $24 \mathrm{~Hz}), 105.4$ (d, $J=29 \mathrm{~Hz}), 73.5,69.8,62.6,59.7,58.8,34.1,33.1,29.6,27.7,24.9$

IR (thin film) 3414, 1660, 1265, $1072 \mathrm{~cm}^{-1}$

Melting Point: $126-127^{\circ} \mathrm{C}$

HRMS (ESI-TOF) m/z: $[\mathrm{M}]^{+}$calcd for $\left[\mathrm{C}_{30} \mathrm{H}_{32} \mathrm{~N}_{4} \mathrm{O}_{2} \mathrm{~F}\right]^{+} 499.2509$, found 499.2502

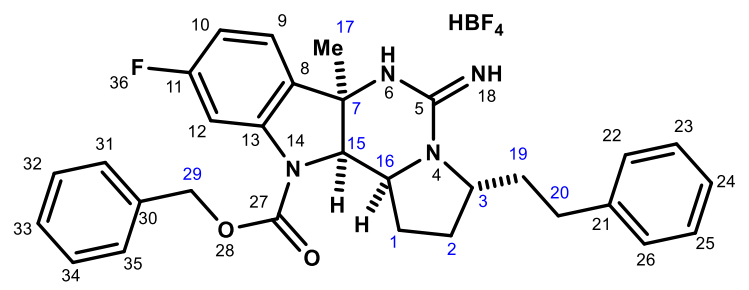

\begin{tabular}{|c|c|c|c|c|c|}
\hline atom & ${ }^{13} \mathrm{C}$ Shift & ${ }^{1} \mathrm{H}$ Shift & atom & ${ }^{13} \mathrm{C}$ Shift & ${ }^{1} \mathrm{H}$ Shift \\
\hline 1 & 24.9 & $2.23-2.09(\mathrm{~m})$ & 17 & 27.7 & $1.61(\mathrm{~s})$ \\
\hline 2 & 29.6 & $1.87-1.73(\mathrm{~m})$ & 19 & 34.1 & $1.87-1.73(\mathrm{~m})$ \\
\hline & & $1.67-1.55(\mathrm{~m})$ & & & $1.67-1.55(\mathrm{~m})$ \\
\hline 3 & 59.7 & $3.61-3.55(\mathrm{~m})$ & 20 & 33.1 & $2.55(\mathrm{ddd}, 5.1,11.7,13.0 \mathrm{~Hz})$ \\
\hline 7 & 62.6 & NA & & & $2.49-2.41(\mathrm{~m})$ \\
\hline 15 & 73.5 & $4.89(\mathrm{~d}, 5.0 \mathrm{~Hz})$ & 29 & 69.8 & $5.29(\mathrm{~d}, 12.0 \mathrm{~Hz})$ \\
\hline 16 & 58.8 & $3.95-3.90(\mathrm{~m})$ & & & $5.25(\mathrm{~d}, 12.0 \mathrm{~Hz})$ \\
\hline
\end{tabular}




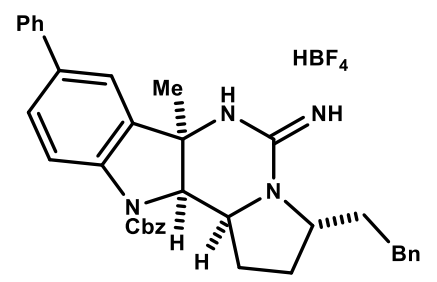

Adduct 12j: According to the representative procedure, $N$-amidinylpyrrolidine salt 10 ( $83 \mathrm{mg}, 0.2$ $\mathrm{mmol}$ ) and indole S26 (204 $\mathrm{mg}, 0.6 \mathrm{mmol}$ ) was used as the dienophile. Purification by flash column chromatography $\left(\mathrm{SiO}_{2}, 1: 0 \rightarrow 95: 5 \mathrm{CH}_{2} \mathrm{Cl}_{2} / \mathrm{MeOH}\right)$ afforded adduct $\mathbf{1 2} \mathbf{j}(84 \mathrm{mg}, 65 \%)$ as a yellow solid. X-Ray quality crystals were obtained by slow evaporation from $\mathrm{CH}_{2} \mathrm{Cl}_{2}$ and $\mathrm{MeOH}$.

${ }^{1}$ H NMR $\left(500 \mathrm{MHz}, \mathrm{CD}_{3} \mathrm{OD}\right) \delta 7.66(\mathrm{~d}, J=8.5 \mathrm{~Hz}, 1 \mathrm{H}), 7.61-7.57(\mathrm{~m}, 2 \mathrm{H}), 7.55(\mathrm{dd}, J=1.9,8.5$ $\mathrm{Hz}, 1 \mathrm{H}), 7.53(\mathrm{~d}, J=1.9 \mathrm{~Hz}, 1 \mathrm{H}), 7.49-7.45(\mathrm{~m}, 2 \mathrm{H}), 7.44-7.34(\mathrm{~m}, 5 \mathrm{H}), 7.33-7.29(\mathrm{~m}, 1 \mathrm{H}), 7.26-$ $7.21(\mathrm{~m}, 2 \mathrm{H}), 7.20-7.17(\mathrm{~m}, 2 \mathrm{H}), 7.16-7.12(\mathrm{~m}, 1 \mathrm{H}), 5.34(\mathrm{~s}, 2 \mathrm{H}), 4.97(\mathrm{~d}, J=5.1 \mathrm{~Hz}, 1 \mathrm{H}), 4.02-$ 4.00 (ddd, $J=3.3,5.1,8.5 \mathrm{~Hz}, 1 \mathrm{H}), 3.68-3.61(\mathrm{~m}, 1 \mathrm{H}), 2.60$ (ddd, $J=5.2,11.4,13.2 \mathrm{~Hz}, 1 \mathrm{H}$ ), 2.52 (ddd, $J=5.2,11.8,13.2 \mathrm{~Hz}, 1 \mathrm{H}), 2.33-2.16(\mathrm{~m}, 2 \mathrm{H}), 1.93-1.79(\mathrm{~m}, 2 \mathrm{H}), 1.74(\mathrm{~s}, 3 \mathrm{H}), 1.73-$ $1.63(\mathrm{~m}, 2 \mathrm{H})$

${ }^{13}$ C NMR (125 MHz, $\left.\mathrm{CD}_{3} \mathrm{OD}\right) \delta 156.4,155.8,142.5,142.1,141.5,139.5,137.4,137.3,130.1$, $130.0,129.9,129.8,129.7,129.6,129.5,128.6,127.9,127.3,121.9,118.0,73.2,69.7,63.1,59.7$, $58.9,34.2,33.1,29.6,27.7,25.0$

IR (thin film) 3415, 1703, 1659, 1265, $1093 \mathrm{~cm}^{-1}$

Melting Point: $132-133{ }^{\circ} \mathrm{C}$

HRMS (ESI-TOF) m/z: $[\mathrm{M}]^{+}$calcd for $\left[\mathrm{C}_{36} \mathrm{H}_{37} \mathrm{~N}_{4} \mathrm{O}_{2}\right]^{+}$557.2916, found 557.2906

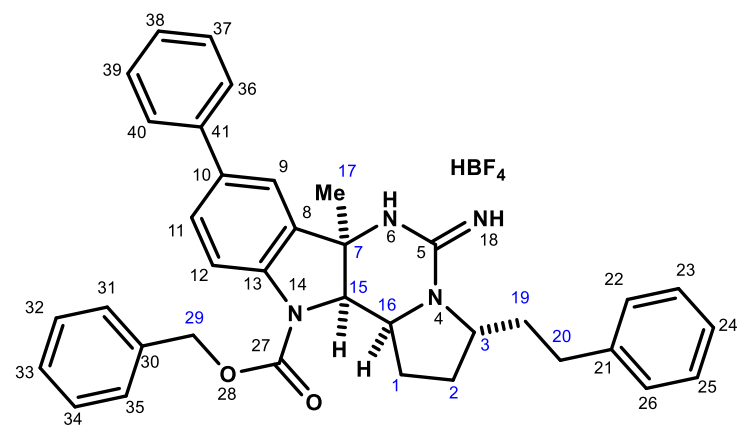

Assignment of Aliphatic Shifts (Solvent: CD3OD)

\begin{tabular}{|c|c|c|c|c|c|}
\hline atom & ${ }^{13} \mathrm{C}$ Shift & ${ }^{1} \mathrm{H}$ Shift & atom & ${ }^{13} \mathrm{C}$ Shift & ${ }^{1} \mathrm{H}$ Shift \\
\hline 1 & 25.0 & $2.33-2.16(\mathrm{~m})$ & 17 & 27.7 & $1.74(\mathrm{~s})$ \\
\hline 2 & 29.6 & $1.93-1.79(\mathrm{~m})$ & 19 & 34.2 & $1.93-1.79(\mathrm{~m})$ \\
\hline & & $1.73-1.63(\mathrm{~m})$ & & & $1.73-1.63(\mathrm{~m})$ \\
\hline 3 & 59.7 & $3.68-3.61(\mathrm{~m})$ & 20 & 33.1 & $2.60(\mathrm{ddd}, 5.2,11.4,13.2 \mathrm{~Hz})$ \\
\hline 7 & 63.1 & NA & & & $2.52(\mathrm{ddd}, 5.2,11.8,13.2 \mathrm{~Hz})$ \\
\hline 15 & 73.2 & $4.97(\mathrm{~d}, 5.1 \mathrm{~Hz})$ & 29 & 69.7 & $5.34(\mathrm{~s})$ \\
\hline 16 & 58.9 & $4.00(\mathrm{ddd}, 3.3,5.1,8.5 \mathrm{~Hz})$ & \multicolumn{2}{r|}{} \\
\cline { 1 - 5 }
\end{tabular}




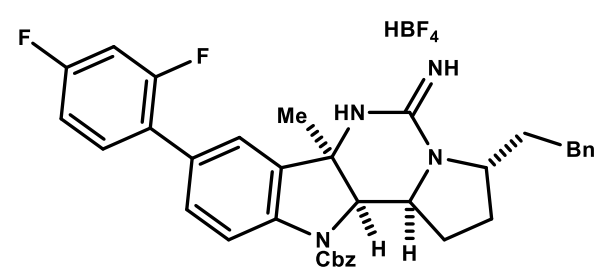

Adduct 12k: According to the representative procedure, $N$-amidinylpyrrolidine salt 10 (56.4 $\mathrm{mg}$, $0.136 \mathrm{mmol}$ ) and indole $\mathbf{S 2 7}(154.6 \mathrm{mg}, 0.41 \mathrm{mmol}, 3$ equiv) were used. Purification by flash column chromatography $\left(\mathrm{SiO}_{2}, 1: 0 \rightarrow 95: 5 \mathrm{CH}_{2} \mathrm{Cl}_{2} / \mathrm{MeOH}\right)$ afforded adduct $12 \mathbf{k}(57.4 \mathrm{mg}, 62 \%)$ as a yellow foam.

${ }^{1} \mathbf{H}$ NMR $\left(600 \mathrm{MHz}, \mathrm{CD}_{3} \mathrm{OD}\right) \delta 7.69(\mathrm{~d}, J=8.2 \mathrm{~Hz}, 1 \mathrm{H}), 7.55-7.36(\mathrm{~m}, 8 \mathrm{H}), 7.26(\mathrm{t}, J=7.5 \mathrm{~Hz}$, 2H), $7.21(\mathrm{~d}, J=7.1 \mathrm{~Hz}, 2 \mathrm{H}), 7.17(\mathrm{t}, J=7.2 \mathrm{~Hz}, 1 \mathrm{H}), 7.06(\mathrm{t}, J=9.0 \mathrm{~Hz}, 2 \mathrm{H}), 5.36(\mathrm{~s}, 2 \mathrm{H}), 4.99$ $(\mathrm{d}, J=5.1 \mathrm{~Hz}, 1 \mathrm{H}), 4.07-4.0(\mathrm{~m}, 1 \mathrm{H}), 3.71-3.64(\mathrm{~m}, 1 \mathrm{H}), 2.67-2.59(\mathrm{~m}, 1 \mathrm{H}), 2.58-2.50(\mathrm{~m}, 1 \mathrm{H})$, 2.33-2.19 (m, 2H), 1.94-1.83 (m, 2H), 1.78-1.66 (m, 2H), 1.75 (s, 3H)

${ }^{13}$ C NMR Peak (150 MHz, CD $\left.{ }_{3} \mathrm{OD}\right) \delta 163.3,163.2,161.64,161.56,160.5,160.4,158.9,158.8$, $154.8,154.2,141.0,140.9,135.7,135.5,131.46,131.42,131.40,131.36,130.14,130.12,128.4$, $128.35,128.3,128.1,127.9,125.7,124.41,124.38,124.32,124.30,124.25,122.5,122.4,116.2$, $111.5,111.49,111.37,111.35,104.0,103.8,103.6,71.6,68.2,61.4,58.2,57.3,32.6,31.6,28.1$, $26.2,23.5$

IR (thin film) 3357, 3031, 2930, 2479, 1707, 1656, 1615, 1584, 1556, 1484, 1454, 1389, 1325, $1275,1263,1221,1140,1091,1018,966 \mathrm{~cm}^{-1}$

HRMS (ESI-TOF) m/z: $[\mathrm{M}]^{+}$calcd for $\left[\mathrm{C}_{36} \mathrm{H}_{35} \mathrm{~F}_{2} \mathrm{~N}_{4} \mathrm{O}_{2}\right]^{+}$593.2728, found 593.2737

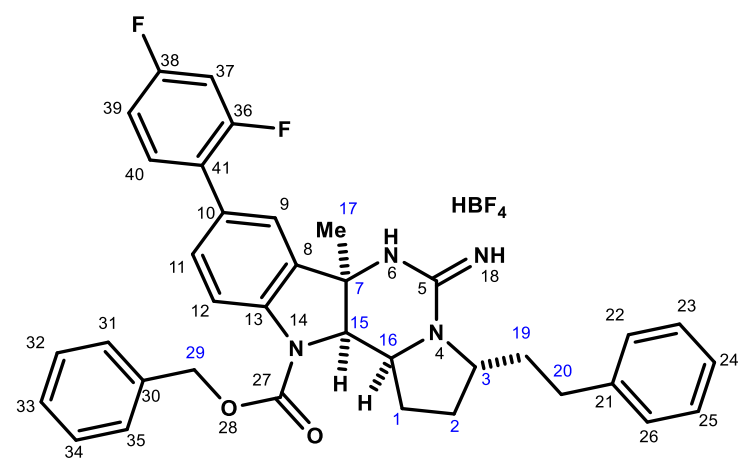

Assignment of Aliphatic Shifts (Solvent: $\mathrm{CD}_{3} \mathrm{OD}$ )

\begin{tabular}{|c|c|c|c|c|c|}
\hline atom & ${ }^{13} \mathrm{C}$ Shift & ${ }^{1} \mathrm{H}$ Shift & atom & ${ }^{13} \mathrm{C}$ Shift & ${ }^{1} \mathrm{H}$ Shift \\
\hline 1 & 23.5 & $2.33-2.19(\mathrm{~m})$ & 17 & 26.2 & $1.75(\mathrm{~s})$ \\
\hline 2 & 28.1 & $1.94-1.83(\mathrm{~m})$ & 19 & 32.6 & $1.94-1.83(\mathrm{~m})$ \\
\hline & & $1.78-1.66(\mathrm{~m})$ & & & $1.78-1.66(\mathrm{~m})$ \\
\hline 3 & 58.2 & $3.71-3.64(\mathrm{~m})$ & 20 & 31.6 & $2.67-2.59(\mathrm{~m})$ \\
\hline 7 & 61.4 & NA & & & $2.58-2.50(\mathrm{~m})$ \\
\hline 15 & 71.6 & $4.99(\mathrm{~d}, \mathrm{~J}=5.1 \mathrm{~Hz})$ & 29 & 68.2 & $5.36(\mathrm{~s})$ \\
\hline 16 & 57.3 & $4.07-4.0(\mathrm{~m})$ & \multicolumn{1}{l}{} \\
\cline { 1 - 5 }
\end{tabular}




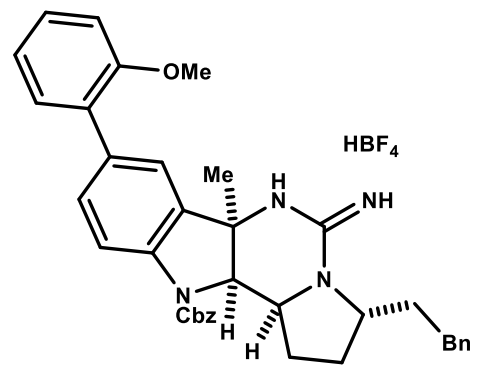

Adduct 121: According to the representative procedure, $N$-amidinylpyrrolidine salt 10 (56 mg, $0.135 \mathrm{mmol}$ ) and indole $\mathbf{S 2 8}$ (150 $\mathrm{mg}, 0.404 \mathrm{mmol}$ ) was used as the dienophile. Purification by flash column chromatography $\left(\mathrm{SiO}_{2}, 1: 0 \rightarrow 95: 5 \mathrm{CH}_{2} \mathrm{Cl}_{2} / \mathrm{MeOH}\right)$ afforded adduct $12 \mathrm{l}(37 \mathrm{mg}, 41 \%)$ as a pale-yellow foam.

${ }^{1} \mathbf{H}$ NMR (600 MHz, CD $\left.3 \mathrm{OD}\right) \delta 7.62(\mathrm{~d}, J=7.7 \mathrm{~Hz}, 1 \mathrm{H}), 7.53-7.47(\mathrm{~m}, 2 \mathrm{H}), 7.45-7.36(\mathrm{~m}, 5 \mathrm{H})$, 7.35-7.25 (m, 4H), 7.24-7.14 (m, 3H), $7.07(\mathrm{~d}, J=8.1 \mathrm{~Hz}, 1 \mathrm{H}), 7.02(\mathrm{t}, J=6.1 \mathrm{~Hz}, 1 \mathrm{H}), 5.36(\mathrm{~s}$, 2H), 4.99-4.95 (m, 1H), 4.06-3.99 (m, 1H), 3.81 (s, 3H), 3.72-3.64 (m, 1H), 2.68-2.59 (m, 1H), 2.58-2.50 (m, 1H), 2.31-2.19 (m, 2H), 1.95-1.89 (m, 1H), 1.88-1.81 (m, 1H), 1.79-1.64 (m, $2 \mathrm{H}), 1.73(\mathrm{~s}, 3 \mathrm{H})$

${ }^{13} \mathrm{C}$ NMR (150 MHz, CD 3 OD) 156.4, 154.9, 154.3, 141.0, 139.9, 135.8, 135.4, 134.9, 134.7, $130.6,130.1,128.6,128.4,128.3,128.2,128.1,127.9,125.7,123.0,120.6,115.6,111.2,71.5$, $68.1,61.5,58.2,57.4,54.7,32.6,31.6,28.1,26.3,23.5$

IR (thin film) 3356, 2931, 2498, 1707, 1656, 1599, 1498, 1481, 1454, 1393, 1325, 1277, 1246, $1117,1089,1055,1024 \mathrm{~cm}^{-1}$

HRMS (ESI-TOF) m/z: $[\mathrm{M}]^{+}$calcd $\left[\mathrm{C}_{37} \mathrm{H}_{39} \mathrm{~N}_{4} \mathrm{O}_{3}\right]^{+}$587.3022, found 587.3041

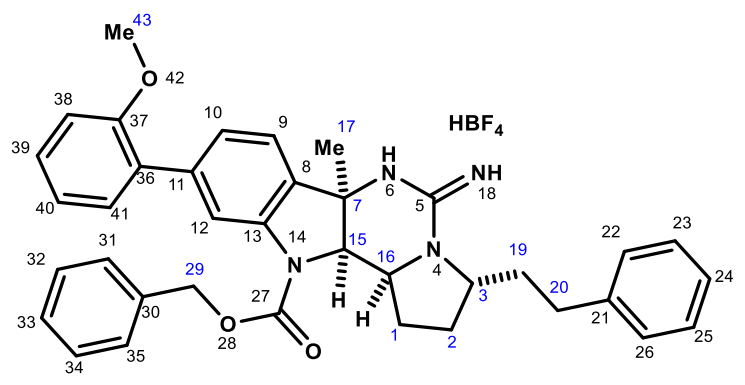

Assignment of Aliphatic Shifts (Solvent: CD3OD)

\begin{tabular}{|c|c|c|c|c|c|}
\hline atom & ${ }^{13} \mathrm{C}$ Shift & ${ }^{1} \mathrm{H}$ Shift & atom & ${ }^{13} \mathrm{C}$ Shift & ${ }^{1} \mathrm{H}$ Shift \\
\hline 1 & 23.5 & $2.31-2.19(\mathrm{~m})$ & 17 & 26.3 & $1.73(\mathrm{~s})$ \\
\hline 2 & 28.1 & $1.95-1.89(\mathrm{~m})$ & 19 & 32.6 & $1.88-1.81(\mathrm{~m})$ \\
\hline & & $1.79-1.64(\mathrm{~m})$ & & & $1.79-1.64(\mathrm{~m})$ \\
\hline 3 & 58.2 & $3.72-3.64(\mathrm{~m})$ & 20 & 31.6 & $2.68-2.59(\mathrm{~m})$ \\
\hline 7 & 61.5 & NA & & & $2.58-2.50(\mathrm{~m})$ \\
\hline 15 & 71.5 & $4.99-4.95(\mathrm{~m})$ & 29 & 68.1 & $5.36(\mathrm{~s})$ \\
\hline 16 & 57.4 & $4.06-3.99(\mathrm{~m})$ & 43 & 54.7 & $3.81(\mathrm{~s})$ \\
\hline
\end{tabular}




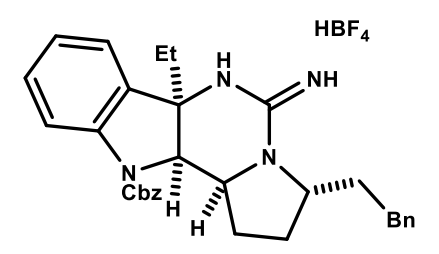

Adduct 12m: According to the representative procedure, $N$-amidinylpyrrolidine salt $10(54.8 \mathrm{mg}$, $0.132 \mathrm{mmol}$ ) and indole $\mathbf{S 2 9}$ (113.2 $\mathrm{mg}, 0.40 \mathrm{mmol}$ ) was used as the dienophile. Purification by flash column chromatography $\left(\mathrm{SiO}_{2}, 1: 0 \rightarrow 95: 5 \mathrm{CH}_{2} \mathrm{Cl}_{2} / \mathrm{MeOH}\right)$ afforded adduct $\mathbf{1 2} \mathbf{m}(42.1 \mathrm{mg}$, $55 \%$ ) as a light orange foam.

${ }^{1}$ H NMR (600 MHz, CDCl $)$ $)$ 7.44-7.33 (m, 7H), 7.25-7.21 (m, 3H), 7.20-7.17 (m, 2H), 7.17$7.13(\mathrm{~m}, 1 \mathrm{H}), 7.08(\mathrm{t}, J=7.5 \mathrm{~Hz}, 1 \mathrm{H}), 6.95(\mathrm{bs}, 1 \mathrm{H}), 6.32(\mathrm{bs}, 1 \mathrm{H}), 6.21$ (bs, $1 \mathrm{H}), 5.33(\mathrm{~d}, J=12.2$ $\mathrm{Hz}, 1 \mathrm{H}), 5.28(\mathrm{~d}, J=12.2 \mathrm{~Hz}, 1 \mathrm{H}), 4.90(\mathrm{~d}, J=5.0 \mathrm{~Hz}, 1 \mathrm{H}), 3.80-3.73(\mathrm{~m}, 1 \mathrm{H}), 3.62-3.55(\mathrm{~m}$, $1 \mathrm{H}), 2.72-2.63(\mathrm{~m}, 1 \mathrm{H}), 2.56-2.46(\mathrm{~m}, 1 \mathrm{H}), 2.31-2.22(\mathrm{~m}, 1 \mathrm{H}), 2.20-2.10(\mathrm{~m}, 1 \mathrm{H}), 2.07-1.98(\mathrm{~m}$, $1 \mathrm{H}), 1.96-1.83(\mathrm{~m}, 2 \mathrm{H}), 1.81(\mathrm{dd}, J=6.9,12.4 \mathrm{~Hz}, 1 \mathrm{H}), 1.73-1.66(\mathrm{~m}, 1 \mathrm{H}), 1.66-1.58(\mathrm{~m}, 1 \mathrm{H})$, $0.84(\mathrm{t}, J=7.3 \mathrm{~Hz}, 3 \mathrm{H})$

${ }^{13}$ C NMR (150 MHz, $\left.\mathrm{CDCl}_{3}\right) \delta 154.9,154.3,141.2,140.5,135.4,133.3,129.9,128.8,128.73$, $128.70,128.5,128.3,126.3,125.6,123.3,116.2,68.59,68.55,65.6,57.74,57.69,33.5,32.6,32.2$, $28.8,23.9,7.6$

IR (thin film) 3432, 3362, 3241, 3065, 3030, 2966, 2932, 1704, 1660, 1632, 1602, 1556, 1496, $1482,1455,1393,1324,1260,1239,1213,1048,1017,908 \mathrm{~cm}^{-1}$

HRMS (ESI-TOF) m/z: $[\mathrm{M}]^{+}$calcd for $\left[\mathrm{C}_{31} \mathrm{H}_{35} \mathrm{~N}_{4} \mathrm{O}_{2}\right]^{+} 495.2760$, found 495.2736

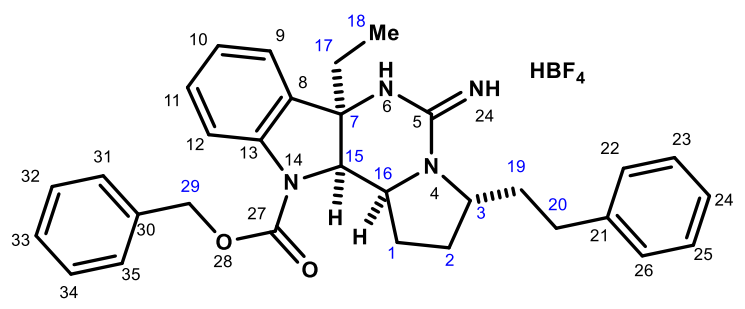

Assignment of Aliphatic Shifts (Solvent: $\mathrm{CDCl}_{3}$ )

\begin{tabular}{|c|c|c|c|c|c|}
\hline atom & ${ }^{13} \mathrm{C}$ Shift & ${ }^{1} \mathrm{H}$ Shift & atom & ${ }^{13} \mathrm{C}$ Shift & ${ }^{1} \mathrm{H}$ Shift \\
\hline 1 & 23.9 & $2.31-2.22(\mathrm{~m})$ & 17 & & $1.96-1.83(\mathrm{~m})$ \\
\hline & & $2.20-2.10(\mathrm{~m})$ & 18 & 7.6 & $0.84(\mathrm{t}, \mathrm{J}=7.3 \mathrm{~Hz})$ \\
\hline 2 & 28.8 & $1.81(\mathrm{dd}, \mathrm{J}=6.9,12.4 \mathrm{~Hz})$ & 19 & 32.2 & $1.96-1.83(\mathrm{~m})$ \\
\hline & & $1.73-1.66(\mathrm{~m})$ & & & $1.66-1.58(\mathrm{~m})$ \\
\hline 3 & 57.74 & $3.62-3.55(\mathrm{~m})$ & 20 & 33.5 & $2.72-2.63(\mathrm{~m})$ \\
\hline 7 & 65.6 & NA & & & $2.56-2.46(\mathrm{~m})$ \\
\hline 15 & 68.59 & $4.90(\mathrm{~d}, \mathrm{~J}=5.0 \mathrm{~Hz})$ & 29 & 68.55 & $5.33(\mathrm{~d}, J=12.2 \mathrm{~Hz})$ \\
\hline 16 & 57.69 & $3.80-3.73(\mathrm{~m})$ & & & $5.28(\mathrm{~d}, J=12.2 \mathrm{~Hz})$ \\
\hline 17 & 32.6 & $2.07-1.98(\mathrm{~m})$ & \multicolumn{3}{|l}{} \\
\hline
\end{tabular}




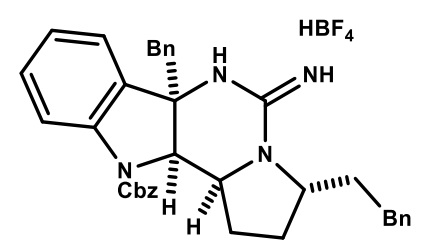

Adduct 12n: According to the representative procedure, $N$-amidinylpyrrolidine salt $10(82 \mathrm{mg}$, $0.2 \mathrm{mmol}, 1.5$ equiv) and indole $\mathbf{S 3 0}$ (45 $\mathrm{mg}, 0.132 \mathrm{mmol}$ ) was used as the dienophile. Purification by flash column chromatography $\left(\mathrm{SiO}_{2}, 1: 0 \rightarrow 9: 1 \mathrm{CH}_{2} \mathrm{Cl}_{2} / \mathrm{MeOH}\right)$ afforded adduct $\mathbf{X}(29 \mathrm{mg}, 34 \%)$ as a colorless solid.

${ }^{1} \mathbf{H}$ NMR $\left(500 \mathrm{MHz}, \mathrm{CD}_{3} \mathrm{OD}\right) \delta$ 7.42-7.30 (m, 6H), 7.29-7.07 (m, 9H), 7.06-7.00 (m, 2H), 6.86$6.82(\mathrm{~m}, 2 \mathrm{H}), 5.08(\mathrm{~d}, \mathrm{~J}=5.3 \mathrm{~Hz}, 1 \mathrm{H}), 5.02(\mathrm{~s}, 2 \mathrm{H}), 3.91-3.84(\mathrm{~m}, 1 \mathrm{H}), 3.58-3.51(\mathrm{~m}, 1 \mathrm{H}), 3.30$ $3.27(\mathrm{~m}, 1 \mathrm{H}), 3.24(\mathrm{~d}, J=13.1 \mathrm{~Hz}, 1 \mathrm{H}), 2.61-2.55(\mathrm{~m}, 1 \mathrm{H}), 2.53-2.46(\mathrm{~m}, 1 \mathrm{H}), 2.22-2.07(\mathrm{~m}$, $2 \mathrm{H}), 1.89-1.79(\mathrm{~m}, 2 \mathrm{H}), 1.72-1.64(\mathrm{~m}, 1 \mathrm{H}), 1.62-1.53(\mathrm{~m}, 1 \mathrm{H})$

${ }^{13} \mathrm{C}$ NMR $\left(125 \mathrm{MHz}, \mathrm{CD}_{3} \mathrm{OD}\right) \delta 156.6,155.0,144.1,142.5,137.1,135.0,134.9,131.4,131.0$, $129.8,129.7,129.68,129.62,129.5,129.3,128.6,127.3,125.9,124.1,117.9,69.9,69.3,66.8$, $59.8,58.7,46.2,34.0,33.1,29.5,24.7$

IR (thin film) 3405, 1709, 1660,1085 $\mathrm{cm}^{-1}$

Melting Point: $143-145^{\circ} \mathrm{C}$

HRMS (ESI-TOF) m/z: $[\mathrm{M}]^{+}$calcd for $\left[\mathrm{C}_{36} \mathrm{H}_{37} \mathrm{~N}_{4} \mathrm{O}_{2}\right]^{+}$557.2916, found 557.2916

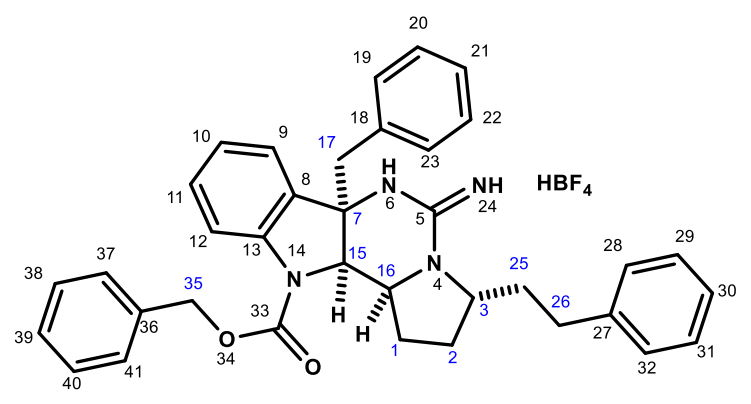

Assignment of Aliphatic Shifts (Solvent: $\mathrm{CD}_{3} \mathrm{OD}$ )

\begin{tabular}{|c|c|c|c|c|c|}
\hline atom & ${ }^{13} \mathrm{C}$ Shift & ${ }^{1} \mathrm{H}$ Shift & atom & ${ }^{13} \mathrm{C}$ Shift & ${ }^{1} \mathrm{H}$ Shift \\
\hline 1 & 24.1 & $2.22-2.07(\mathrm{~m})$ & 17 & 46.2 & $3.27(\mathrm{~m})$ \\
\hline 2 & 29.5 & $1.89-1.79(\mathrm{~m})$ & & & $3.24(\mathrm{~d}, 13.1 \mathrm{~Hz})$ \\
\hline & & $1.62-1.53(\mathrm{~m})$ & 25 & 34.0 & $1.89-1.79(\mathrm{~m})$ \\
\hline 3 & 59.8 & $3.58-3.51(\mathrm{~m})$ & & & $1.72-1.64(\mathrm{~m})$ \\
\hline 7 & 66.8 & NA & 26 & 33.1 & $2.61-2.55(\mathrm{~m})$ \\
\hline 15 & 69.9 & $5.08(\mathrm{~d}, 5.3 \mathrm{~Hz})$ & & & $2.53-2.46(\mathrm{~m})$ \\
\hline 16 & 58.7 & $3.91-3.84(\mathrm{~m})$ & 35 & 69.3 & $5.02(\mathrm{~s})$ \\
\hline
\end{tabular}




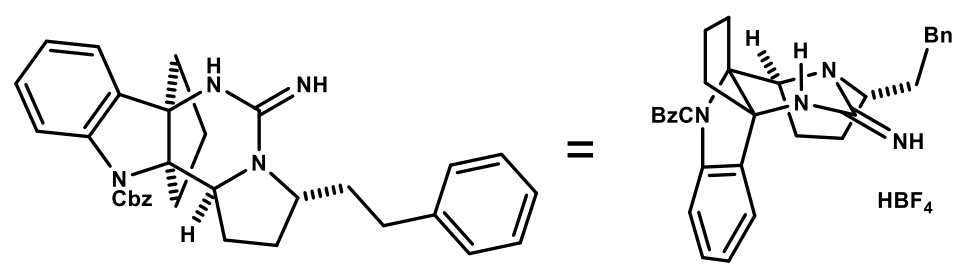

Adduct 12p: According to the representative procedure, $N$-amidinylpyrrolidine salt 10 (55.8 $\mathrm{mg}$, $0.135 \mathrm{mmol})$ and benzyl 2,3-dihydrocyclopenta[b]indole-4(1H)-carboxylate $(60.6 \mathrm{mg}, 0.206$ mmol, 1.5 equiv) were used. Purification by flash column chromatography $\left(\mathrm{SiO}_{2}, 1: 0 \rightarrow 9: 1\right.$ $\left.\mathrm{CH}_{2} \mathrm{Cl}_{2} / \mathrm{MeOH}\right)$ afforded adduct $12 p(59.6 \mathrm{mg}, 74 \%)$.

${ }^{1} \mathbf{H}$ NMR $\left(500 \mathrm{MHz}, \mathrm{CD}_{3} \mathrm{OD}\right) \delta 7.51(\mathrm{~d}, J=7.6 \mathrm{~Hz}, 1 \mathrm{H}), 7.49-7.45(\mathrm{~m}, 2 \mathrm{H}), 7.42-7.34(\mathrm{~m}, 3 \mathrm{H})$, $7.27-7.19(\mathrm{~m}, 5 \mathrm{H}), 7.15(\mathrm{t}, J=7.2 \mathrm{~Hz}, 1 \mathrm{H}), 7.07(\mathrm{t}, J=7.6 \mathrm{~Hz}, 1 \mathrm{H}), 5.68-5.12(\mathrm{~m}, 2 \mathrm{H}), 3.95$ (app $\mathrm{d}, J=9.8 \mathrm{~Hz}, 1 \mathrm{H}), 3.73-3.64(\mathrm{~m}, 1 \mathrm{H}), 2.69-2.59(\mathrm{~m}, 2 \mathrm{H}), 2.58-2.43(\mathrm{~m}, 3 \mathrm{H}), 2.33(\mathrm{dt}, J=6.7$, $13.7 \mathrm{~Hz}, 1 \mathrm{H}), 2.22(\mathrm{dt}, J=6.7,13.7 \mathrm{~Hz}, 1 \mathrm{H}), 2.06-1.96(\mathrm{~m}, 1 \mathrm{H}), 1.93-1.83(\mathrm{~m}, 3 \mathrm{H}), 1.75$ (ddd, $J=5.4,11.4,13.3 \mathrm{~Hz}, 1 \mathrm{H}), 1.63-1.54(\mathrm{~m}, 1 \mathrm{H}), 1.54-1.45(\mathrm{~m}, 1 \mathrm{H})$

${ }^{13} \mathrm{C}$ NMR (150 MHz, CD $\left.3 \mathrm{OD}\right) \delta 153.7,152.7,142.0,141.0,135.8,129.4,129.0,128.4,128.3$, $128.2,128.1,127.9,125.8,125.0,123.7,114.2$, 88.7, 67.8, 61.7 (2C), 58.6, 40.1 (2C), .5, 31.6, $27.9,23.2,23.1$

IR (thin film) 3359, 3062, 3032, 2953, 2871, 2485, 1708, 1657, 1544, 1483, 1454, 1394, 1354, $1307,1288,1261,1234,1187,1142,1063,1027 \mathrm{~cm}^{-1}$

HRMS (ESI-TOF) m/z: $[\mathrm{M}]^{+}$calcd for $\left[\mathrm{C}_{32} \mathrm{H}_{35} \mathrm{~N}_{4} \mathrm{O}_{2}\right]^{+}$507.2760, found 507.2739

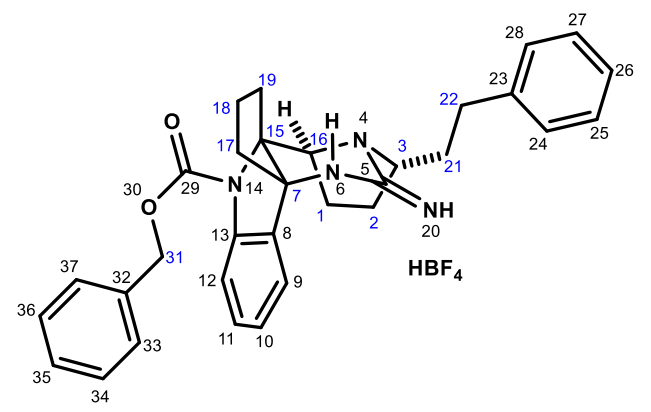

\section{Assignment of Aliphatic Shifts (Solvent: $\mathrm{CD}_{3} \mathrm{OD}$ )}

\begin{tabular}{|c|c|c|c|c|c|}
\hline atom & ${ }^{13} \mathrm{C}$ Shift & ${ }^{1} \mathrm{H}$ Shift & atom & ${ }^{13} \mathrm{C} \mathrm{Shift}$ & ${ }^{1} \mathrm{H}$ Shift \\
\hline 1 & 23.1 & $2.69-2.59(\mathrm{~m})$ & 18 & 23.2 & $1.93-1.83(\mathrm{~m})$ \\
\hline & & $2.58-2.43(\mathrm{~m})$ & & & $1.63-1.54(\mathrm{~m})$ \\
\hline 2 & 27.9 & $1.93-1.83(\mathrm{~m})$ & 19 & 40.1 & $2.58-2.43(\mathrm{~m})$ \\
\hline & & $1.54-1.45(\mathrm{~m})$ & & & $2.33(\mathrm{dt}, 6.7,13.7 \mathrm{~Hz})$ \\
\hline 3 & 58.6 & $3.73-3.64(\mathrm{~m})$ & 21 & 32.5 & $1.93-1.83(\mathrm{~m})$ \\
\hline 7 & 61.7 & NA & & & $1.75(\mathrm{ddd}, 5.4,11.4,13.3 \mathrm{~Hz})$ \\
\hline 15 & 88.7 & NA & 22 & 23.1 & $2.69-2.59(\mathrm{~m})$ \\
\hline 16 & 61.7 & $3.95(\mathrm{app} \mathrm{d}, 9.8 \mathrm{~Hz})$ & & & $2.58-2.43(\mathrm{~m})$ \\
\hline 17 & 40.1 & $2.22(\mathrm{dt}, 6.7,13.7 \mathrm{~Hz})$ & 31 & 67.8 & $5.68-5.12(\mathrm{~m})$ \\
\hline & \multicolumn{3}{|r|}{$2.06-1.96(\mathrm{~m})$} & &
\end{tabular}




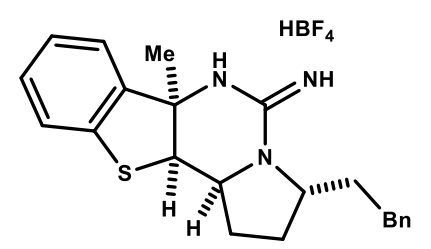

Adduct 120: According to the representative procedure, $N$-amidinylpyrrolidine salt $10(55 \mathrm{mg}$, $0.132 \mathrm{mmol}$ ) and benzothiophene $(59 \mathrm{mg}, 0.396 \mathrm{mmol}$ ) was used as the dienophile. Purification by flash column chromatography $\left(\mathrm{SiO}_{2}, 1: 0 \rightarrow 95: 5 \mathrm{CH}_{2} \mathrm{Cl}_{2} / \mathrm{MeOH}\right)$ afforded adduct $\mathbf{1 2 0}$ (43 mg, $72 \%)$ as a clear oil.

${ }^{1}$ H NMR $\left(500 \mathrm{MHz}, \mathrm{CDCl}_{3}\right) \delta$ 7.39-7.12 (m, 9H), $6.88(\mathrm{~s}, 1 \mathrm{H}), 5.98(\mathrm{~s}, 2 \mathrm{H}), 4.40-4.33(\mathrm{~m}, 1 \mathrm{H})$, 4.23-4.13 (m, 1H), 4.12-4.02 (m, 1H), 2.83-2.73 (m, 1H), 2.71-2.60 (m, 1H), 2.55-2.42 (m, 1H), $2.31-2.20(\mathrm{~m}, 1 \mathrm{H}), 2.13-2.03(\mathrm{~m}, 1 \mathrm{H}), 1.97-1.80(\mathrm{~m}, 3 \mathrm{H}), 1.75(\mathrm{~s}, 3 \mathrm{H})$

${ }^{1} \mathbf{H}$ NMR $\left(500 \mathrm{MHz}, \mathrm{CD}_{3} \mathrm{OD}\right) \delta 7.42(\mathrm{~d}, J=7.6 \mathrm{~Hz}, 1 \mathrm{H}), 7.37-7.27(\mathrm{~m}, 6 \mathrm{H}), 7.26-7.19(\mathrm{~m}, 2 \mathrm{H})$, $4.66(\mathrm{~d}, J=3.3 \mathrm{~Hz}, 1 \mathrm{H}), 4.40(\mathrm{dt}, J=3.2,7.2 \mathrm{~Hz}, 1 \mathrm{H}), 4.19-4.11(\mathrm{~m}, 1 \mathrm{H}), 2.80-2.65(\mathrm{~m}, 2 \mathrm{H})$, 2.62-2.51 (m, 1H), 2.37-2.27 (m, 1H), 2.14-1.99 (m, 2H), 1.98-1.91 (m, 1H), 1.89-1.78 (m, 1H), $1.82(\mathrm{~s}, 3 \mathrm{H})$

${ }^{13}$ C NMR $\left(125 \mathrm{MHz}, \mathrm{CDCl}_{3}\right) \delta 151.5,140.9,140.7,138.0,130.4,128.8,128.6,126.4,126.1$, 124.6, 123.2, 64.4, 59.1, 57.6, 53.1, 35.4, 31.8, 29.4, 28.5, 25.5

IR (thin film) 3369, 2304, 1642, 1590, $1265 \mathrm{~cm}^{-1}$

HRMS (ESI-TOF) m/z: $[\mathrm{M}]^{+}$calcd for $\left[\mathrm{C}_{22} \mathrm{H}_{26} \mathrm{~N}_{3} \mathrm{~S}\right]^{+} 364.1848$, found 364.1839

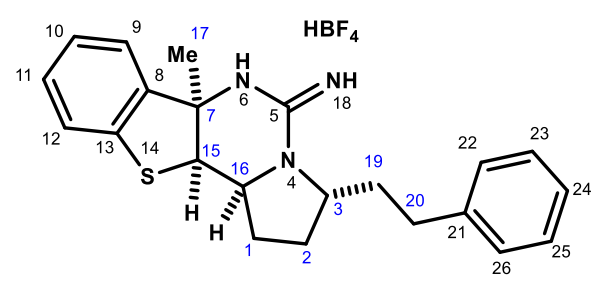

Assignment of Aliphatic Shifts (Solvent: $\left.\mathrm{CDCl}_{3}\right)$

\begin{tabular}{|c|c|c|c|c|c|}
\hline atom & ${ }^{13} \mathrm{C}$ Shift & ${ }^{1} \mathrm{H}$ Shift & atom & ${ }^{13} \mathrm{C}$ Shift & ${ }^{1} \mathrm{H}$ Shift \\
\hline 1 & 29.4 & $2.55-2.42(\mathrm{~m})$ & 16 & 53.1 & $4.23-4.13(\mathrm{~m})$ \\
\hline & & $1.97-1.80(\mathrm{~m})$ & 17 & 25.5 & $1.75(\mathrm{~s})$ \\
\hline 2 & 28.5 & $2.31-2.20(\mathrm{~m})$ & 19 & 35.4 & $2.13-2.03(\mathrm{~m})$ \\
\hline & & $1.97-1.80(\mathrm{~m})$ & & & $1.97-1.80(\mathrm{~m})$ \\
\hline 3 & 59.1 & $4.12-4.02(\mathrm{~m})$ & 20 & 31.8 & $2.83-2.73(\mathrm{~m})$ \\
\hline 7 & 64.4 & NA & & & $2.71-2.60(\mathrm{~m})$ \\
\hline 15 & 57.6 & $4.40-4.33(\mathrm{~m})$ & \multicolumn{3}{l}{} \\
\cline { 1 - 5 }
\end{tabular}




\section{F. Scope of N-Amidinyliminium Ion Precursors}

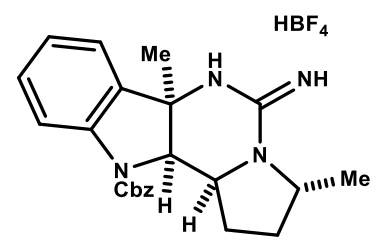

Adduct 12q: According to the representative procedure, $N$-amidinylpyrrolidine salt $\mathbf{S 5}$ (43.1 $\mathrm{mg}$, $0.133 \mathrm{mmol}$ ) and benzyl 3-methyl-1H-indole-1-carboxylate $(106.4 \mathrm{mg}, 0.401 \mathrm{mmol})$ was used as the dienophile. Purification by flash column chromatography $\left(\mathrm{SiO}_{2}, 1: 0 \rightarrow 95: 5 \mathrm{CH}_{2} \mathrm{Cl}_{2} / \mathrm{MeOH}\right)$ afforded adduct $\mathbf{1 2 q}(37.2 \mathrm{mg}, 58 \%)$ as a green oil.

${ }^{1}$ H NMR $\left(600 \mathrm{MHz}, \mathrm{CD}_{3} \mathrm{OD}\right) \delta 7.60(\mathrm{~d}, J=7.6 \mathrm{~Hz}, 1 \mathrm{H}), 7.47(\mathrm{~d}, J=6.8 \mathrm{~Hz}, 2 \mathrm{H}), 7.41(\mathrm{t}, J=7.0$ $\mathrm{Hz}, 2 \mathrm{H}), 7.39-7.35(\mathrm{~m}, 1 \mathrm{H}), 7.31-7.26(\mathrm{~m}, 2 \mathrm{H}), 7.13(\mathrm{t}, J=7.2 \mathrm{~Hz}, 1 \mathrm{H}), 5.33(\mathrm{~s}, 2 \mathrm{H}), 4.94(\mathrm{~d}, J=$ $2.8 \mathrm{~Hz}, 1 \mathrm{H}), 4.06-3.98(\mathrm{~m}, 1 \mathrm{H}), 3.76-3.68(\mathrm{~m}, 1 \mathrm{H}), 2.32-2.20(\mathrm{~m}, 2 \mathrm{H}), 1.80-1.72(\mathrm{~m}, 1 \mathrm{H}), 1.70$ $(\mathrm{s}, 3 \mathrm{H}), 1.66-1.60(\mathrm{~m}, 1 \mathrm{H}), 1.16(\mathrm{~d}, J=5.6 \mathrm{~Hz}, 3 \mathrm{H})$

${ }^{13} \mathrm{C}$ NMR (150 MHz, CD 3 OD) $\delta 154.9,154.3,141.3,135.8,135.0,129.3,128.4,128.3,128.2$, $124.4,122.0,116.2,71.5,68.1,61.6,57.1,53.6,31.4,26.2,23.3,16.7$

IR (thin film) 3360, 3034, 2976, 2472, 1706, 1629, 1567, 1482, 1455, 1396, 1325, 1282, 1260, $1214,1057,1030 \mathrm{~cm}^{-1}$

HRMS (ESI-TOF) m/z: $[\mathrm{M}]^{+}$calcd for $\left[\mathrm{C}_{23} \mathrm{H}_{27} \mathrm{~N}_{4} \mathrm{O}_{2}\right]^{+}$391.2134, found 391.2144

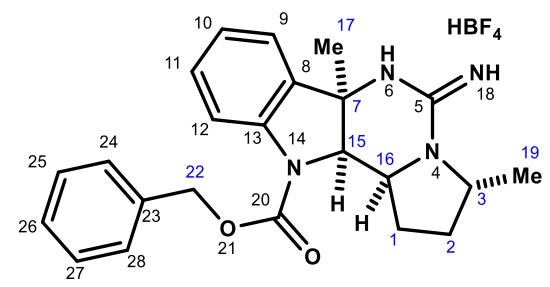

\section{Assignment of Aliphatic Shifts (Solvent: $\mathrm{CD}_{3} \mathrm{OD}$ )}

\begin{tabular}{|c|c|c|}
\hline atom & ${ }^{13} \mathrm{C}$ Shift & ${ }^{1} \mathrm{H}$ Shift \\
\hline 1 & 23.3 & $2.32-2.20(\mathrm{~m})$ \\
\hline 2 & 31.4 & $1.80-1.72(\mathrm{~m})$ \\
\hline & & $1.66-1.60(\mathrm{~m})$ \\
\hline 3 & 53.6 & $3.76-3.68(\mathrm{~m})$ \\
\hline 7 & 61.6 & NA \\
\hline 15 & 71.5 & $4.94(\mathrm{~d}, \mathrm{~J}=2.8 \mathrm{~Hz})$ \\
\hline 16 & 57.1 & $4.06-3.98(\mathrm{~m})$ \\
\hline 17 & 26.2 & $1.70(\mathrm{~s})$ \\
\hline 19 & 16.7 & $1.16(\mathrm{~d}, \mathrm{~J}=5.6 \mathrm{~Hz})$ \\
\hline 22 & 68.1 & $5.33(\mathrm{~s})$ \\
\hline
\end{tabular}




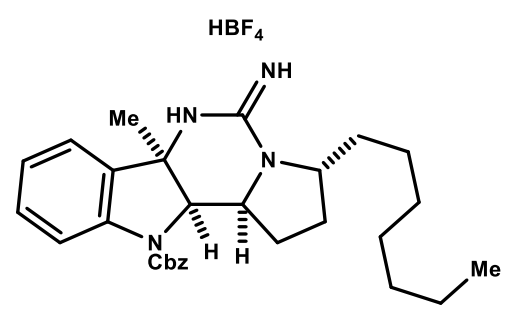

Adduct 12r: According to the representative procedure, $N$-amidinylpyrrolidine salt $\mathbf{S 8}(53.6 \mathrm{mg}$, $0.132 \mathrm{mmol}$ ) and benzyl 3-methyl- $1 H$-indole-1-carboxylate $(104.4 \mathrm{mg}, 0.40 \mathrm{mmol})$ was used as the dienophile. Purification by flash column chromatography $\left(\mathrm{SiO}_{2}, 1: 0 \rightarrow 9: 1 \mathrm{CH}_{2} \mathrm{Cl}_{2} / \mathrm{MeOH}\right)$ afforded adduct $12 \mathrm{r}(64 \mathrm{mg}, 86 \%)$ as a brown oil.

${ }^{1} \mathbf{H}$ NMR $\left(600 \mathrm{MHz}, \mathrm{CD}_{3} \mathrm{OD}\right) \delta 7.59(\mathrm{~d}, J=7.5 \mathrm{~Hz}, 1 \mathrm{H}), 7.50-7.44(\mathrm{~m}, 2 \mathrm{H}), 7.43-7.36(\mathrm{~m}, 3 \mathrm{H})$, $7.32-7.24(\mathrm{~m}, 2 \mathrm{H}), 7.13(\mathrm{t}, J=7.2 \mathrm{~Hz}, 1 \mathrm{H}), 5.33(\mathrm{~s}, 2 \mathrm{H}), 4.96-4.92(\mathrm{~m}, 1 \mathrm{H}), 4.00$ (dd, $J=4.8,7.7$ $\mathrm{Hz}, 1 \mathrm{H}), 3.53-3.47(\mathrm{~m}, 1 \mathrm{H}), 2.28-2.21(\mathrm{~m}, 1 \mathrm{H}), 2.21-2.13(\mathrm{~m}, 1 \mathrm{H}), 1.85-1.77(\mathrm{~m}, 1 \mathrm{H}), 1.70(\mathrm{~s}$, $3 \mathrm{H}), 1.67-1.58(\mathrm{~m}, 1 \mathrm{H}), 1.56-1.49(\mathrm{~m}, 1 \mathrm{H}), 1.45-1.37(\mathrm{~m}, 1 \mathrm{H}), 1.36-1.24(\mathrm{~m}, 9 \mathrm{H}), 1.24-1.15(\mathrm{~m}$, $1 \mathrm{H}), 0.90(\mathrm{t}, J=6.4 \mathrm{~Hz}, 3 \mathrm{H})$

${ }^{13}$ C NMR (150 MHz, CD $\left.{ }_{3} \mathrm{OD}\right) \delta 154.8,154.3,141.2,135.8,135.0,129.3,128.4,128.3,128.2$, $124.4,122.0,116.2,71.3,68.1,61.5,58.4,57.3,31.6,30.9,29.3,29.0,27.9,26.2,25.6,23.4,22.3$, 13.0

IR (thin film) 3356, 2928, 2856, 2492, 1715, 1652, 1635, 1603, 1558, 1482, 1455, 1397, 1326, $1282,1246,1216,1027,974 \mathrm{~cm}^{-1}$

HRMS (ESI-TOF) m/z: $[\mathrm{M}]^{+}$calcd for $\left[\mathrm{C}_{29} \mathrm{H}_{39} \mathrm{~N}_{4} \mathrm{O}_{2}\right]^{+} 475.3073$, found 475.3072

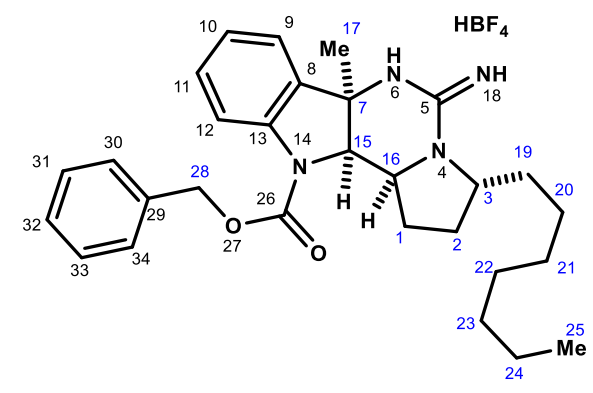

Assignment of Aliphatic Shifts (Solvent: $\mathrm{CD}_{3} \mathrm{OD}$ )

\begin{tabular}{|c|c|c|c|c|c|}
\hline atom & ${ }^{13} \mathrm{C}$ Shift & ${ }^{1} \mathrm{H}$ Shift & atom & ${ }^{13} \mathrm{C}$ Shift & ${ }^{1} \mathrm{H}$ Shift \\
\hline 1 & 23.4 & $2.28-2.21(\mathrm{~m})$ & 19 & 30.9 & $1.56-1.49(\mathrm{~m})$ \\
\hline & & $2.21-2.13(\mathrm{~m})$ & & & $1.45-1.37(\mathrm{~m})$ \\
\hline 2 & 27.9 & $1.85-1.77(\mathrm{~m})$ & 20 & 25.6 & $1.36-1.24(\mathrm{~m})$ \\
\hline & & $1.67-1.58(\mathrm{~m})$ & & & $1.24-1.15(\mathrm{~m})$ \\
\hline 3 & 58.4 & $3.53-3.47(\mathrm{~m})$ & 21 & 31.6 & $1.36-1.24(\mathrm{~m})$ \\
\hline 7 & 61.5 & NA & 22 & 29.0 & $1.36-1.24(\mathrm{~m})$ \\
\hline 15 & 71.3 & $4.96-4.92(\mathrm{~m})$ & 23 & 29.3 & $1.36-1.24(\mathrm{~m})$ \\
\hline 16 & 57.3 & $4.00(\mathrm{dd}, \mathrm{J}=4.8,7.7 \mathrm{~Hz})$ & 24 & 22.3 & $1.36-1.24(\mathrm{~m})$ \\
\hline 17 & 26.2 & $1.70(\mathrm{~s})$ & 25 & 13.0 & $0.90(\mathrm{t}, \mathrm{J}=6.4 \mathrm{~Hz})$ \\
\hline & & & 28 & 68.1 & $5.33(\mathrm{~s})$ \\
\hline
\end{tabular}




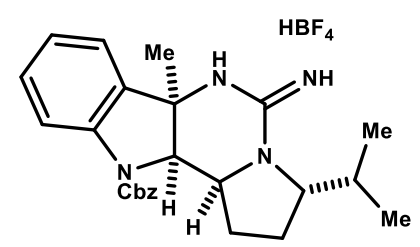

Adduct 12s: According to the representative procedure, $N$-amidinylpyrrolidine salt $\mathbf{S 1 2}(17.0 \mathrm{mg}$, $0.048 \mathrm{mmol}$ ) and benzyl 3-methyl- $1 H$-indole-1-carboxylate $(38.9 \mathrm{mg}, 0.146 \mathrm{mmol})$ was used as the dienophile. Purification by flash column chromatography $\left(\mathrm{SiO}_{2}, 1: 0 \rightarrow 9: 1 \mathrm{CH}_{2} \mathrm{Cl}_{2} / \mathrm{MeOH}\right)$ afforded adduct $12 \mathrm{~s}(9.7 \mathrm{mg}, 39 \%)$ as a brown oil.

${ }^{1} \mathbf{H}$ NMR $\left(600 \mathrm{MHz}, \mathrm{CD}_{3} \mathrm{OD}\right) \delta 7.61(\mathrm{~d}, J=7.7 \mathrm{~Hz}, 1 \mathrm{H}), 7.51-7.46(\mathrm{~m}, 2 \mathrm{H}), 7.45-7.36(\mathrm{~m}, 3 \mathrm{H})$, $7.33-7.27(\mathrm{~m}, 2 \mathrm{H}), 7.14(\mathrm{t}, J=7.0 \mathrm{~Hz}, 1 \mathrm{H}), 5.38-5.29(\mathrm{~m}, 2 \mathrm{H}), 4.95(\mathrm{~d}, J=3.1 \mathrm{~Hz}, 1 \mathrm{H}), 4.13-$ $4.06(\mathrm{~m}, 1 \mathrm{H}), 3.59-3.51(\mathrm{~m}, 1 \mathrm{H}), 2.18-2.05(\mathrm{~m}, 3 \mathrm{H}), 1.92-1.82(\mathrm{~m}, 1 \mathrm{H}), 1.76-1.63(\mathrm{~m}, 1 \mathrm{H}), 1.72$ (s, $3 \mathrm{H}), 0.91(\mathrm{~d}, J=5.5 \mathrm{~Hz}, 3 \mathrm{H}), 0.88(\mathrm{~d}, J=6.0 \mathrm{~Hz}, 3 \mathrm{H})$

${ }^{13}$ C NMR (150 MHz, $\left.\mathrm{CD}_{3} \mathrm{OD}\right) \delta 155.0,154.3,141.1,135.8,135.0,129.4,128.36,128.30,128.26$, $124.4,121.9,116.2,70.2,68.1,63.4,61.2,58.6,30.2,26.3,25.4,24.6,18.0,15.5$

IR (thin film) 3367, 2965, 2926, 2461, 1712, 1629, 1550, 1482, 1456, 1396, 1325, 1288, 1259, $1085,1030 \mathrm{~cm}^{-1}$

HRMS (ESI-TOF) m/z: $[\mathrm{M}]^{+}$calcd for $\left[\mathrm{C}_{25} \mathrm{H}_{31} \mathrm{~N}_{4} \mathrm{O}_{2}\right]^{+} 419.2447$, found 419.2463

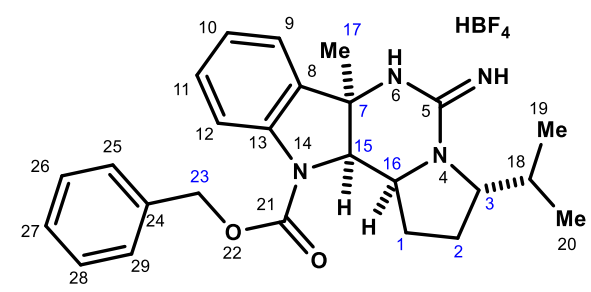

Assignment of Aliphatic Shifts (Solvent: $\mathrm{CD}_{3} \mathrm{OD}$ )

\begin{tabular}{|c|c|c|c|c|c|}
\hline atom & ${ }^{13} \mathrm{C}$ Shift & ${ }^{1} \mathrm{H}$ Shift & atom & ${ }^{13} \mathrm{C}$ Shift & ${ }^{1} \mathrm{H} \mathrm{Shift}$ \\
\hline 1 & 25.4 & $2.18-2.05(\mathrm{~m})$ & 16 & 58.6 & $4.13-4.06(\mathrm{~m})$ \\
\hline 2 & 24.6 & $1.92-1.82(\mathrm{~m})$ & 17 & 26.3 & $1.72(\mathrm{~s})$ \\
\hline & & $1.76-1.63(\mathrm{~m})$ & 18 & 30.2 & $2.18-2.05(\mathrm{~m})$ \\
\hline 3 & 63.4 & $3.59-3.51(\mathrm{~m})$ & 19 & 18.0 & $0.91(\mathrm{~d}, \mathrm{~J}=5.5 \mathrm{~Hz})$ \\
\hline 7 & 61.2 & NA & 20 & 15.5 & $0.88(\mathrm{~d}, \mathrm{~J}=6.0 \mathrm{~Hz})$ \\
\hline 15 & 70.2 & $4.95(\mathrm{~d}, \mathrm{~J}=3.1 \mathrm{~Hz})$ & 23 & 68.1 & $5.38-5.29(\mathrm{~m})$ \\
\hline
\end{tabular}




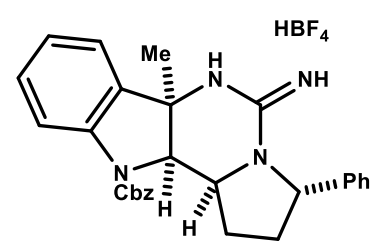

Adduct 12t: According to the representative procedure, $N$-amidinylpyrrolidine salt $\mathbf{S 1 5}$ (50.5 $\mathrm{mg}$, $0.131 \mathrm{mmol}$ ) and benzyl 3-methyl-1H-indole-1-carboxylate $(106.1 \mathrm{mg}, 0.40 \mathrm{mmol})$ was used as the dienophile. Purification by flash column chromatography $\left(\mathrm{SiO}_{2}, 1: 0 \rightarrow 9: 1 \mathrm{CHCl}_{3} / \mathrm{MeOH}\right)$ afforded adduct $\mathbf{1 2 t}$ (44.8 $\mathrm{mg}, 63 \%$ ) as a brown foam.

${ }^{1}$ H NMR (600 MHz, CD $\left.{ }_{3} \mathrm{OD}\right) \delta 7.67(\mathrm{~d}, J=8.0 \mathrm{~Hz}, 1 \mathrm{H}), 7.47(\mathrm{~d}, J=7.1 \mathrm{~Hz}, 2 \mathrm{H}), 7.42-7.38$ (m, $3 \mathrm{H}), 7.37-7.29(\mathrm{~m}, 5 \mathrm{H}), 7.16(\mathrm{~d}, J=7.5 \mathrm{~Hz}, 1 \mathrm{H}), 7.14-7.10(\mathrm{~m}, 2 \mathrm{H}), 5.34(\mathrm{~s}, 2 \mathrm{H}), 5.06(\mathrm{~d}, J=4.9$ $\mathrm{Hz}, 1 \mathrm{H}), 4.74(\mathrm{~d}, J=6.6 \mathrm{~Hz}, 1 \mathrm{H}), 4.60(\mathrm{bs}, 1 \mathrm{H}), 4.40-4.36(\mathrm{~m}, 1 \mathrm{H}), 2.31-2.23(\mathrm{~m}, 1 \mathrm{H}), 2.20-2.12$ $(\mathrm{m}, 1 \mathrm{H}), 2.05-1.96(\mathrm{~m}, 1 \mathrm{H}), 1.86-1.81(\mathrm{~m}, 1 \mathrm{H}), 1.78(\mathrm{~s}, 3 \mathrm{H})$

${ }^{13}$ C NMR (150 MHz, CD 3 OD) $\delta$ 155.5, 154.2, 141.2, 138.8, 135.7, 135.0, 129.5, 128.8, 128.4, $128.31,128.29,127.8,125.0,124.5,122.1,116.2,71.7,68.1,61.9,61.3,59.2,34.8,26.2,23.4$

IR (thin film) 3347, 3029, 2970, 2919, 2850, 2479, 1737, 1723, 1651, 1603, 1558, 1482, 1453, $1397,1365,1325,1282,1228,1216,1052,1028,972 \mathrm{~cm}^{-1}$

HRMS (ESI-TOF) m/z: $[\mathrm{M}]^{+}$calcd for $\left[\mathrm{C}_{28} \mathrm{H}_{29} \mathrm{~N}_{4} \mathrm{O}_{2}\right]^{+} 453.2291$, found 453.2285

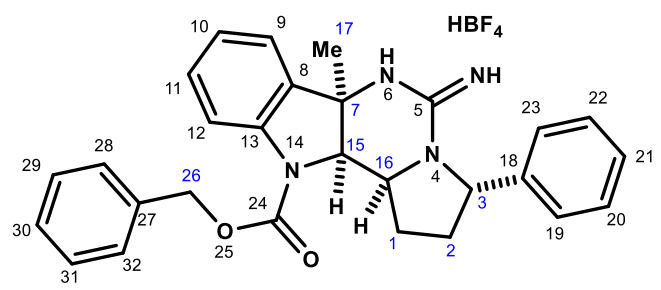

Assignment of Aliphatic Shifts (Solvent: CD $30 D$ )

\begin{tabular}{|c|c|c|c|c|c|}
\hline atom & ${ }^{13}$ C Shift & ${ }^{1} \mathrm{H}$ Shift & atom & ${ }^{13} \mathrm{C} \mathrm{Shift}$ & ${ }^{1} \mathrm{H}$ Shift \\
\hline 1 & 23.4 & $2.31-2.23(\mathrm{~m})$ & 7 & 61.9 & $\mathrm{NA}$ \\
\hline & & $2.05-1.96(\mathrm{~m})$ & 15 & 71.7 & $5.06(\mathrm{~d}, 4.9 \mathrm{~Hz})$ \\
\hline 2 & 34.8 & $2.20-2.12(\mathrm{~m})$ & 16 & 59.2 & $4.40-4.36(\mathrm{~m})$ \\
\hline & & $1.86-1.81(\mathrm{~m})$ & 17 & 26.2 & $1.78(\mathrm{~s})$ \\
\hline 3 & 61.3 & $4.74(\mathrm{~d}, \mathrm{~J}=6.6 \mathrm{~Hz})$ & 26 & 68.1 & $5.34(\mathrm{~s})$ \\
\hline
\end{tabular}




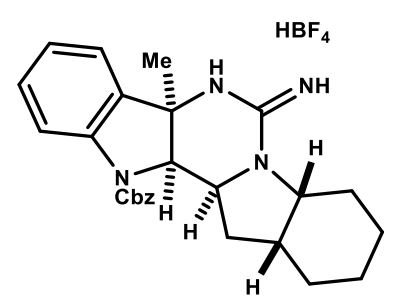

Adduct 12u: According to the representative procedure, $N$-amidinylpyrrolidine salt $\mathbf{S 2 0}$ (46.5 mg, $0.128 \mathrm{mmol}$ ) and benzyl 3-methyl- $1 H$-indole-1-carboxylate $(107.9 \mathrm{mg}, 0.41 \mathrm{mmol}, 3$ equiv) were used. Purification by flash column chromatography $\left(\mathrm{SiO}_{2}, 1: 0 \rightarrow 95: 5 \mathrm{CHCl}_{3} / \mathrm{MeOH}\right)$ afforded adduct $\mathbf{1 2 u}(57.9 \mathrm{mg}, 87 \%)$ as a brown foam.

${ }^{1} \mathbf{H}$ NMR $\left(600 \mathrm{MHz}, \mathrm{CDCl}_{3}\right) \delta 7.61(\mathrm{~d}, J=7.6 \mathrm{~Hz}, 1 \mathrm{H}), 7.50-7.45(\mathrm{~m}, 2 \mathrm{H}), 7.42-7.34(\mathrm{~m}, 3 \mathrm{H})$, $7.32-7.25(\mathrm{~m}, 2 \mathrm{H}), 7.12(\mathrm{t}, J=7.2 \mathrm{~Hz}, 1 \mathrm{H}), 5.35(\mathrm{~d}, J=11.6 \mathrm{~Hz}, 1 \mathrm{H}), 5.32(\mathrm{~d}, J=11.6 \mathrm{~Hz}, 1 \mathrm{H})$, 4.96-4.92 (m, 1H), 4.06-3.98 (m, 1H), 3.43-3.36 (m, 1H), 2.24-2.14 (m, 1H), 2.11-1.99 (m, 2H), $1.97-1.89(\mathrm{~m}, 1 \mathrm{H}), 1.73-1.62(\mathrm{~m}, 1 \mathrm{H}), 1.69(\mathrm{~s}, 3 \mathrm{H}), 1.59-1.51(\mathrm{~m}, 2 \mathrm{H}), 1.48-1.40(\mathrm{~m}, 1 \mathrm{H}), 1.35-$ $1.27(\mathrm{~m}, 1 \mathrm{H}), 1.24-1.07(\mathrm{~m}, 2 \mathrm{H})$

${ }^{13} \mathrm{C}$ NMR $\left(150 \mathrm{MHz}, \mathrm{CDCl}_{3}\right) \delta 154.9,154.3,141.4,135.9,135.1,129.3,128.4,128.3,128.3$, $124.5,122.0,116.2,71.9,67.9,61.7,56.9,56.7,37.0,26.4,26.2,24.9,24.8,22.7,19.0$

IR (thin film) 3359, 3246, 2930, 2860, 1704, 1660, 1602, 1584, 1557, 1482, 1454, 1394, 1326, $1279,1262,1013 \mathrm{~cm}^{-1}$

HRMS (ESI-TOF) m/z: $[\mathrm{M}]^{+}$calcd for $\left[\mathrm{C}_{26} \mathrm{H}_{31} \mathrm{~N}_{4} \mathrm{O}_{2}\right]^{+} 431.2447$, found 431.2457

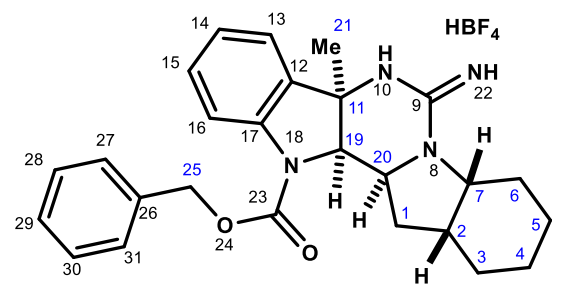

Assignment of Aliphatic Shifts (Solvent: $\left.\mathrm{CD}_{3} \mathrm{OD}\right)$

\begin{tabular}{|c|c|c|c|c|c|}
\hline atom & ${ }^{13} \mathrm{C}$ Shift & ${ }^{1} \mathrm{H}$ Shift & atom & ${ }^{13} \mathrm{C}$ Shift & ${ }^{1} \mathrm{H}$ Shift \\
\hline 1 & 26.4 & $2.24-2.14(\mathrm{~m})$ & 6 & 24.9 & $1.97-1.89(\mathrm{~m})$ \\
\hline & & $2.11-1.99(\mathrm{~m})$ & & & $1.24-1.07(\mathrm{~m})$ \\
\hline 2 & 37.0 & $2.11-1.99(\mathrm{~m})$ & 7 & 56.7 & $3.43-3.36(\mathrm{~m})$ \\
\hline 3 & 24.8 & $1.59-1.51(\mathrm{~m})$ & 11 & 61.7 & NA \\
\hline 4 & 19.0 & $1.48-1.40(\mathrm{~m})$ & 19 & 71.9 & $4.96-4.92(\mathrm{~m})$ \\
\hline & & $1.35-1.27(\mathrm{~m})$ & 20 & 56.9 & $4.06-3.98(\mathrm{~m})$ \\
\hline 5 & 22.7 & $1.73-1.62(\mathrm{~m})$ & 21 & 26.2 & $1.69(\mathrm{~s})$ \\
\hline & & $1.24-1.07(\mathrm{~m})$ & 25 & 67.9 & $5.35(\mathrm{~d}, \mathrm{~J}=11.6 \mathrm{~Hz})$ \\
\hline & & & & & $5.32(\mathrm{~d}, \mathrm{~J}=11.6 \mathrm{~Hz})$ \\
\hline
\end{tabular}




\section{G. Large Scale Cycloaddition Procedure}

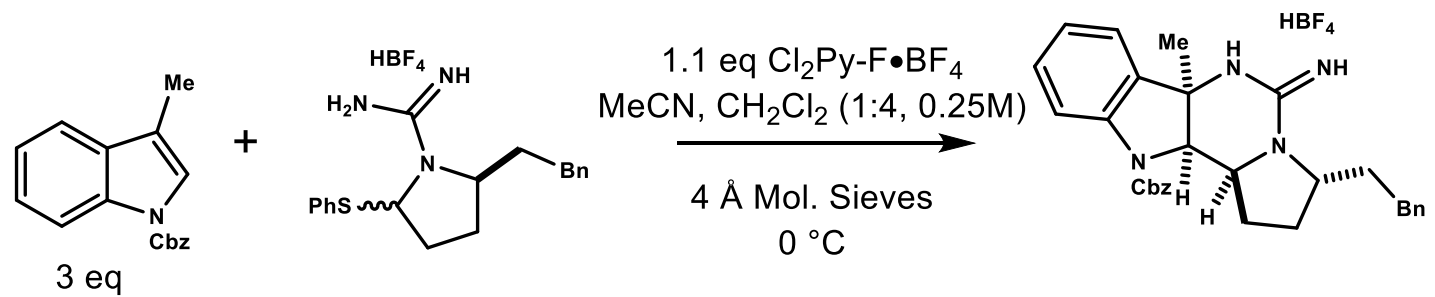

Adduct 12a: A $15 \mathrm{~mL}$ round bottom flask was charged with $4 \AA$ molecular sieves $(\sim 300 \mathrm{mg})$ and the system was flame-dried under vacuum. The system was then allowed to cool to room temperature under vacuum and then backfilled with argon. The flask was then charged with $N$ amidinylpyrrolidine salt 10 (413.7 $\mathrm{mg}, 1.001 \mathrm{mmol}, 1$ equiv) and benzyl 3-methyl- $H$-indole-1carboxylate (795.7 mg, $3.0 \mathrm{mmol}, 3$ equiv.) followed by the addition of $\mathrm{CH}_{2} \mathrm{Cl}_{2}(3.2 \mathrm{~mL})$ and $\mathrm{MeCN}\left(0.8 \mathrm{~mL}, 0.25 \mathrm{M}\right.$ final concentration). The system was then cooled to $0{ }^{\circ} \mathrm{C}$ and vigorously stirred for 15 minutes. Then 2,6-dichloro-1-fluoropyridinium tetrafluoroborate $(280.4 \mathrm{mg}, 1.10$ mmol, 1.1 equiv.) was added in one portion. The reaction was then allowed to slowly warm to room temperature overnight and progressed from a heterogenous white mixture to a deep purple heterogeneous solution. The reaction was then filtered and the solids were washed with $\mathrm{CH}_{2} \mathrm{Cl}_{2}$ $(20 \mathrm{~mL})$. The filtrate was then concentrated to a dark purple oil. The crude residue was then purified by flash column chromatography $\left(\mathrm{SiO}_{2}, 1: 0 \rightarrow 95: 5 \mathrm{CH}_{2} \mathrm{Cl}_{2} / \mathrm{MeOH}\right)$ to afford the desired cycloadduct 12a (551 mg, 97\%) as a light orange foam.

The spectroscopic properties of the product matched those reported above. 


\section{H. Deprotection of Cyclization Product}

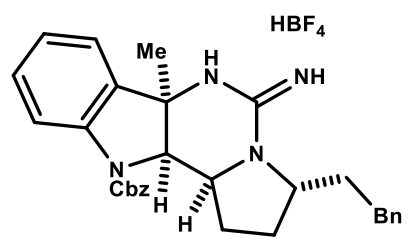

$12 \mathrm{a}$

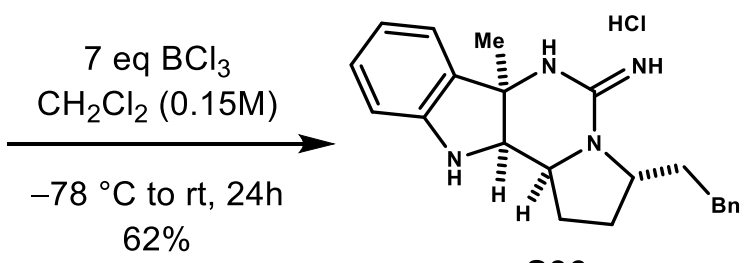

S36

Preparation of Cyclic Guanidine S36: A flame dried $25 \mathrm{~mL}$ round-bottom flask was charged with protected indoline 12a $(34 \mathrm{mg}, 0.06 \mathrm{mmol})$ in $\mathrm{CH}_{2} \mathrm{Cl}_{2}(7.5 \mathrm{~mL}, 0.15 \mathrm{M})$. The solution was then cooled to $-78{ }^{\circ} \mathrm{C}$ and $\mathrm{BCl}_{3}\left(0.42 \mathrm{~mL}, 0.42 \mathrm{mmol}, 7\right.$ equiv, $1.0 \mathrm{M}$ in $\left.\mathrm{CH}_{2} \mathrm{Cl}_{2}\right)$ was added. The reaction was then allowed to warm slowly to room temperature over $22 \mathrm{~h}$. The reaction was then quenched by the addition of sat. aq. $\mathrm{NaHCO}_{3}(5 \mathrm{~mL})$ and vigorously stirred for $2 \mathrm{~h}$. The mixture was then diluted with $\mathrm{CH}_{2} \mathrm{Cl}_{2}(5 \mathrm{~mL})$ and poured into a separatory funnel. The aqueous layer was then washed with $\mathrm{CH}_{2} \mathrm{Cl}_{2}(2 \times 25 \mathrm{~mL})$ and the combined organic layers were then dried over $\mathrm{Na}_{2} \mathrm{SO}_{4}$, filtered, and concentrated. The crude residue was then purified by flash column chromatography $\left(\mathrm{SiO}_{2}, 1: 0 \rightarrow 95: 5 \rightarrow 9: 1 \mathrm{CHCl}_{3} / \mathrm{MeOH}\right)$ to afford cyclic guanidine 13 (16 mg, $70 \%)$.

${ }^{1} \mathbf{H}$ NMR $\left(500 \mathrm{MHz}, \mathrm{CDCl}_{3}\right) \delta 7.25-7.10(\mathrm{~m}, 6 \mathrm{H}), 6.97(\mathrm{t}, J=7.5 \mathrm{~Hz}, 1 \mathrm{H}), 6.66(\mathrm{t}, J=7.5 \mathrm{~Hz}$, $1 \mathrm{H}), 6.61(\mathrm{~d}, J=7.7 \mathrm{~Hz}, 1 \mathrm{H}), 3.96-3.80(\mathrm{~m}, 2 \mathrm{H}), 3.75-3.64(\mathrm{~m}, 1 \mathrm{H}), 2.72-2.55(\mathrm{~m}, 2 \mathrm{H}), 2.34-$ $2.20(\mathrm{~m}, 1 \mathrm{H}), 2.19-2.08(\mathrm{~m}, 1 \mathrm{H}), 2.07-1.98(\mathrm{~m}, 1 \mathrm{H}), 1.95-1.85(\mathrm{~m}, 1 \mathrm{H}), 1.78-1.65(\mathrm{~m}, 2 \mathrm{H}), 1.25$ $(\mathrm{s}, 3 \mathrm{H})$

${ }^{13}$ C NMR $\left(125 \mathrm{MHz}, \mathrm{CDCl}_{3}\right) \delta 152.4,149.0,141.1,132.6,129.6,128.8,128.5,126.4,123.5$, $120.0,111.1,77.4,66.4,60.3,58.5,35.5,32.1,28.3,26.8,25.7$

IR (thin film) 3314, 3180, 3027, 2958, 2925, 2855, 1644, 1598, 1581, 1484, 1454, 1383, 1308, $1224,1181,1070,1030 \mathrm{~cm}^{-1}$

HRMS (ESI-TOF) m/z: [M] $]^{+}$calcd for $\left[\mathrm{C}_{22} \mathrm{H}_{27} \mathrm{~N}_{4}\right]^{+} 347.2236$, found 347.2225 


\section{Unsuccessful or Low Yielding Indole Dienophile Examples}

Table S3. Scope of unsuccessful or low yielding examples for the $\mathrm{N}$-amidinyliminium ion cycloaddition reaction. Potential reasons for low yield or failure are also included.

Unreactive Dienophiles (0-10\%)

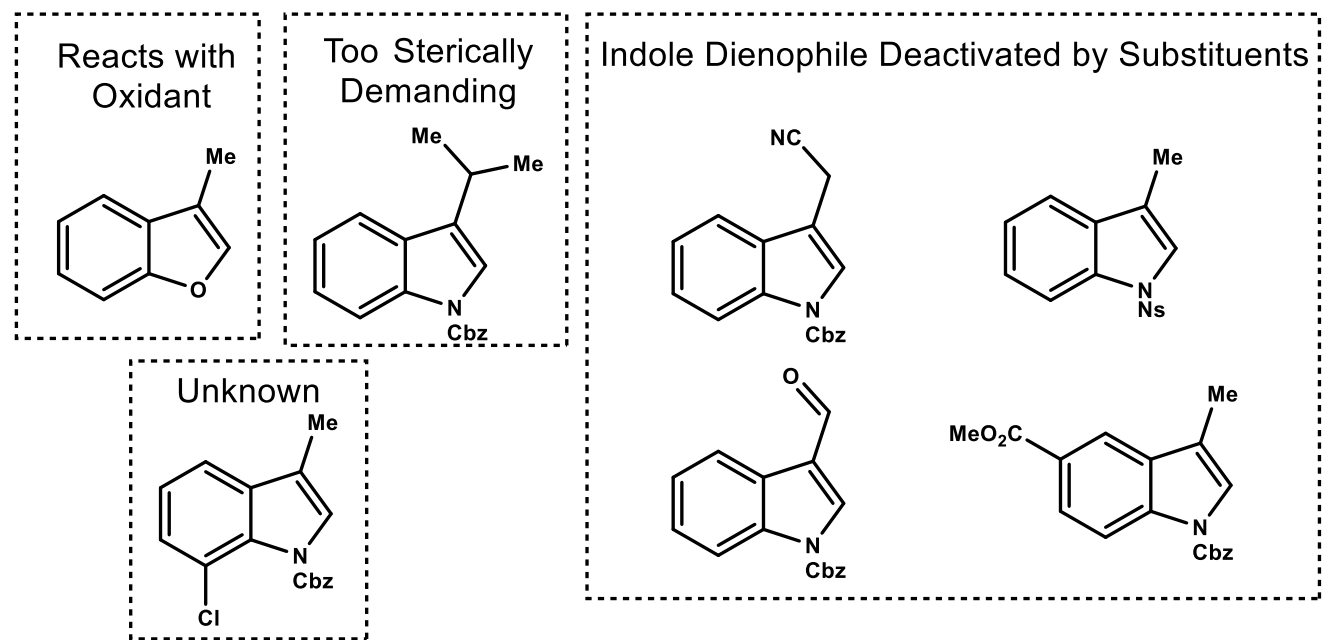

Low Yielding Acceptors (<25\%)
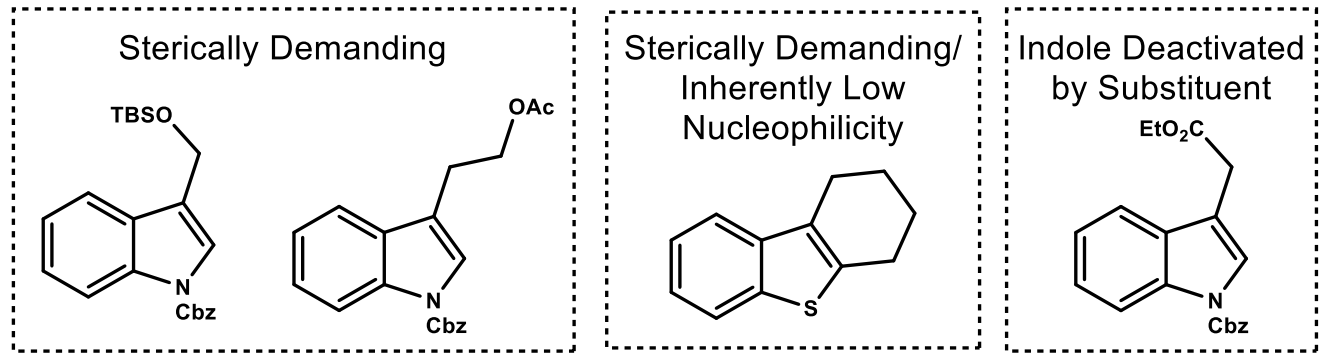


\section{J. X-Ray Structure and Analysis of NAIC Adduct 12j:}

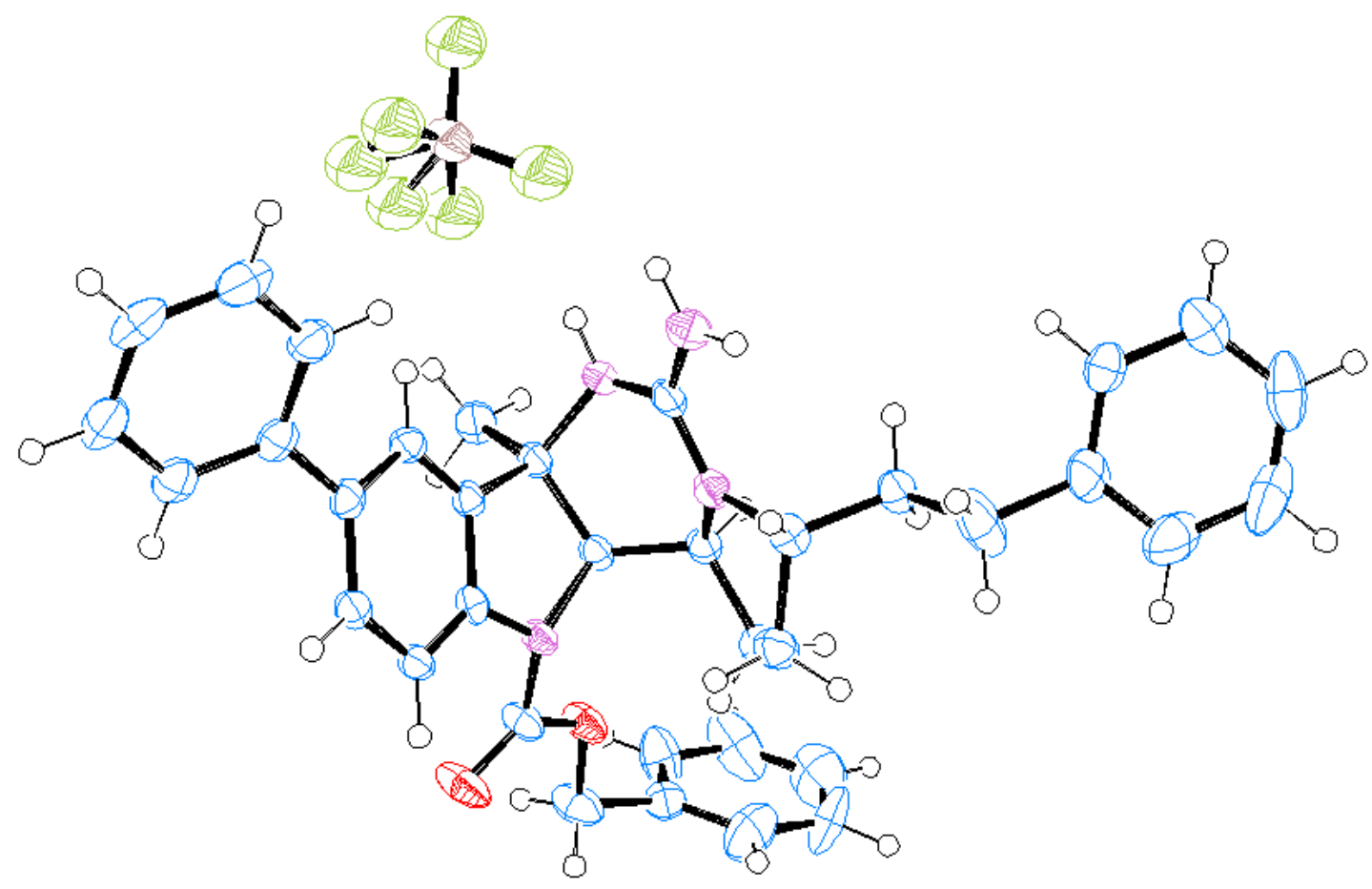

The thermal ellipsoid plot is shown at the $50 \%$ probability level and $\mathrm{H}$ atoms are shown as small spheres of arbitrary radii; Blue $=$ Carbon, Red $=$ Oxygen, Purple $=$ Nitrogen, Brown $=$ Boron, Green $=$ Fluorine

Table S4. Crystal data and structure refinement for leo273.

Identification code

Empirical formula

Formula weight

Temperature

Wavelength

Crystal system

Space group

Unit cell dimensions leo273

$\mathrm{C}_{36} \mathrm{H}_{37} \mathrm{~B} \mathrm{~F}_{4} \mathrm{~N}_{4} \mathrm{O}_{2}$

644.50

143(2) K

$0.71073 \AA$

Monoclinic

$\mathrm{C} 2 / \mathrm{c}$

$\mathrm{a}=34.971(2) \AA \quad \square=90^{\circ}$.

$\mathrm{b}=8.8637(5) \AA \quad \square=108.6202(8)^{\circ}$.

$\mathrm{c}=23.3261(14) \AA \quad \square=90^{\circ}$.

Volume

$6851.9(7) \AA^{3}$ 
Density (calculated)

Absorption coefficient

$\mathrm{F}(000)$

Crystal color

Crystal size

Theta range for data collection

Index ranges

Reflections collected

Independent reflections

Completeness to theta $=25.500^{\circ}$

Absorption correction

Max. and min. transmission

Refinement method

Data / restraints / parameters

Goodness-of-fit on $\mathrm{F}^{2}$

Final $\mathrm{R}$ indices $[\mathrm{I}>2 \operatorname{sigma}(\mathrm{I})=5324$ data $]$

$\mathrm{R}$ indices (all data, $0.82 \AA$ )

Largest diff. peak and hole
$1.250 \mathrm{Mg} / \mathrm{m}^{3}$

$0.093 \mathrm{~mm}^{-1}$

2704

colorless

$0.428 \times 0.293 \times 0.110 \mathrm{~mm}^{3}$

1.229 to $25.716^{\circ}$

$-42 \leq h \leq 42,-10 \leq k \leq 10,-28 \leq l \leq 28$

34671

$6524[\mathrm{R}(\mathrm{int})=0.0305]$

$100.0 \%$

Semi-empirical from equivalents

0.8620 and 0.8214

Full-matrix least-squares on $\mathrm{F}^{2}$

6524 / 0 / 417

1.034

$\mathrm{R} 1=0.0858, \mathrm{wR} 2=0.2247$

$\mathrm{R} 1=0.0997, \mathrm{wR} 2=0.2370$

1.561 and -1.341 e. $\AA^{-3}$ 


\section{Computational Procedures}

\section{K. Computational Methods}

All density functional theory (DFT) calculations were conducted with Gaussian $16 .{ }^{12}$ Geometry optimizations of all the intermediates and transition states were performed at the B3LYP ${ }^{13}$ level of theory with def2-SVP ${ }^{14}$ basis set, including solvation energy corrections and Grimme's D3 dispersion corrections. ${ }^{15}$ Based on the optimized structures, vibrational frequencies were calculated at the same level of theory to evaluate its zero-point vibrational energy (ZPVE) and thermal corrections at $298 \mathrm{~K}$. The single-point energies were computed with $\omega \mathrm{B}^{2} 7 \mathrm{X}-\mathrm{D}^{16}$ functional and def2-TZVPP ${ }^{14,17}$ basis set, including solvation energy corrections. Solvation energy corrections were evaluated with the CPCM model. ${ }^{18}$ Frontier molecular orbitals were calculated on DFT-optimized structures at the HF level of theory with the $6-31 \mathrm{G}(\mathrm{d})$ basis set and visualized with Multiwfn ${ }^{19}$ and VMD. ${ }^{20}$ The orbital $^{2}$ coefficients were calculated using the $\mathrm{NBO}^{21}$ module of Gaussian 16 and Multiwfn. Extensive conformational searches for the intermediates and transition states have been conducted to ensure that the lowest energy conformers were located. Intrinsic reaction coordinate (IRC) calculations of the transition states were performed to verify their positions in the free energy surface. The $3 \mathrm{D}$ diagrams of molecules were generated using CYLView. ${ }^{22}$ 


\section{Origins of the regioselectivity for the cycloaddition of $A$ and $C$}

To explore the origins of the regioselectivity for the cycloaddition of $\mathbf{A}$ and $\mathbf{C}$, we studied the orbital interaction between the reactants. Figure 5d suggests that the competition between TS5 and TS6 determines the regioselectivity, and TS5 is $3.7 \mathrm{kcal} / \mathrm{mol}$ more favorable than TS6. As shown in Figure S1, for benzothiophene (C), the HOMO-1 and HOMO are very similar in energy $(\Delta \Delta \mathrm{E}=0.6 \mathrm{eV})$. In addition, the orbital coefficients for HOMO-1 and HOMO indicate that $\mathrm{C} 2$ should be more nucleophilic compared to $\mathrm{C} 3$. Because the orbital coefficients for $\mathrm{C} 2$ and $\mathrm{C} 3$ are similar in HOMO (13.9\% vs. 17.8\%), while in HOMO-1, the coefficient for $\mathrm{C} 2$ is much bigger compared to that of $\mathrm{C} 3(11.0 \%$ vs. $0.0 \%)$.

The orbital interaction diagram (Figure S1) indicates that the dominant interaction is from HOMO (and/or HOMO-1) of $\mathbf{C}$ to the LUMO of $\mathbf{A}$. The interactions between HOMO of $\mathbf{C}$ and LUMO of $\mathbf{A}$ not only produce the primary orbital interaction (highlighted in green), but also the favorable secondary orbital interaction (SOI, grey line) between sulfur and nitrogen. The difference in nucleophilicity between C2 and C3 (orbital coefficients for $\mathrm{C} 2$ and $\mathrm{C} 3$ in HOMO and HOMO-1) of $\mathbf{C}$ determines the regioselectivity, making TS5 is more favorable compared to TS6.

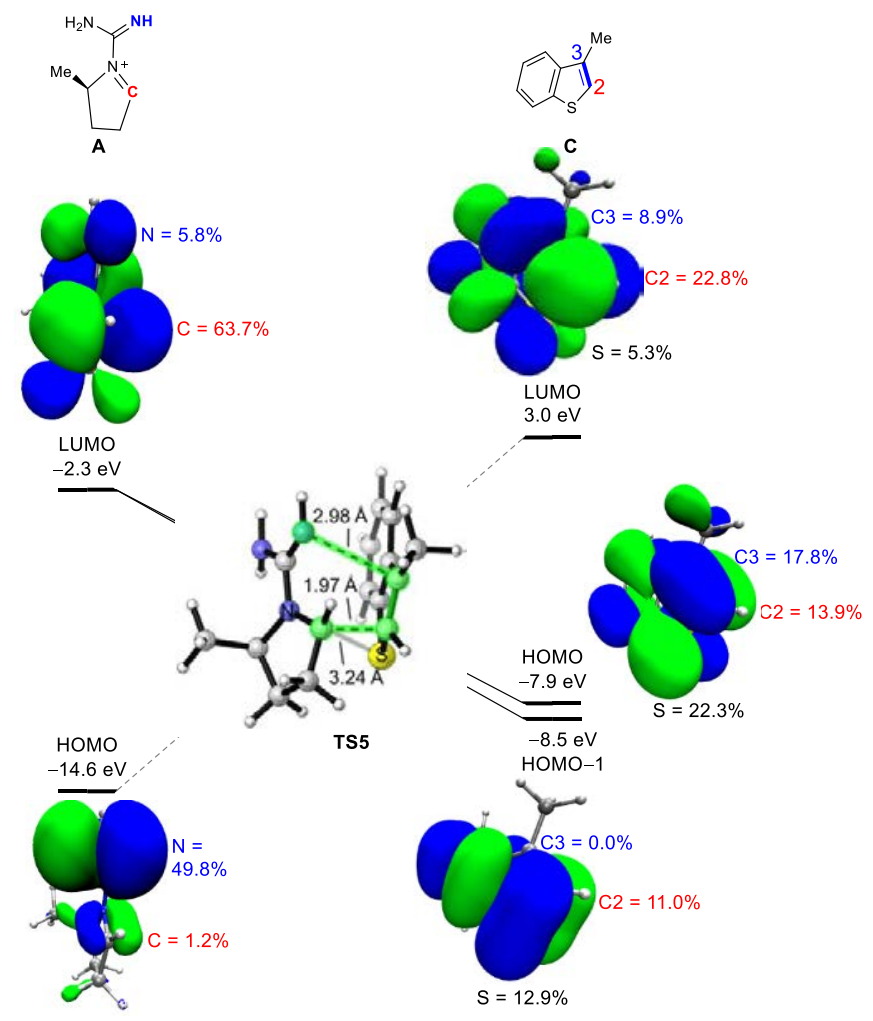

Figure S1. Orbital interaction diagram for the cycloaddition of $\mathbf{A}$ and C. Percentages given are the fraction of electron density at that position in that orbital. 


\section{Table of energies.}

Table S5. Energies in Figure 5, Figure 6, Figure 7 and Figure S1. Zero-point vibrational energy $(Z P V E)$, thermal correction to enthalpy $(T C H)$, thermal correction to Gibbs free energy $(T C G)$, energies $(E)$, enthalpies $(H)$, and Gibbs free energies $(G)$ (in Hartree) of the structures calculated at the $\omega$ B97X-D/def2-TZVPPCPCM(chloroform)//B3LYP-D3/def2-SVP-CPCM(chloroform) level of theory.

\begin{tabular}{cccccccc}
\hline Structures & $\boldsymbol{Z P V} \boldsymbol{T}$ & $\boldsymbol{T C H}$ & $\boldsymbol{T C G}$ & $\boldsymbol{E}$ & $\boldsymbol{H}$ & $\boldsymbol{G}$ & Imaginary Frequency \\
$\mathbf{A}$ & 0.184993 & 0.195187 & 0.150658 & -399.972008 & -399.473215 & -399.517744 & \\
$\mathbf{B}$ & 0.281633 & 0.299291 & 0.233537 & -862.106829 & -861.192062 & -861.257816 & \\
TS1 & 0.469743 & 0.496527 & 0.413497 & -1262.082608 & -1260.677863 & -1260.760893 & $350.7 i$ \\
TS2 & 0.470314 & 0.496773 & 0.415789 & -1262.082933 & -1260.676291 & -1260.757275 & $318.9 i$ \\
TS3 & 0.469692 & 0.496395 & 0.413466 & -1262.074915 & -1260.671029 & -1260.753958 & $354.8 i$ \\
TS4 & 0.470465 & 0.496932 & 0.415037 & -1262.074426 & -1260.668592 & -1260.750487 & $362.9 i$ \\
C & 0.141717 & 0.150789 & 0.108765 & -745.991479 & -745.482637 & -745.524660 & \\
TS5 & 0.328803 & 0.347477 & 0.283868 & -1145.955757 & -1144.957597 & -1145.021206 & $383.0 i$ \\
TS6 & 0.329454 & 0.347662 & 0.285972 & -1145.951963 & -1144.949044 & -1145.010734 & $293.4 i$ \\
TS7 & 0.328816 & 0.347444 & 0.283914 & -1145.952961 & -1144.954394 & -1145.017924 & $372.6 i$ \\
TS8 & 0.329419 & 0.347763 & 0.285265 & -1145.945786 & -1144.944573 & -1145.007071 & $371.8 i$ \\
TS9 & 0.329292 & 0.347810 & 0.284409 & -1145.953111 & -1144.953117 & -1145.016519 & $358.3 i$ \\
D & 0.330409 & 0.349088 & 0.285264 & -1145.960060 & -1144.955333 & -1145.019158 & $35.2 i$ \\
TS10 & 0.329803 & 0.347862 & 0.285671 & -1145.957213 & -1144.952237 & -1145.014428 & \\
PAC & 0.334251 & 0.352041 & 0.291070 & -1146.033369 & -1145.023344 & -1145.084316 & \\
\hline
\end{tabular}




\section{N. Cartesian coordinates of the structures}

A

$\begin{array}{lrrr}\mathrm{C} & -2.13822300 & 0.24610200 & -0.48351000 \\ \mathrm{C} & -1.97357600 & -1.21031900 & 0.00047100 \\ \mathrm{C} & -0.50877900 & -1.40396300 & 0.01169800 \\ \mathrm{C} & -0.75691900 & 0.90348400 & -0.26057000 \\ \mathrm{H} & -2.92752600 & 0.78372800 & 0.05672000 \\ \mathrm{H} & -2.47266000 & -1.96706300 & -0.62455100 \\ \mathrm{H} & -2.33684800 & -1.38458100 & 1.03156200 \\ \mathrm{H} & 0.03688900 & -2.33752600 & 0.17466700 \\ \mathrm{H} & -0.44278100 & 1.48153100 & -1.13978200 \\ \mathrm{H} & -2.39279700 & 0.25711500 & -1.55221200 \\ \mathrm{C} & -0.62988400 & 1.75615300 & 0.99786100 \\ \mathrm{H} & 0.40213800 & 2.11043800 & 1.13307500 \\ \mathrm{H} & -1.28384000 & 2.63427500 & 0.89727600 \\ \mathrm{H} & -0.93670200 & 1.19159300 & 1.89148800 \\ \mathrm{C} & 1.60184400 & -0.23208300 & -0.05291500 \\ \mathrm{~N} & 2.14142200 & -1.07643500 & 0.72469300 \\ \mathrm{H} & 3.16239900 & -1.02174300 & 0.71333100 \\ \mathrm{~N} & 2.12305600 & 0.79002400 & -0.78139900 \\ \mathrm{H} & 1.64615400 & 1.13010700 & -1.60948000 \\ \mathrm{H} & 3.13283200 & 0.88692300 & -0.79703700 \\ \mathrm{~N} & 0.14209000 & -0.30231000 & -0.15090400\end{array}$

B

C

$\begin{array}{llll}-3.20648900 & 0.83161100 & -0.00054600\end{array}$ $\begin{array}{lll}-2.55441800 & -0.42899300 & -0.00001500\end{array}$ $\begin{array}{llll}-3.26878400 & -1.63216100 & 0.00073100\end{array}$ $\begin{array}{llll}-4.66393300 & -1.54948800 & 0.00093800\end{array}$ $\begin{array}{llll}-5.32907500 & -0.30765300 & 0.00042000\end{array}$ $\begin{array}{llll}-4.60924200 & 0.88737300 & -0.00032500\end{array}$ $\begin{array}{llll}-2.18302300 & 1.86155400 & -0.00126500\end{array}$ $\begin{array}{llll}-0.98024100 & 1.21830600 & -0.00116400\end{array}$ $\begin{array}{llll}-2.75052000 & -2.58880800 & 0.00112600\end{array}$ $\begin{array}{llll}-5.24966500 & -2.47223900 & 0.00151400\end{array}$ $\begin{array}{llll}-6.42169900 & -0.28328800 & 0.00060300\end{array}$ $\begin{array}{llll}-5.12315400 & 1.85183300 & -0.00073000\end{array}$ $\begin{array}{llll}0.02838600 & 1.62067900 & -0.00156200\end{array}$ $\begin{array}{llll}-2.42628900 & 3.33740100 & -0.00196800\end{array}$ $\begin{array}{llll}-3.00865600 & 3.64425900 & -0.88739200\end{array}$ $\begin{array}{llll}-3.00829600 & 3.64515900 & 0.88338100\end{array}$ $\begin{array}{llll}-1.48201400 & 3.90127700 & -0.00244600\end{array}$ C $\quad-0.17171200-1.14094900 \quad-0.00007000$ 


$\begin{array}{lrrr}\mathrm{O} & -0.37193100 & -2.33506200 & 0.00103100 \\ \mathrm{O} & 1.03822000 & -0.56796400 & -0.00032500 \\ \mathrm{C} & 2.17428600 & -1.46610500 & 0.00061600 \\ \mathrm{H} & 2.11354100 & -2.11070000 & -0.88922600 \\ \mathrm{H} & 2.11289000 & -2.10952300 & 0.89127400 \\ \mathrm{C} & 3.41905600 & -0.62440200 & 0.00052400 \\ \mathrm{C} & 3.99660000 & -0.21019100 & -1.20918100 \\ \mathrm{C} & 3.99228300 & -0.20384700 & 1.21012600 \\ \mathrm{C} & 5.13001200 & 0.60800100 & -1.21081200 \\ \mathrm{H} & 3.55448500 & -0.53317700 & -2.15580700 \\ \mathrm{C} & 5.12562500 & 0.61439500 & 1.21152600 \\ \mathrm{H} & 3.54680400 & -0.52192900 & 2.15683800 \\ \mathrm{C} & 5.69593100 & 1.02190100 & 0.00028900 \\ \mathrm{H} & 5.57397200 & 0.92197400 & -2.15884100 \\ \mathrm{H} & 5.56618300 & 0.93336500 & 2.15947500 \\ \mathrm{H} & 6.58361100 & 1.65961700 & 0.00021200\end{array}$

\section{TS1}

$\begin{array}{lll}4.28867800 & -1.39562100 & -1.48262800\end{array}$

$\begin{array}{llll}3.16108700 & -1.71688600 & -2.28619500\end{array}$

$\begin{array}{llll}1.86371600 & -1.71105300 & -1.78822000\end{array}$

$\begin{array}{lll}1.70234100 & -1.36421900 & -0.43554200\end{array}$

$\begin{array}{llll}2.82408100 & -1.03437900 & 0.38761700\end{array}$

$\begin{array}{llll}4.13294100 & -1.05922600 & -0.15215500\end{array}$

$\begin{array}{llll}5.28395300 & -1.41447200 & -1.93075700\end{array}$

$\begin{array}{llll}3.31995100 & -1.97803200 & -3.33519200\end{array}$

$\begin{array}{llll}1.00548100 & -1.96468200 & -2.40580400\end{array}$

$\begin{array}{lll}4.98948100 & -0.79697300 & 0.47127200\end{array}$

$\begin{array}{llll}2.33889300 & -0.68830100 & 1.67194300\end{array}$

$\begin{array}{lll}0.91382400 & -0.67086800 & 1.58394300\end{array}$

$\begin{array}{lll}0.26703800 & -0.86800200 & 2.43773800\end{array}$

$\begin{array}{lll}0.58430700 & 1.23174900 & 1.31347500\end{array}$

$\begin{array}{lll}1.03348900 & 1.61296800 & 0.07297900\end{array}$

$\begin{array}{lll}3.11891800 & -0.30932000 & 2.87724700\end{array}$

$\begin{array}{lll}2.63642200 & -0.67281000 & 3.79605900\end{array}$

$\begin{array}{lll}4.14355500 & -0.70232300 & 2.82417600\end{array}$

$\begin{array}{lll}3.19176000 & 0.79006500 & 2.93302200\end{array}$

$\begin{array}{lll}3.11181400 & 2.26524100 & 0.86747500\end{array}$

$\begin{array}{llll}4.08698800 & 2.42148000 & 0.60569000\end{array}$

$\begin{array}{llll}2.39011500 & 1.97059300 & -0.14788000\end{array}$

$\begin{array}{lll}1.16806700 & 1.59603700 & 2.16149500\end{array}$

$\begin{array}{lll}-0.92042500 & 1.42567700 & 1.32751600\end{array}$

$\begin{array}{lll}-1.10417700 & 2.41303500 & 1.78466900\end{array}$

$\begin{array}{lll}-1.46307400 & 0.68529700 & 1.92564200\end{array}$

$\begin{array}{llll}-1.31164500 & 1.42241300 & -0.15634400\end{array}$ 


$\begin{array}{lrrr}\mathrm{H} & -2.19344000 & 2.04420500 & -0.35747300 \\ \mathrm{H} & -1.56529300 & 0.41457600 & -0.50119700 \\ \mathrm{C} & -0.06330600 & 1.94519600 & -0.88649900 \\ \mathrm{~N} & 2.74656400 & 1.97551500 & -1.47063700 \\ \mathrm{H} & 2.26286900 & 1.35912900 & -2.11441000 \\ \mathrm{H} & 3.72496100 & 2.12575000 & -1.68663400 \\ \mathrm{C} & -0.11431500 & 3.44796500 & -1.16734700 \\ \mathrm{H} & 0.82513900 & 3.80429700 & -1.61165700 \\ \mathrm{H} & -0.93131000 & 3.65699900 & -1.87420900 \\ \mathrm{H} & -0.30413000 & 4.01211200 & -0.24074400 \\ \mathrm{H} & 0.09136900 & 1.39688900 & -1.82841700 \\ \mathrm{~N} & 0.55843800 & -1.27421600 & 0.34088000 \\ \mathrm{C} & -0.69440600 & -1.80419800 & 0.01191100 \\ \mathrm{O} & -0.96186700 & -2.25013400 & -1.07798100 \\ \mathrm{O} & -1.50937700 & -1.74758200 & 1.06347900 \\ \mathrm{C} & -2.89012900 & -2.18660300 & 0.87134700 \\ \mathrm{H} & -2.88590000 & -3.07630600 & 0.22877800 \\ \mathrm{H} & -3.21590400 & -2.46180700 & 1.88153600 \\ \mathrm{C} & -3.72816800 & -1.07663900 & 0.29464100 \\ \mathrm{C} & -3.93929300 & -0.98531000 & -1.09005800 \\ \mathrm{C} & -4.24847800 & -0.08004100 & 1.13514900 \\ \mathrm{C} & -4.65424200 & 0.08998600 & -1.62536500 \\ \mathrm{H} & -3.52183500 & -1.75125600 & -1.74689700 \\ \mathrm{C} & -4.96028100 & 0.99693800 & 0.60057300 \\ \mathrm{H} & -4.09320800 & -0.14785500 & 2.21555500 \\ \mathrm{C} & -5.16191000 & 1.08431000 & -0.78186800 \\ \mathrm{H} & -4.81402700 & 0.15333200 & -2.70447700 \\ \mathrm{H} & -5.36126400 & 1.76798600 & 1.26288900 \\ \mathrm{H} & -5.71947300 & 1.92553600 & -1.20107900 \\ \mathrm{~T} & & & \end{array}$

\section{TS2}

C

C

C

C

C

C

$\mathrm{H}$

$\mathrm{H}$

$\mathrm{H}$

$\mathrm{H}$

C

C

$\mathrm{H}$

$\mathrm{N}$

$\begin{array}{rrr}-3.15050800 & -2.00999500 & -2.21601400 \\ -4.23208100 & -1.55072200 & -1.44742300 \\ -4.02987700 & -1.02021800 & -0.16848100 \\ -2.72727300 & -0.96783200 & 0.32877900 \\ -1.66537700 & -1.48752300 & -0.43350900 \\ -1.84096500 & -1.99434000 & -1.72026700 \\ -3.33194200 & -2.40856200 & -3.21655200 \\ -5.24398700 & -1.60715900 & -1.85419300 \\ -4.87167400 & -0.65669100 & 0.42371600 \\ -1.00354900 & -2.37528300 & -2.29985700 \\ -2.15574900 & -0.40201200 & 1.57604800 \\ -0.81283800 & -0.90373600 & 1.59371100 \\ -0.09894500 & -0.89869700 & 2.40840700 \\ 0.83649100 & 1.23638300 & 1.62513100\end{array}$ 


$\begin{array}{lrrr}\mathrm{H} & 1.82954000 & 1.01414300 & 1.70785200 \\ \mathrm{C} & 0.49903900 & 1.44264500 & 0.39621100 \\ \mathrm{C} & -1.80166200 & 1.44048400 & 1.13340100 \\ \mathrm{~N} & -0.86384400 & 1.61739800 & 0.12828800 \\ \mathrm{~N} & 1.32805700 & 1.48852400 & -0.68602100 \\ \mathrm{H} & 0.98108900 & 1.37230700 & -1.62893800 \\ \mathrm{H} & 2.32011500 & 1.33758400 & -0.55150800 \\ \mathrm{C} & -2.94838500 & -0.32190600 & 2.86355900 \\ \mathrm{H} & -3.25989200 & -1.32829600 & 3.18005300 \\ \mathrm{H} & -2.34837100 & 0.12500700 & 3.66960100 \\ \mathrm{H} & -3.85560300 & 0.28449000 & 2.73407800 \\ \mathrm{C} & -1.41379600 & 2.21380300 & -1.11916000 \\ \mathrm{H} & -1.10482100 & 1.60427200 & -1.98362600 \\ \mathrm{C} & -2.93050400 & 2.09557000 & -0.89486000 \\ \mathrm{H} & -3.47362700 & 2.92754500 & -1.36296000 \\ \mathrm{H} & -3.30679600 & 1.16280600 & -1.33137700 \\ \mathrm{C} & -3.08563500 & 2.07884100 & 0.63364000 \\ \mathrm{H} & -3.99769300 & 1.57575700 & 0.97690500 \\ \mathrm{H} & -3.11658200 & 3.10139200 & 1.04562200 \\ \mathrm{C} & -0.94635700 & 3.66062500 & -1.30041500 \\ \mathrm{H} & 0.14873000 & 3.73152900 & -1.35020500 \\ \mathrm{H} & -1.36120300 & 4.06331800 & -2.23658200 \\ \mathrm{H} & -1.29784200 & 4.28844500 & -0.46675300 \\ \mathrm{H} & -1.43988600 & 1.70184100 & 2.13074000 \\ \mathrm{~N} & -0.50027300 & -1.43325000 & 0.39343200 \\ \mathrm{C} & 0.79564400 & -1.89147800 & 0.00931200 \\ \mathrm{O} & 1.04077000 & -2.26782200 & -1.10579100 \\ \mathrm{O} & 1.62246600 & -1.82461700 & 1.03709600 \\ \mathrm{C} & 3.02624200 & -2.16196800 & 0.79694400 \\ \mathrm{H} & 3.06627200 & -3.02434900 & 0.11932600 \\ \mathrm{H} & 3.39550000 & -2.45048700 & 1.78775200 \\ \mathrm{C} & 3.76128300 & -0.97023100 & 0.24535800 \\ \mathrm{C} & 3.93218000 & -0.81176500 & -1.13862600 \\ \mathrm{C} & 4.22575600 & 0.03080300 & 1.11491400 \\ \mathrm{C} & 4.55652200 & 0.33277200 & -1.64573300 \\ \mathrm{H} & 3.55792200 & -1.58056400 & -1.81746100 \\ \mathrm{C} & 4.84178500 & 1.17923000 & 0.60829100 \\ \mathrm{H} & 4.10722400 & -0.09216800 & 2.19519900 \\ \mathrm{C} & 5.00673700 & 1.33188900 & -0.77425500 \\ \mathrm{H} & 4.68640800 & 0.44829200 & -2.72425300 \\ \mathrm{H} & 5.19680100 & 1.95388200 & 1.29170100 \\ \mathrm{H} & 5.48882400 & 2.22816500 & -1.17148600\end{array}$

TS3

$\mathrm{C}$

$\begin{array}{lll}-2.87300000 & -1.17363200 & 0.29286400\end{array}$ 


\begin{tabular}{|c|c|c|c|}
\hline $\mathrm{C}$ & -1.79227500 & -2.01148700 & -0.11301500 \\
\hline $\mathrm{C}$ & -2.00853100 & -3.15743700 & -0.89181000 \\
\hline $\mathrm{C}$ & -3.32524300 & -3.44443400 & -1.24095700 \\
\hline $\mathrm{C}$ & -4.41225000 & -2.62529900 & -0.84449600 \\
\hline $\mathrm{C}$ & -4.19788400 & -1.49052900 & -0.08156000 \\
\hline $\mathrm{H}$ & -1.17928100 & -3.78956200 & -1.20016400 \\
\hline $\mathrm{H}$ & -3.52664200 & -4.33246900 & -1.84489800 \\
\hline $\mathrm{H}$ & -5.42440200 & -2.89583300 & -1.15166500 \\
\hline $\mathrm{H}$ & -5.02537500 & -0.84682700 & 0.22213500 \\
\hline $\mathrm{C}$ & -2.33492400 & -0.10872000 & 1.06310100 \\
\hline $\mathrm{C}$ & -0.91718600 & -0.22510300 & 1.01698200 \\
\hline $\mathrm{H}$ & -0.26480300 & 0.11204800 & 1.82133800 \\
\hline $\mathrm{C}$ & -3.06695400 & 0.90787800 & 1.85659300 \\
\hline $\mathrm{H}$ & -2.49886500 & 1.84616800 & 1.92918700 \\
\hline $\mathrm{H}$ & -4.06489800 & 1.11416100 & 1.45122600 \\
\hline $\mathrm{H}$ & -3.18075000 & 0.53144000 & 2.88925600 \\
\hline $\mathrm{C}$ & -0.43040700 & 1.08343900 & -0.40387300 \\
\hline $\mathrm{H}$ & -0.95157500 & 0.52987500 & -1.18858300 \\
\hline $\mathrm{C}$ & 1.07659300 & 1.16292700 & -0.37175400 \\
\hline $\mathrm{H}$ & 1.40096300 & 1.40209400 & -1.39966900 \\
\hline $\mathrm{H}$ & 1.58387500 & 0.24091200 & -0.08641600 \\
\hline $\mathrm{N}$ & -0.89583900 & 2.34958600 & -0.17108000 \\
\hline $\mathrm{C}$ & 1.33314200 & 2.33383100 & 0.58099700 \\
\hline $\mathrm{H}$ & 1.29587400 & 1.98604000 & 1.62513500 \\
\hline $\mathrm{H}$ & 2.31040700 & 2.80847500 & 0.42393700 \\
\hline $\mathrm{C}$ & 0.16585600 & 3.29510300 & 0.28632000 \\
\hline $\mathrm{H}$ & -0.16633800 & 3.80024300 & 1.20716600 \\
\hline $\mathrm{C}$ & 0.50147800 & 4.33140400 & -0.78962700 \\
\hline $\mathrm{H}$ & 0.88949500 & 3.84187000 & -1.69615600 \\
\hline $\mathrm{H}$ & -0.37875400 & 4.92625000 & -1.06805100 \\
\hline $\mathrm{H}$ & 1.27425500 & 5.01472400 & -0.40698600 \\
\hline $\mathrm{N}$ & -2.90510600 & 1.84439700 & -1.17839600 \\
\hline $\mathrm{H}$ & -3.86692000 & 2.15511800 & -1.32475100 \\
\hline $\mathrm{C}$ & -2.21854800 & 2.70755800 & -0.52290000 \\
\hline $\mathrm{N}$ & -2.60531200 & 3.95513200 & -0.11567100 \\
\hline $\mathrm{H}$ & -2.13193300 & 4.42628000 & 0.64533000 \\
\hline $\mathrm{H}$ & -3.57638300 & 4.21134100 & -0.24610700 \\
\hline $\mathrm{N}$ & -0.61645300 & -1.48347500 & 0.41321300 \\
\hline $\mathrm{C}$ & 0.60317600 & -2.16478700 & 0.49580100 \\
\hline $\mathrm{O}$ & 0.85720200 & -3.15944300 & -0.13949400 \\
\hline $\mathrm{O}$ & 1.40468100 & -1.56778500 & 1.38110800 \\
\hline $\mathrm{C}$ & 2.78963800 & -2.02229100 & 1.46545700 \\
\hline $\mathrm{H}$ & 3.06671700 & -1.84970500 & 2.51224300 \\
\hline $\mathrm{H}$ & 2.81901900 & -3.09713900 & 1.24612100 \\
\hline
\end{tabular}




$\begin{array}{lrrr}\mathrm{C} & 3.64045400 & -1.21962900 & 0.51789400 \\ \mathrm{C} & 3.74536800 & -1.59609600 & -0.83112000 \\ \mathrm{C} & 4.24805100 & -0.03032500 & 0.94832600 \\ \mathrm{C} & 4.44133300 & -0.78948100 & -1.73570900 \\ \mathrm{H} & 3.26313500 & -2.51602600 & -1.16986800 \\ \mathrm{C} & 4.94538700 & 0.77600000 & 0.04416300 \\ \mathrm{H} & 4.16621900 & 0.27023000 & 1.99650500 \\ \mathrm{C} & 5.03915700 & 0.39905600 & -1.30032500 \\ \mathrm{H} & 4.51800300 & -1.08877300 & -2.78384600 \\ \mathrm{H} & 5.41560200 & 1.70033800 & 0.38862100 \\ \mathrm{H} & 5.58266800 & 1.02940700 & -2.00851300\end{array}$

\section{TS4}

$\mathrm{C}$

$\begin{array}{lll}4.35865500 & -2.45509600 & -0.81562000\end{array}$

$\begin{array}{llll}\mathrm{C} & 2.03554700 & -3.21450300 & -0.88277900\end{array}$

$\begin{array}{llll}\mathrm{C} & 1.71981200 & -2.11593900 & -0.08452900\end{array}$

$\begin{array}{llll}\mathrm{C} & 2.67996000 & -1.16990600 & 0.32934700\end{array}$

$\begin{array}{llll}\mathrm{C} & 4.01857800 & -1.34907300 & -0.02900900\end{array}$

$\mathrm{H} \quad 5.40179600 \quad-2.61556100 \quad-1.09666200$

$\mathrm{H} \quad 3.66915500 \quad-4.21749300 \quad-1.86269900$

$\mathrm{H} \quad \begin{array}{llll}1.27101400 & -3.92035900 & -1.20075600\end{array}$

$\begin{array}{llll}\mathrm{H} & 4.78993800 & -0.65535800 & 0.31167600\end{array}$

$\begin{array}{llll}\mathrm{C} & 1.97531500 & -0.09655800 & 1.07132400\end{array}$

$\begin{array}{llll}\mathrm{C} & 0.64767900 & -0.60105900 & 1.21986000\end{array}$

$\begin{array}{llll}\mathrm{H} & -0.14684500 & -0.22988800 & 1.85603200\end{array}$

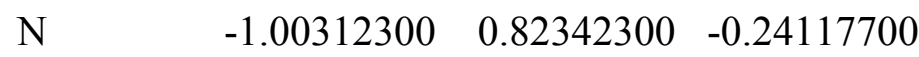

$\mathrm{H} \quad-1.99763800 \quad 0.70414400 \quad-0.04877600$

$\begin{array}{llll}\mathrm{C} & -0.58570800 & 2.00239200 & 0.06976500\end{array}$

$\begin{array}{llll}\mathrm{N} & -1.34420000 & 3.05688100 & 0.49632400\end{array}$

$\mathrm{H} \quad-2.32008700 \quad 2.86956000 \quad 0.69675500$

$\mathrm{H} \quad-0.93084500 \quad 3.81399900 \quad 1.02677400$

$\begin{array}{llll}\mathrm{N} & 0.79080200 & 2.23882800 & -0.05651700\end{array}$

$\begin{array}{llll}\mathrm{C} & 1.64073400 & 1.22495400 & -0.42774100\end{array}$

$\begin{array}{llll}\mathrm{H} & 1.21157900 & 0.47681000 & -1.09649300\end{array}$

$\begin{array}{llll}\mathrm{C} & 1.44423700 & 3.57605500 & 0.02124300\end{array}$

$\begin{array}{llll}\mathrm{H} & 1.18977300 & 4.05269100 & 0.98110400\end{array}$

$\begin{array}{llll}\mathrm{C} & 2.93849800 & 3.21046200 & -0.02723800\end{array}$

$\mathrm{H} \quad 3.52612100 \quad 3.98630100 \quad-0.53570800$

$\begin{array}{llll}\mathrm{H} & 3.34045000 & 3.10394200 & 0.98838200\end{array}$

$\begin{array}{llll}\mathrm{C} & 2.96712000 & 1.86616000 & -0.77082300\end{array}$

$\begin{array}{llll}\mathrm{H} & 3.83699300 & 1.24167400 & -0.53983600\end{array}$

$\begin{array}{llll}\mathrm{H} & 2.97459000 & 2.01260200 & -1.86468100\end{array}$

$\begin{array}{llll}\mathrm{C} & 1.01191400 & 4.47239300 & -1.14316800\end{array}$

$\mathrm{H} \quad-0.07583000 \quad 4.62366600 \quad-1.15752100$ 


$\begin{array}{lrrr}\mathrm{H} & 1.49756900 & 5.45480500 & -1.04590900 \\ \mathrm{H} & 1.31334500 & 4.03003400 & -2.10541600 \\ \mathrm{C} & 2.62224800 & 0.69403900 & 2.18519200 \\ \mathrm{H} & 2.76623900 & 0.05683900 & 3.07110600 \\ \mathrm{H} & 1.99797000 & 1.55110900 & 2.47753900 \\ \mathrm{H} & 3.61105700 & 1.06488800 & 1.88426300 \\ \mathrm{~N} & 0.48420300 & -1.73950200 & 0.51064700 \\ \mathrm{C} & -0.77238300 & -2.36516600 & 0.28391100 \\ \mathrm{O} & -0.95332100 & -3.15281100 & -0.60657500 \\ \mathrm{O} & -1.64751400 & -1.94784100 & 1.18522200 \\ \mathrm{C} & -3.05600600 & -2.28792100 & 0.99734800 \\ \mathrm{H} & -3.44093900 & -2.42385600 & 2.01500700 \\ \mathrm{H} & -3.11906300 & -3.23446500 & 0.44592800 \\ \mathrm{C} & -3.73969900 & -1.15633800 & 0.27900100 \\ \mathrm{C} & -4.25324000 & -0.06915400 & 1.00429400 \\ \mathrm{C} & -3.77147200 & -1.12321000 & -1.12404300 \\ \mathrm{C} & -4.78307500 & 1.03991100 & 0.33651900 \\ \mathrm{H} & -4.22952300 & -0.08847700 & 2.09716900 \\ \mathrm{C} & -4.30007500 & -0.01474800 & -1.79186000 \\ \mathrm{H} & -3.36016000 & -1.96175000 & -1.69117300 \\ \mathrm{C} & -4.80254900 & 1.06949100 & -1.06352300 \\ \mathrm{H} & -5.18456400 & 1.88012400 & 0.90864500 \\ \mathrm{H} & -4.31604800 & 0.00515800 & -2.88401000 \\ \mathrm{H} & -5.21309800 & 1.93666800 & -1.58628800 \\ \mathrm{C} & & & \\ \mathrm{C} & 0.01576600 & 0.56842000 & 0.00005400 \\ \mathrm{C} & 0.40707900 & -0.79684900 & -0.00004100 \\ \mathrm{C} & 1.75823000 & -1.17390500 & 0.00003100 \\ \mathrm{C} & 2.72635000 & -0.17161800 & 0.00019800 \\ \mathrm{C} & 2.35714900 & 1.18961700 & 0.00029200 \\ \mathrm{C} & 1.01627600 & 1.56192900 & 0.00022300 \\ \mathrm{C} & -1.42547100 & 0.73359900 & -0.00004800 \\ \mathrm{C} & -2.06688900 & -0.47212300 & -0.00020300 \\ \mathrm{H} & 2.04446700 & -2.22811400 & -0.00004200 \\ \mathrm{H} & 3.78439400 & -0.44528700 & 0.00025700 \\ \mathrm{H} & 3.13422500 & 1.95797800 & 0.00042200 \\ \mathrm{H} & 0.73375400 & 2.61753400 & 0.00029500 \\ \mathrm{~S} & -0.99206600 & -1.85306500 & -0.00024100 \\ \mathrm{H} & -3.14491600 & -0.63675400 & -0.00030100 \\ \mathrm{C} & -2.09965500 & 2.07429700 & 0.00001500 \\ \mathrm{H} & -1.80866400 & 2.66403400 & 0.88582500 \\ \mathrm{H} & -1.80853500 & 2.66418200 & -0.88565400 \\ \mathrm{H} & -3.19468100 & 1.97526200 & -0.00007300\end{array}$

TS5 


$\begin{array}{lrrr}\mathrm{C} & 3.50595200 & 1.19753500 & 0.07757700 \\ \mathrm{C} & 3.12766300 & 1.48678600 & -1.25777700 \\ \mathrm{C} & 2.11541700 & 0.78064700 & -1.89542700 \\ \mathrm{C} & 1.46549300 & -0.23846800 & -1.17936700 \\ \mathrm{C} & 1.82125300 & -0.53355300 & 0.17099700 \\ \mathrm{C} & 2.86690000 & 0.19917600 & 0.78804400 \\ \mathrm{H} & 4.30600100 & 1.77502600 & 0.54482900 \\ \mathrm{H} & 3.64441000 & 2.28353200 & -1.79806800 \\ \mathrm{H} & 1.83446900 & 1.00823900 & -2.92514400 \\ \mathrm{H} & 3.14595200 & -0.01544500 & 1.82101300 \\ \mathrm{C} & 1.00367800 & -1.55088600 & 0.73845600 \\ \mathrm{C} & -0.06507600 & -1.91975200 & -0.12958600 \\ \mathrm{H} & -0.49089700 & -2.92488300 & -0.06812400 \\ \mathrm{C} & -1.59460000 & -0.93797400 & 0.62473400 \\ \mathrm{~S} & 0.20212000 & -1.27806300 & -1.77730300 \\ \mathrm{~N} & -1.43867600 & 0.40814600 & 0.45194700 \\ \mathrm{C} & 1.22239700 & -2.20698800 & 2.05692400 \\ \mathrm{H} & 1.88317600 & -3.08038200 & 1.90936900 \\ \mathrm{H} & 1.69555800 & -1.53432000 & 2.78205400 \\ \mathrm{H} & 0.28065100 & -2.58216300 & 2.48181300 \\ \mathrm{~N} & -0.22996000 & 0.59547300 & 2.40524300 \\ \mathrm{H} & 0.45952600 & 1.15907100 & 2.90587000 \\ \mathrm{C} & -0.58624800 & 1.14874600 & 1.30346200 \\ \mathrm{H} & -1.44830700 & -1.30040700 & 1.64387800 \\ \mathrm{C} & -2.82868400 & -1.35518700 & -0.14517800 \\ \mathrm{H} & -3.66192700 & -1.34586200 & 0.57877100 \\ \mathrm{H} & -2.77500600 & -2.37157400 & -0.55587500 \\ \mathrm{C} & -2.99771200 & -0.25636200 & -1.20655600 \\ \mathrm{H} & -4.05279800 & -0.07087200 & -1.44899500 \\ \mathrm{H} & -2.48767400 & -0.53625300 & -2.13679500 \\ \mathrm{C} & -2.33703000 & 0.99366800 & -0.58476600 \\ \mathrm{~N} & -0.25310100 & 2.38990100 & 0.83600300 \\ \mathrm{H} & -0.27178900 & 2.57858100 & -0.15936500 \\ \mathrm{H} & 0.43763600 & 2.91548400 & 1.35817900 \\ \mathrm{C} & -3.34353500 & 1.96084500 & 0.04132100 \\ \mathrm{H} & -2.83839900 & 2.79196000 & 0.55215100 \\ \mathrm{H} & -3.98541800 & 2.37926600 & -0.74834400 \\ \mathrm{H} & -3.98336400 & 1.43722100 & 0.76870200 \\ \mathrm{H} & -1.72876900 & 1.51876800 & -1.33856500 \\ \mathrm{~T} & & & \end{array}$

\section{TS6}

$\begin{array}{lrrr}C & -3.25554900 & 0.87240300 & -1.55736600 \\ \mathrm{C} & -3.37834300 & 1.29053200 & -0.22318500 \\ \mathrm{C} & -2.56675400 & 0.74802000 & 0.77759500 \\ \mathrm{C} & -1.62288900 & -0.22846000 & 0.44060800\end{array}$




$\begin{array}{lrrr}\mathrm{C} & -1.56540300 & -0.67878200 & -0.89116900 \\ \mathrm{C} & -2.34908300 & -0.13206600 & -1.90837500 \\ \mathrm{H} & -3.89144300 & 1.31514400 & -2.32690300 \\ \mathrm{H} & -4.11924500 & 2.04896600 & 0.03824500 \\ \mathrm{H} & -2.67418500 & 1.08307300 & 1.81056500 \\ \mathrm{H} & -2.27629900 & -0.49171200 & -2.93632600 \\ \mathrm{C} & -0.59528500 & -0.87076100 & 1.30914300 \\ \mathrm{C} & -0.08496900 & -2.02646500 & 0.61017000 \\ \mathrm{H} & 0.45513200 & -2.85011000 & 1.06880900 \\ \mathrm{~S} & -0.47381600 & -2.07002600 & -1.03334200 \\ \mathrm{~N} & 2.53429200 & -1.66001800 & 0.54914700 \\ \mathrm{H} & 3.06016400 & -2.44688300 & 0.16384400 \\ \mathrm{C} & 2.38000700 & -0.70950600 & -0.31531800 \\ \mathrm{C} & 0.87120200 & 0.29192900 & 1.27481900 \\ \mathrm{~N} & 1.54615800 & 0.34382300 & 0.05635800 \\ \mathrm{~N} & 2.93635700 & -0.63108100 & -1.55867900 \\ \mathrm{H} & 2.53073200 & -0.03672200 & -2.27109600 \\ \mathrm{H} & 3.44710400 & -1.43560900 & -1.90082200 \\ \mathrm{C} & -0.88180600 & -1.03584600 & 2.79548000 \\ \mathrm{H} & -1.79481100 & -1.63152300 & 2.93994000 \\ \mathrm{H} & -0.04876800 & -1.54531600 & 3.30075400 \\ \mathrm{H} & -1.03176500 & -0.06209600 & 3.28227000 \\ \mathrm{C} & 1.47274600 & 1.66459200 & -0.62404500 \\ \mathrm{H} & 1.16605600 & 1.51942800 & -1.67243600 \\ \mathrm{C} & 0.36112200 & 2.37134600 & 0.16938100 \\ \mathrm{H} & 0.51634400 & 3.45804500 & 0.20453700 \\ \mathrm{H} & -0.61512100 & 2.18762500 & -0.29700800 \\ \mathrm{C} & 0.42880600 & 1.71902200 & 1.55756900 \\ \mathrm{H} & -0.50254500 & 1.79755600 & 2.12957800 \\ \mathrm{H} & 1.21647600 & 2.18059400 & 2.17586300 \\ \mathrm{C} & 2.81212900 & 2.40531400 & -0.56578900 \\ \mathrm{H} & 3.61761900 & 1.82984900 & -1.04171400 \\ \mathrm{H} & 2.71884200 & 3.36773400 & -1.09079700 \\ \mathrm{H} & 3.10033000 & 2.60613200 & 0.47777800 \\ \mathrm{H} & 1.43320100 & -0.23045400 & 2.05348800 \\ \mathrm{H} & & & \end{array}$

\section{TS7}

$\begin{array}{lrrr}\mathrm{C} & -2.07355700 & 0.39143900 & 0.45018600 \\ \mathrm{C} & -2.50293700 & -0.84712000 & -0.11135800 \\ \mathrm{C} & -3.71473700 & -0.94578400 & -0.81097900 \\ \mathrm{C} & -4.50004000 & 0.19636700 & -0.92717700 \\ \mathrm{C} & -4.09702200 & 1.43163700 & -0.36577500 \\ \mathrm{C} & -2.89743700 & 1.53540400 & 0.31631300 \\ \mathrm{H} & -4.03572100 & -1.89373800 & -1.24625900 \\ \mathrm{H} & -5.44970200 & 0.13794700 & -1.46425400\end{array}$




$\begin{array}{lrrr}\mathrm{H} & -4.73910600 & 2.30736500 & -0.47883800 \\ \mathrm{H} & -2.58015500 & 2.48901800 & 0.74184500 \\ \mathrm{C} & -0.81249400 & 0.28183700 & 1.10561400 \\ \mathrm{C} & -0.21987800 & -0.99569100 & 0.92887600 \\ \mathrm{H} & 0.44610700 & -1.39718000 & 1.69661700 \\ \mathrm{~S} & -1.37761500 & -2.14501100 & 0.20196900 \\ \mathrm{C} & -0.19963900 & 1.31929100 & 1.98017500 \\ \mathrm{H} & 0.89517600 & 1.22858300 & 2.00628800 \\ \mathrm{H} & -0.47141000 & 2.33794500 & 1.67798900 \\ \mathrm{H} & -0.55424700 & 1.16015700 & 3.01452700 \\ \mathrm{C} & 1.16309100 & -0.74987800 & -0.51434100 \\ \mathrm{H} & 0.42119800 & -0.54940100 & -1.28840000 \\ \mathrm{C} & 1.96080900 & -2.03212900 & -0.47357500 \\ \mathrm{H} & 2.33450800 & -2.20736500 & -1.49768500 \\ \mathrm{H} & 1.37775600 & -2.91699600 & -0.19142900 \\ \mathrm{~N} & 2.02631700 & 0.27062300 & -0.24809800 \\ \mathrm{C} & 3.10571200 & -1.68603600 & 0.48724400 \\ \mathrm{H} & 2.78583600 & -1.83033200 & 1.53060000 \\ \mathrm{H} & 4.00383500 & -2.29689000 & 0.32733800 \\ \mathrm{C} & 3.36981100 & -0.19355900 & 0.20896900 \\ \mathrm{H} & 3.64303200 & 0.33202700 & 1.13754200 \\ \mathrm{C} & 4.43762700 & 0.03959300 & -0.86325700 \\ \mathrm{H} & 4.20510800 & -0.53006200 & -1.77608200 \\ \mathrm{H} & 4.51856600 & 1.10166000 & -1.13127300 \\ \mathrm{H} & 5.41352500 & -0.29705900 & -0.48306100 \\ \mathrm{~N} & 0.52788800 & 1.79658800 & -1.09587600 \\ \mathrm{H} & 0.30499300 & 2.78810600 & -1.19494900 \\ \mathrm{C} & 1.65898800 & 1.61179500 & -0.51986200 \\ \mathrm{~N} & 2.57130200 & 2.55264300 & -0.13114200 \\ \mathrm{H} & 3.27259500 & 2.34807400 & 0.56960400 \\ \mathrm{H} & 2.30160800 & 3.52634500 & -0.20212500 \\ \mathrm{~T} & & & \end{array}$

\section{TS8}

$\mathrm{C}$

$$
\begin{array}{rrr}
-3.58455500 & -1.88217600 & 0.01725800 \\
-4.26129900 & -0.92598700 & -0.75599300 \\
-3.74011100 & 0.36050700 & -0.91120300 \\
-2.52680900 & 0.65155700 & -0.28366900 \\
-1.80488600 & -0.30476100 & 0.45854600 \\
-2.36090600 & -1.58086900 & 0.62127700 \\
-4.02182900 & -2.87346000 & 0.15446100 \\
-5.21405700 & -1.18064700 & -1.22520300 \\
-4.27445700 & 1.11874600 & -1.48658800 \\
-1.85478400 & -2.33231500 & 1.23054300 \\
-0.49640400 & 0.20841700 & 0.94379100 \\
-0.47848100 & 1.63333600 & 0.75182700
\end{array}
$$




\begin{tabular}{lrrr}
$\mathrm{H}$ & 0.16836400 & 2.32512400 & 1.28316800 \\
$\mathrm{~S}$ & -1.74140800 & 2.23876600 & -0.20617700 \\
$\mathrm{~N}$ & 1.58714800 & 2.16427300 & -0.65417800 \\
$\mathrm{H}$ & 1.91244100 & 3.12617100 & -0.54060800 \\
$\mathrm{C}$ & 2.43763000 & 1.29906700 & -0.20607300 \\
$\mathrm{~N}$ & 3.70292000 & 1.54027800 & 0.24496600 \\
$\mathrm{H}$ & 3.99972800 & 2.50439200 & 0.33330000 \\
$\mathrm{H}$ & 4.18063100 & 0.87897500 & 0.84425500 \\
$\mathrm{~N}$ & 2.01806700 & -0.03124300 & -0.19372600 \\
$\mathrm{C}$ & 0.71407000 & -0.36073300 & -0.50596900 \\
$\mathrm{H}$ & 0.24955100 & 0.25737900 & -1.27825900 \\
$\mathrm{C}$ & 2.89994300 & -1.20609000 & 0.05449500 \\
$\mathrm{H}$ & 3.42659700 & -1.07354500 & 1.01333700 \\
$\mathrm{C}$ & 1.88857000 & -2.36195900 & 0.14581500 \\
$\mathrm{H}$ & 2.30978800 & -3.29710300 & -0.24656700 \\
$\mathrm{H}$ & 1.60252400 & -2.54268900 & 1.19029100 \\
$\mathrm{C}$ & 0.68757400 & -1.86648800 & -0.67163500 \\
$\mathrm{H}$ & -0.26558000 & -2.33027000 & -0.39908400 \\
$\mathrm{H}$ & 0.82918400 & -2.06408900 & -1.74816600 \\
$\mathrm{C}$ & 3.91204700 & -1.38314300 & -1.08107500 \\
$\mathrm{H}$ & 4.54554300 & -0.49386100 & -1.20216500 \\
$\mathrm{H}$ & 4.56132900 & -2.24331700 & -0.85984300 \\
$\mathrm{H}$ & 3.39494700 & -1.57363400 & -2.03424900 \\
$\mathrm{C}$ & 0.10944900 & -0.33474600 & 2.22519100 \\
$\mathrm{H}$ & -0.46158800 & 0.02029800 & 3.09618000 \\
$\mathrm{H}$ & 1.15174500 & -0.00330100 & 2.33838300 \\
$\mathrm{H}$ & 0.09051400 & -1.43186700 & 2.24072500 \\
$\mathrm{~T}$ & & & \\
\hline
\end{tabular}

\section{TS9}

C

C

C

C

C

C

$\mathrm{H}$

$\mathrm{H}$

$\mathrm{H}$

$\mathrm{H}$

C

C

$\mathrm{H}$

C

S

$\mathrm{N}$

$$
\begin{array}{rrr}
-4.03910400 & -1.46130100 & 0.62743500 \\
-4.55580100 & -0.55503800 & -0.33188200 \\
-3.81431500 & 0.52955600 & -0.78755300 \\
-2.52519800 & 0.71421800 & -0.26699100 \\
-1.98058400 & -0.19508900 & 0.69485300 \\
-2.76743000 & -1.29044600 & 1.14050900 \\
-4.65452700 & -2.30007900 & 0.95797600 \\
-5.56333400 & -0.71232500 & -0.72405900 \\
-4.22626900 & 1.21963700 & -1.52568600 \\
-2.36613100 & -1.98674200 & 1.87887200 \\
-0.66561000 & 0.15847400 & 1.09175800 \\
-0.13905800 & 1.24162900 & 0.32928200 \\
0.56783400 & 1.93785000 & 0.79414300 \\
1.13421500 & 0.51183900 & -0.92225300 \\
-1.42298700 & 2.00409100 & -0.65789400 \\
2.21584500 & -0.01884400 & -0.26263400
\end{array}
$$




\begin{tabular}{|c|c|c|c|}
\hline $\mathrm{C}$ & 0.10262300 & -0.50129800 & 2.18345500 \\
\hline $\mathrm{H}$ & 1.17995000 & -0.30204900 & 2.12039800 \\
\hline $\mathrm{H}$ & -0.24663600 & -0.08756200 & 3.14712100 \\
\hline $\mathrm{H}$ & -0.07056700 & -1.58612200 & 2.21868800 \\
\hline $\mathrm{N}$ & 3.76901900 & 0.08136300 & 1.43700700 \\
\hline $\mathrm{H}$ & 4.42766300 & 0.69118300 & 1.92535700 \\
\hline $\mathrm{C}$ & 3.12168900 & 0.71513500 & 0.53354100 \\
\hline $\mathrm{H}$ & 1.27915300 & 1.47719600 & -1.41194000 \\
\hline $\mathrm{C}$ & 0.51847500 & -0.59860400 & -1.74229200 \\
\hline $\mathrm{H}$ & 0.97732700 & -0.55788500 & -2.74465500 \\
\hline $\mathrm{H}$ & -0.56438400 & -0.48498700 & -1.88273700 \\
\hline $\mathrm{C}$ & 0.92858200 & -1.86799800 & -0.98322300 \\
\hline $\mathrm{H}$ & 1.01659300 & -2.74323000 & -1.64068600 \\
\hline $\mathrm{H}$ & 0.17721300 & -2.11049300 & -0.21866900 \\
\hline $\mathrm{C}$ & 2.27736400 & -1.50781400 & -0.32964800 \\
\hline $\mathrm{N}$ & 3.11336900 & 2.07574900 & 0.28694100 \\
\hline $\mathrm{H}$ & 3.06674200 & 2.37737600 & -0.68281600 \\
\hline $\mathrm{H}$ & 3.78372600 & 2.61637300 & 0.82480100 \\
\hline $\mathrm{C}$ & 3.49450400 & -1.95341200 & -1.13906400 \\
\hline $\mathrm{H}$ & 4.42107500 & -1.58476800 & -0.67751700 \\
\hline $\mathrm{H}$ & 3.53554500 & -3.05291900 & -1.16611500 \\
\hline $\mathrm{H}$ & 3.43836600 & -1.58220300 & -2.17475500 \\
\hline $\mathrm{H}$ & 2.35872600 & -1.90068200 & 0.69182700 \\
\hline \multicolumn{4}{|l|}{ D } \\
\hline $\mathrm{C}$ & -4.17884300 & -1.37282400 & 0.71771200 \\
\hline $\mathrm{C}$ & -4.67331200 & -0.43300200 & -0.22834600 \\
\hline $\mathrm{C}$ & -3.87988600 & 0.58236800 & -0.74627800 \\
\hline $\mathrm{C}$ & -2.54782300 & 0.66777300 & -0.31291100 \\
\hline $\mathrm{C}$ & -2.02349900 & -0.27583800 & 0.64032100 \\
\hline $\mathrm{C}$ & -2.87590200 & -1.30068200 & 1.15424600 \\
\hline $\mathrm{H}$ & -4.84282800 & -2.15319000 & 1.09313600 \\
\hline $\mathrm{H}$ & -5.71182500 & -0.51313700 & -0.55859300 \\
\hline $\mathrm{H}$ & -4.28152500 & 1.29430500 & -1.46878600 \\
\hline $\mathrm{H}$ & -2.48872700 & -2.01868100 & 1.87837600 \\
\hline $\mathrm{C}$ & -0.68473100 & -0.03093800 & 0.96763900 \\
\hline $\mathrm{C}$ & -0.07353800 & 1.04445500 & 0.16746600 \\
\hline $\mathrm{H}$ & 0.43539400 & 1.78999700 & 0.80334700 \\
\hline $\mathrm{C}$ & 1.09929700 & 0.56344500 & -0.85803400 \\
\hline $\mathrm{S}$ & -1.39628000 & 1.86288500 & -0.79445800 \\
\hline $\mathrm{N}$ & 2.24343300 & 0.03376600 & -0.17593900 \\
\hline $\mathrm{C}$ & 0.06919100 & -0.70157400 & 2.05316900 \\
\hline $\mathrm{H}$ & 1.15112700 & -0.71496500 & 1.87217500 \\
\hline $\mathrm{H}$ & -0.07519800 & -0.09580900 & 2.96856100 \\
\hline $\mathrm{H}$ & -0.29841200 & -1.71353300 & 2.26573000 \\
\hline
\end{tabular}




\begin{tabular}{|c|c|c|c|}
\hline $\mathrm{N}$ & 3.74839000 & 0.08705100 & 1.57334800 \\
\hline $\mathrm{H}$ & 4.31076300 & 0.71881200 & 2.14623800 \\
\hline $\mathrm{C}$ & 3.02107800 & 0.73907400 & 0.73913500 \\
\hline $\mathrm{H}$ & 1.32937100 & 1.48874000 & -1.40653600 \\
\hline $\mathrm{C}$ & 0.67729200 & -0.56184400 & -1.81210900 \\
\hline $\mathrm{H}$ & 1.20563800 & -0.42015400 & -2.76694600 \\
\hline $\mathrm{H}$ & -0.39869100 & -0.55334200 & -2.03246100 \\
\hline $\mathrm{C}$ & 1.15674000 & -1.83725200 & -1.11026000 \\
\hline $\mathrm{H}$ & 1.31228500 & -2.67235700 & -1.80722100 \\
\hline $\mathrm{H}$ & 0.41379900 & -2.17009600 & -0.36901100 \\
\hline $\mathrm{C}$ & 2.46185000 & -1.41301900 & -0.41547800 \\
\hline $\mathrm{N}$ & 2.83383800 & 2.11900000 & 0.69492800 \\
\hline $\mathrm{H}$ & 2.79068800 & 2.54368300 & -0.22849800 \\
\hline $\mathrm{H}$ & 3.43459900 & 2.64657000 & 1.32103600 \\
\hline $\mathrm{C}$ & 3.71664600 & -1.65002400 & -1.25672300 \\
\hline $\mathrm{H}$ & 4.59742700 & -1.23046100 & -0.75020700 \\
\hline $\mathrm{H}$ & 3.87939200 & -2.73027100 & -1.39494100 \\
\hline $\mathrm{H}$ & 3.62325900 & -1.18250200 & -2.25004500 \\
\hline $\mathrm{H}$ & 2.58696600 & -1.91919100 & 0.55233100 \\
\hline \multicolumn{4}{|c|}{ TS10 } \\
\hline $\mathrm{C}$ & -4.72975500 & -0.00481700 & 0.45994100 \\
\hline $\mathrm{C}$ & -4.49498000 & -1.21035800 & -0.26236900 \\
\hline $\mathrm{C}$ & -3.25489500 & -1.52062000 & -0.79870300 \\
\hline $\mathrm{C}$ & -2.20074300 & -0.60968800 & -0.60812800 \\
\hline $\mathrm{C}$ & -2.41649400 & 0.62076300 & 0.11452000 \\
\hline $\mathrm{C}$ & -3.71458900 & 0.90134800 & 0.64874100 \\
\hline $\mathrm{H}$ & -5.72620200 & 0.19309500 & 0.85838600 \\
\hline $\mathrm{H}$ & -5.32072400 & -1.91333700 & -0.39680200 \\
\hline $\mathrm{H}$ & -3.09794200 & -2.45025700 & -1.34760300 \\
\hline $\mathrm{H}$ & -3.88929800 & 1.82949800 & 1.19438300 \\
\hline $\mathrm{C}$ & -1.27248300 & 1.41276400 & 0.18686900 \\
\hline $\mathrm{C}$ & -0.09701800 & 0.83105900 & -0.50568600 \\
\hline $\mathrm{H}$ & 0.06588500 & 1.43156200 & -1.42135500 \\
\hline $\mathrm{C}$ & 1.26808900 & 0.87734200 & 0.32645900 \\
\hline $\mathrm{S}$ & -0.58554700 & -0.81428200 & -1.15217200 \\
\hline $\mathrm{N}$ & 2.07064000 & -0.30822600 & 0.15341100 \\
\hline $\mathrm{C}$ & -1.15447800 & 2.71945400 & 0.87716700 \\
\hline $\mathrm{H}$ & -0.48778500 & 2.60679600 & 1.75213300 \\
\hline $\mathrm{H}$ & -0.67690000 & 3.46660200 & 0.22404200 \\
\hline $\mathrm{H}$ & -2.11808100 & 3.10737500 & 1.22656300 \\
\hline $\mathrm{N}$ & 0.78701800 & -1.37067600 & 1.74098700 \\
\hline $\mathrm{H}$ & 0.61130800 & -2.27822100 & 2.17528700 \\
\hline $\mathrm{C}$ & 1.76425500 & -1.43962300 & 0.89854000 \\
\hline $\mathrm{H}$ & 0.98282100 & 0.97167900 & 1.38073600 \\
\hline
\end{tabular}




\begin{tabular}{|c|c|c|c|}
\hline $\mathrm{C}$ & 2.18212500 & 2.00872200 & -0.16607500 \\
\hline $\mathrm{H}$ & 2.80601700 & 2.35311900 & 0.67212500 \\
\hline $\mathrm{H}$ & 1.63140000 & 2.88131400 & -0.54451800 \\
\hline $\mathrm{C}$ & 3.03800600 & 1.31179400 & -1.23150400 \\
\hline $\mathrm{H}$ & 3.96357100 & 1.85417100 & -1.46891900 \\
\hline $\mathrm{H}$ & 2.46745700 & 1.19970200 & -2.16802400 \\
\hline $\mathrm{C}$ & 3.31446400 & -0.07043100 & -0.61178700 \\
\hline $\mathrm{N}$ & 2.56864000 & -2.53583300 & 0.66289500 \\
\hline $\mathrm{H}$ & 3.00476000 & -2.63289200 & -0.24742600 \\
\hline $\mathrm{H}$ & 2.24993900 & -3.41432300 & 1.05512900 \\
\hline $\mathrm{C}$ & 4.57261900 & -0.08932900 & 0.26407600 \\
\hline $\mathrm{H}$ & 4.67982500 & -1.05162400 & 0.78267900 \\
\hline $\mathrm{H}$ & 5.46438700 & 0.06903500 & -0.36174800 \\
\hline $\mathrm{H}$ & 4.53573800 & 0.70690500 & 1.02368900 \\
\hline $\mathrm{H}$ & 3.41374400 & -0.82882600 & -1.40740800 \\
\hline \multicolumn{4}{|c|}{$\mathbf{P}_{\text {AC }}$} \\
\hline $\mathrm{C}$ & 4.37394700 & 0.52323100 & -0.40113300 \\
\hline $\mathrm{C}$ & 4.29749800 & -0.73451900 & -1.00973600 \\
\hline $\mathrm{C}$ & 3.11706300 & -1.48554700 & -0.95658400 \\
\hline $\mathrm{C}$ & 2.01155800 & -0.95096700 & -0.28933700 \\
\hline $\mathrm{C}$ & 2.07391000 & 0.31570400 & 0.31622200 \\
\hline $\mathrm{C}$ & 3.25898500 & 1.05044100 & 0.26382500 \\
\hline $\mathrm{H}$ & 5.30402000 & 1.09427200 & -0.43908100 \\
\hline $\mathrm{H}$ & 5.16999900 & -1.14452400 & -1.52411300 \\
\hline $\mathrm{H}$ & 3.06618000 & -2.47391700 & -1.41798600 \\
\hline $\mathrm{H}$ & 3.32345900 & 2.03266900 & 0.73774500 \\
\hline $\mathrm{C}$ & 0.75081700 & 0.78256000 & 0.91403800 \\
\hline $\mathrm{C}$ & -0.08557700 & -0.49734400 & 1.16079400 \\
\hline $\mathrm{H}$ & 0.17855900 & -0.90000700 & 2.14865700 \\
\hline $\mathrm{C}$ & -1.58229700 & -0.19314700 & 1.13188900 \\
\hline $\mathrm{S}$ & 0.43876100 & -1.74944000 & -0.10829900 \\
\hline $\mathrm{N}$ & -1.93910900 & 0.44009800 & -0.14926200 \\
\hline $\mathrm{C}$ & 0.90992200 & 1.61384300 & 2.18967900 \\
\hline $\mathrm{H}$ & 1.50021600 & 1.05964400 & 2.93292700 \\
\hline $\mathrm{H}$ & 1.42668400 & 2.56059400 & 1.97341400 \\
\hline $\mathrm{H}$ & -0.07055900 & 1.86242300 & 2.62096900 \\
\hline $\mathrm{N}$ & 0.05872800 & 1.60578300 & -0.11776300 \\
\hline $\mathrm{H}$ & 0.61485900 & 2.35488400 & -0.51739100 \\
\hline $\mathrm{C}$ & -1.11264900 & 1.32457200 & -0.70950500 \\
\hline $\mathrm{H}$ & -1.77740900 & 0.51653100 & 1.95196500 \\
\hline $\mathrm{C}$ & -2.54321300 & -1.39744900 & 1.23103900 \\
\hline $\mathrm{H}$ & -3.37119500 & -1.14818000 & 1.90944200 \\
\hline $\mathrm{H}$ & -2.04112600 & -2.28423600 & 1.64212400 \\
\hline $\mathrm{C}$ & -3.05873600 & -1.61669400 & -0.20119400 \\
\hline
\end{tabular}




$\begin{array}{lrrr}\mathrm{H} & -4.04699900 & -2.09601700 & -0.22611900 \\ \mathrm{H} & -2.36209600 & -2.24901500 & -0.77026300 \\ \mathrm{C} & -3.09055200 & -0.20809700 & -0.81907700 \\ \mathrm{~N} & -1.43877000 & 1.94290900 & -1.85545100 \\ \mathrm{H} & -2.38618400 & 1.93132200 & -2.20971800 \\ \mathrm{H} & -0.77485400 & 2.54371400 & -2.32868700 \\ \mathrm{C} & -4.39642300 & 0.54855100 & -0.56755500 \\ \mathrm{H} & -4.34229700 & 1.58754400 & -0.92791000 \\ \mathrm{H} & -5.22346300 & 0.04848100 & -1.09322400 \\ \mathrm{H} & -4.63572100 & 0.57757700 & 0.50665100 \\ \mathrm{H} & -2.88370600 & -0.25509600 & -1.89946900\end{array}$




\section{References}

1. Overman, L.E.; Wolfe, J.P. J. Org. Chem. 2001, 66, 3167-3175.

2. Stowell, J.C.; Polito, M.A. J. Org. Chem. 1992, 57, 2195-2196.

3. (a) Hoffman, H.M.R. \& Rabe, J. J. Org. Chem. 1985, 50(20), 3849-3859. (b) "2-Hydroxy-5alkyltetrahydrofuran and its production intermediates/ Production of 2-Hydroxy-5-alkyltetrahydrofuran and its novel intermediate” Jpn. Kokai Tokkyo Koho (1981), JP 56071085 A 19810613.

4. Loozen, H.J.J. J. Org. Chem. 1975, 40, 520-521.

5. Arizpe, A.; Sayago, F.J.; Jiménez, A.I.; Ordóñez, M.; Cativiela, C. Eur. J. Org. Chem. 2011, 2011(16), 3074-3081.

6. Heller, S.T.; Schultz, E.E.; Sarpong, R. Angew. Chem. Int. Ed. 2012, 51, 8304-8308.

7. Sharma, S.K.; Miller, M.J.; Payne, S.M. J. Med. Chem. 1989, 32, 357-367.

8. Prieto, M.; Zurita, E.; Rosa, E.; Muñoz, L.; Lloyd-Williams, P.; Giralt, E. J. Org. Chem. 2004, 69(20), 6812-6820.

9. Jia, Y.; Zhu, J. J. Org. Chem. 2006, 71, 7826-7834.

10. Zhang, Z.; Smal, V.; Retailleau, P.; Voituriez, A.; Frison, G.; Marinetti, A.; Guinchard, X. J. Am. Chem. Soc. 2020, 142(8), 3797-3805.

11. Kimura, R.; Nagano, T.; Kinoshita, H. Bull. Chem. Soc. Jpn. 2002, 75(11), 2517-2525.

12. Frisch, M. J.; Trucks, G. W.; Schlegel, H. B.; Scuseria, G. E.; Robb, M. A.; Cheeseman, J. R.; Scalmani, G.; Barone, V.; Petersson, G. A.; Nakatsuji, H.; Li, X.; Caricato, M.; Marenich, A. V.; Bloino, J.; Janesko, B. G.; Gomperts, R.; Mennucci, B.; Hratchian, H. P.; Ortiz, J. V.; Izmaylov, A. F.; Sonnenberg, J. L.; Williams; Ding, F.; Lipparini, F.; Egidi, F.; Goings, J.; Peng, B.; Petrone, A.; Henderson, T.; Ranasinghe, D.; Zakrzewski, V. G.; Gao, J.; Rega, N.; Zheng, G.; Liang, W.; Hada, M.; Ehara, M.; Toyota, K.; Fukuda, R.; Hasegawa, J.; Ishida, M.; Nakajima, T.; Honda, Y.; Kitao, O.; Nakai, H.; Vreven, T.; Throssell, K.; Montgomery, J. A., Jr.; Peralta, J. E.; Ogliaro, F.; Bearpark, M. J.; Heyd, J. J.; Brothers, E. N.; Kudin, K. N.; Staroverov, V. N.; Keith, T. A.; Kobayashi, R.; Normand, J.; Raghavachari, K.; Rendell, A. P.; Burant, J. C.; Iyengar, S. S.; Tomasi, J.; Cossi, M.; Millam, J. M.; Klene, M.; Adamo, C.; Cammi, R.; Ochterski, J. W.; Martin, R. L.; Morokuma, K.; Farkas, O.; Foresman, J. B.; Fox, D. J. Gaussian 16, Rev. A.03, Wallingford, CT, 2016.

13. (a) Becke, A. D. Density Functional Thermochemistry. III. The Role of Exact Exchange. J. Chem. Phys. 1993, 98, 5648-5652. (b) Lee, C.; Yang, W.; Parr, R. G. Development of the Colle-Salvetti CorrelationEnergy Formula into a Functional of the Electron Density. Phys. Rev. B: Condens. Matter Mater. Phys. 1988, 37, 785-789.

14. Weigend, F.; Ahlrichs, R. Balanced Basis Sets of Split Valence, Triple Zeta Valence and Quadruple Zeta Valence Quality for H to Rn: Design and Assessment of Accuracy. Phys. Chem. Chem. Phys. 2005, 7, 3297-3305.

15. Grimme, S.; Antony, J.; Ehrlich, S.; Krieg, H. A Consistent and Accurate Ab Initio Parametrization of Density Functional Dispersion Correction (DFT-D) for the 94 Elements H-Pu. J. Chem. Phys. 2010, 132, 154104.

16. Chai, J.-D.; Head-Gordon, M. Long-Range Corrected Hybrid Density Functionals with Damped AtomAtom Dispersion Corrections. Phys. Chem. Chem. Phys. 2008, 10, 6615-6620.

17. Weigend, F. Accurate Coulomb-Fitting Basis Sets for H to Rn. Phys. Chem. Chem. Phys. 2006, 8, 10571065 .

18. Cossi, M.; Rega, N.; Scalmani, G.; Barone, V. Energies, Structures, and Electronic Properties of Molecules in Solution with the C-PCM Solvation Model. J. Comput. Chem. 2003, 24, 669-681. 
19. (a) Lu, T.; Chen, F. Multiwfn: A Multifunctional Wavefunction Analyzer. J. Comput. Chem. 2012, 33, 580-592. (b) Lu, T.; Chen, F. Quantitative Analysis of Molecular Surface Based on Improved Marching Tetrahedra Algorithm. J. Mol. Graphics Model. 2012, 38, 314-323.

20. Humphrey, W.; Dalke, A.; Schulten, K. VMD: Visual Molecular Dynamics. J. Mol. Graphics. 1996, 14, 33-38. VMD Official website. http://www.ks.uiuc.edu/Research/ vmd/.

21. (a) Foster, J. P.; Weinhold, F. Natural Hybrid Orbitals. J. Am. Chem. Soc. 1980, 102, 7211-7218. (b) Reed, A. E.; Weinstock, R. B.; Weinhold, F. Natural Population Analysis. J. Chem. Phys. 1985, 83, 735-746.

22. Legault, C. Y. CYLview; version 1.0b; Universitéde Sherbrooke: 2009 (http://www.cylview.org). 


\section{Spectral Data}


E2 - Acquisition Parane

Date

INSTRUM

PROBHD

PULPROG

TD

SOLVENT

NS

SWH

FIDRE

AQ
DW

$\mathrm{DE}$
$\mathrm{TE}$

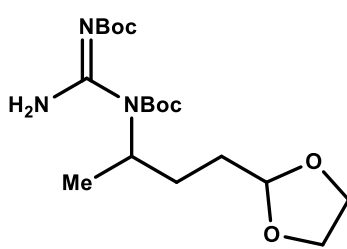

D1

20210501

8.10

$5 \mathrm{~mm}$ CPBBO BB-

zg30
98074

98074

16
2

9615.385 5.0998478

52.000

14.23

0.10000000

$=======$ CHANNEL $\mathrm{f} 1 \mathrm{l}===$

$\begin{array}{lr}\text { SFO1 } & 600.1342009 \\ \text { NUC1 } & 1 \mathrm{H}\end{array}$

$\begin{array}{ll}\mathrm{P} 1 & 9.50\end{array}$

S4

F2 - Processing paramet
SI

SF $\quad 600.1300346$

WDW

I

GB

0.30

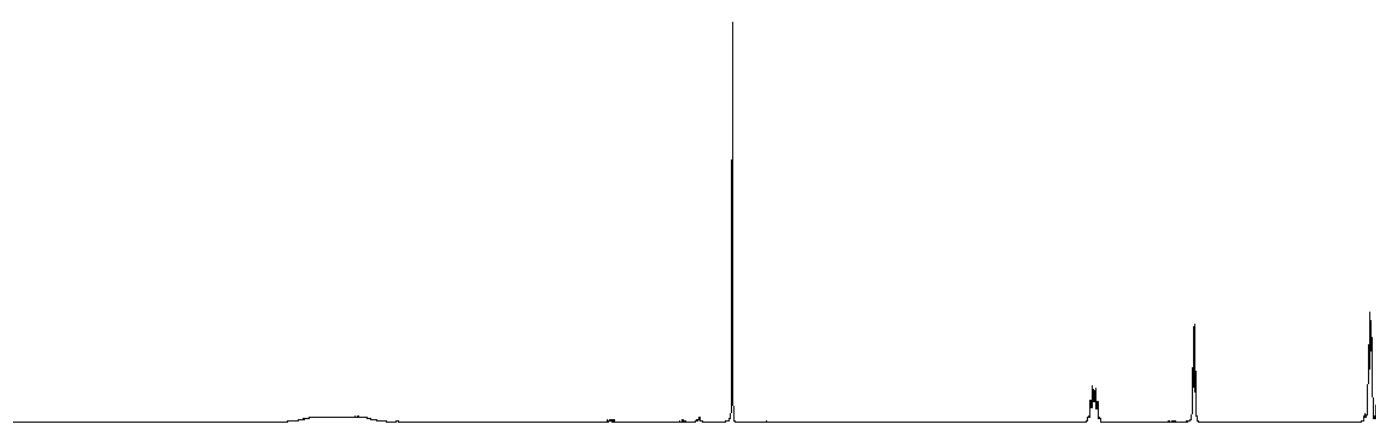

$M$

1.00

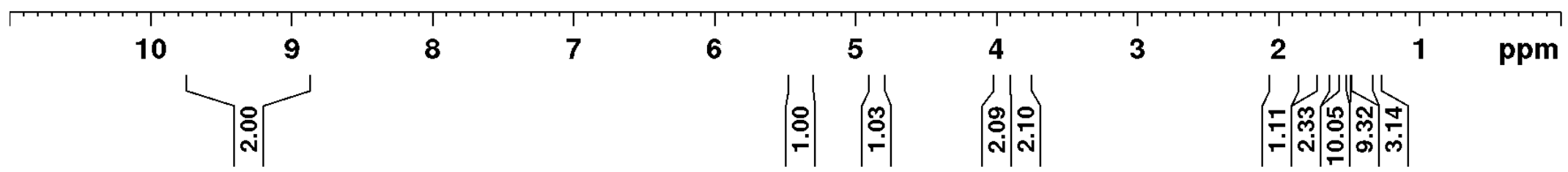



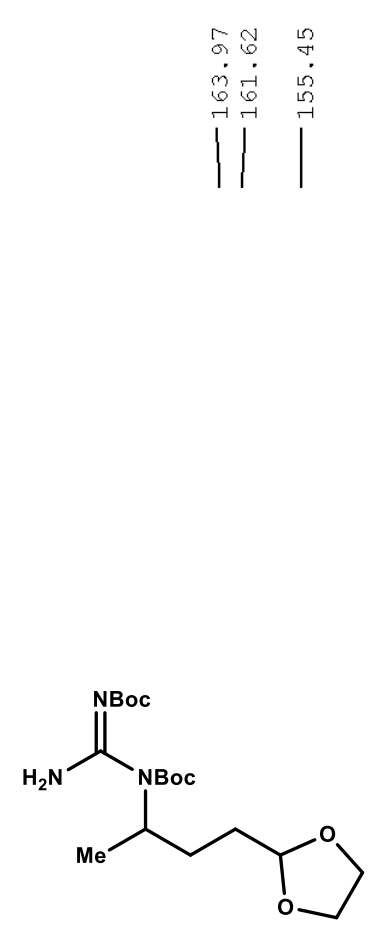

S4

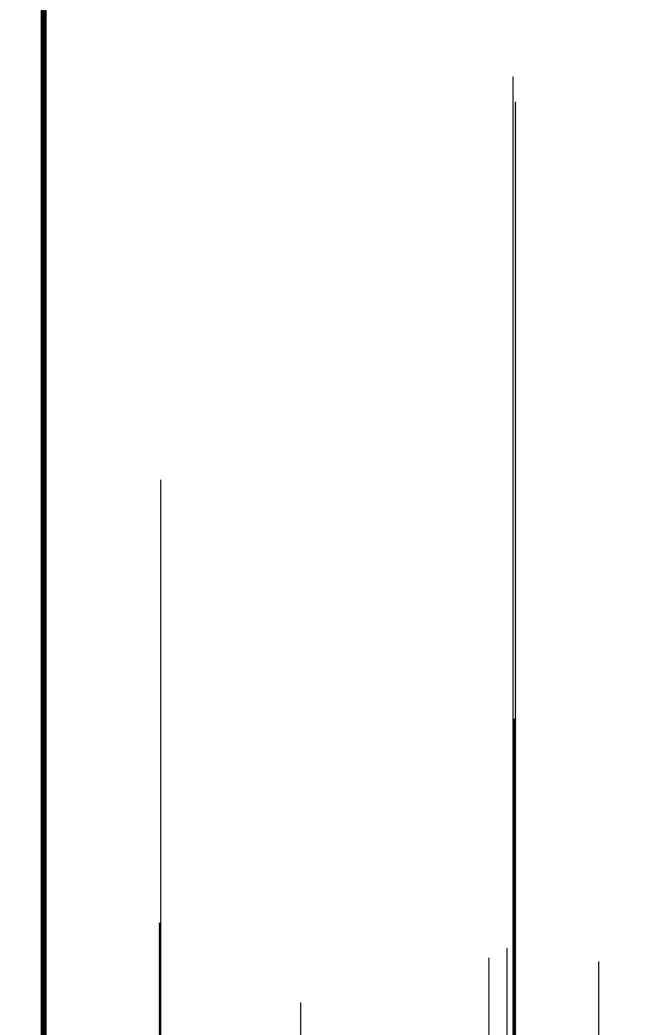

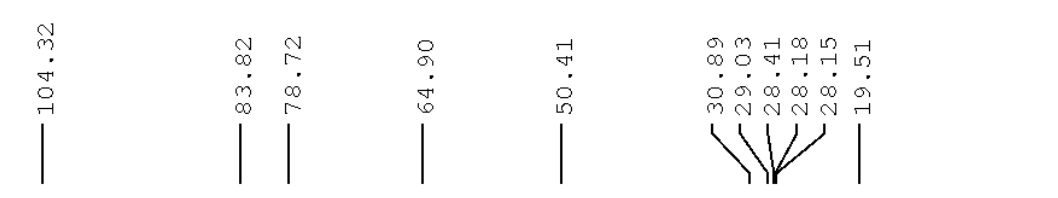

AME

PROCNO

Dime

INSTRUM

PROBHD

PULPROG

TD

SOLVENT

NS

SWH

FIDRES

FIDP
AQ
RG
DW

DW

TE

D1
D11

D11
TD0

$===$
SFO1

SFO1

P1

PLW1

$====$
$\mathrm{SFO} 2$

$\mathrm{SFO} 2$
$\mathrm{NUC2}$

CPDPRG [

PCPD2

PLW12

F2 - Processing paramet

SI

SF

SSB

$\mathrm{LB}$
$\mathrm{GB}$

GB
PC

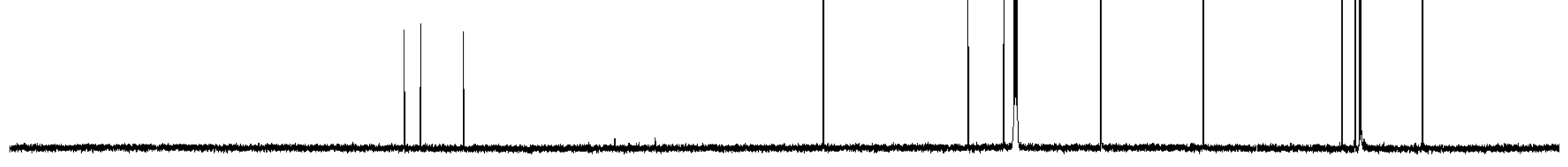

\begin{tabular}{rrrrrrrrrr|r|r|}
\hline 200 & 180 & 160 & 140 & 120 & 100 & 80 & 60 & 40 & 20 & ppm
\end{tabular}




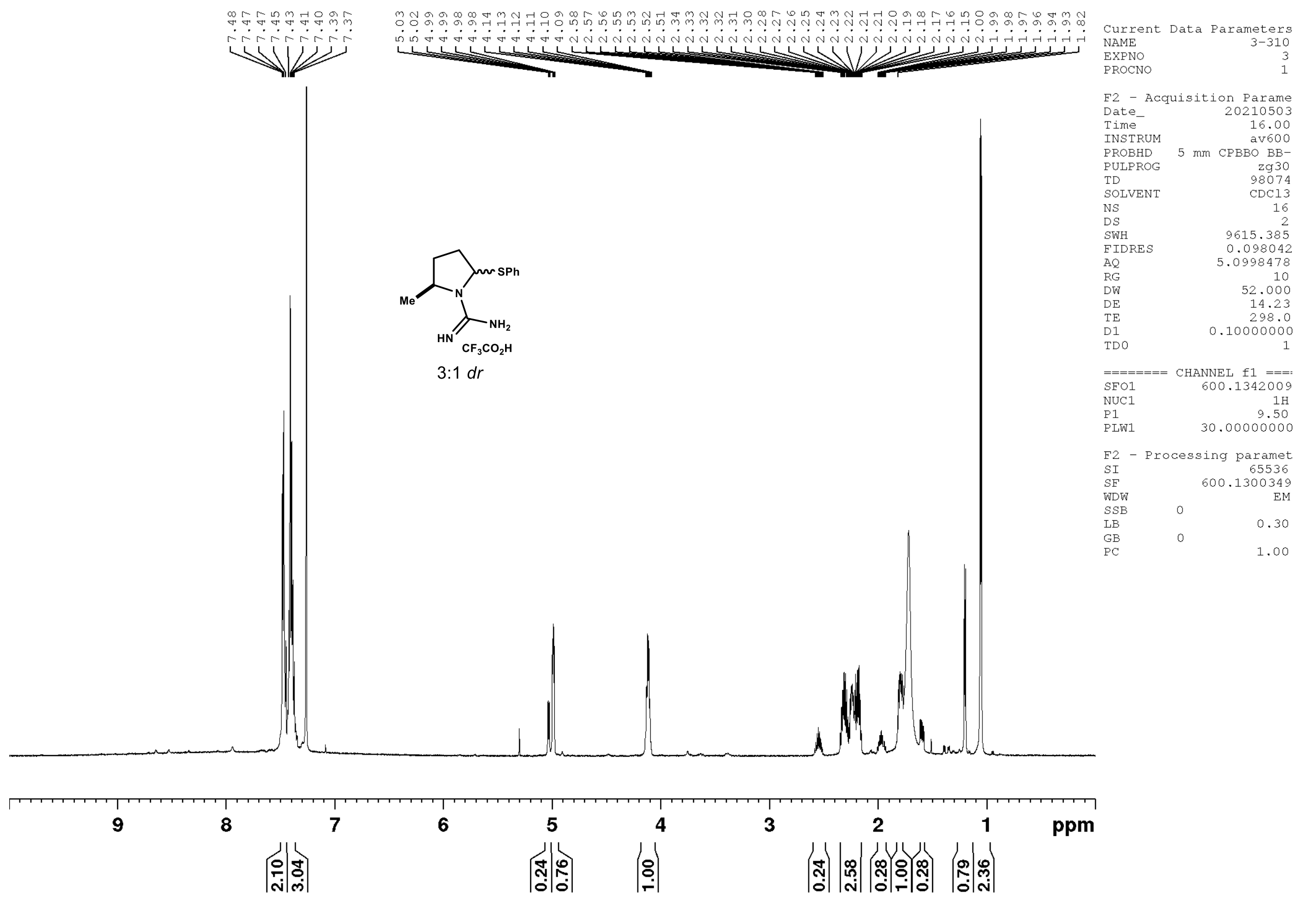




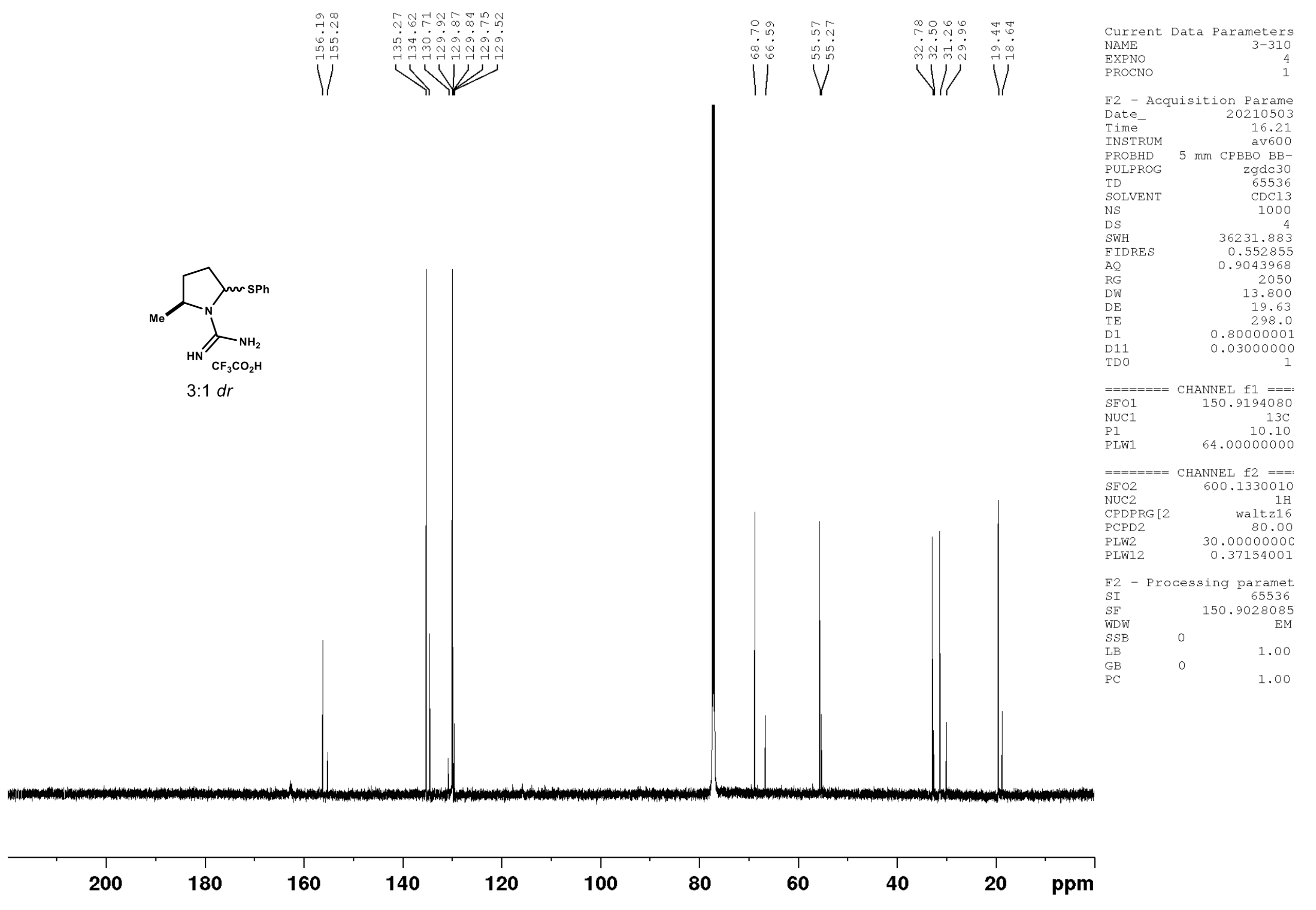


F2 - Acquisition Parane
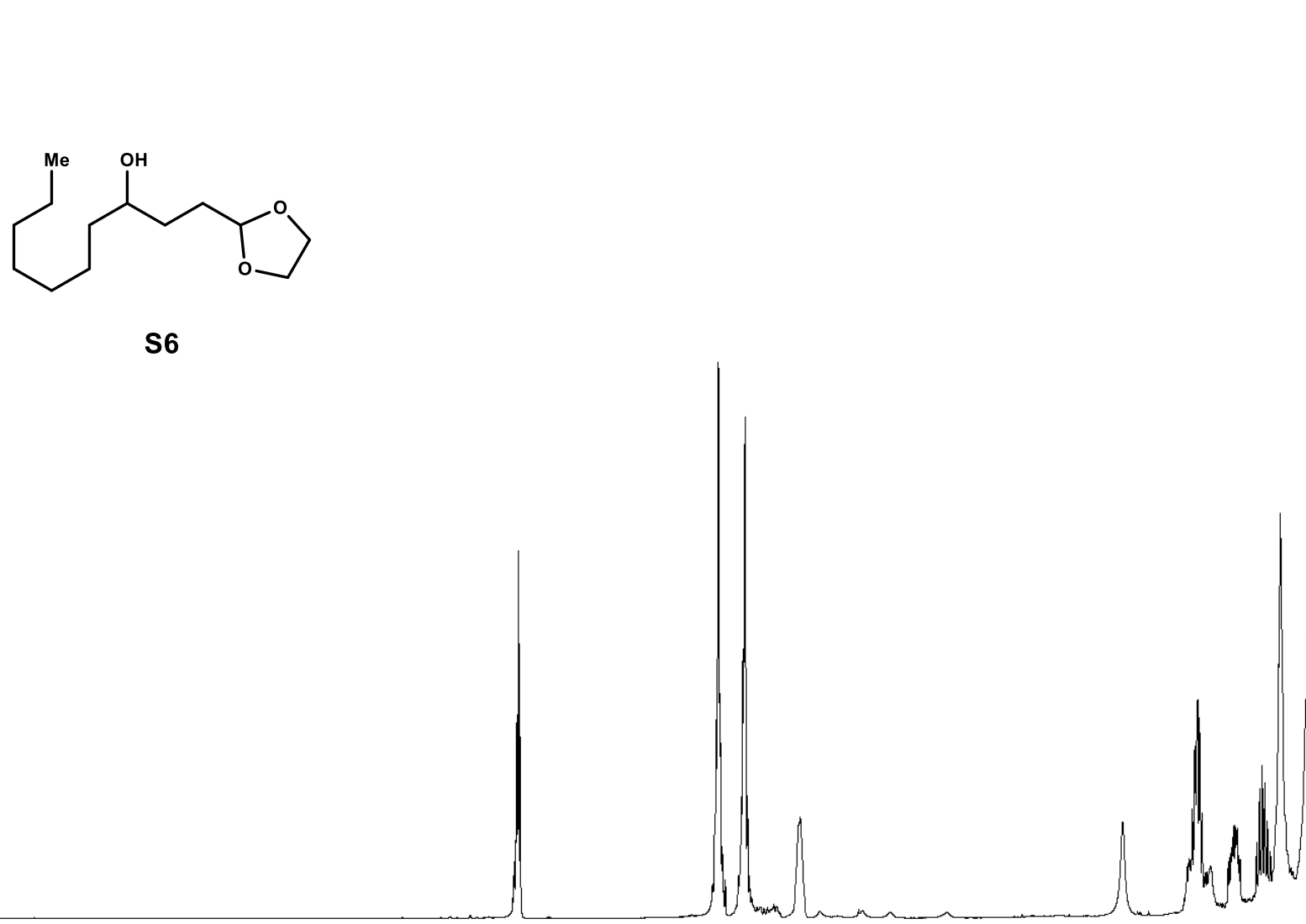

s6

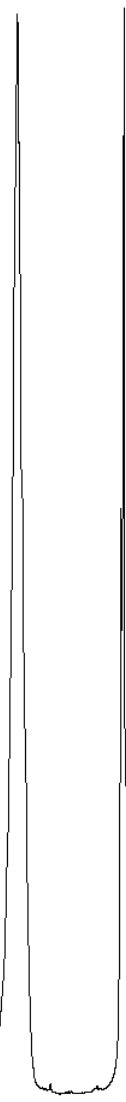

NS

DS

FIDRE S

$\mathrm{AQ}$

RG

DW

TE

D1

$\mathrm{mm}$ CPBBO BB-

$\mathrm{zg} 30$
98074

CDCl3

16
2

915.385 5.0998478

52.000

14.23

0.10000000

$=======$ CHANNEL $\mathrm{f} 1===$ :

$\begin{array}{rr}\text { SFO1 } & 600.1342009 \\ \text { NUC1 } & 1 \mathrm{H}\end{array}$

P1 $\quad 9.50$

PLW1 $\quad 30.00000000$

F2 - Processing paramet

SI $\quad 65536$

SF $\quad 600.1300345$

WSW

0.30

$\mathrm{PC}$

1.00
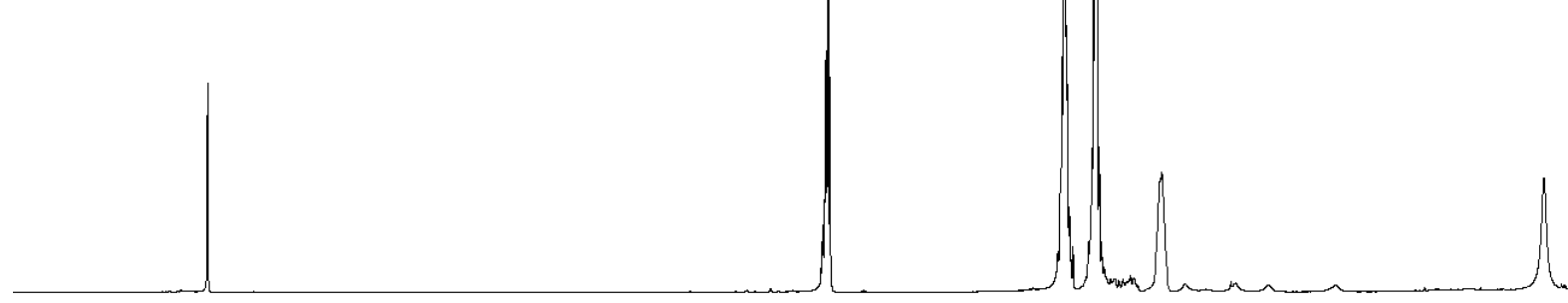

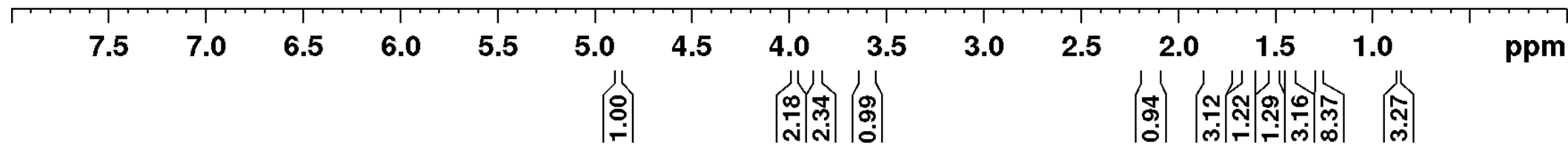




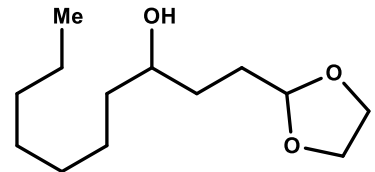

s6
की

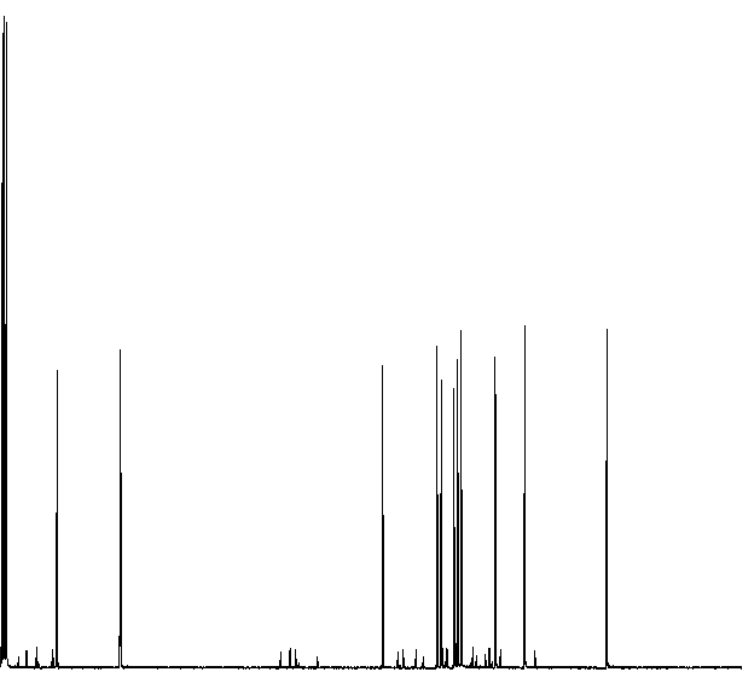

200

180

160

140

80

60

40

20

ppm
Current Data Parameters NAME

EXPNO

F2 - Acquisition Parame

Dime

INSTRUM

PROBHD

PROBHD

TD

SOLVEN

DS

SWH

FIDRE

AQ

DW

$\mathrm{DE}$

TE

D1 11

TDO

9.43
av 600

mm $\mathrm{CPBBO} B \mathrm{BB}-$

65536

5613

568
4

36231.883

0.552855

0.9043968

2050
13.800

19.63

$0.0300000 \mathrm{C}$

$========$ CHANNEL $\mathrm{f} 1===$

NUC

P1

150.9194080
$13 \mathrm{C}$

LW1

$=======$ CHANNEL $\mathrm{f2}===$

$\mathrm{SFO} 2$

CPDPRG [2

PCPD2

600.1330010

F2 - Processing paramet

SI 65536

$\begin{array}{ll}\mathrm{SF} & 150.9028085\end{array}$

WDW EM

$\begin{array}{ll}\mathrm{BB} & 1.00\end{array}$

1.00 
F2 - Acquisition Parame 20210321
12.02

INSTRUM

PROBHD

PULPROG

$\mathrm{mm} \mathrm{CPBBO} \mathrm{BB}-$

PU

SOLVENT

NS

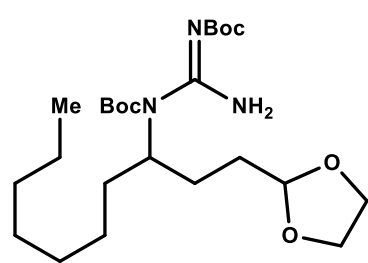

DS

FIDRES

$\mathrm{AQ}$

RG

$\mathrm{DW}$
$\mathrm{DE}$

$\mathrm{DE}$

D1
TDO

S7

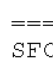

SFO1

$\mathrm{zg} 30$
98074

98074

16 5.0998478

10

52.000
14.23

0.10000000

PLWI

CHANNEL $\mathrm{f} 1===$

$1 \mathrm{H}$

F2 - Processing paramet

SI $\quad 65536$

600.1300342

30

PC

1.00

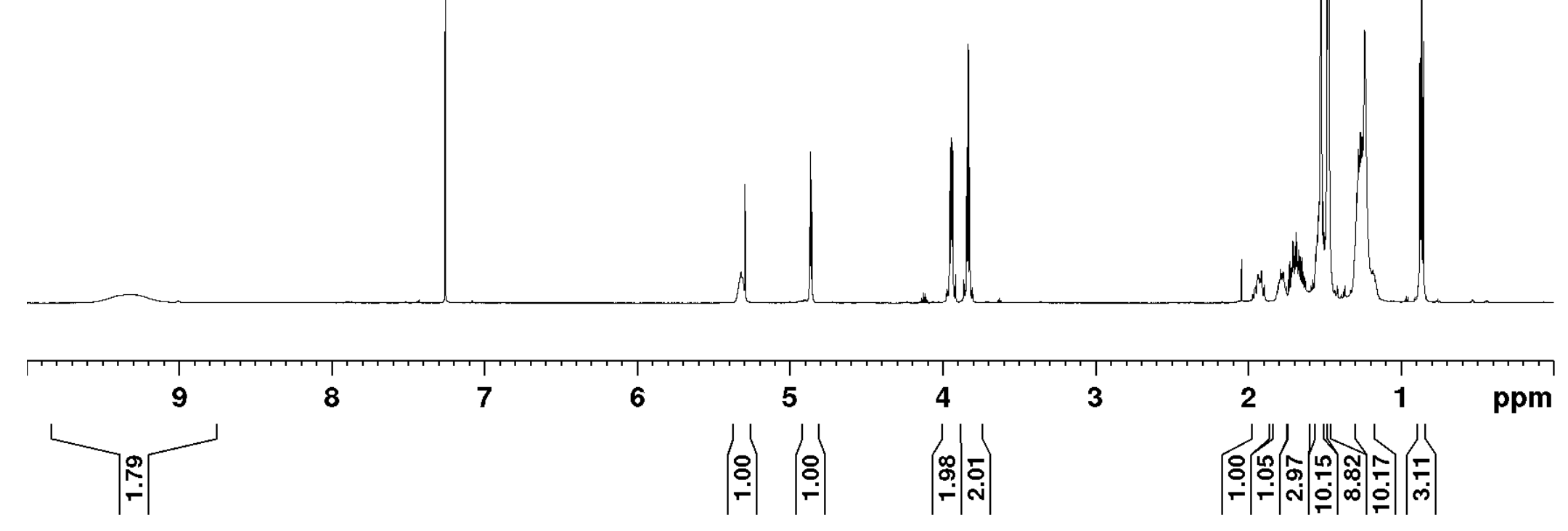




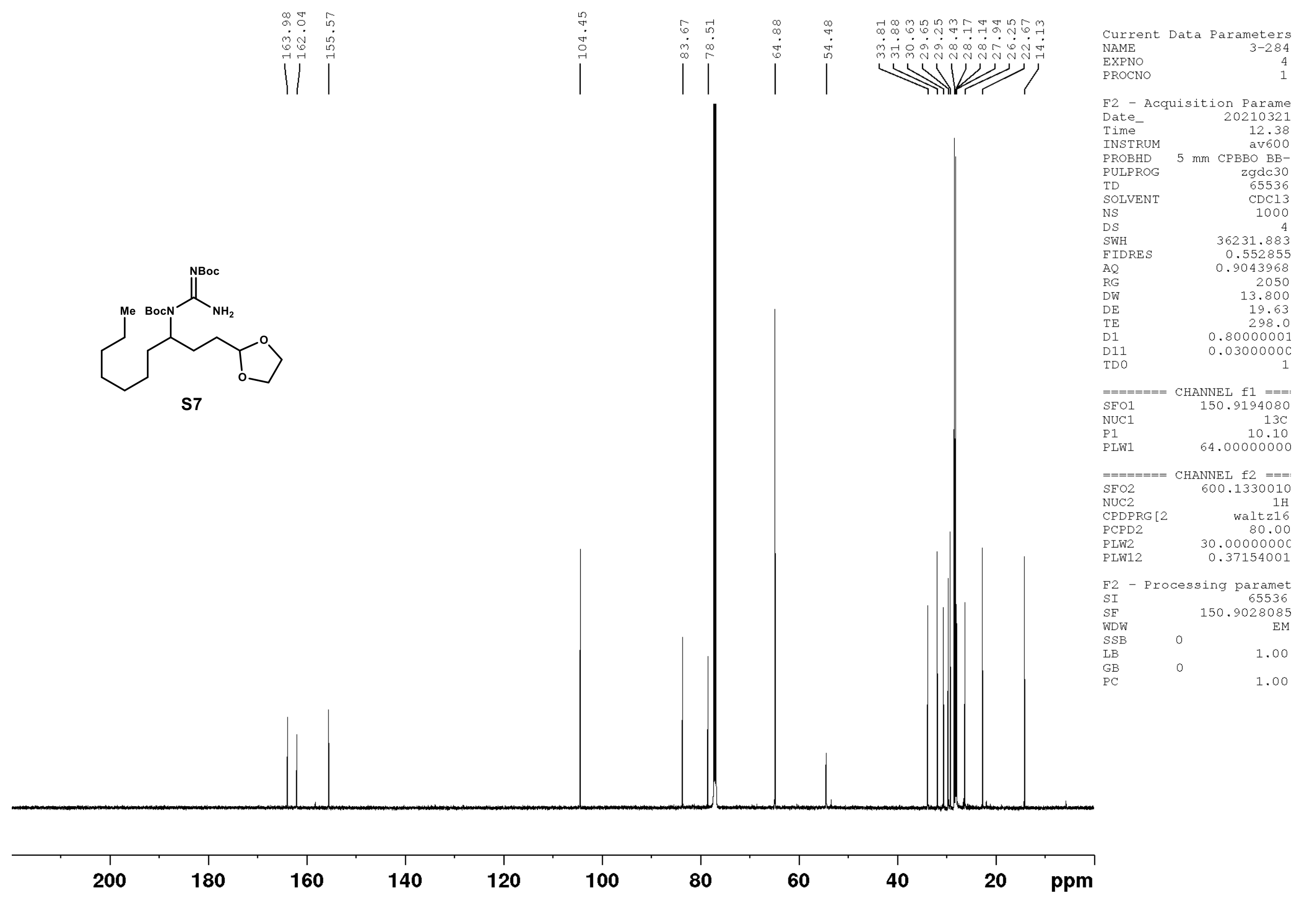




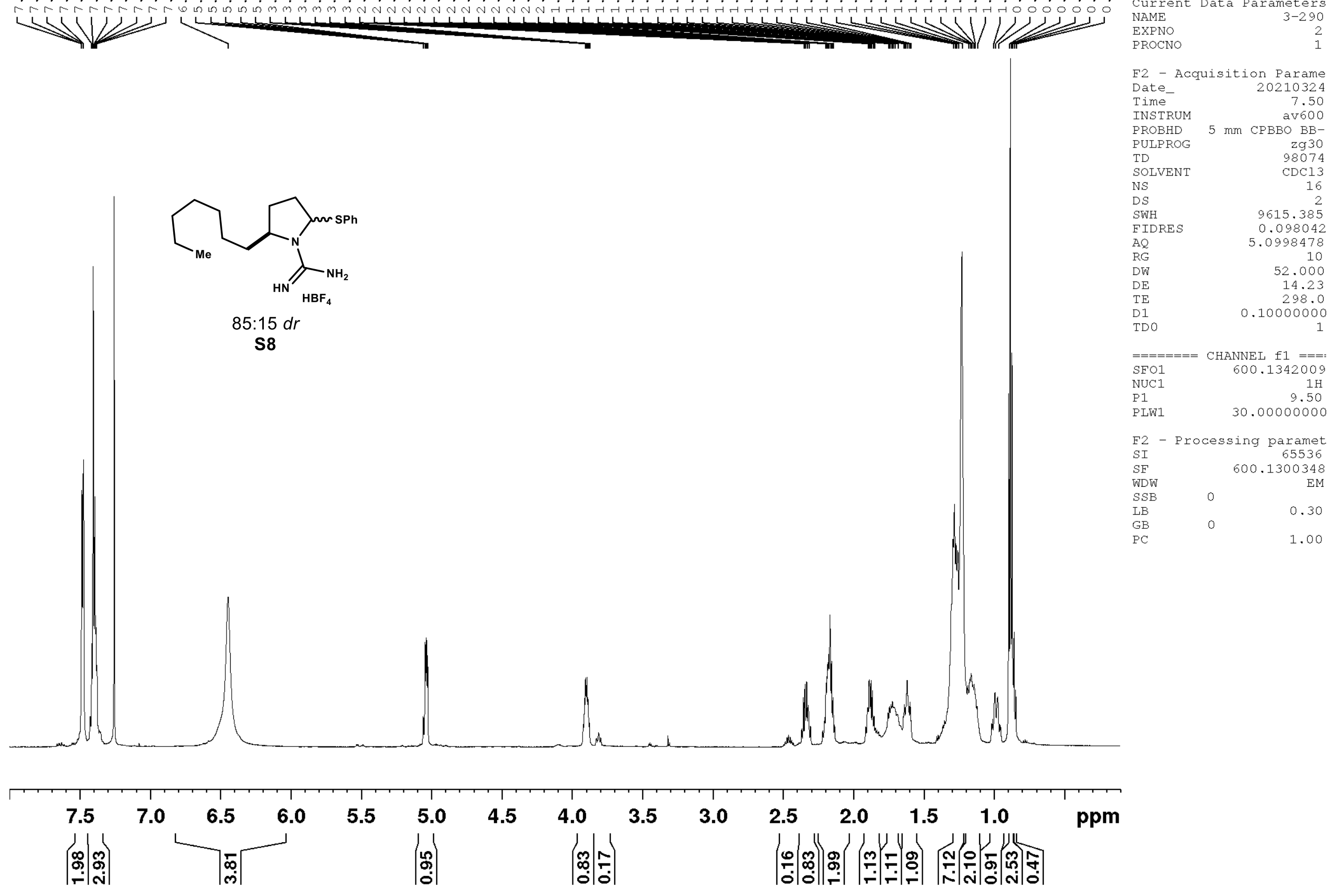




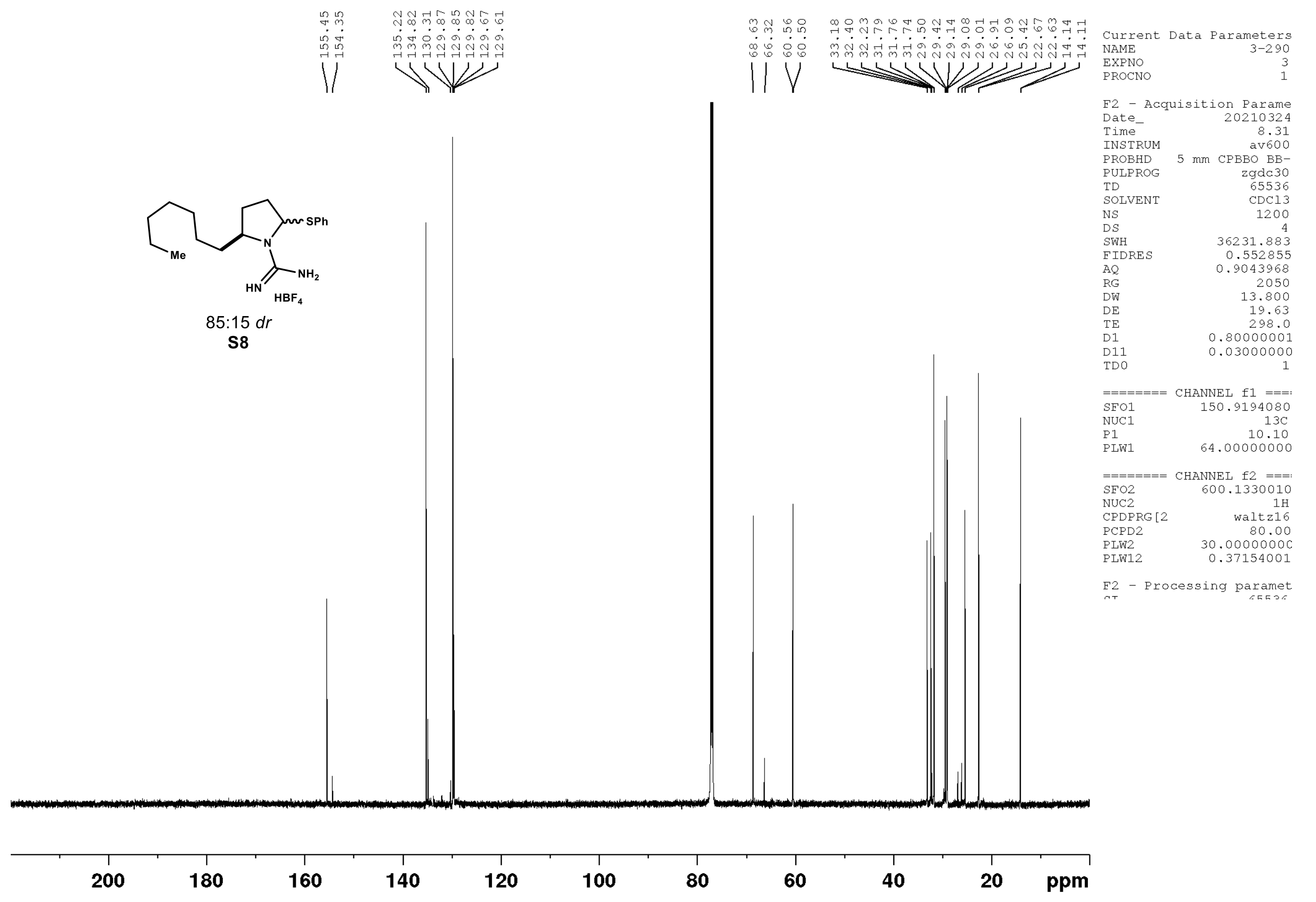




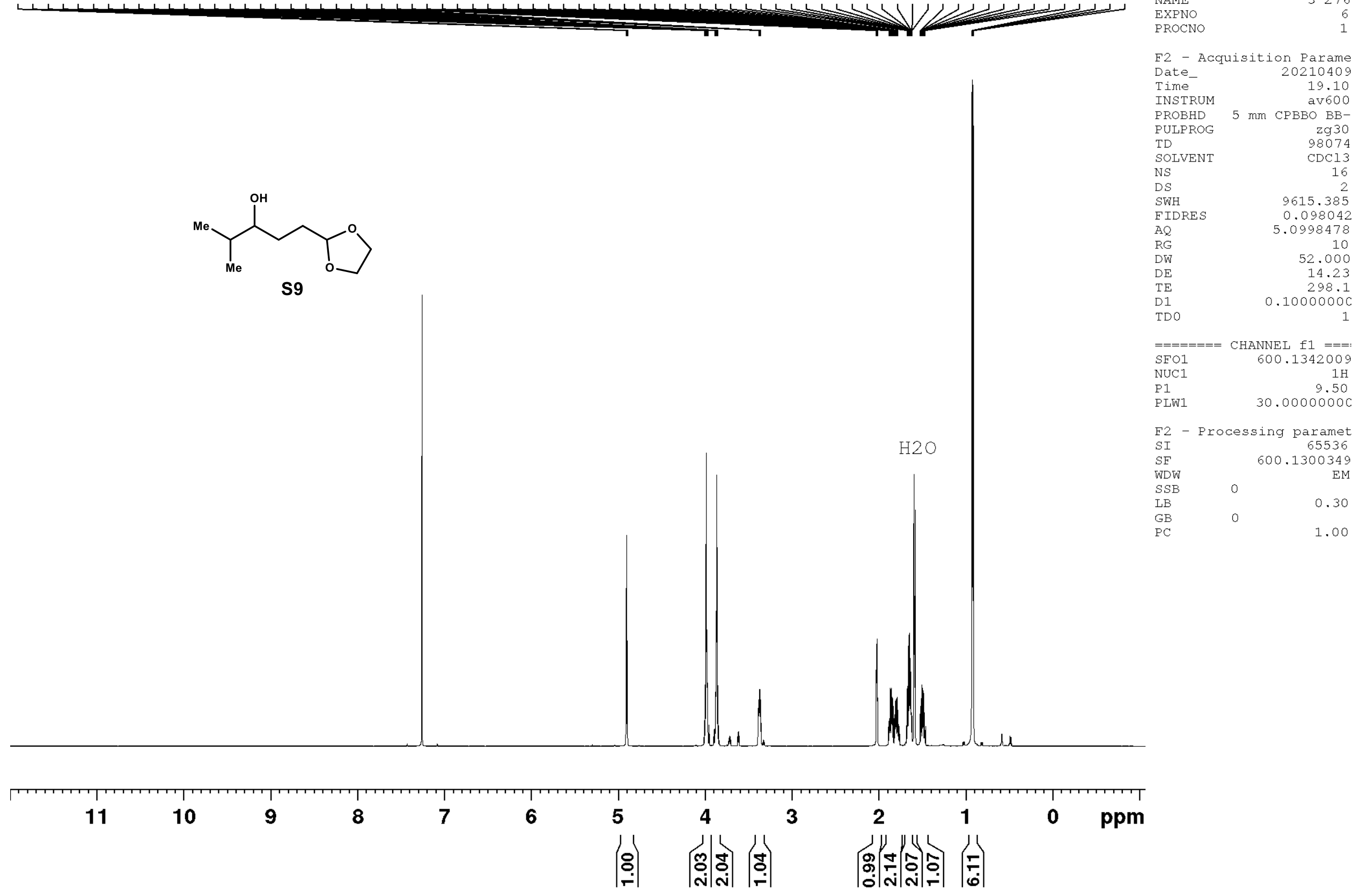




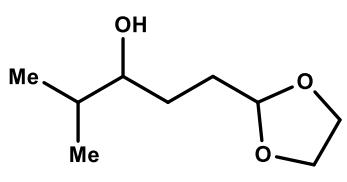

S9

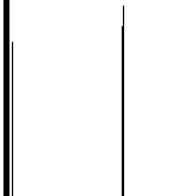

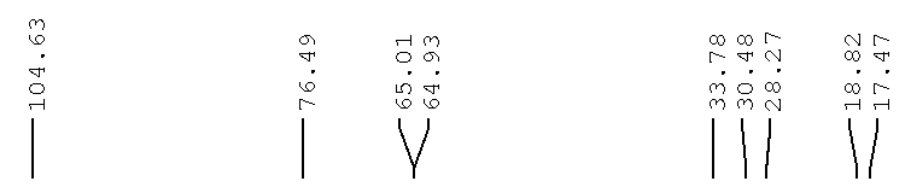
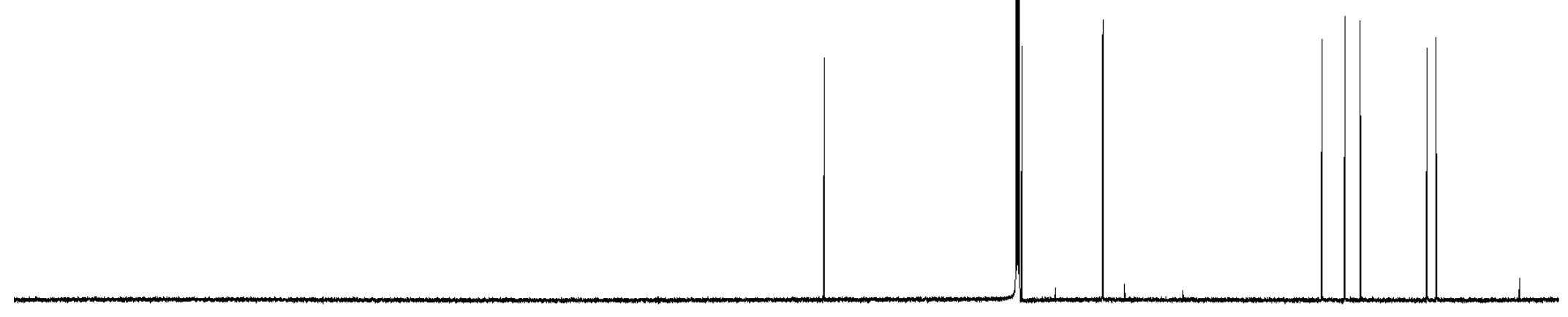

F2 - Acouisition Parame

Date

INSTRUM

PROBHD

PROBHD

PUT

SOLVENT

NS

SWH

FIDRES

$\mathrm{AQ}$
$\mathrm{RG}$
$\mathrm{DW}$

$\mathrm{DW}$

TE

D1

D11
TD0

$=====$

SFO1

P1

PLW1

$=====$
$\mathrm{SFO} 2$

NUC2

CPDPRG
PCPD2

PLW2

PLW12

F2 - Processing paramet

SI 65536

$\begin{array}{lr}\text { SF } & 150.9028085\end{array}$ 
F2 - Acquisition Parame

Date

Date

INSTRUM

PROBHD

PULPROG

TD
SOLVENT

SOLV
NS
DS

DS

FIDRES
AQ

RG

$\mathrm{DW}$

$\mathrm{DE}$

D1

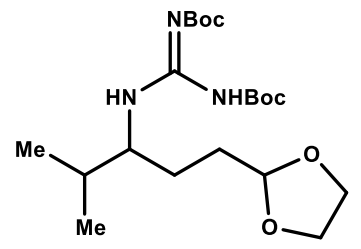

$====$
SFO1
NUC1
P1

P1

PLW1

20210503

9.00
av 600

mm CPBBO BB-

$\mathrm{zg} 30$
98074

CDCl3

16
2

9615.385

5.0998478

10

52.000
14.23

0.10000000

F2 - Processing

SI Processing paramet

SF $\quad 600.1300344$

s11

WD

ISB

GB

0.30

1.00

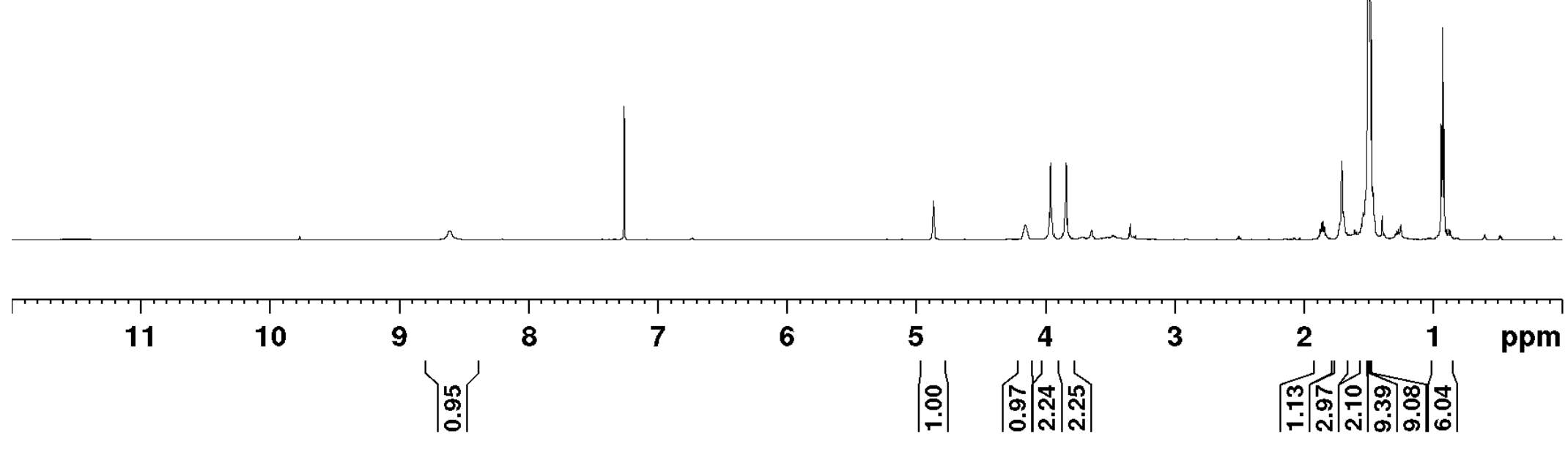




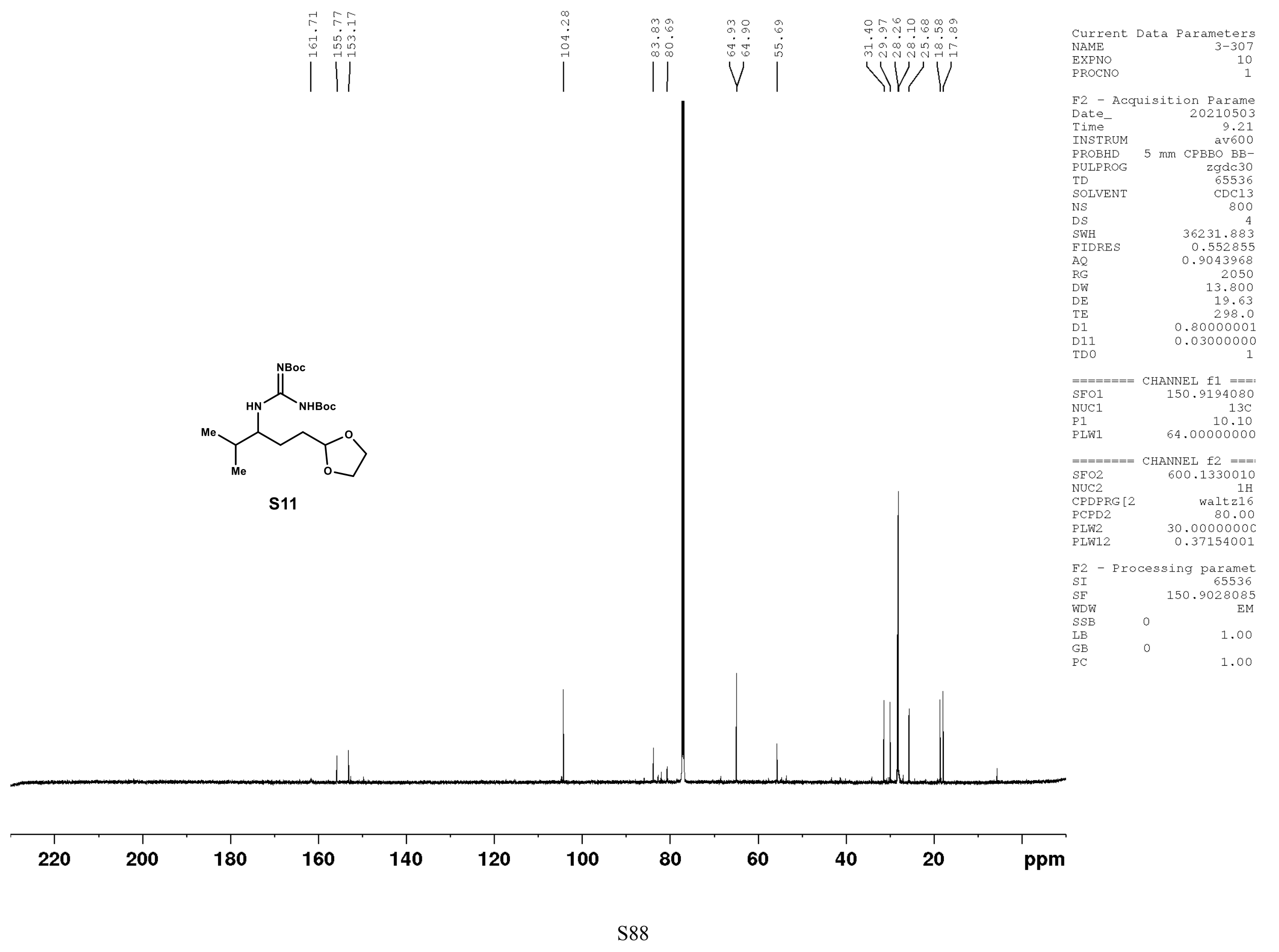




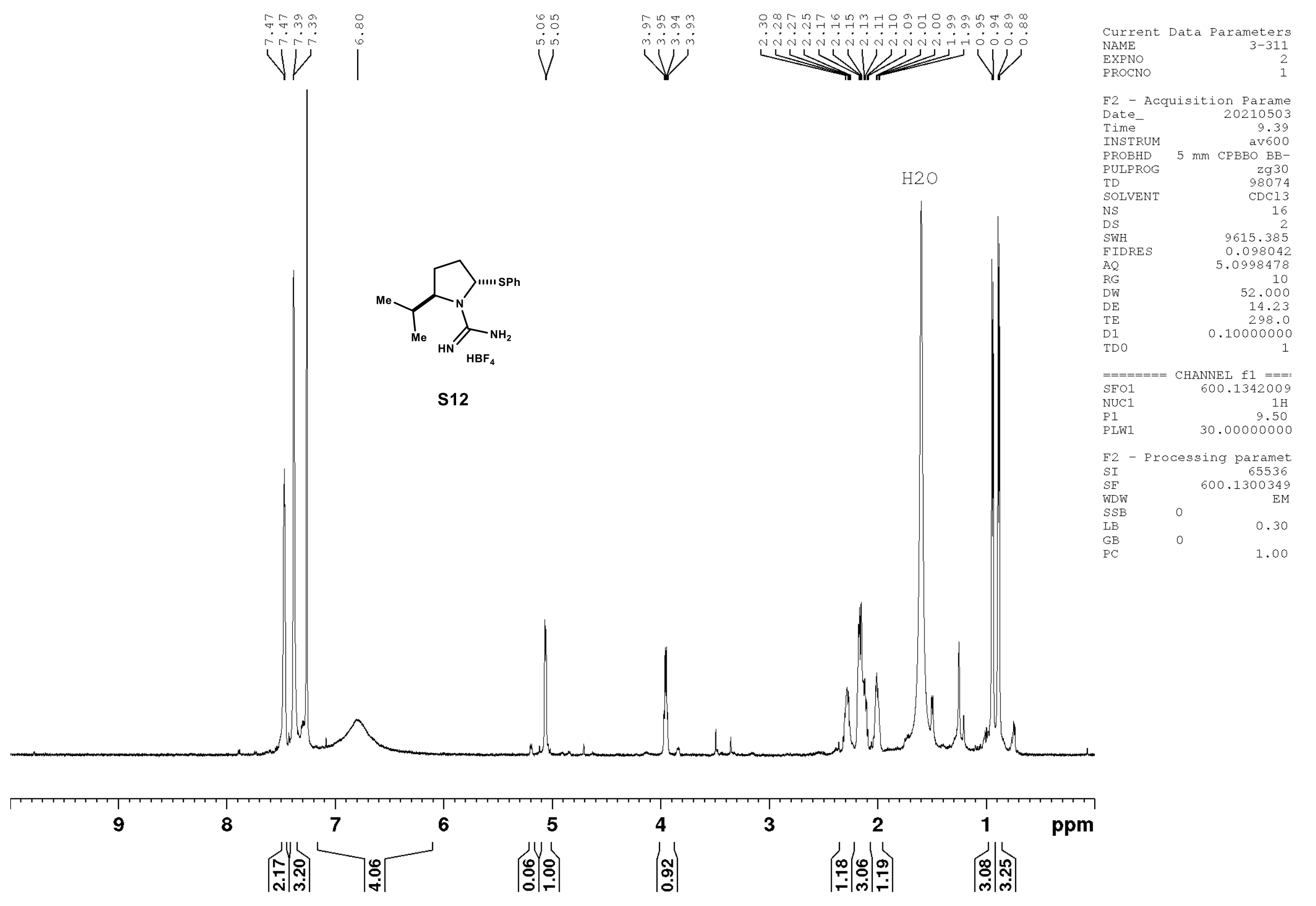




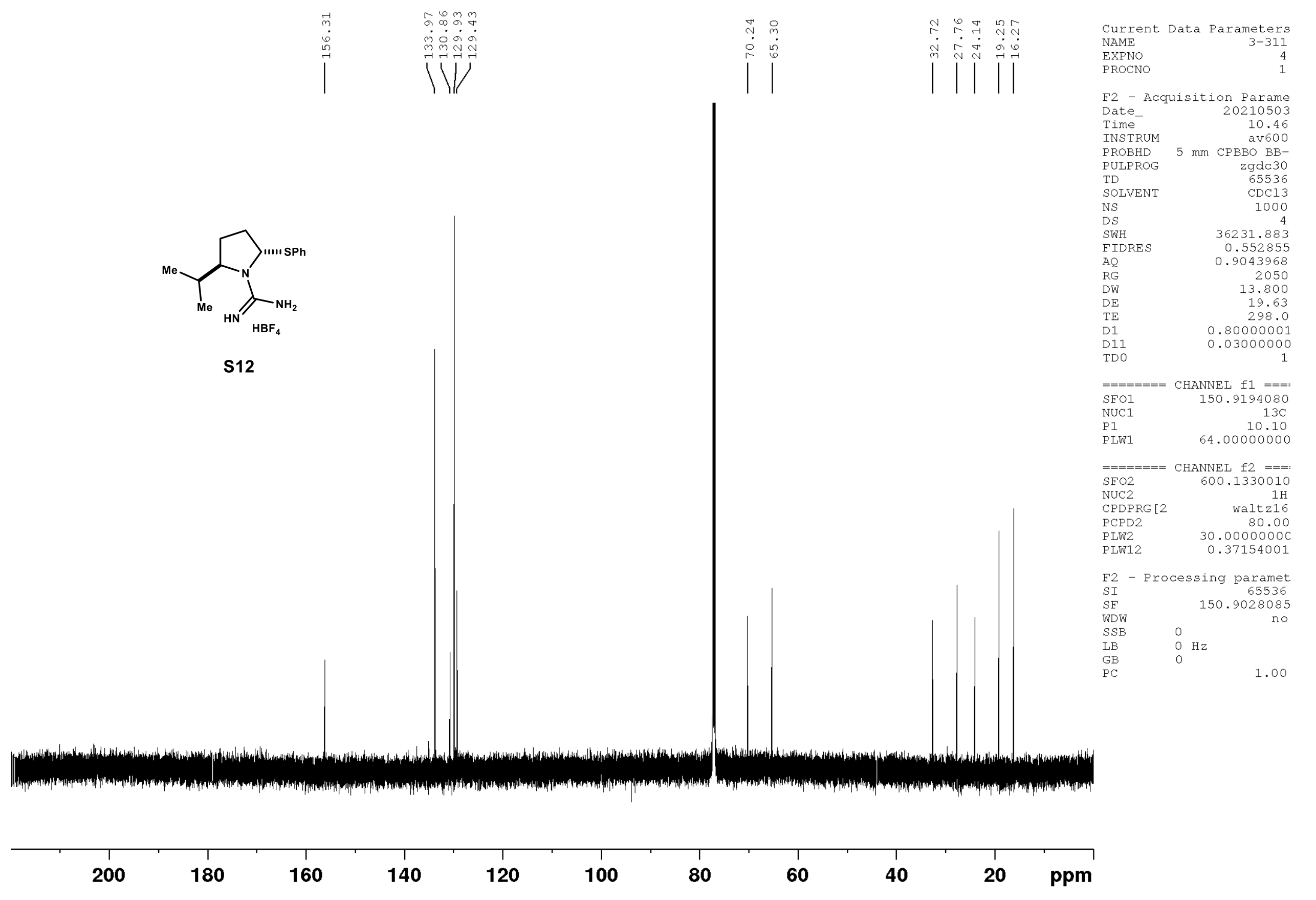




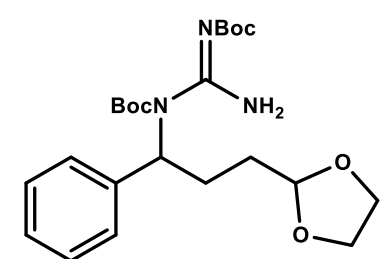

s14
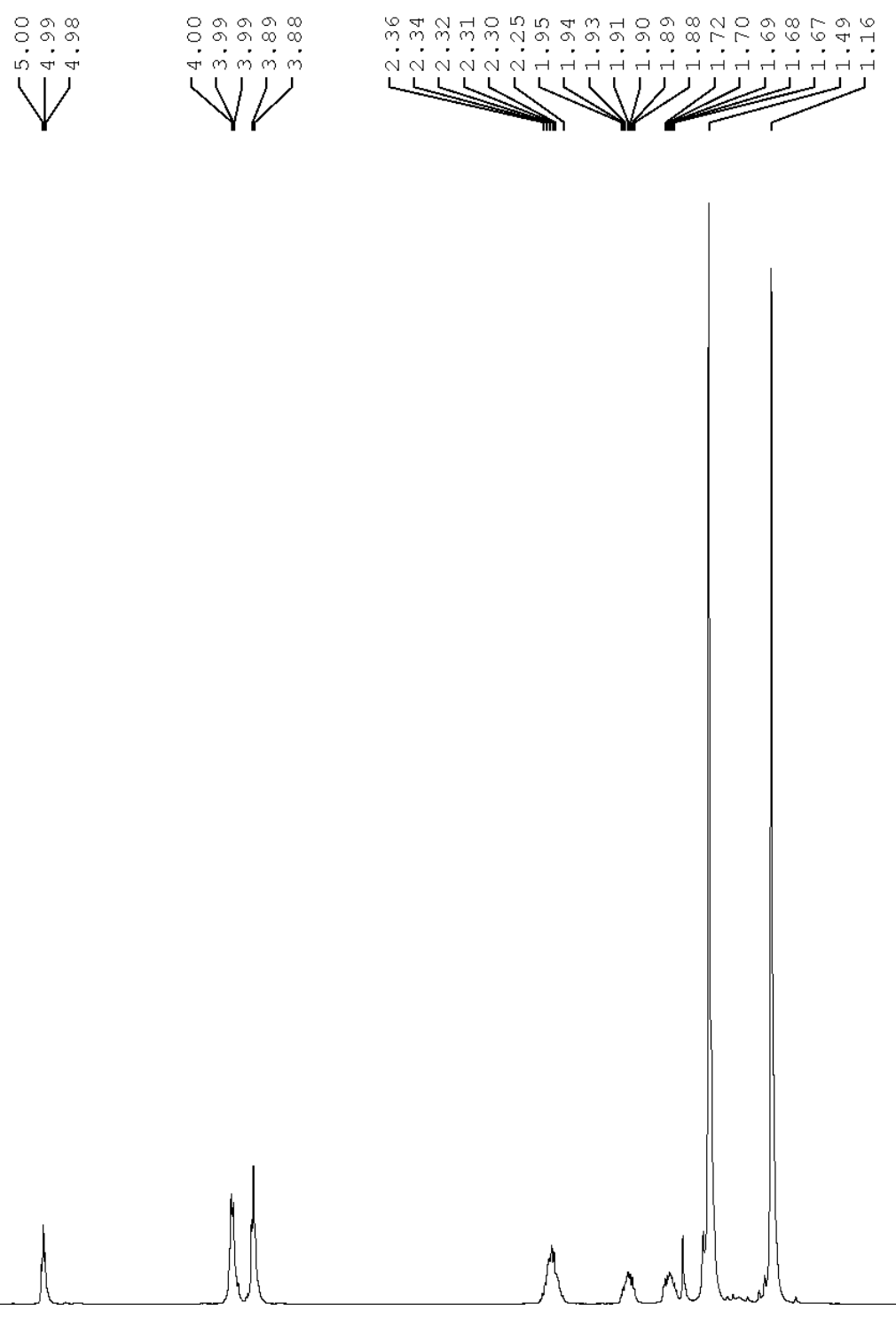

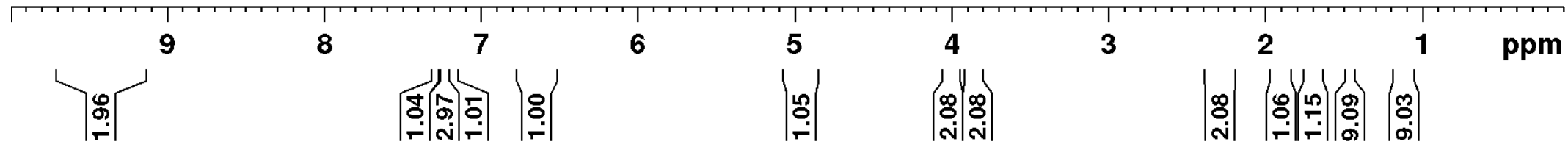

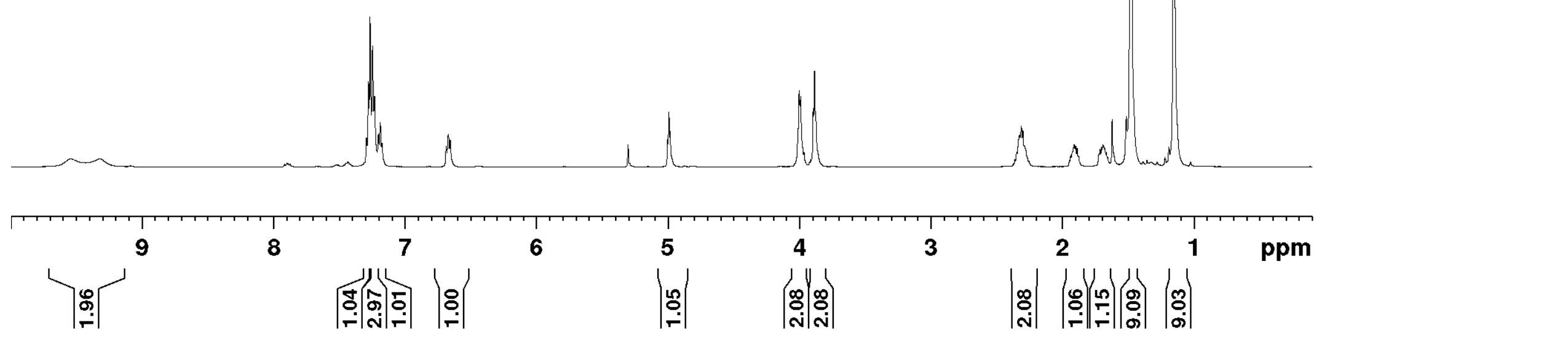

Current Data Parameters

F2 - Acquisition Parame

Date

INSTRUM

PROBHD

PULPROG

SOLVENT

NS

SWH

FIDRES $\quad 8012.820$

$\begin{array}{lr}0.098043 \\ \text { AO } & 0.0998273\end{array}$

RG

DW

DE

D1 $\quad 0.10000000$

MCRE

MCWR

0.01500000

$===$

NUC1

P1

$1 \mathrm{H}$
7.50

$\begin{array}{lr} & 1.60 \\ \text { SFO1 } & 500.2235015\end{array}$

F2 - Processing paramet

SI $\quad 500.2200305$

500.2200305

SSB 0

$\mathrm{P}$

4.00 
F2 - Acquisition Parame 


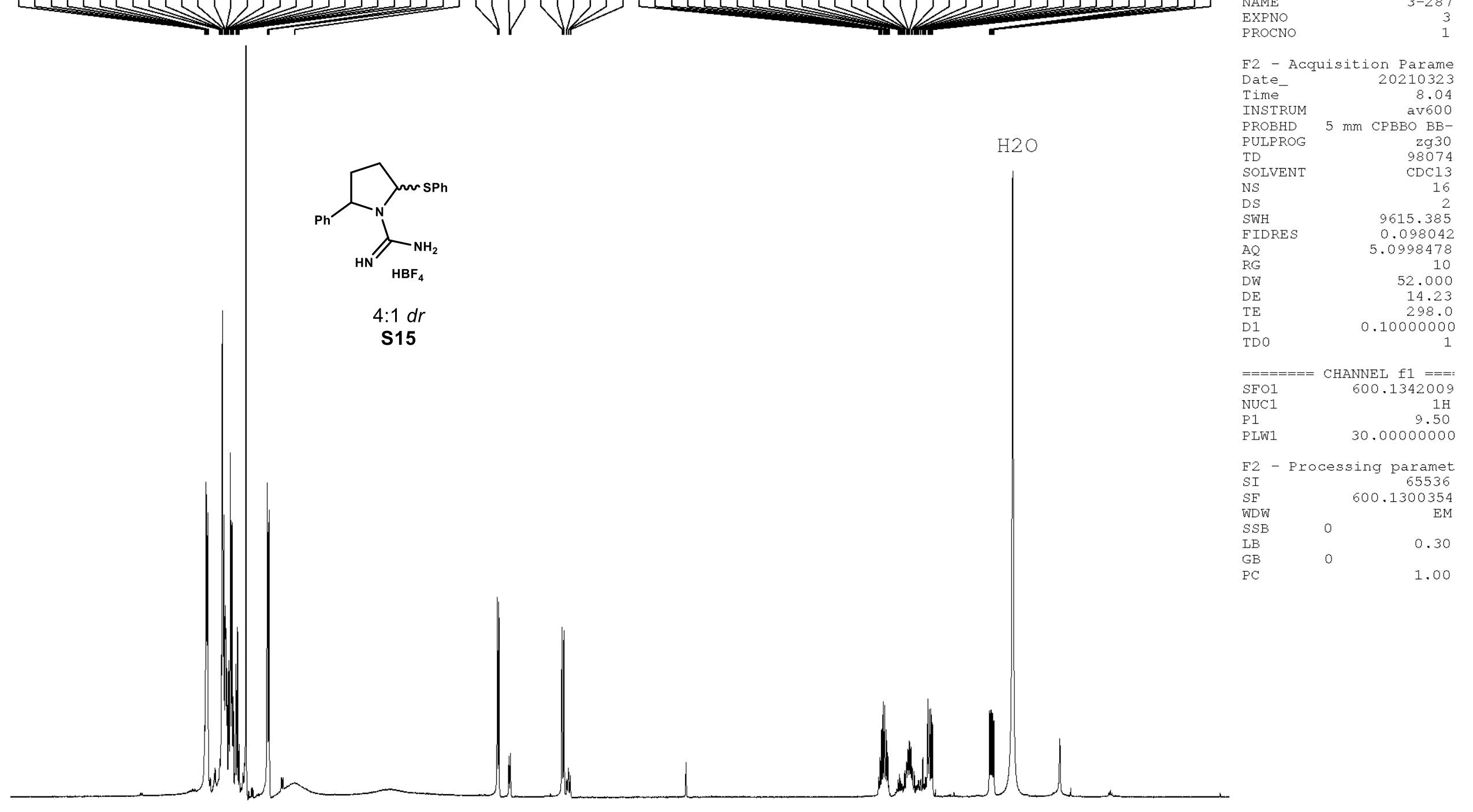

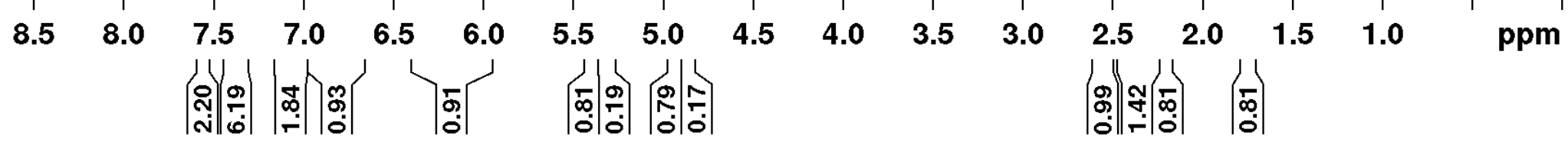




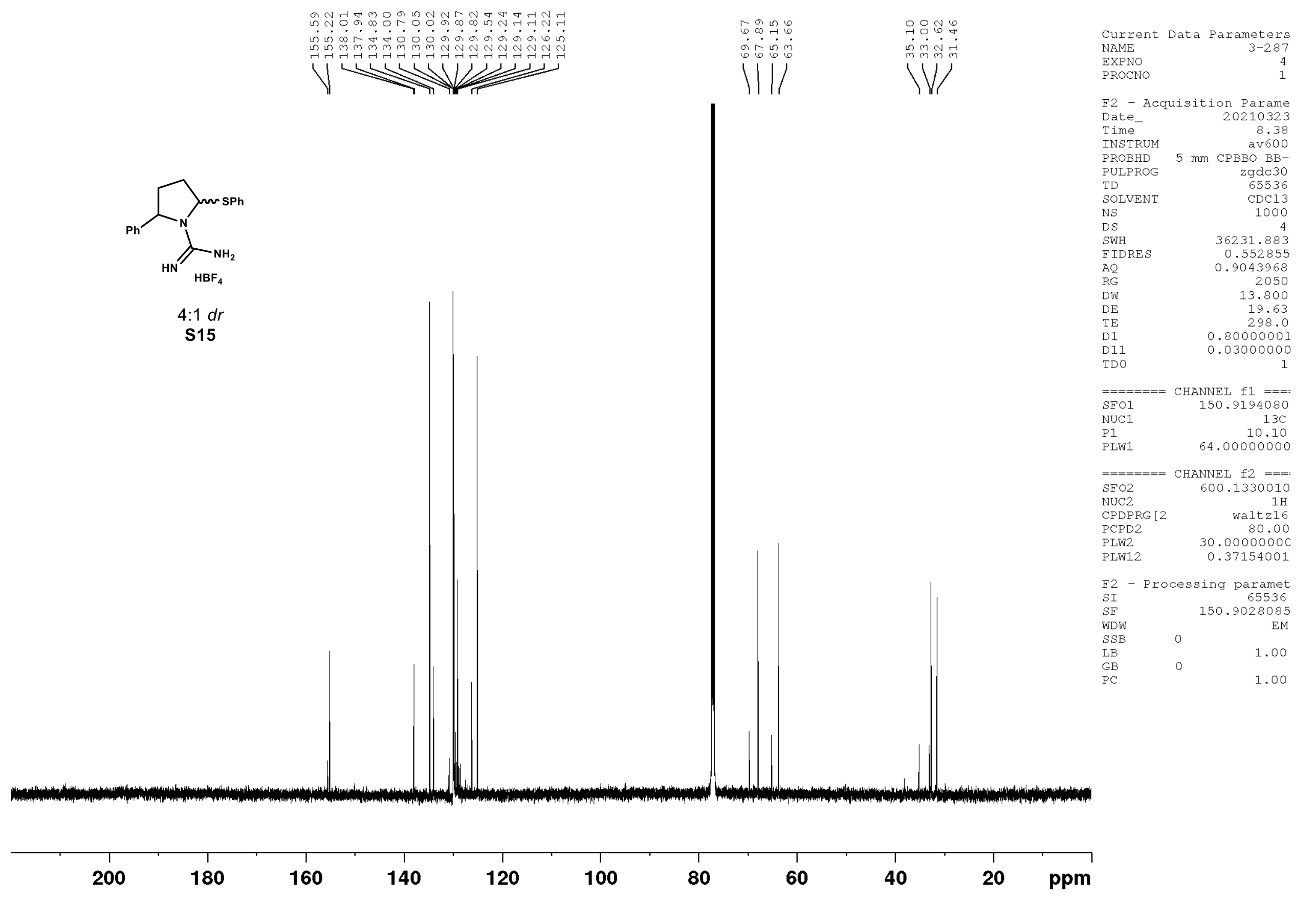



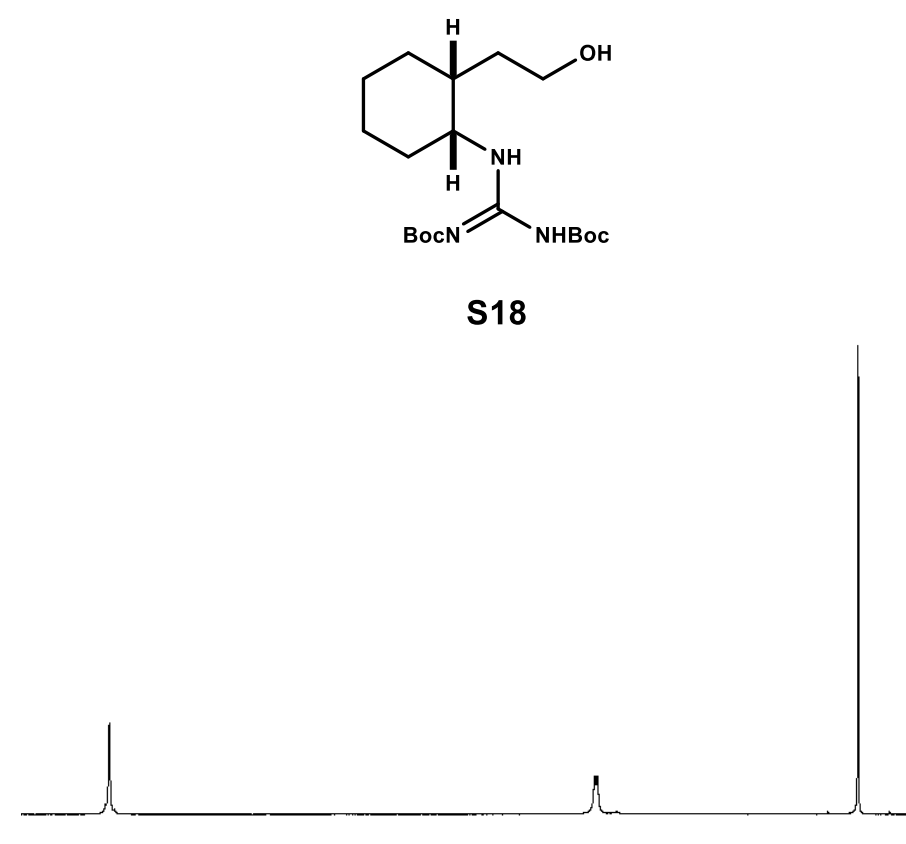

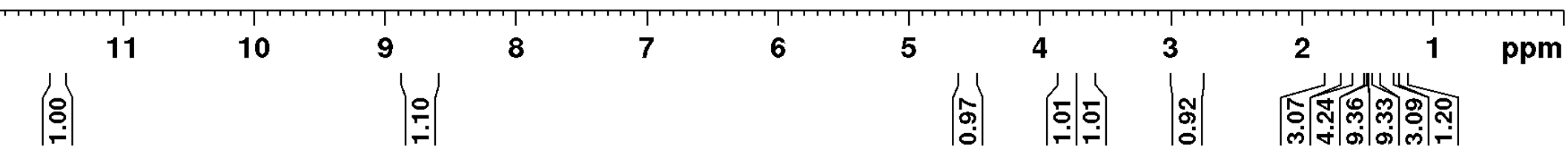



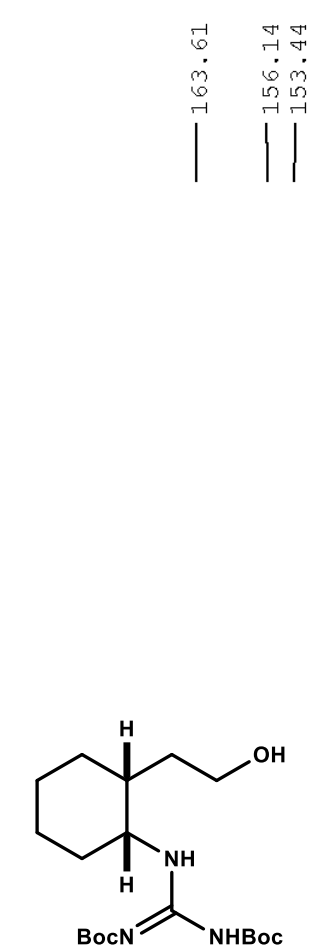

S18

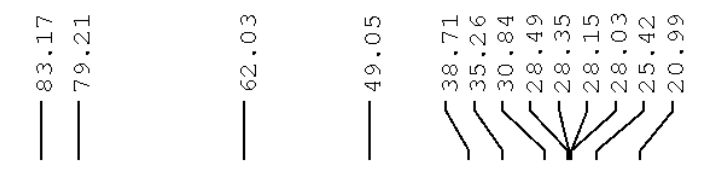

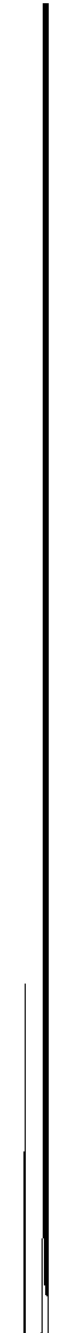

Current Data Parameters NAME

EXPNO

F2 - Accuisition Parame$$
\text { Date }
$$

Time

PROBHD

PULPROG

TD

SOLVENT

NS

SWH

FIDRES

$\mathrm{AQ}$

RG

RG
DW
DE
TE

D1
D11

D11

$====$
SFO1
NUC1

P1

20210423

9.26
av 600

mm $\mathrm{CPBBO}$ BB-

zgde 30

65536

520

36231.883

0.552855
0.9043968

9043968
2050

13.800

19.63
298.1

0.80000001

$0.0300000 \mathrm{C}$

$=$ CHANNEL $\mathrm{f} 1===$

$======$
SFO2
NUC2

CPDPRG

PCPD 2

PLW12

150.9194080
$13 \mathrm{C}$

64.000 .10

CHANNEL $f 2===$
600.1330010

F2 - Processing paramet

SI $\quad 150.902536$

$\begin{array}{lr}\text { SF } & 150.9028085 \\ \text { WDW } & \end{array}$

WDW

SSB

$\mathrm{LB}$
$\mathrm{GB}$

1.00

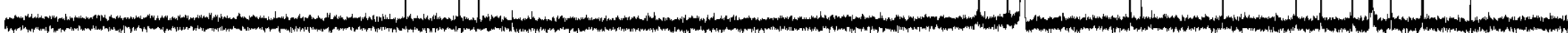

\begin{tabular}{rrrrrrrrrrr|}
\hline 200 & 180 & 160 & 140 & 120 & 100 & 80 & 60 & 40 & 20 & ppm
\end{tabular}




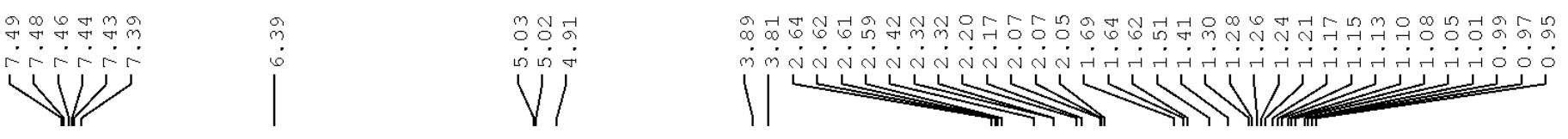

Current Data Parameters

F2 - Acquisition Parane

Date

INSTRUM

on Parame
20210524

INSTRUM

$5 \mathrm{~mm}$ CPBBO BB-

PULPROG

SOLVENT

DS

DS
SWH
FIDRE

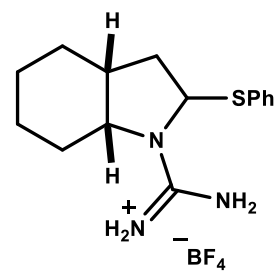

RG

DW

$\mathrm{DE}$

D1

$\mathrm{zg} 30$
98074

CDCl3

16
2

9615.385 5.0998478

10
52.000

14.23

0.10000000

$=======$ CHANNEL $\mathrm{f} 1 \mathrm{=}==$ :

$\begin{array}{lr}\text { SFO1 } & 600.1342009 \\ \text { NUC1 } & 1 \mathrm{H}\end{array}$

$\mathrm{P} 1$
$\mathrm{P}$

$\mathbf{S 2 0}$

PLW

30.00000000

F2 - Processing paramet

$\begin{array}{ll}\text { SI } & 65536 \\ \text { SF } & 600.1300354\end{array}$

SF

$S S B$

$G B$
$P C$

0.30
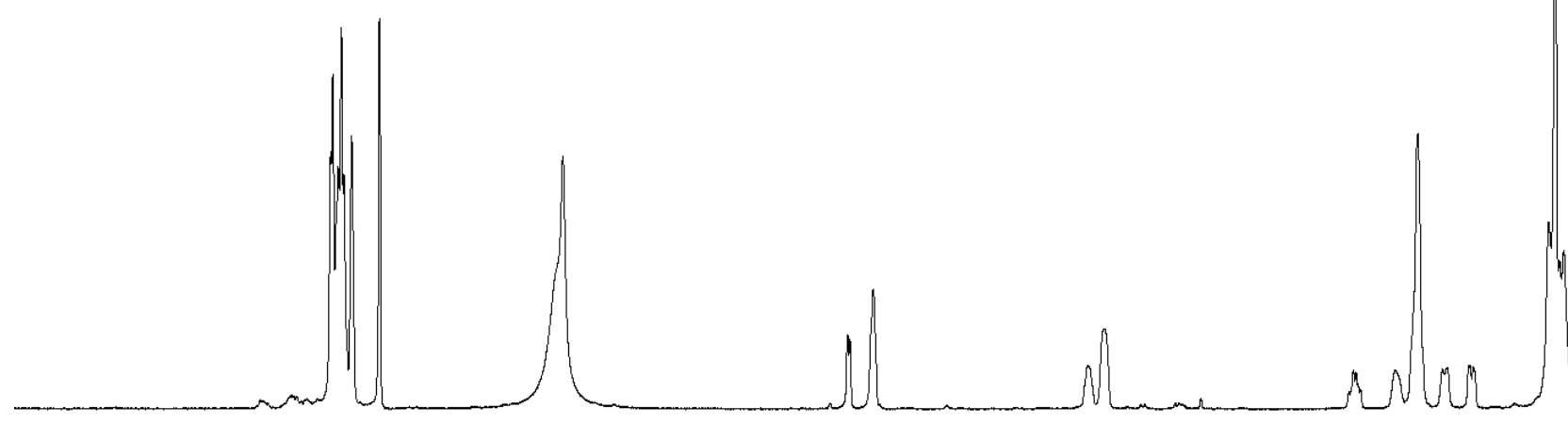

(1) wh

1.00 


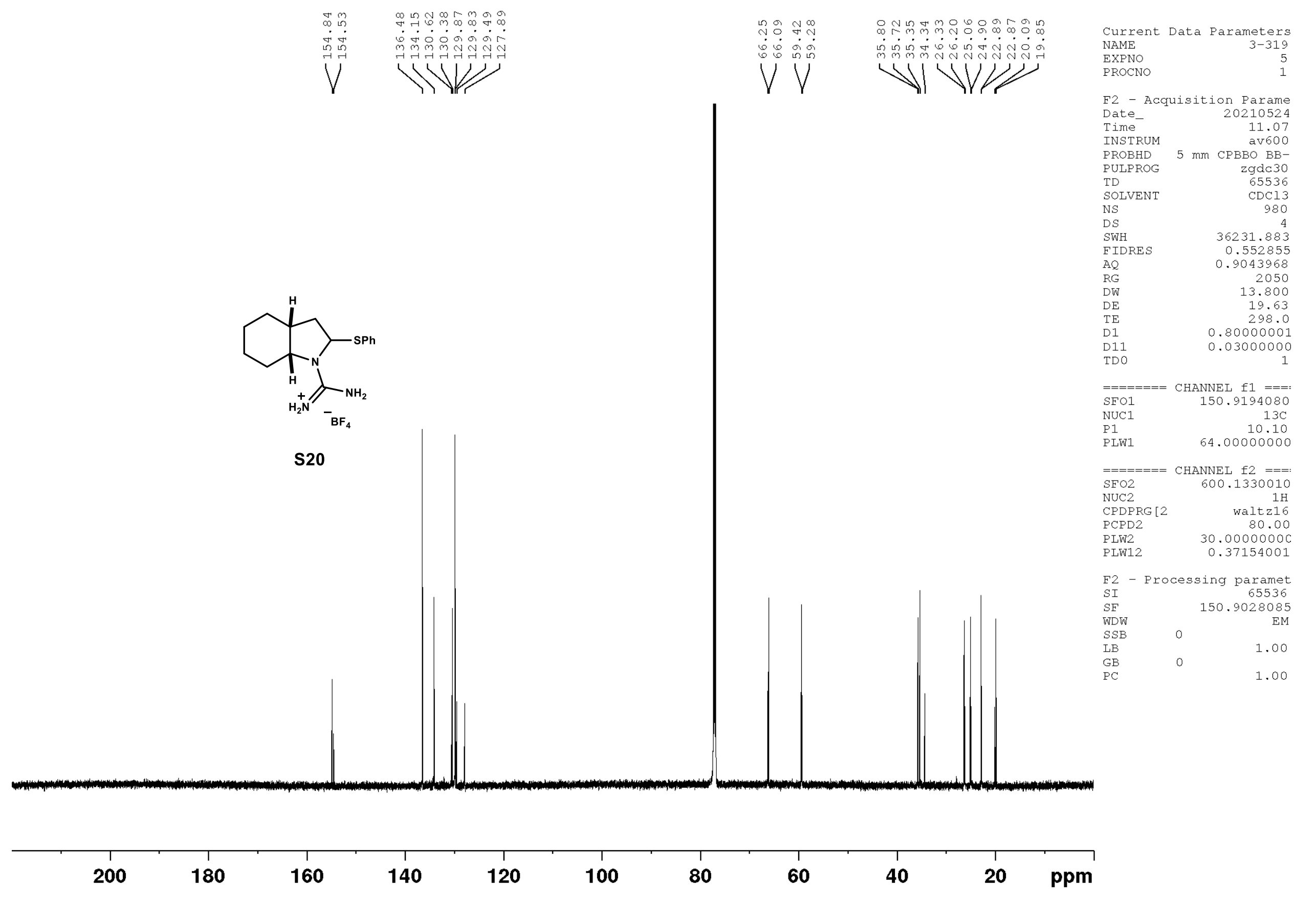




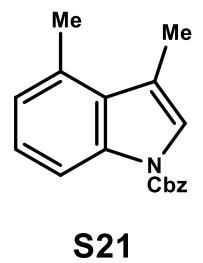

Current Data Parameters

F2 - Acquisition Parame Date 20130624 Time

INSTRUM $\begin{array}{rr}\text { PROBHD } & 5 \mathrm{~mm} \text { CPTCI } 1 \mathrm{H}- \\ \text { PULPROG } & \mathrm{zg} 30\end{array}$

SOLVENT

NS

$\begin{array}{lr}\text { NS } & 8 \\ \text { DS } & 2 \\ \text { SWH } & 8012.820\end{array}$

FIDRES $\quad 0.098043$

AQ $\quad 5.0998273$

DW

$\mathrm{DE}$

TE

298.0
D1

MCREST O sec

0.01500000 NUC 1

P1

PL1
SFO1 CHANNEL $\mathrm{f} 1===$

500.2235015

F2 - Processing paramet

SI 65536

$\begin{array}{lr}\text { SF } & 500.2200301 \\ \text { WDW } & \text { EM }\end{array}$

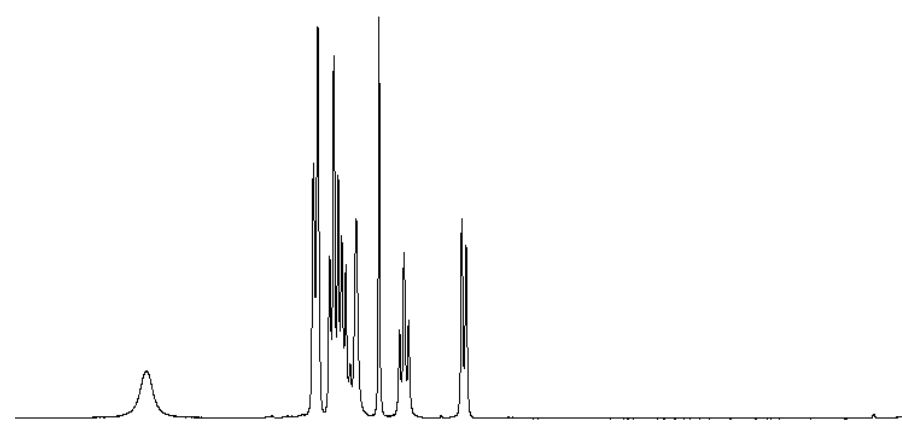

SSB $\quad 0$

0.30

4.00

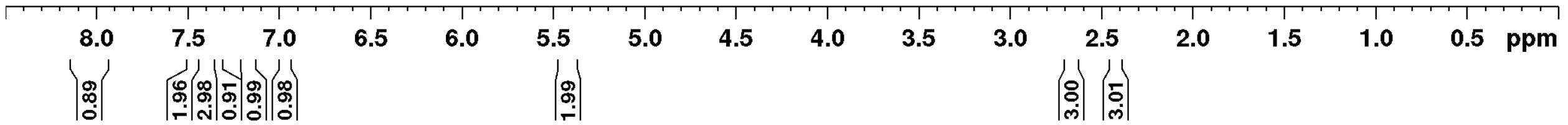




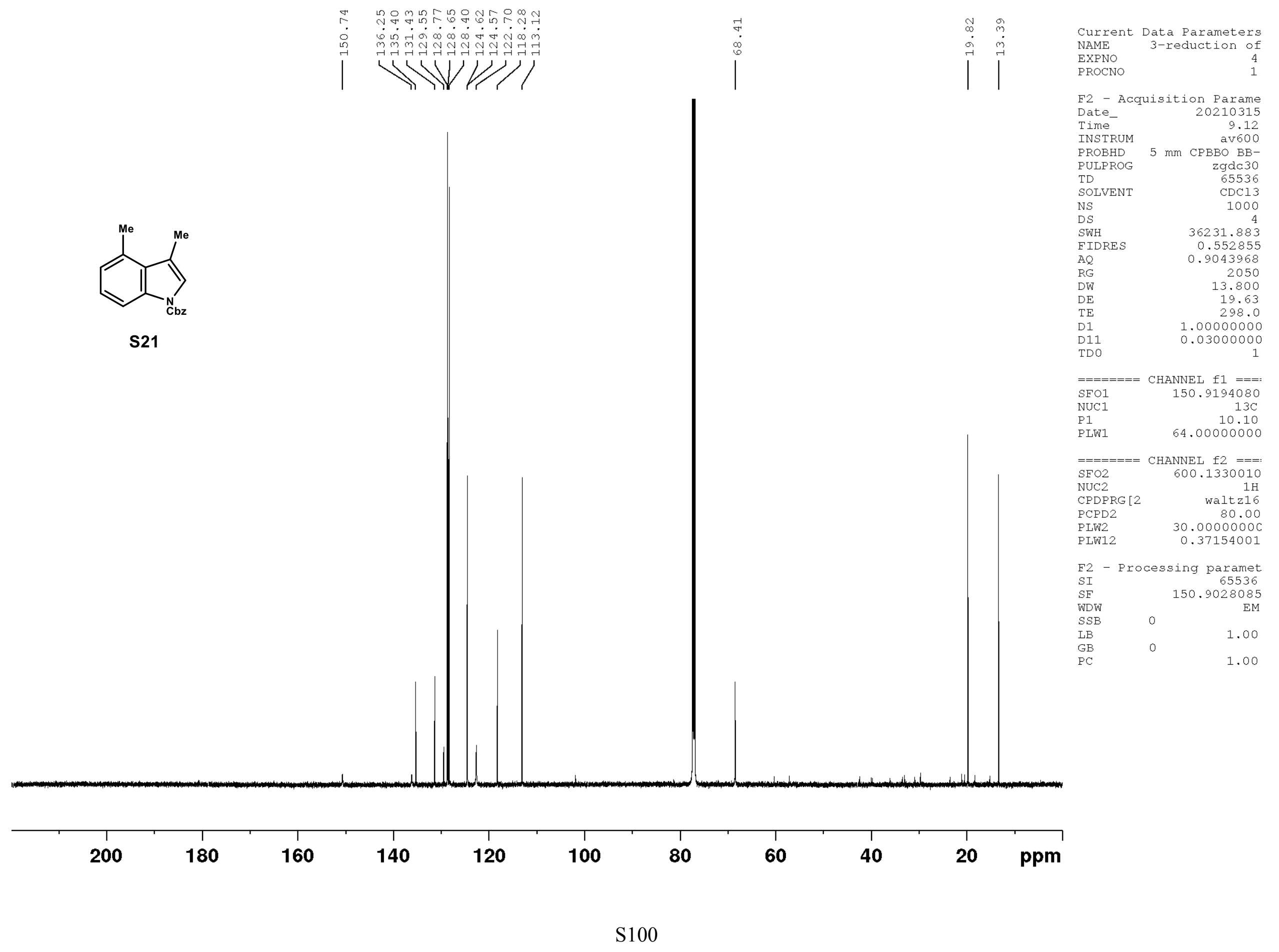




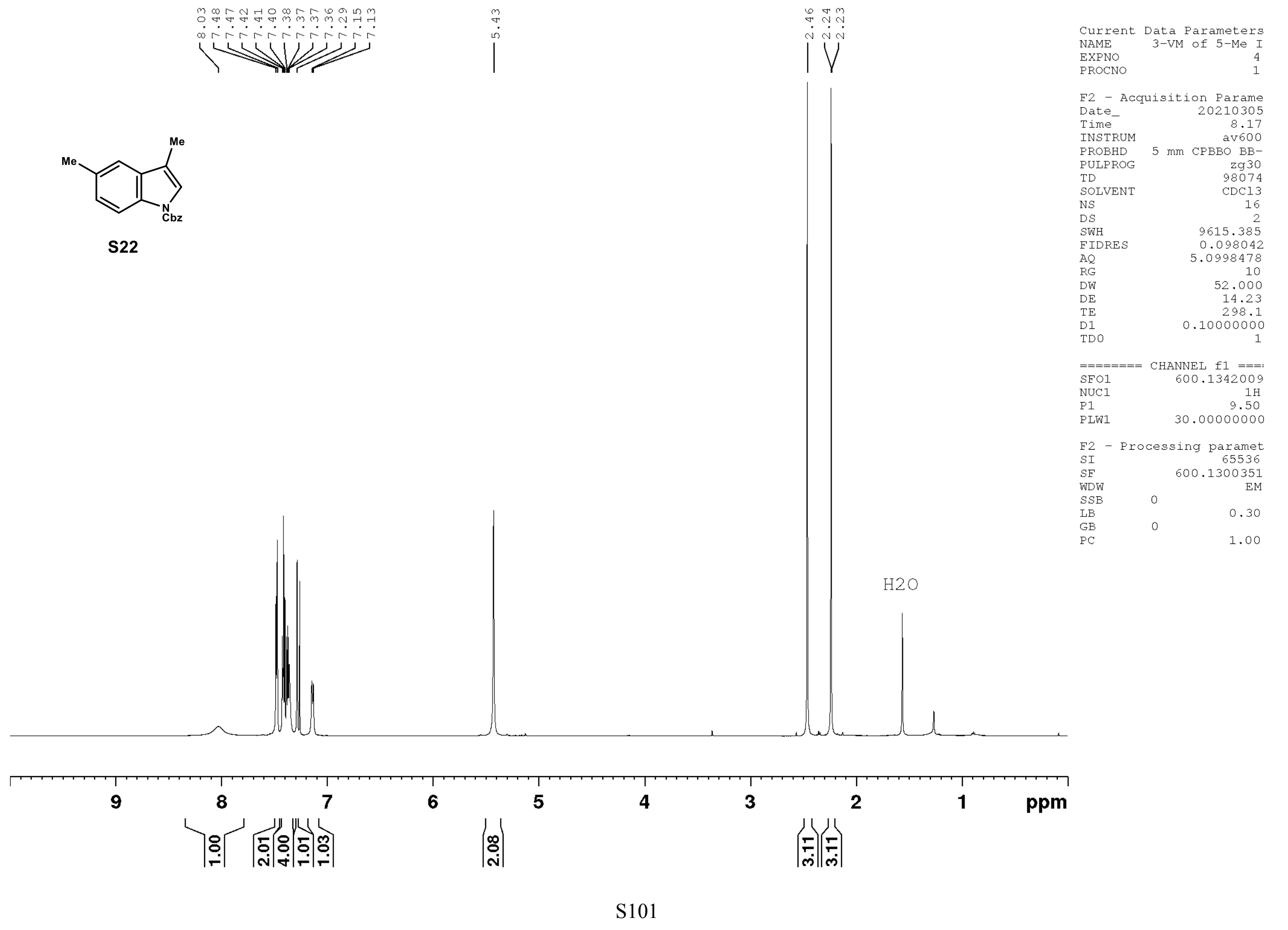




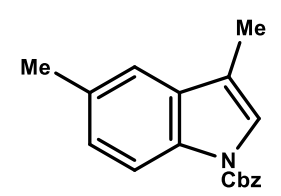

S22

\section{(n)}

22

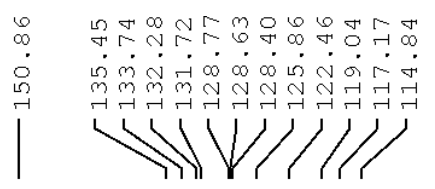

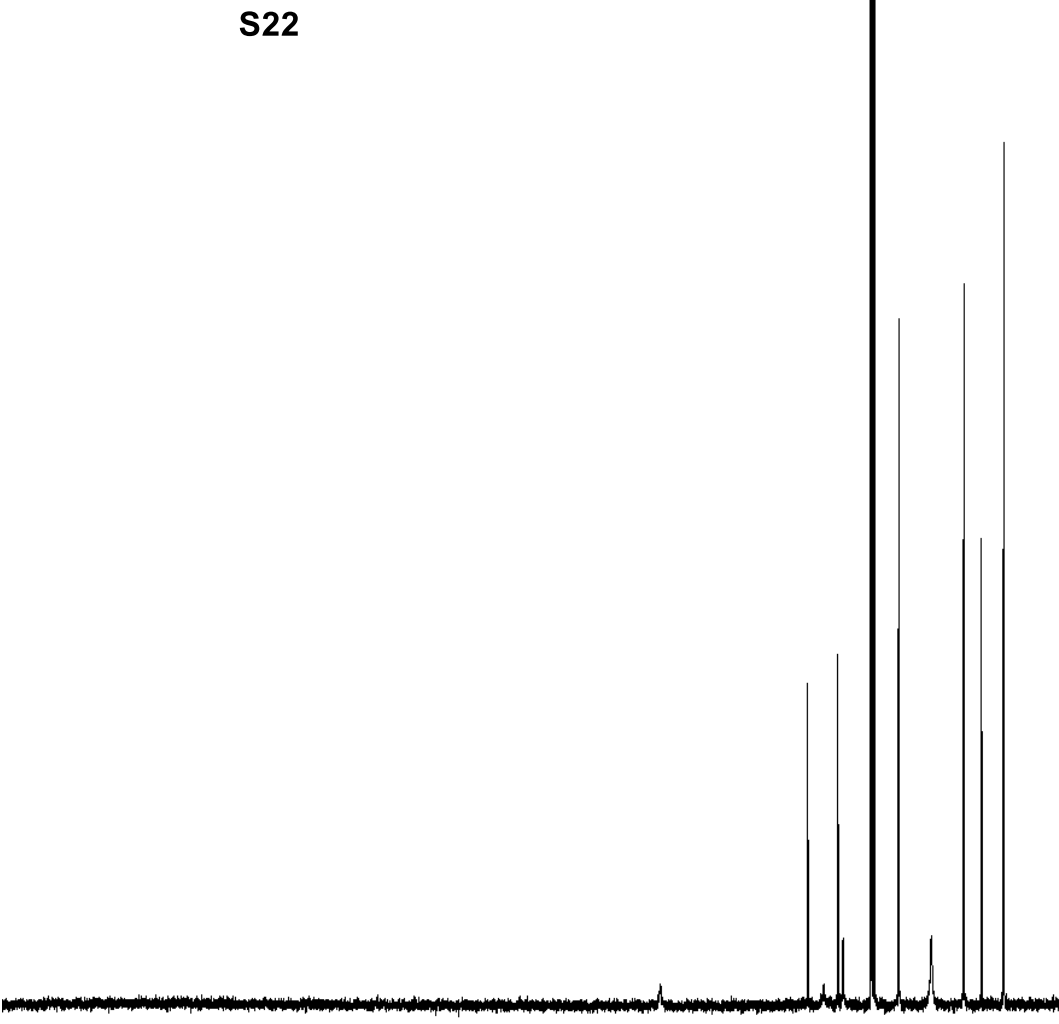

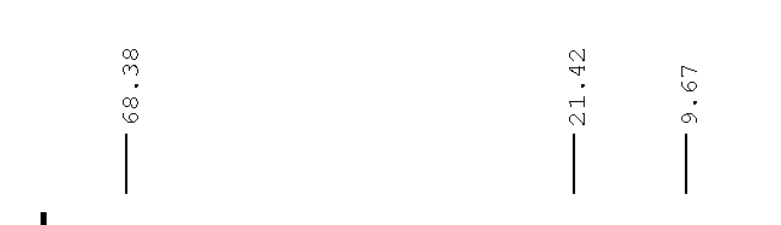

Current Data Parameters

EXPNO
PROCNO

F2 - Acquisition Parame

Date_

Time

5 mm CPBBO

PUIPROG 5 -

TD $\quad 65536$

SOLVENT

DS

DS

$\begin{array}{lr}4 \\ \text { SWH } & 36231.883\end{array}$

0.552855

RG

DW

DE

D1

D11

$=====$

$\mathrm{SFO1}$
NUC1

2050
13.800

19.63

1.00000000

0.03000000

CHANNEL $f 1===$

PLW1

10.10
64.00000000

$==$

ANNEL $12===$

$\mathrm{SFO} 2$

CHANNEL $\mathrm{f2}===$
600.1330010

NUC2 $1 \mathrm{H}$

PLW2 $\quad 30.00000000$

PLW12

F2 - Processing paramet

SI

SF $\quad 150.9028085$

WDW

$\mathrm{LB}$
$\mathrm{GB}$

$\mathrm{PC}$

1.00

1.00

\begin{tabular}{rrrrrrrrr|r|r|r|}
\hline 200 & 180 & 160 & 140 & 120 & 100 & 80 & 60 & 40 & 20 & ppm
\end{tabular}




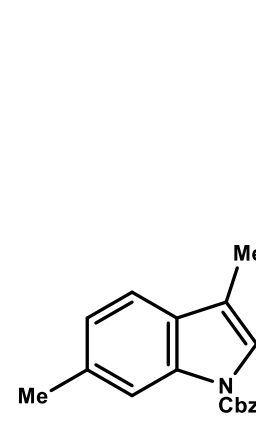

S23

$\begin{array}{lr}\text { F2 - Acquisition Parame } \\ \text { Date_- } \\ \text { Time } & 910315 \\ \text { Iime } & 9.23\end{array}$

Time

INSTRUM av600

PROBHD $5 \mathrm{~mm}$ CPBBO $\mathrm{BB}-$

PULPROG 2930

TD

DS

$\begin{array}{lr}\text { DS } & 2 \\ \text { SWH } & 9615.385 \\ \text { FTDRES } & 0.098042\end{array}$

AO $\quad 0.098042$
R.

AQ

RG

$\mathrm{DE}$

D1

10

52.000

14.23

0.10000000

$=======$ CHAnNEL $\mathrm{fl}===$

SFO1 $\quad 600.1342009$

NUC1 $\quad 1 \mathrm{H}$

$\begin{array}{lr}\text { P1 } & 9.50 \\ \text { PLW1 } & 30.00000000\end{array}$

F2 - Processing paramet

ST Processing paramet

SI 65536

$\begin{array}{lr}\text { SF } & 600.1300340 \\ \text { WDW } & \text { EM }\end{array}$

SSB $\quad 0$

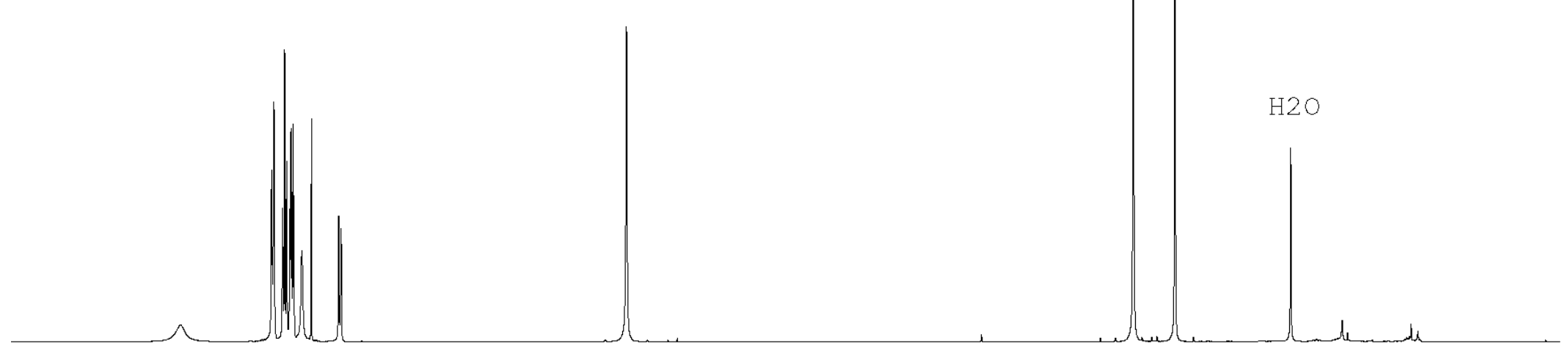

0.30

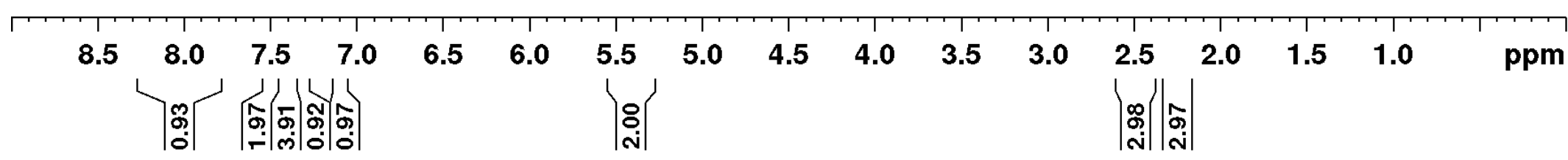




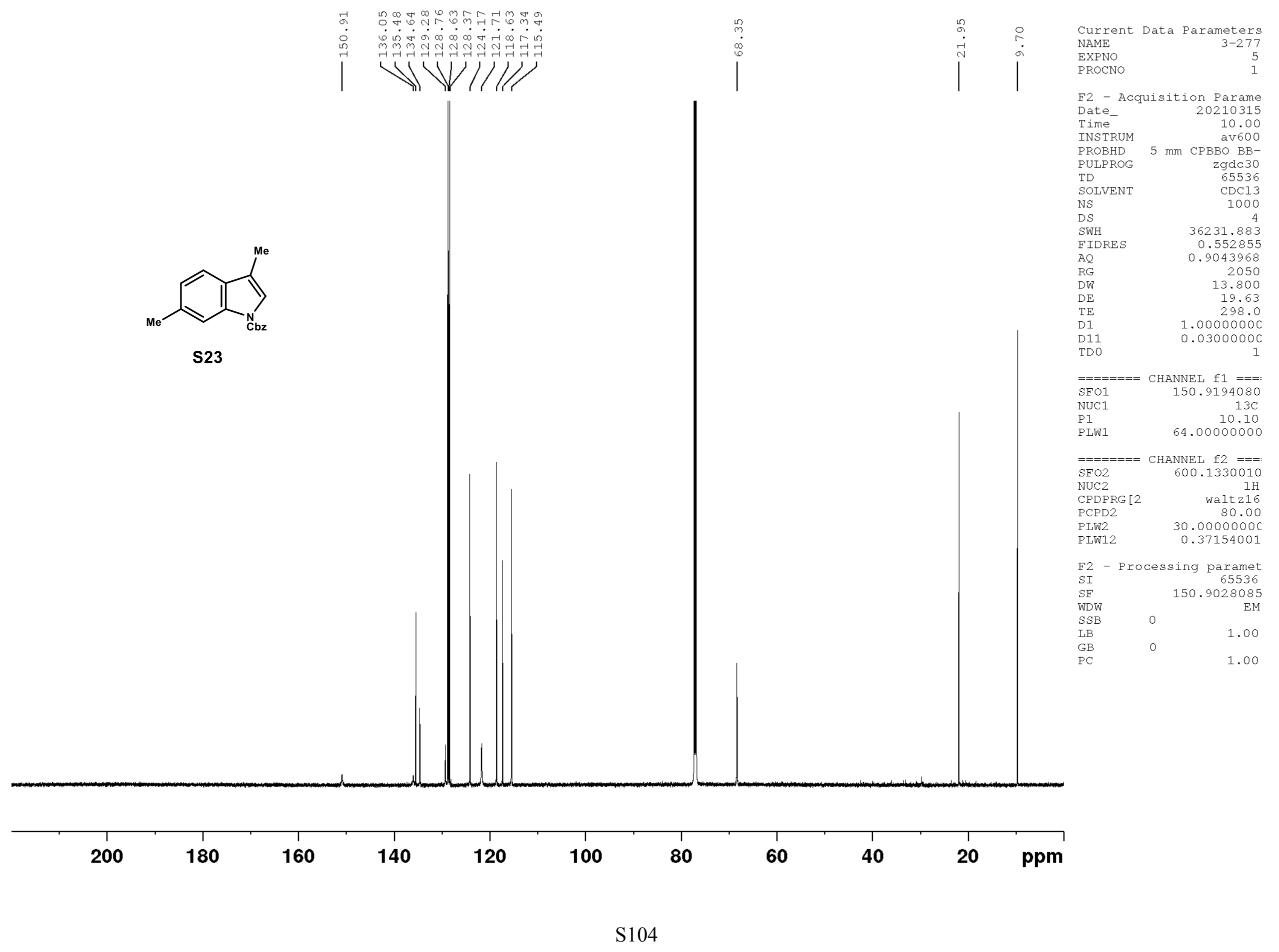




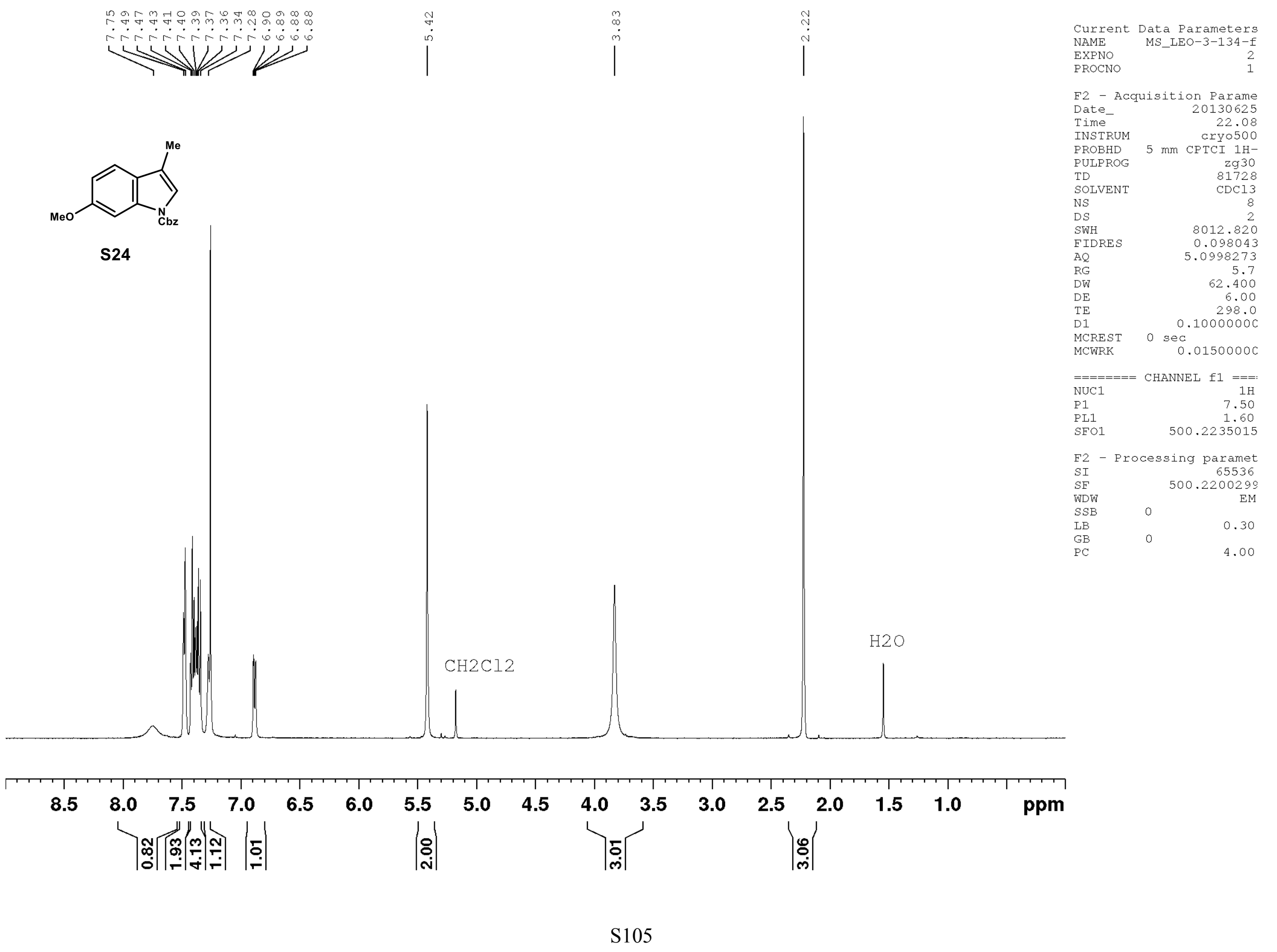




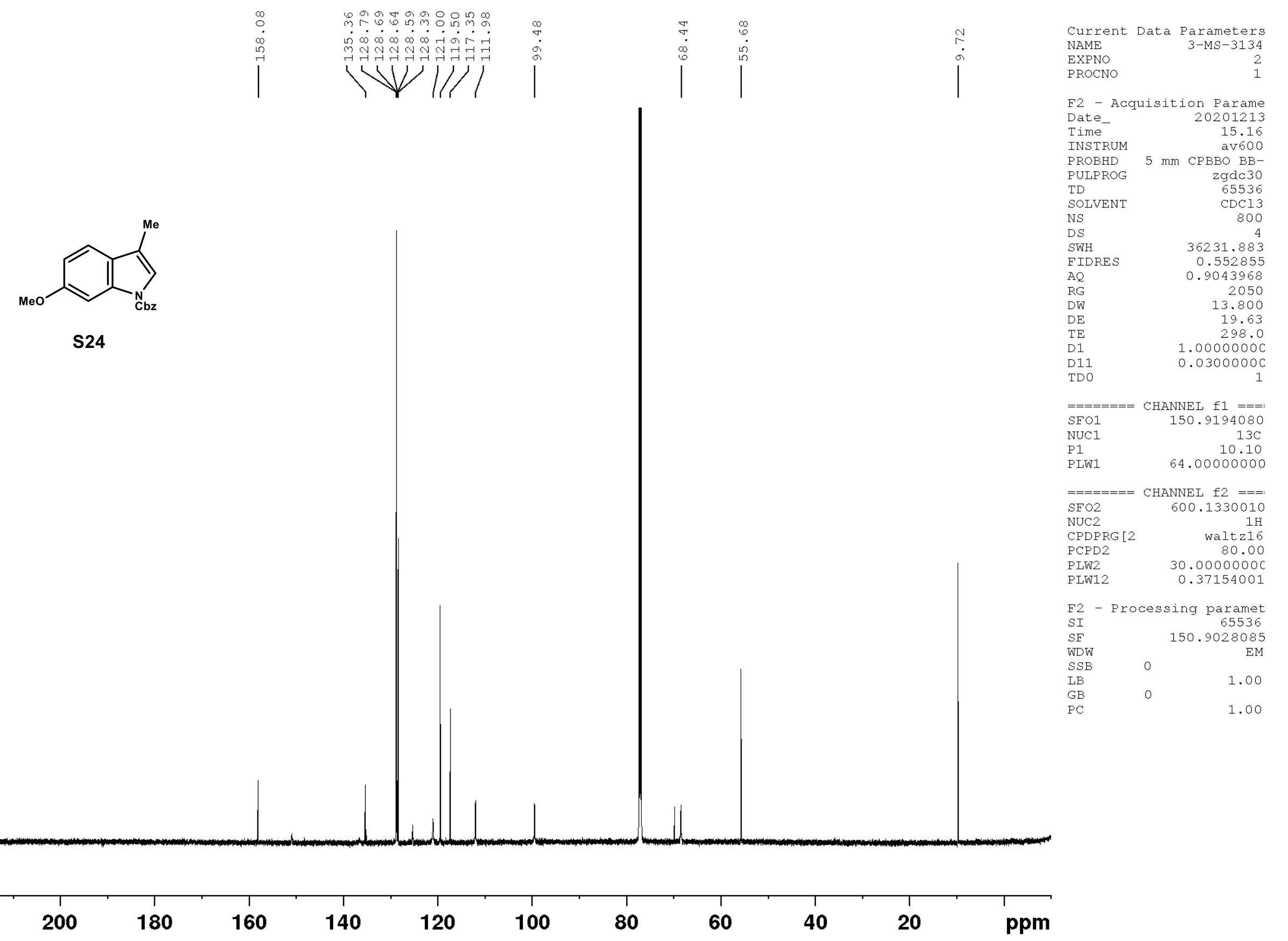


F2 - Acquisition Parame

Date

INSTRUM

PROBHD

PULPROG

TD

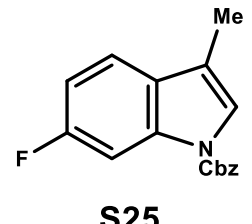

NS

DS

SWH $\quad 8012.820$

FIDRES

RG

RG

$\mathrm{DE}$

D1

MCREST

MCWR

23.44

cryo500

CI $1 \mathrm{H}-$

$=======$ CHANNEL $\mathrm{f} 1===$

NUC

$\mathrm{P} 1$

$\begin{array}{lr}\text { PL1 } & 1.60 \\ \text { SFO1 } & 500.2235015\end{array}$

F2 - Processing paramet
SI

$\begin{array}{ll}\text { SF } & 500.2200298\end{array}$
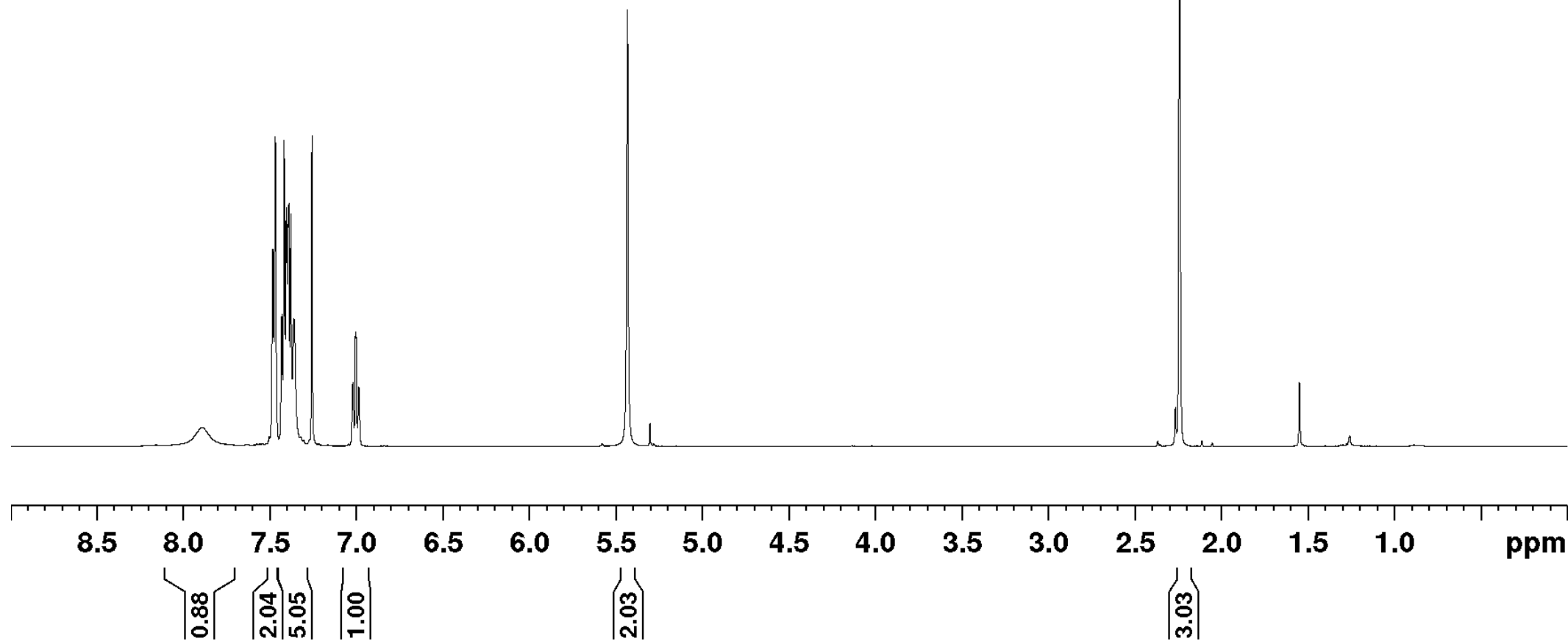


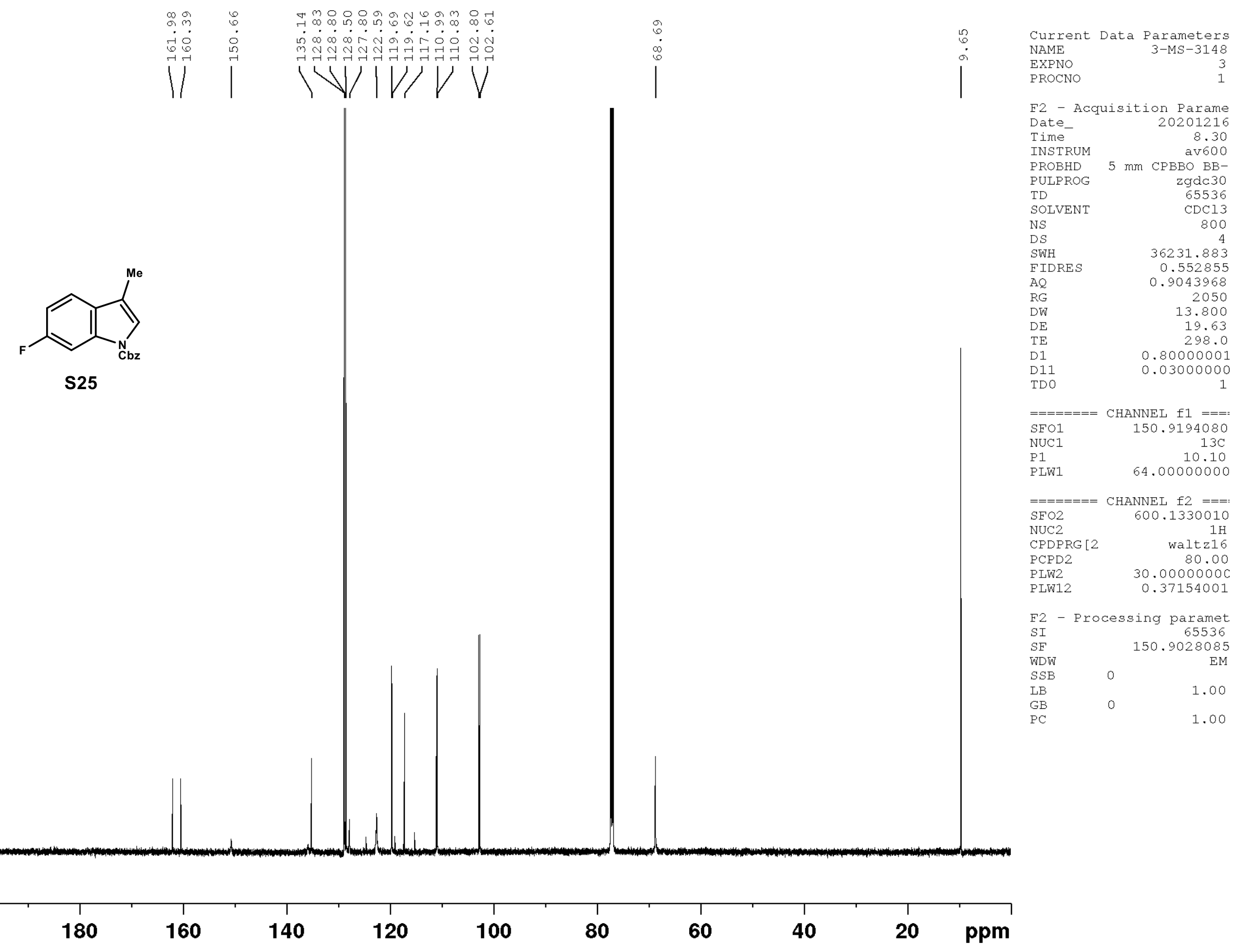



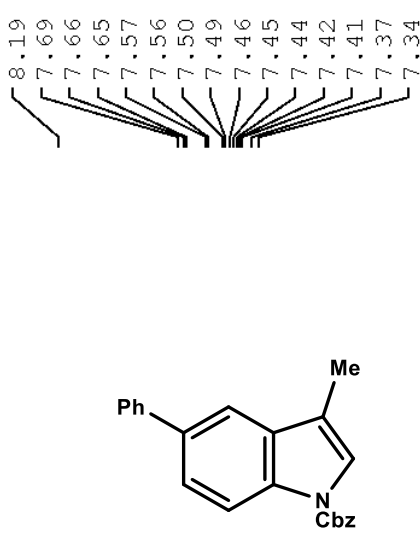

526

contaminated $\mathrm{w} / \sim 30 \% \mathrm{CO}(\mathrm{OBn})_{2}$

\section{$\left.\right|^{\stackrel{\infty}{n}} \stackrel{\infty}{+\infty}$}

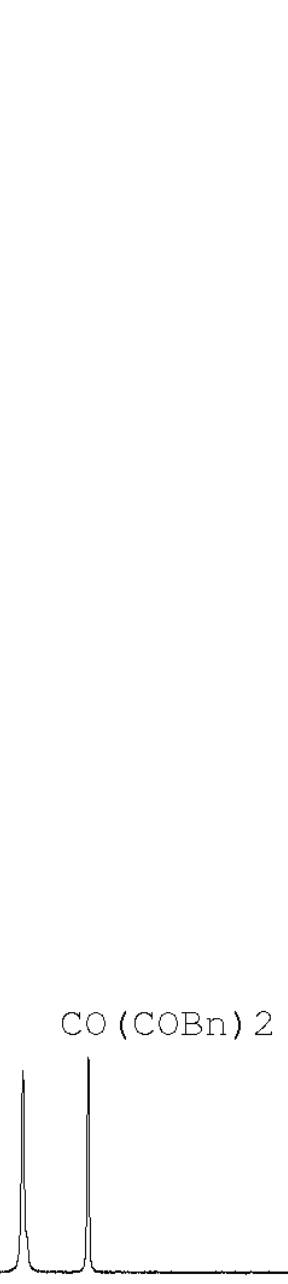

?

120
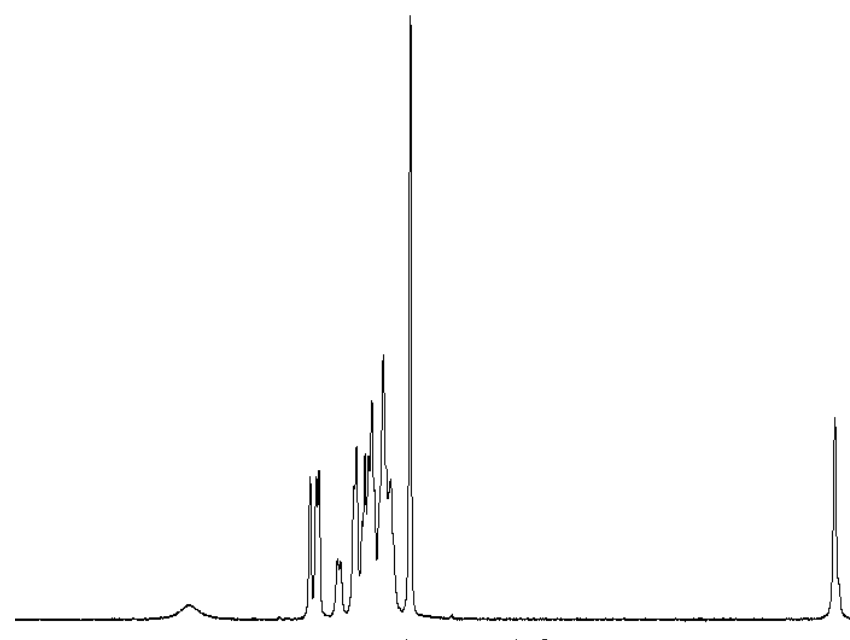

$\mathrm{CO}(\mathrm{COBn}) 2$

Current Data Parameters NAME

PROCNO

F2 - Acquisition Parame

Date

Time

INSTRUM

PROBHD

PROBHD

TD

SOLVENT

DS

SWH

AQ

RG

DW

DE

D1

$===$

SFO1

$\mathrm{P} 1$

PLW1

8.16

mm. $\mathrm{CPBBO}$ BB-

zg30

2930
98074

CDCl3

2
.385

5.0998478

10

52.000
14.23

0.10000000

30.00000000

F2 - Processing paramet

SI

SF $\quad 600.1300000$

WDW

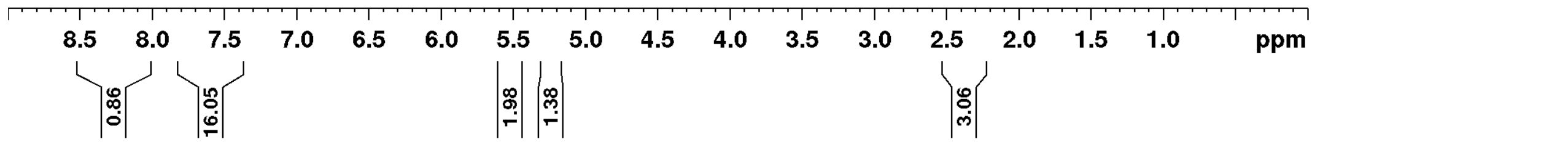




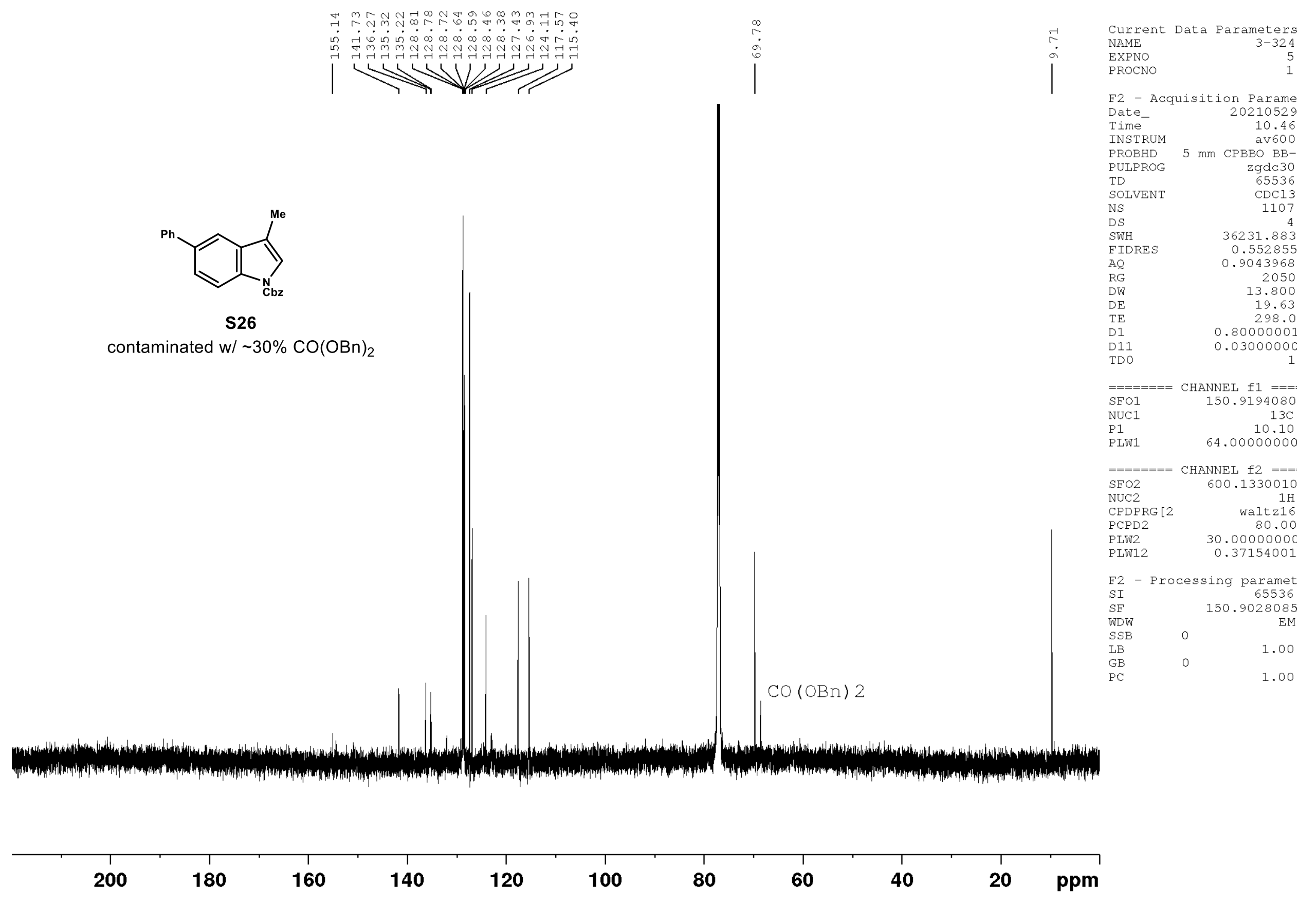



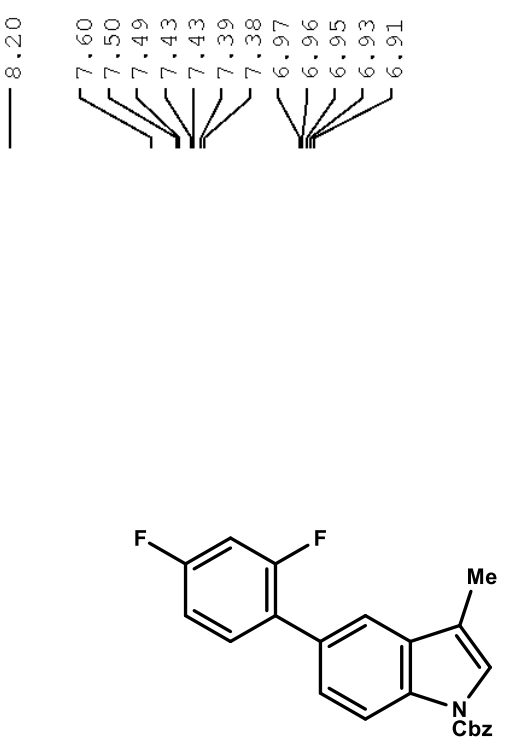

S27
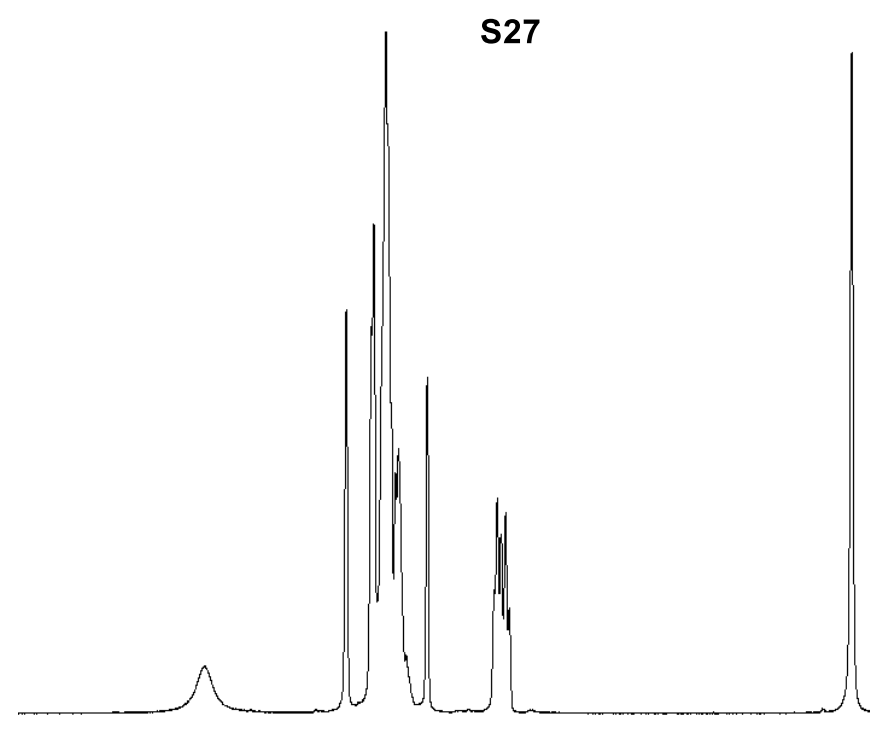

$\mathrm{CO}(\mathrm{OBn}) 2$
E2 - Acquisition Parame

Date

Time

PROBHD

PULPRO

TD

SOLVENT

NS

SWH

FIDRES

$\mathrm{AQ}$

RG

DW

$\mathrm{DE}$

D1

$===$
SFO

SFO

P1
PLW1

20210515
9.17

9.17
$2 \mathrm{v} 600$

mm $\mathrm{CPBBO}$ BBzg30
98074 CDC13

16
2
385

9615.385
0.098042 5.0998478

10

52.000
14.23

298.0

0.10000000

CHANNEL f1 $===$

F2 - Processing paramet

SI Processing

SF $\quad 600.1300364$

WDW

$S: S$

GB

0.30

1.00

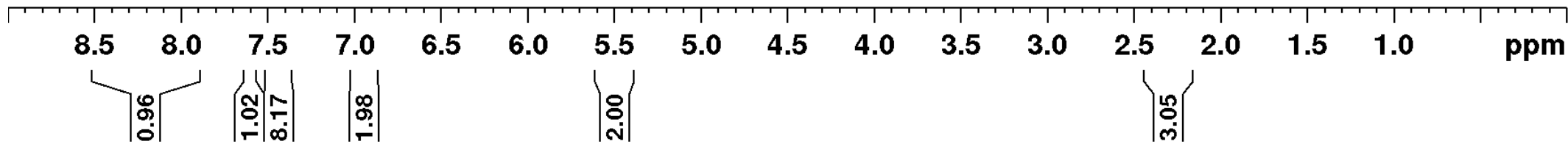




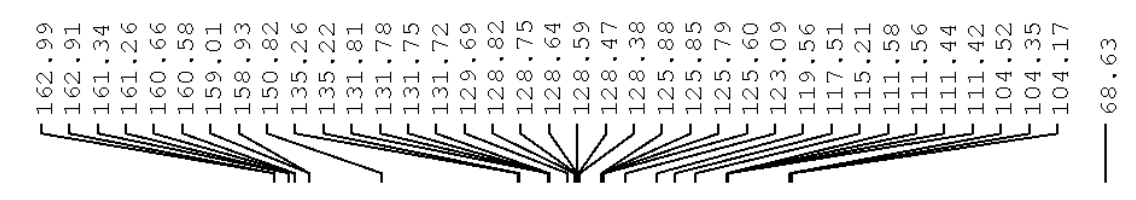

Current Data Parameters

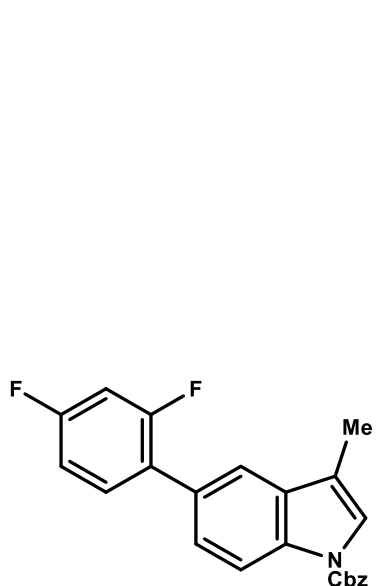

S27

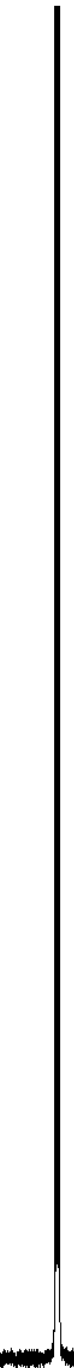

F2 - Acquisition Parame

Date__ 20210515

Time

av600

5 mm $\mathrm{CPBBO} \mathrm{BB}-$

PULPROG $\quad$ zgdc30

TD

NS

DS

SWH $\quad 36231.883$

FIDRES $\quad 0.552855$

$\mathrm{AQ} \quad 0.9043968$

$\begin{array}{lr}\text { RG } & 2050 \\ \text { DWT } & 13.800\end{array}$

$\begin{array}{lr}\mathrm{DE} & 19.63 \\ \mathrm{TE} & 298.0\end{array}$

$\begin{array}{lr}\text { TE } & 298.0 \\ \text { D1 } & 0.40000001\end{array}$

D11 $\quad 0.03000000$

TDO

$========$ CHANNEL $\mathrm{f1}===$ :

SFO1 $\quad 150.9194080$

NUC1 150.9194080

P1

64.00000000

$=======$ CHANNEL $f 2===$

$\mathrm{SFO} 2$

600.1330010

CPDPRG [2 waltz16

PCPD2 $\quad 80.00$

$\begin{array}{lr}\text { PLW2 } & 30.00000000 \\ \text { PIW12 } & 0.37154001\end{array}$

F2 - Processing paramet
SI

$\begin{array}{ll}\text { SF } & 150.9028085\end{array}$

WDW

LB 1.00

GB $\quad 0$

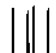

$\mathrm{CO}(\mathrm{OBn}) 2$

\begin{tabular}{rrrrrrrrrrr|r}
\hline 220 & 200 & 180 & 160 & 140 & 120 & 100 & 80 & 60 & 40 & 20 & ppm
\end{tabular}


F2 - Acquisition Parame

Date

INSTRUM

PROBHD

PULPROG

TD
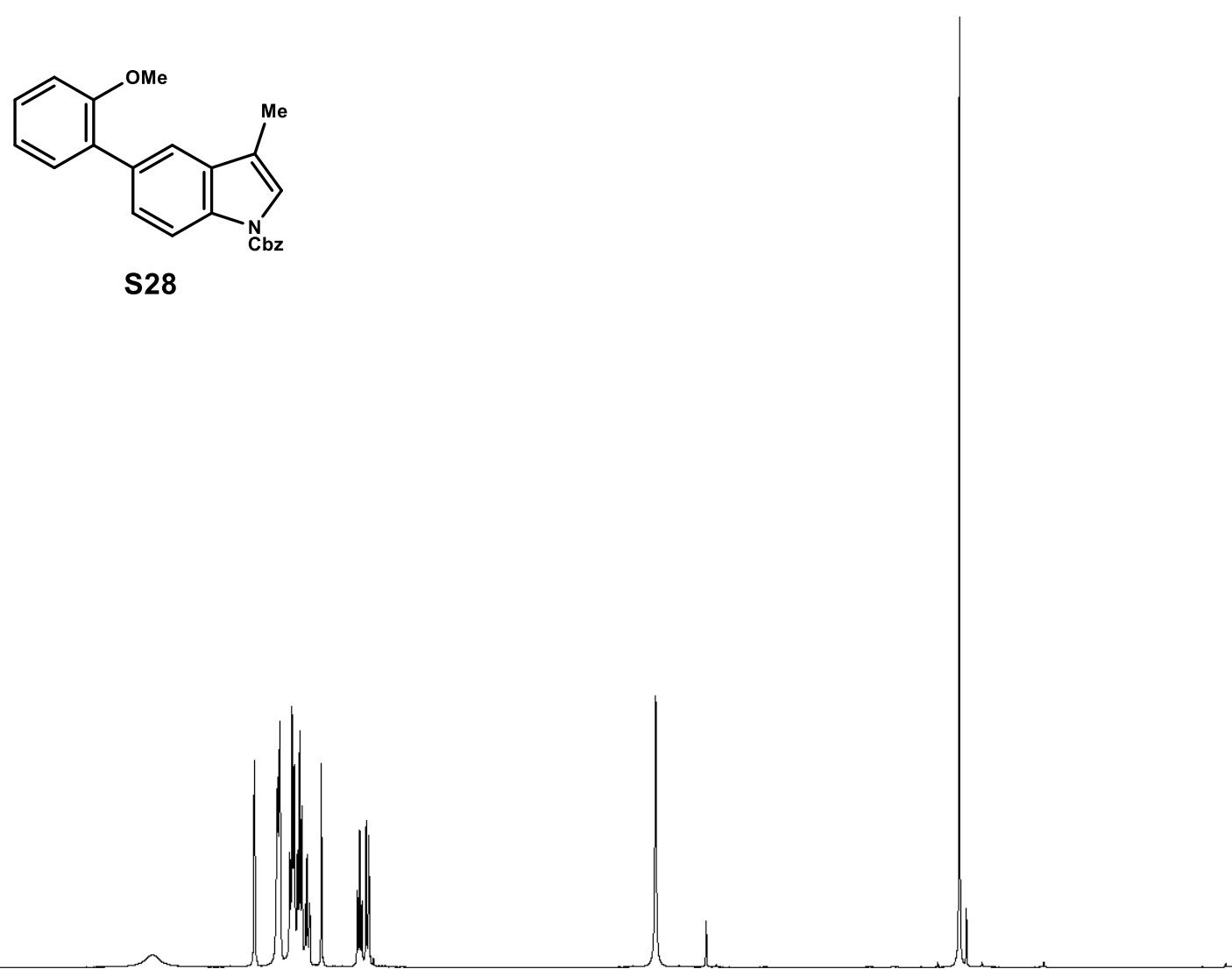

SO

DS

SWH

FIDRE

$\mathrm{AQ}$
$\mathrm{RG}$
$\mathrm{DW}$

$\mathrm{DE}$
$\mathrm{TE}$

TE

TDO

20210412

8.30
av600

$\mathrm{mm} \mathrm{CPBBO} \mathrm{BB}-$

$\mathrm{zg} 30$
98074

CDC13

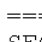

SFO1

NUC1

PLW1

9615.385

0.098042 5.0998478

52.000

14.23

0.10000000

$1 \mathrm{H}$
3.50
30.00000000

F2 - Processing paramet

$\begin{array}{lr}\text { SI } & 65536 \\ \text { SF } & 600.1300345\end{array}$

WDW

SSB

$L B$

GB

0.30

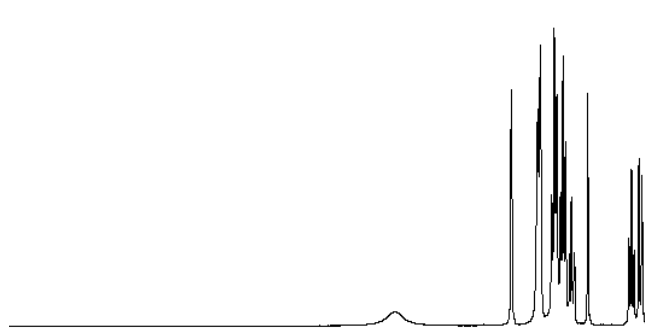

7

ר:

$\mid \begin{aligned} & \text { 옹 } \\ & \mid\end{aligned}$

5
4

$\left|\begin{array}{l}\frac{0}{m} \\ \text { in }\end{array}\right|$
3

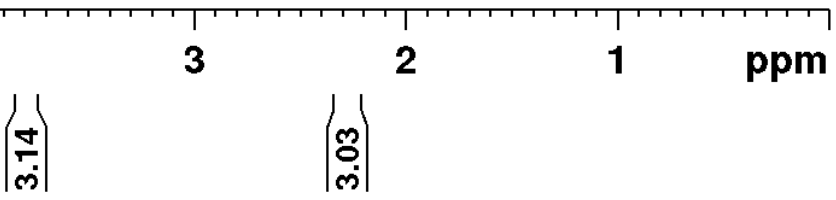




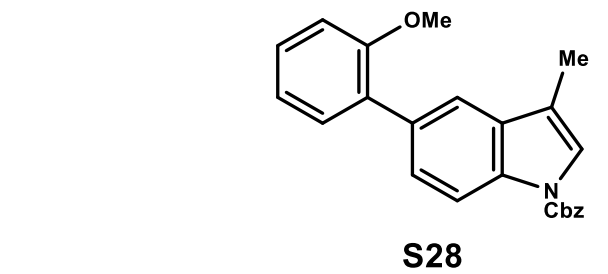

S28

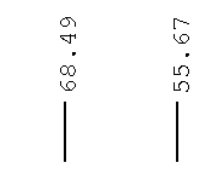

$\stackrel{N}{a}$ 


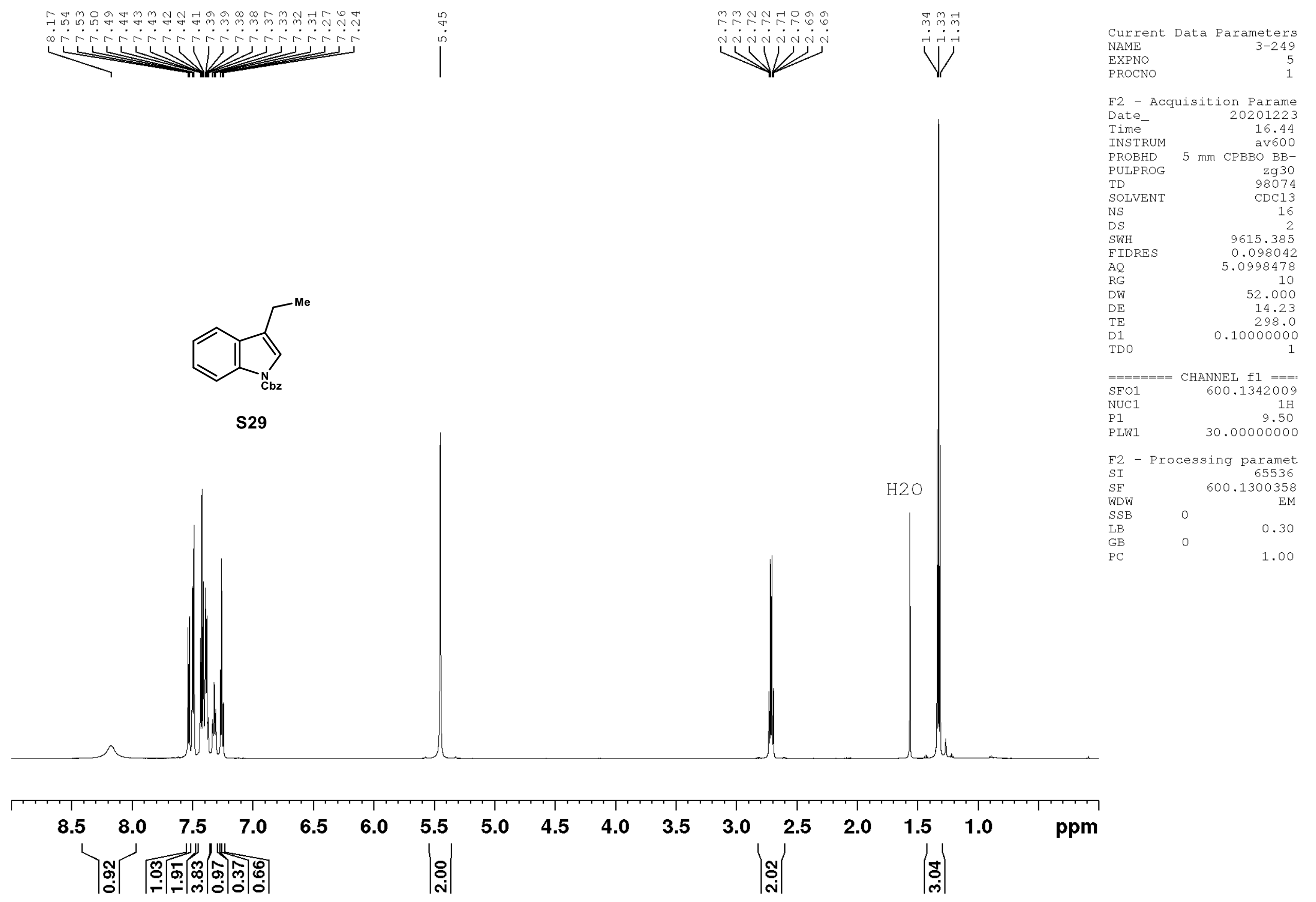




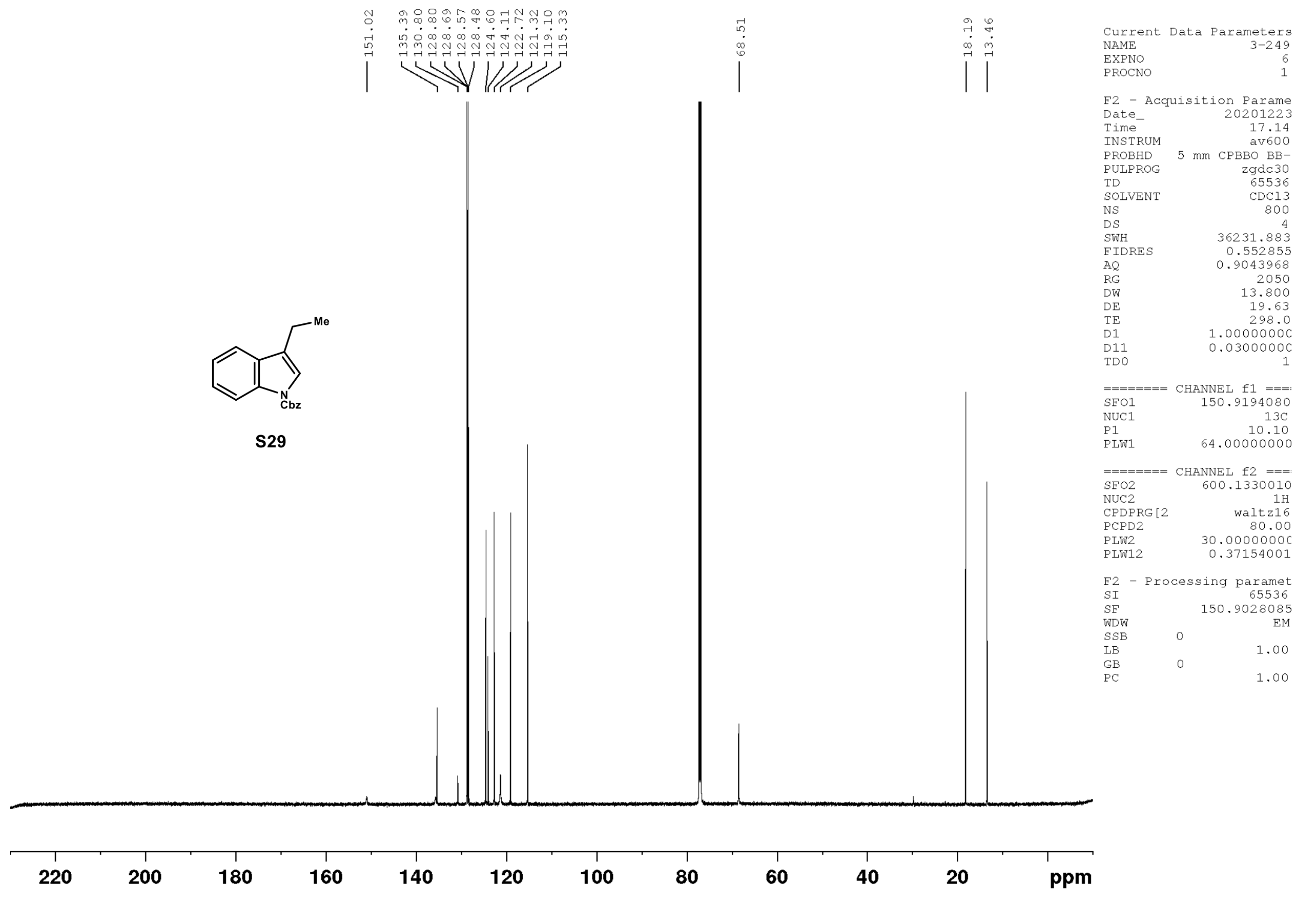


F2 - Acqui Parame Time-

THSTUM 16.15
$2 \mathrm{~V} 600$ $5 \mathrm{~mm}$ CPBBO BBzg30 TD 98074

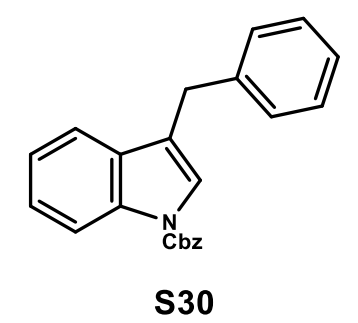

$\begin{array}{lr}\text { DS } & 2 \\ \text { SWH } & 9615.385 \\ \text { FIDRES } & 0.098042\end{array}$ AQ

RG

$\mathrm{DE}$
$\mathrm{TE}$

D1
TDO

$===$
SFO SFO1
NUC1 NUC1
P1

P1 5.0998478 10
52.000 14.23 0.10000000 600.1342009 9.50

F2 - Processing paramet SI 65536 $\begin{array}{ll}\text { SF } & 600.1300344\end{array}$ WSW $\quad 0 \quad$ EM

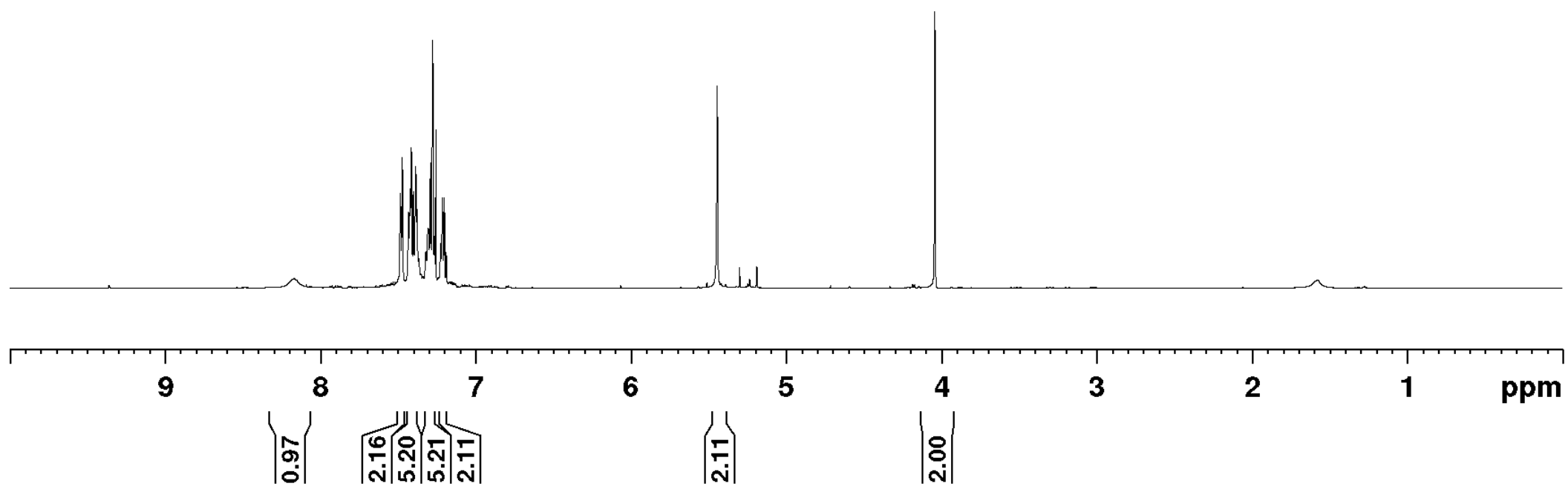




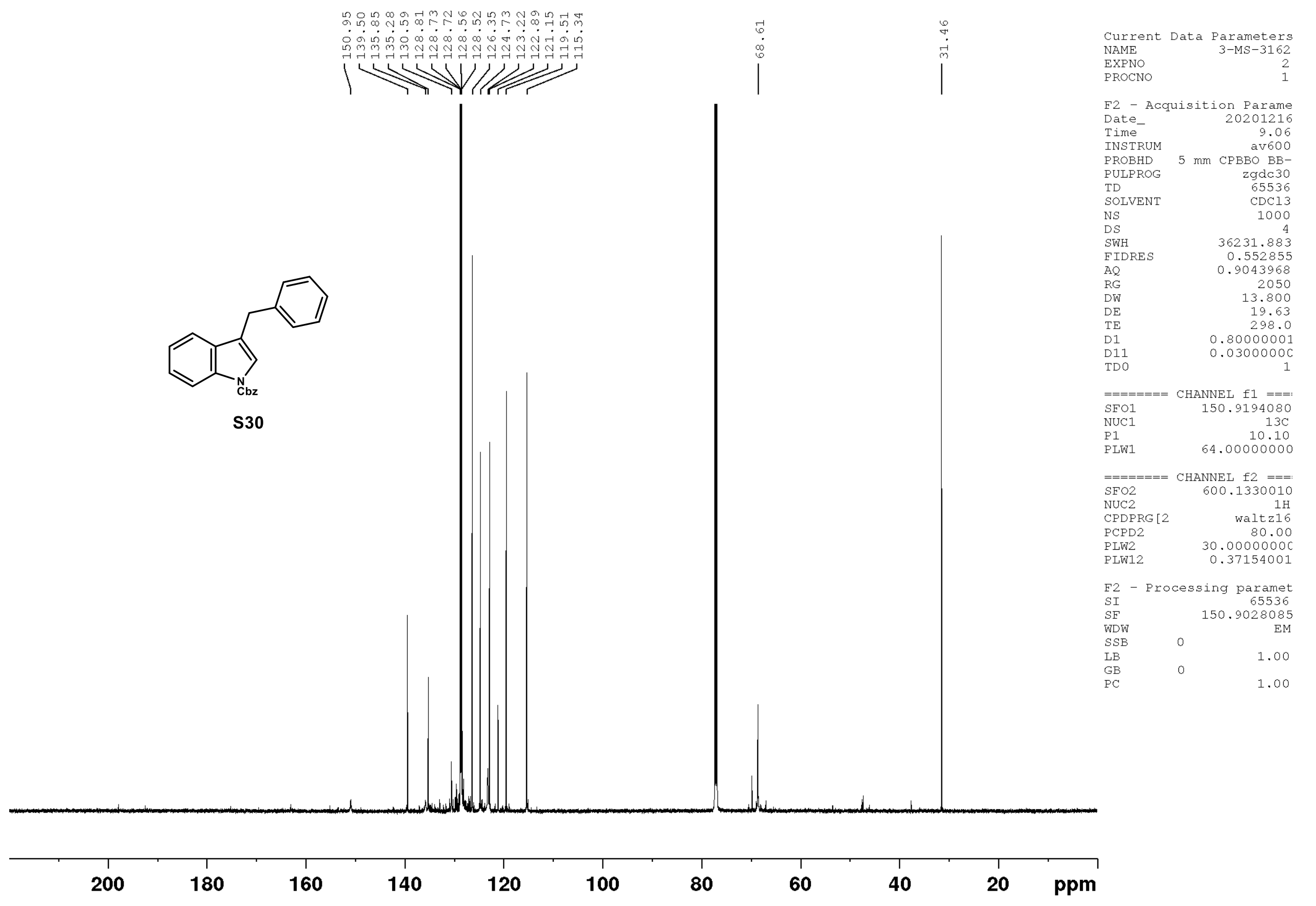


F2 - Acquisition Parane
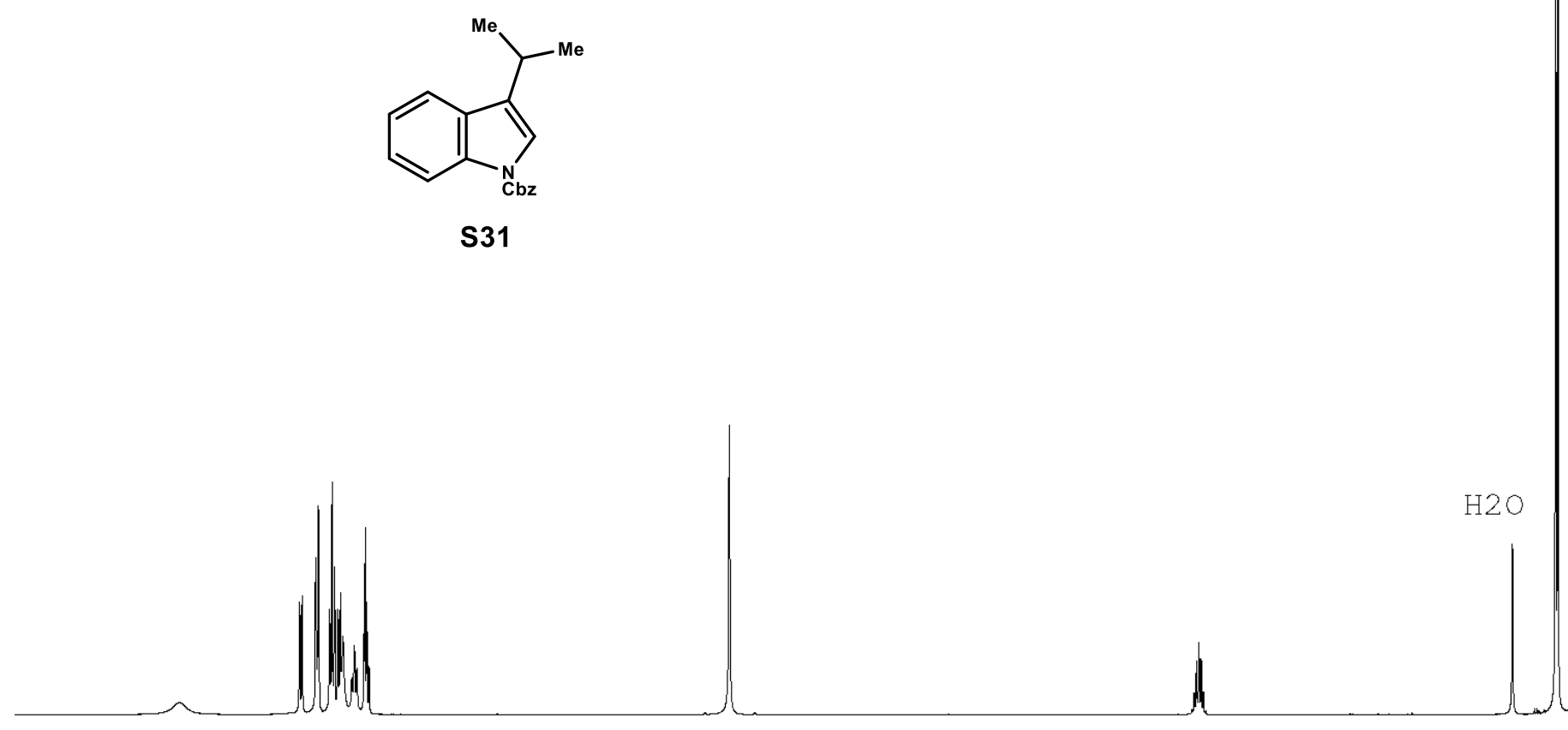

20201223 17.31
17.62 mm $\mathrm{CPBBO}$ BBzg 30 $\mathrm{zg} 30$
98074 $\mathrm{CDCl} 3$ 16
2

F2 - Processing paramet

1.00

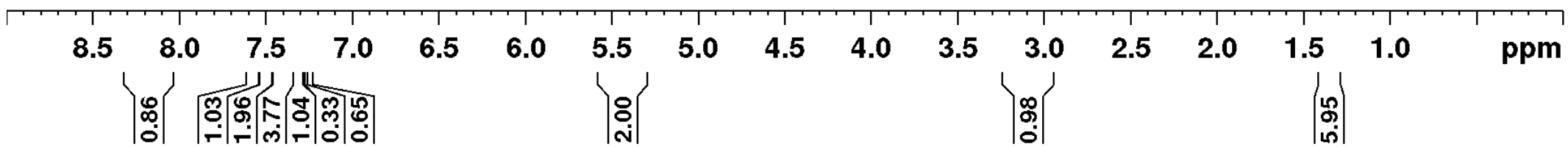




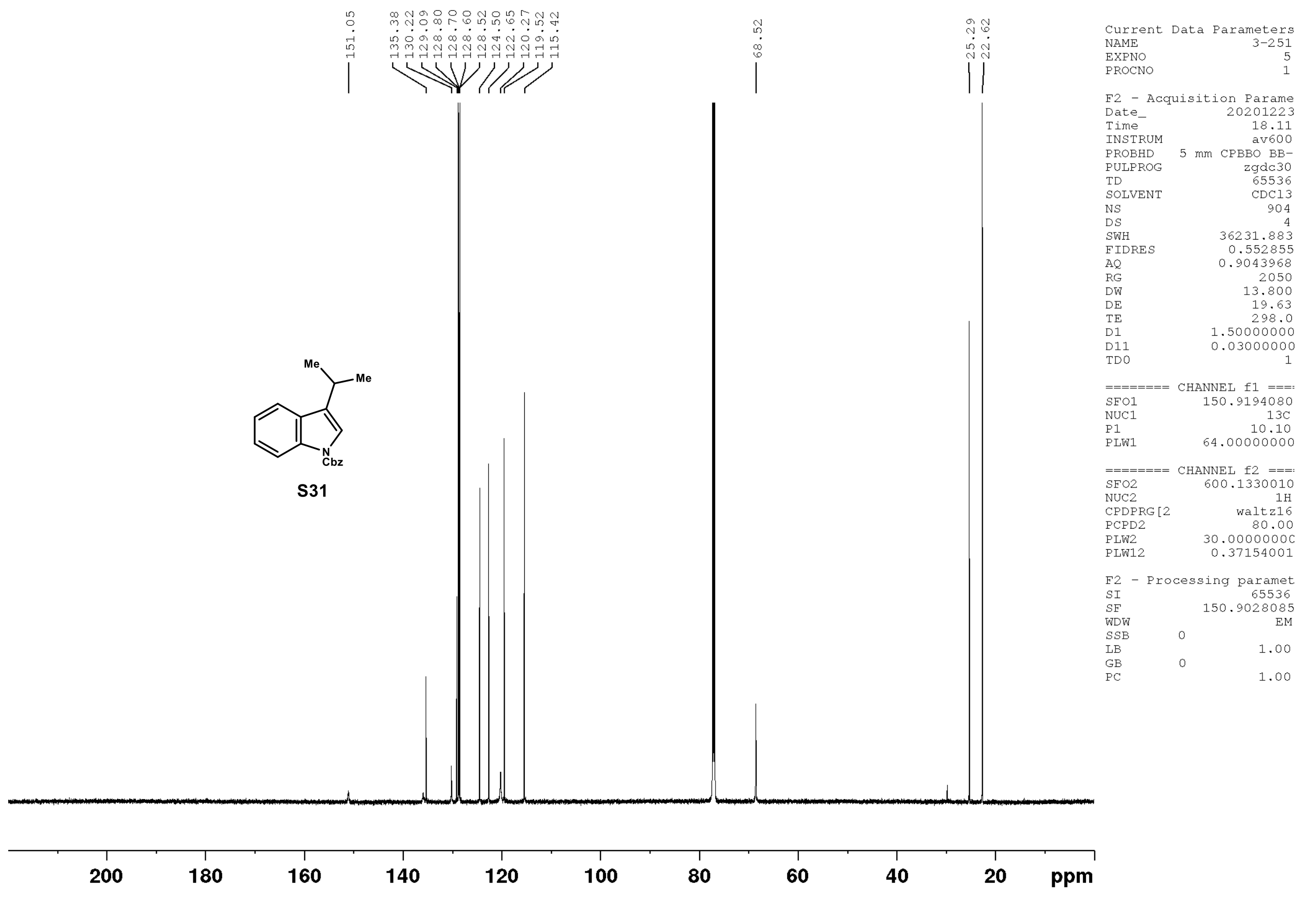




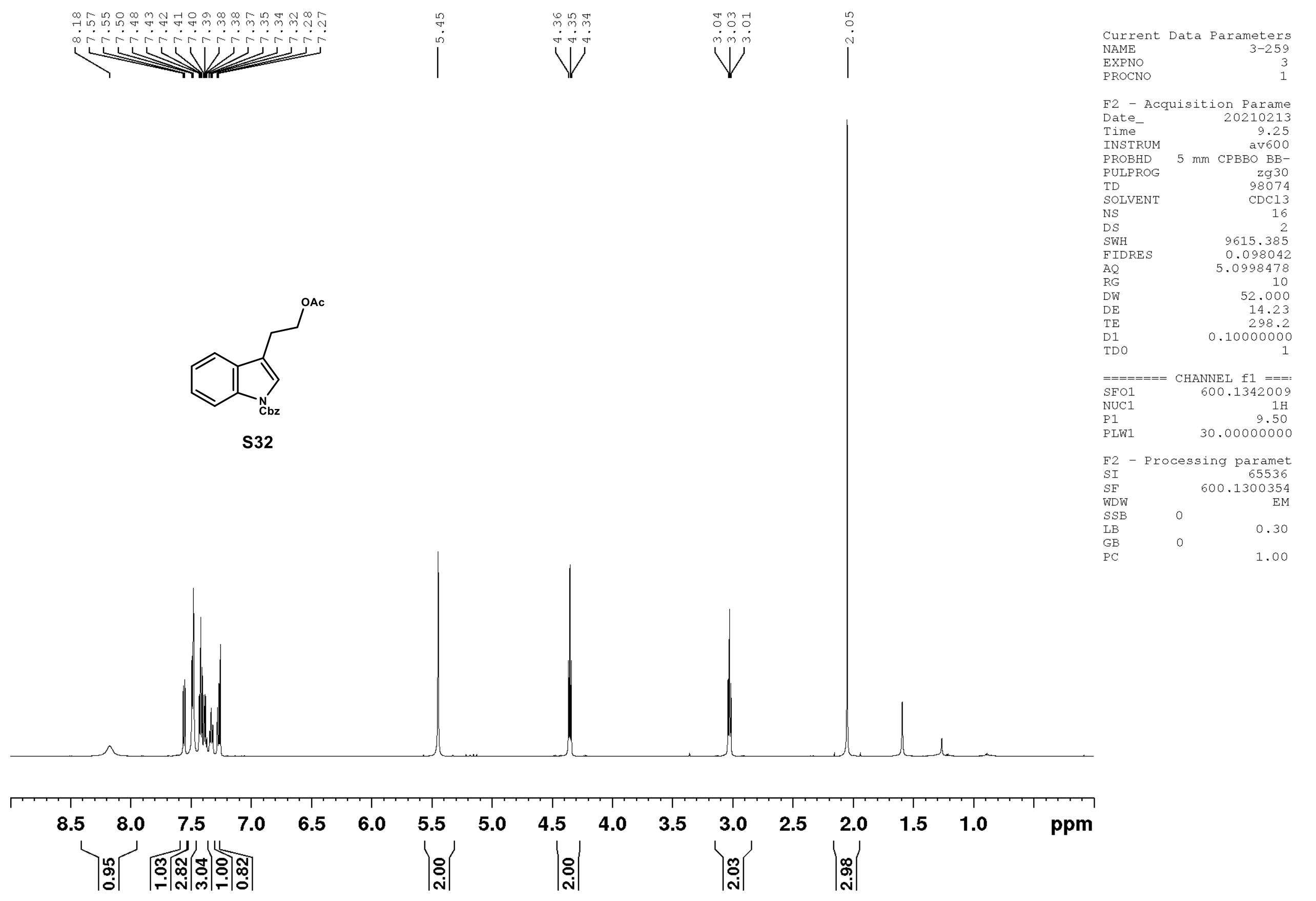




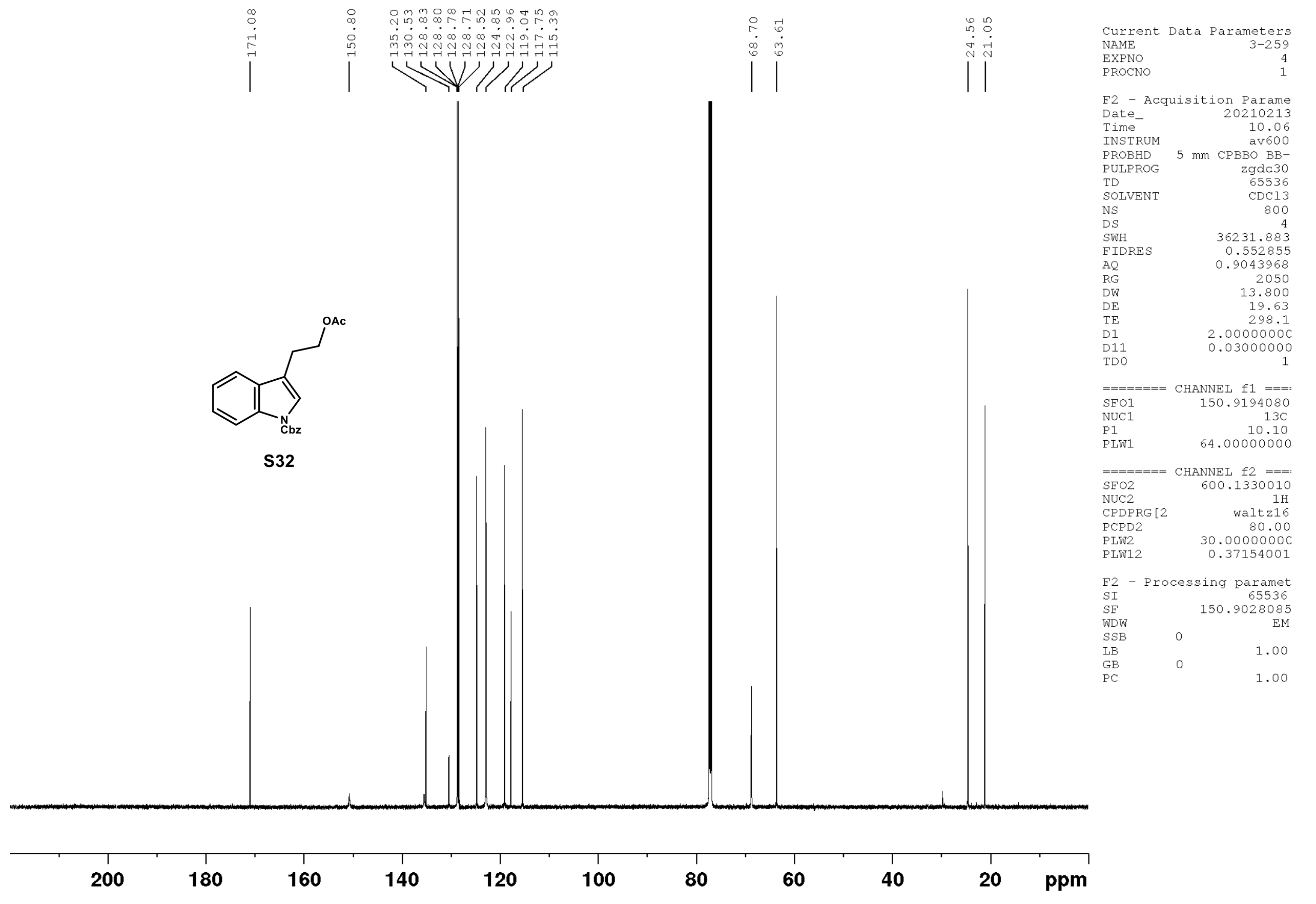




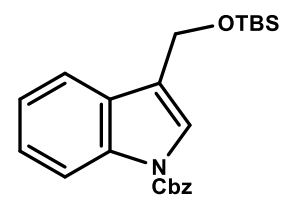

S33
Current Data Parameters NAME

PROCNO

F2 - Acquisition Parame

Date

INSTRUM

PROBHD

PULPRO

PU

SOLVENT

NS

SWH

FIDRES

RG

$\mathrm{DW}$
$\mathrm{DE}$
$\mathrm{TE}$

TE

TDO

$=======$ CHANNEL $\mathrm{fl}===$ :

$\mathrm{SFO1}$

$\begin{array}{lr}\text { PUC1 } & 1 \mathrm{H} \\ \mathrm{P} 1 & 9.50\end{array}$

9.46 $\mathrm{zg} 30$
98074 $\mathrm{CDCl} 3$ 16
2

9615.385 5.0998478 52.000 14.23

$0.1000000 \mathrm{C}$

FI - Processing paramet

SF $\quad 600.1300354$

WDW

$S: S$

PC

0.30

1.00

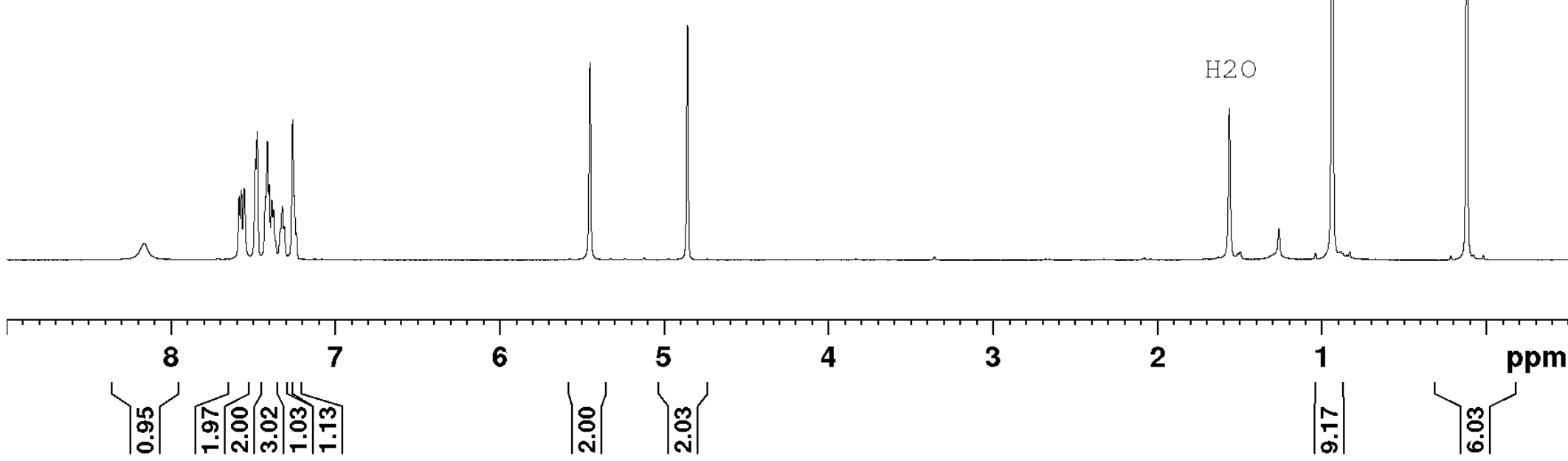




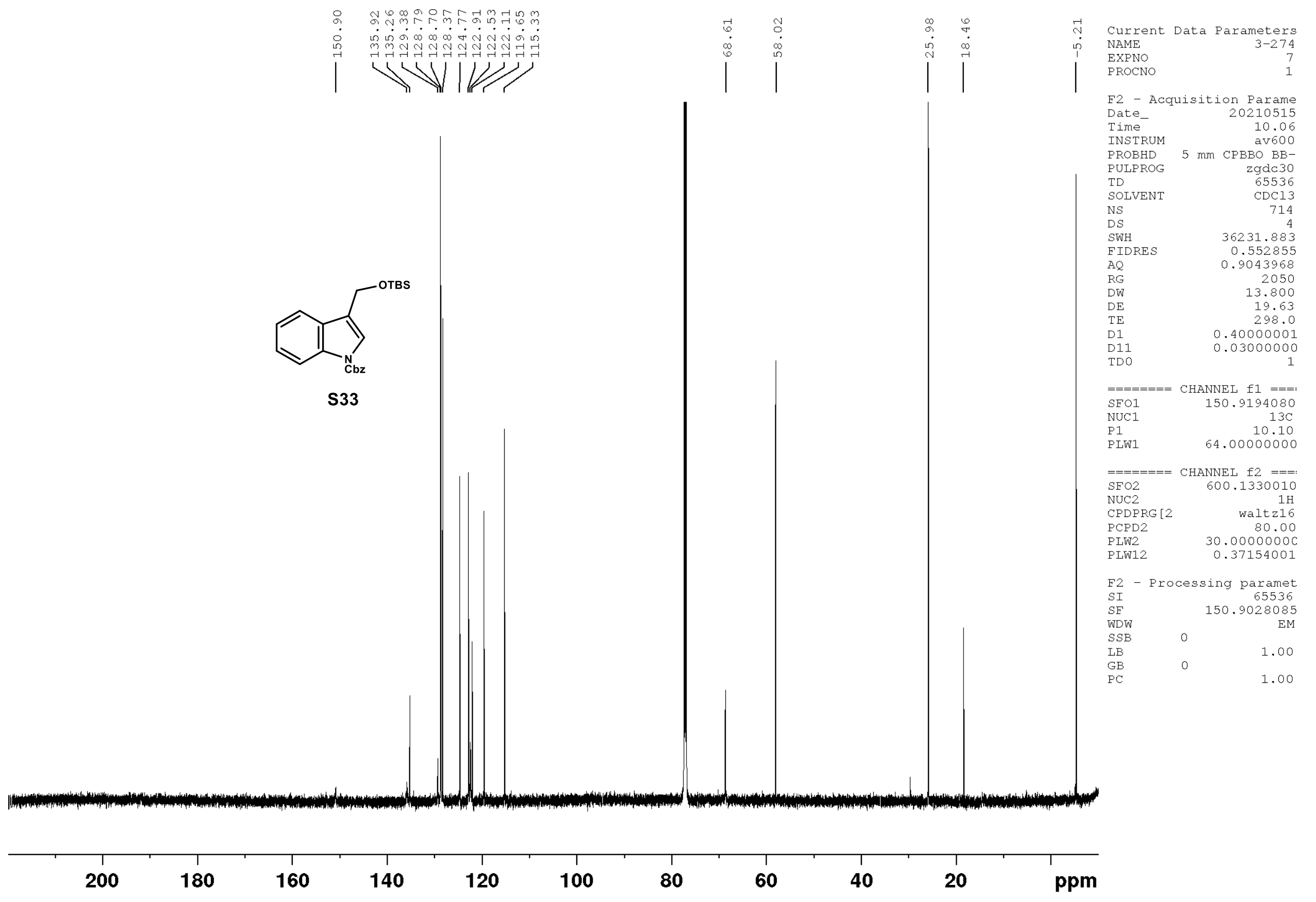



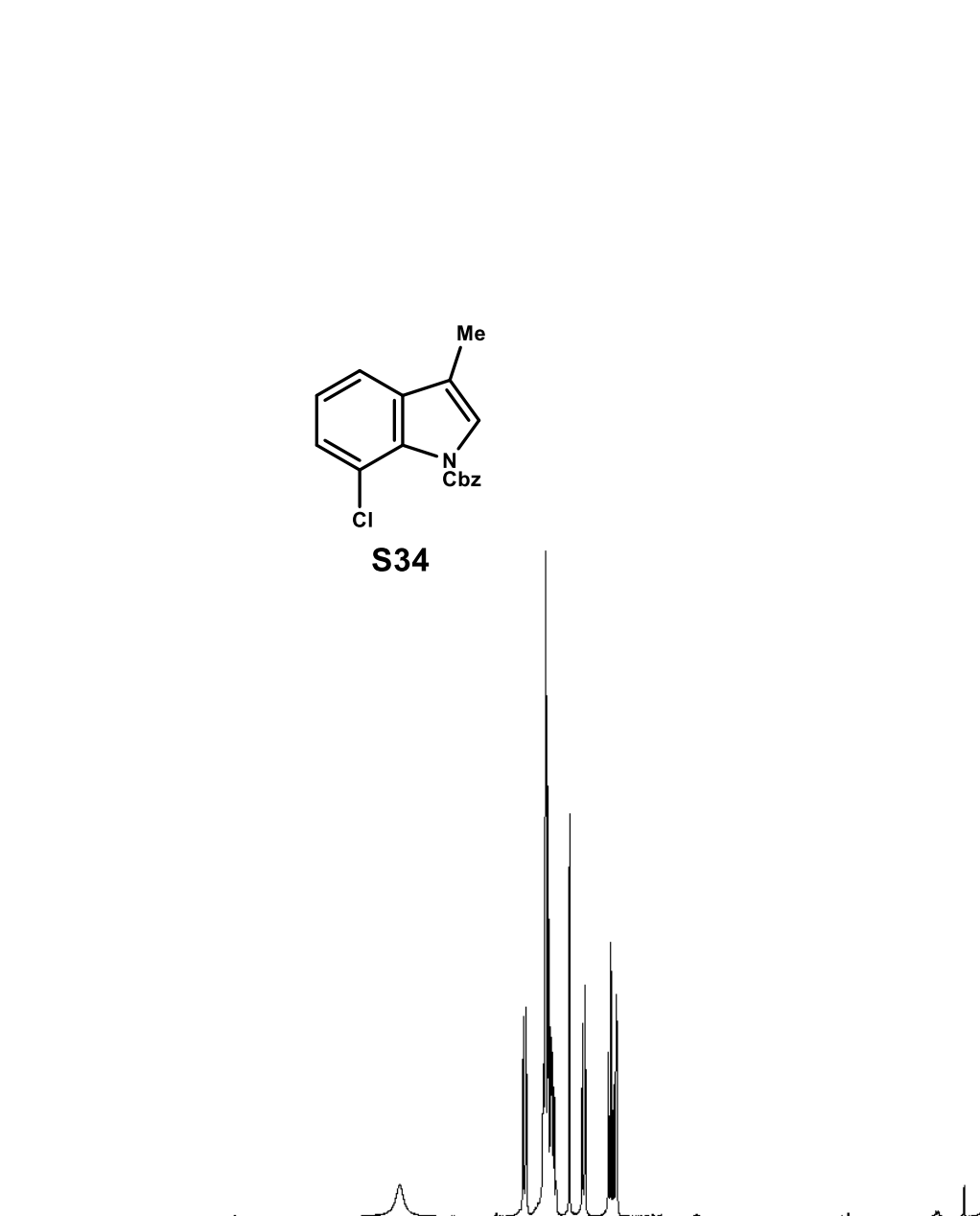

F2 - Acquisition Parame Date

Date

Time

$5 \mathrm{~mm} \mathrm{CPBBO} \mathrm{BB}-$

SOLVENT

NS

DS

SWH

9615.385

$\begin{array}{lr}\text { RG } & 10 \\ \text { DW } & 52.000\end{array}$

$\mathrm{DE} \quad 14.23$

0.10000000

TDO

$=====$

CHANNET 11

SFO1 $\quad 600.1342009$

NUC1

600.1342009
$1 \mathrm{H}$

P1

F2 - Processing paramet

SF $\quad 600.130028$

SF

SSB

$\begin{array}{lll}\mathrm{LB} & 0 & 0.30\end{array}$

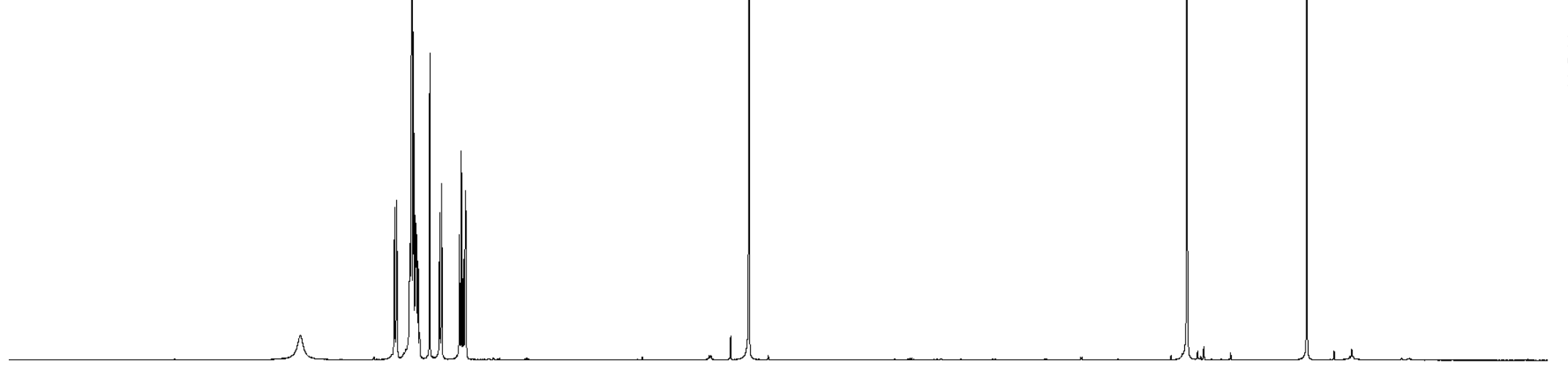

1.00

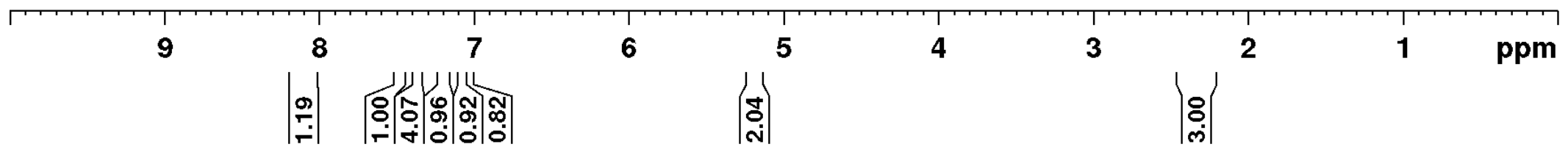




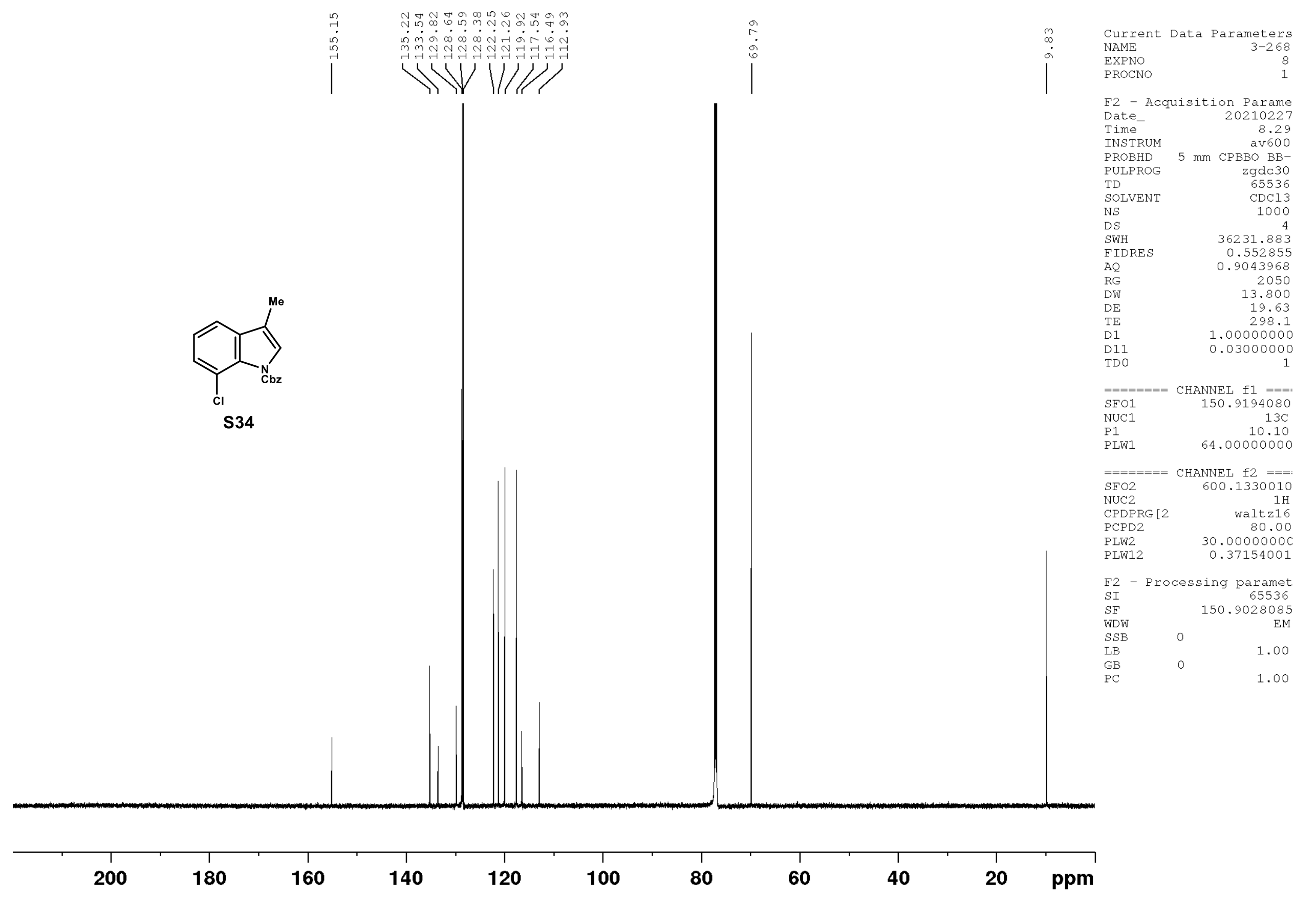


F2 - Acquisition Parame

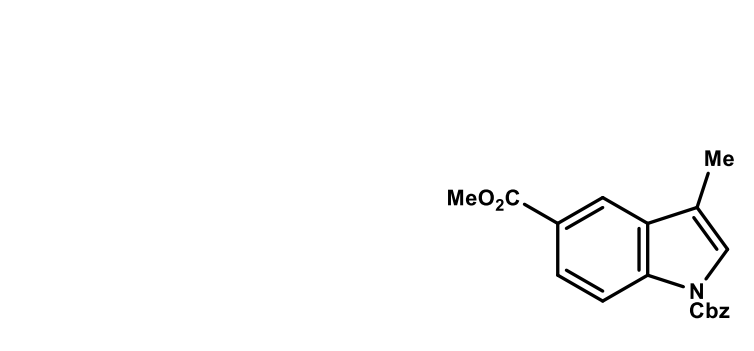

S35

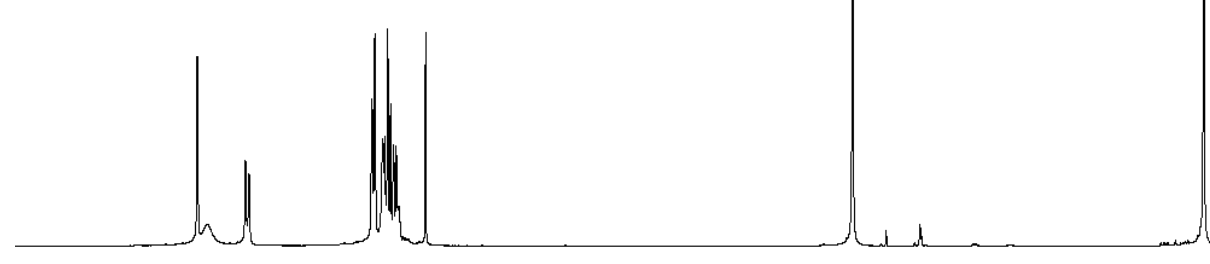




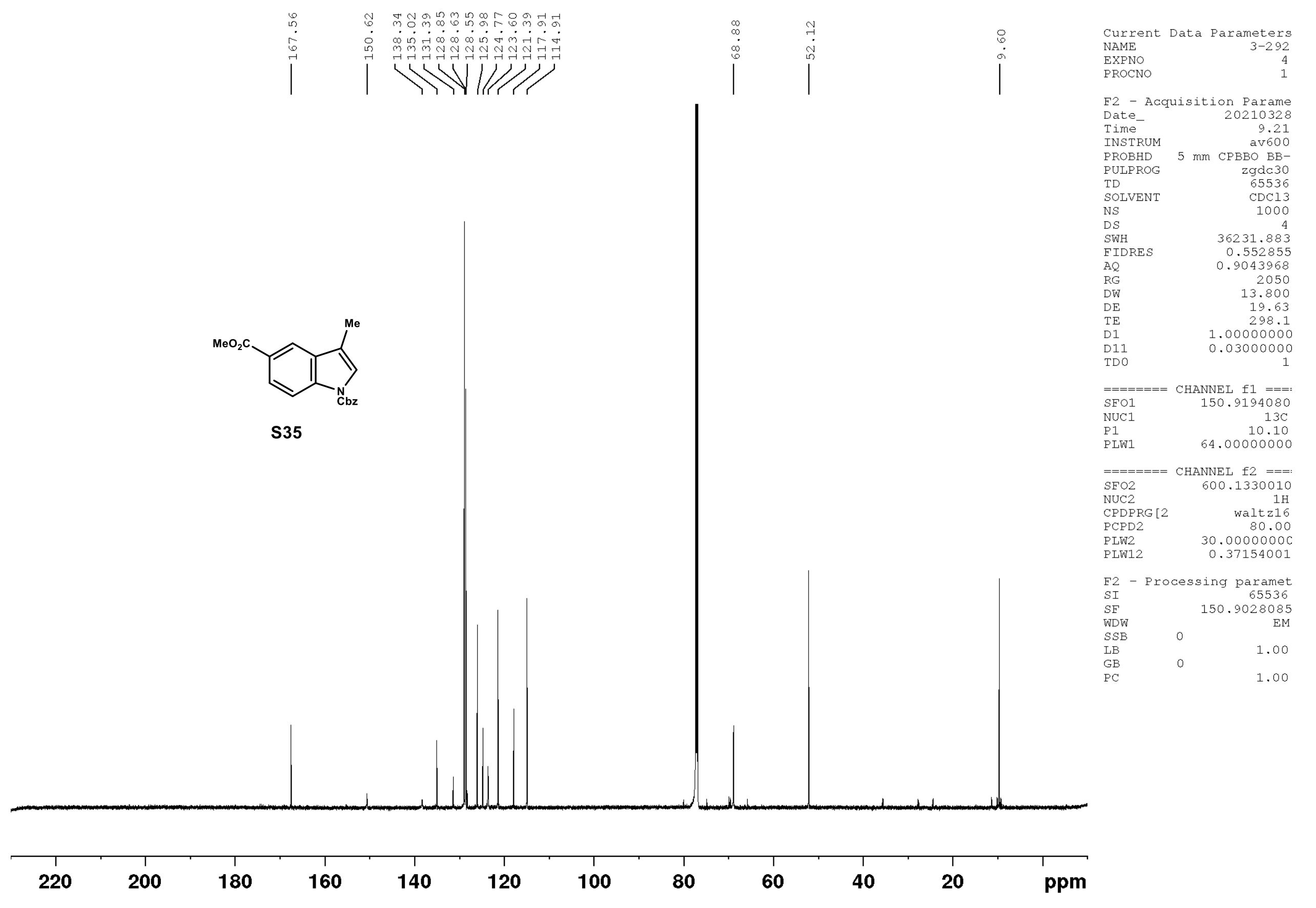



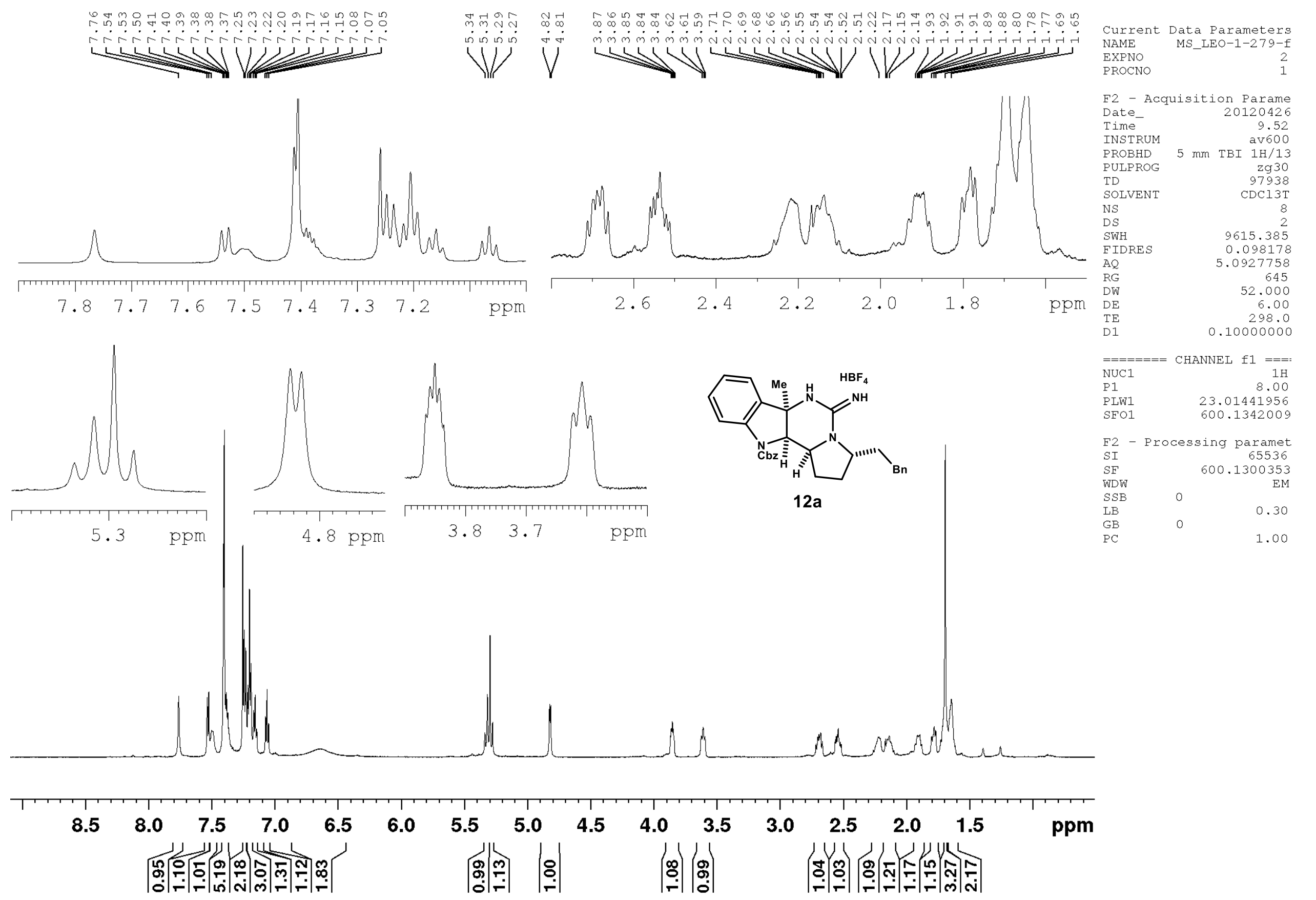


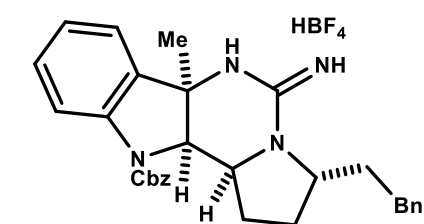

$12 a$
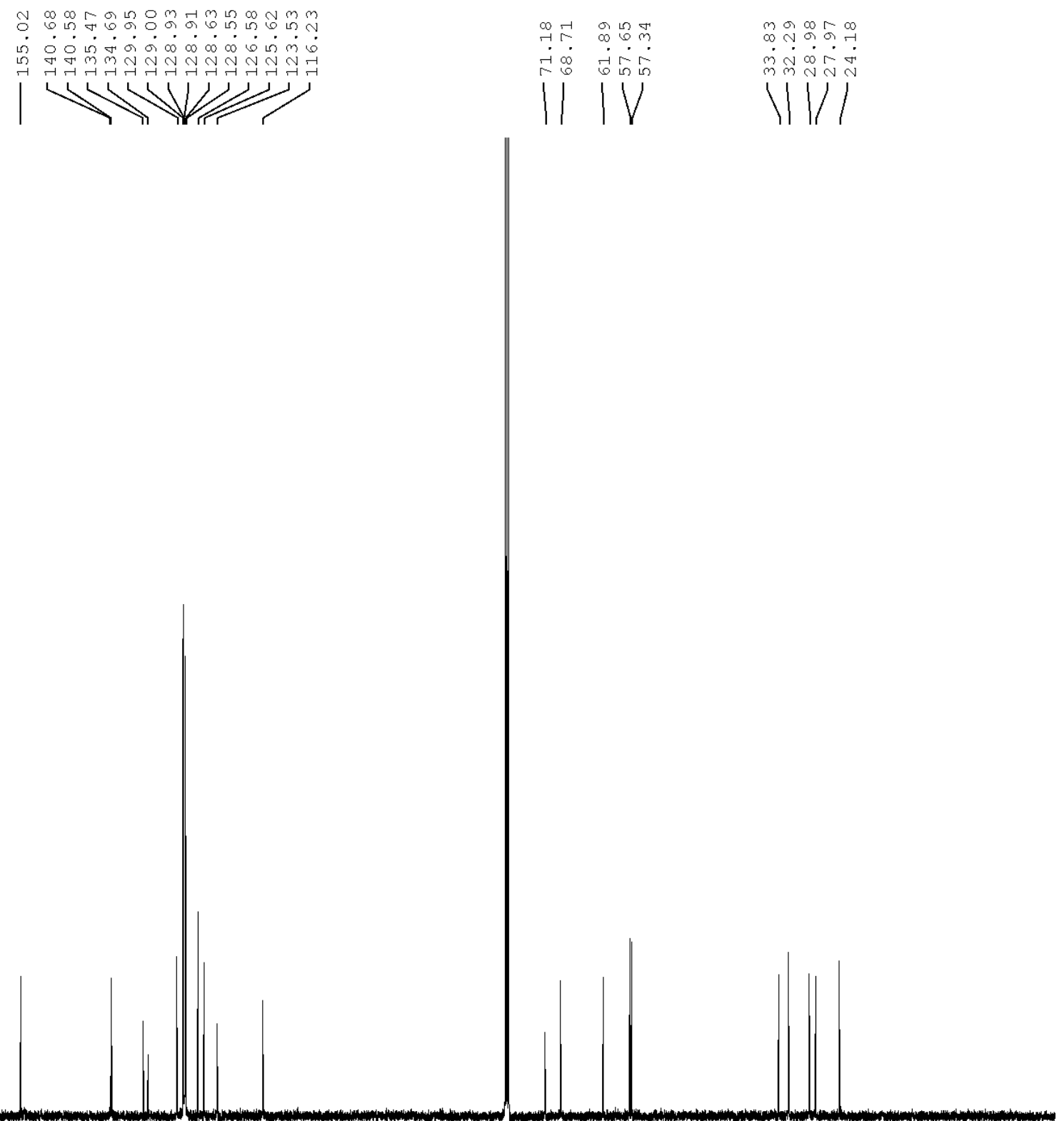

Current Data Parameters NAME MS_LEO-1-279-f ExPNO

D2 - Acquisition Parame sition Parame

INSTRUM

PUTPROC SPIN CPCI $1 \mathrm{H}-$

SOLVENT CDCl3T

NS

$\begin{array}{lr}\text { SWH } & 16 \\ & 30303.031\end{array}$

0.462388

AQ $\quad 1.0813440$

$\begin{array}{lr}\text { RG } & 3251 \\ \text { DW } & 16.500\end{array}$

$\begin{array}{lr}\mathrm{DE} & 6.00 \\ \mathrm{TE} & 298.0\end{array}$

D1 $\quad 0.25000000$

0.03000000

0.00020000

d17 0.00019600

$\begin{array}{lll}\text { MCREST } & \text { Sec } \\ \text { MCWRK } & 0.01500000\end{array}$

31.00

$========$ CHANNEL $\mathrm{f} 1 \mathrm{=}==$ :

NuC1 $13 \mathrm{C}$

$\begin{array}{lr}\mathrm{P} 1 & 15.50 \\ \mathrm{P} 11 & 500.00\end{array}$

500.00
$P 12 \quad 500.00$

PLO

\begin{tabular}{lr} 
& -1.00 \\
\hline & 125.7942548
\end{tabular}

SP2 $\quad 3.20$

SPNAM [1] Crp60, 0.5,20.1 SPNAM[2] Crp60comp.4

SPOFFI $0 \mathrm{~Hz}$

SPOFF2 $0 \mathrm{~Hz}$

CHANNEL $f 2=$ CPDPRG[2 waltzl6 NUC2

PL2

PL12

$\mathrm{SFO} 2$
220
200
180
160
140
120
100
80
60
40
20
ppm 


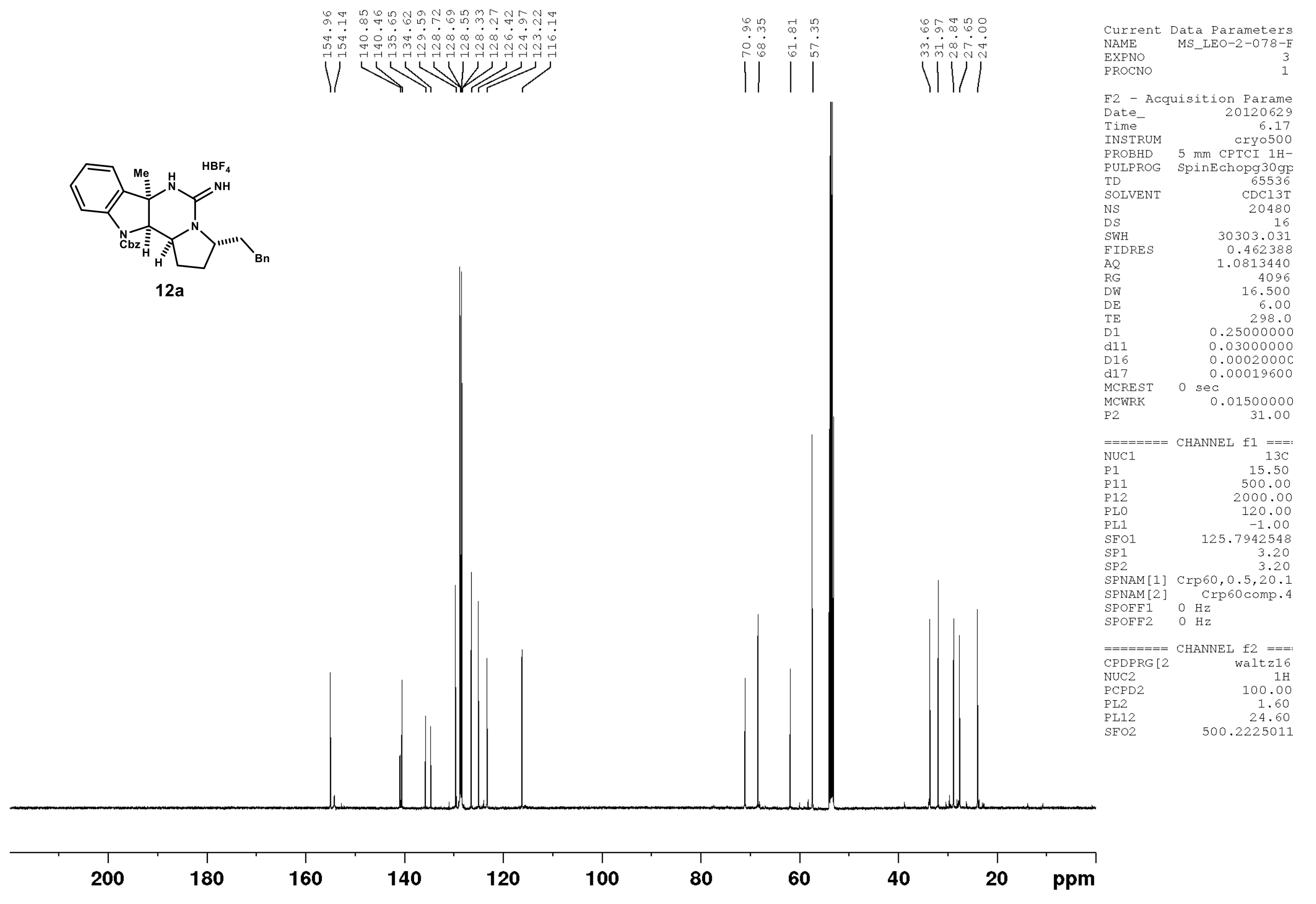




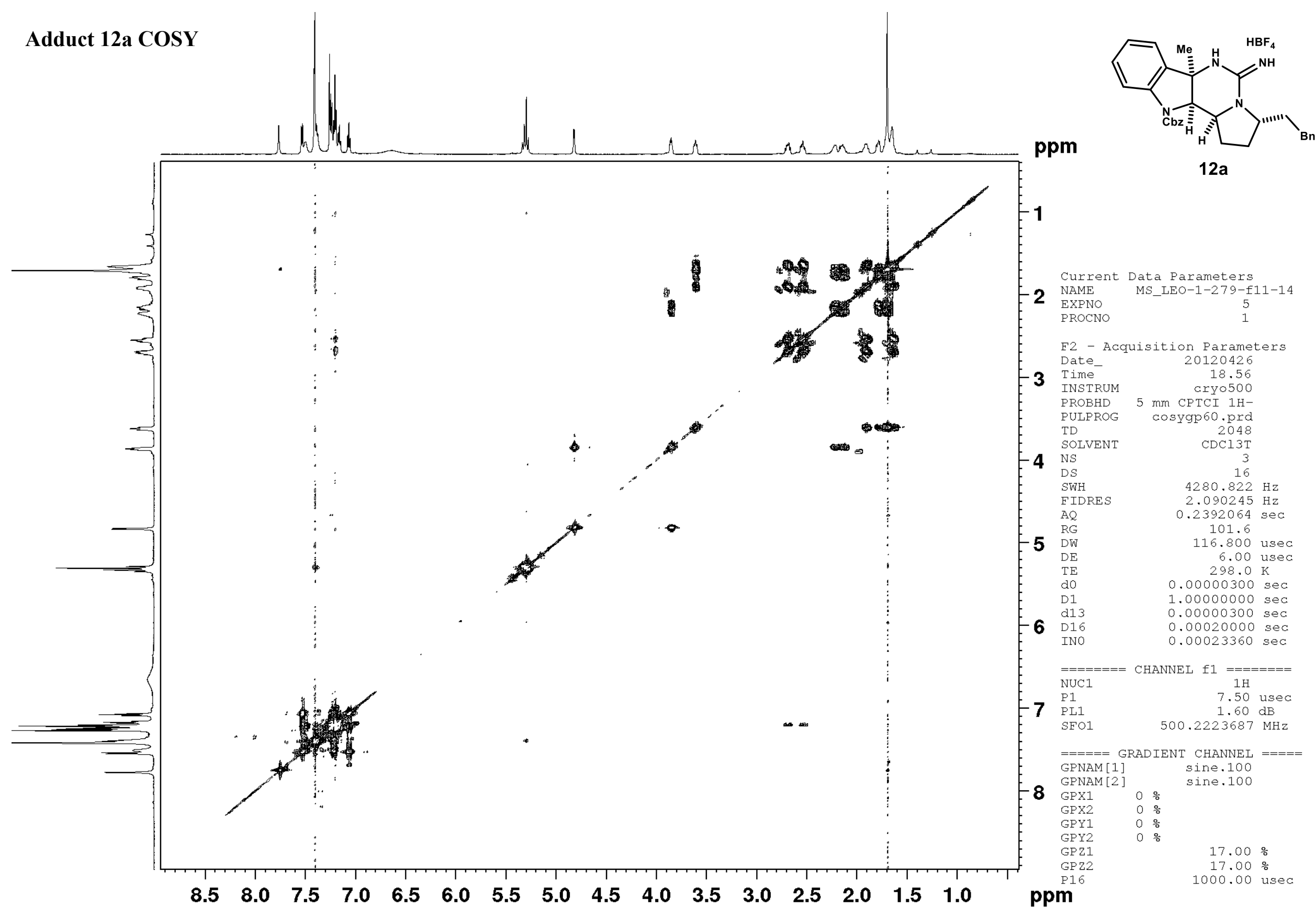




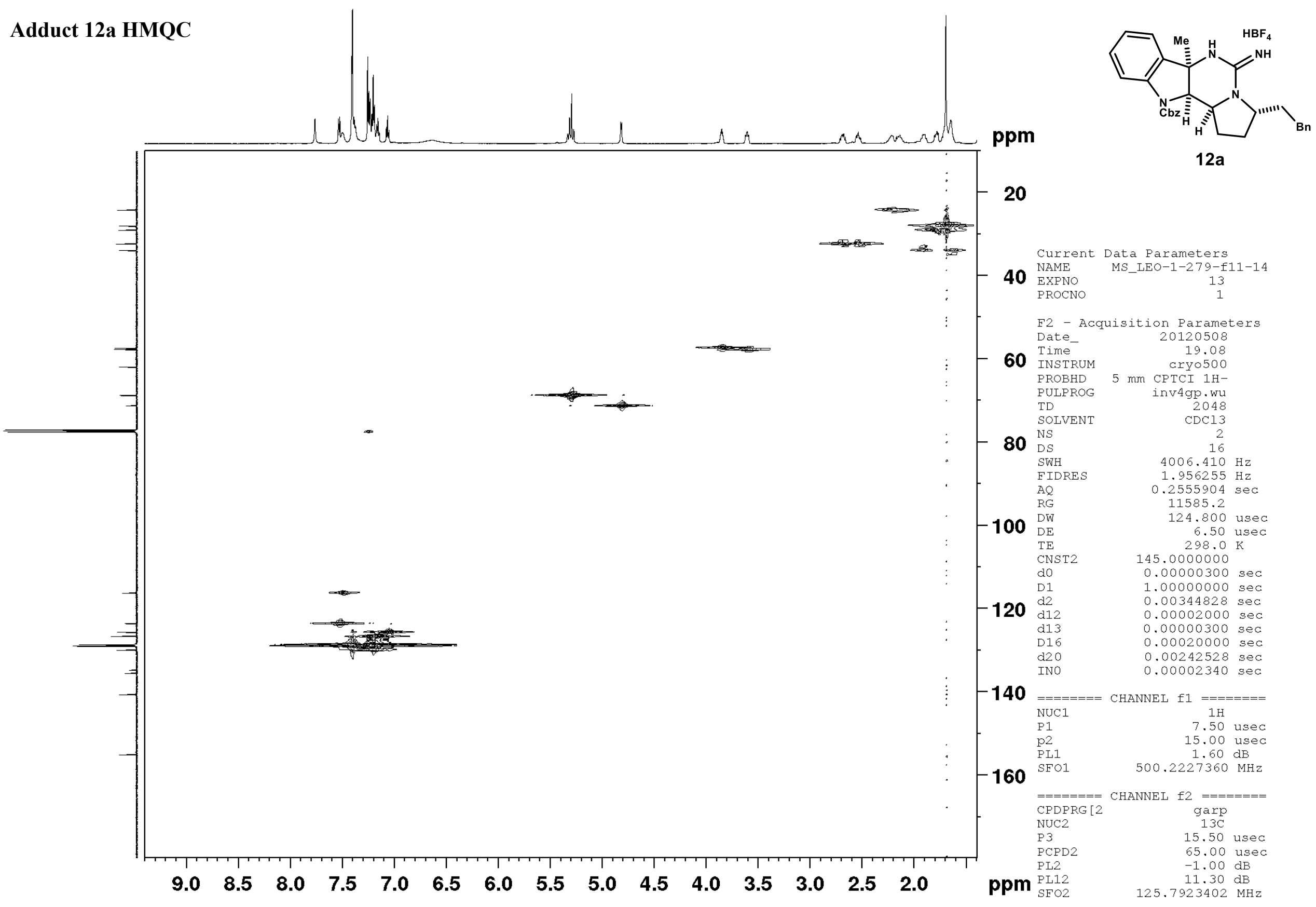



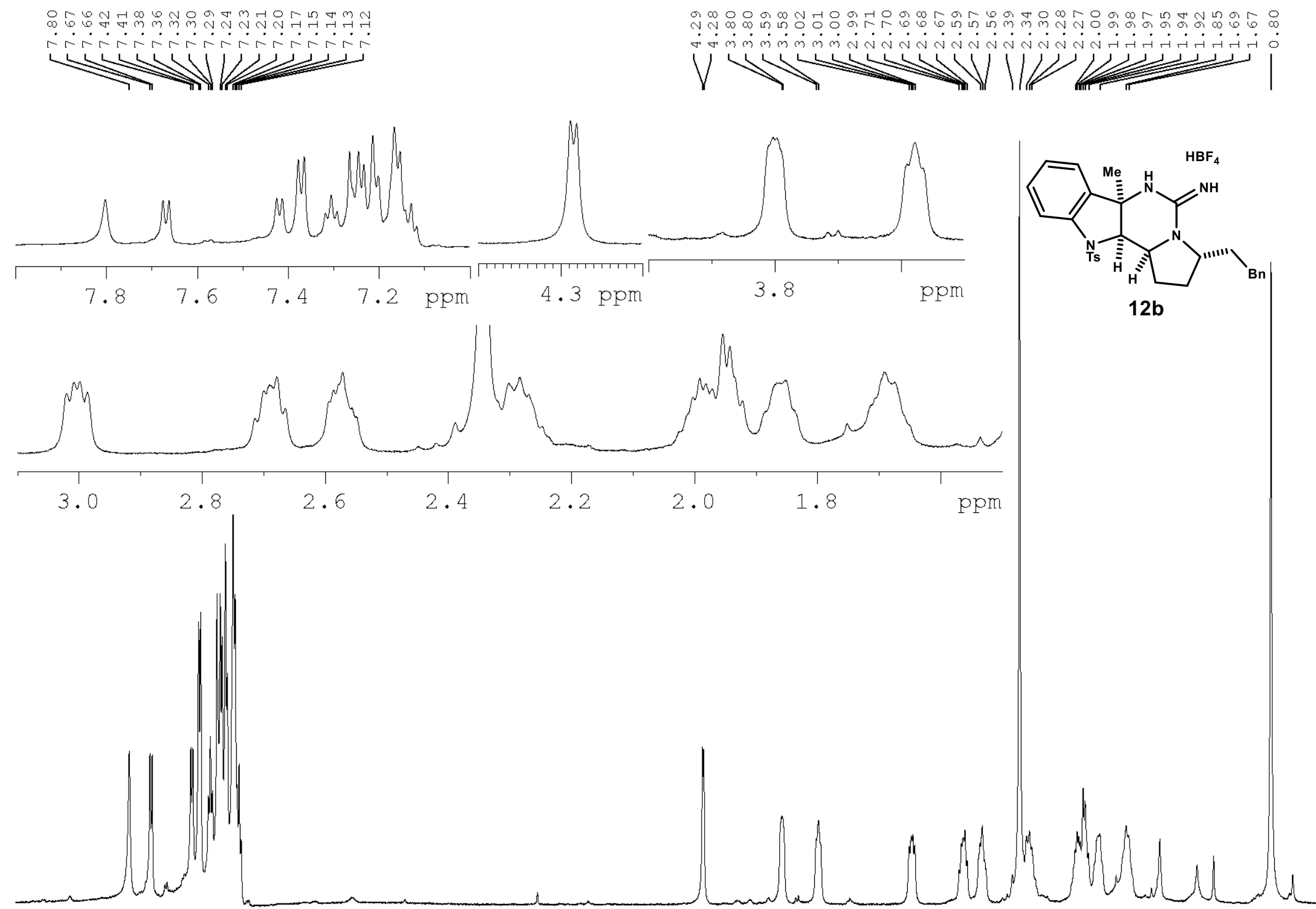

ppm

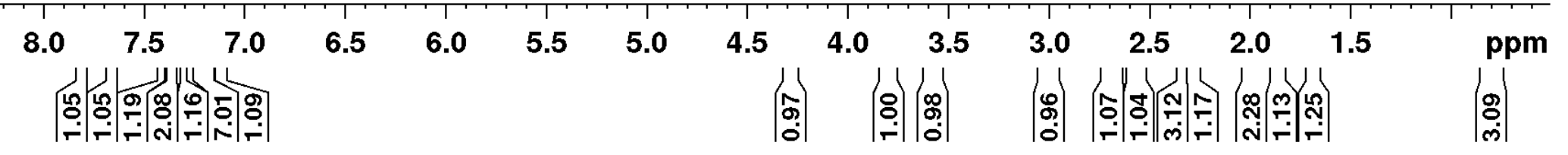




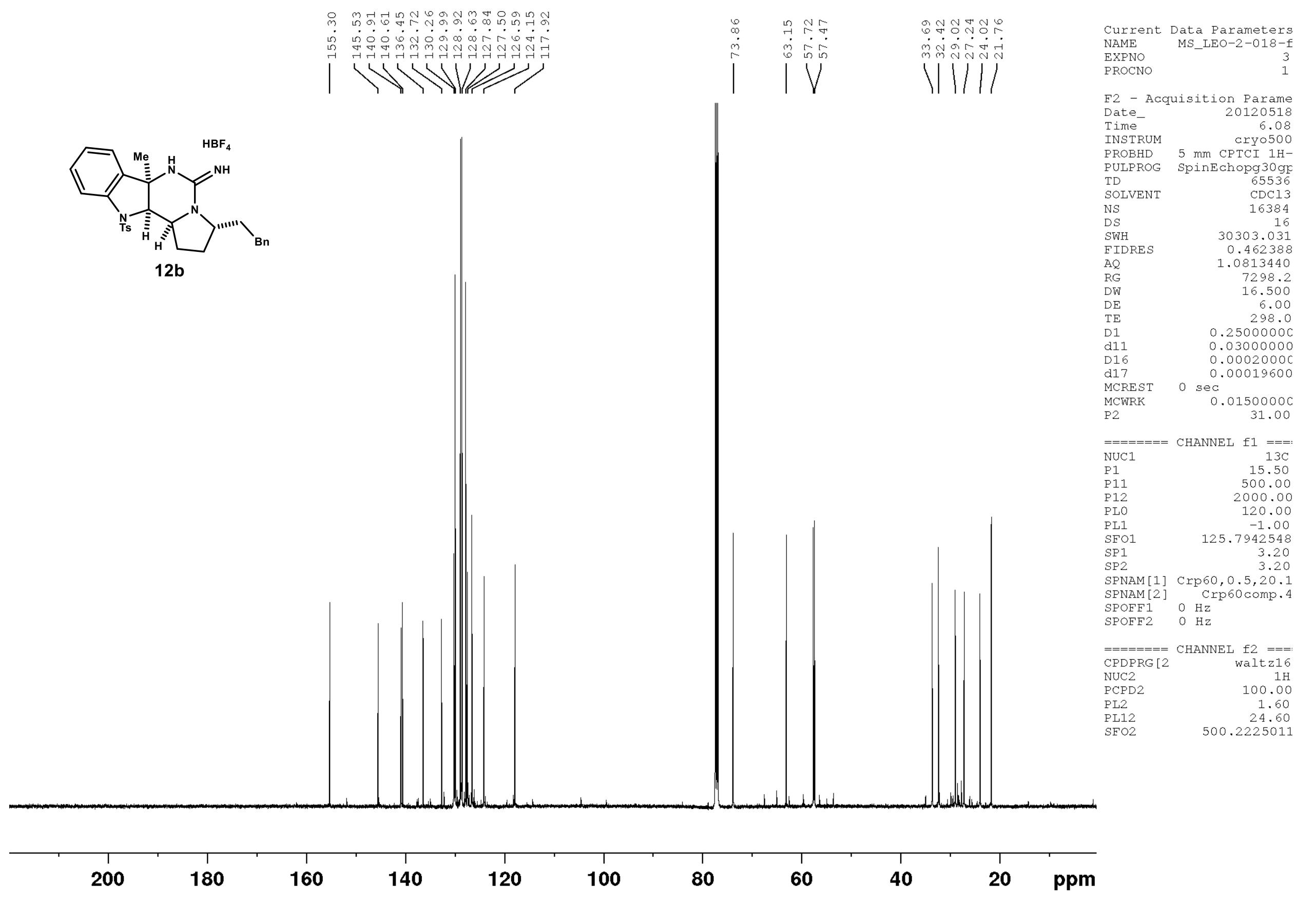




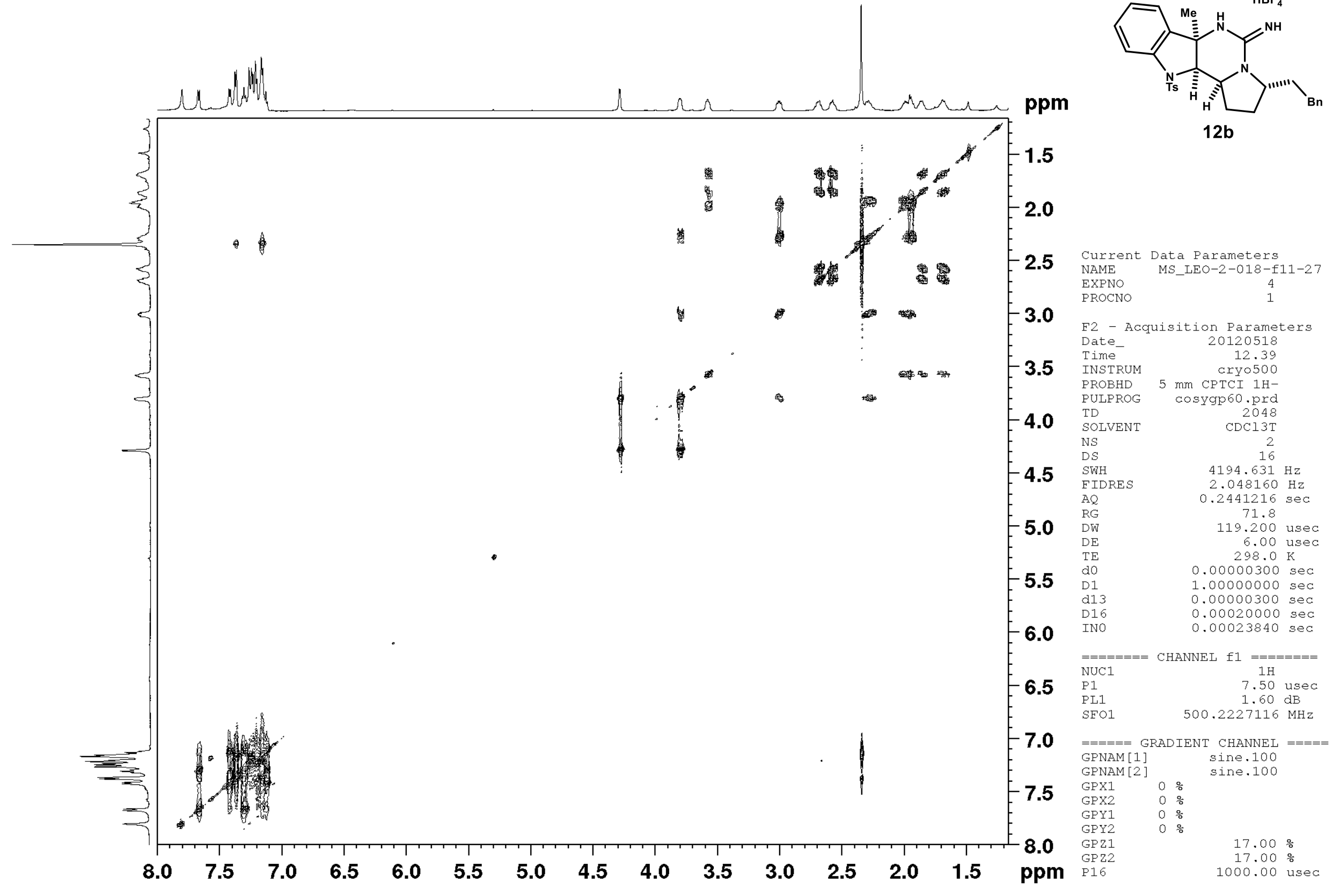


Adduct 12b HMQC

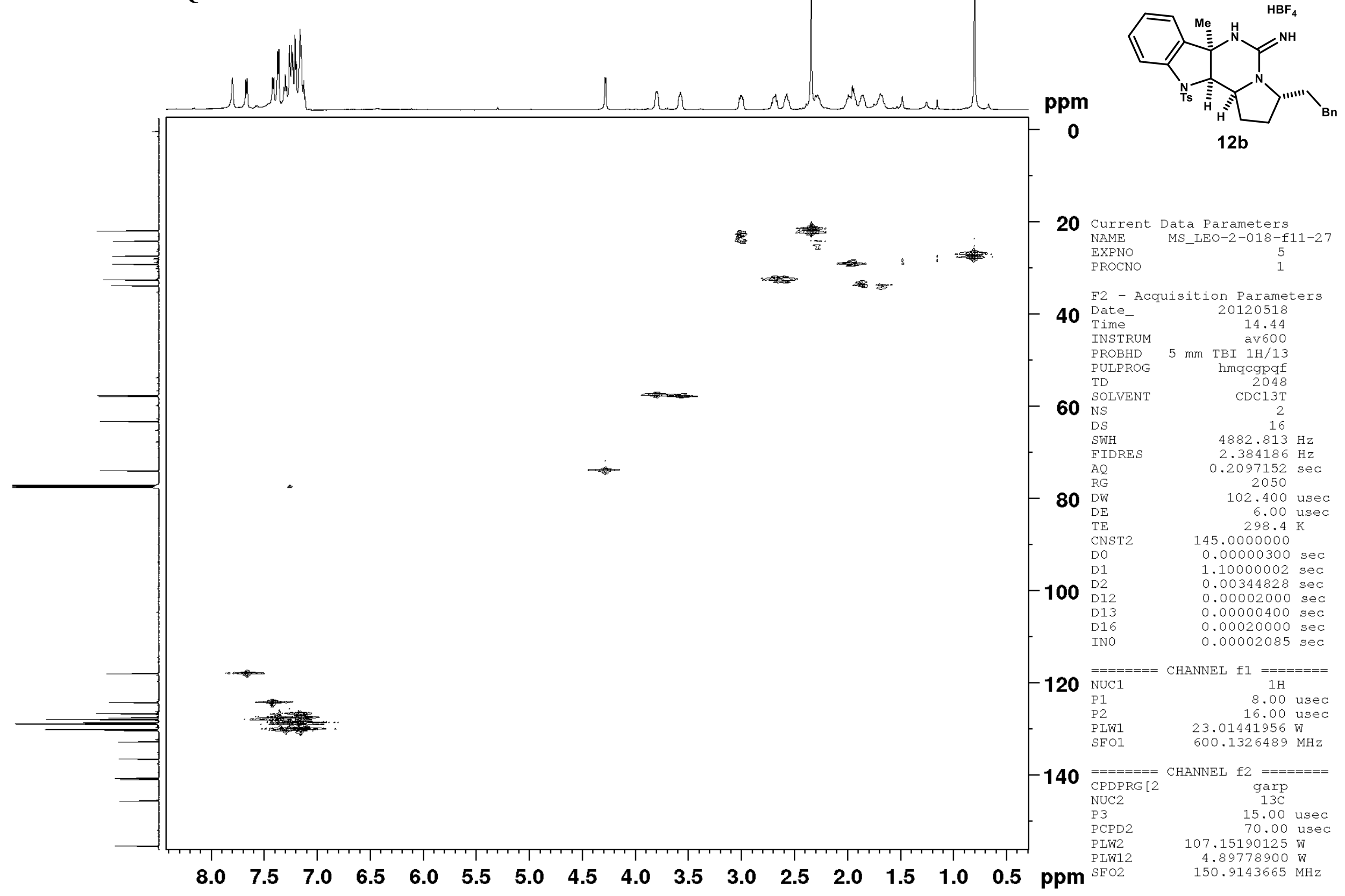




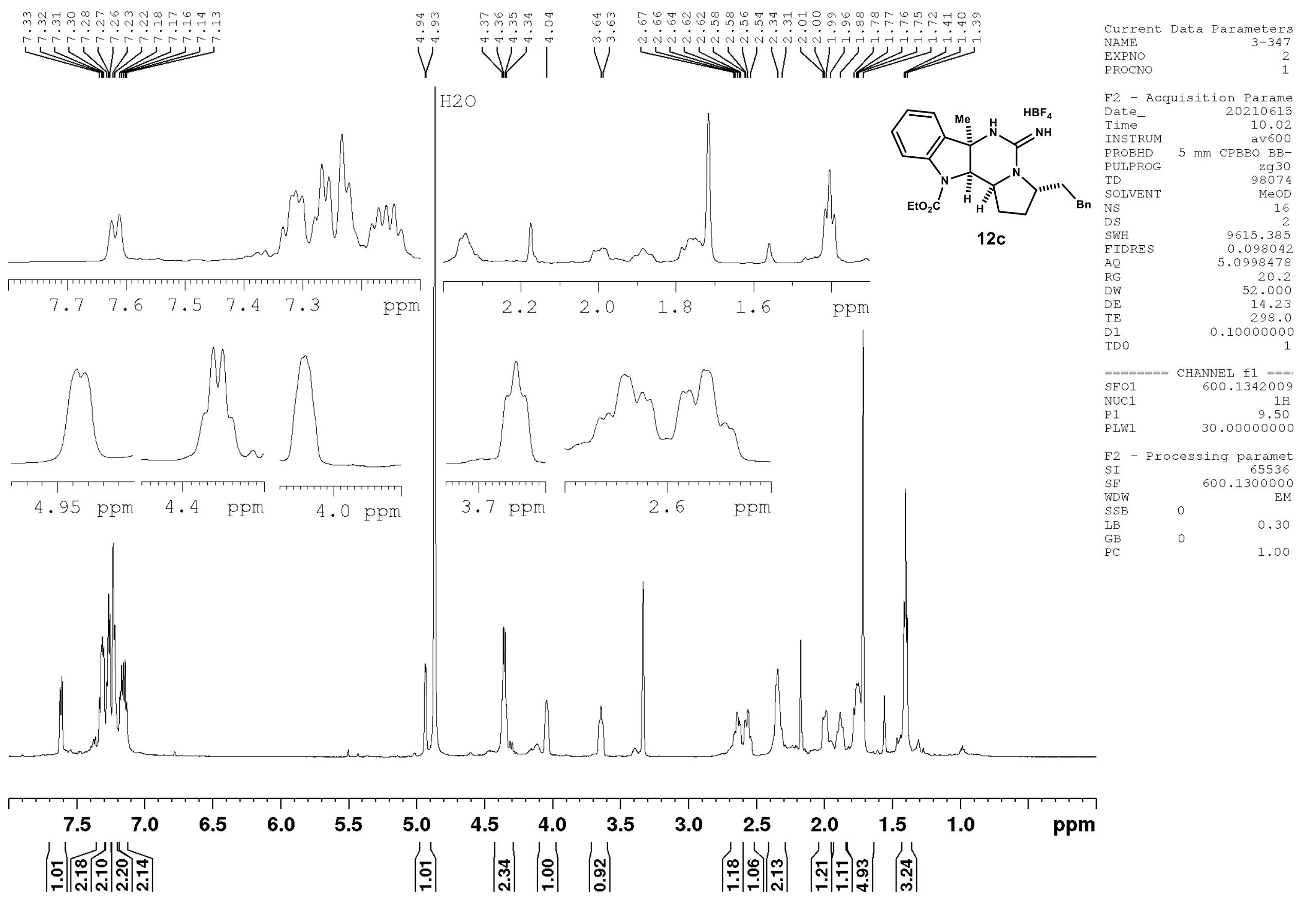



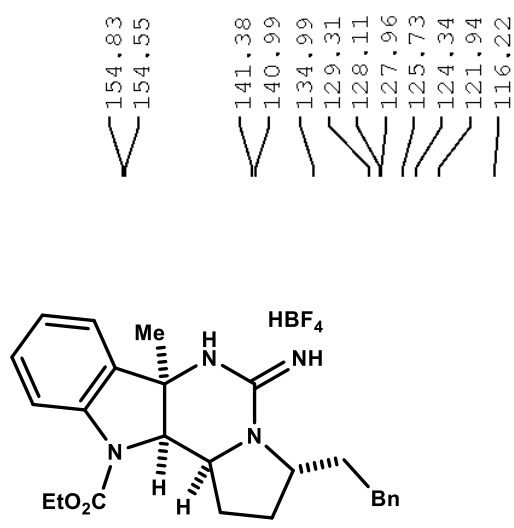

12c

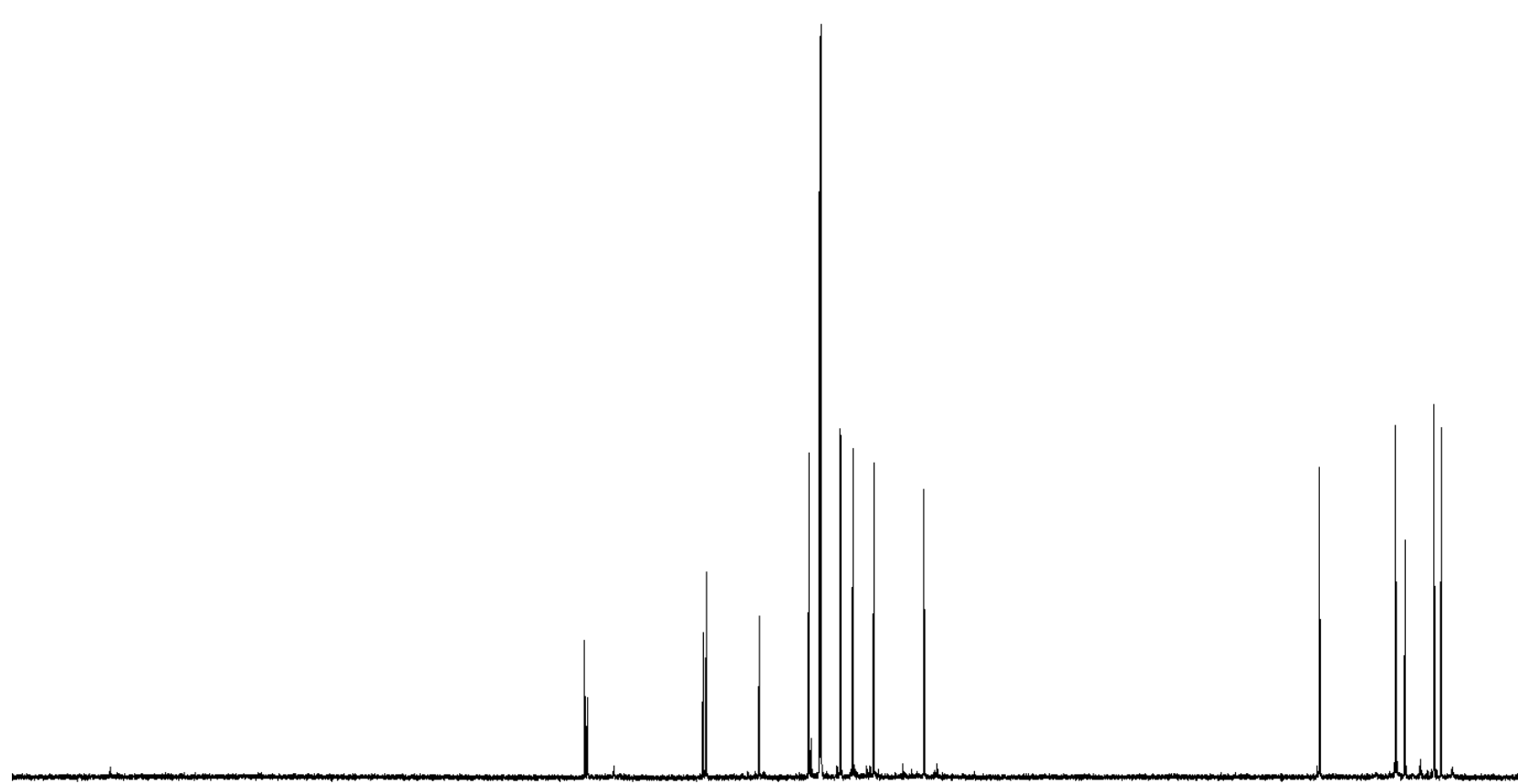

200 


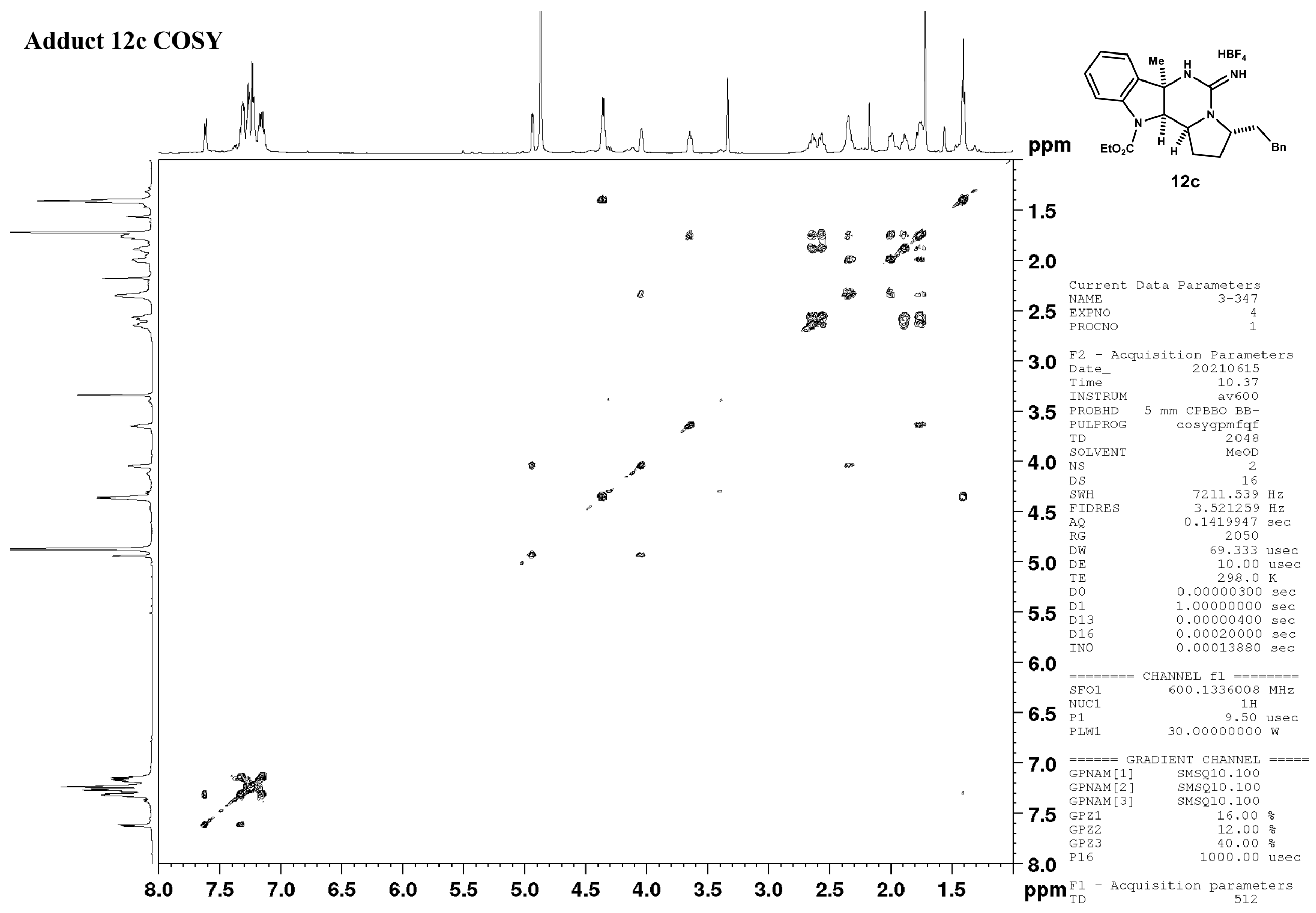




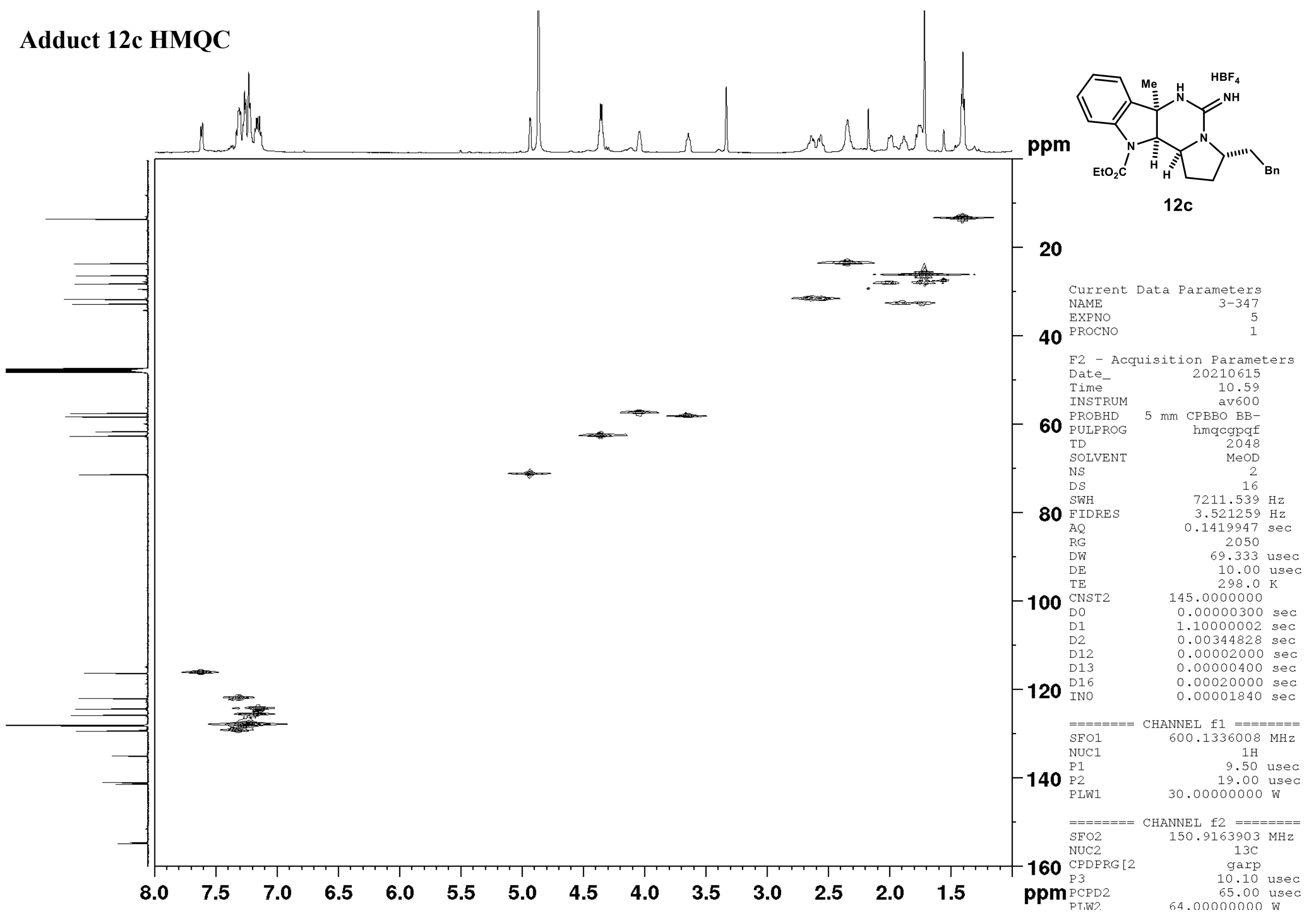



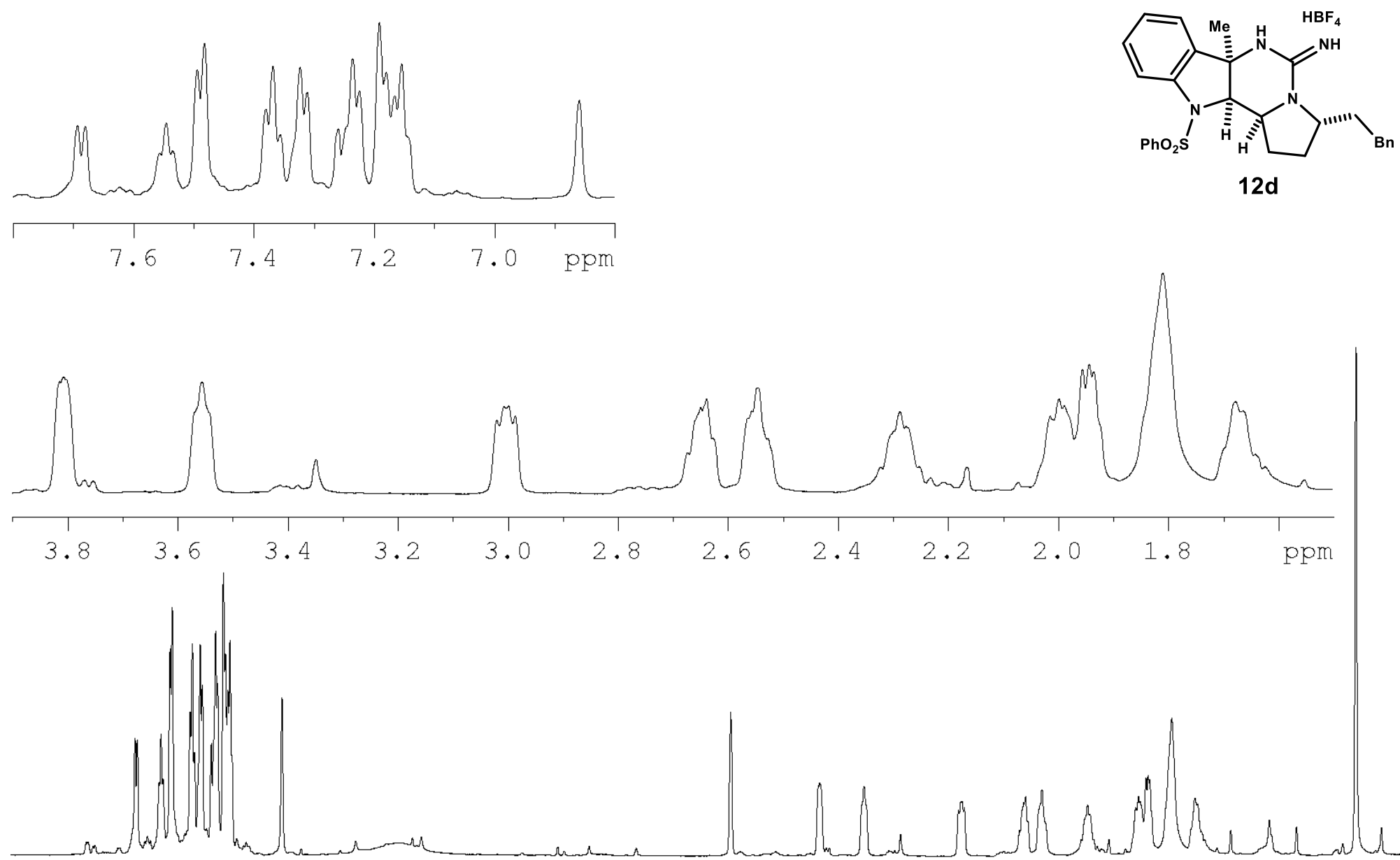

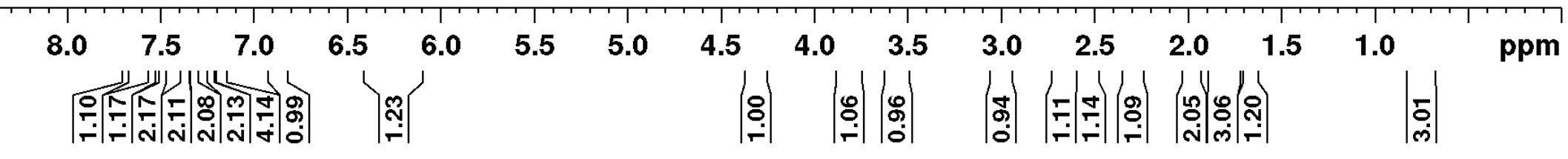

F2 - Accuisition Parame$$
\text { Date }
$$

Date_-
Time

INSTRUM

PROBHD

TD

SOLVENT

NS

SWH

$F I D R E$
$A Q$

$\mathrm{AQ}$
$\mathrm{RG}$

RG

$\mathrm{DE}$
$\mathrm{TE}$
$\mathrm{D} 1$
$\mathrm{TD}$

TDO

$====$
SFO1

SFO1

P 1

8.59
-600

$5 \mathrm{~mm}$ CPBBO $\mathrm{BB}-$ av 600 zg30
98074 CDCl3 16
2
385

9615.385 0.098042
5.0998478 10 14.23 $1.1000000 \mathrm{C}$
1 CHANNEL $\mathrm{f} 1===$ $1 \mathrm{H}$
9.50 F2 - Processing paramet $\begin{array}{ll}\text { SF } & 600.1300342\end{array}$ WDW SS PC

.00 


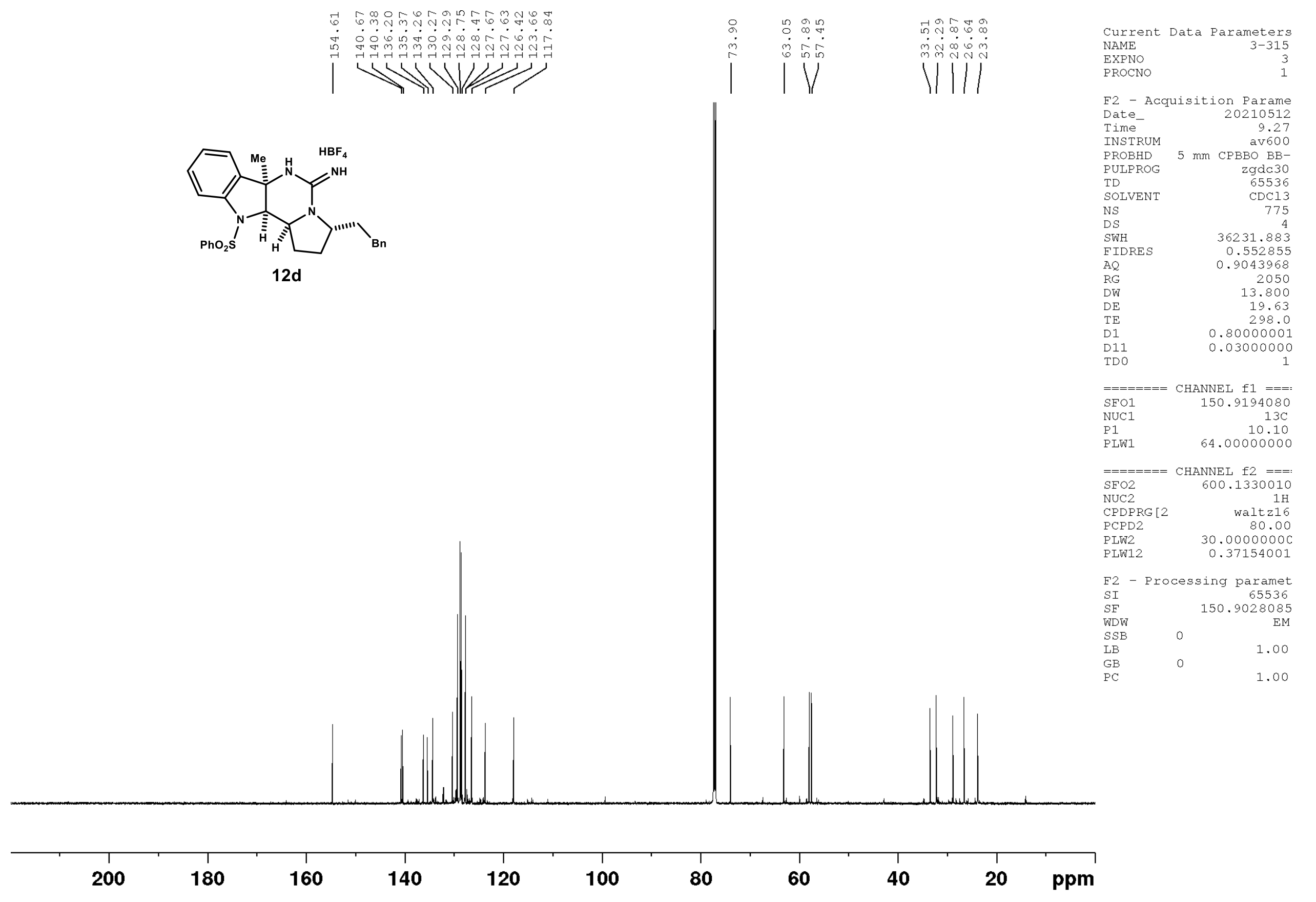




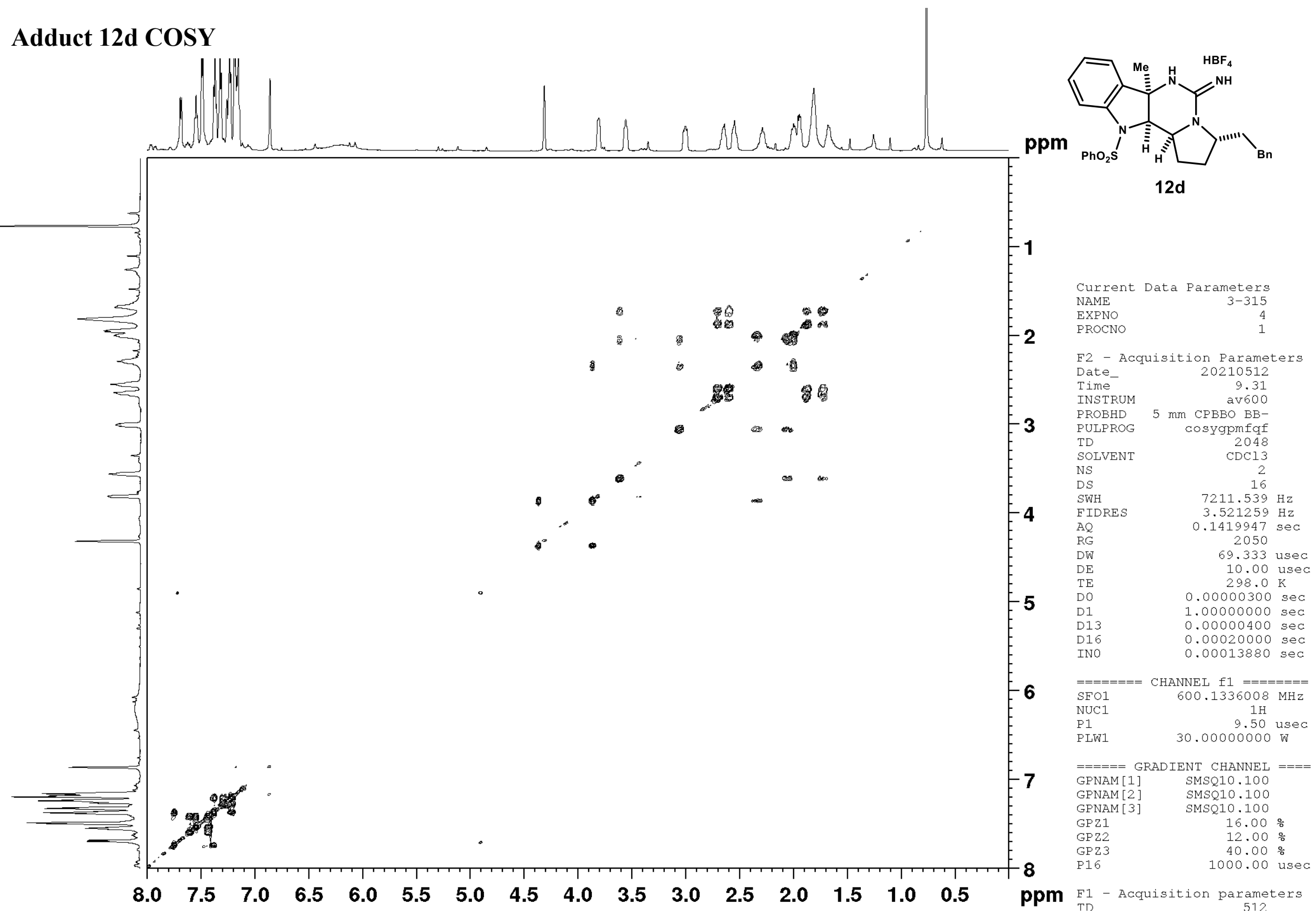




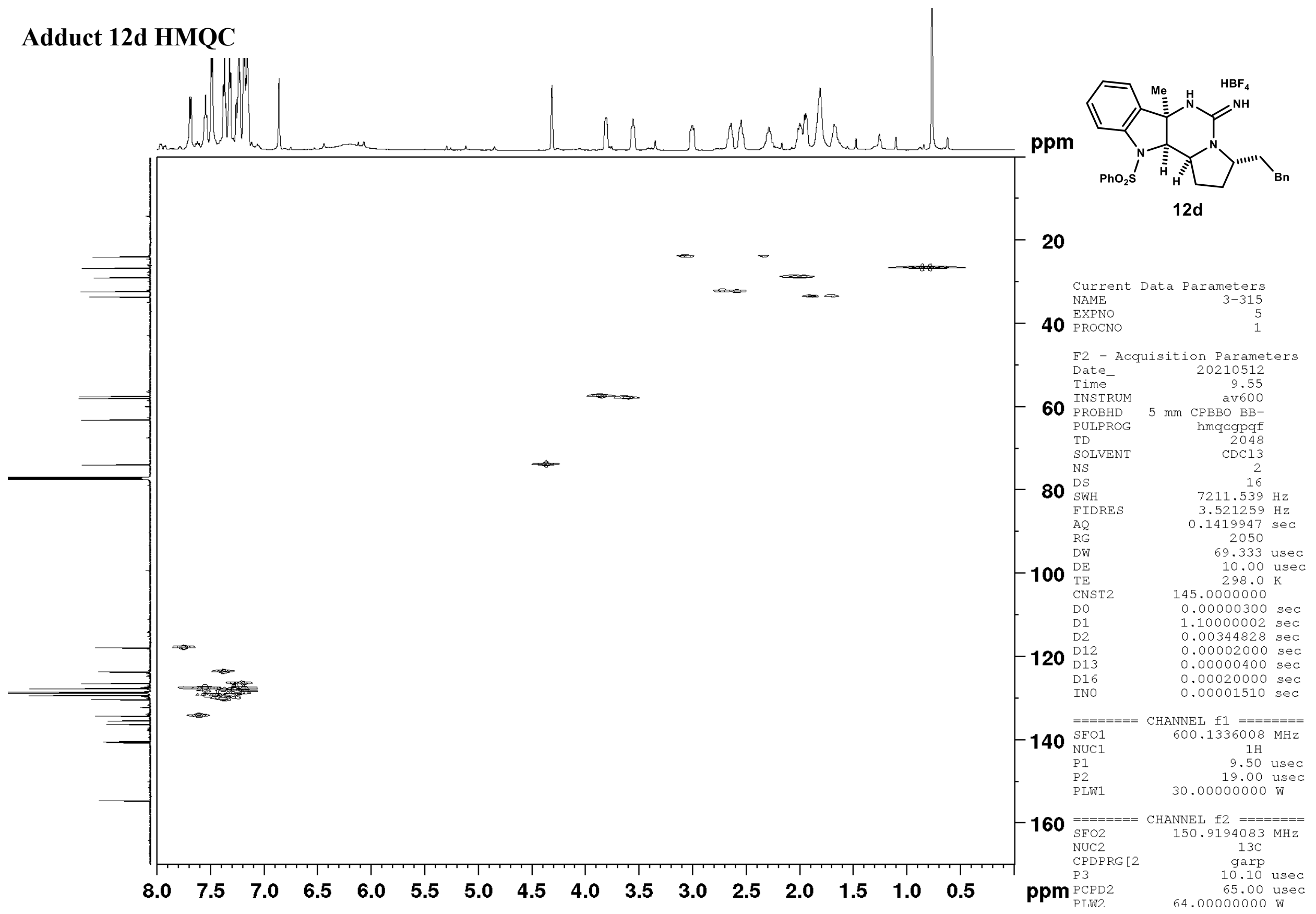




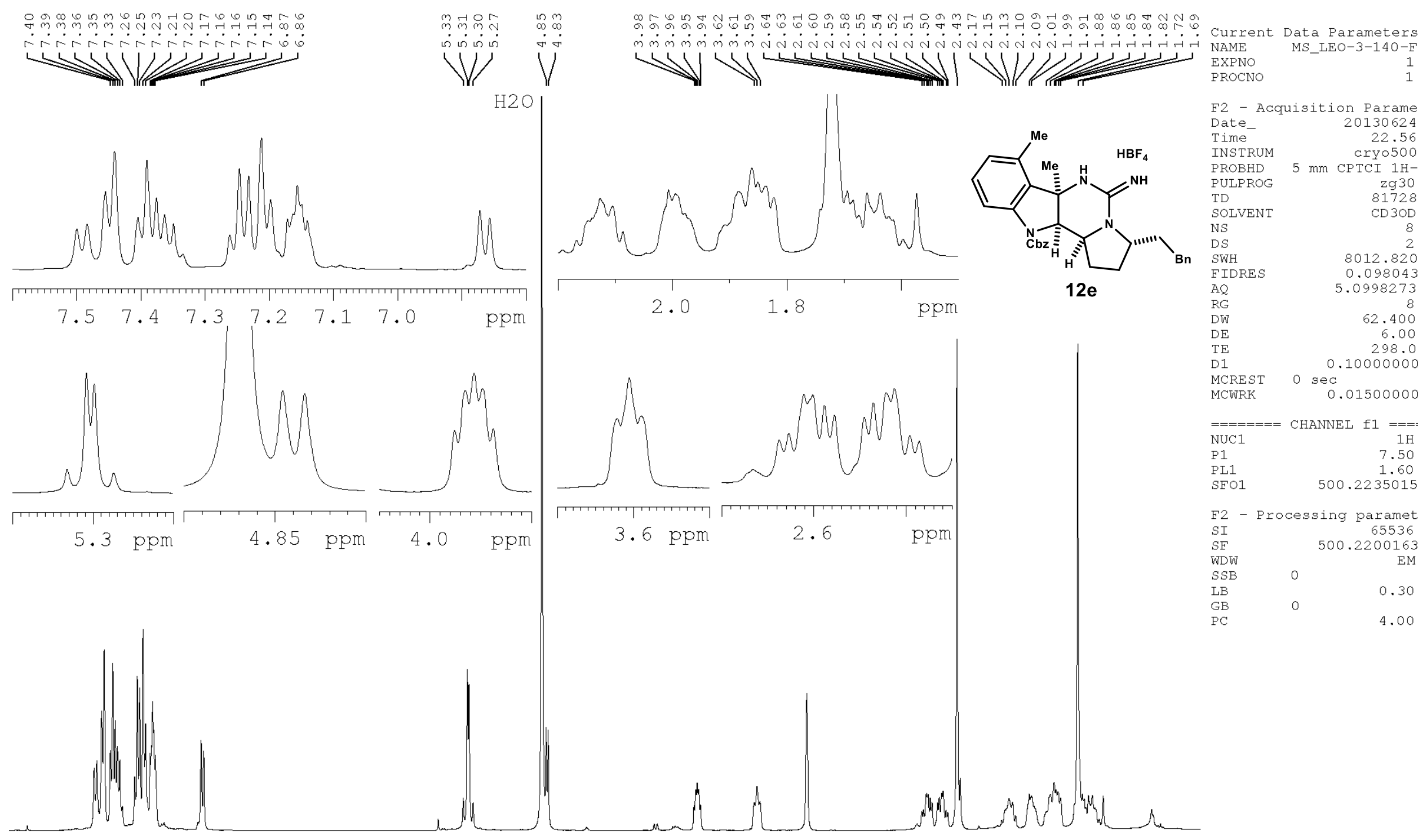

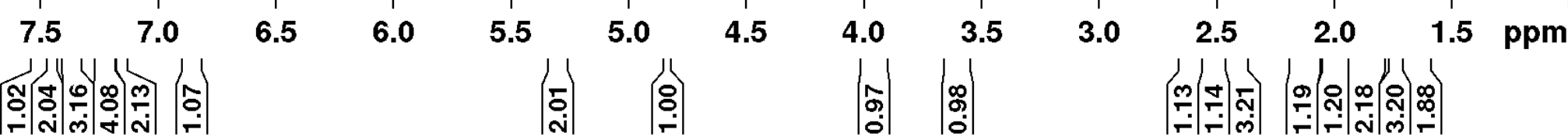




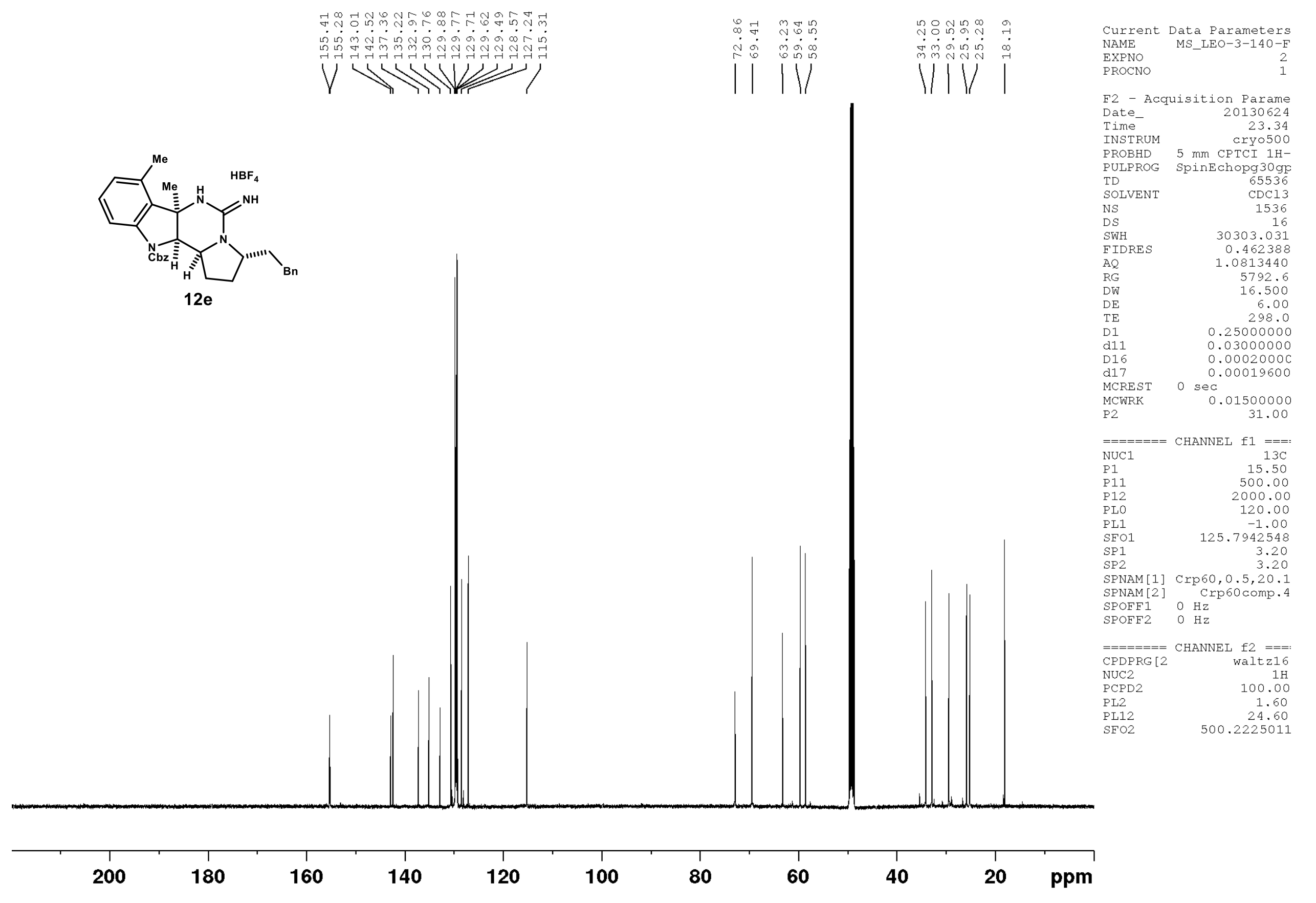




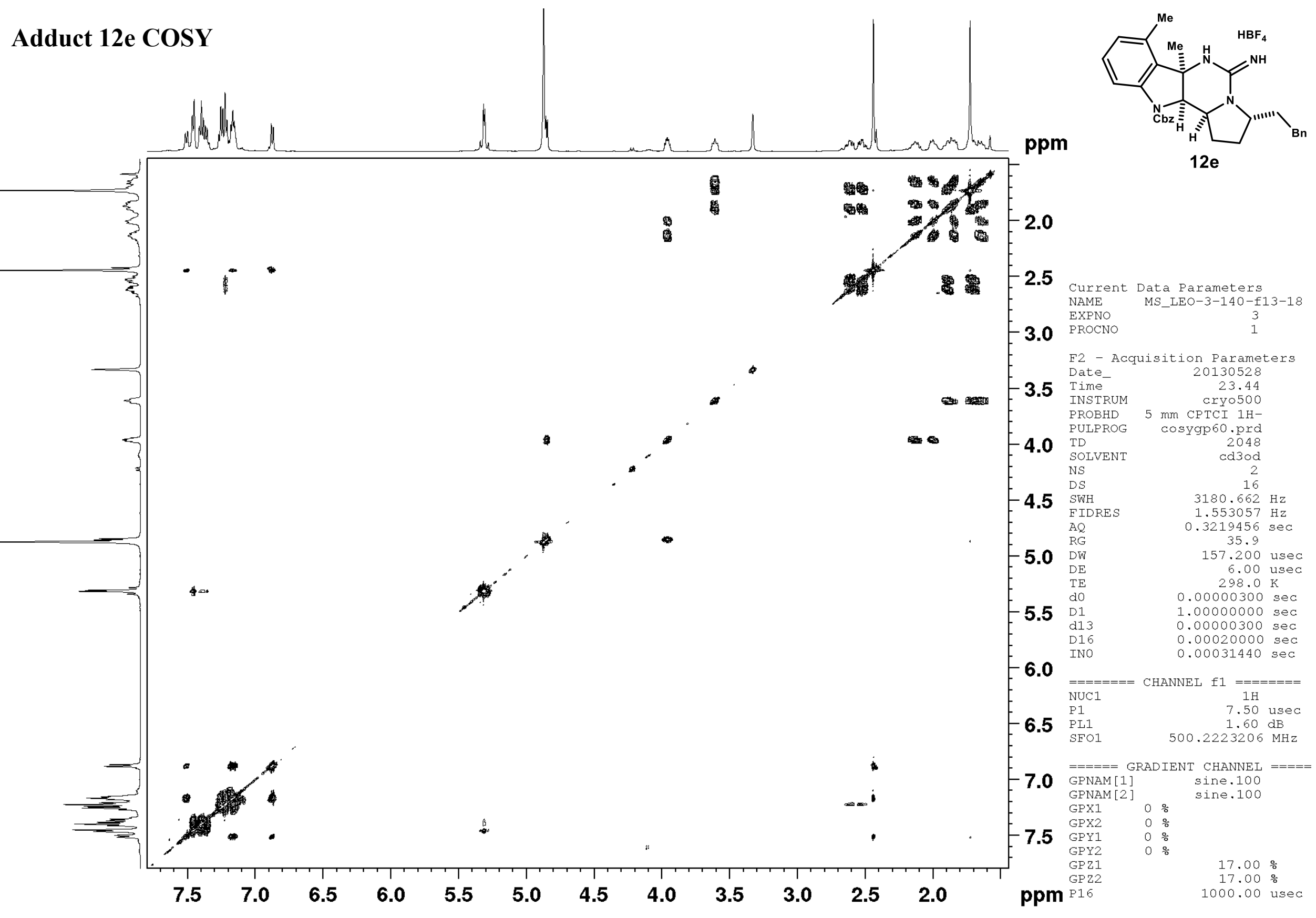




\section{Adduct 12e HMQC}

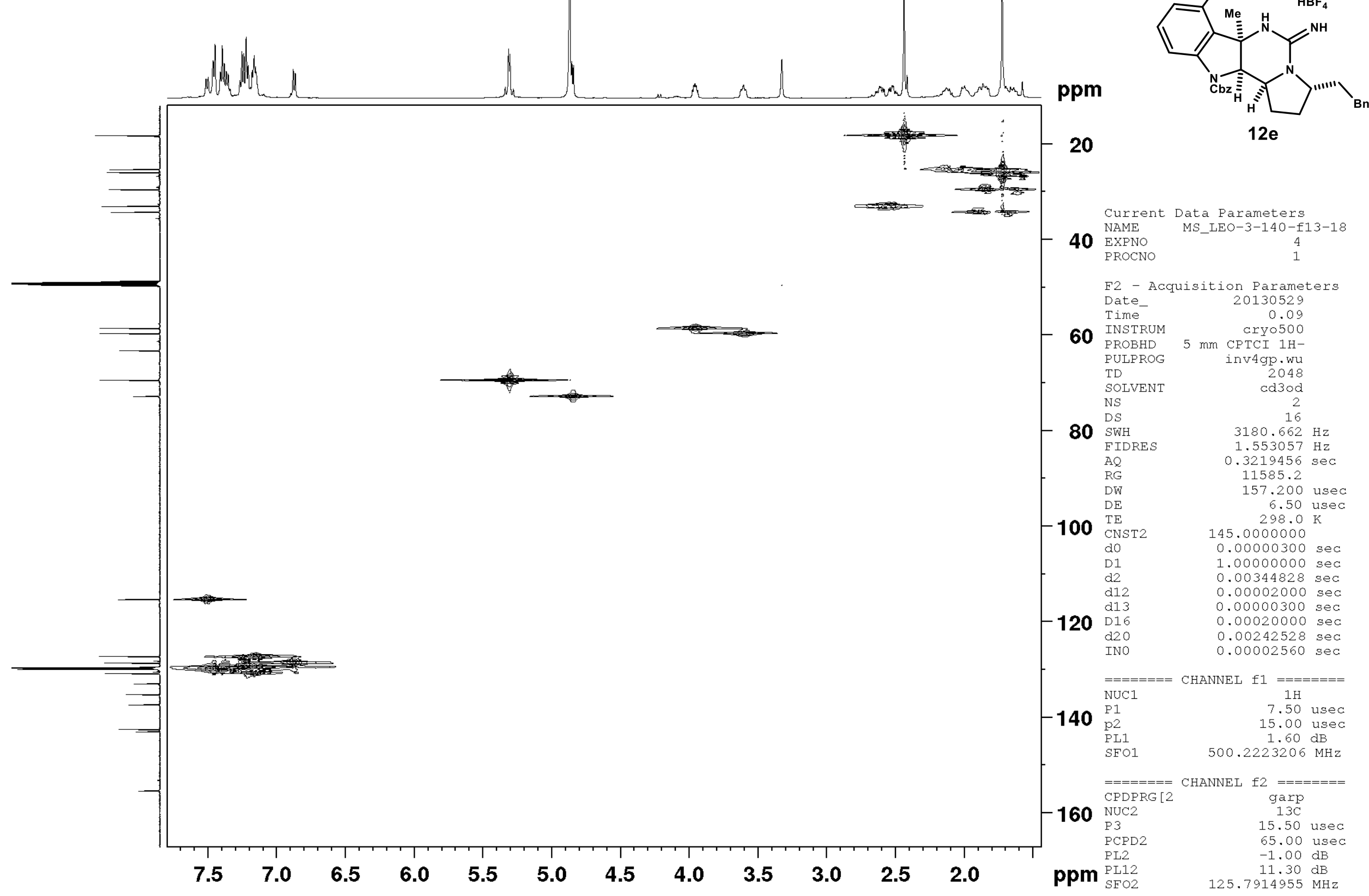




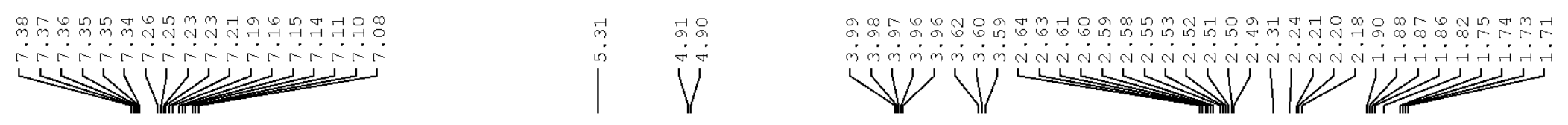

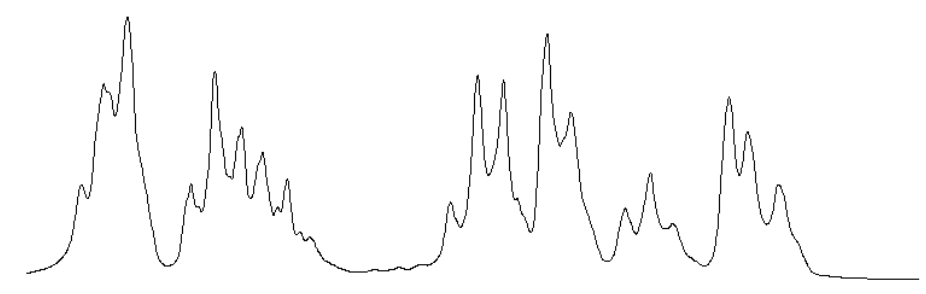
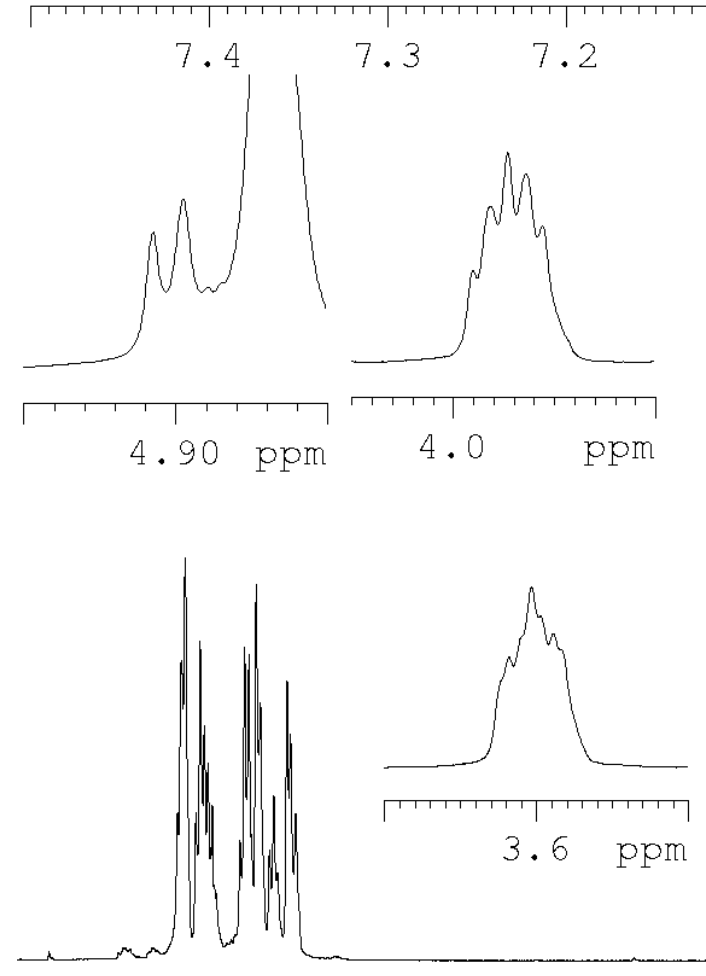
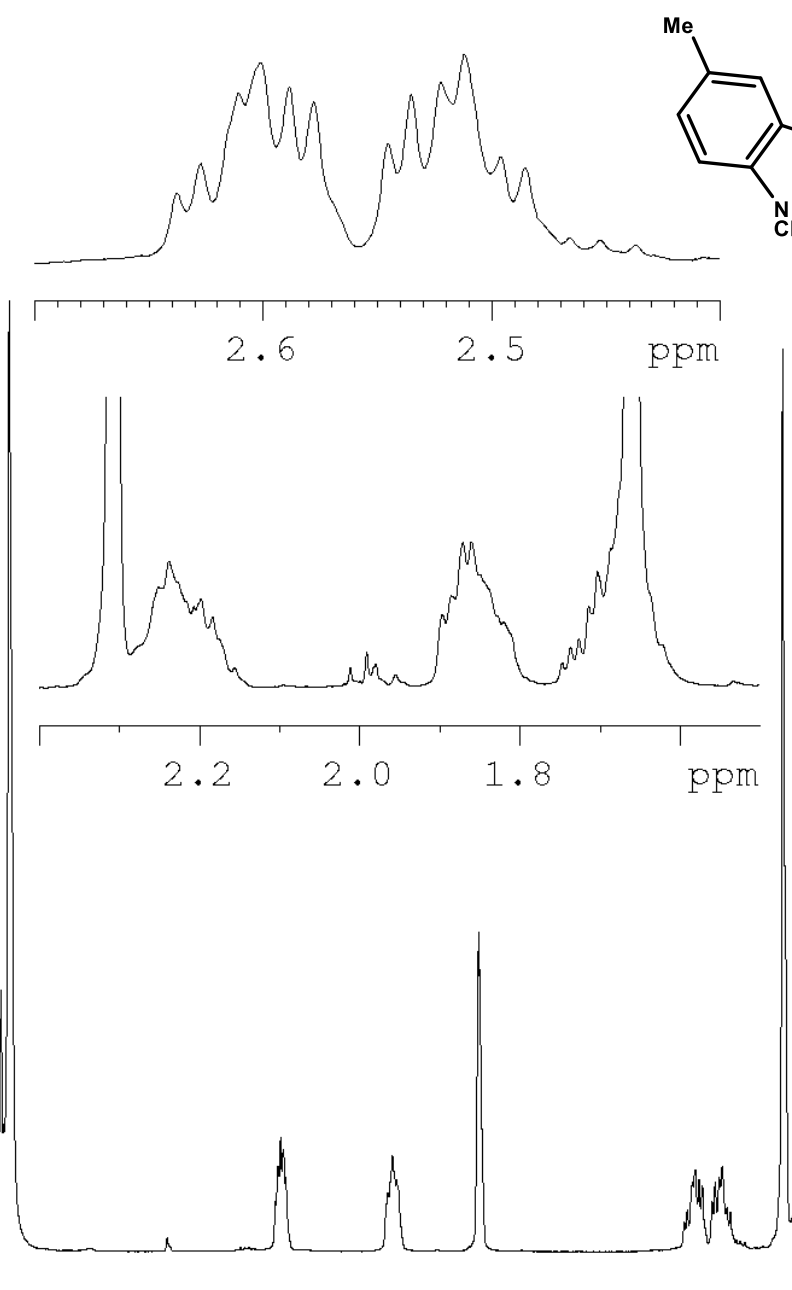

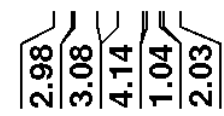

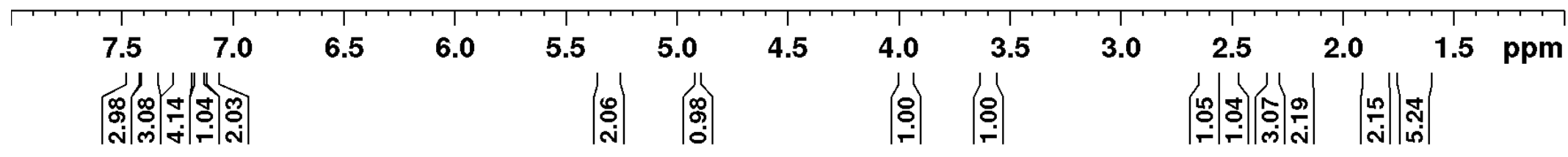

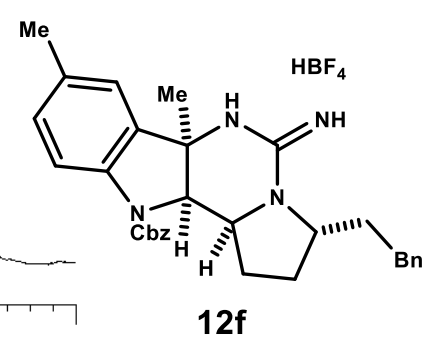

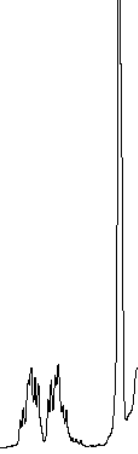

Current Data Parameters NAME PROCNO Date__ 20130602 INSTRUM PROBHD $5 \mathrm{~mm}$ CPTCI $1 \mathrm{H}-$ PULPROG SOLVENT NS SWH FIDRES $\quad 8012.820$ $\begin{array}{ll}\text { AQ } & 5.0998273 \\ \text { RG } & 5\end{array}$ DE TE $\quad 6.00$ 0.1000000 MCREST 0.100 .00000 MCWRK $\quad 0.0150000 \mathrm{C}$ $=======$ CHANNEL $\mathrm{fl}===$ P1 1.60
500.2235015 ramet SI SF $\quad 500.2200151$ $S S B$ LB $\mathrm{GB}$
$\mathrm{PC}$ 0.30 4.00 


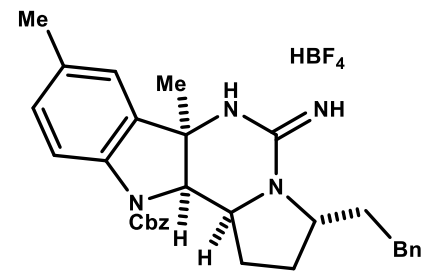

$12 f$

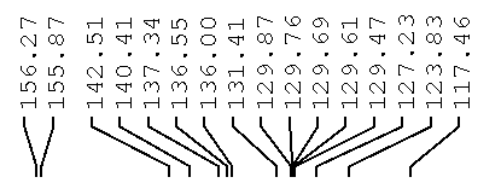

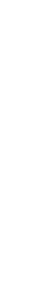

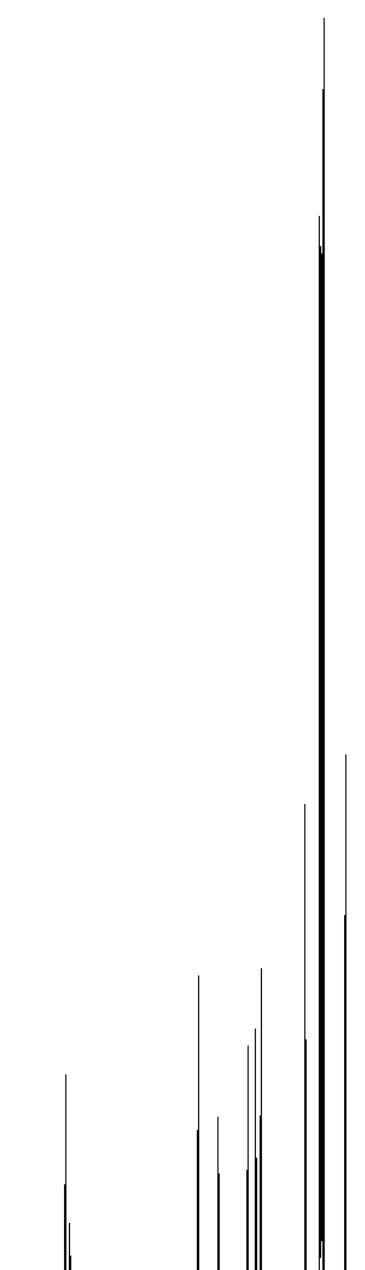

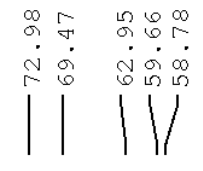
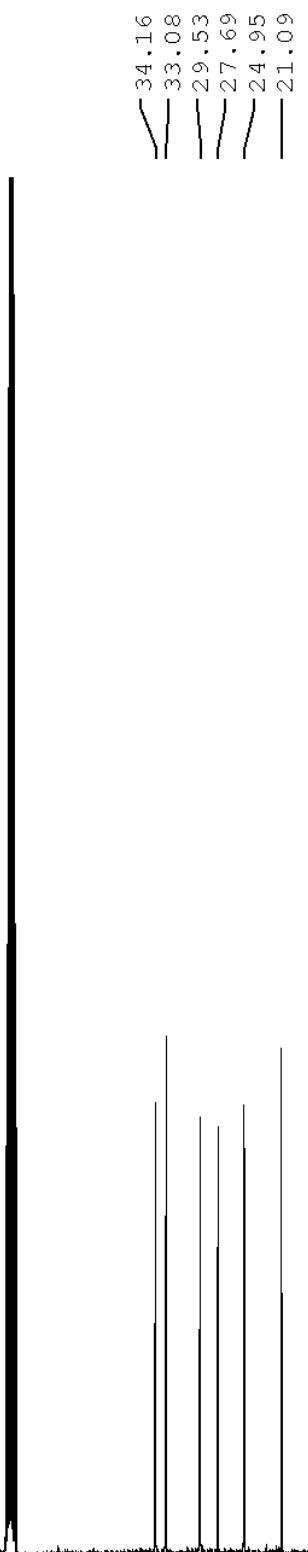

Current Data Parameters NAME MS IFO-3-147-f EXPNO PROCNO

F2 - Acquisition Parame

Time

PROBHD PULPROG SpinEchopg30gk SOLVENT NS

DS SWH

FIDRES

$\mathrm{AQ}$
$\mathrm{RG}$
$\mathrm{DW}$
$\mathrm{DE}$

DW

TE

D1 11

D16

d17

MCWRK

P2

$====$

NUC1

P1

P12

PLO

SFO1

SP1

SP2

SPNAM [2] Crp60comp.4

SPOFF2

$0 \mathrm{~Hz}$
$\mathrm{O} \mathrm{Hz}$

$=======$ CHANNEL $f 2===$ CPDPRG [2 waltz16 NUC2

PL2

$\mathrm{PL} 12$
$\mathrm{SFO} 2$

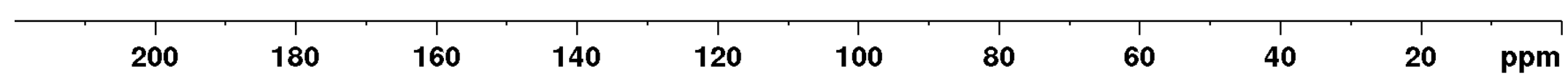




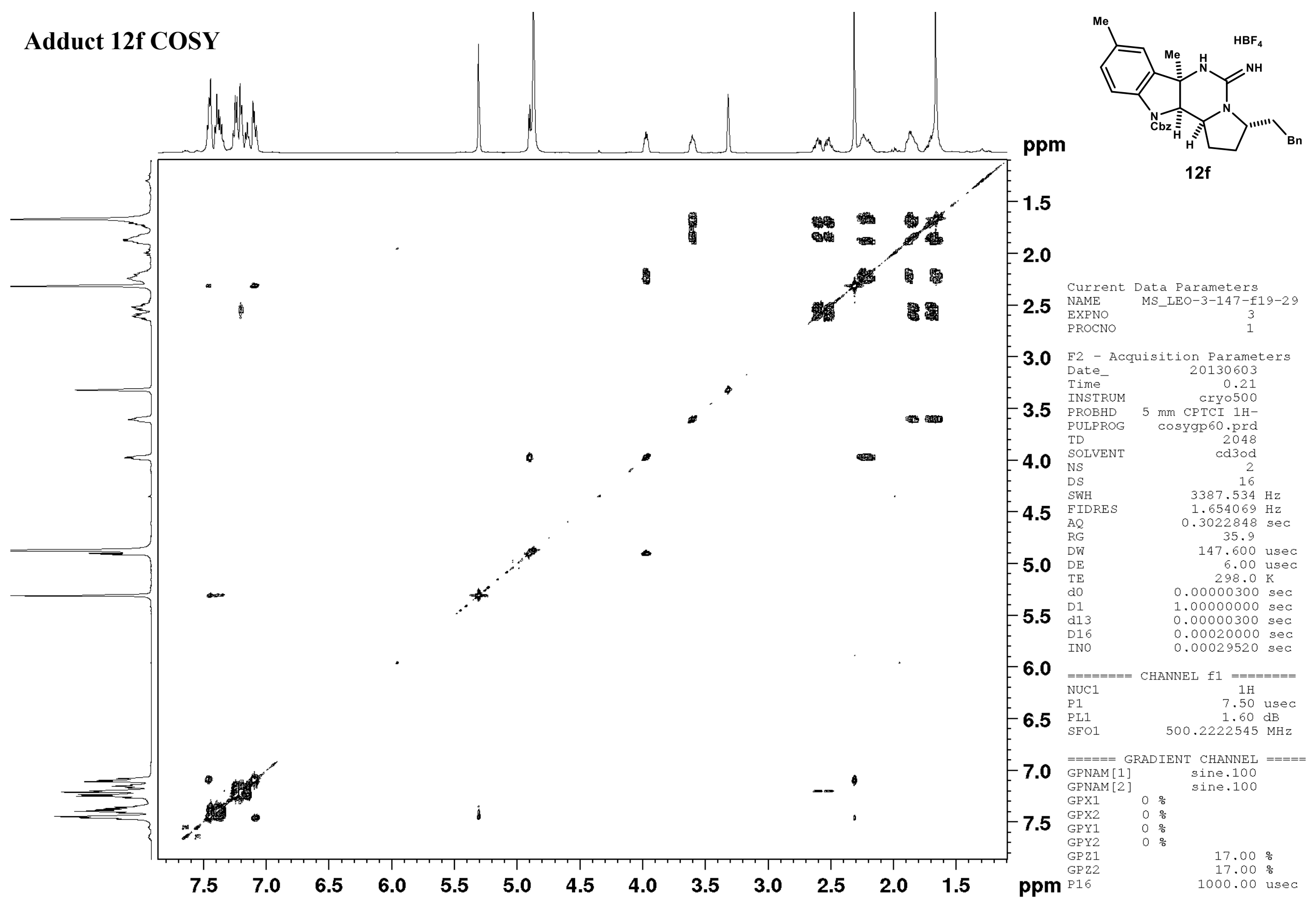




\section{Adduct 12f HMQC}

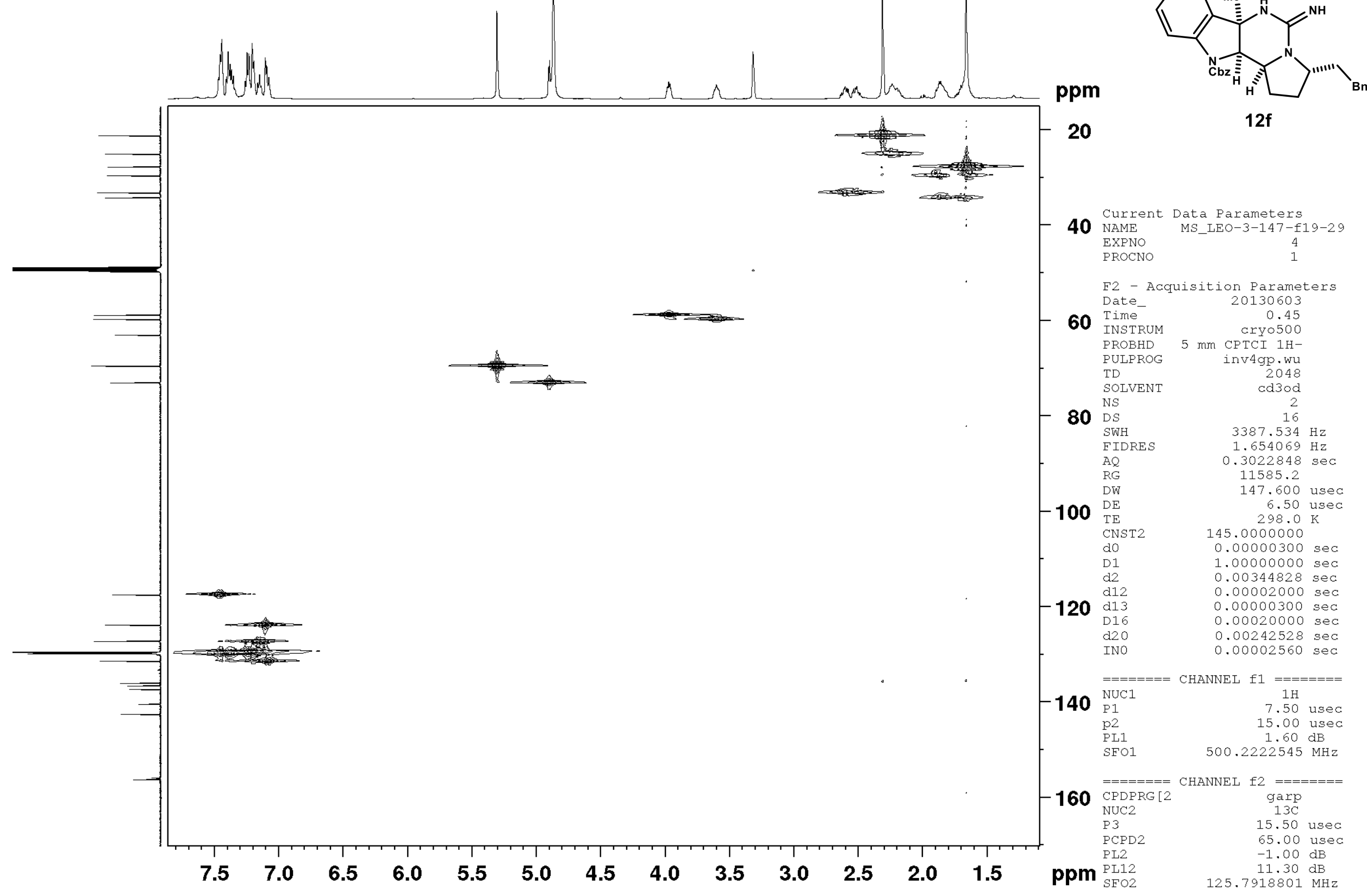



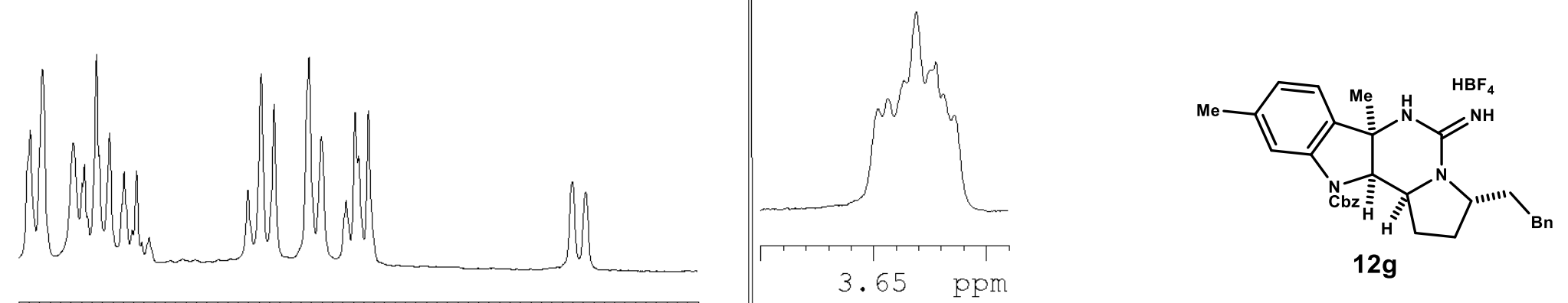

$12 \mathrm{~g}$
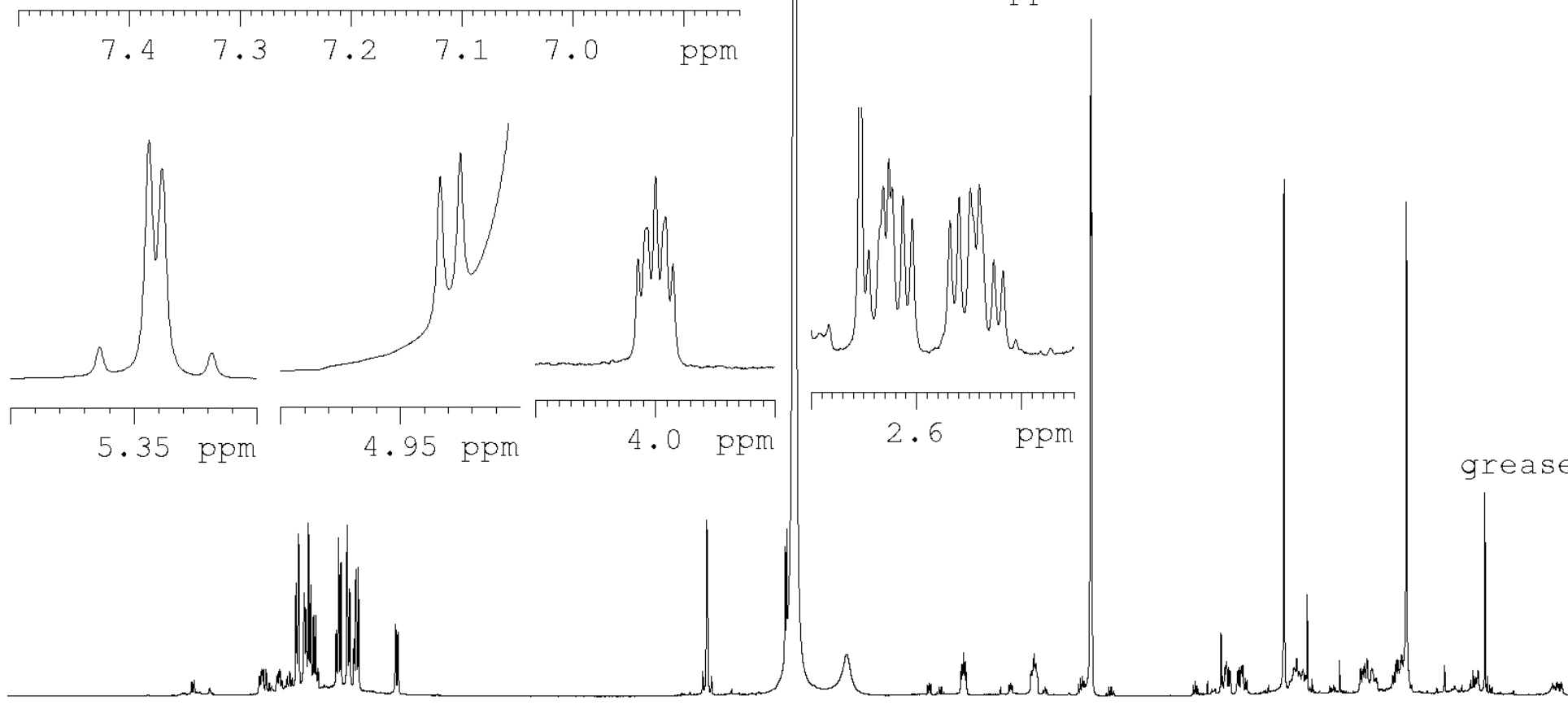

F2 - Acquisition Parame

Date_ 20210328

20210328
TNSTRUM

PROBHD $5 \mathrm{~mm}$ CPBBO BB-

PULPROG

TD

SOLVENT

NS

SWH

FIDRES

$\mathrm{AQ}$

RG

$\mathrm{DW}$
$\mathrm{DE}$

$\mathrm{DE}$
$\mathrm{TE}$
$\mathrm{D} 1$

D1

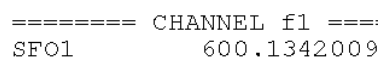

SFO1

P1

$\begin{array}{lr}\text { P1 } & 9.50 \\ \text { PLW1 } & 30.00000000\end{array}$

1H

F2 - Processing paramet

SI 65536

SF $\quad 600.1300000$

WSB

LB

GB

0.30

1.00

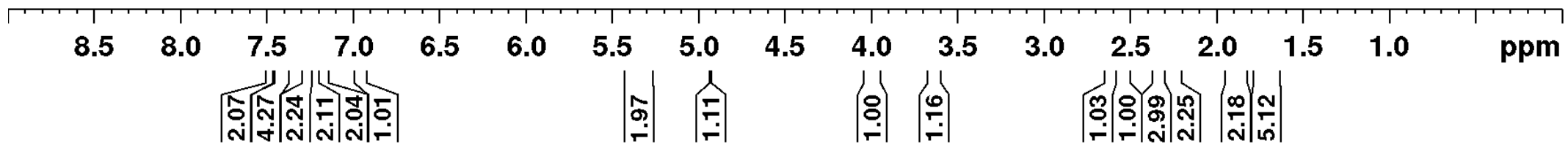




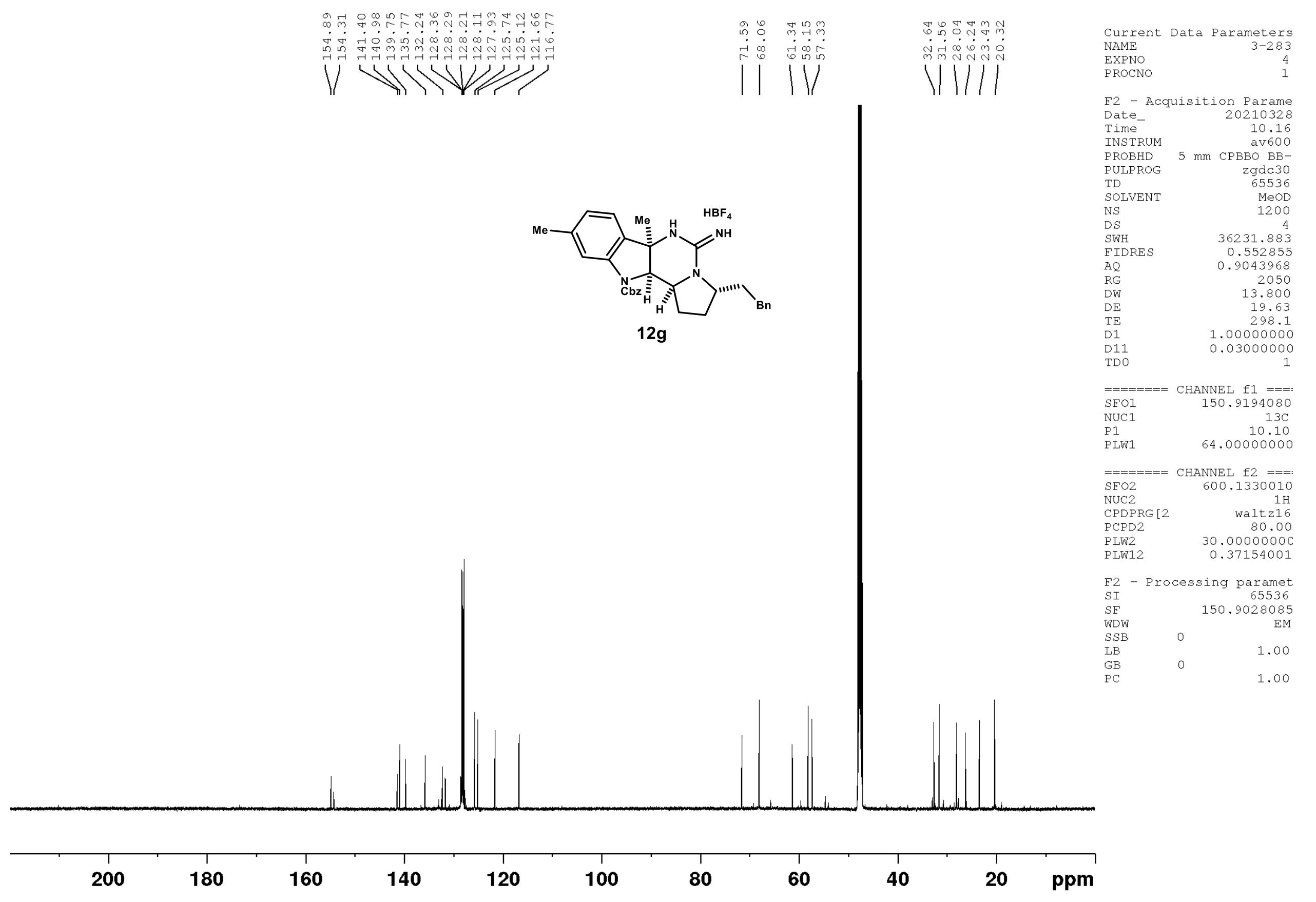




\section{Adduct 12g COSY}

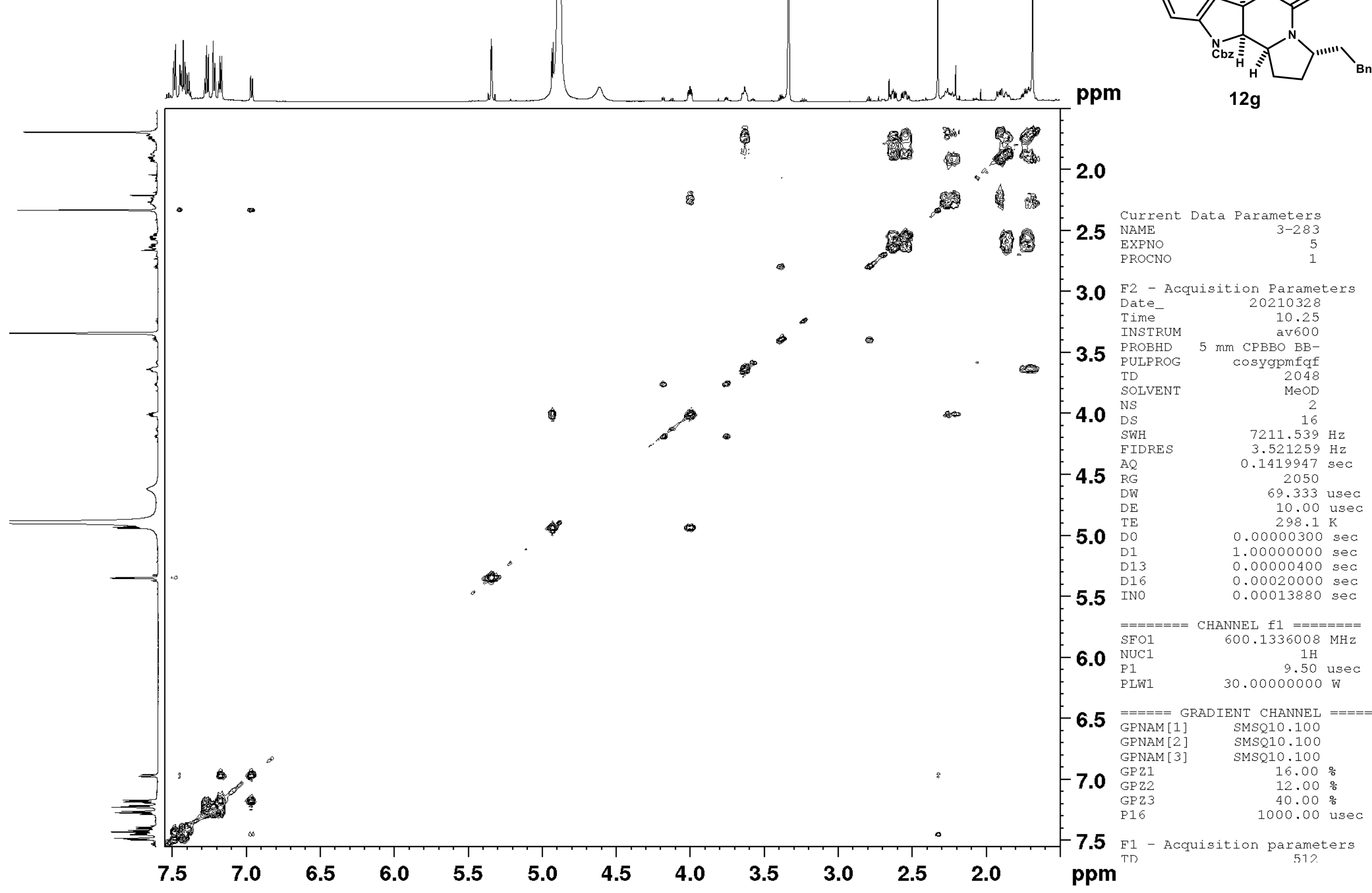


Adduct 12g HMQC

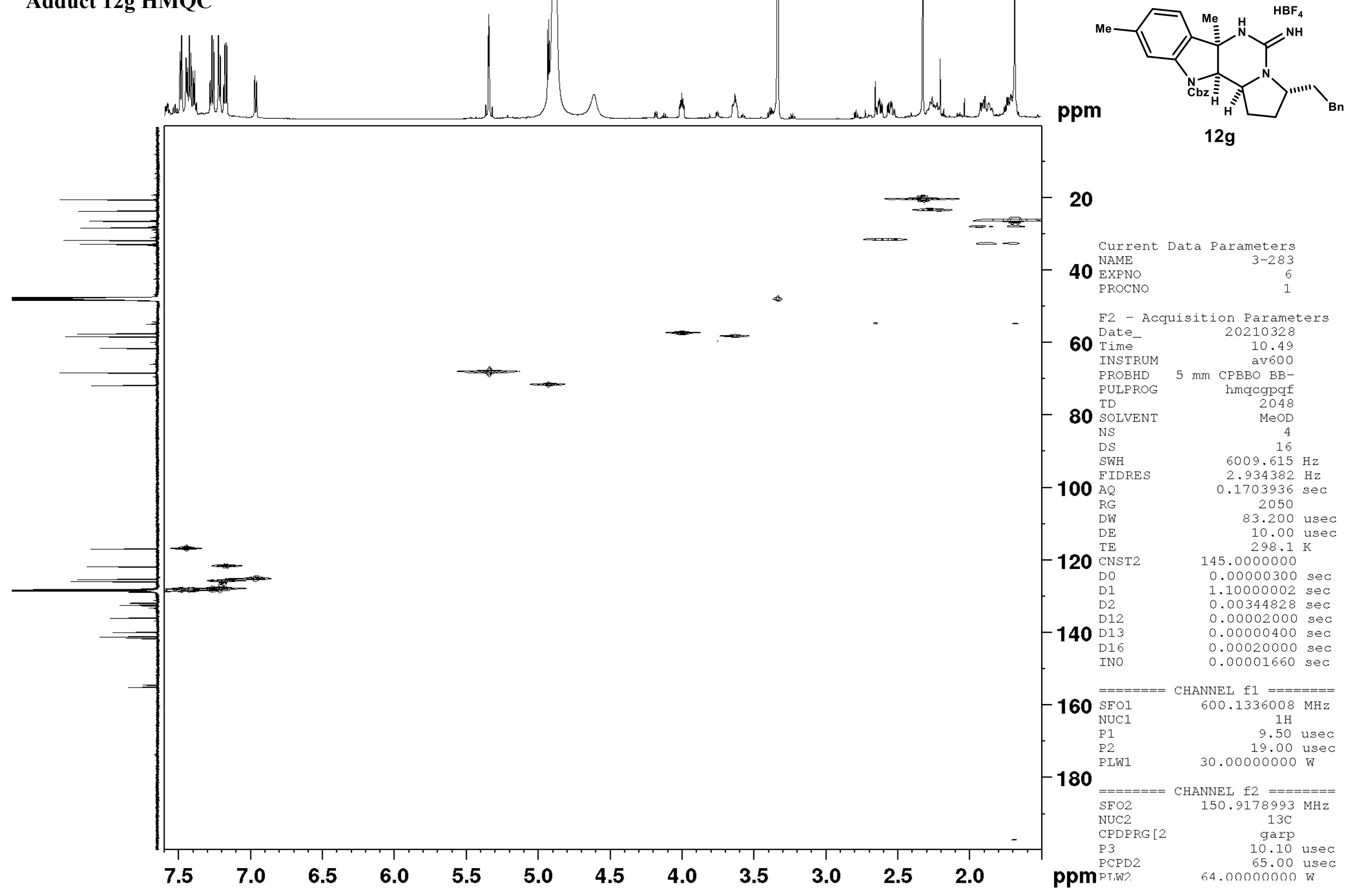




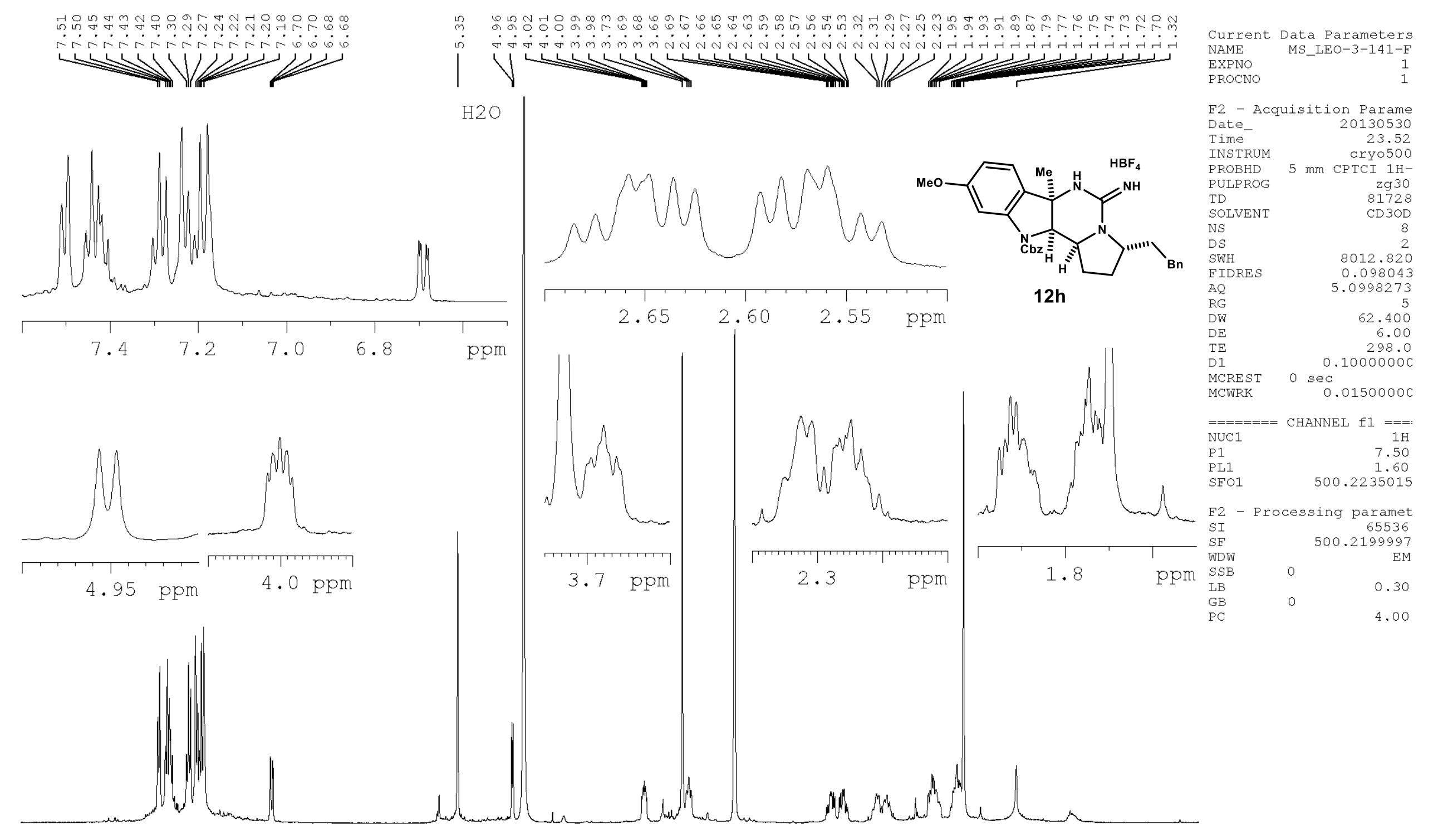

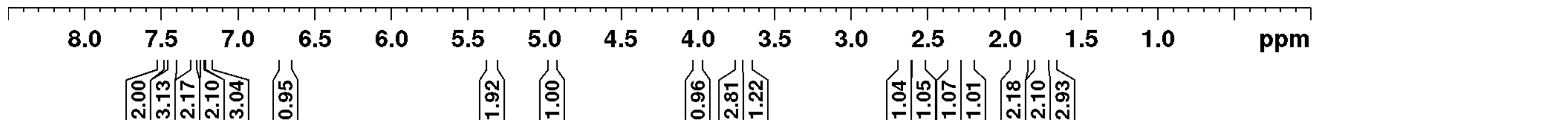




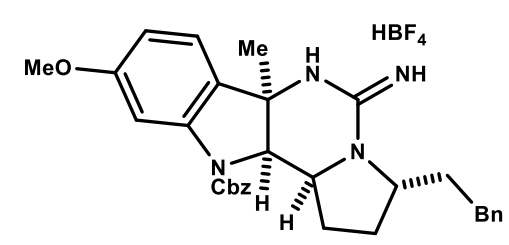

$12 \mathrm{~h}$
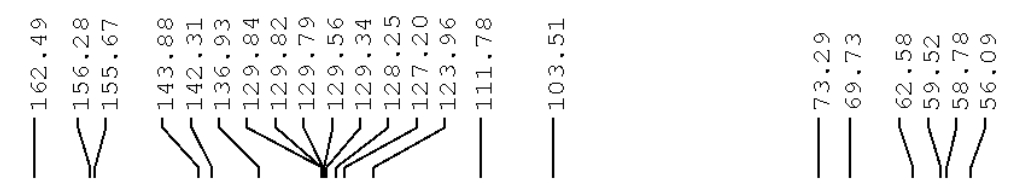

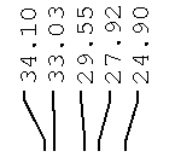

ที่

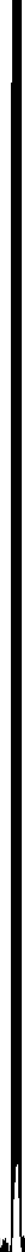

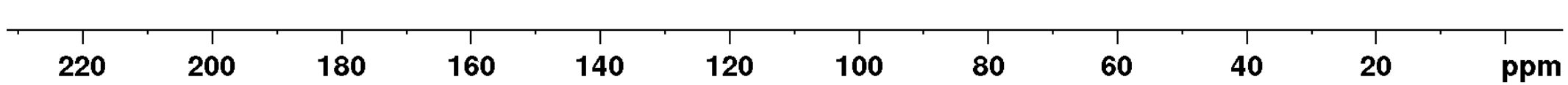

Current Data Parameters NAME PROCNO

F2 - Acquisition Parame

Date

Time

PROBHD

65536

NS

SWH

FIDRES

RG

$\mathrm{RG}$
$\mathrm{DW}$
$\mathrm{DE}$

TE
D1

D1
d11
D16

D16
d17

$0.0002000 \mathrm{C}$
0.00019600

MCWRK $\mathrm{sec} .01500000$

P2

0.01500000
31.00

$=======$ CHANNEL $\mathrm{f} 1===$

Nuc1

P1

P11

PLO

SFO1

SP1

SPNAM [1] Crp60, 0.5,20.1

SPNAM [2] Crp60comp.4

SPOFF1 0

SPOFF2

$\mathrm{Hz}$

CHANNEL $f 2===$ CPDPRG [2 waltz16 NUC2 2

$\mathrm{PL} 2$
$\mathrm{PL} 12$

100.00

1.60
24.60

500.2225011

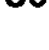

10

80

60

pm 


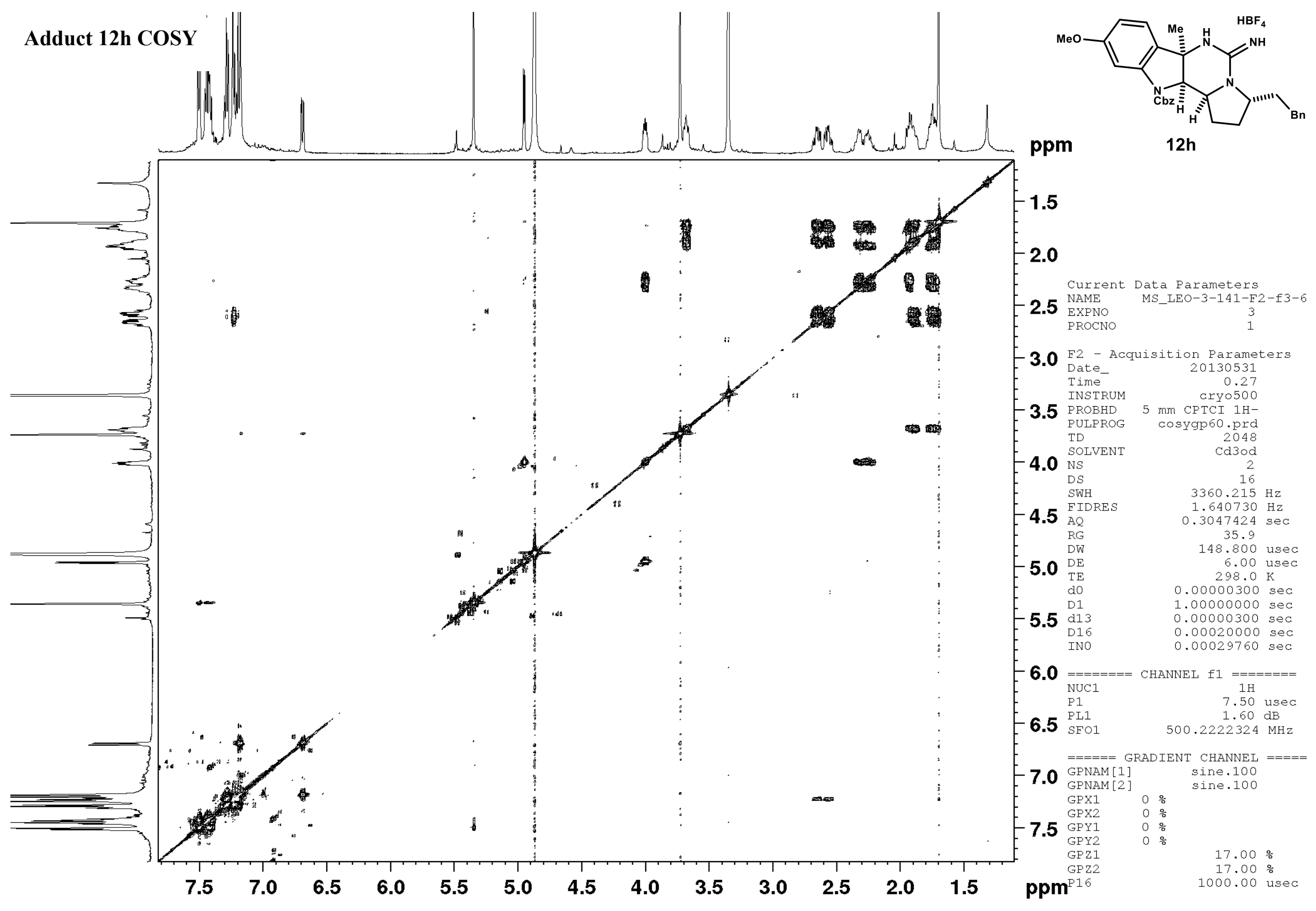




\section{Adduct 12h HMQC}

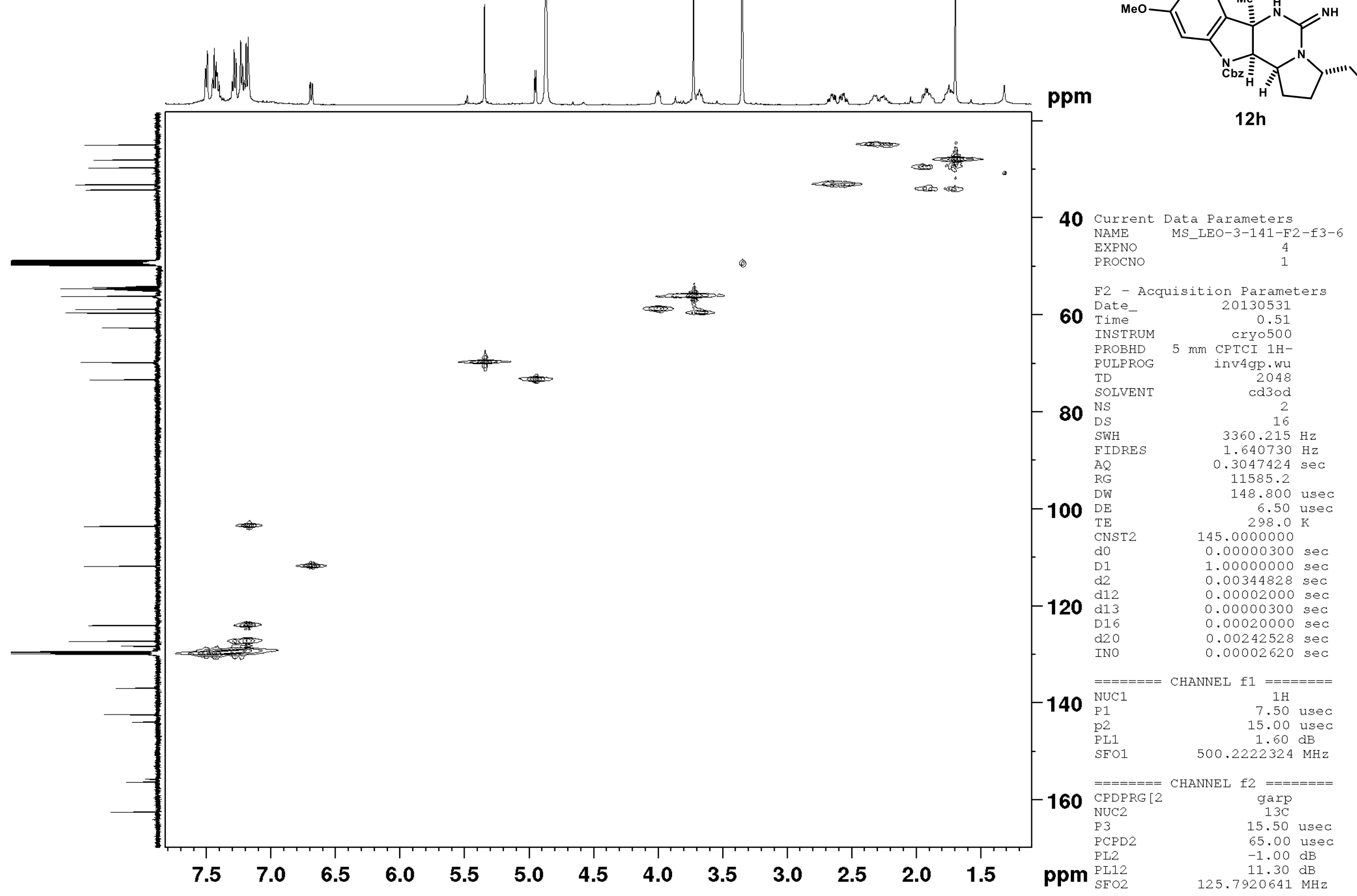




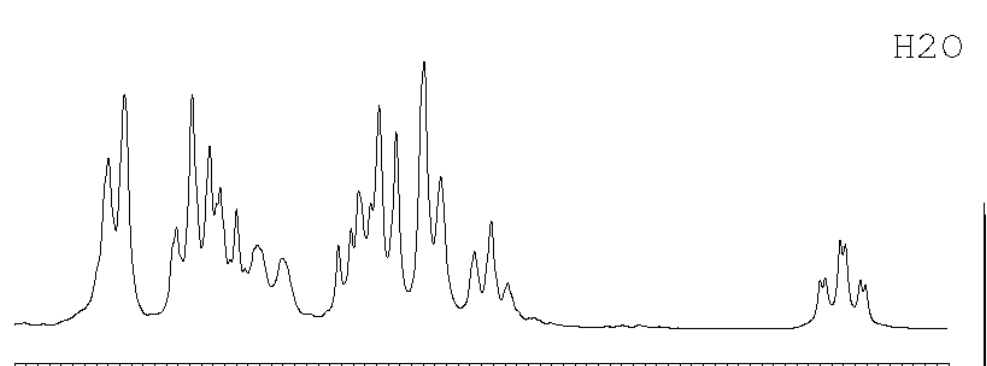

20
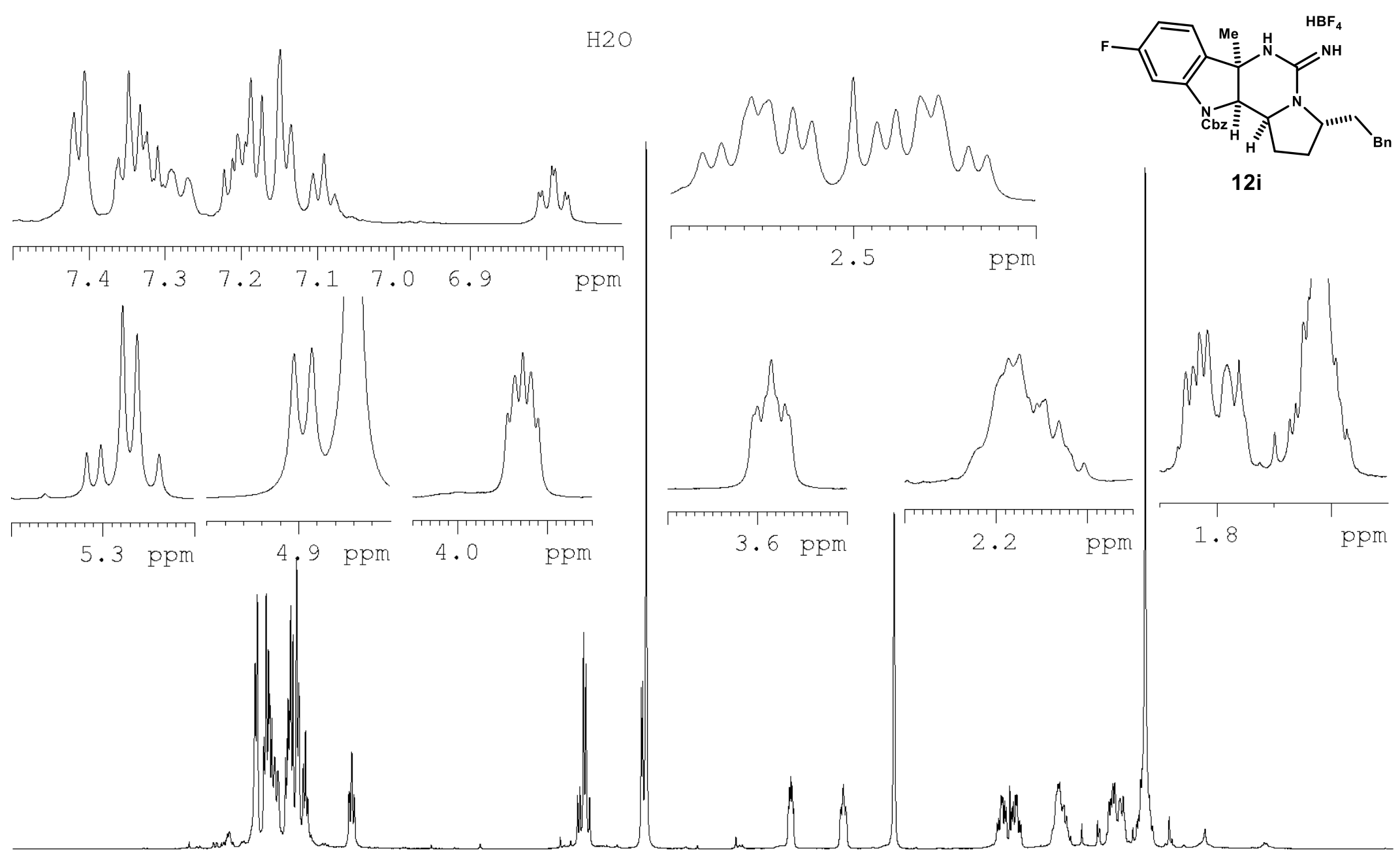

F2 - Acquisition Parame

Date

Time-

PROBHD $5 \mathrm{~mm}$ CPTCI $1 \mathrm{H}-$

PULPROG 5 mn CPTCI 1 30

TD

SOLVENT

zg30
81728
CD $30 \mathrm{D}$

$12 \mathrm{i}$

DS

$\begin{array}{ll}\text { DS } & 8012.820 \\ \text { SWH } & 0.098043 \\ \text { FIDRES } & 5.0998273\end{array}$

$\begin{array}{lr} & 0.098043 \\ & 5.0998273\end{array}$

$\begin{array}{lr}\text { RG } & 6.3 \\ \text { DW } & 62.400\end{array}$

$\begin{array}{lr}D E & 6.00 \\ T E & 291.4\end{array}$

$\begin{array}{ll}\text { TE } & 0.10000000\end{array}$

MCREST 0 sec

DCREST O sec 0.01500000

(c)

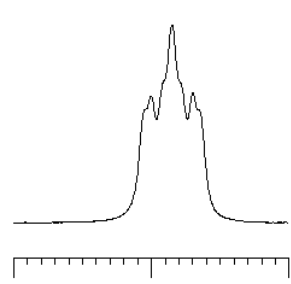

$3.6 \mathrm{ppm}$
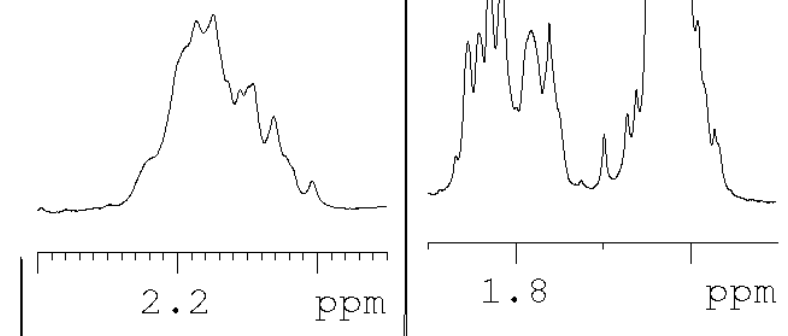

NUC

$\mathrm{P} 1$

7.50

SFO1 500.2235015

2 - Processing paramet SI $\quad 500.2200536$ WDW $\begin{array}{lll}S S B & 0 & 0.30\end{array}$

Muther
8.58 .0

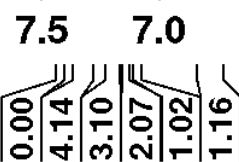
$6.5 \quad 6.0 \quad 5.5$

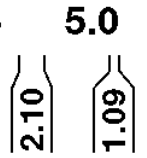
$\begin{array}{lll}4.5 & 4.0 & 3.5\end{array}$

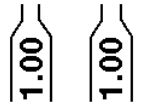

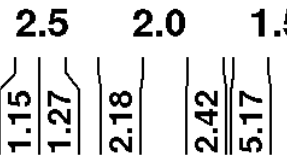




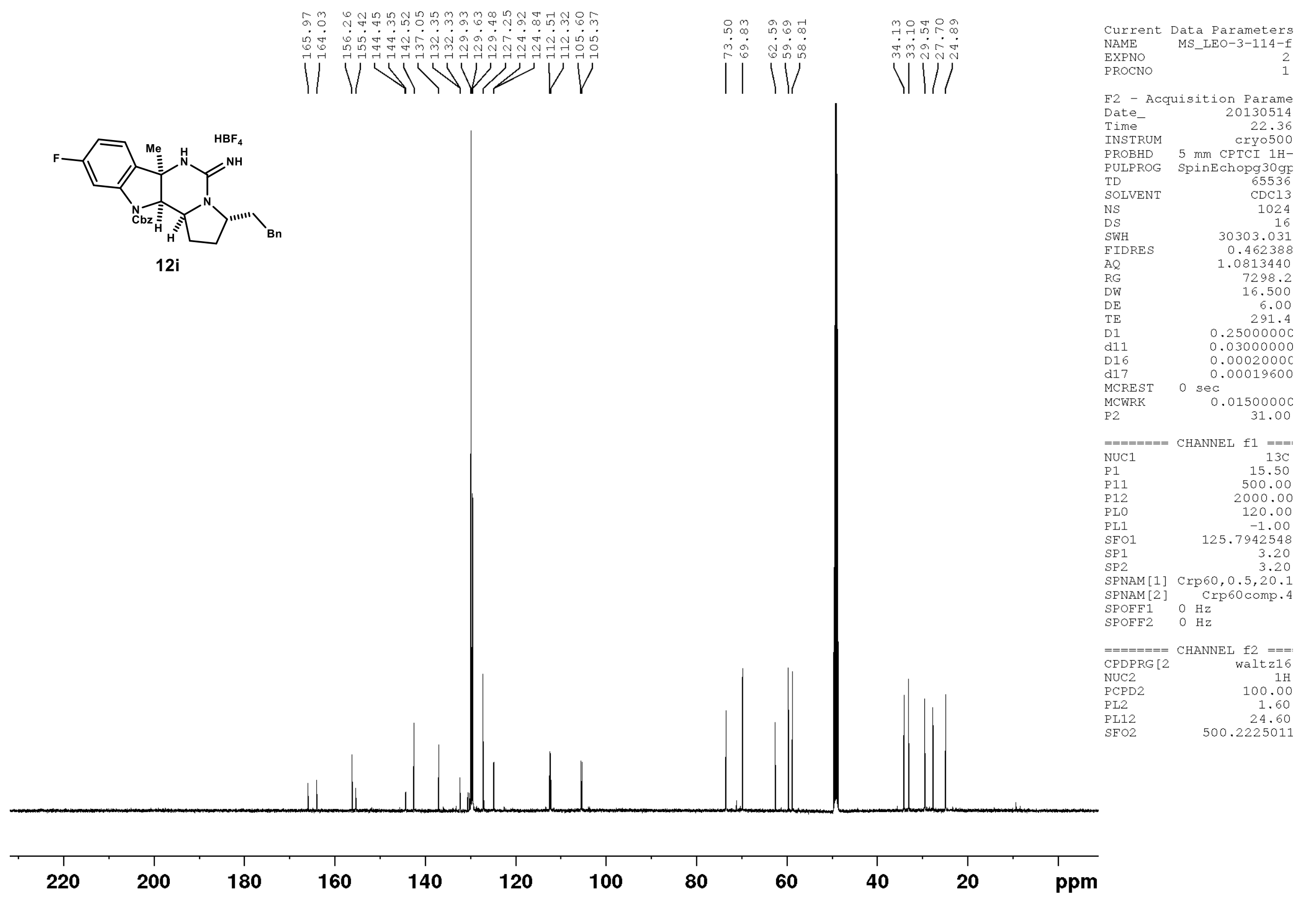




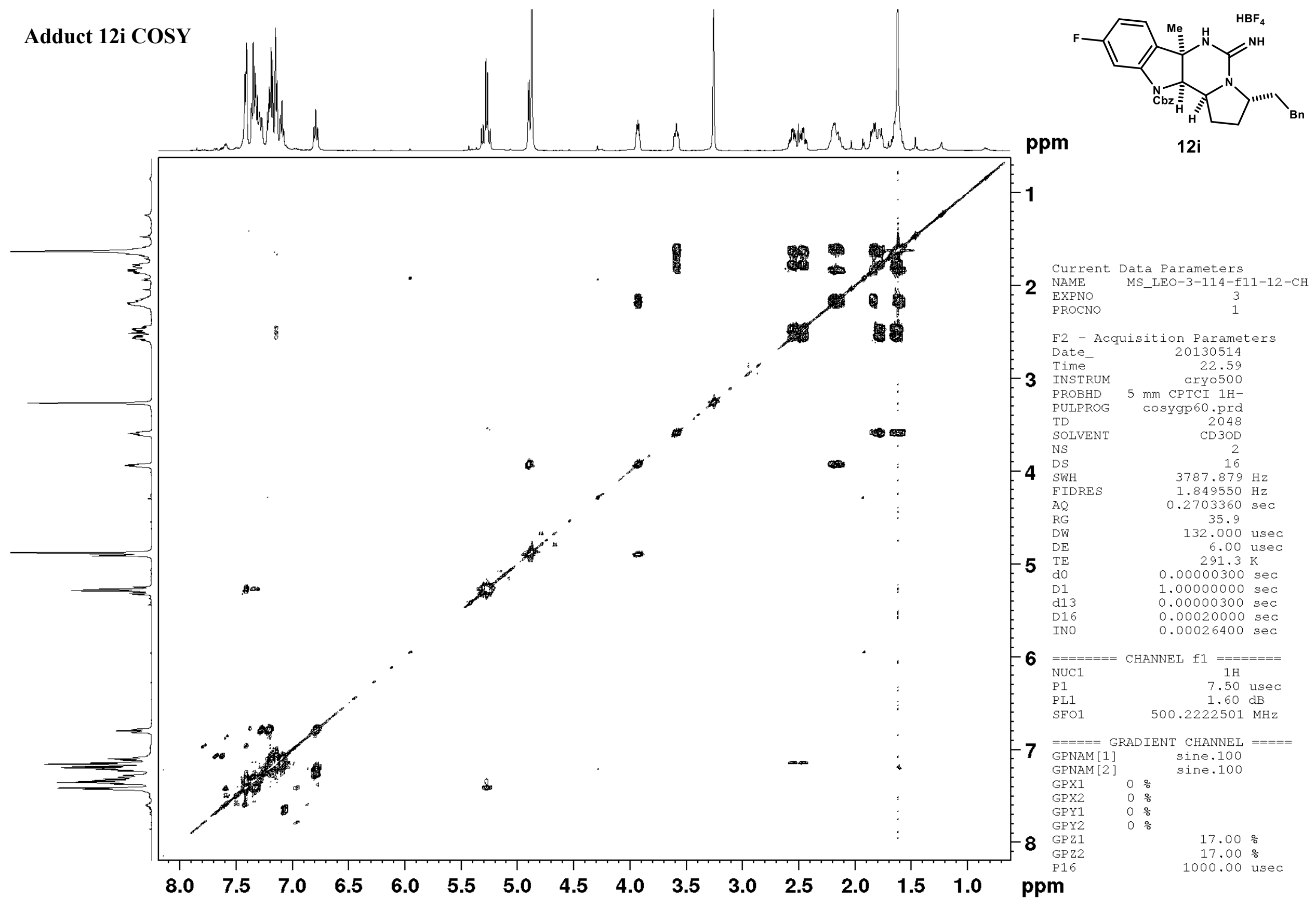




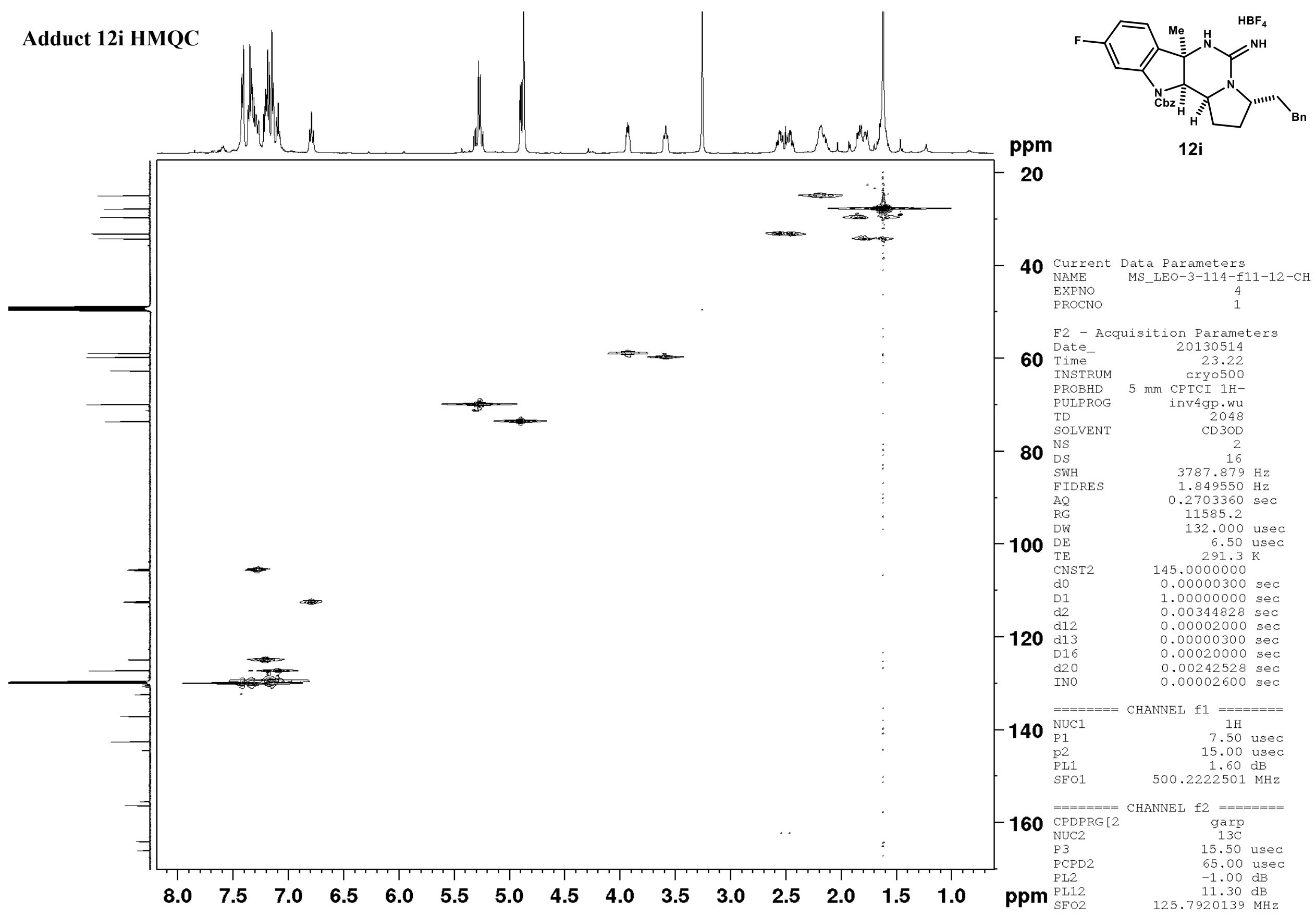



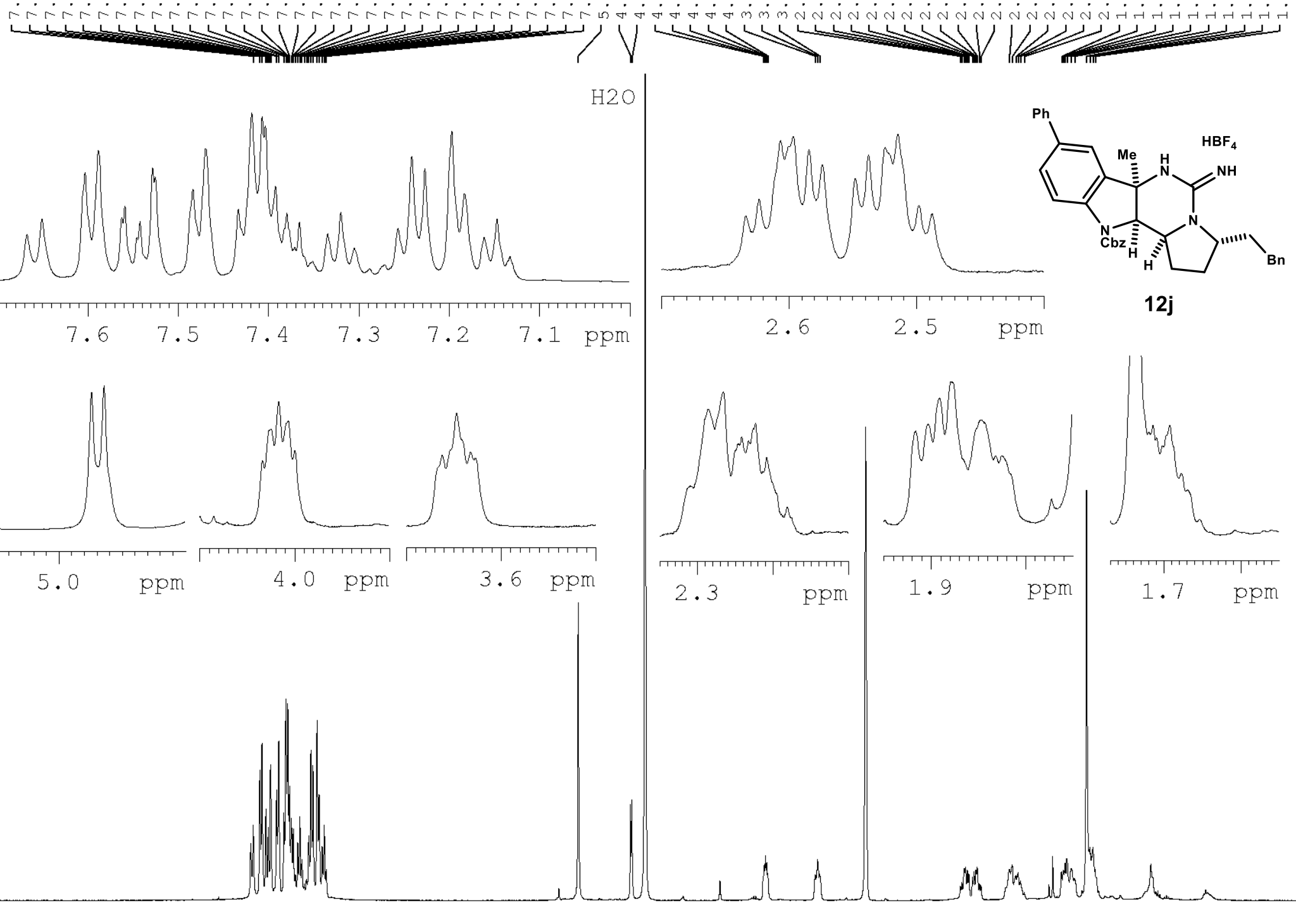

Current Data Parameters

F2 - Acquisition Parame

Date

Time-

INSTRUM

PULPROG

TD

NS

DS

FIDRES

$\mathrm{AQ}$

AQ
DW

12j

$\mathrm{DE}$
$\mathrm{TE}$

$\mathrm{TE}$
$\mathrm{D} 1$

298.0

MCRES

$===$

P1

$1 \mathrm{H}$
7.50

$\begin{array}{lr}\text { SEO1 } & 1.60 \\ & 500.2235015\end{array}$

F2 - Processing paramet

$\begin{array}{ll}\text { SF } & 500.2200211\end{array}$

WSB

$\begin{array}{lll}\mathrm{LB} & 0 & 0.30\end{array}$

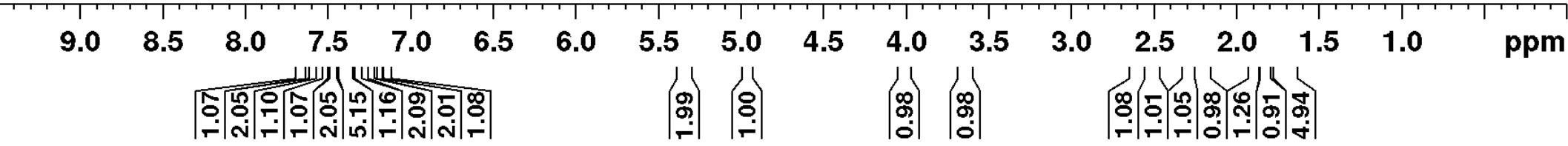




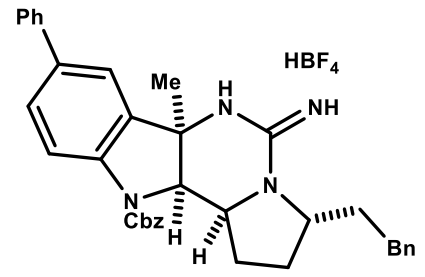

12j
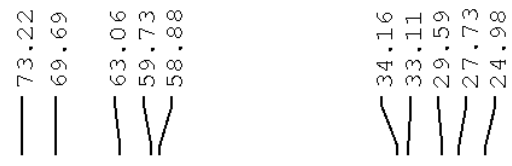

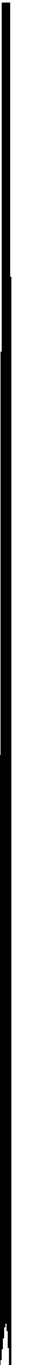
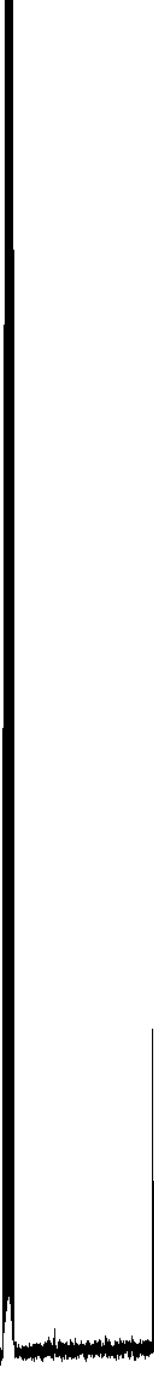

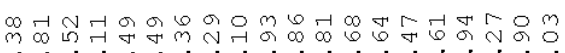
sty

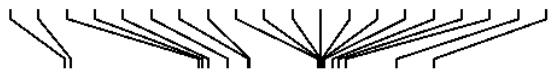

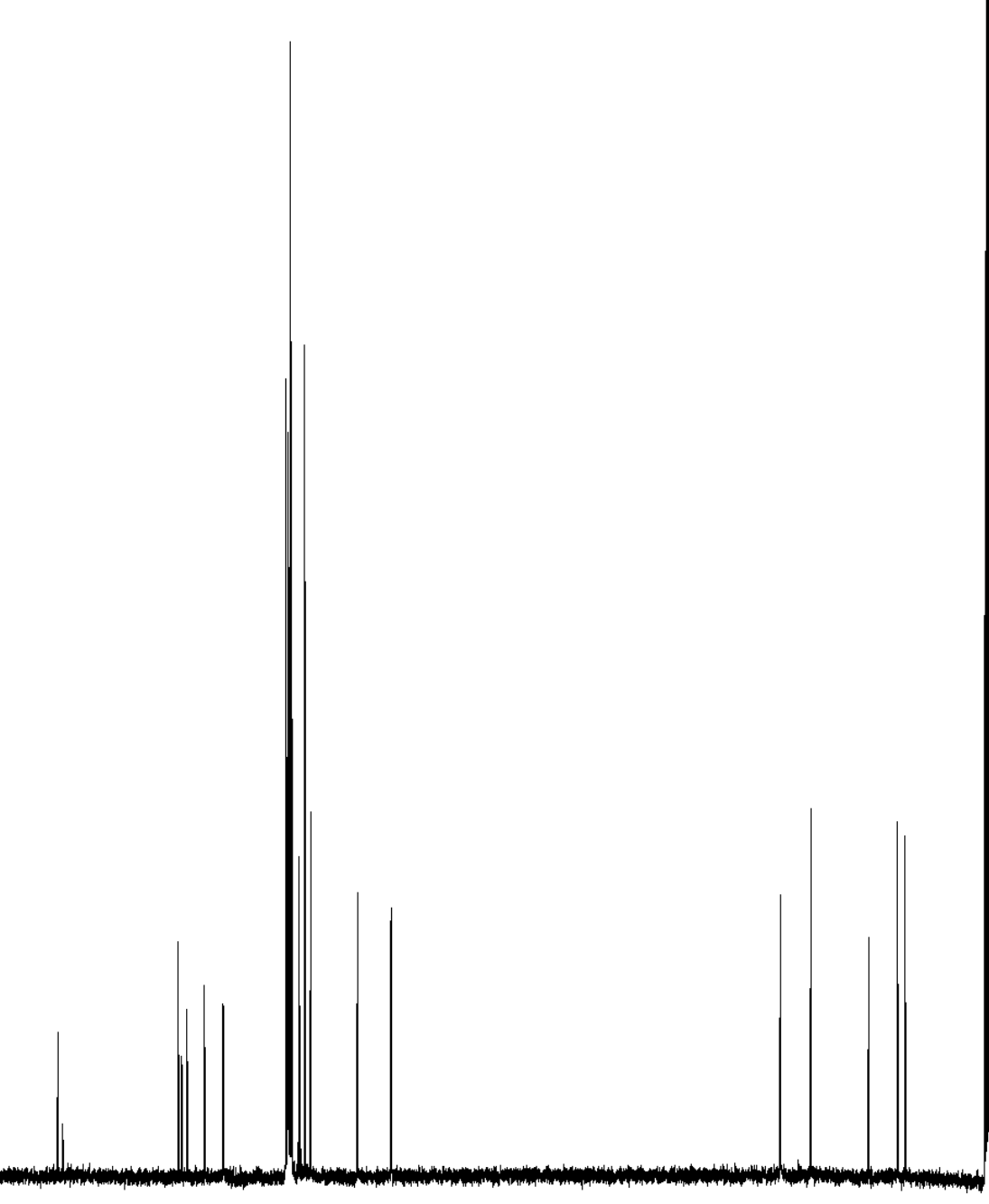

Current Data Parameters

F2 - Acquisition Parame

Date

Dime-

INSTRUM

PROBHD

PULPROG SPIn $1 \mathrm{CPCI}$ SOI

NS

SWH

FIDRE

$\mathrm{AQ}$

RG

$\mathrm{DE}$
$\mathrm{TE}$

TE

d11

d17

MCREST
MCWRK

M2

P2

$====$
NUC1

NUC1

P1

P12

PLO

SFO1

SP1

SP2

SPNAM [1] Crp60, 0.5,20.1

SPNAM [2] Crp60comp. 4

SPOFF 1

$\begin{array}{ll}0 & \mathrm{~Hz} \\ 0 & \mathrm{~Hz}\end{array}$

f2 $===$ CPDPRG [2 waltz16 NUC2 2

PCPD2

$\mathrm{PL} 2$

$\mathrm{PL} 12$
$\mathrm{SFO} 2$

\begin{tabular}{rrrrrrrrrr|r}
\hline 200 & 180 & 160 & 140 & 120 & 100 & 80 & 60 & 40 & 20 & ppm
\end{tabular}




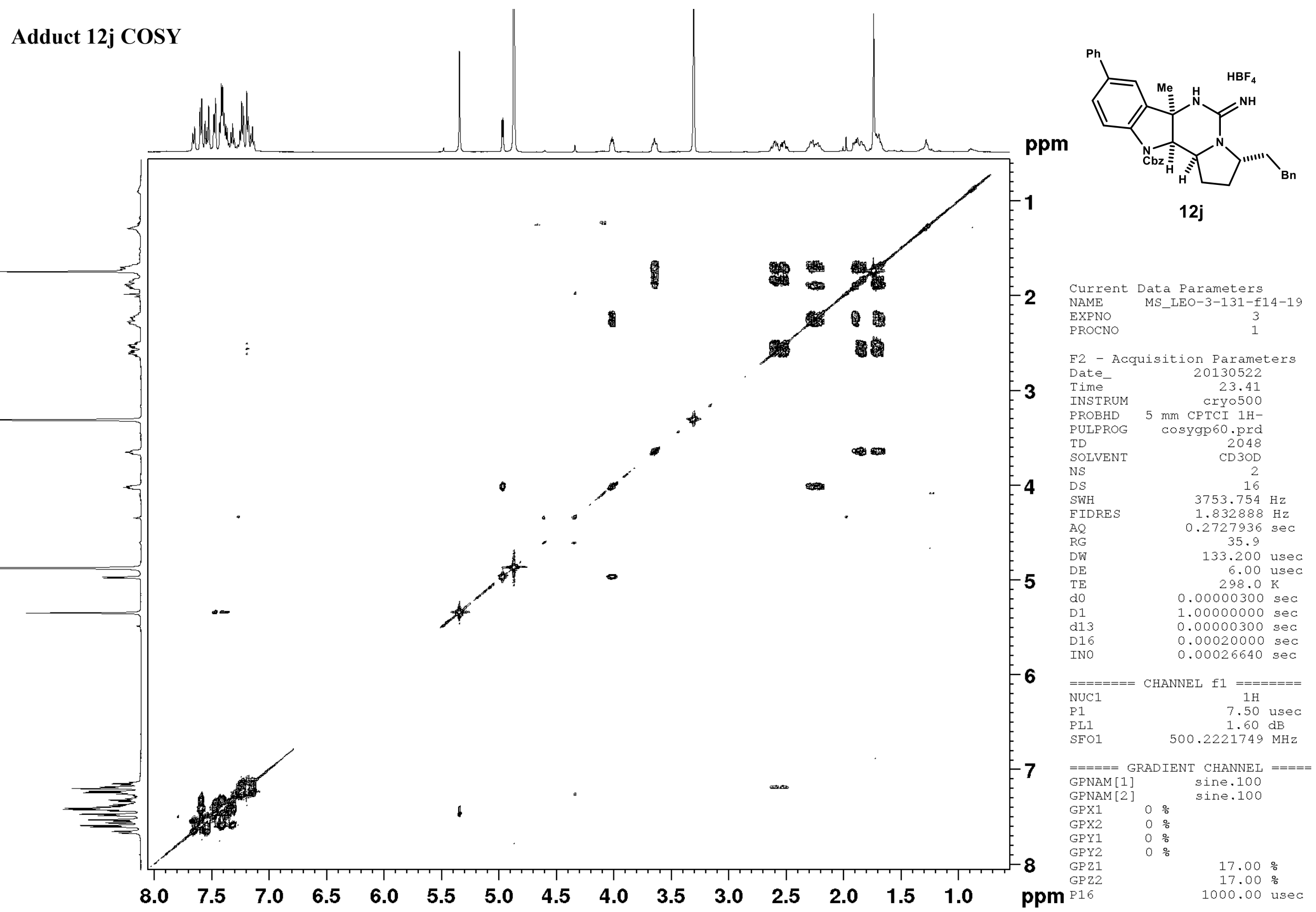




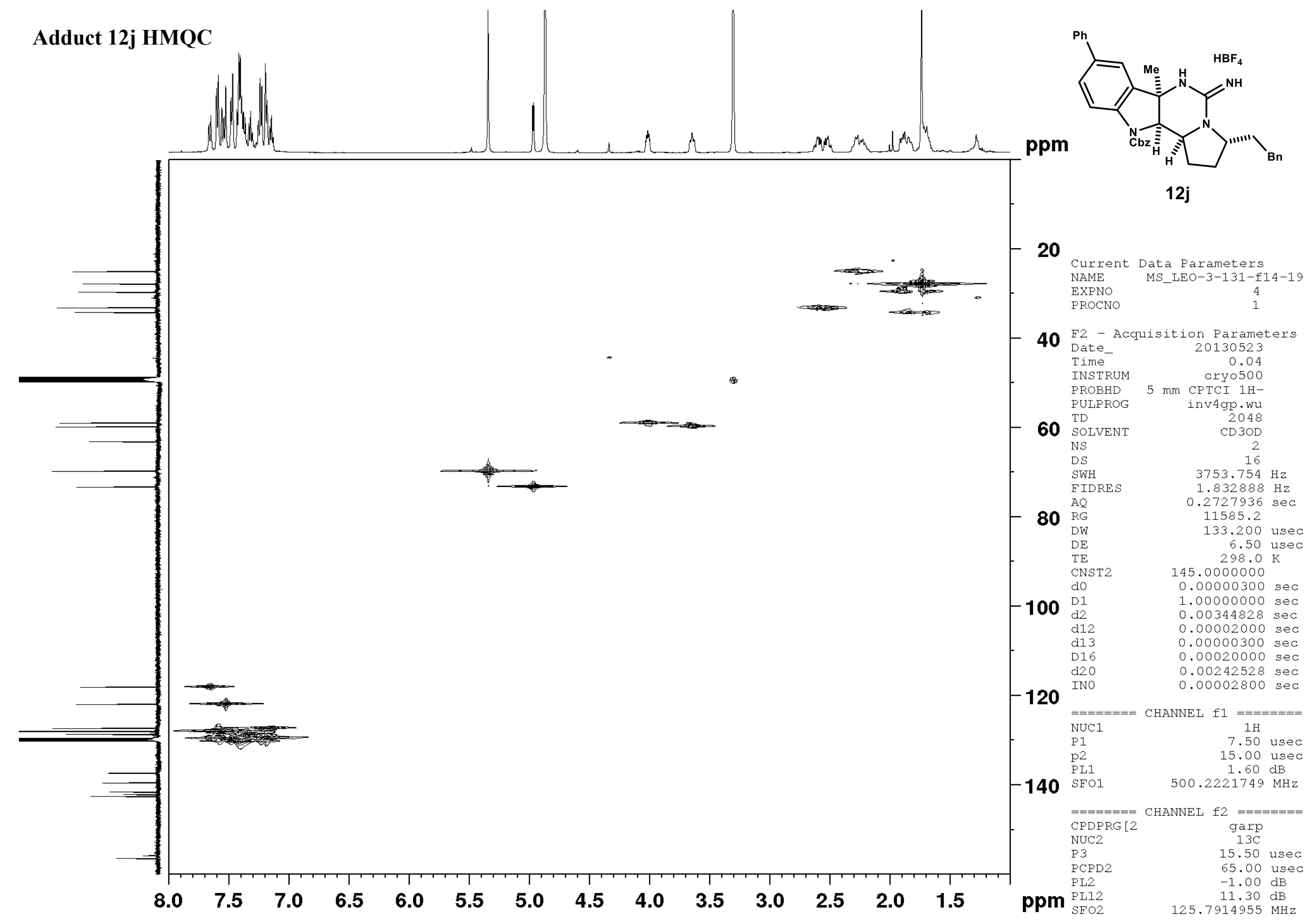




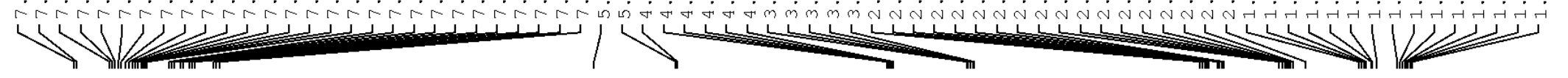

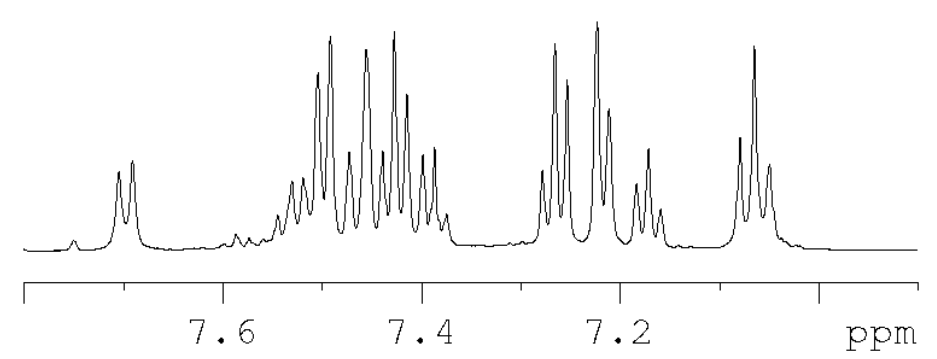
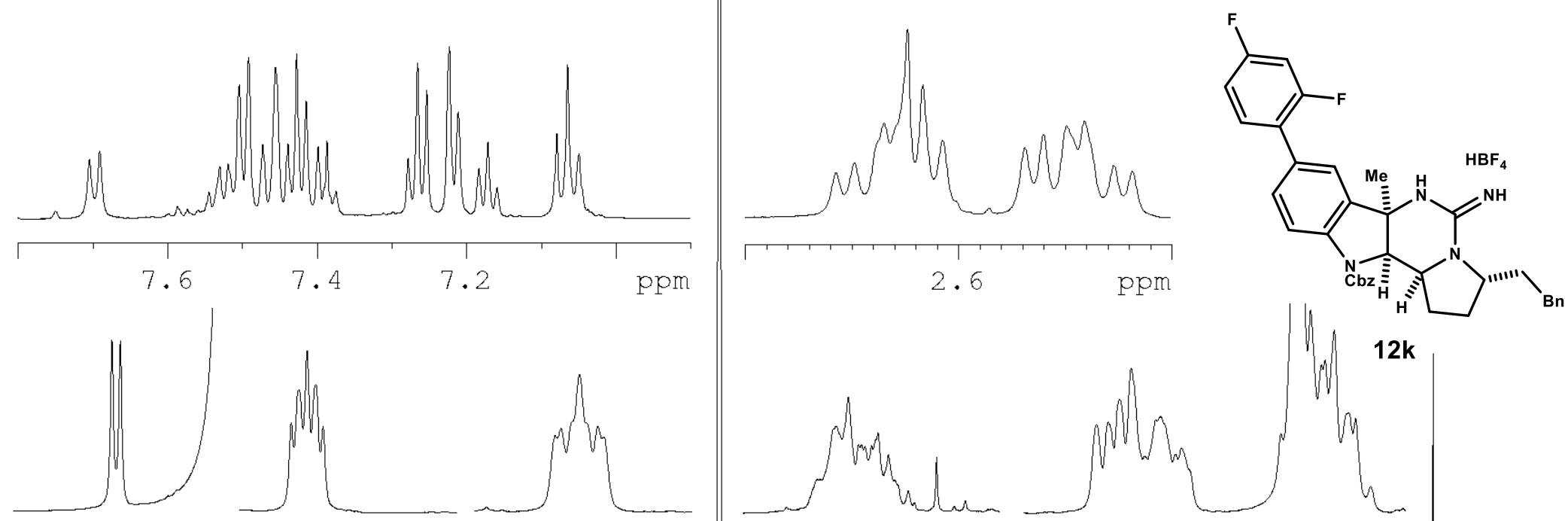

5. 0 pom
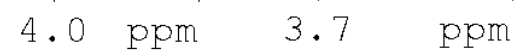

pm
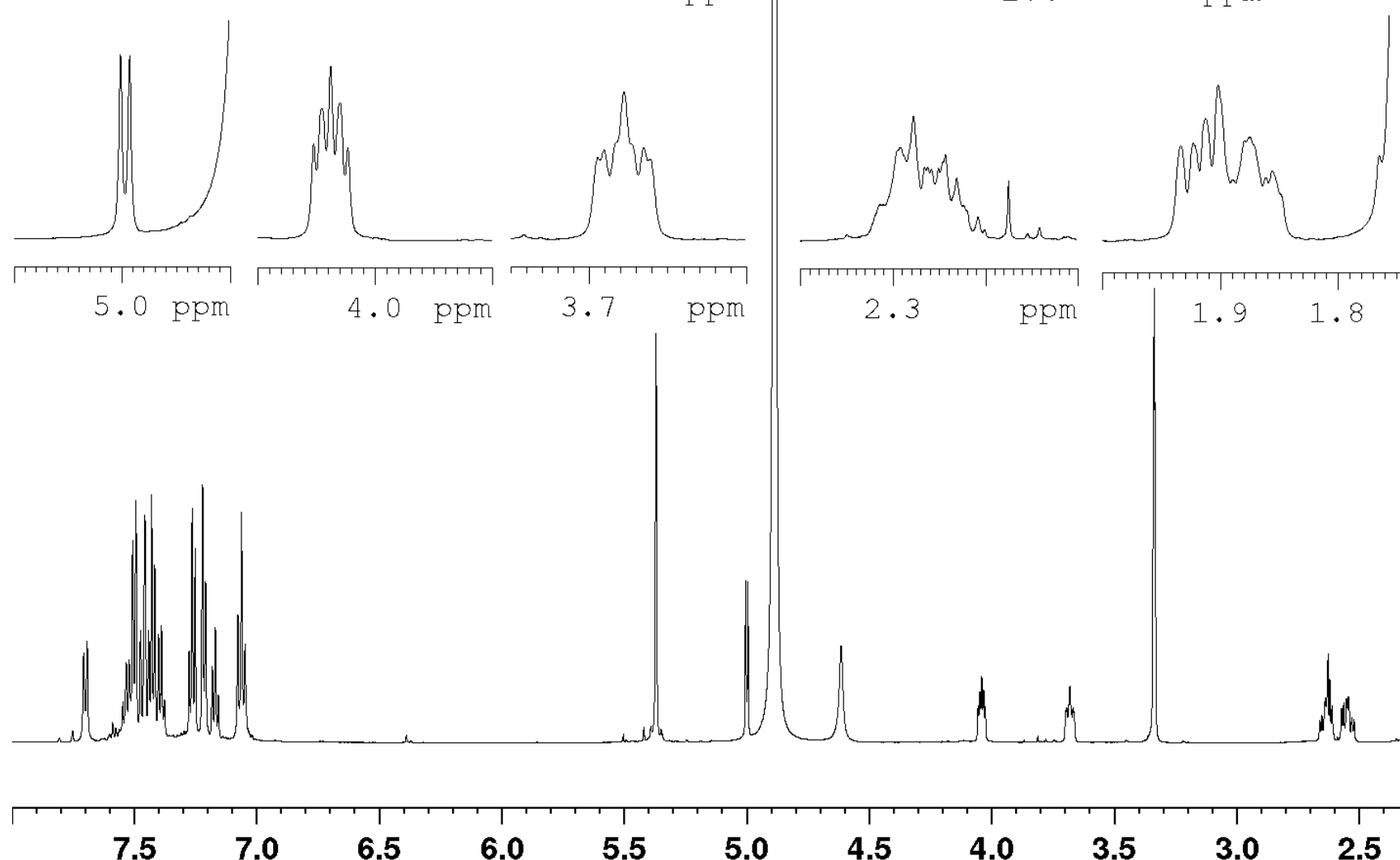

7.5

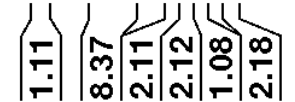

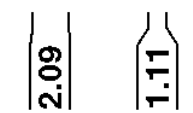

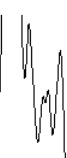

$12 k$

1.8

ppm

\section{PROCNO}

F2 - Acquisition Parame

Date

Time

PROBHD

PULPROG

SOLVENT

NS

SWH

FIDRES

RG

$\mathrm{RG}$
$\mathrm{DE}$

$\mathrm{DE}$

D1

SFO1

SFO

P1

PLW1

20210414
7.57

$\mathrm{zg} 30$
98074

MeOD

16
2

9615.385
0.098042 5.0998478

10

14.23

0.10000000

F

SI

WDW

LB

$\mathrm{PC}$

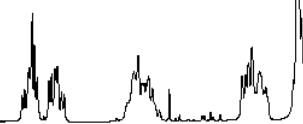

CHANNEL $\mathrm{f} 1===$

$1 \mathrm{H}$

Processing paramet 600.1300000

0.30

1.00 


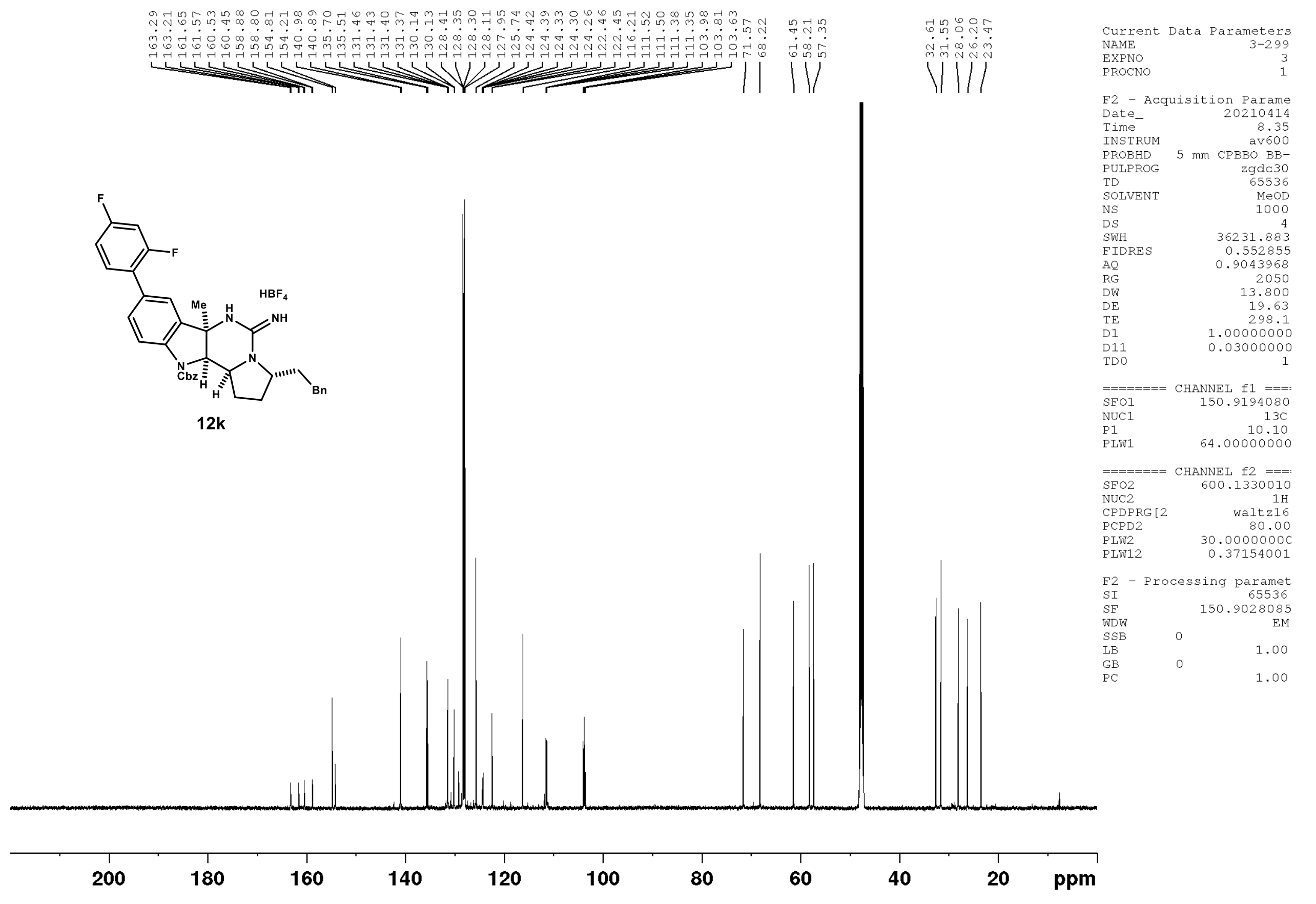




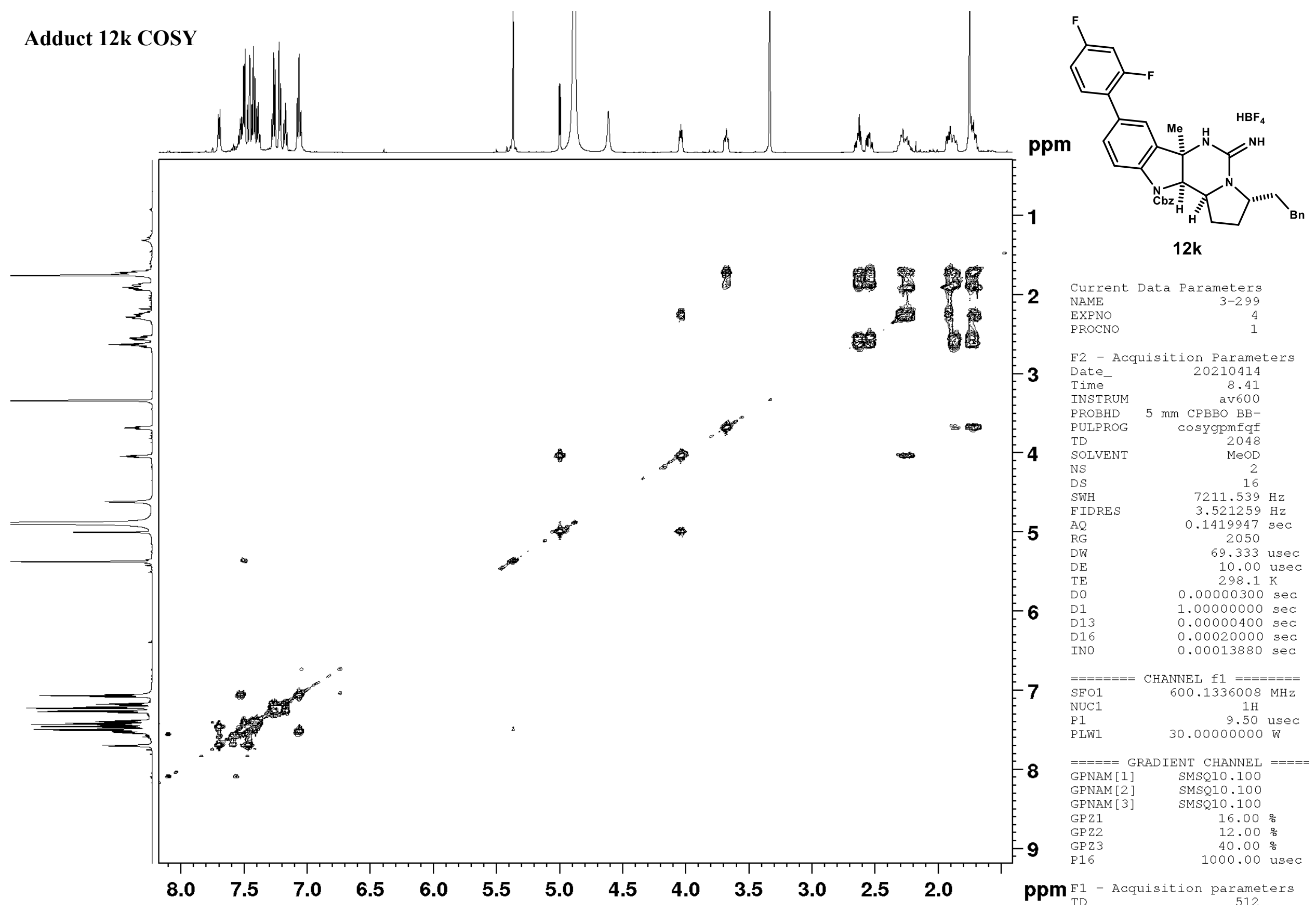




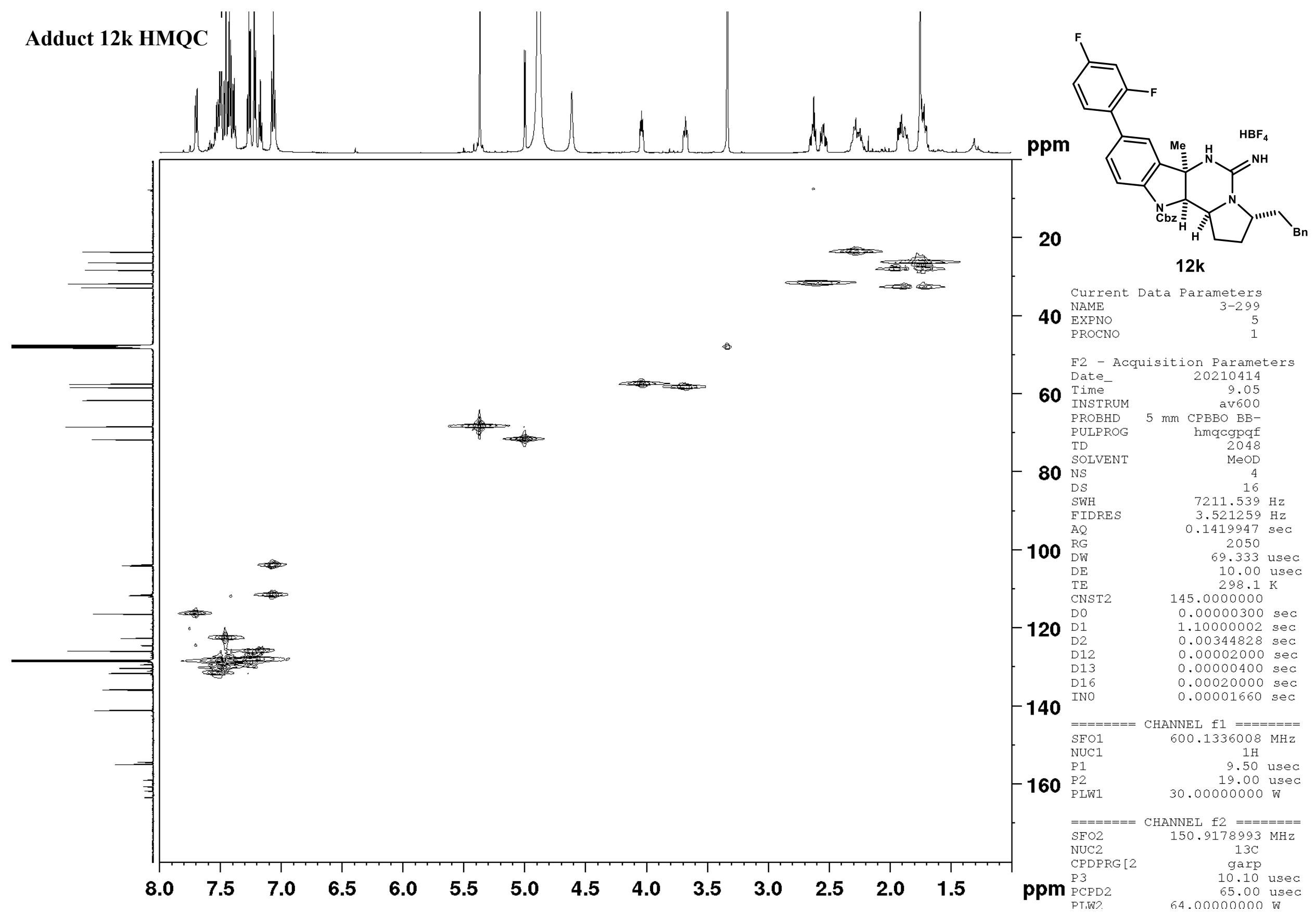




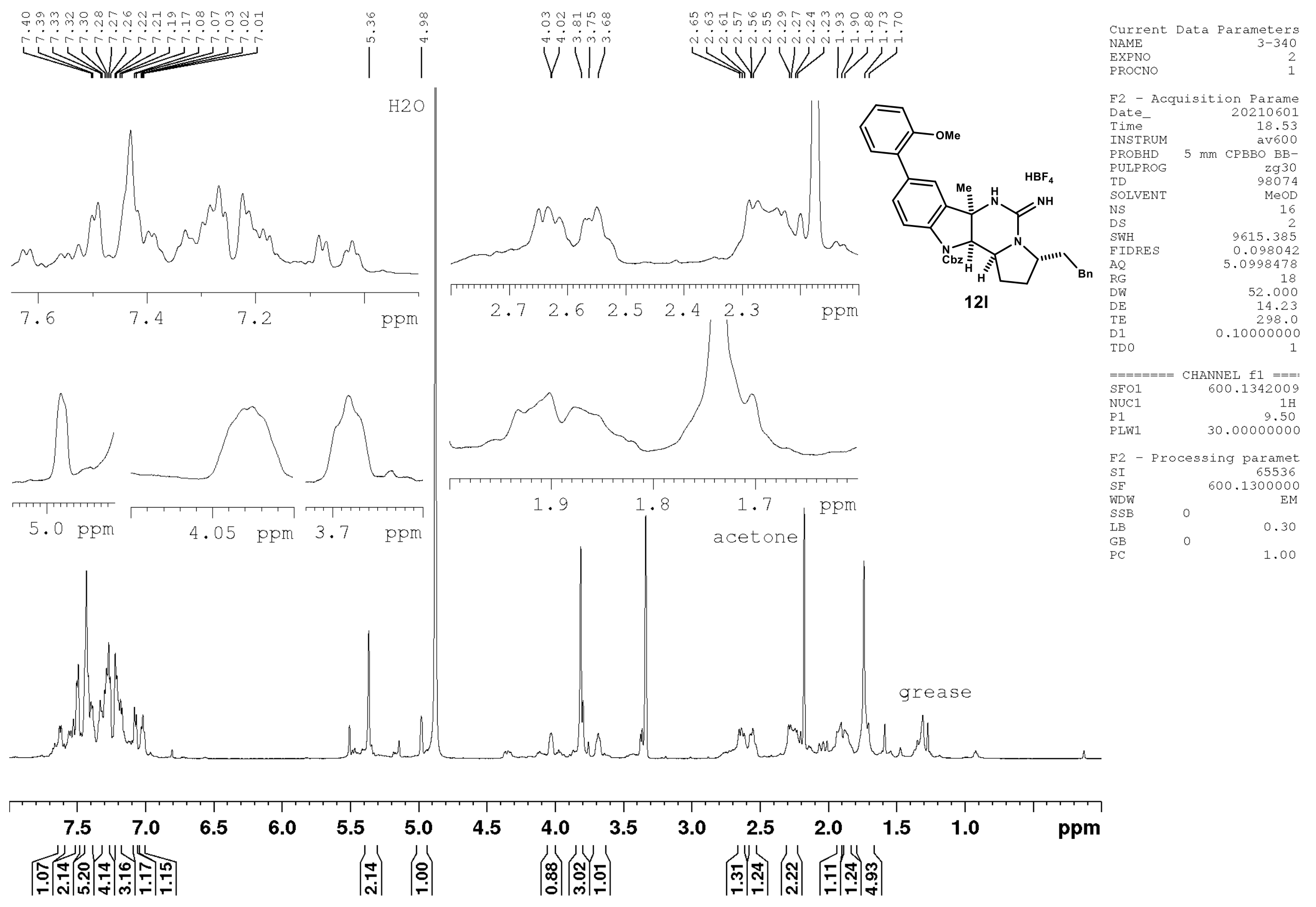




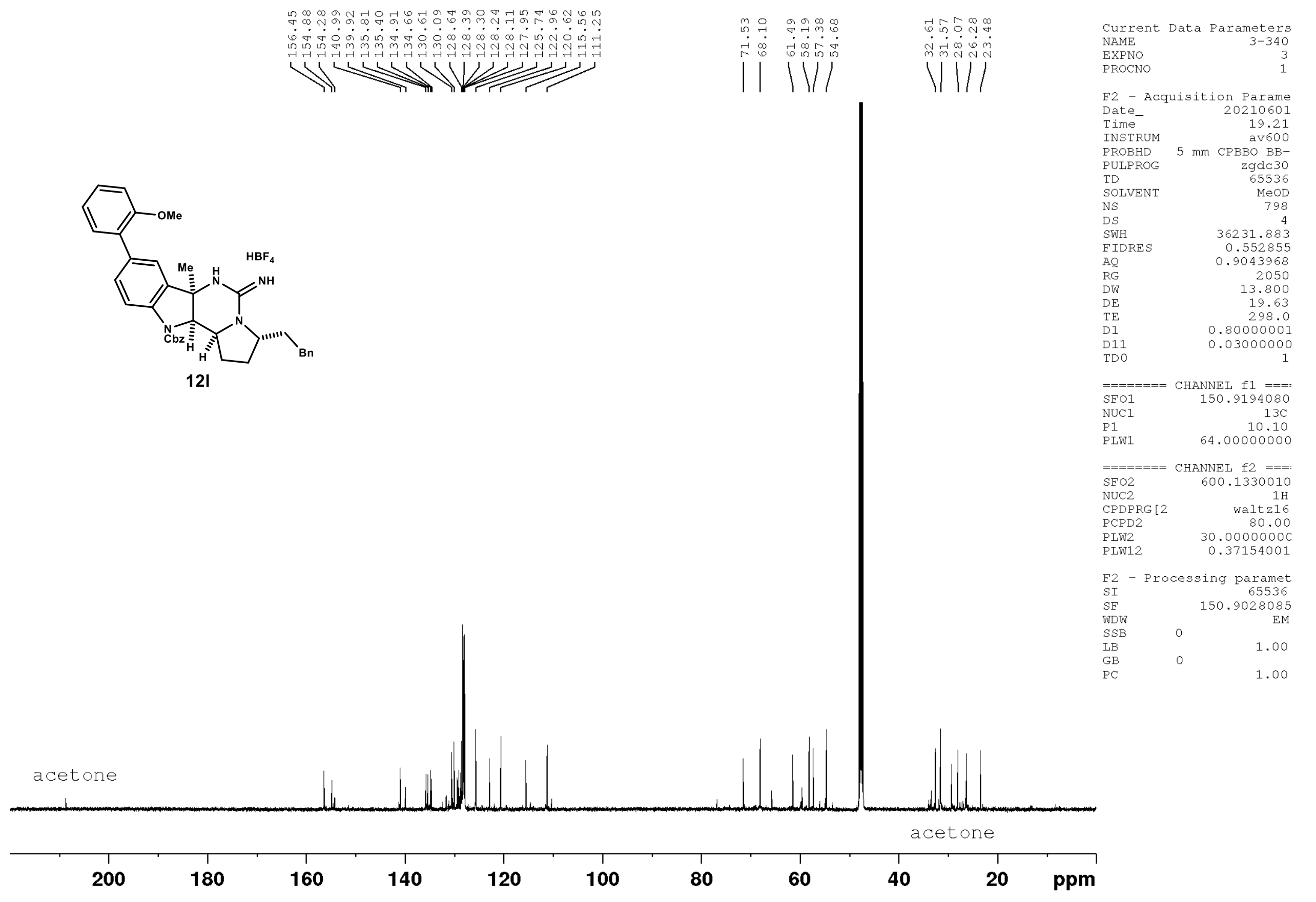




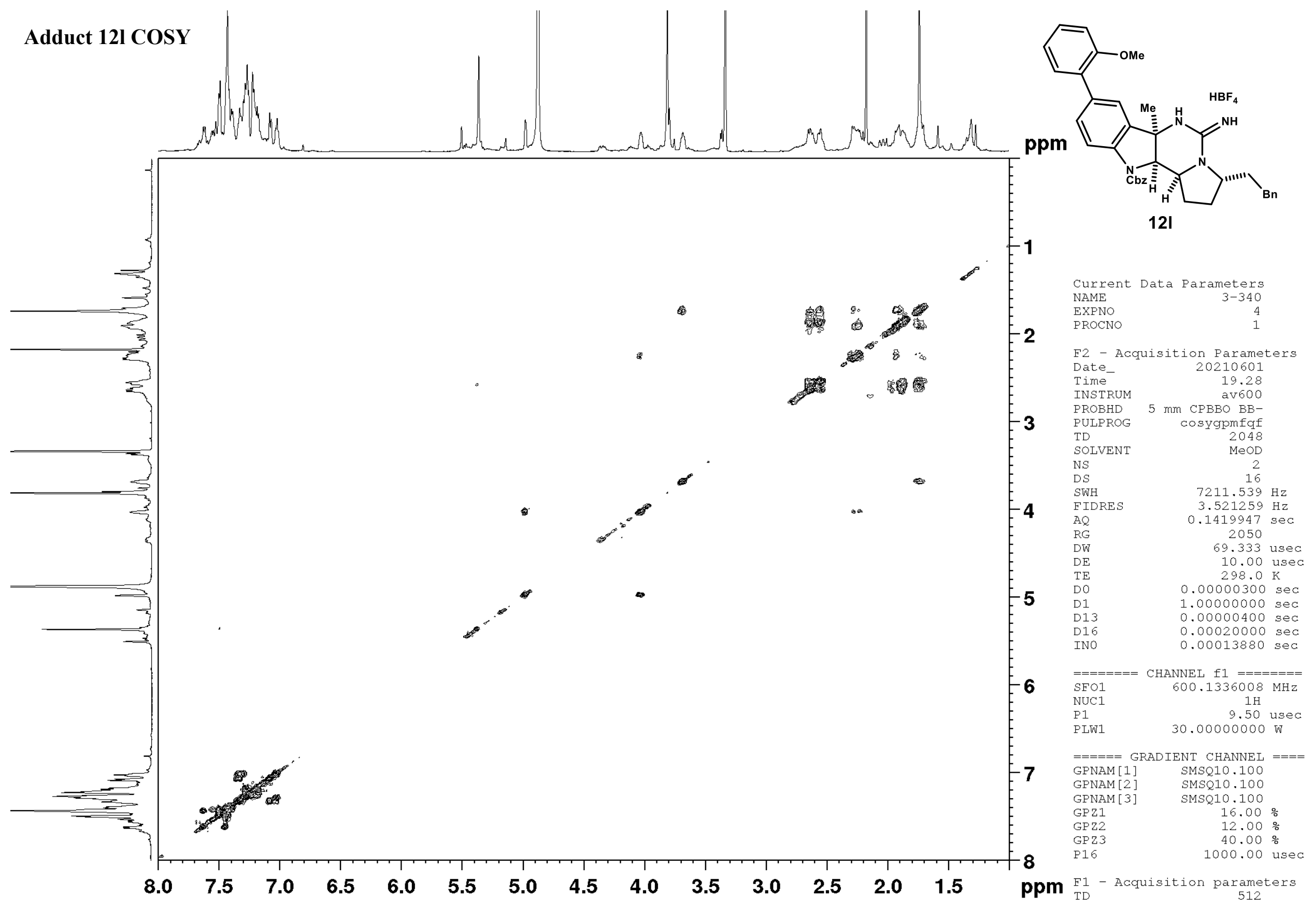




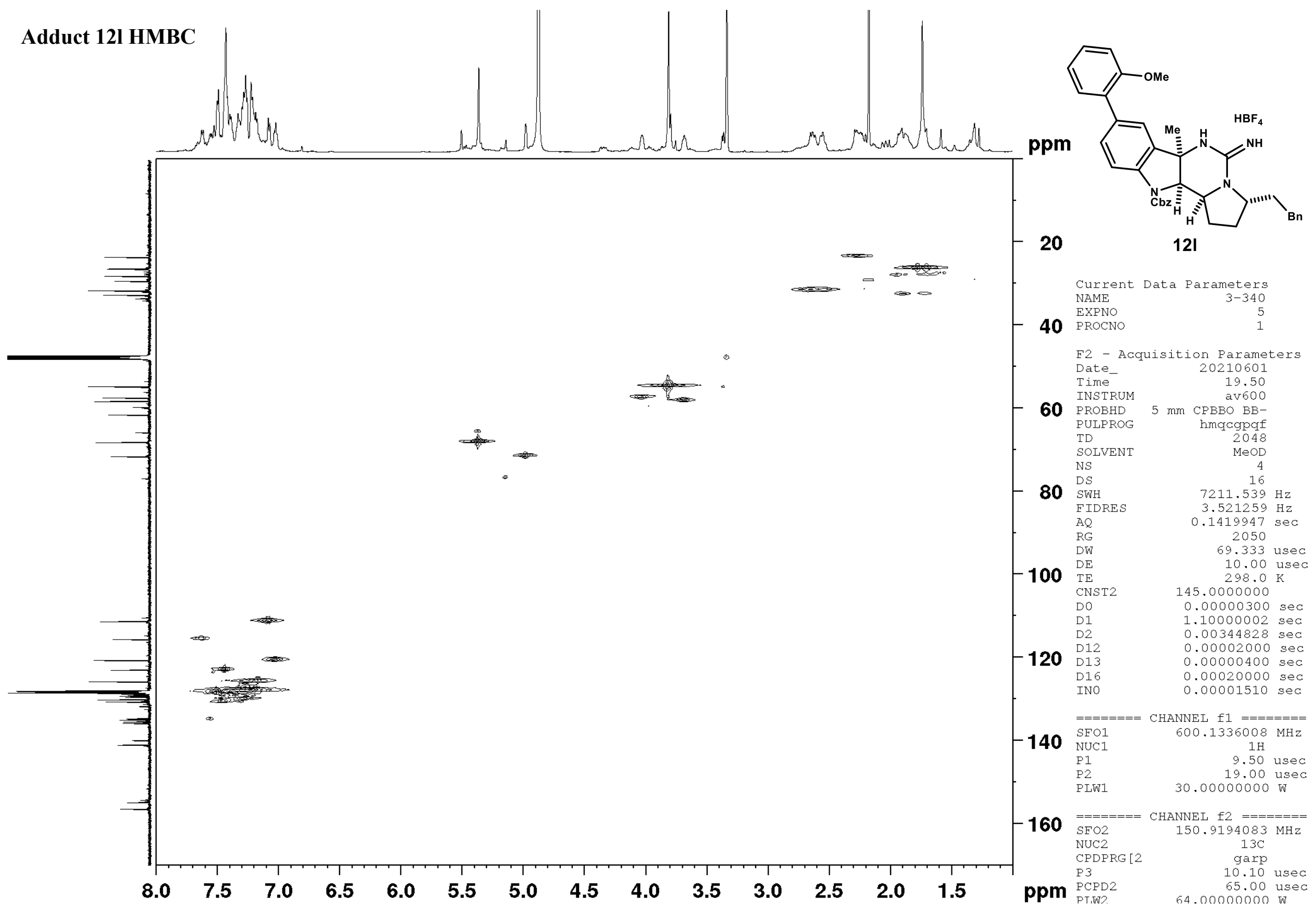

S177 

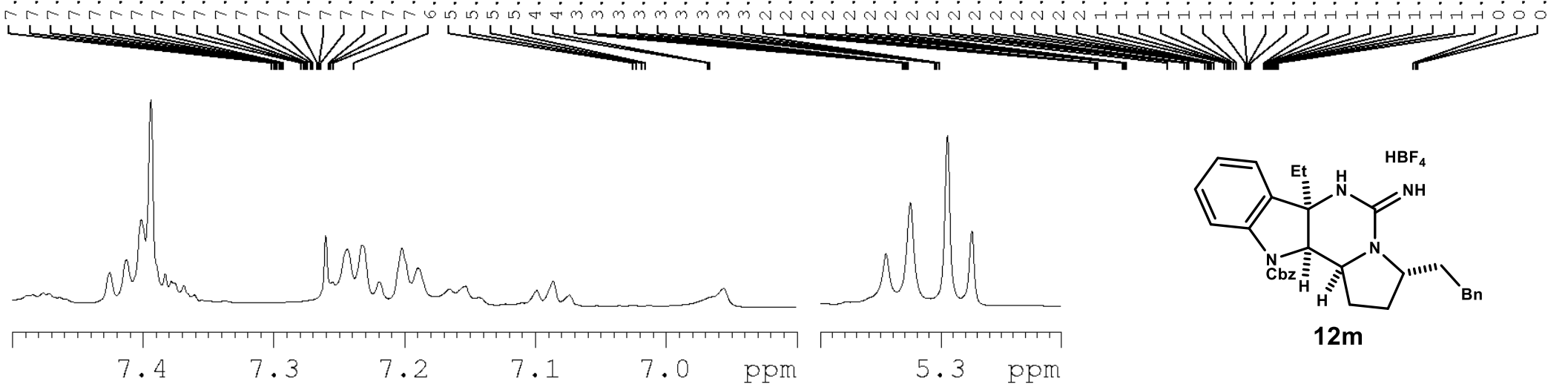

F2 - Acquisition Parame ton Parame $\begin{array}{lr}20201229 \\ \text { Time } & 11.11\end{array}$ $5 \mathrm{~mm}$ CPBBO $\mathrm{BB}-$ PULPROG $\quad$ zg30 TD 98074 SOLVENT NS DS FIDRES $\quad 9615.385$ FIDRES $\quad 0.098042$ $\begin{array}{rr}\text { AQ } & 5.0998478 \\ \text { RG } & 10\end{array}$ $\begin{array}{rr}\text { DW } & 52.000 \\ \text { DE } & 14.23\end{array}$ $\begin{array}{lr}\mathrm{DE} & 14.23 \\ \mathrm{TE} & 298.0\end{array}$
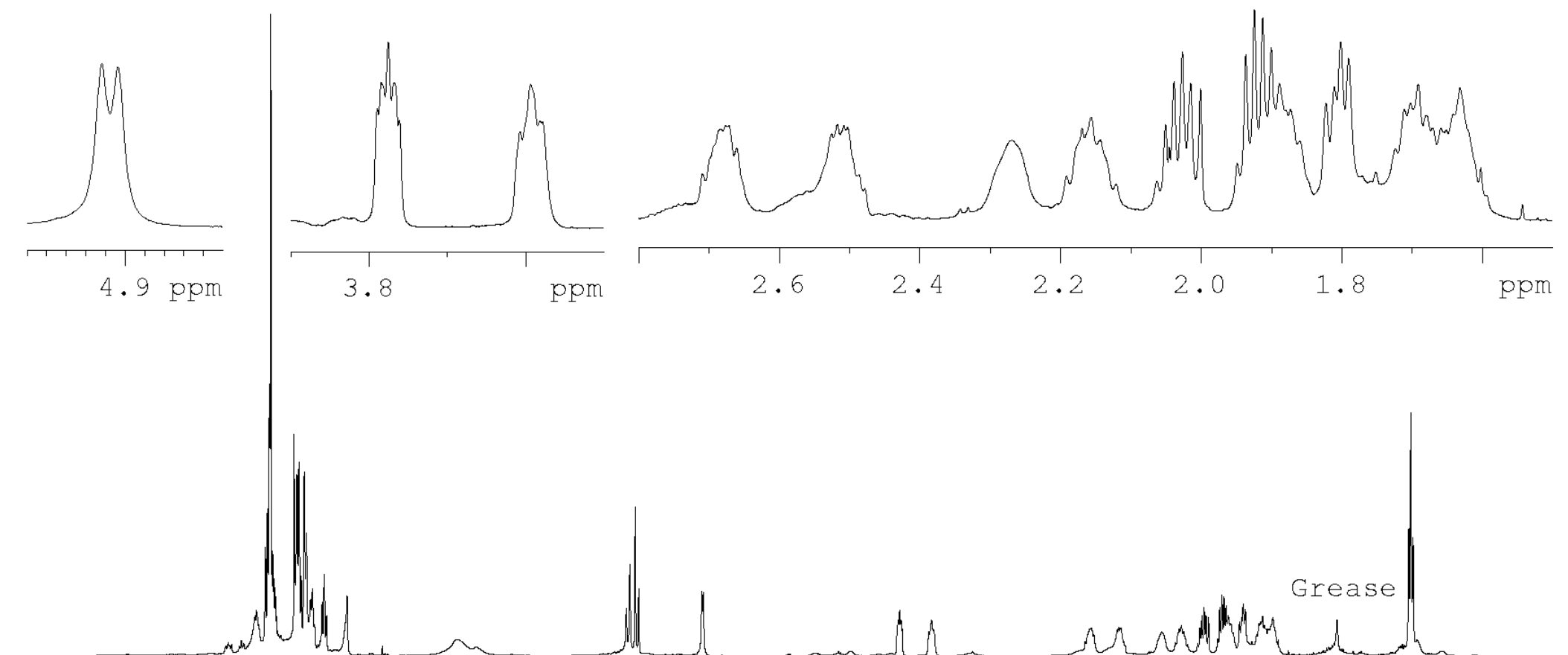
TDO

0.10000000
1
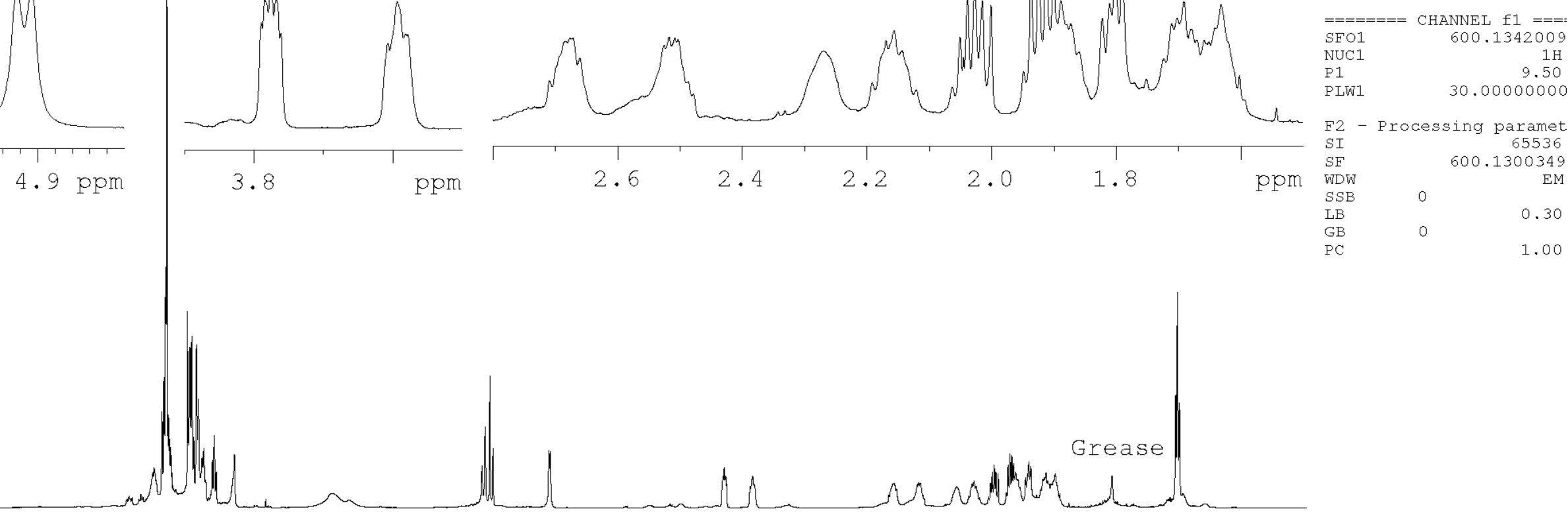

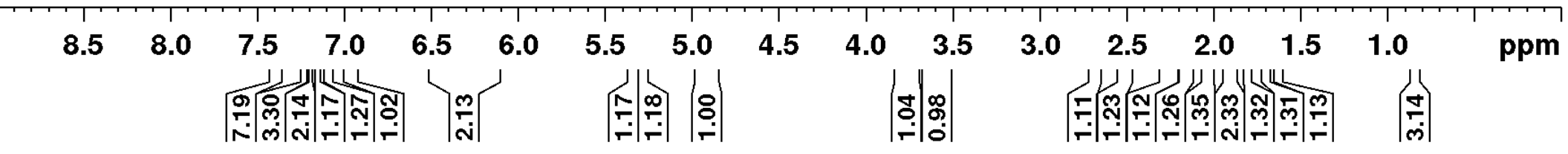




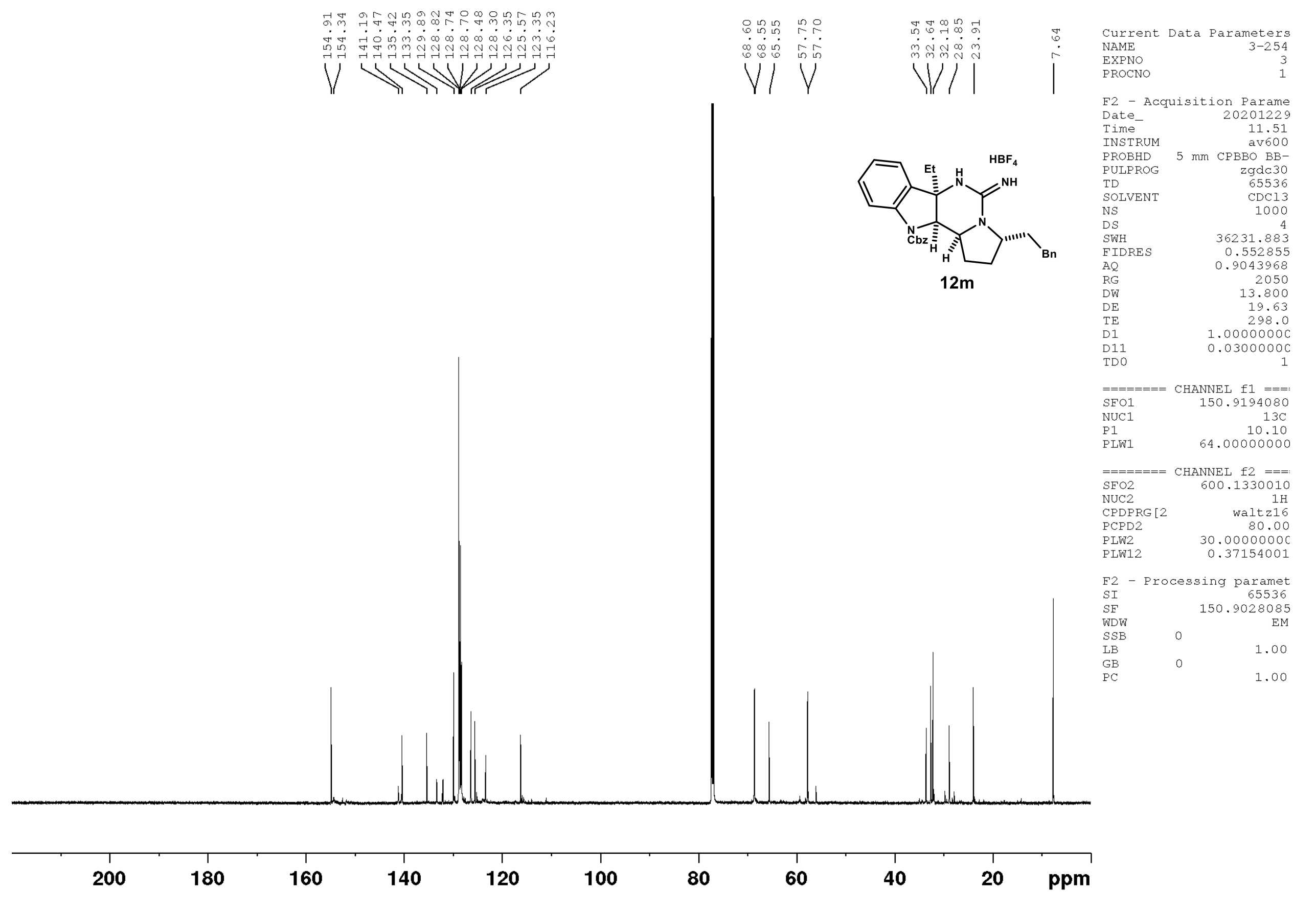




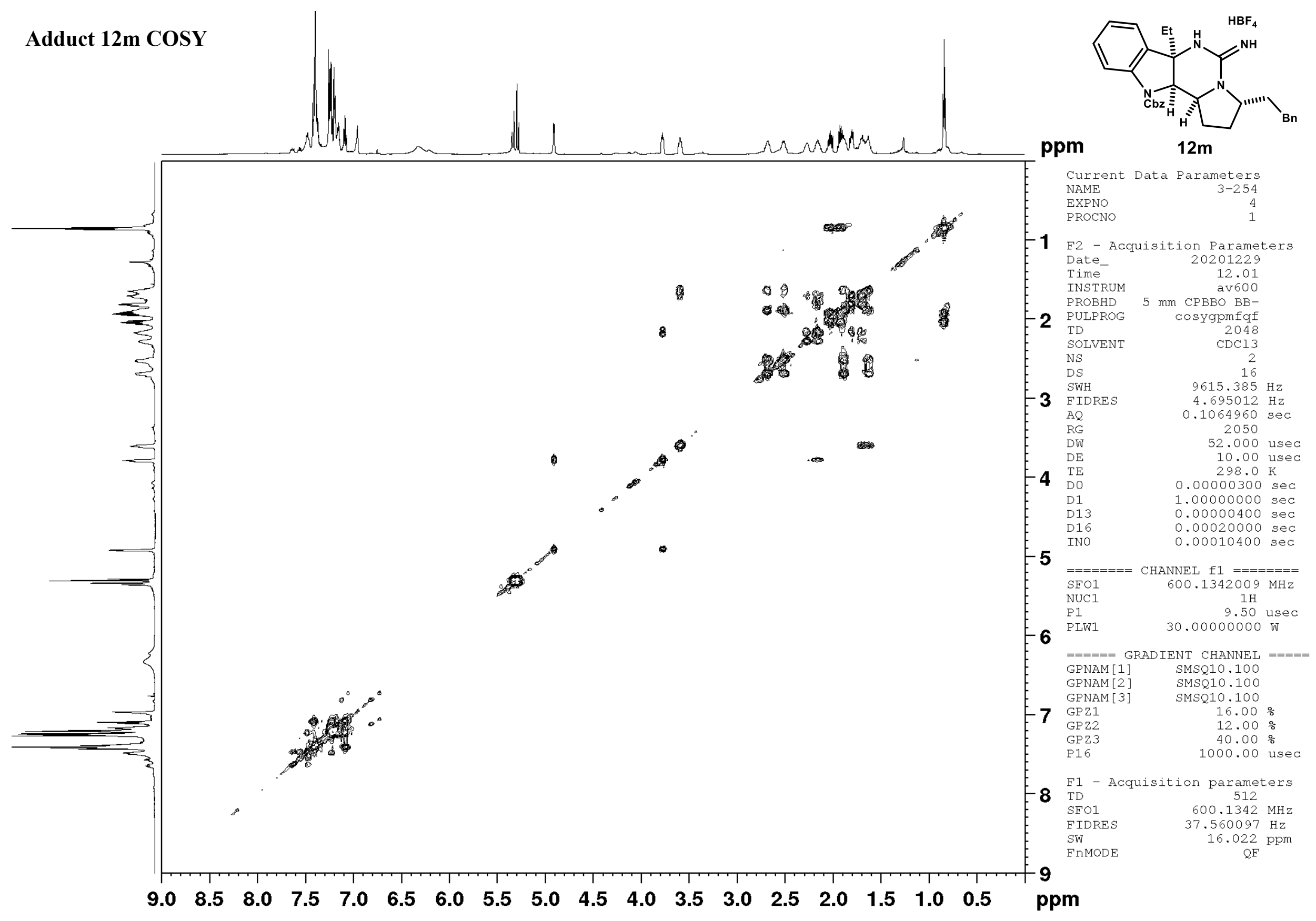




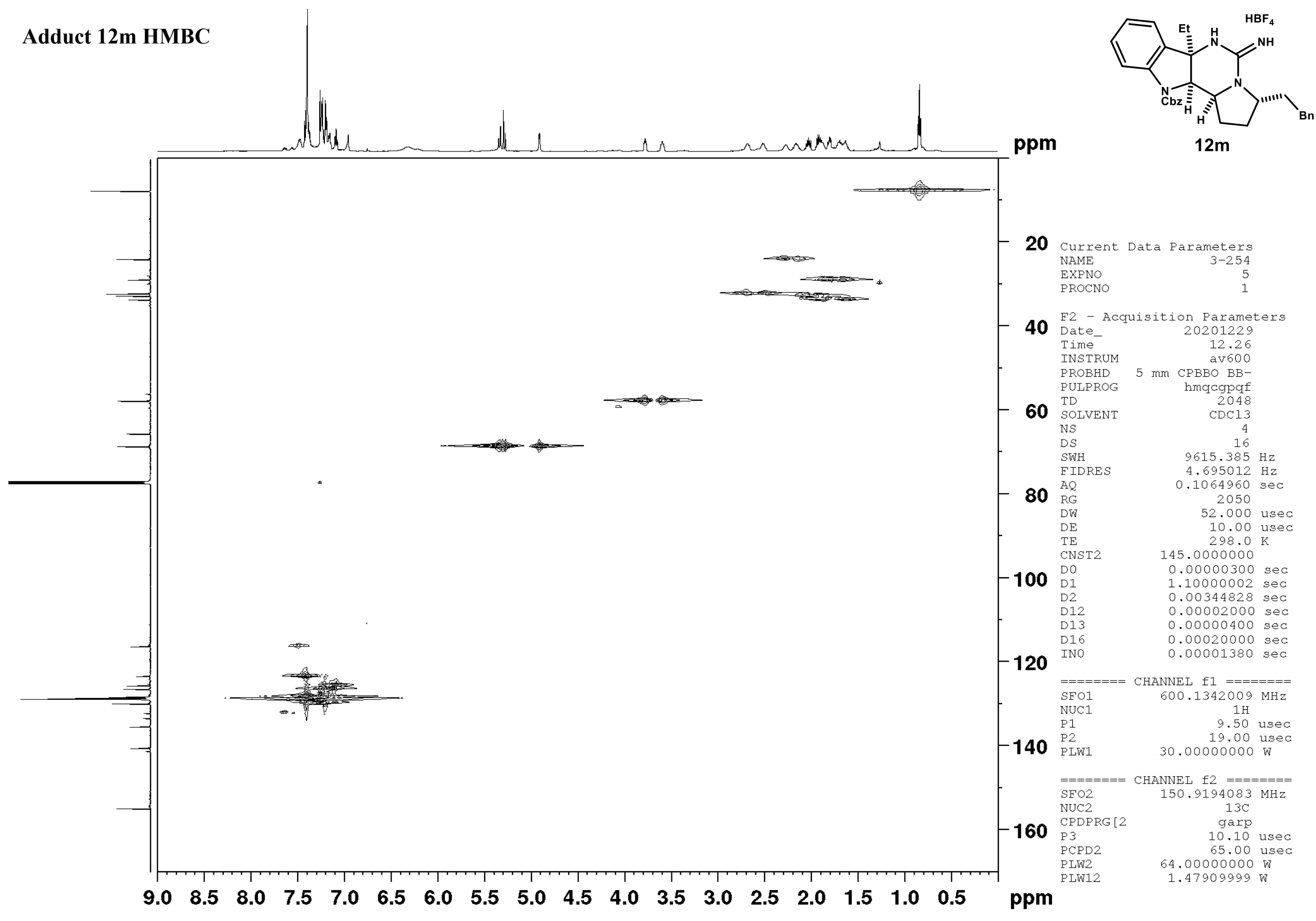



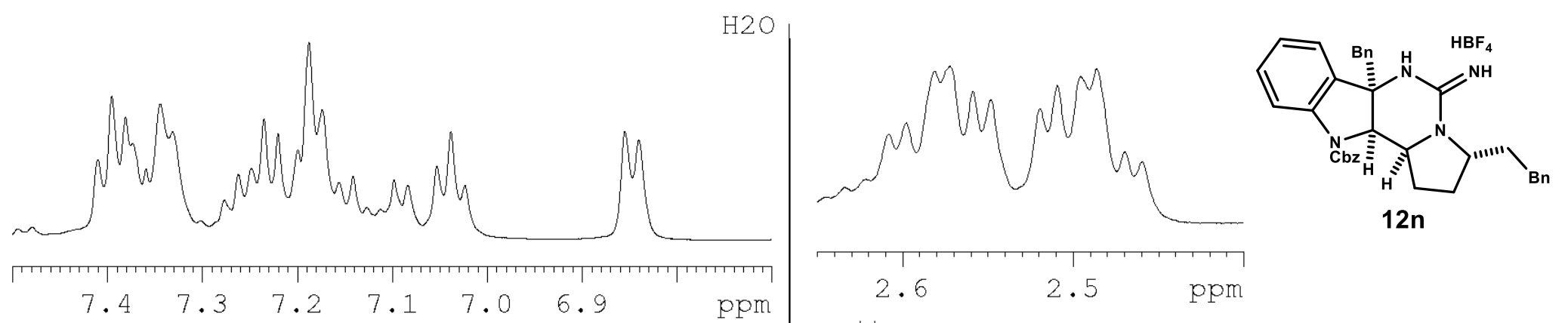

F2 - Acquisition Parame Date_r 20130620

INSTRUM $5 \mathrm{~mm}$ CPTCT $1 \mathrm{H}-$ PULPROG TD

SOLVENT

NS
DS
SWH

$\begin{array}{ll}0 \\ \text { SWH } & 8012.820\end{array}$ $\begin{array}{lr}\text { FIDRES } & 0.098043 \\ \text { AQ } & 5.0998273 \\ \text { RG } & 5\end{array}$

$\mathrm{DW}$

TE

6.00

0.10000000 MCREST O sec

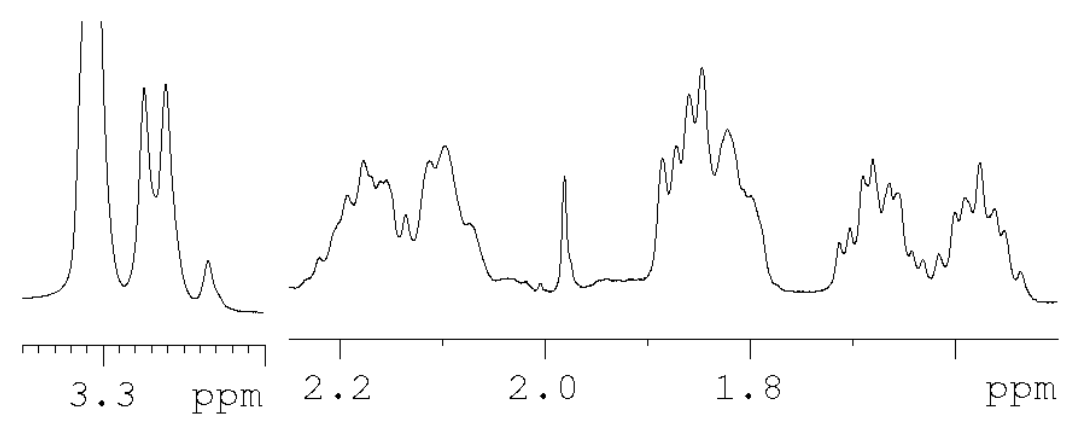

0.01500000

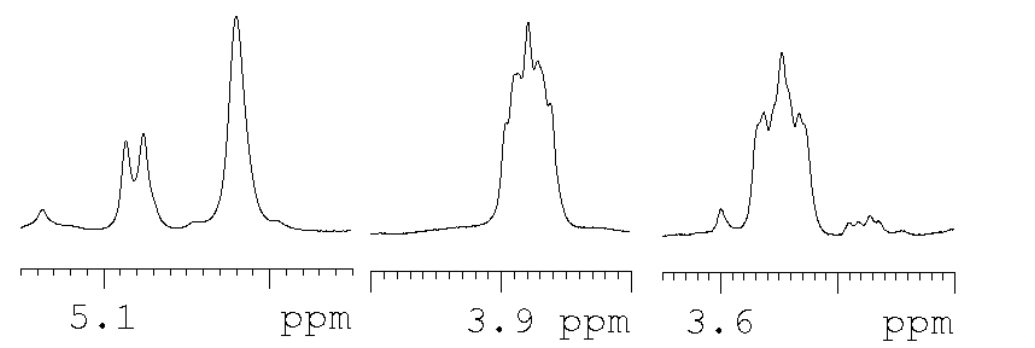

3.3 ppm 2.2

$====$

P1

SFO1 500.2235015

F2 - Processing paramet SI $\quad 65536$ DFW $\quad 500.2200189$
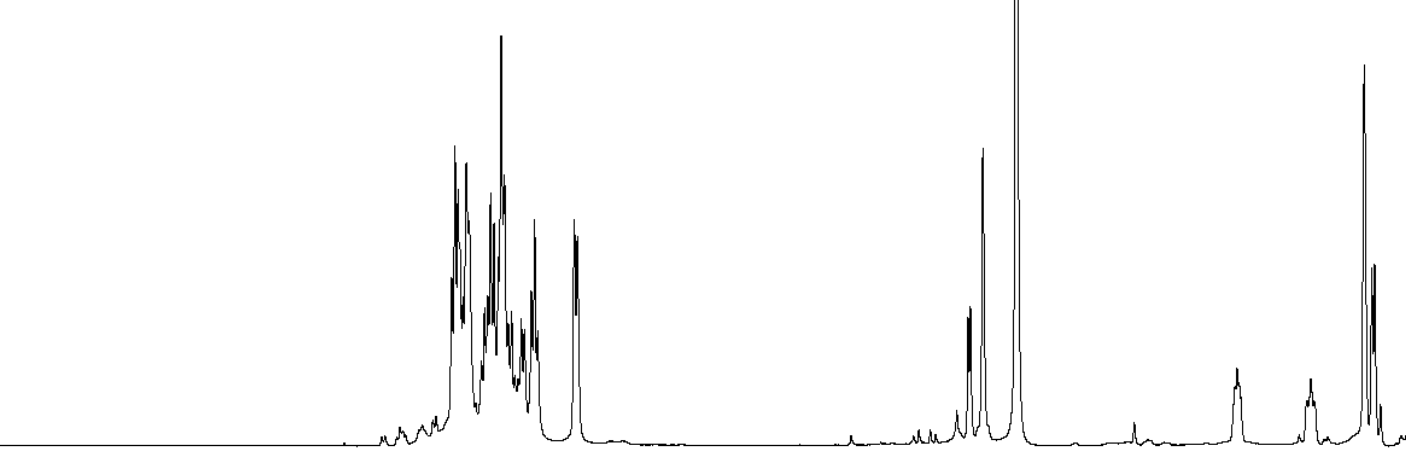

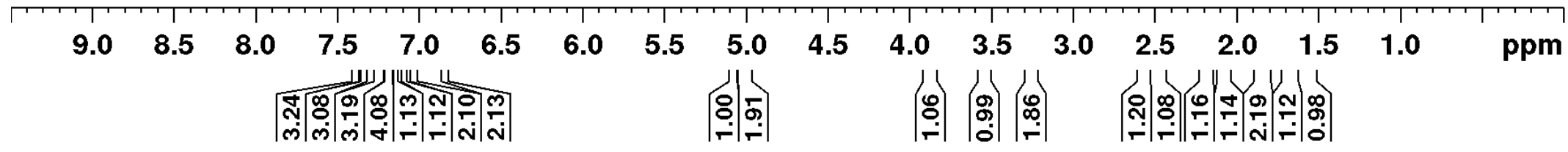




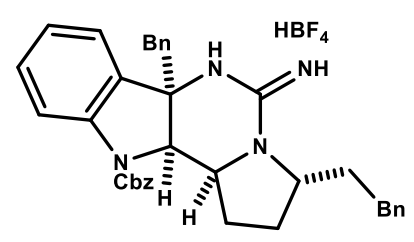

12n

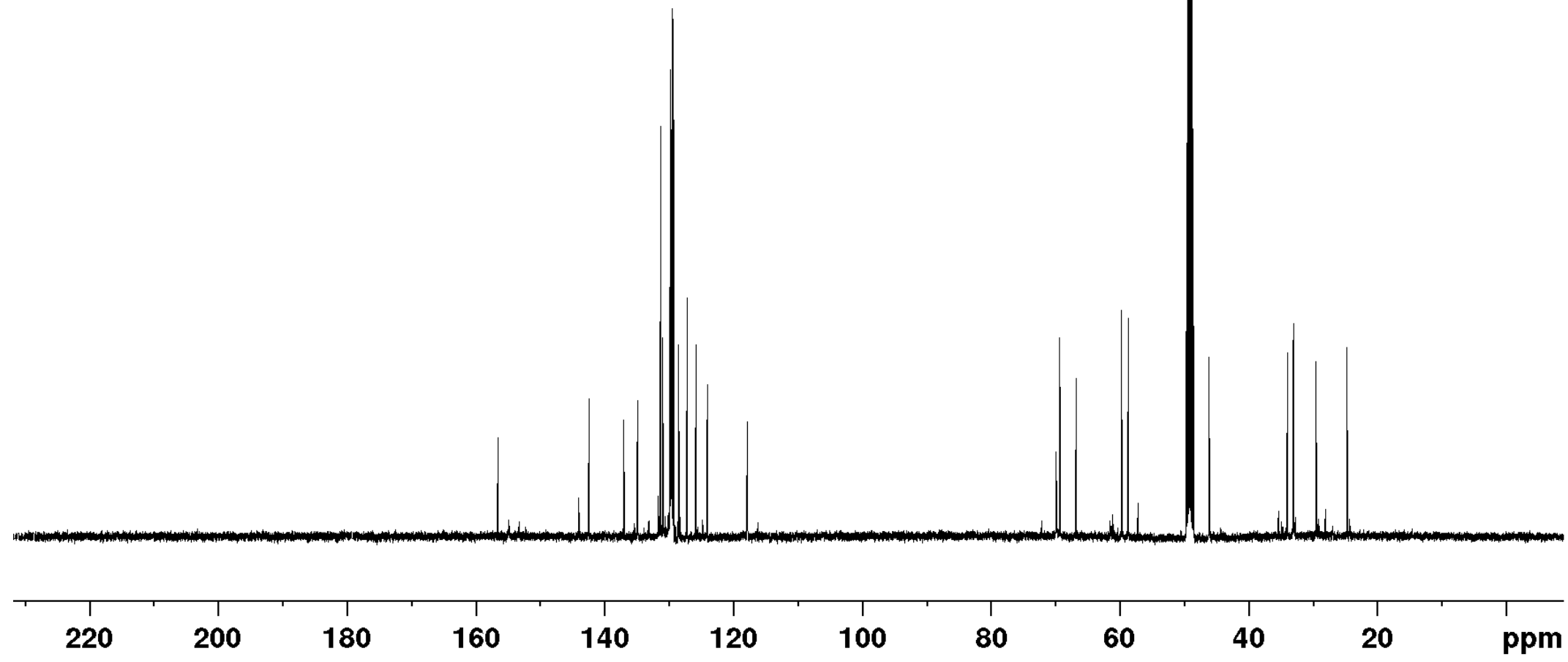

F2 - Acquisition Parame

Date__ 20130620

Time

INSTRUM cryo500

PROBHD $5 \mathrm{~mm}$ CPTCI $1 \mathrm{H}-$

PULPROG SpinEchopg30gr

TD 65536

NS

DS

SWH $\quad 30303.031$

FIDRES

AQ $\quad 1.0813440$

$\begin{array}{ll}\text { RG } & 7298.2 \\ \text { DW } & 16.500\end{array}$

$\begin{array}{lr}\mathrm{DE} & 6.00 \\ \mathrm{TE} & 298.0\end{array}$

$\begin{array}{lr}\text { TE } & 298.0 \\ \text { D1 } & 0.25000000\end{array}$

$\begin{array}{ll}\text { D1 } & 0.25000000 \\ \text { d11 } & 0.03000000\end{array}$

D16 $\quad 0.00020000$

MCREST O 0.00019600

$\begin{array}{ll}\text { MCREST } & \text { Sec } \\ \text { MCWRK } & 0.01500000\end{array}$

P2

31.00

$=======$ CHANNEL $\mathrm{f} 1===$ :

$\begin{array}{lr}\text { NUC1 } & 13 \mathrm{C} \\ \mathrm{P} 1 & 15.50\end{array}$

$\begin{array}{lr}\text { P11 } & 15.50 \\ \text { P12 } & 500.00\end{array}$

P12 2000.00

PLO $\quad 120.00$

$\begin{array}{lr}\text { PLI } & -1.00 \\ & 125.7942548\end{array}$

$\begin{array}{lr}\text { SP1 } & 125.7942548 \\ \text { SP1 } & 3.20\end{array}$

SP2 $\quad 3.20$

SPNAM [1] Crp60,0.5,20.1

SPNAM [2] Crp60comp.4

SPOFF2 $0 \mathrm{~Hz}$

CHANNEL $\mathrm{f} 2===$

CPDPRG[2 waltz16

NUC2

$\mathrm{PL} 2$

$\mathrm{PL} 12$

100.00

24.60 


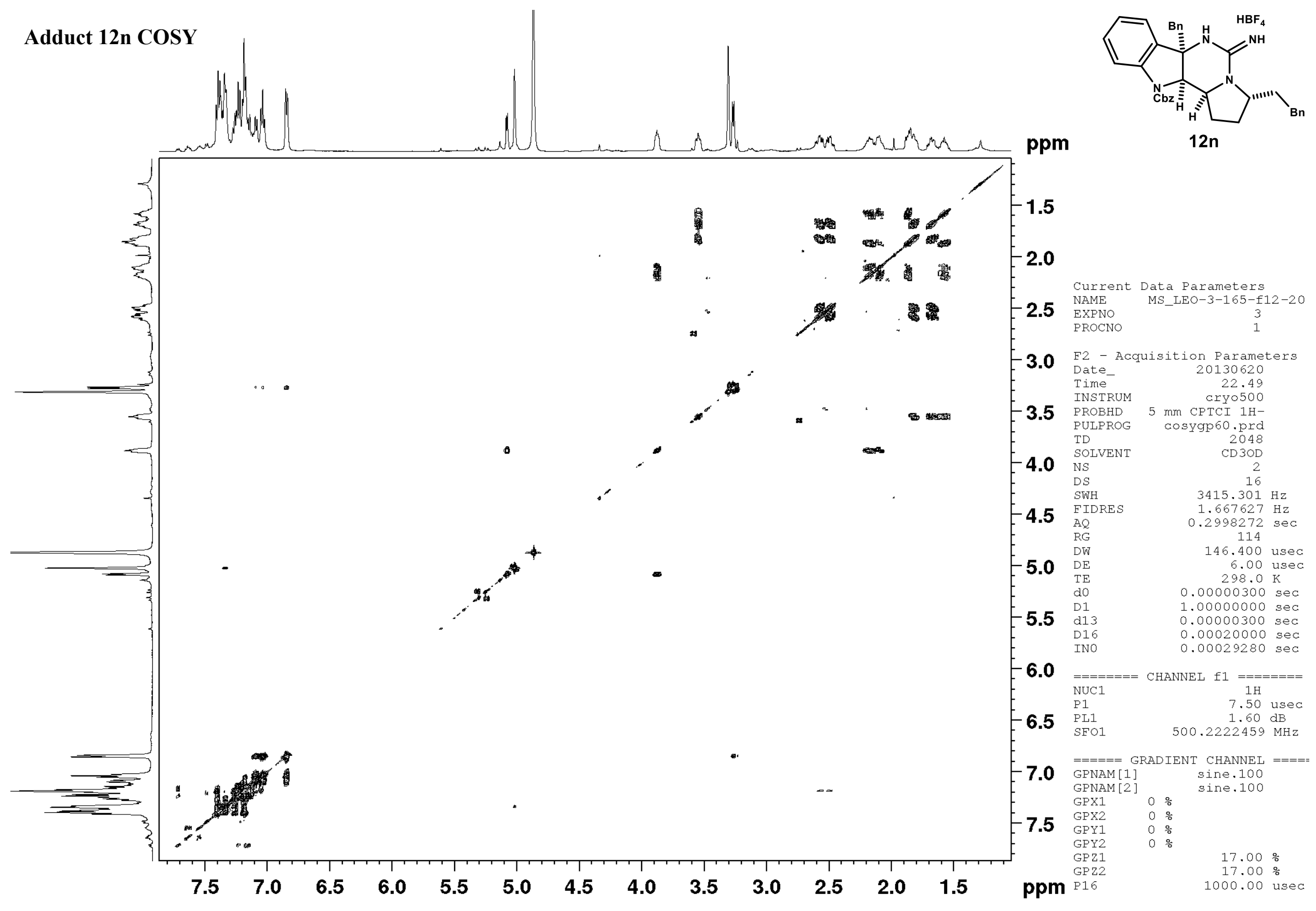




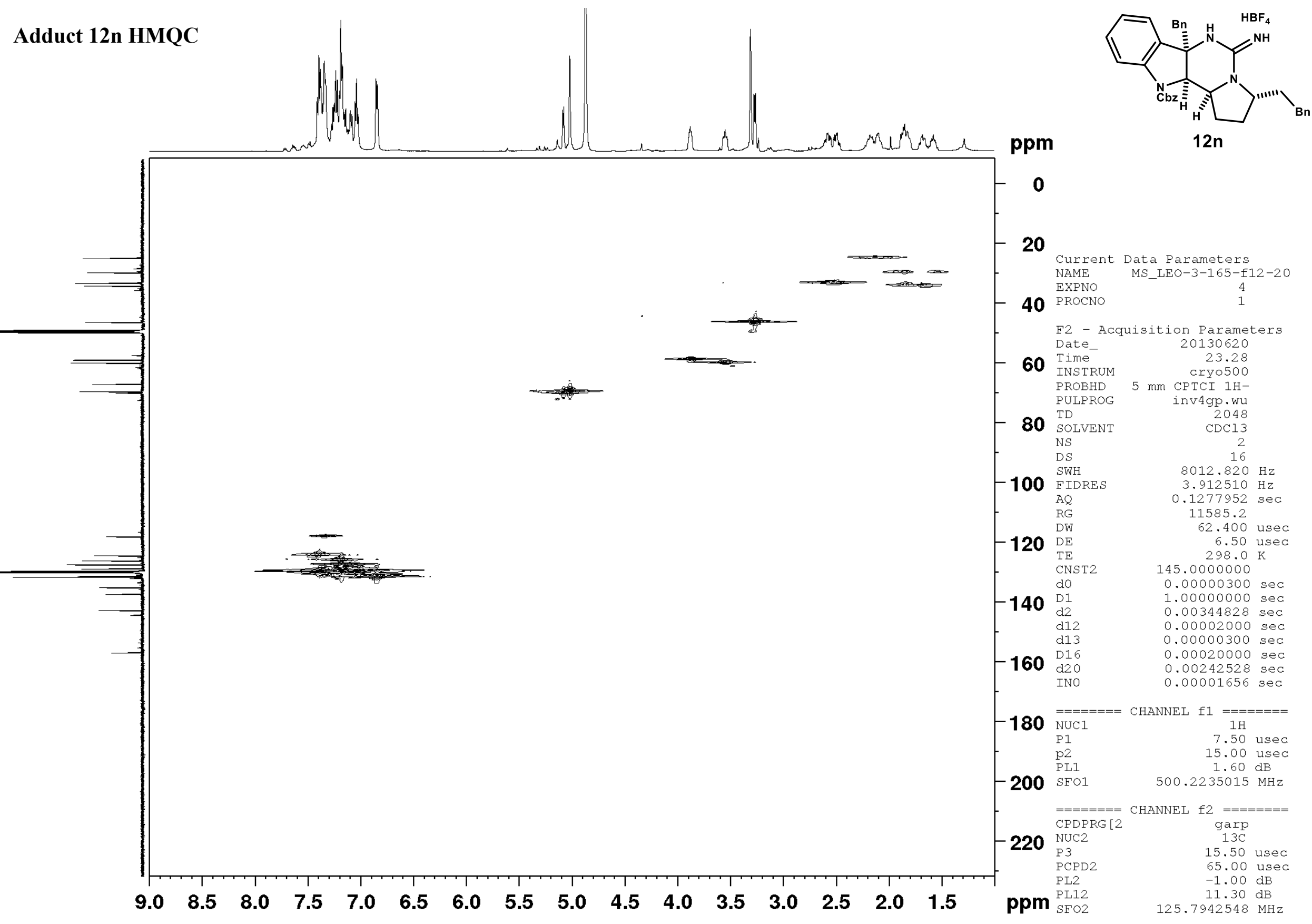




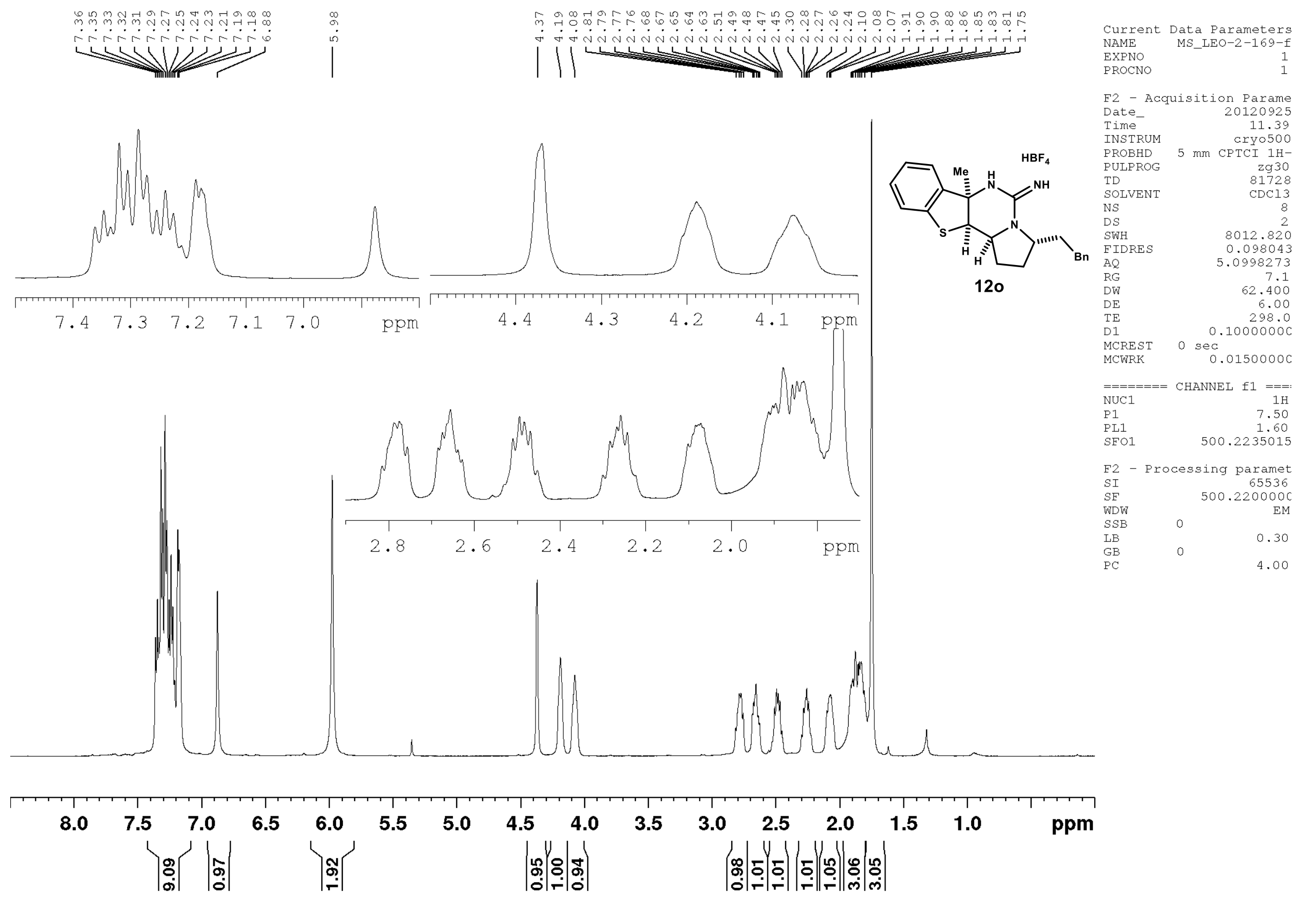




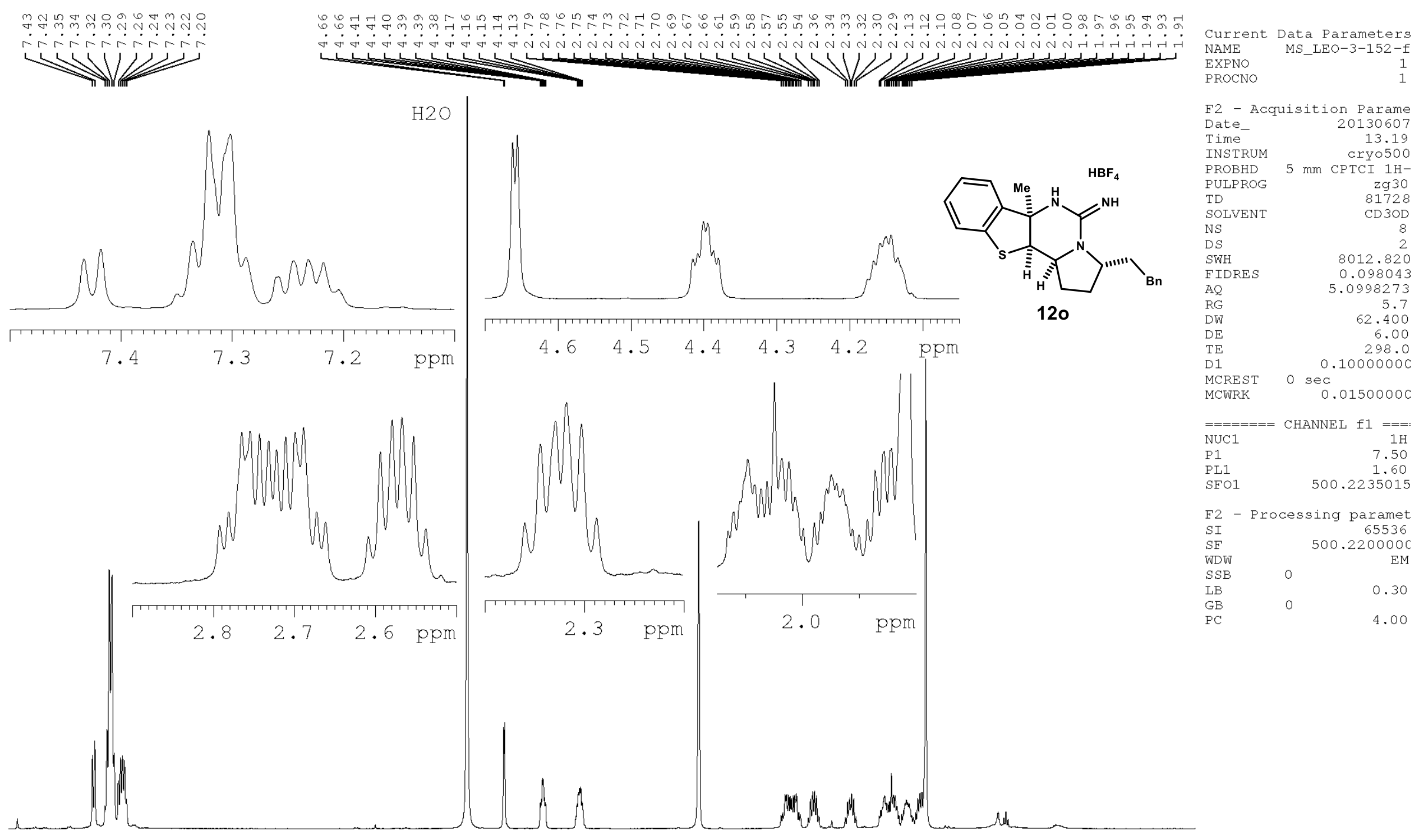

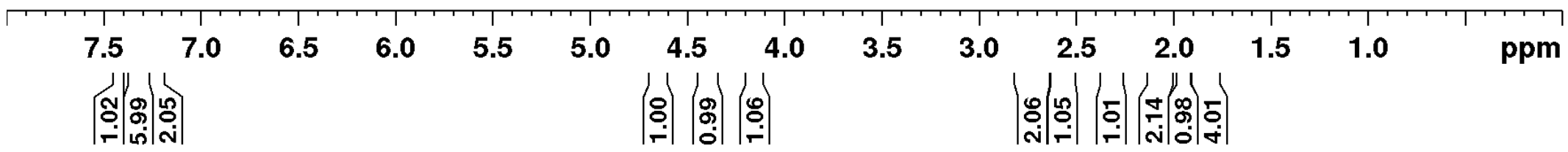




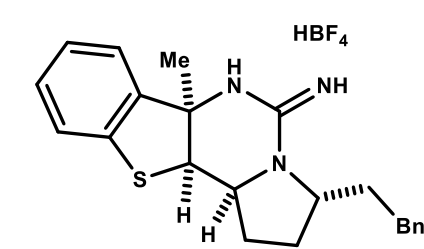

120
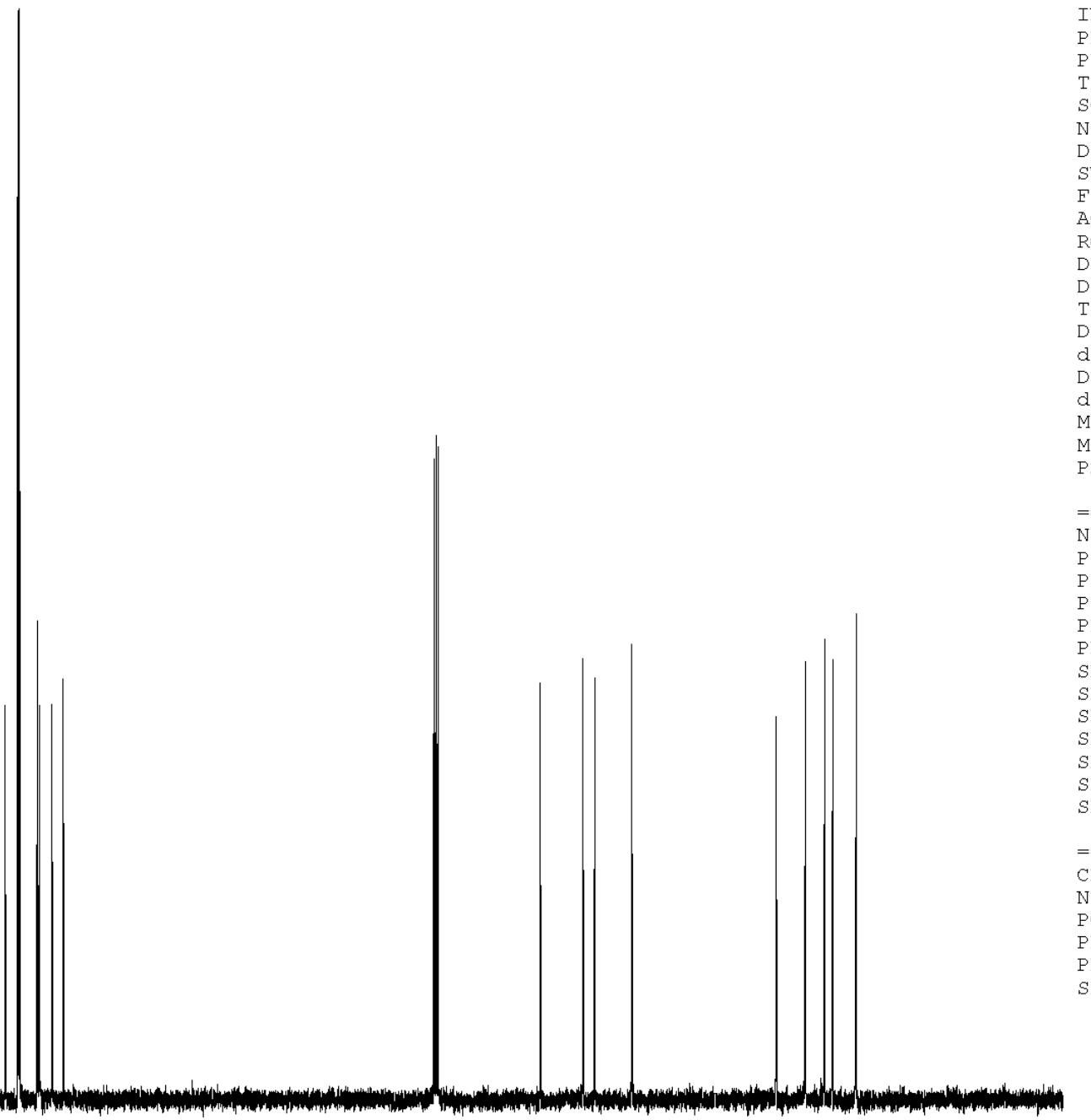

urrent Data Parameters

F2 - Acquisition Parame

$$
\text { Date }
$$

INSTRUM

PROBHD

PULPROG SPInTCh $1 \mathrm{H}-$

SOLVENT

NS

SWH

AQ $\quad 1.0813440$

7298.2
DW
DW

$\begin{array}{lr}\mathrm{DE} & 6.00 \\ \mathrm{TE} & 298.0\end{array}$

$\begin{array}{lr}\mathrm{TE} & 298.0 \\ \mathrm{D} 1 & 0.25000000\end{array}$

$\begin{array}{ll}\text { D1 } & 0.25000000 \\ \text { d11 } & 0.03000000\end{array}$

0.00020000

MCREST O sec 0.00019600

$\begin{array}{ll}\text { MCREST } & 0 \text { sec } \\ \text { MCWRK } & 0.01500000\end{array}$

P2

31.00

$=======$ CHANnEL $\mathrm{fl}===$ :

$\mathrm{P} 1$

120.00

-1.00
125.7942548

3.20
3.20

SPNAM [1] Crp60,0.5,20.1

SPNAM[2] Crp60comp.4

SPOFF1 0

$========$ CHANNEL $\mathrm{f} 2===$ CPDPRG[2 waltz16 NUC2 2

PCPD 2

$\mathrm{PL} 2$

$\mathrm{PL} 12$
$\mathrm{SFO} 2$

$1 \mathrm{H}$
100.00

1.60
24.60

500.2225011

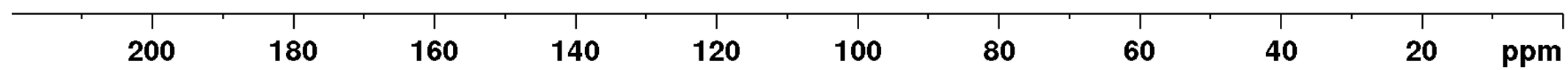




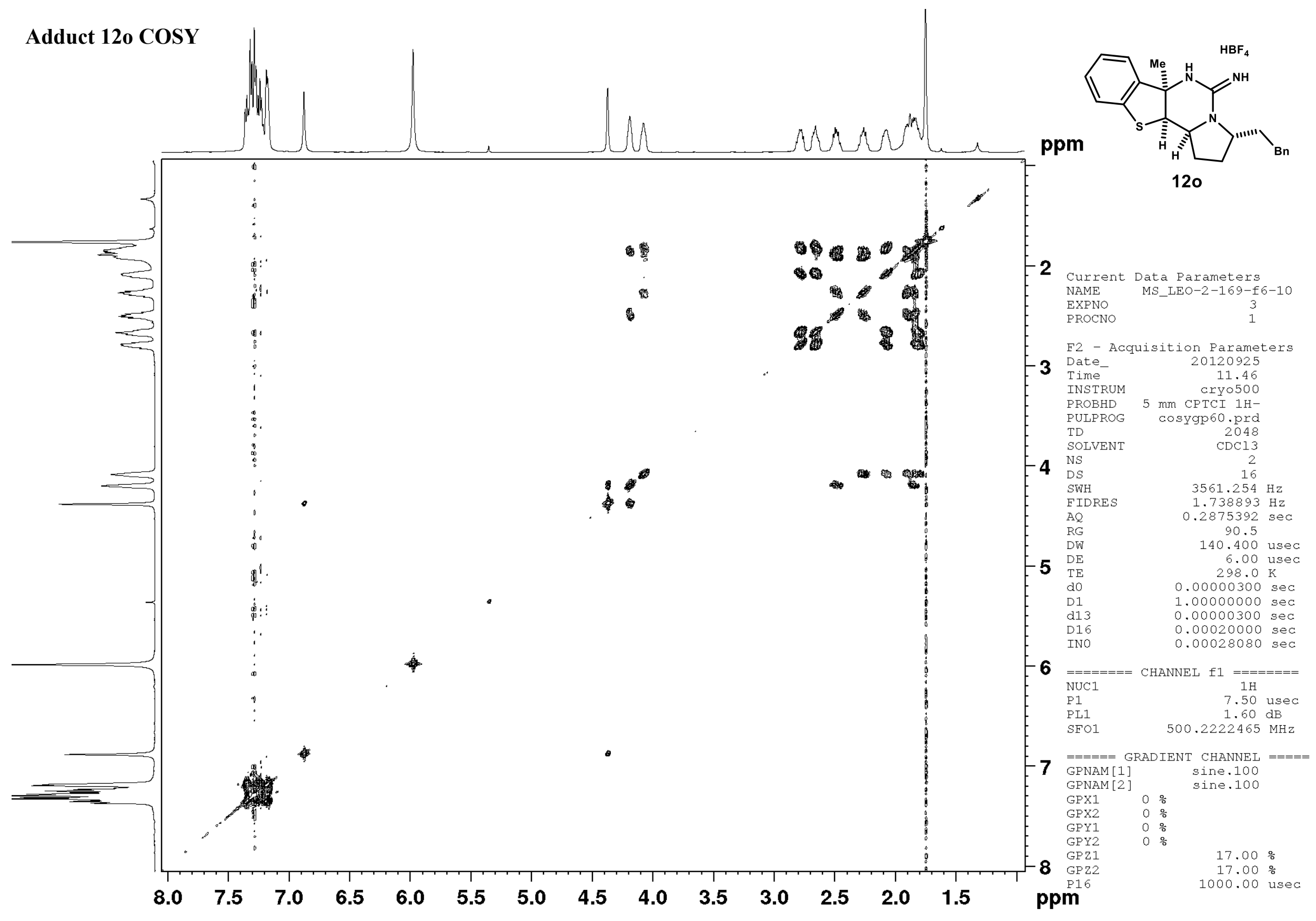




\section{Adduct 12o HMQC}

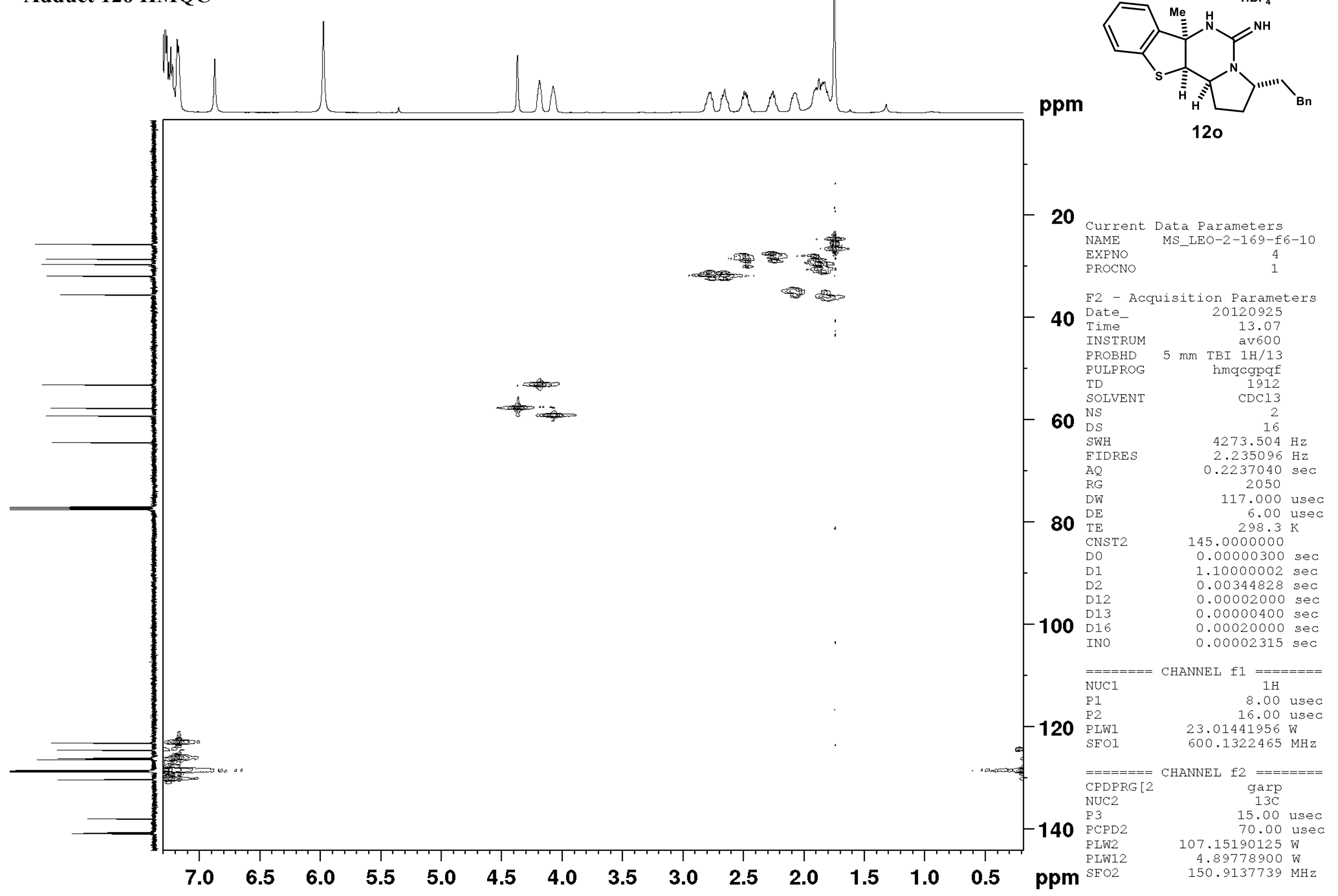




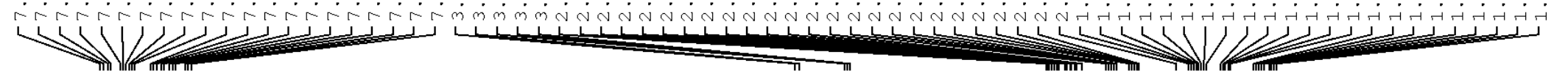

Urrent Data Parameters
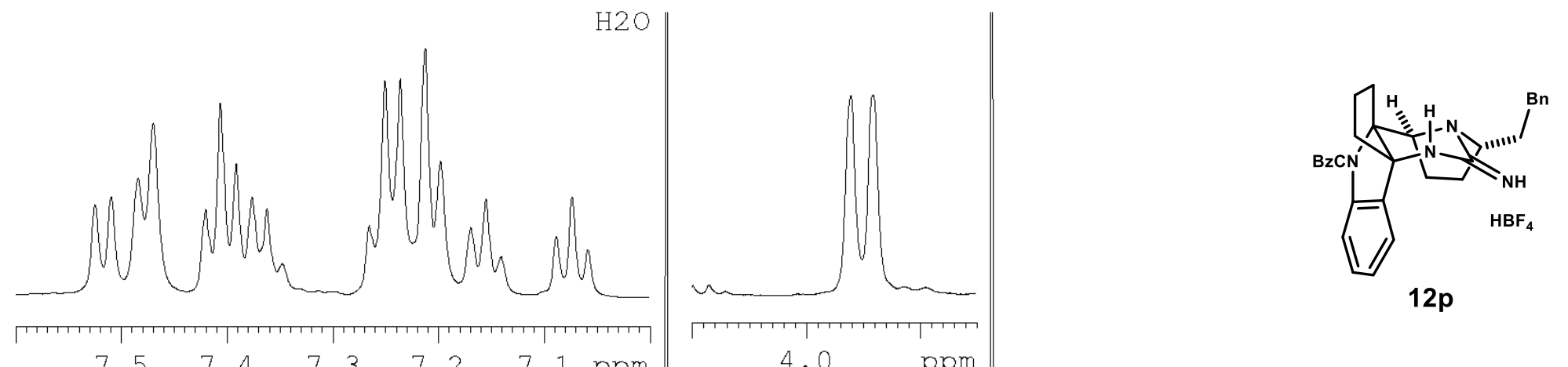

F2 - Acquisition Parame Date_ 20130610 22.48 PROBHD $5 \mathrm{~mm}$ CPTCI $1 \mathrm{H}$ $1 \mathrm{H}-$ P $\begin{array}{lr}\text { TD } & 81728 \\ \text { SOLVENT } & \text { CDCl3 }\end{array}$ NS

DS

SWH

FIDRE
AQ

AQ
DW

$\mathrm{DW}$

DE

MCREST 0 sec

MCWRK $\quad 0.01500000$

$=======$ CHANNEL $\mathrm{f1}===$ :

NUC1

P1

1.60
PFO1

F2 - Processing paramet

$\begin{array}{lr}\text { SI } & 65536 \\ \text { SF } & 500.2200223\end{array}$
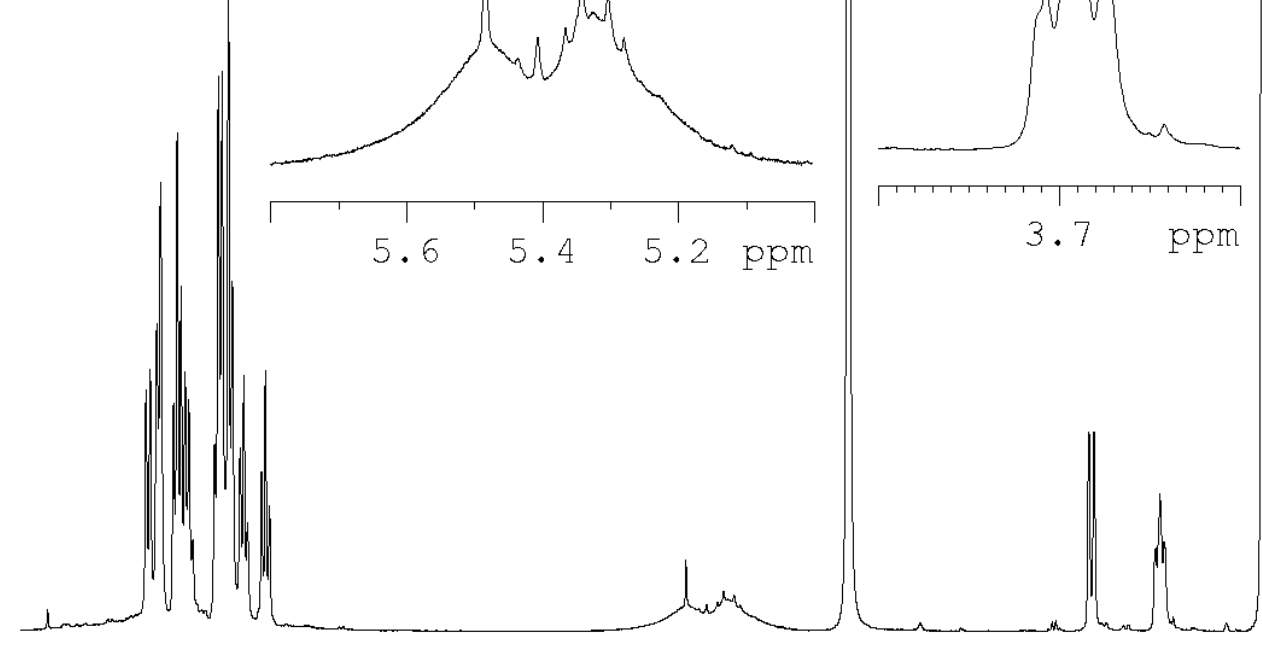

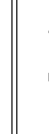

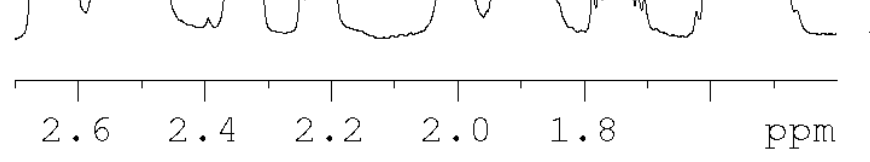

WSB

500.2200223
EM

3. 7 ppm

2.62

0.30

4.00

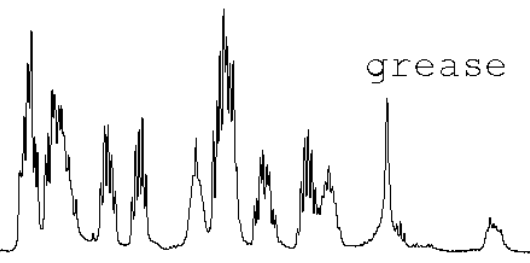

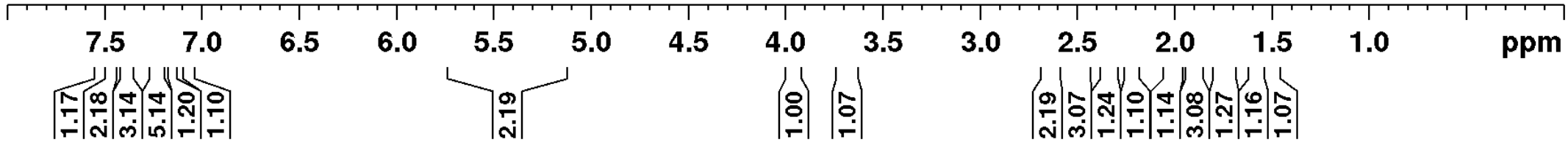




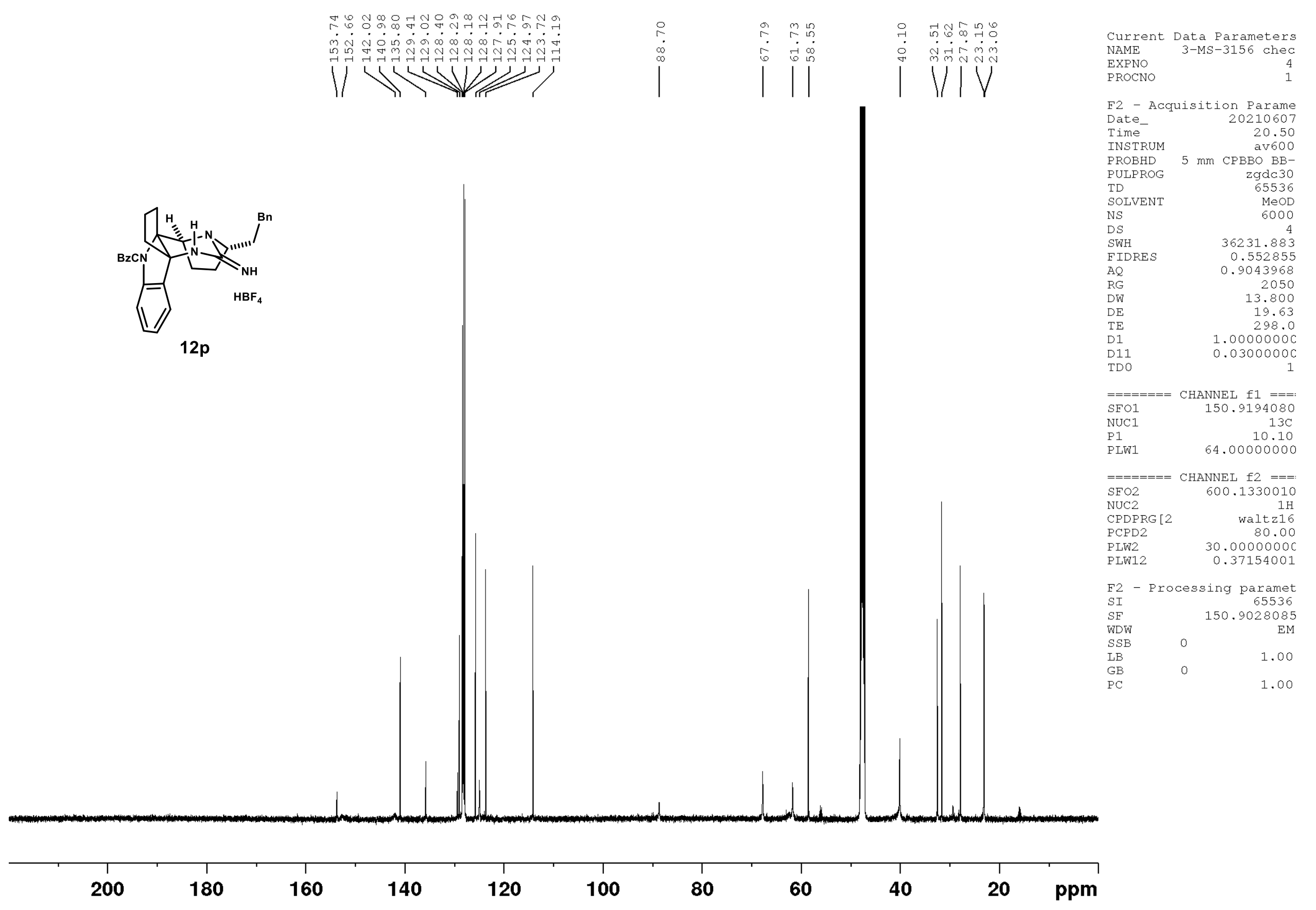




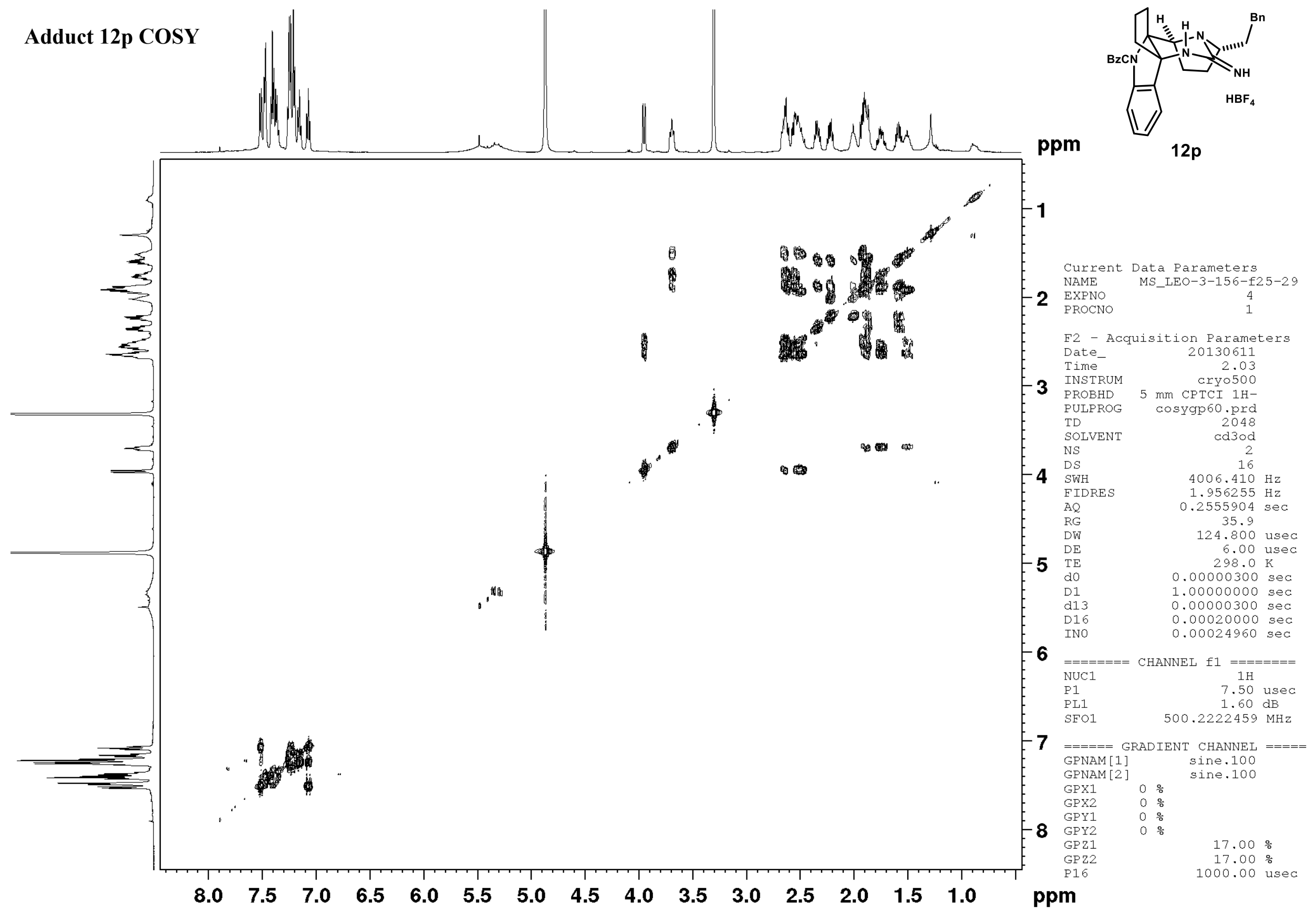




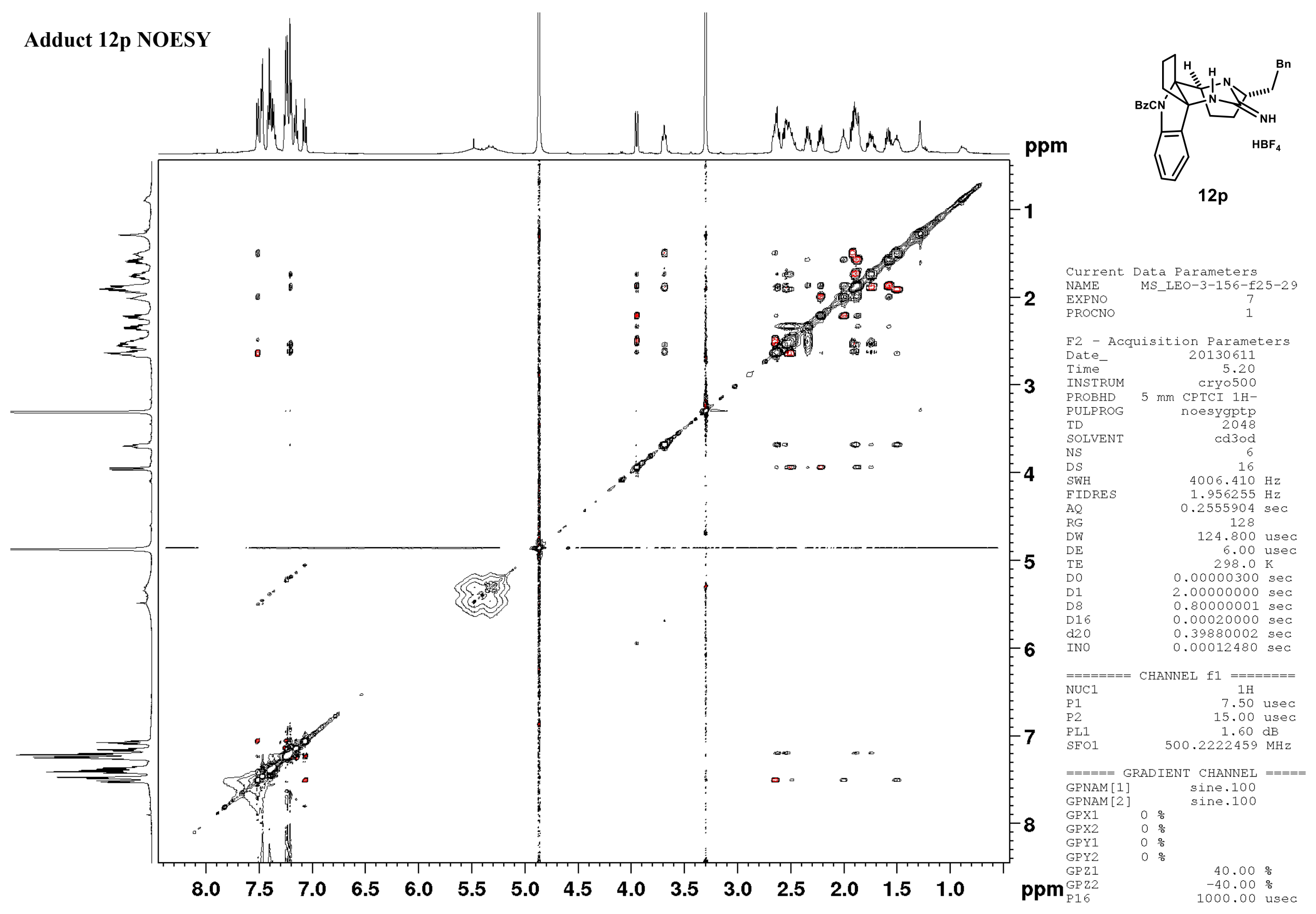




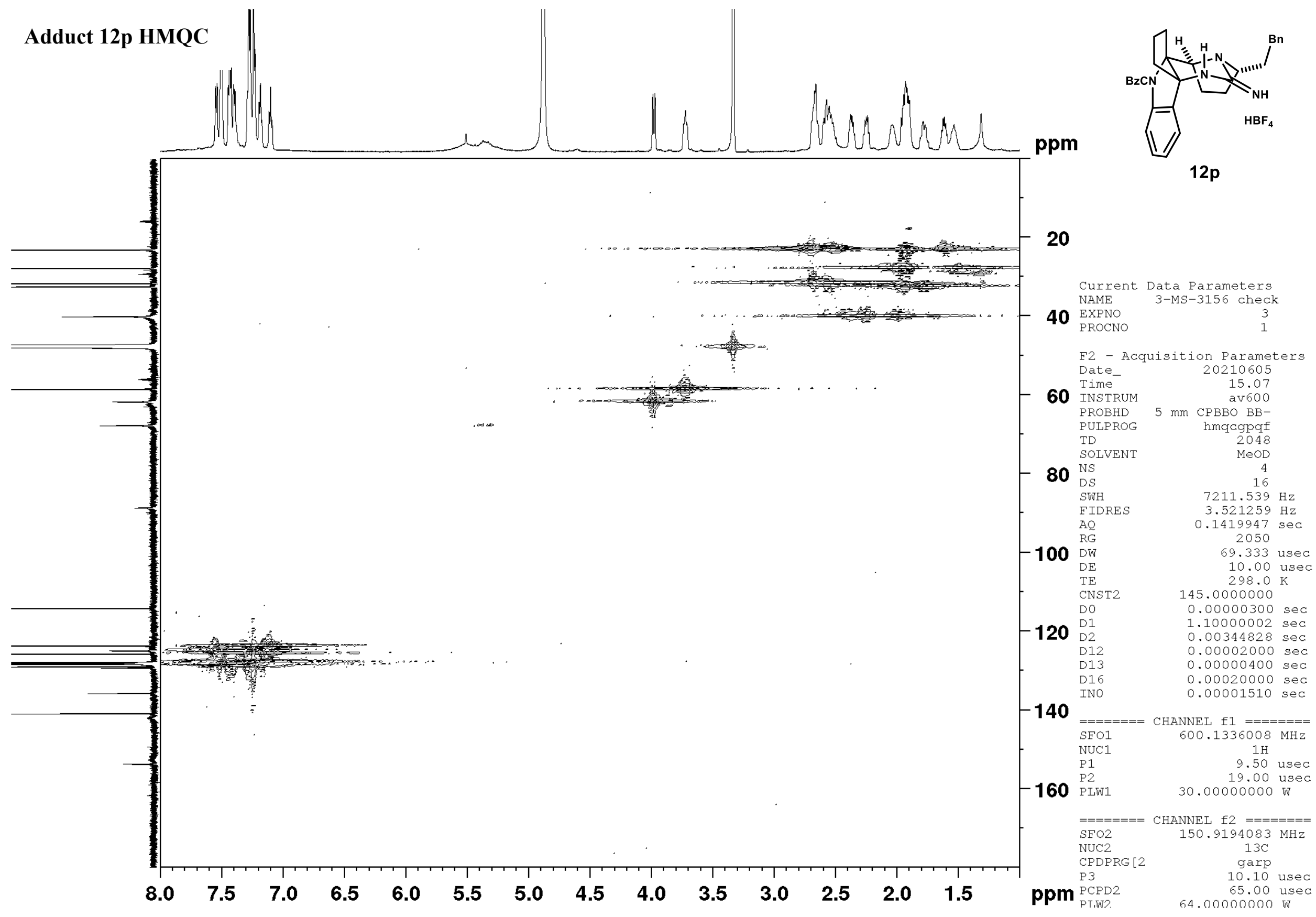




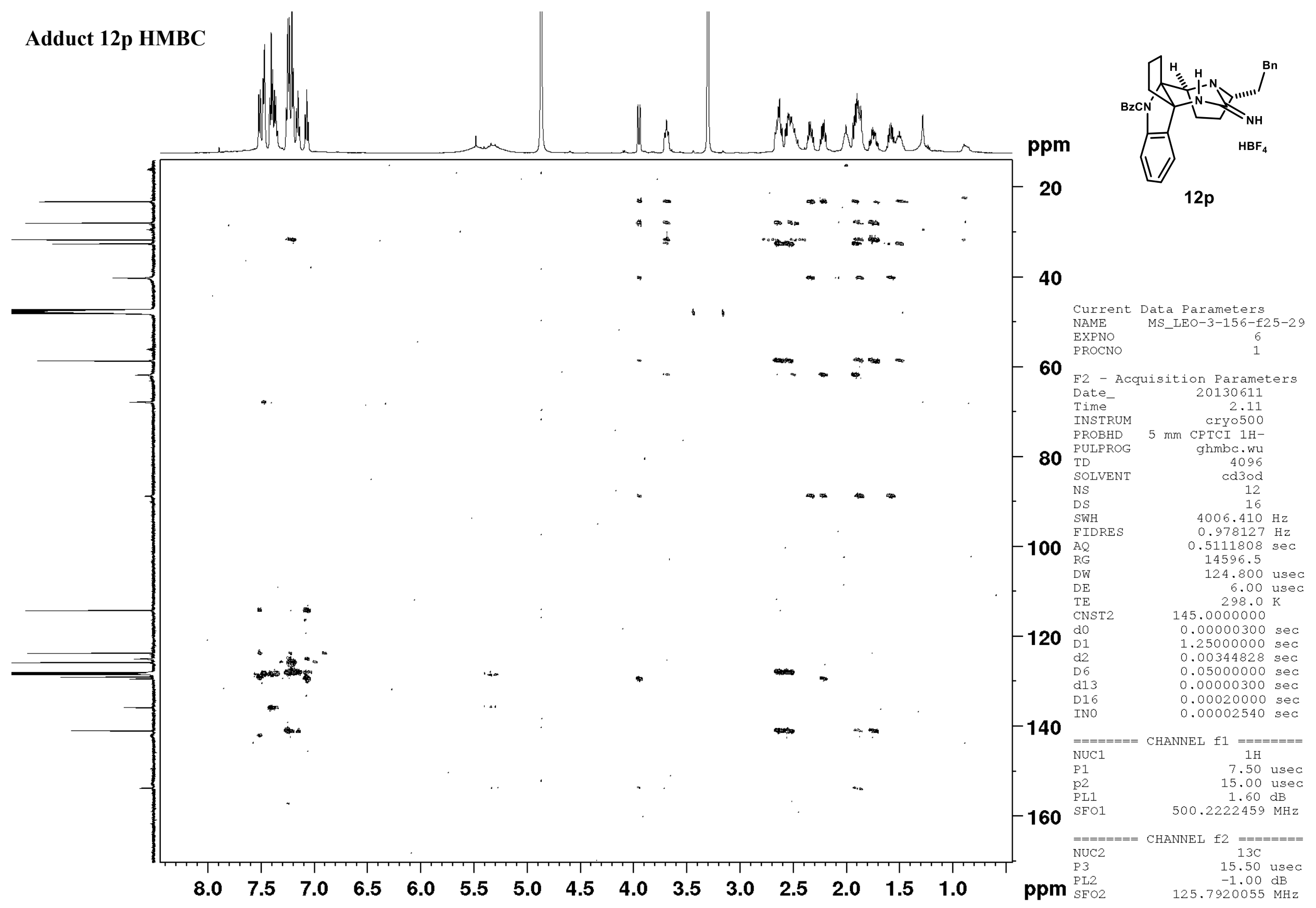




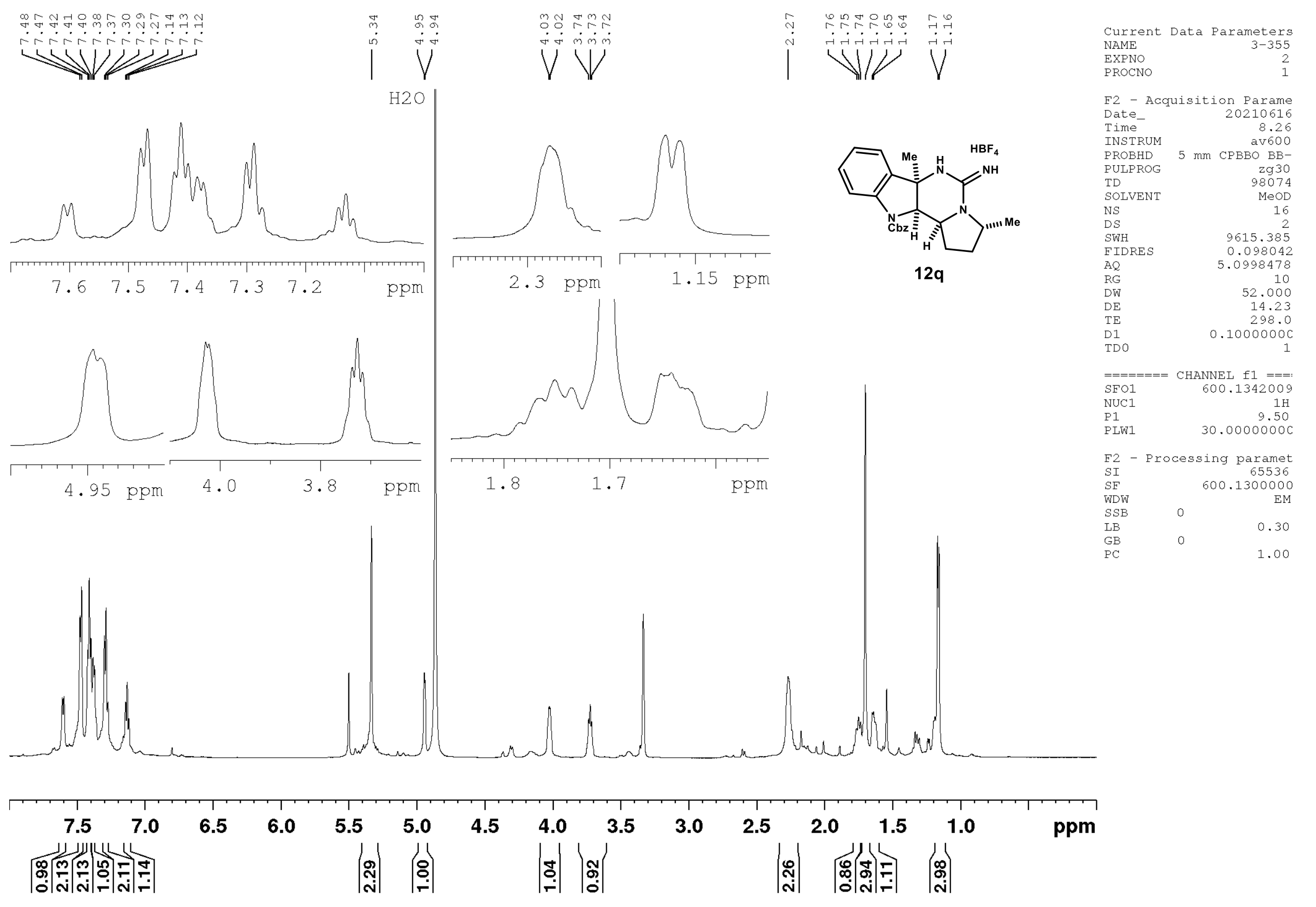



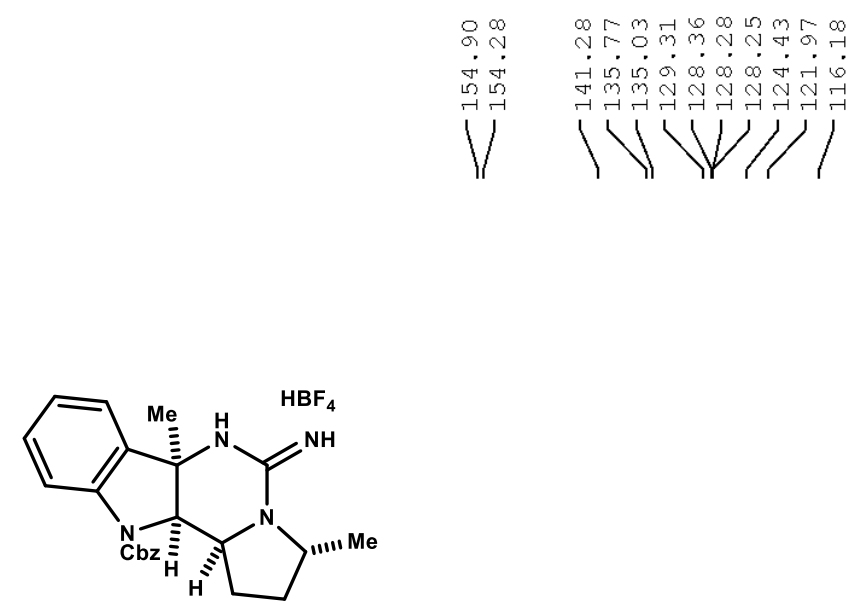

$12 q$

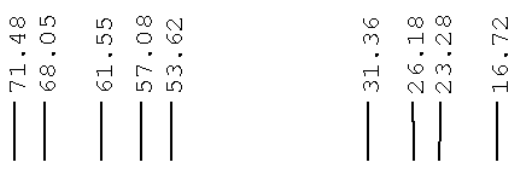

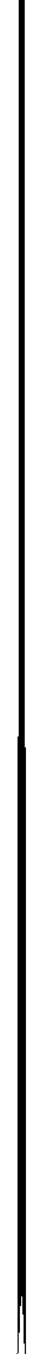

Current Data Parameters NAME

PROCNO

F2 - Acquisition Parame Date

INSTRUM

PROBHD

PULPROG

TD
SOLVENT

NS

DS

0.552855

AQ $\quad 0.9043968$

$\begin{array}{rr}\text { RG } & 2050 \\ \text { DW } & 13.800\end{array}$

$\begin{array}{lr}\mathrm{DE} & 19.63 \\ \mathrm{TE} & 298.0\end{array}$

$\begin{array}{ll}\mathrm{TE} & 1.00000000 \\ \mathrm{D} 1 & 0.03000000\end{array}$

$\begin{array}{lr}\text { D1 } & 1.00000000 \\ \text { D11 } & 0.03000000 \\ \text { TD0 } & 1\end{array}$

$=======$ CHANNEL $\mathrm{f} 1===$ :

SFO1 $\quad 150.9194080$

NUC1 $13 \mathrm{C}$

$\begin{array}{ll}P 1 & 10.10\end{array}$

$=======$ CHANNEL $\mathrm{f} 2===$

$\mathrm{SFO} 2$

NUC2

CPDPRG 2
PCPD2

PLW2 2

PLW12

$1 \mathrm{H}$
waltz16
80.00

$30.0000000 \mathrm{c}$

F2 - Processing paramet

SI 150.902836

WDW $\quad 150.9028085$

SSB $\quad 0$

$\mathrm{LB}$
$\mathrm{GB}$

PC 


\section{Adduct 12q COSY}

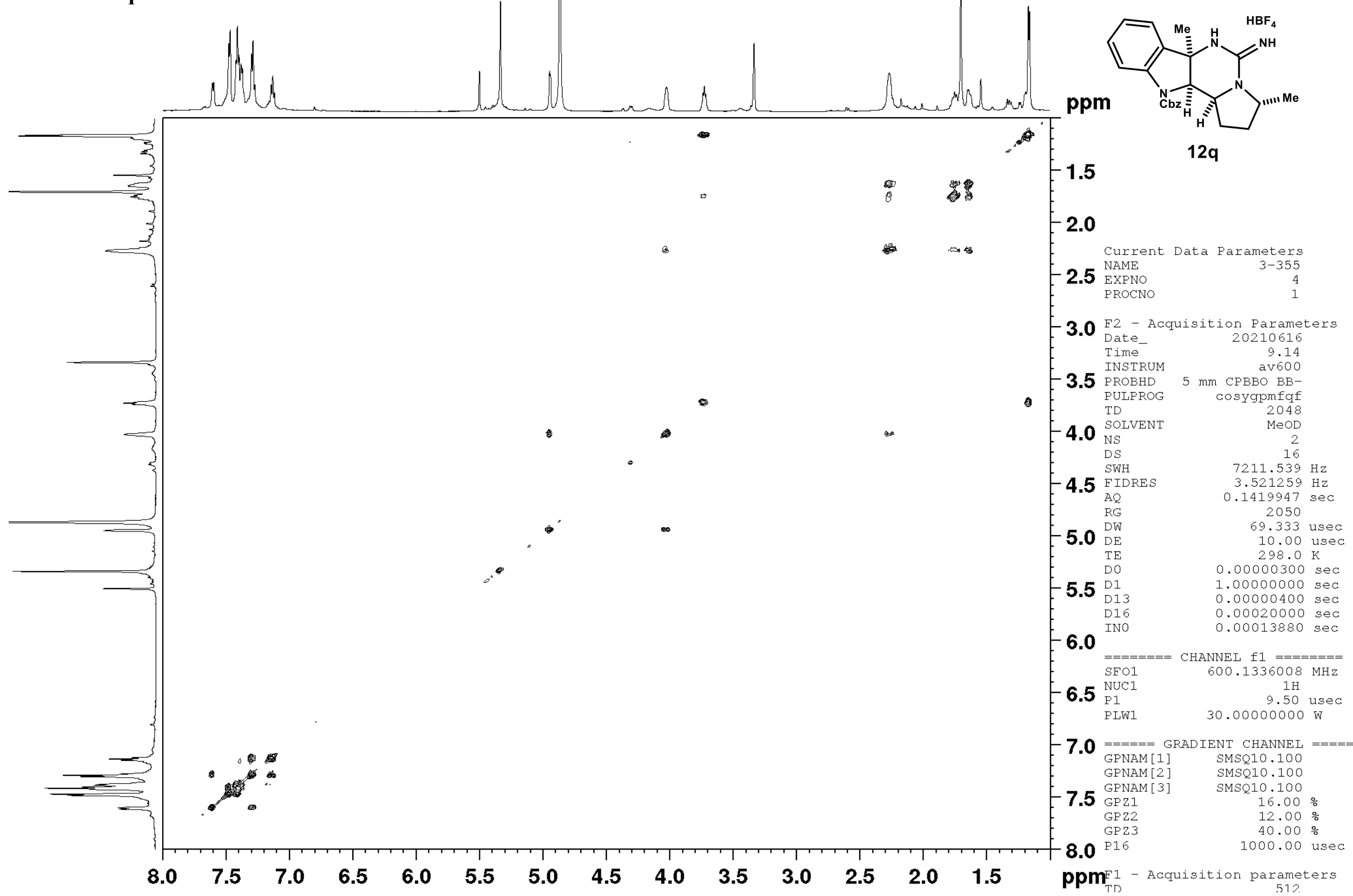




\section{Adduct 12q HMQC}

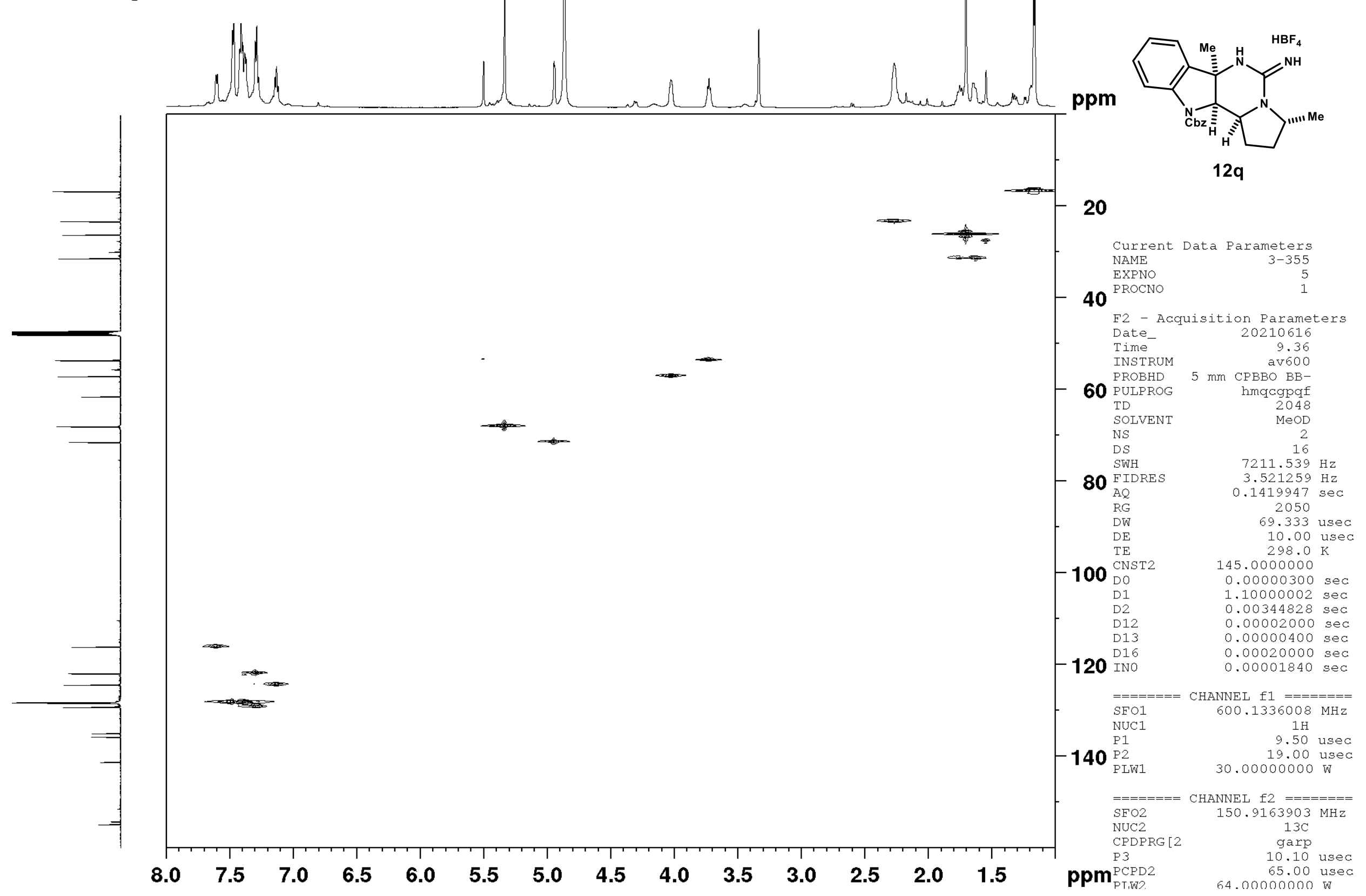



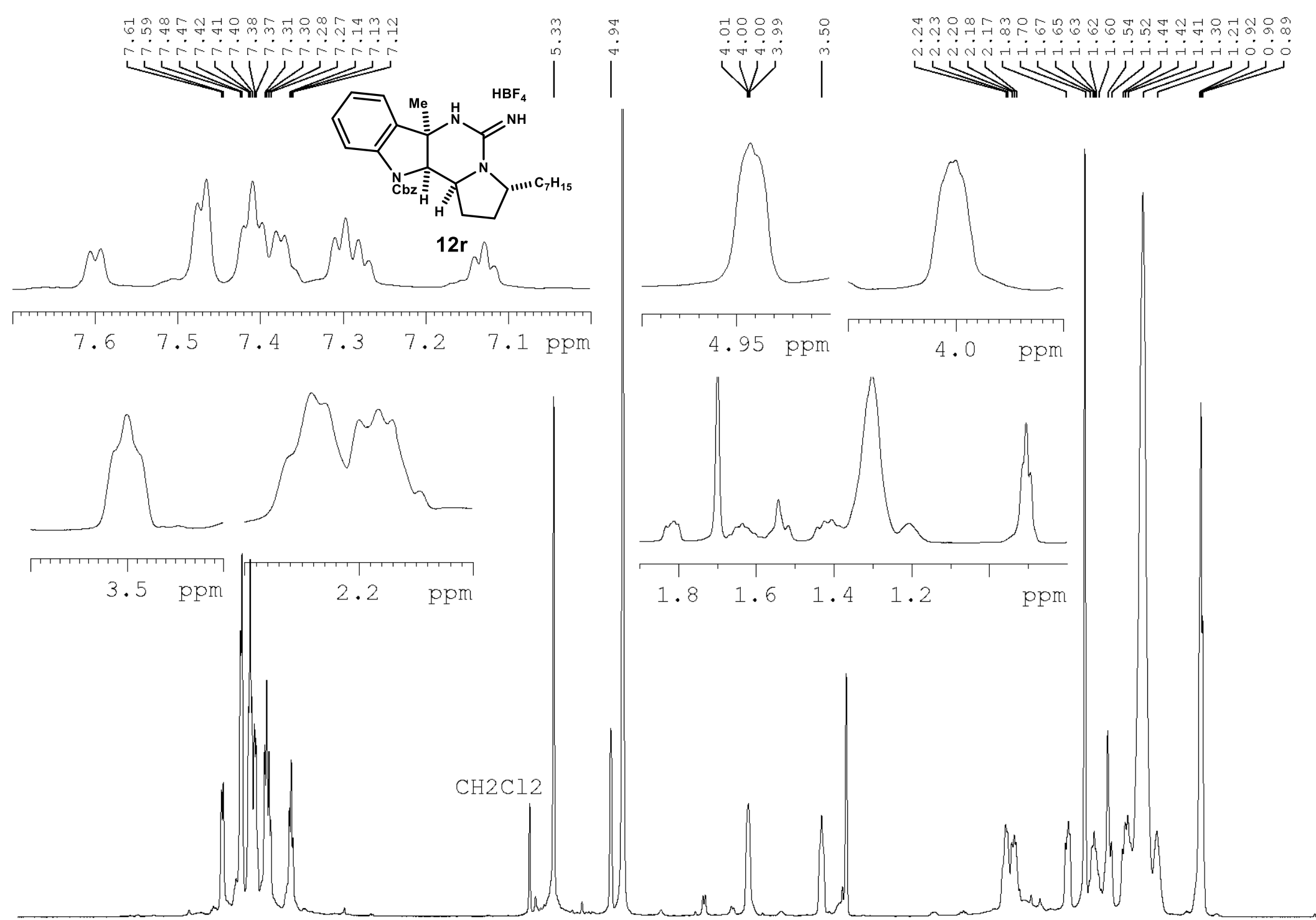

Current Data Parameters NAME

PROCNO

F2 - Acquisition Parame

Date__ 20210529

Time

av 600

$5 \mathrm{~mm}$ CPBBO BB-

PULPROG

TD 98074

SOLVENT

NS

SWH

F IDRE

RG

DW

$\mathrm{DE}$

TE

TD

$========$ CHANNEL $\mathrm{fl}===$

$\begin{array}{lr}\text { SFO1 } & 600.1342009 \\ \text { NUC1 } & 1 \mathrm{H}\end{array}$

P1 $\quad 9.50$

PLW

30.00000000

F2 - Processing paramet SI $\quad 600.1300000$

WDW

SSB

LB

0

0.30

PC

1.00

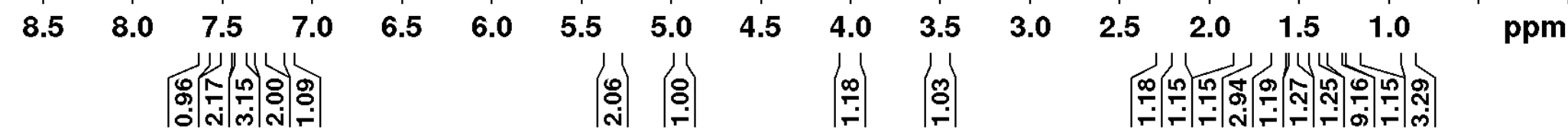



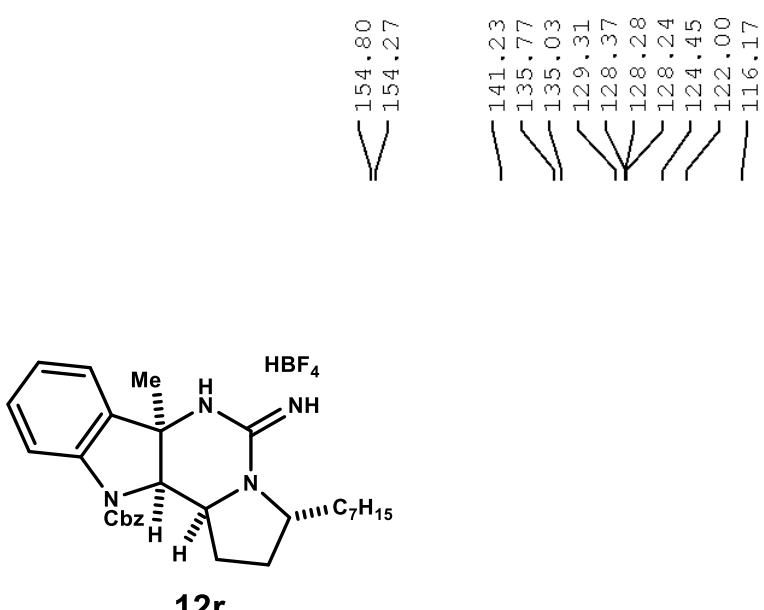

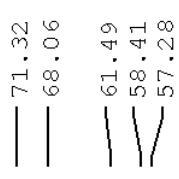

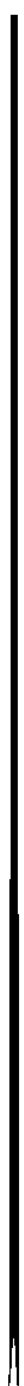

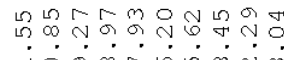

mon

Willt
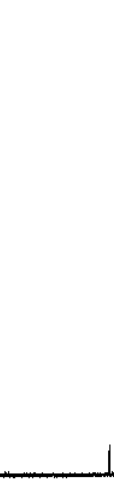

Current Data Parameters NAME

EXPNO

Time

PROBHD

PROBHD

PU

TD
SOLVENT

NS

DS

RG
DW

$\mathrm{DE}$

TE

D1

D11

$====$

$\mathrm{SFO1}$

NuC1
P1

PLW1

0.9043968

2050
13.800

19.63

0.80000001

0.03000000

CHANNEL $\mathrm{f} 1===$

150.9194080
$13 \mathrm{C}$

10.10
64.00000000$$
===
$$

CHANNEL $\mathrm{f2}===$
600.1330010

$\mathrm{SFO} 2$

NUC2 $1 \mathrm{H}$

PCPD2

PLW2 30.00000000

PLW12 $\quad 0.37154001$

F2 - Processing paramet

SI 65536

$\begin{array}{lr}\text { SF } & 150.9028085 \\ \text { WDW } & \text { EM }\end{array}$

WDW

$S S B$
$L B$

LB

$\mathrm{PC}$

1.00

1.00 


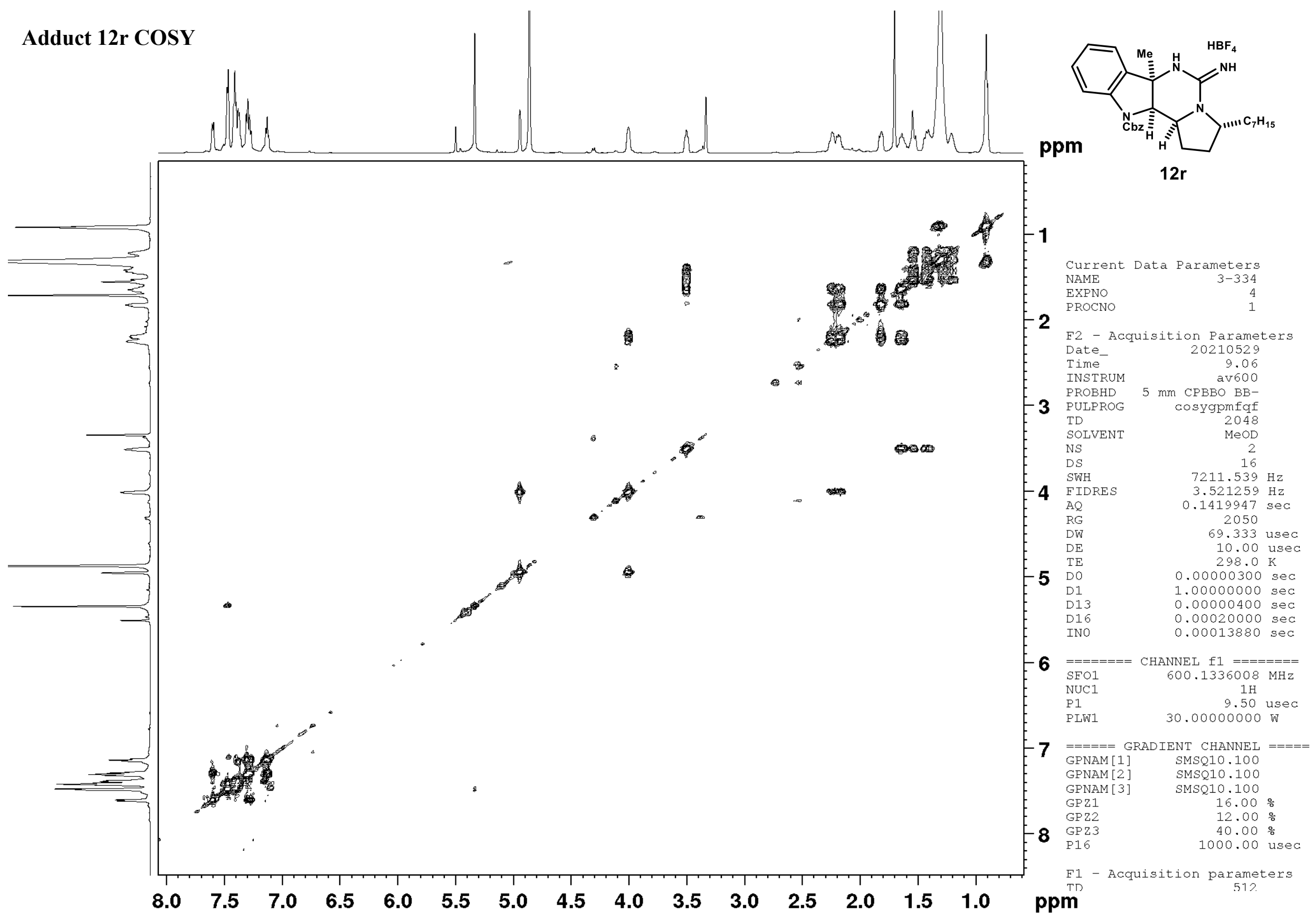




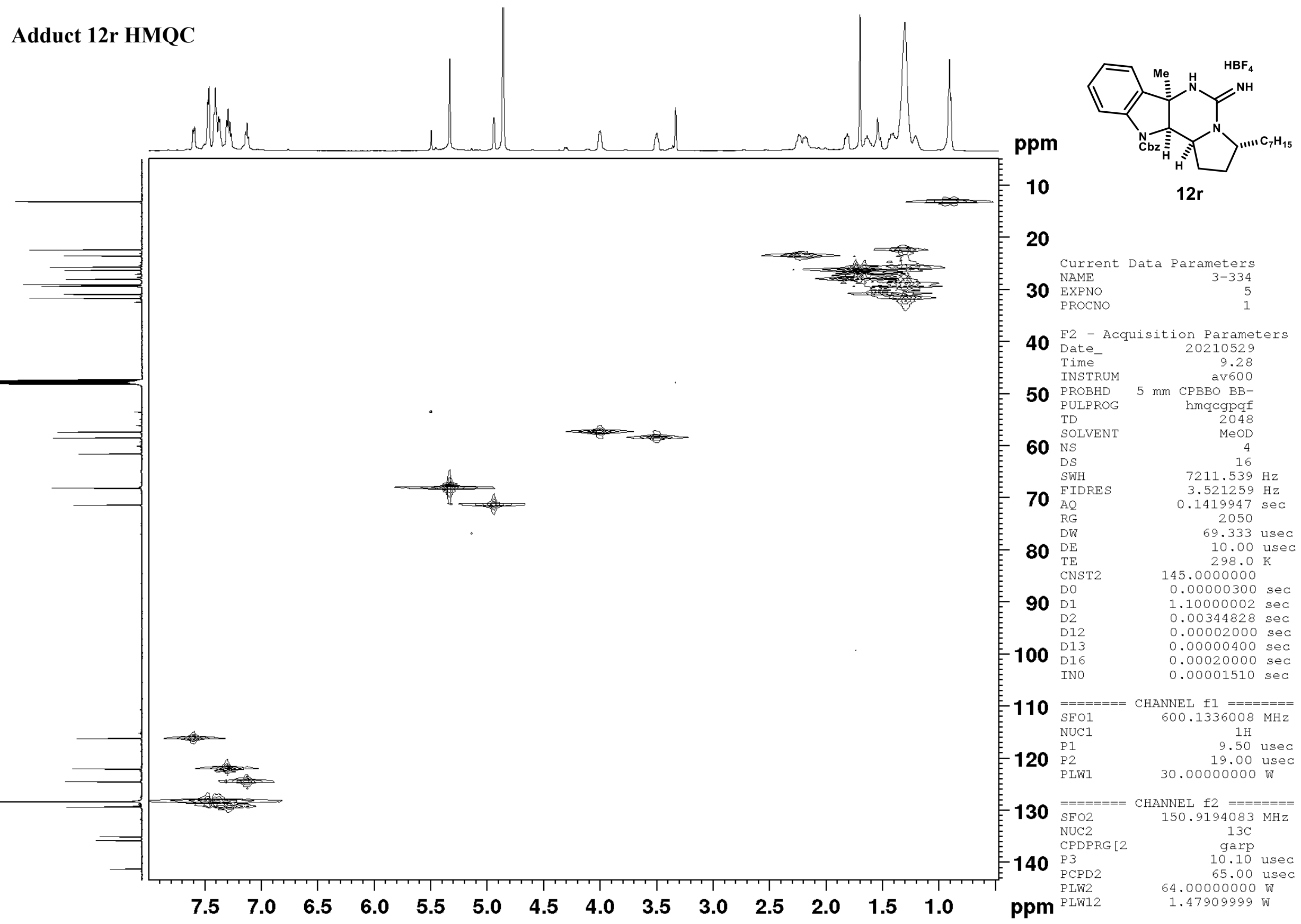




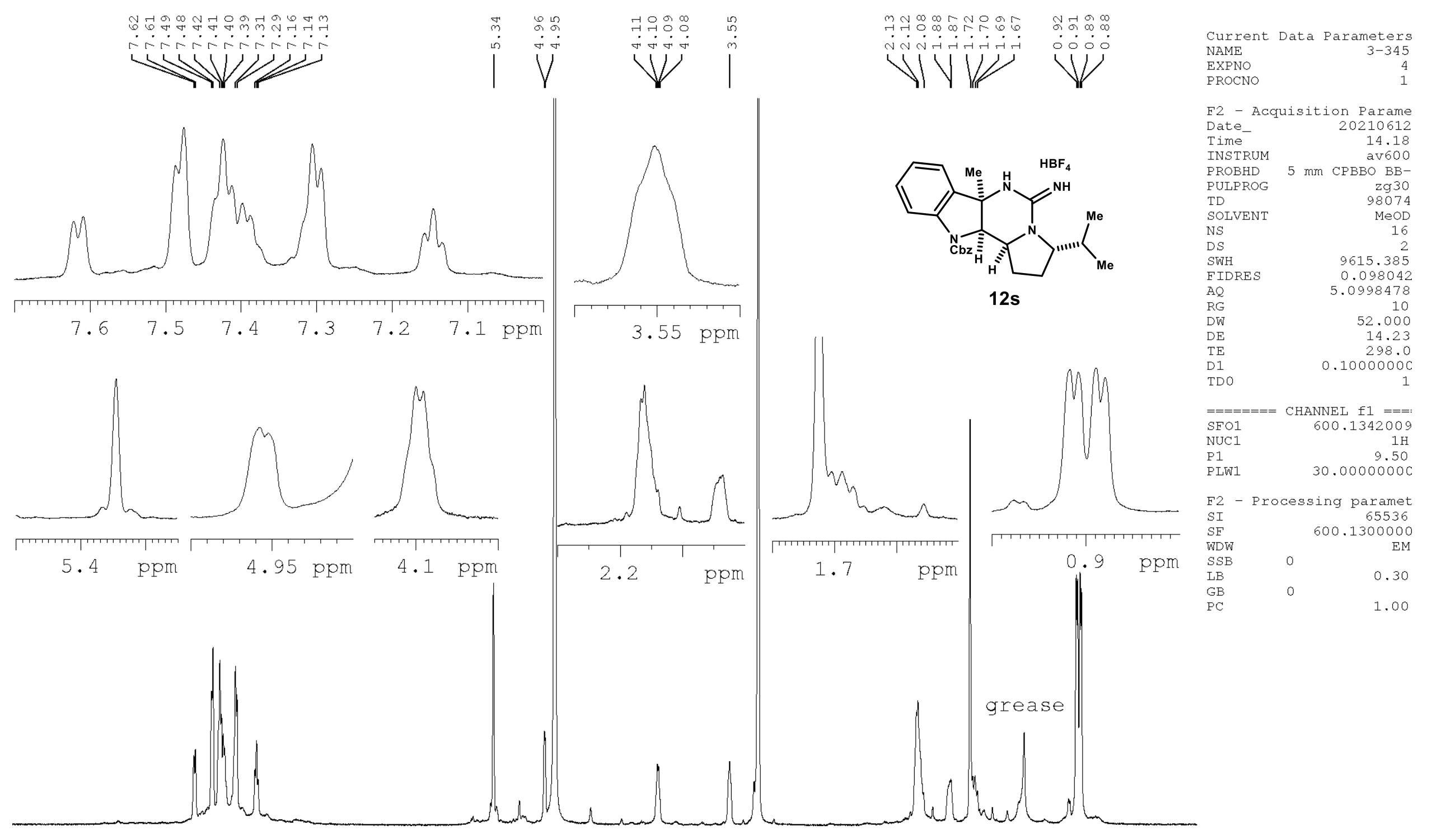

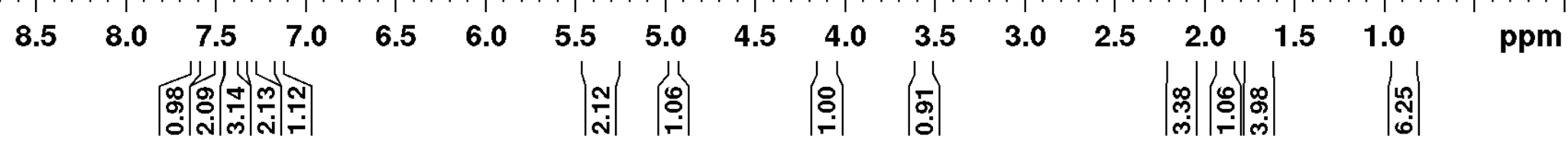



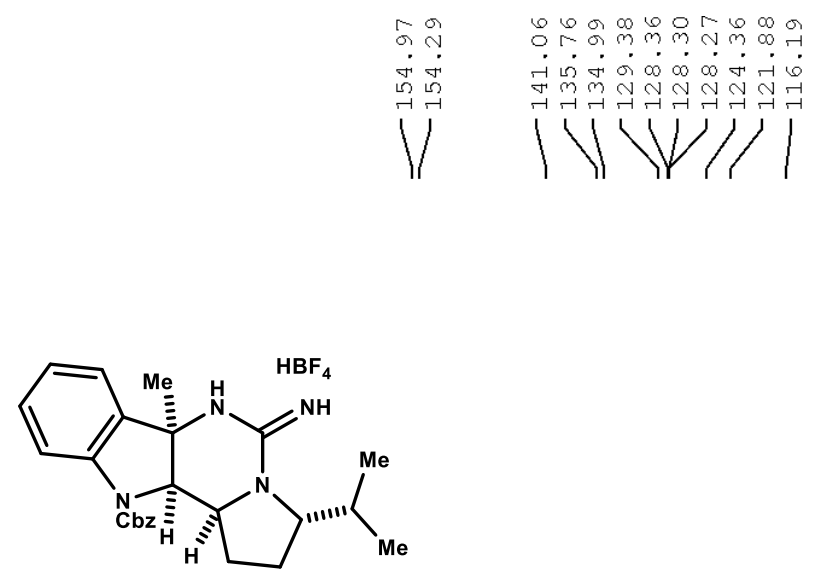

12s

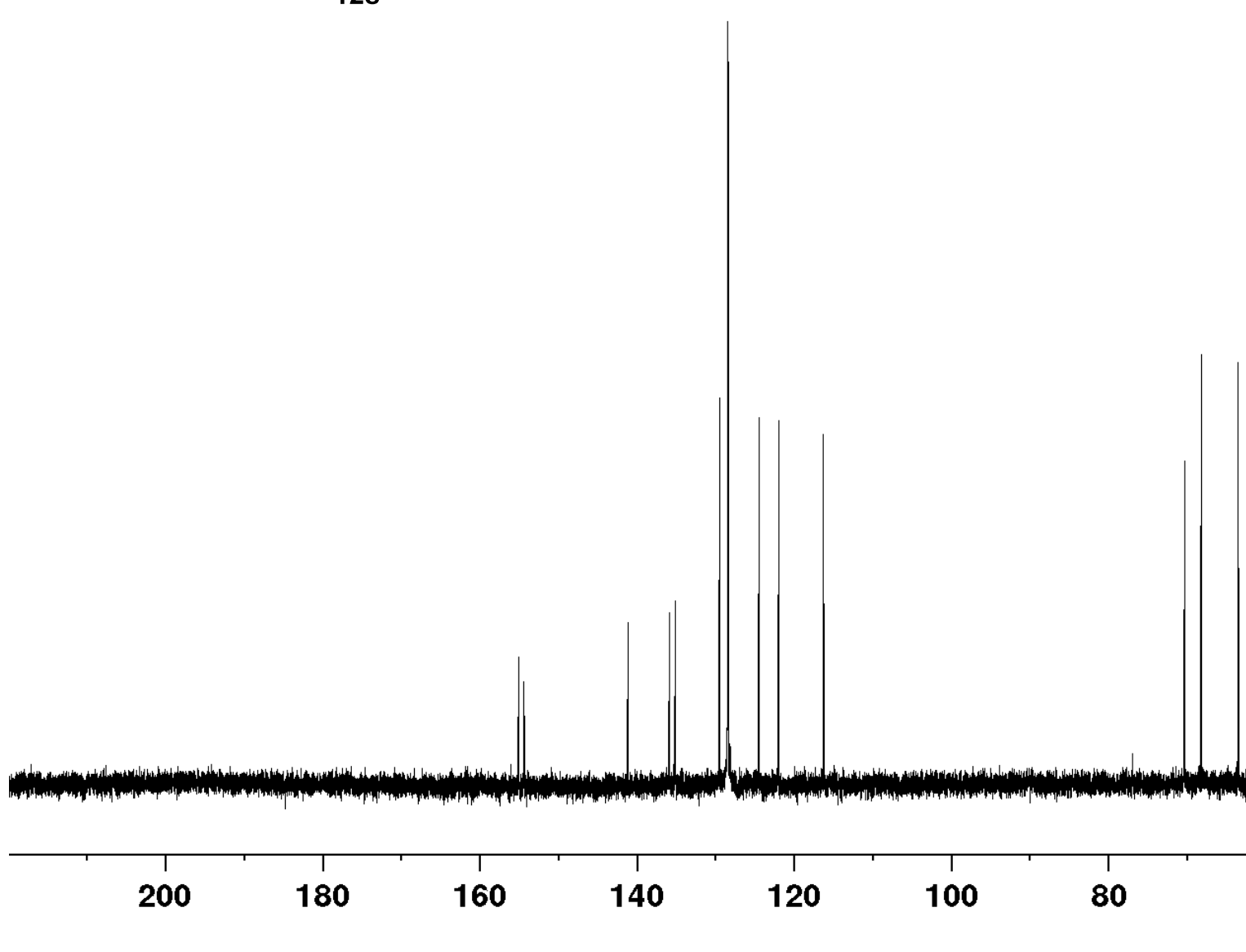

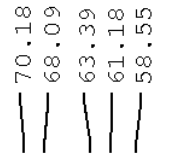

$\left.\right|_{1} ^{1}$

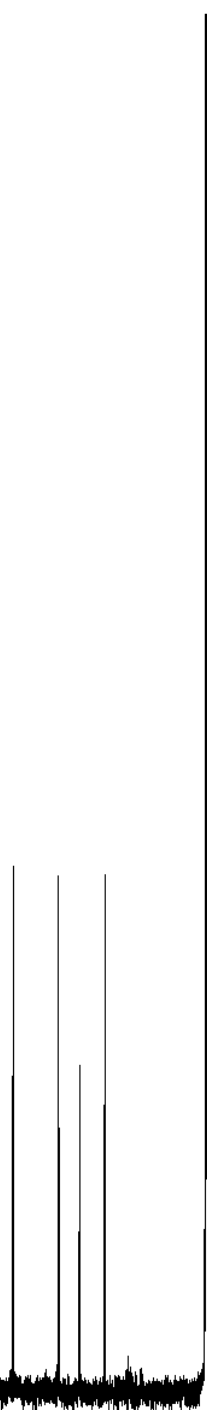

งิن

1111

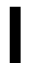
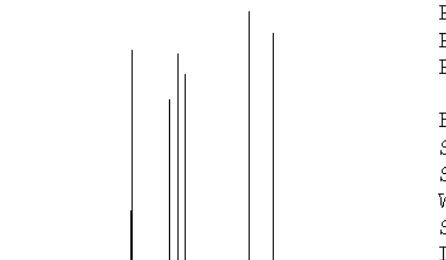

Current Data Parameters NAME

PROCNO

Date

Time

INSTRUM

PROBHD

TD

SOLVENT

NS

SWH

FIDRES

AQ

RG

DW

TE

D1

D11

$===$
SFO1

SFO1

$\mathrm{P} 1$

PLW1

$===$

$\mathrm{SFO} 2$

CPDPRG [

PCPD 2

PLW12

Processing paramet

150.9028085

DW $\quad 150.9028085$

SB $\quad 0=-2$

GB $\begin{array}{r}20210612 \\ 14.43 \\ \hline\end{array}$

$\mathrm{mm}$ CPBBO BB-

zgda 30

MeOD

36231.883

2050

19.63

1.00000000

0.03000000

HANNEL $\mathrm{f} 1===$

$13 \mathrm{C}$
10.10

HANNEL $12===$

600.1330010

waltz16
80.00 0

1.00

1.00 


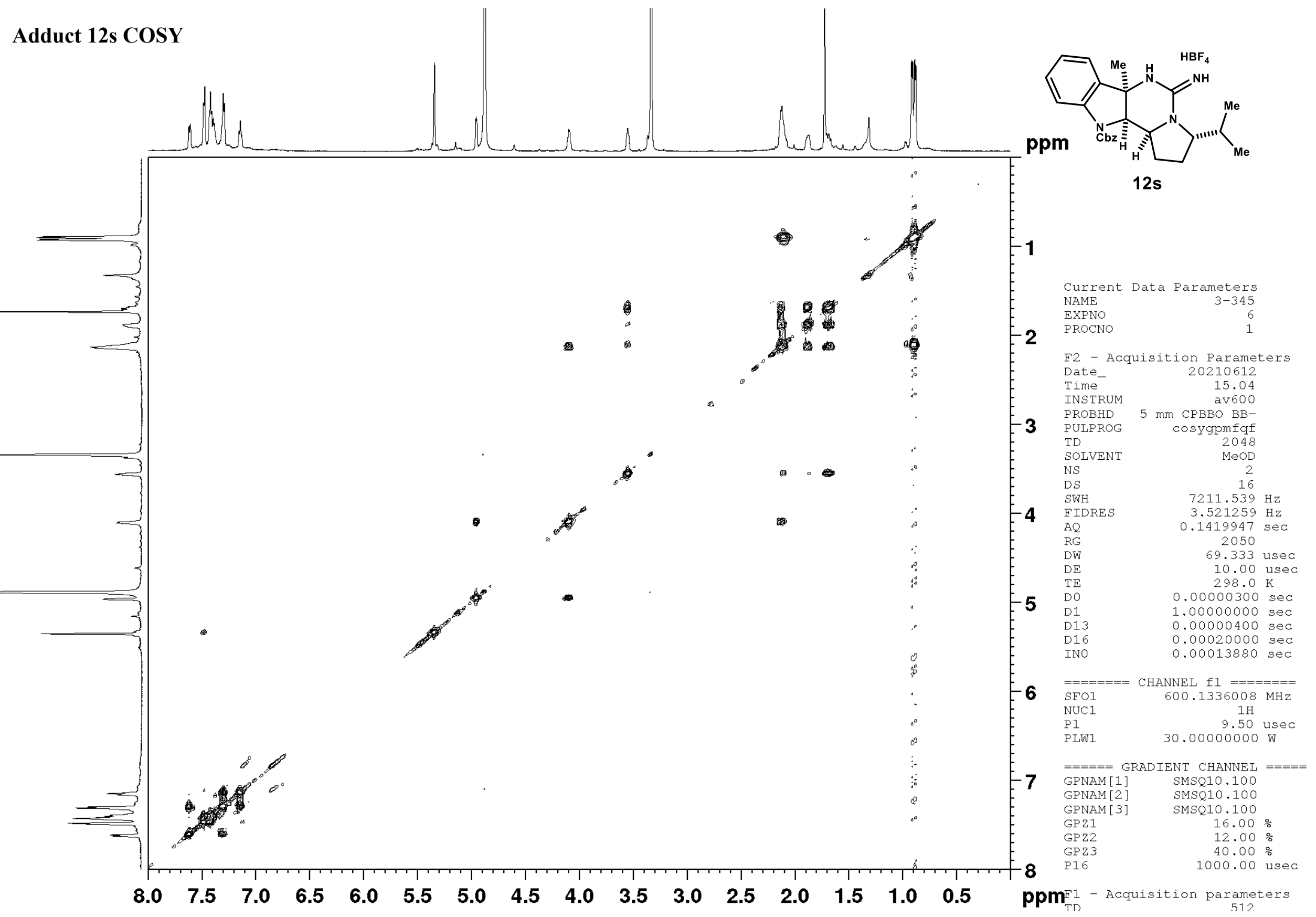




\section{Adduct 12s HMQC}

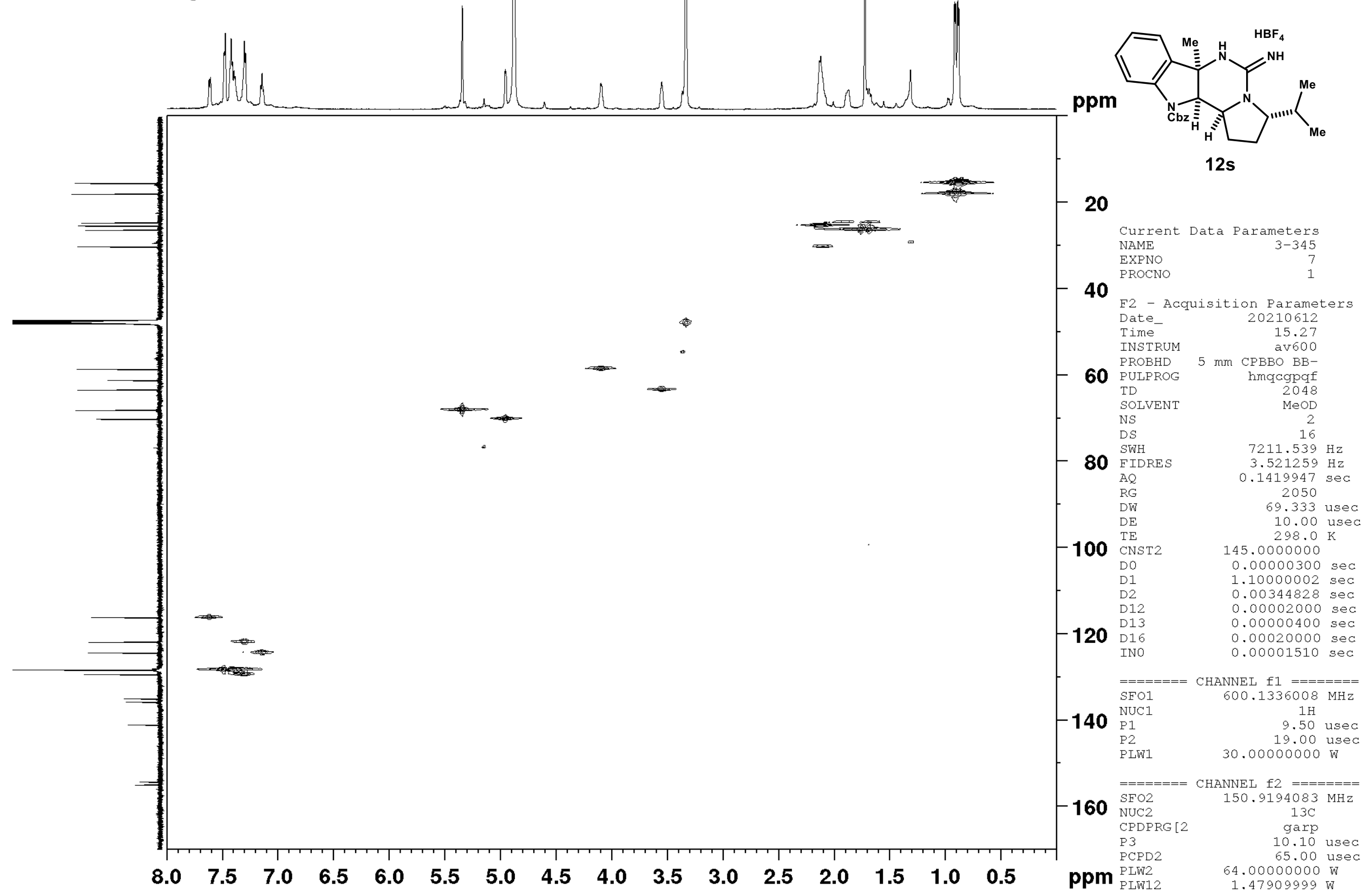



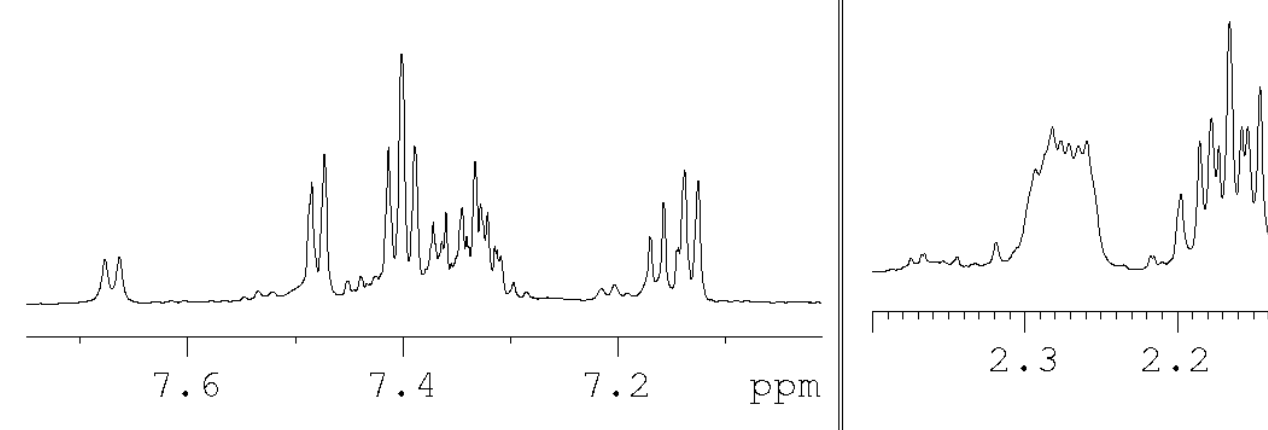$$
\Gamma
$$
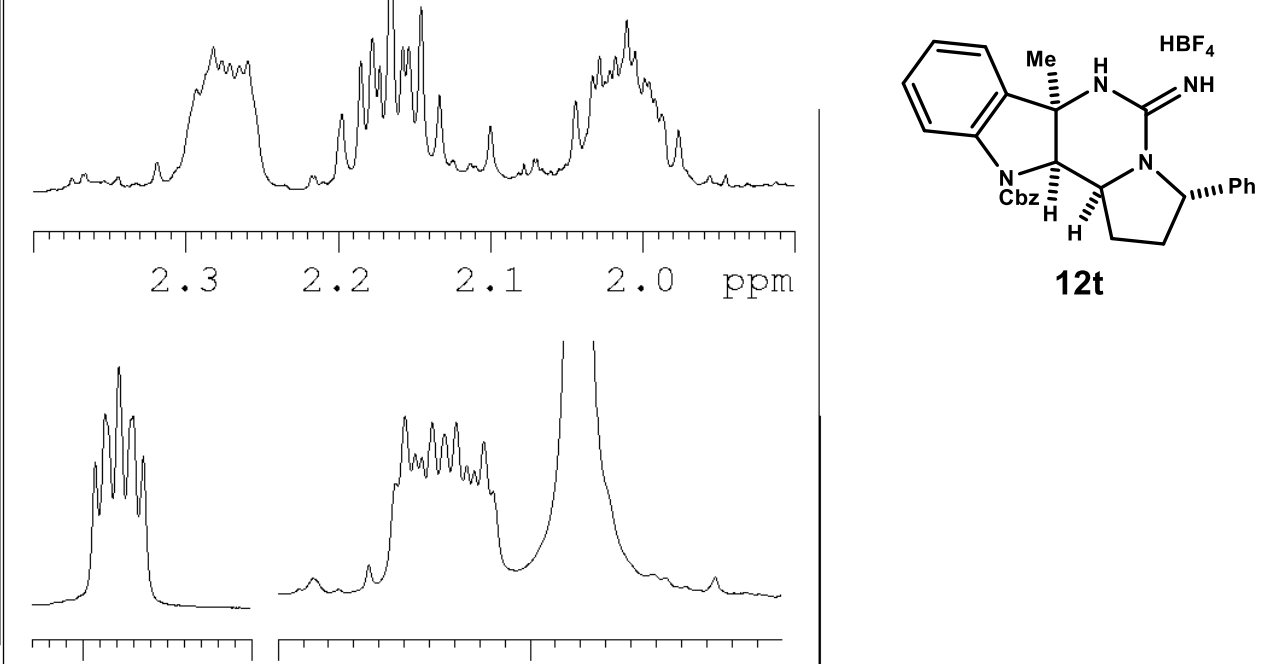

$12 t$

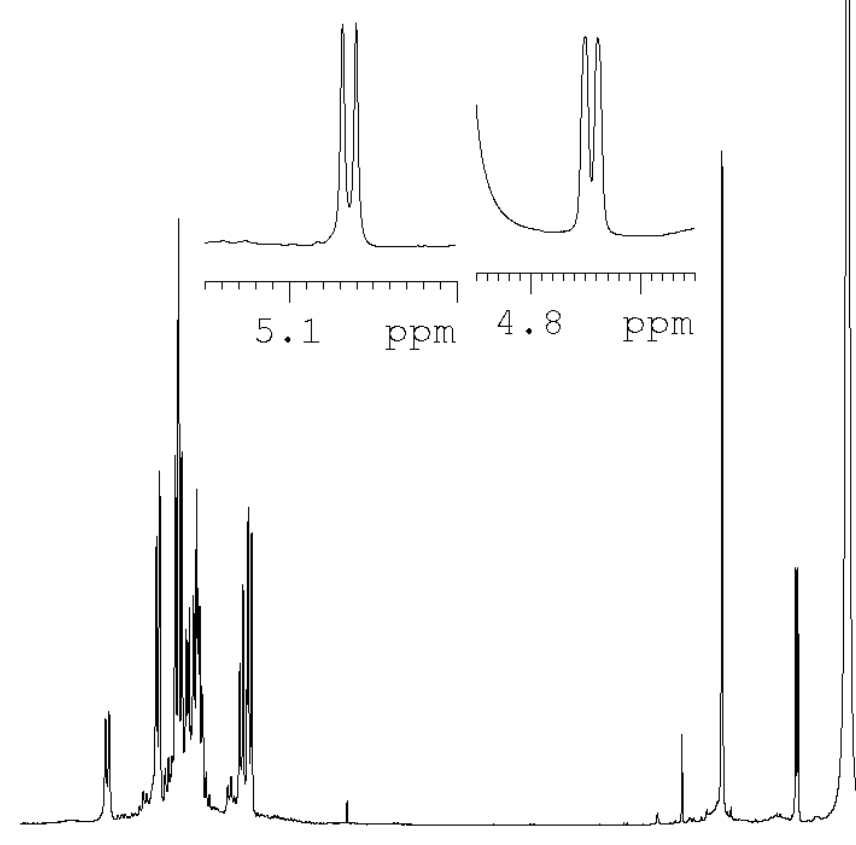

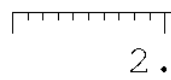
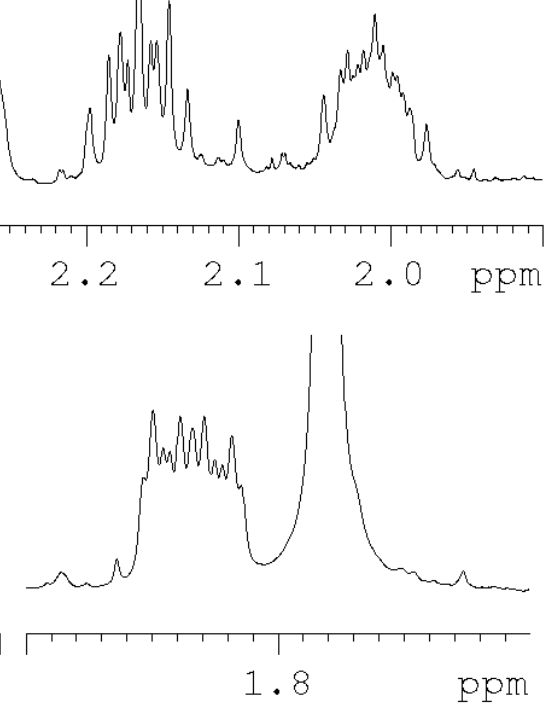

2 .

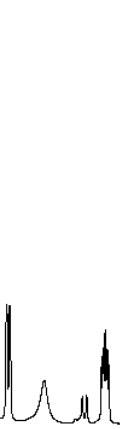

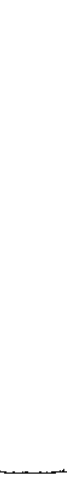

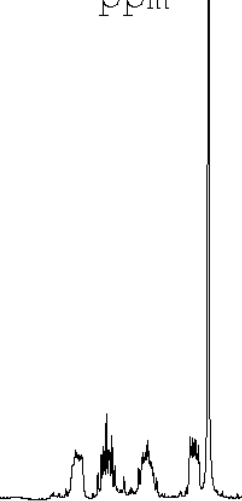
F2 - Acquisition Parame
Time
INSTRUM

$5 \mathrm{~mm} \mathrm{CPBBO} \mathrm{BB}-$

PULPROG

TD

SOLVENT

DS

SWH
FIDRE

$\mathrm{AQ}$

RG

DW
DE
TE
D1

D1
TD 0

SFO1

$\mathrm{SFO} 1$
NUC1

P1

zg30
98074
MeOD

16
2

9615.385

0.098042

5.0998478

52.000

14.23

0.10000000

PI

CHANNEL $\mathrm{f} 1===$

30.00000000

F2 - Processing paramet

SI 65536

$\begin{array}{ll}\text { SF } & 600.1300000\end{array}$

WS

L

GB

0.30

1.00

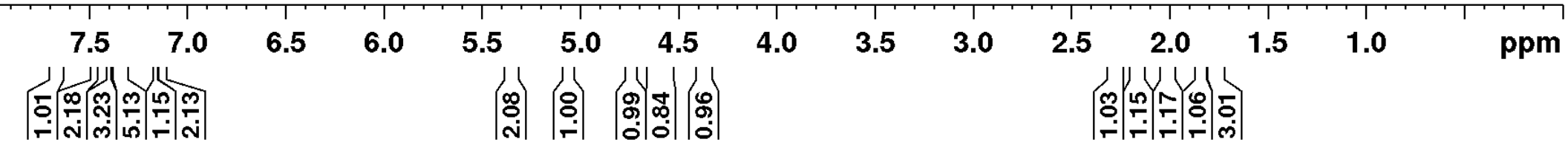



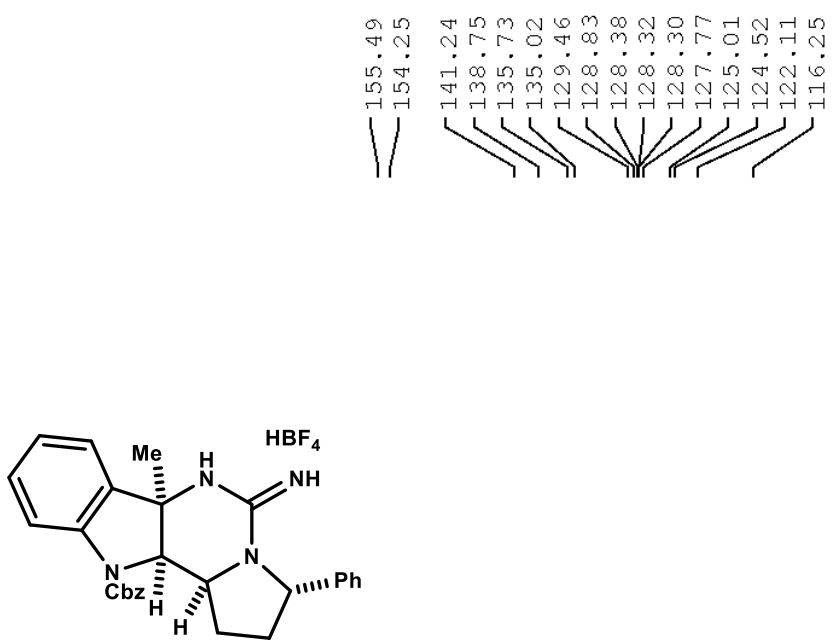

$12 t$

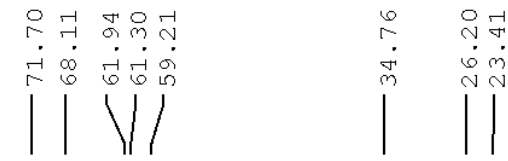

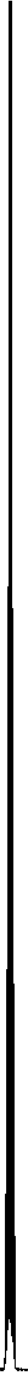

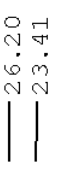

Current Data Parameters NAME

EXPNO

F2 - Acquisition Parame

Date

INSTRUM

PROBHD

PULPROG

TD
SOLVENT

NS
DS
SWH

FIDRES $\quad 0.552855$

AQ $\quad 0.9043968$

RG

DW

$\mathrm{DE}$

1.00000000

0.03000000

$=======$ CHANNEL $\mathrm{f1}===$

SFO1 $\quad 150.9194080$

$13 \mathrm{C}$

$\begin{array}{lr}\text { P1 } & 10.10\end{array}$

$========$ CHANNEL $f 2===$

$=======$ CHANLL $12===$

$\mathrm{SFO} 2$

CPDPRG [

PCPD2

PLW12

$1 \mathrm{H}$
waltz16
80.00

30.00000000

0.37154001

F2 - Processing paramet

SI 65536

SF $\quad 150.9028085$

WDW EM

LB

GB

1.00

1.00 


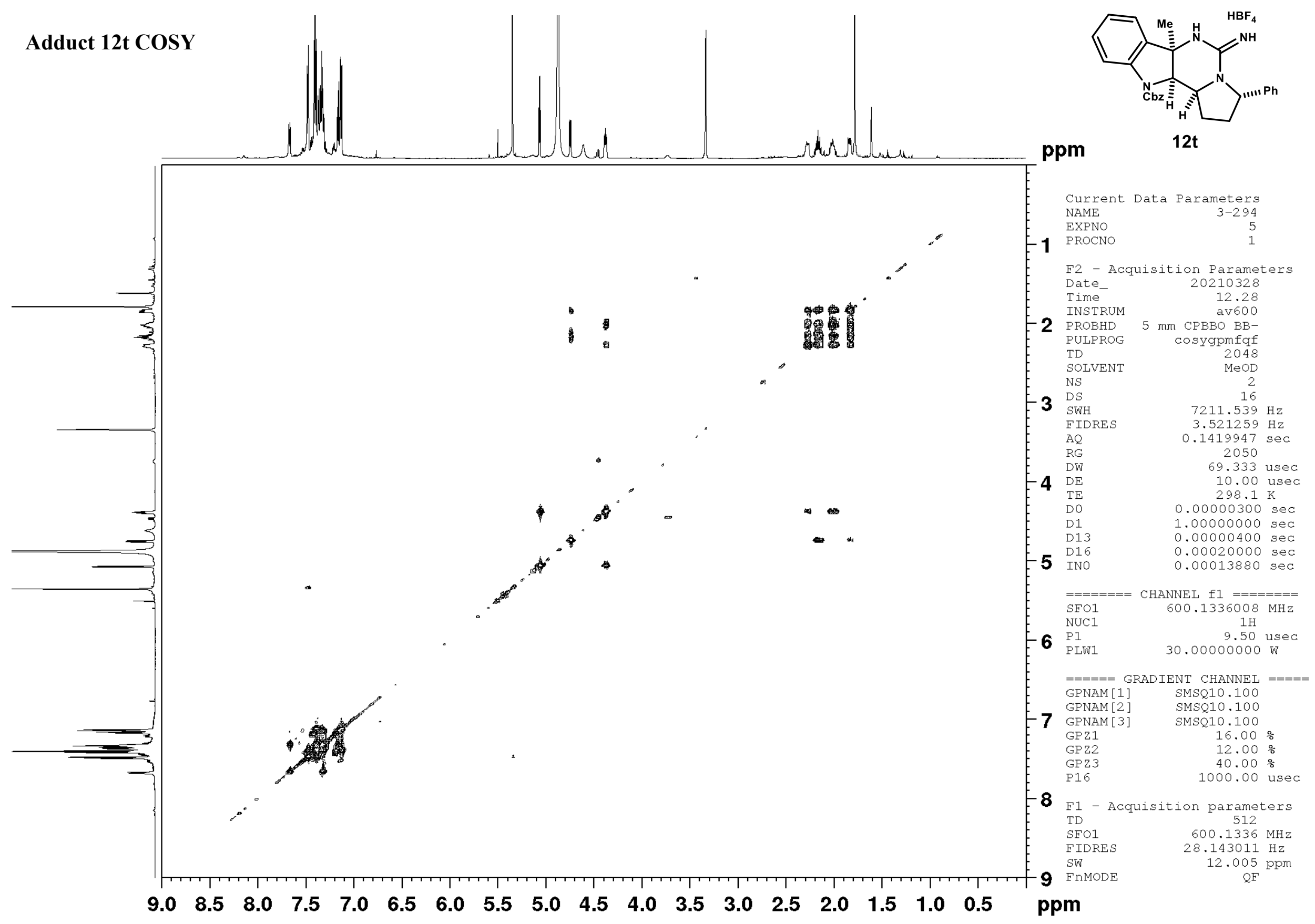




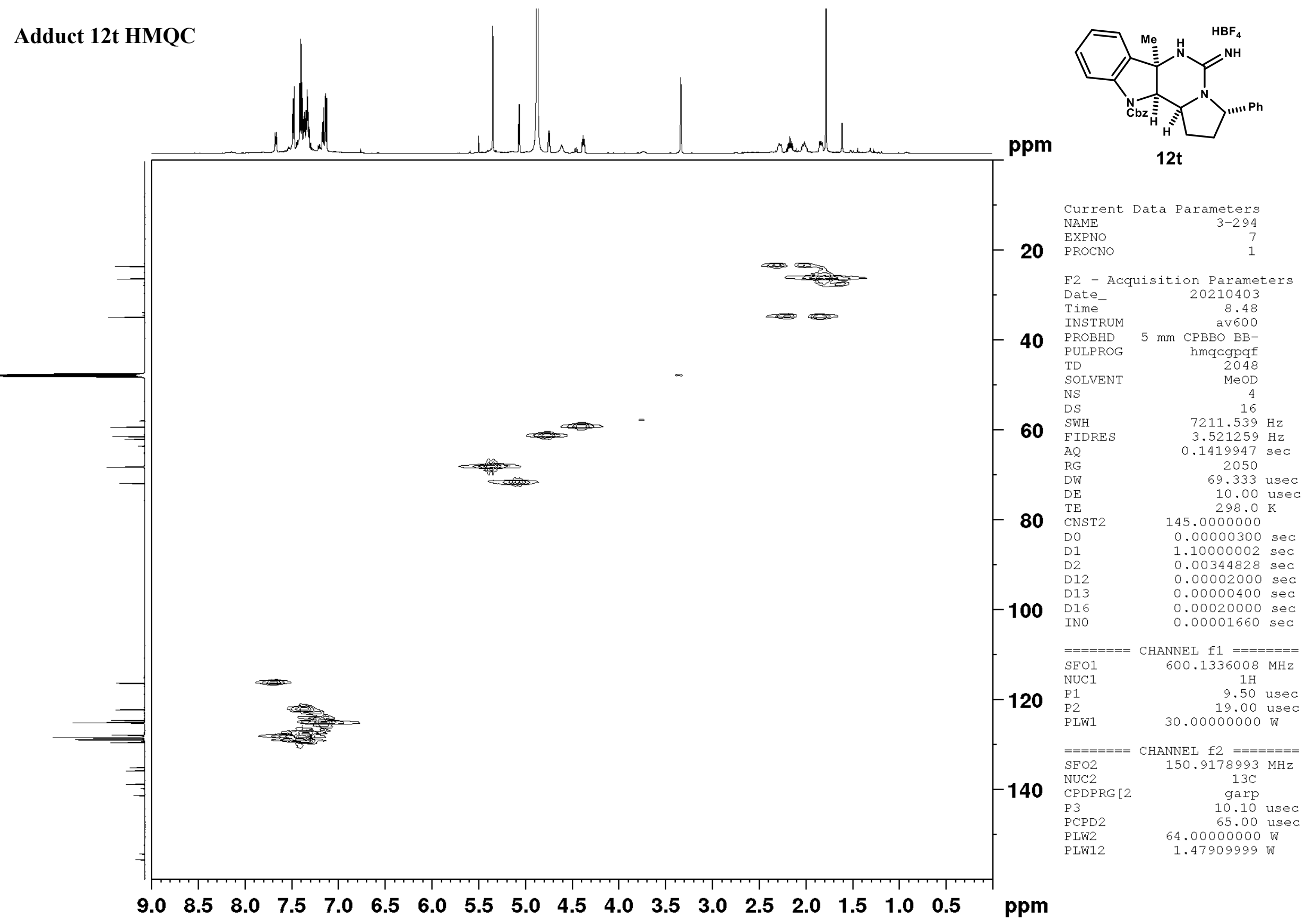



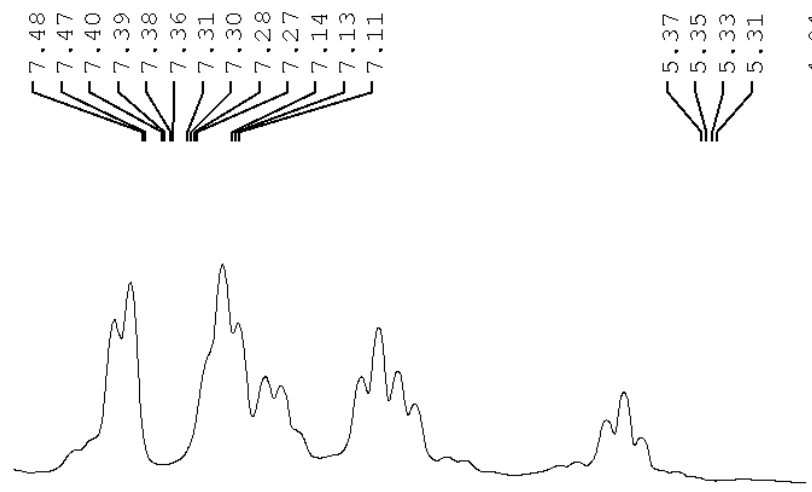

$\begin{array}{lllllll}7.5 & 7.4 & 7.3 & 7.2 & 7.1 & \mathrm{ppm}\end{array}$

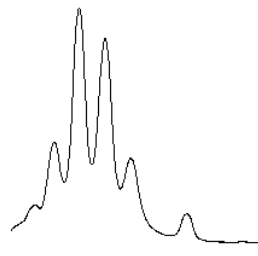

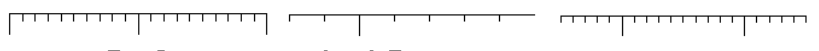

$5.3 \mathrm{ppm} 4.95 \mathrm{ppm}$
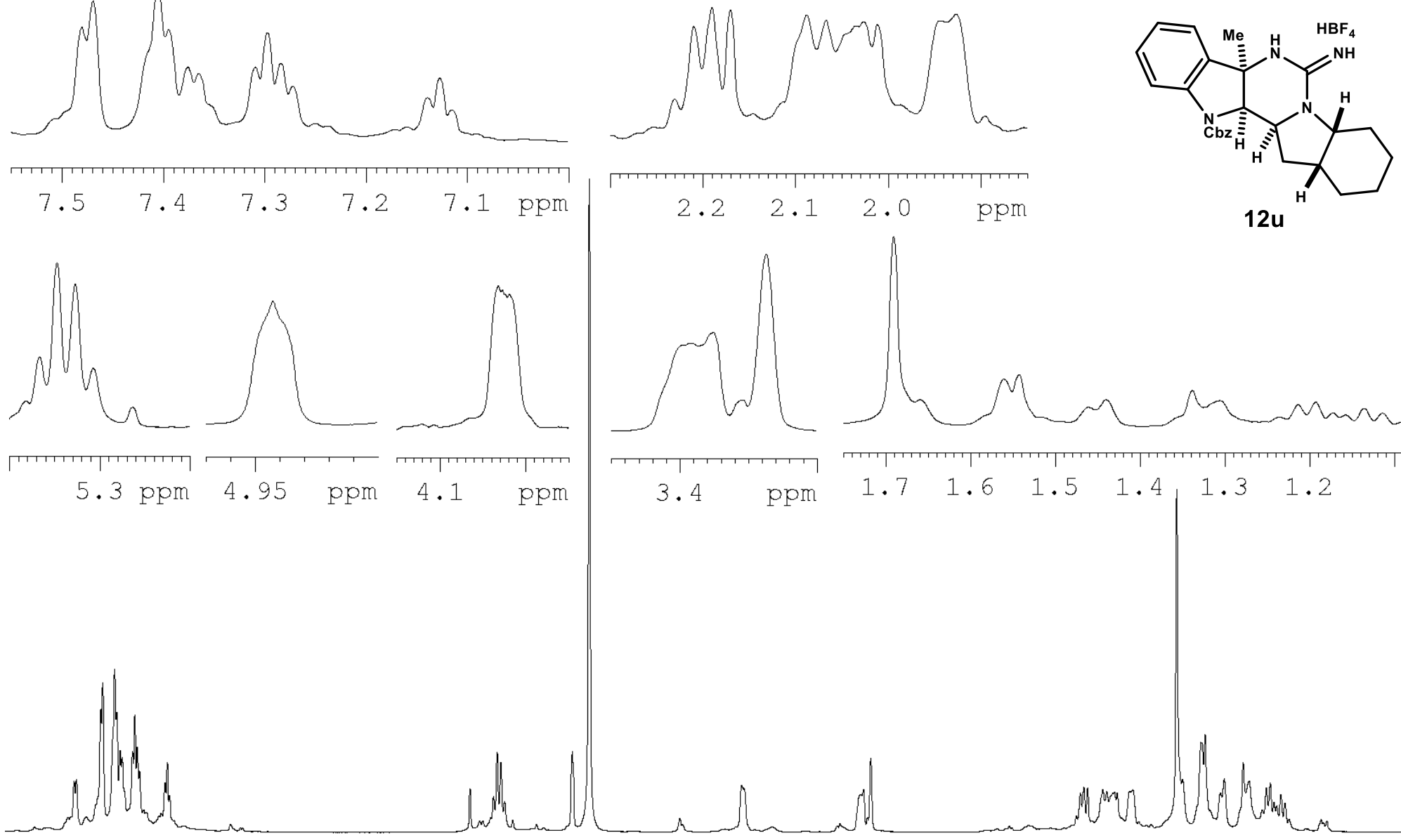

3.4 ppm

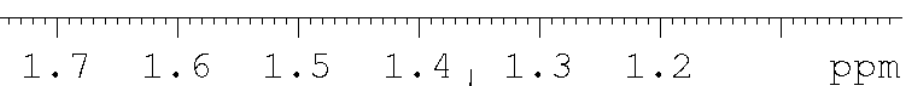

$4 \cdot 1$
Current Data Parameters NAME

EXPNO

PROCNO

F2 - Acquisition Parame

Date_

Time

INSTRUM

PROBHD

PULPROG

TD

SOLVENT

NS

DS

FIDRES

$\mathrm{AQ}$

RG

DW

DE

TE

TDO

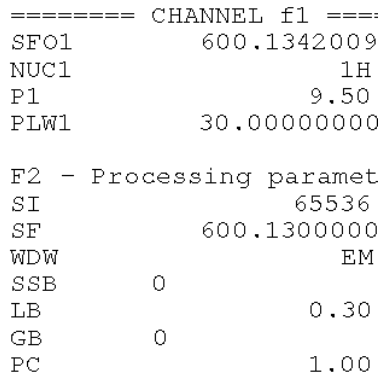

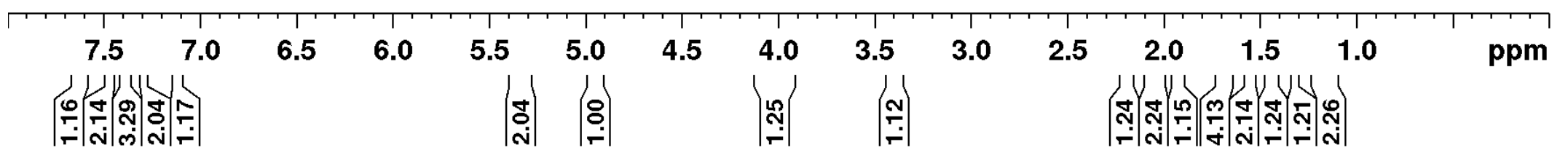

S213 
F2 - Acquisition Parame

Date

Time

PROBHD

PULPROG

20210608
10.34

PU

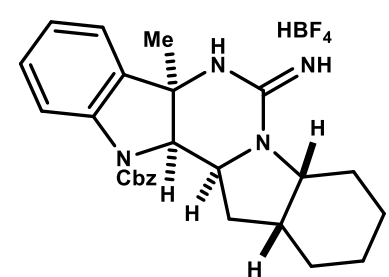

SOLVENT

NS
DS
SWH

FIDRES

RG
DW

$\mathrm{DE}$

TE

1.00000000

$\begin{array}{lr}\text { D11 } & 0.03000000 \\ \text { TD0 } & 1\end{array}$

$12 \mathrm{u}$

$=======$ CHANnEL $\mathrm{fl}===$

$\begin{array}{lr}\text { SFO1 } & 150.9194080 \\ \text { NUC1 } & 13 \mathrm{C}\end{array}$

$\begin{array}{lr}\text { NUC1 } & 13 \mathrm{C} \\ \text { P1 } & 10.10 \\ \text { PLW1 } & 64.00000000\end{array}$

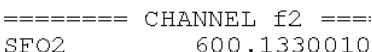

CPDPRG [

PCPD2

600.1330010
$1 \mathrm{H}$

0.00000000

F2 - Processing paramet

SI 65536

SF $\quad 150.9028085$

WDW EM
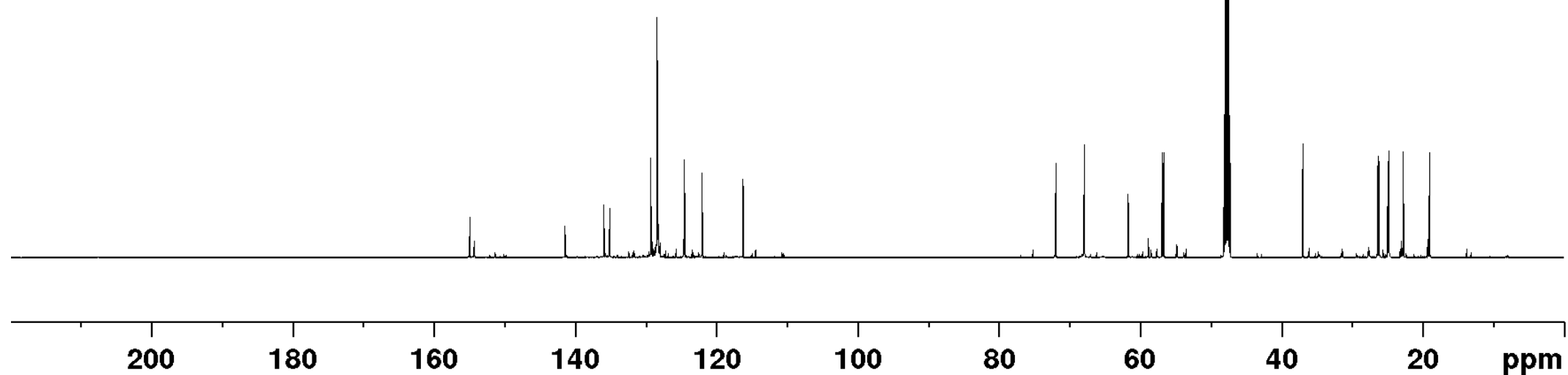


\section{Adduct 12u COSY}

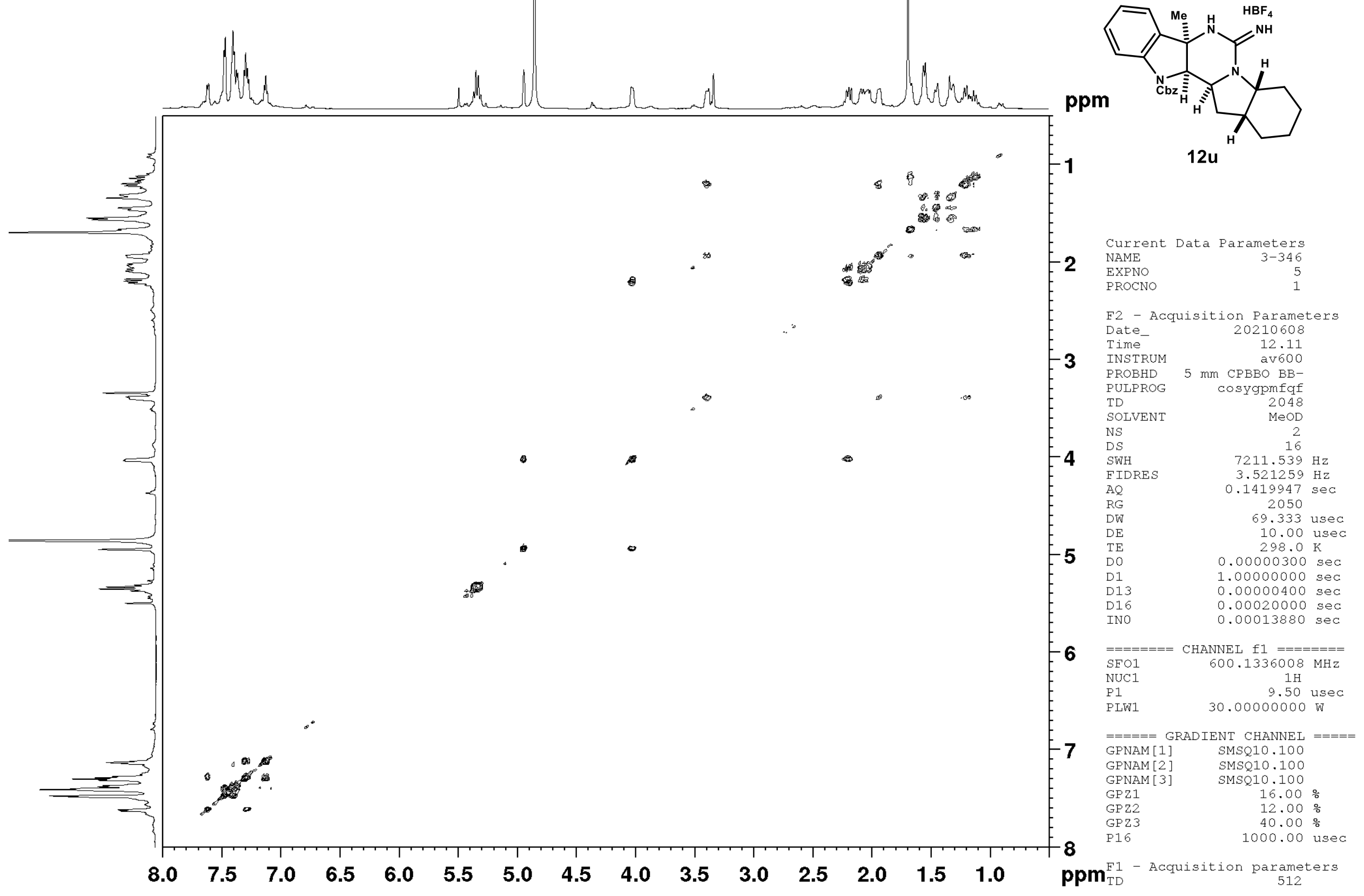




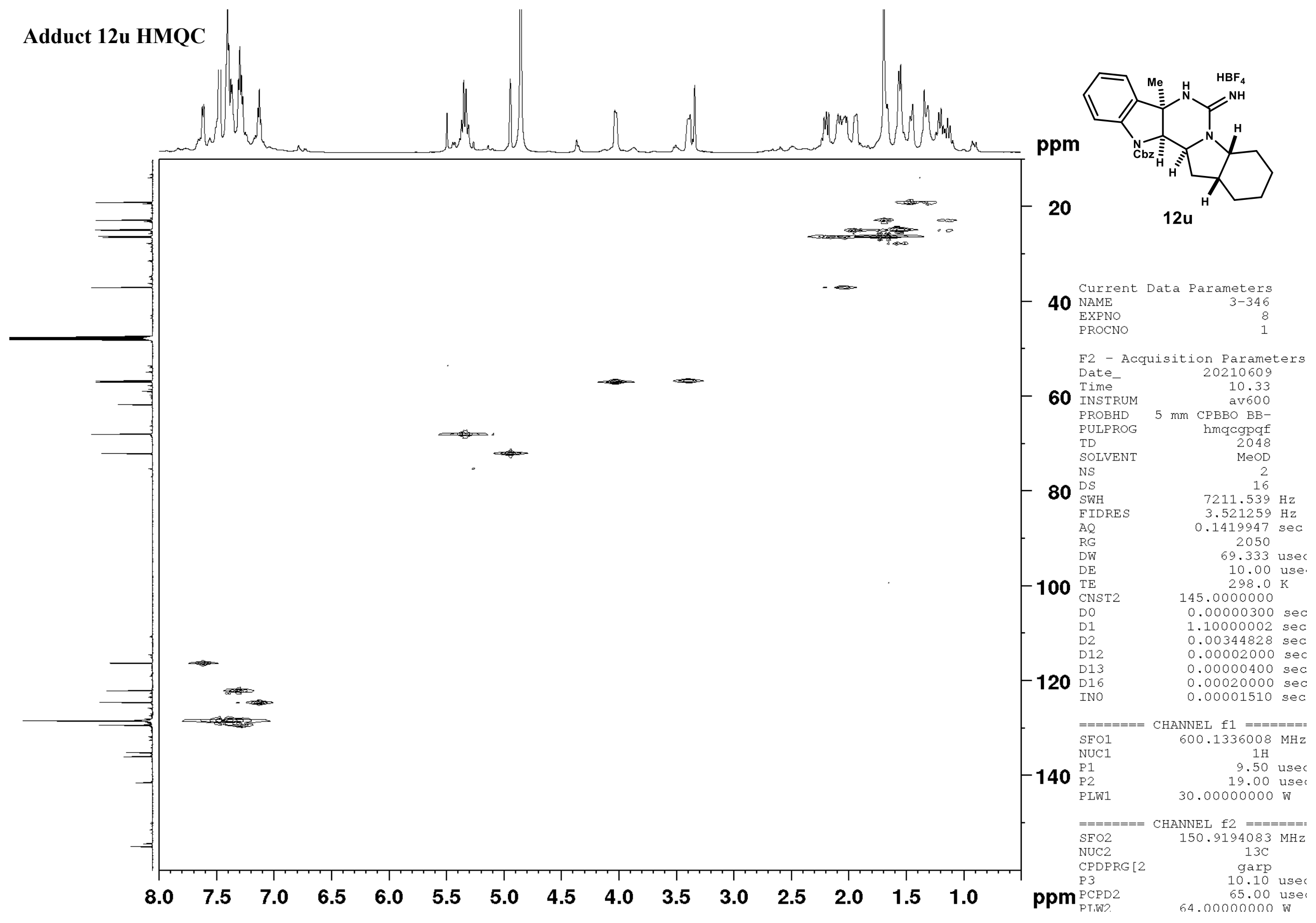



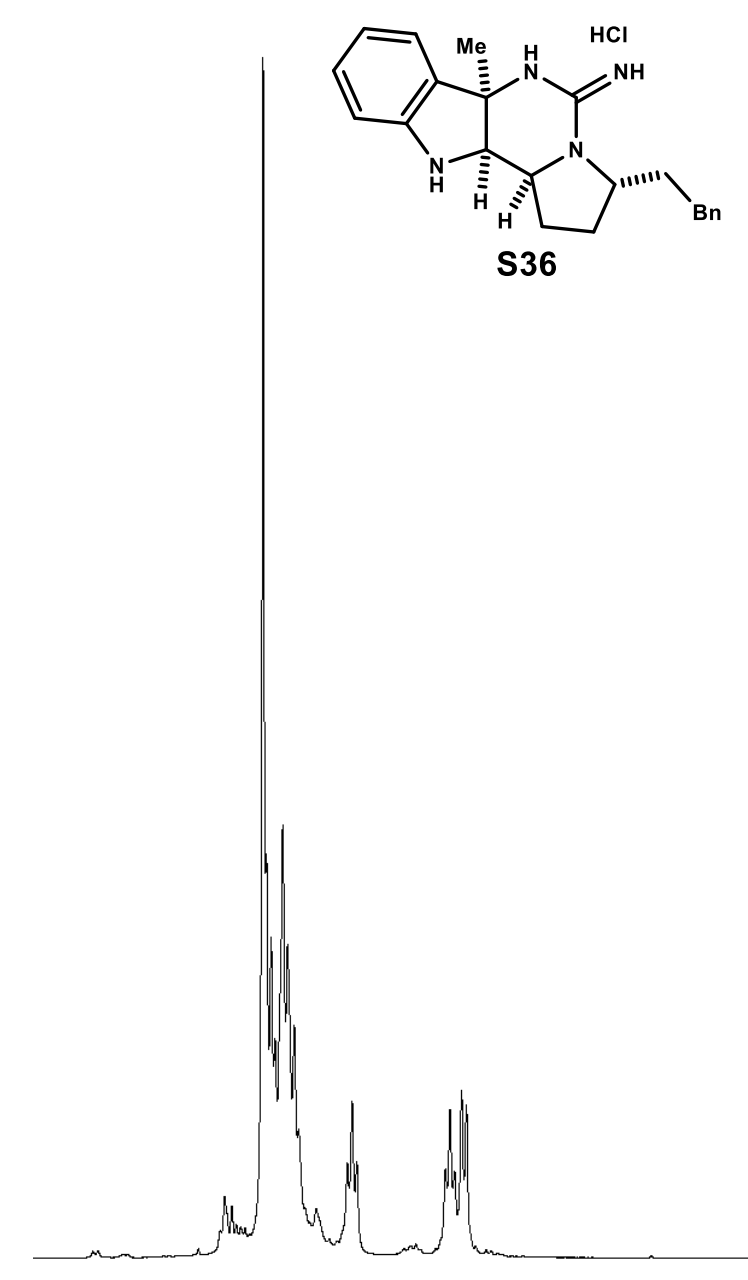

Current Data Parameters

NAME MS_LEO-3-160-p

NAME

PROCNO

F2 - Acquisition Parame Date_ Time

INSTRUM

PROBHD

PULPROG

SOLVENT

NS

DS

FIDRES

$\mathrm{AQ}$

\section{$\mathrm{CH} 2 \mathrm{Cl} 2$}

RG
$\mathrm{DW}$
$\mathrm{DE}$

0.25
aryo 500

$5 \mathrm{~mm}$ CPTCI $1 \mathrm{H}-$

0.10000000

MCWRK

0.01500000

$====$ CHANNEL $\mathrm{f} 1===$

$\mathrm{P} 1$
$\mathrm{PL} 1$

SFO1

500.2235015

F2 - Processing paramet

SI 65536

$\begin{array}{lr}\text { SF } & 500.2200313 \\ \text { WDW } & \text { EM }\end{array}$

SSB $\quad 0$

$\begin{array}{ll}\text { LB } & 0.30\end{array}$

then

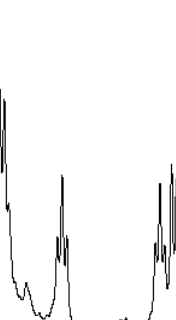

7.5

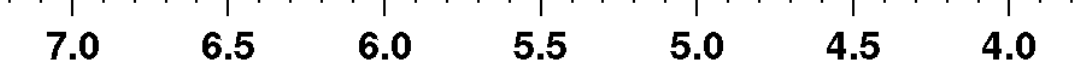

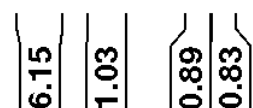

|ர்!

2.5

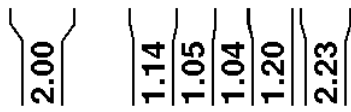

1.5

(̇)

$1.0 \quad 0.5$ ppm 

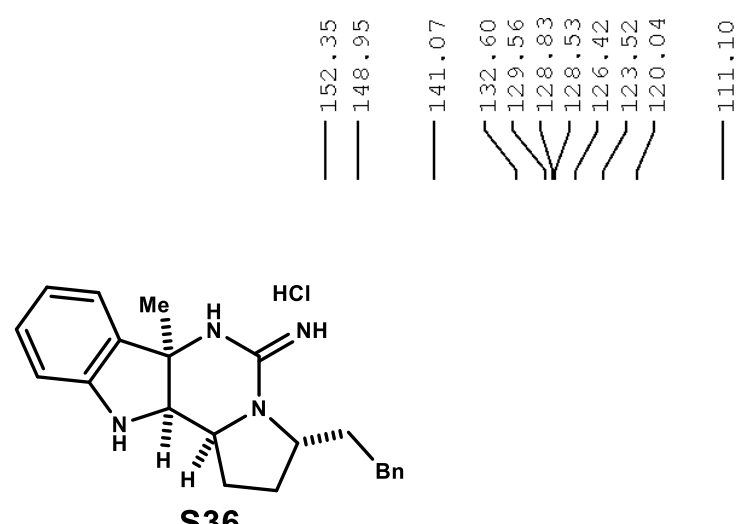

S36
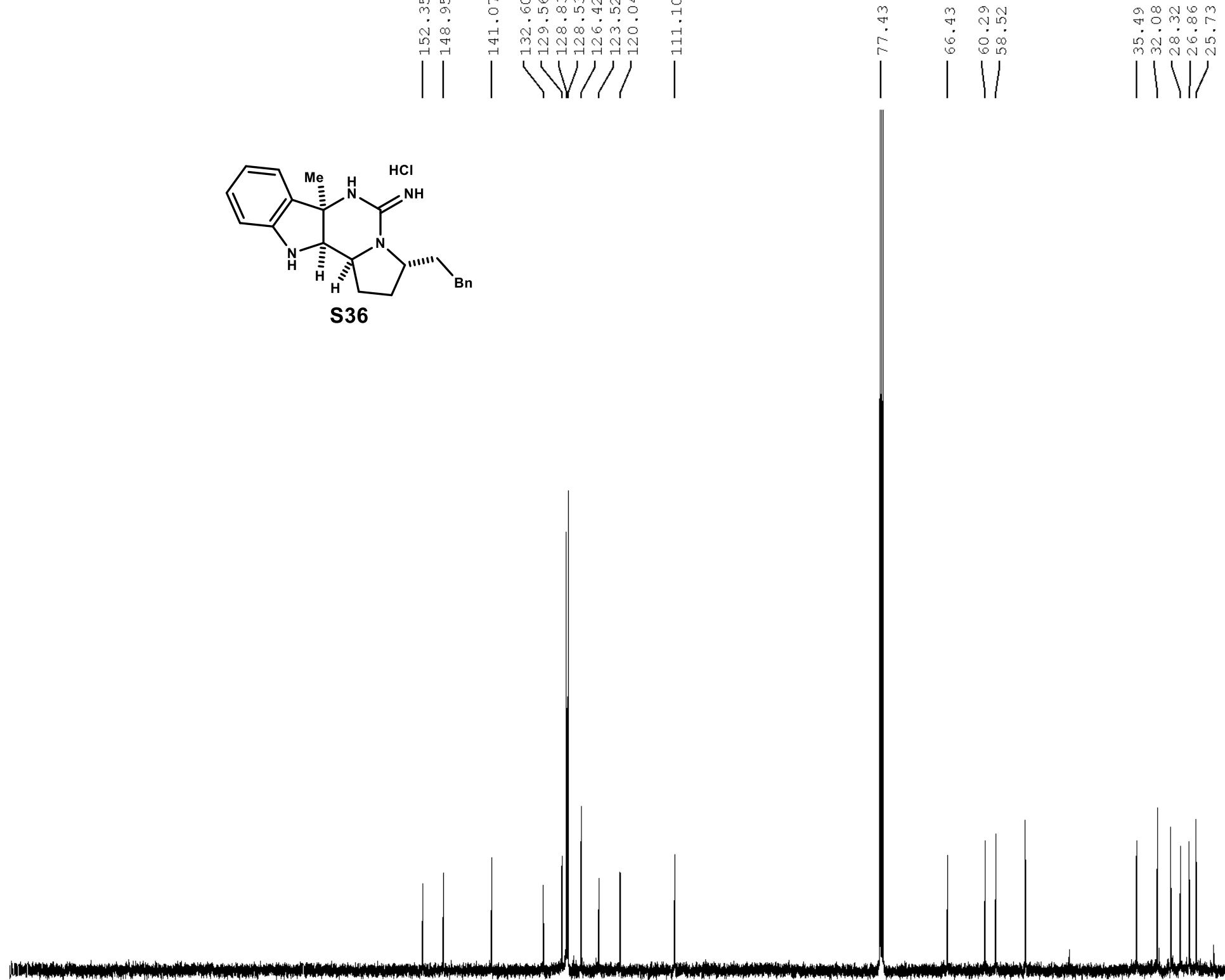

(n)
$\mathrm{CH} 2 \mathrm{Cl} 2$
Current Data Parameters NAME PROCNO

F2 - Acquisition Parame

Date

INSTRUM

PROBHD

aryo500

PULPROG SPInEChOP 30 -

NS

DS

SWH

FID

$\mathrm{AQ}$

RG

DW

TE

D1
d11
D16

d17

MCREST
MCWRK

$\mathrm{MCT}$

$===$
NUC 1

NUC1
P11

P11

P11
PLO

PL1

SFO1

SP1
SP2

SPNAM [1] Crp60, 0.5,20.1 SPNAM [2] Crp60comp.4

SPOFF1 $1 \mathrm{~Hz}$

SPOFF2 O Hz

CHANEL $=$ CPDPRG [2 waltz16 NUC2

PL2

$\mathrm{PL} 2$
$\mathrm{PL} 12$

$\mathrm{SFO} 2$

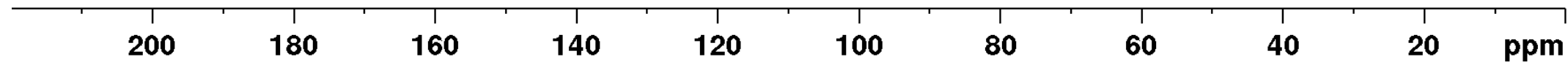

